\title{
On the Plasticity of Human Mating Strategies: Experimental Evidence for Mating Strategy Change in Response to Evolutionarily Relevant Stimuli
}

\section{Thomas, Andrew G.}

How to cite:

Thomas, Andrew G. (2015) On the Plasticity of Human Mating Strategies: Experimental Evidence for Mating Strategy Change in Response to Evolutionarily Relevant Stimuli. Doctoral thesis, Swansea University.

http://cronfa.swan.ac.uk/Record/cronfa41184

Use policy:

This item is brought to you by Swansea University. Any person downloading material is agreeing to abide by the terms of the repository licence: copies of full text items may be used or reproduced in any format or medium, without prior permission for personal research or study, educational or non-commercial purposes only. The copyright for any work remains with the original author unless otherwise specified. The full-text must not be sold in any format or medium without the formal permission of the copyright holder. Permission for multiple reproductions should be obtained from the original author.

Authors are personally responsible for adhering to copyright and publisher restrictions when uploading content to the repository.

Please link to the metadata record in the Swansea University repository, Cronfa (link given in the citation reference above.) 


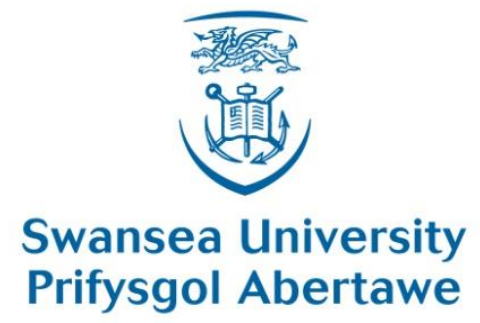

On the Plasticity of Human Mating Strategies: Experimental Evidence for Mating Strategy Change in Response to Evolutionarily Relevant Stimuli

by

Andrew George Thomas

BSc (Hons), Cardiff University, 2008

Submitted to Swansea University in

Fulfilment of the Requirements for the

Degree of Doctor of Philosophy

Swansea University

June, 2015 


\begin{abstract}
Evolutionary psychological theory explains the large variance found in human mating behaviour through the use of a mating strategies perspective. Specifically, both sexes have short-term and long-term mating strategies containing sets of evolved psychological adaptations which guide mating effort. Individuals vary in their mating behaviour due to the differential activation of these two strategies which are thought to be activated conditionally. That is, an individual is hypothesized to engage in a shortterm mating strategy over a long-term one in circumstances where, ancestrally, a shortterm strategy would have led to the best fitness outcome.

Despite a large body of evidence for the existence of mating strategies in humans, evidence for the ability to conditionally switch between strategies is less robust. To date, such evidence is either in the form of correlational studies, or experimental studies which demonstrate changes to behaviours only partly related to mating strategies. The aim of this thesis was to fill the gap in this literature by demonstrating that participants can change their mating strategies in response to evolutionarily relevant stimuli. A novel measure of mating strategies was developed in order to capture a participants' propensity towards short- and long-term mating before and after exposure to cues hypothesized to have affected the effectiveness of the two mating strategies in the ancestral environment. These included cues related to a skewed local sex-ratio, self-perceived dominance, and environmental danger. Of the ten experimental hypotheses tested, support (or partial support) was found for seven and the experimental effects were typically small-to-medium in size. Thus, moderate support was found that humans are flexible in their mating strategy implementation and respond to evolutionarily relevant cues, although it was concluded that marked changes in an individual's environment would be required for any lasting effect on their mating behaviour to occur.
\end{abstract}




\section{Declaration and Statements}

This work has not previously been accepted in substance for any degree and is not being concurrently submitted in candidature for any degree.

Signed: (candidate)

Date:

\section{Statement 1}

This thesis is the result of my own investigations, except where otherwise stated. Other sources are acknowledged by footnotes giving explicit references. A bibliography is appended.

Signed: (candidate)

Date:

\section{Statement 2}

I hereby give consent for my thesis, if accepted, to be available for photocopying and for inter-library loan, and for the title and summary to be made available to outside organisations.

Signed: (candidate)

Date:

\section{Statement of Ethics}

All experiments within this thesis were conducted in accordance with the ethical standards of the British Psychological Society, and were approved by the Department of Psychology Ethics Board at Swansea University.

Signed: (candidate)

Date: 


\section{Table of Contents}

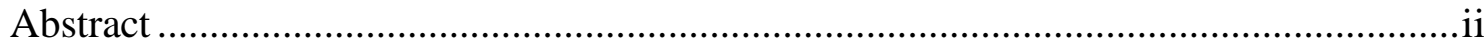

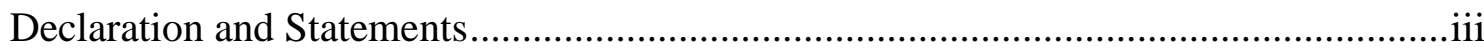

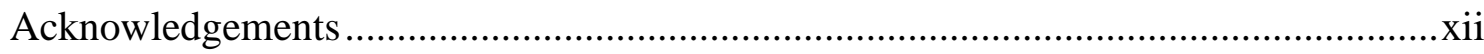

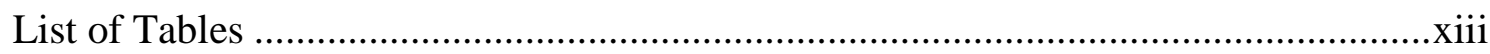

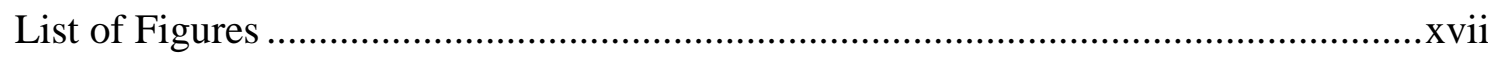

Chapter 1: Literature Review and Introduction ........................................................ 1

Section I: Mating Behaviour as Explained by Sexual Selection .............................. 1

Males Compete, Females Choose …............................................................ 4

Within-Species Variation and Mating Strategies .............................................. 7

Section II: Mating Strategies in the Animal Kingdom....................................... 8

The Development of Mating Strategies ........................................................ 9

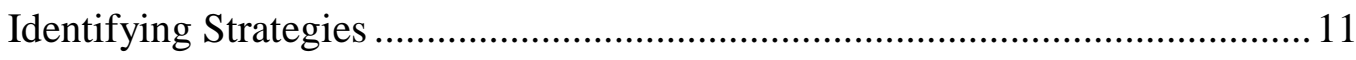

Conditional Mating Strategies ...................................................................... 13

Section III: Typical Human Mating Behaviour..................................................... 14

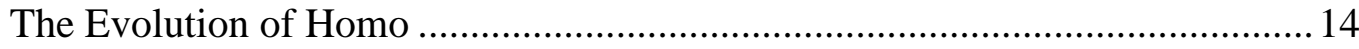

The Large Problem with a Large Brain ........................................................ 17

Typical Levels of Parental Investment in Humans .......................................... 18

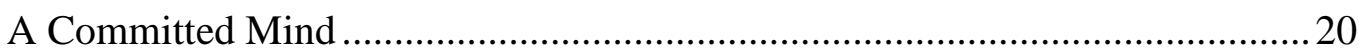

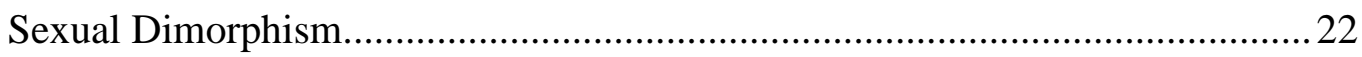

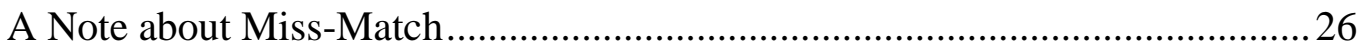

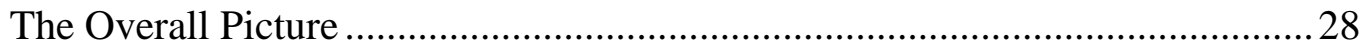

Section IV: Human Mating Strategies and Their Potential Flexibility ................... 29

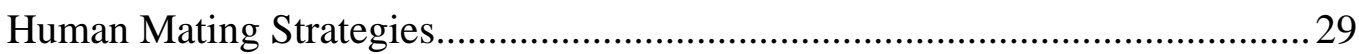

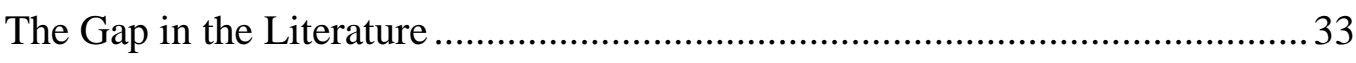

Factors Influencing Strategy Choice in Humans ............................................. 34

Existing Support for the Working Hypothesis ................................................ 40

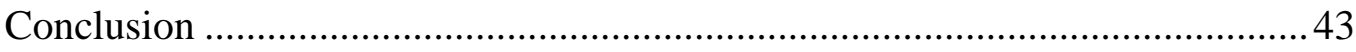

Chapter 2: Snog, Marry, Avoid (SMA) - A Novel Tool for Measuring Mating

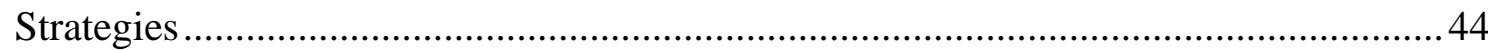

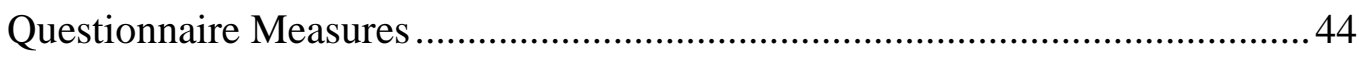

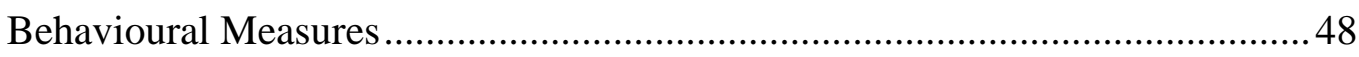

An Alternative Measure - The SMA Task …….............................................50 


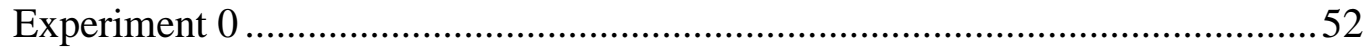

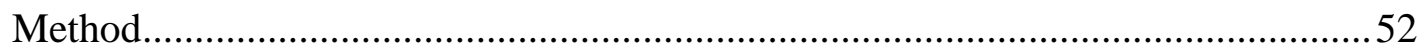

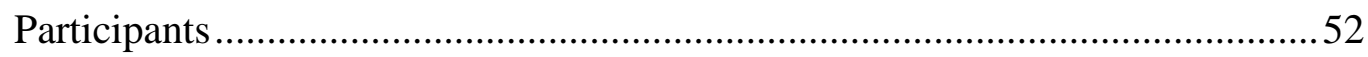

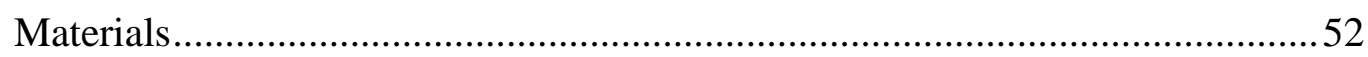

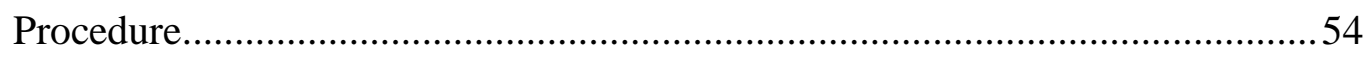

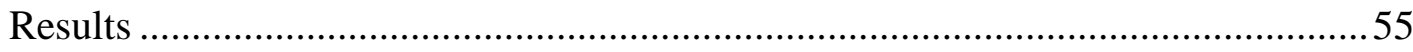

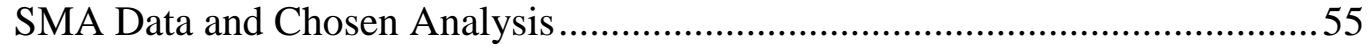

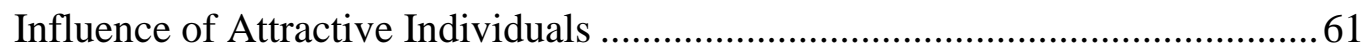

Influence of Unattractive Individuals............................................................6 63

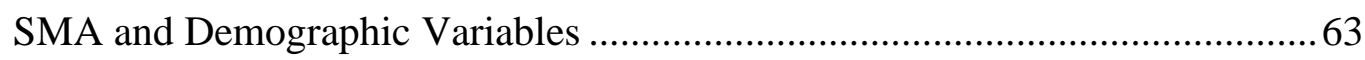

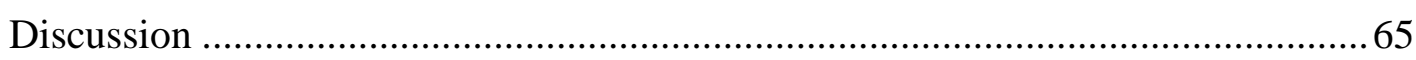

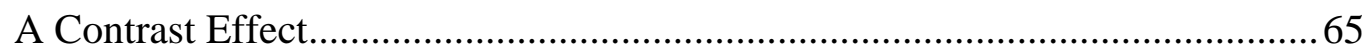

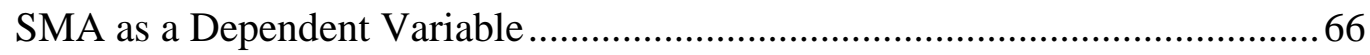

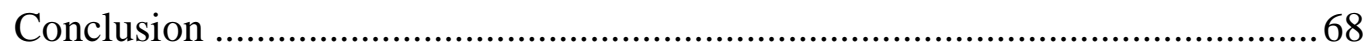

Chapter 3: Relative Attractiveness and Mating Strategies in Men - An Investigation

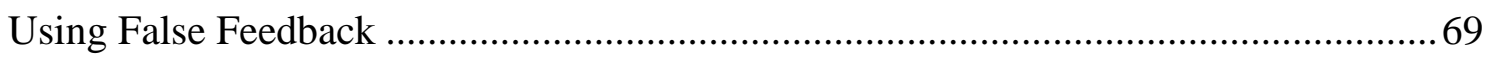

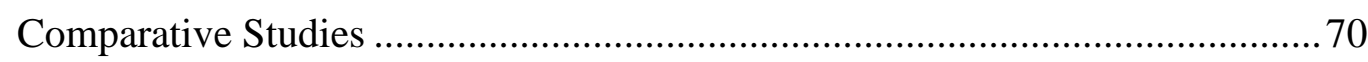

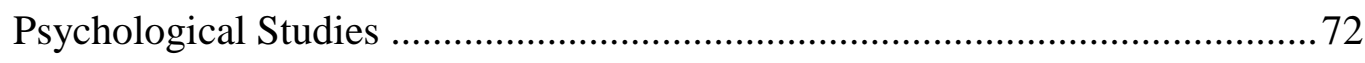

False Feedback Experiments in Psychology ................................................... 74

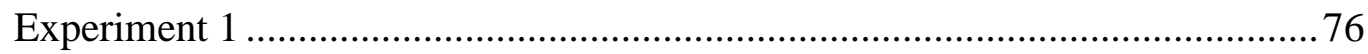

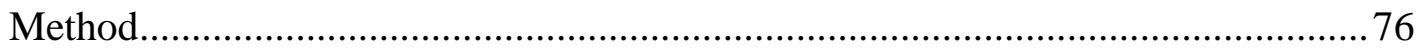

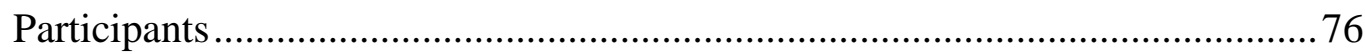

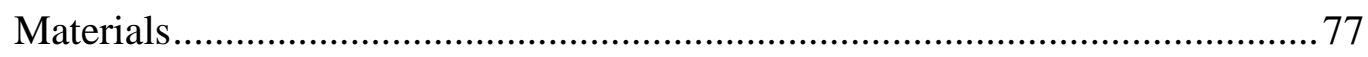

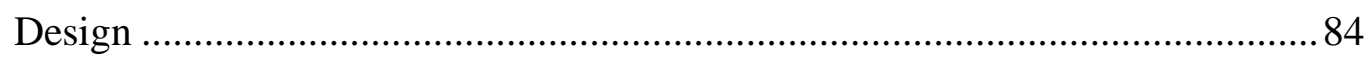

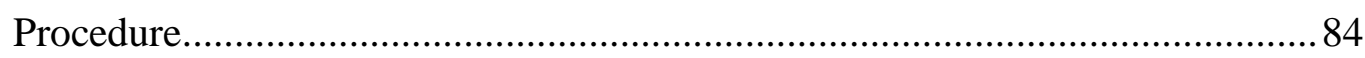

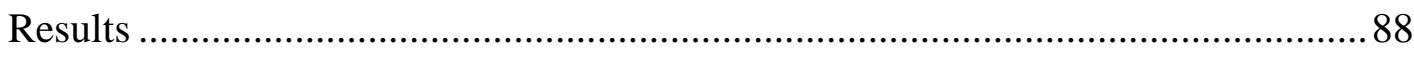

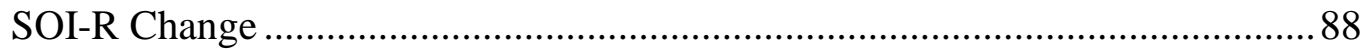

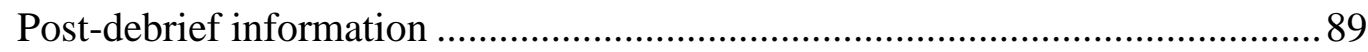

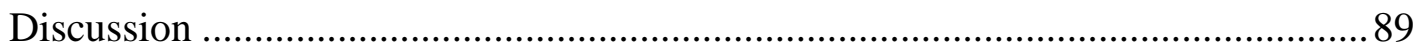

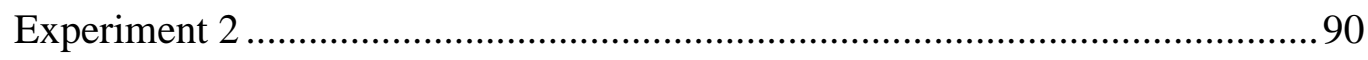

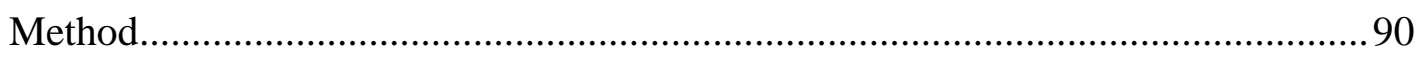

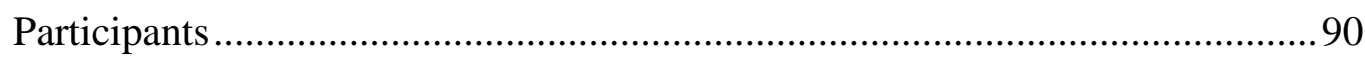

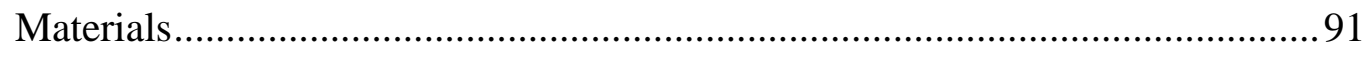

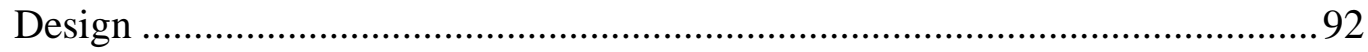




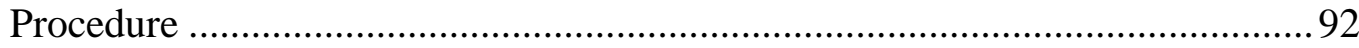

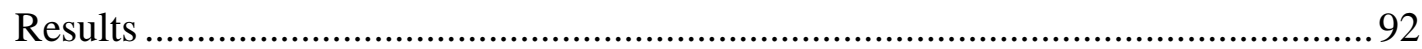

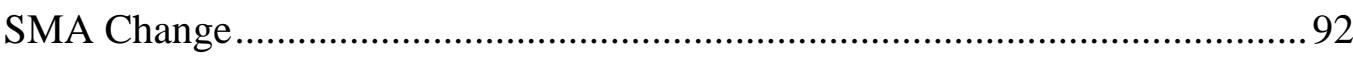

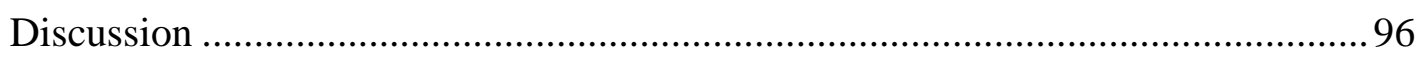

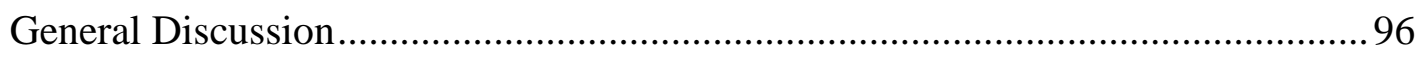

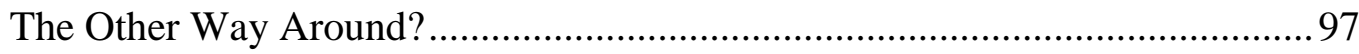

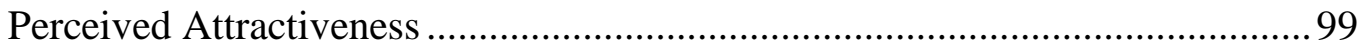

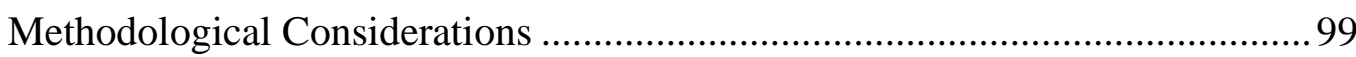

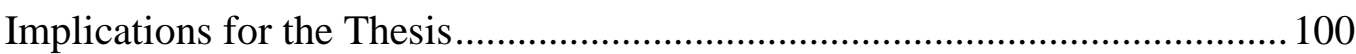

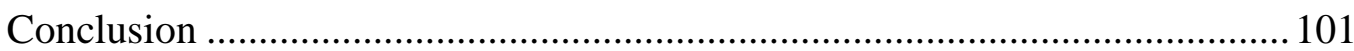

Chapter 4: Parental and Wealth Stimuli Influence Human Mating Strategies. (Are

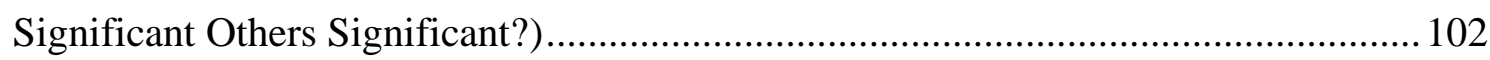

Parental Care and Mating Strategies ............................................................ 102

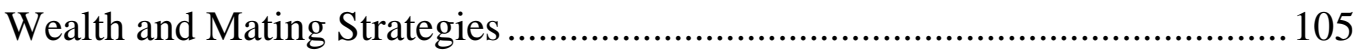

Overt Stimuli Exposure and Behavioural Change .......................................... 108

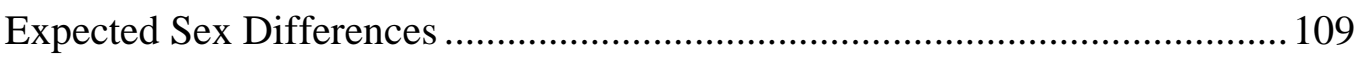

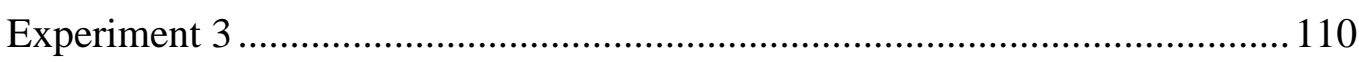

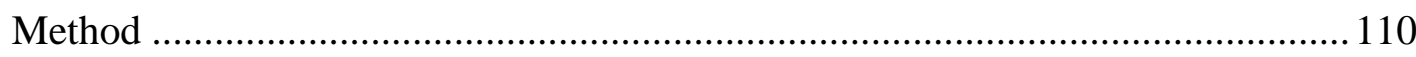

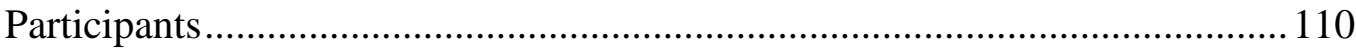

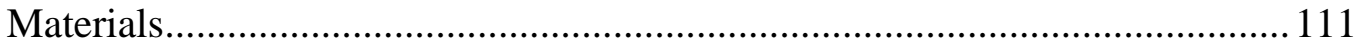

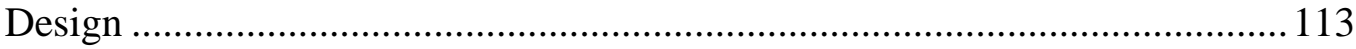

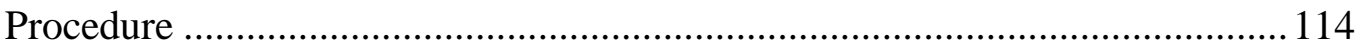

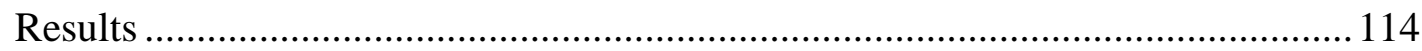

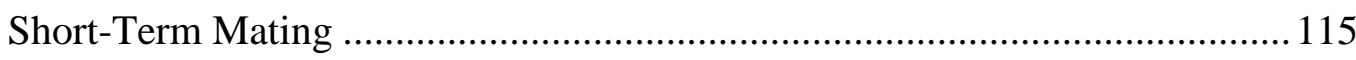

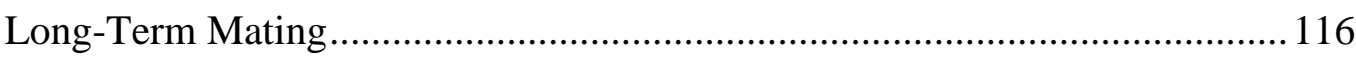

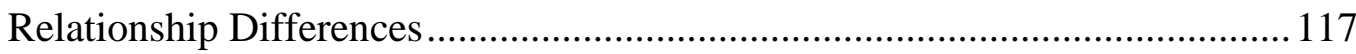

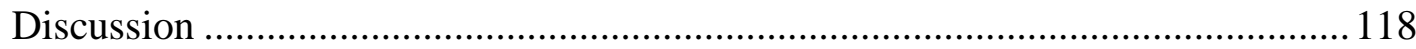

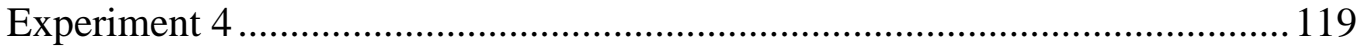

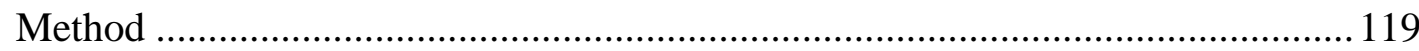

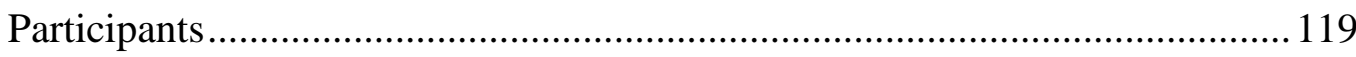

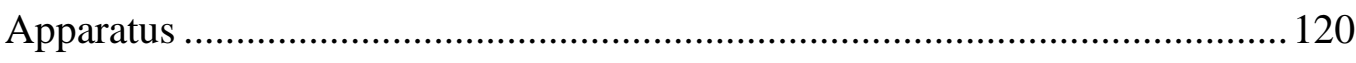

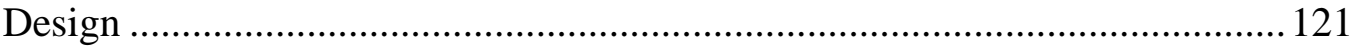

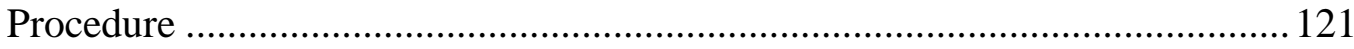

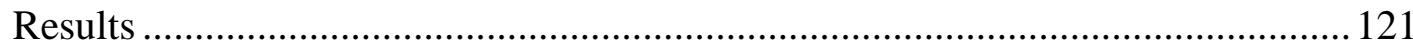




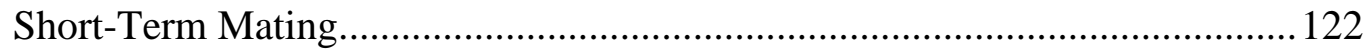

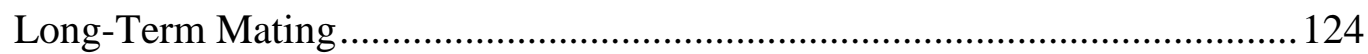

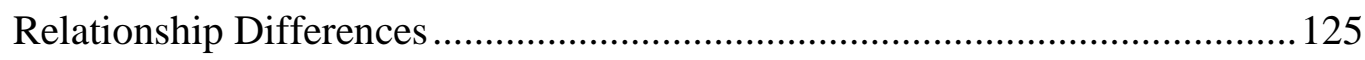

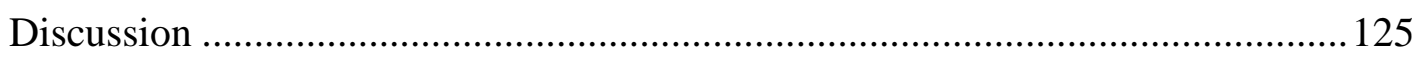

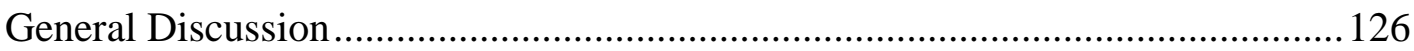

Mating Strategy Plasticity and the Pair Bond ................................................ 126

Sex Differences and Socio-Sexuality ....................................................... 128

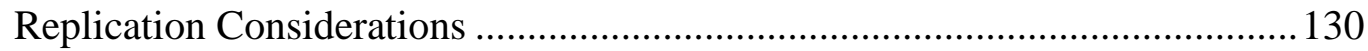

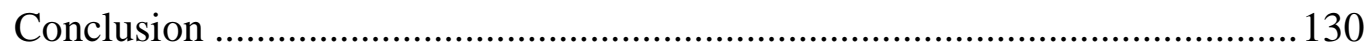

Chapter 5: Danger, Charles Darwin, Danger! ........................................................ 132

Life History and Human Mating ................................................................ 133

Quinlan's Model - a Reconciliation ........................................................... 135

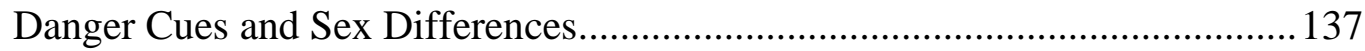

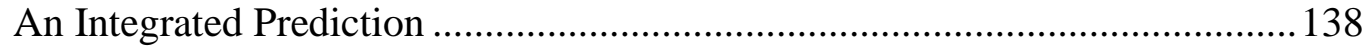

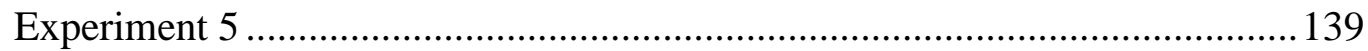

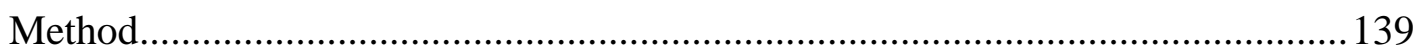

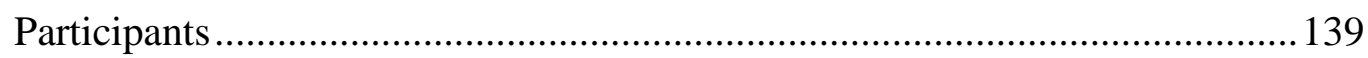

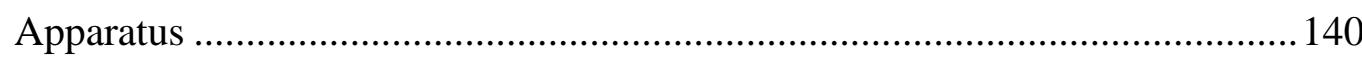

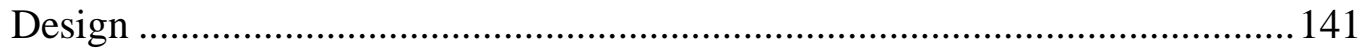

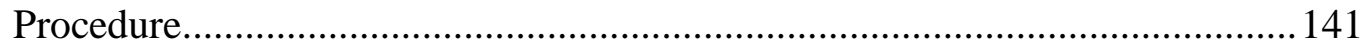

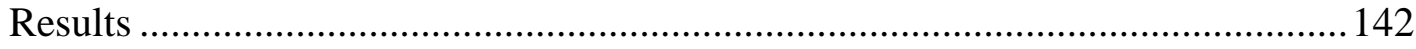

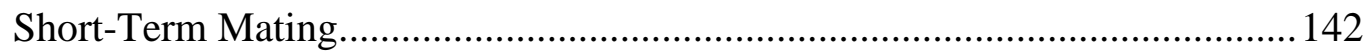

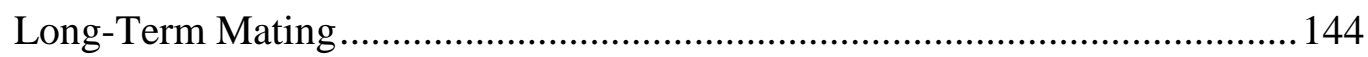

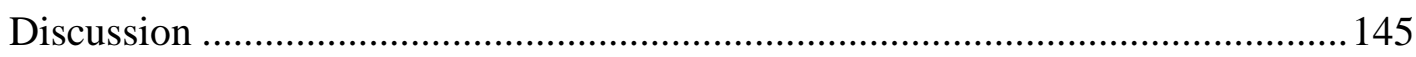

Creativity Tasks as a Manipulation............................................................. 146

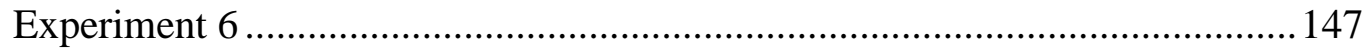

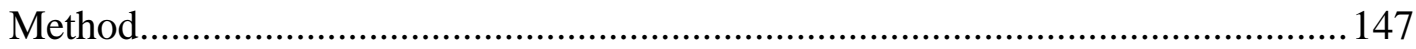

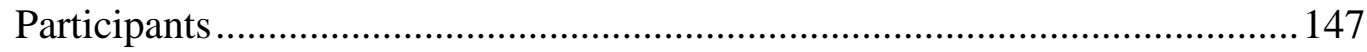

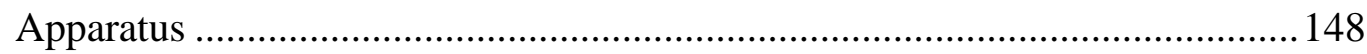

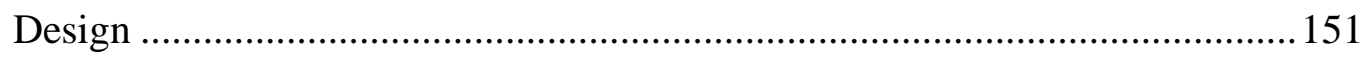

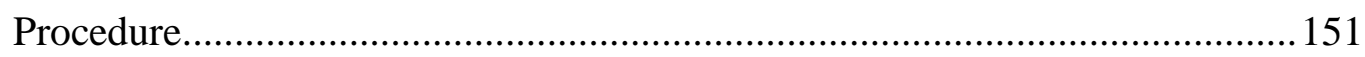

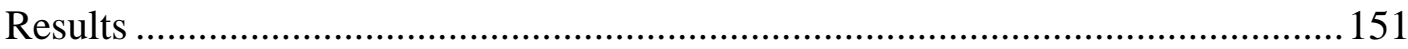

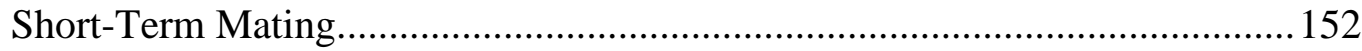

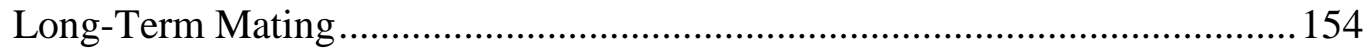




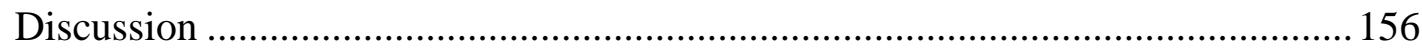

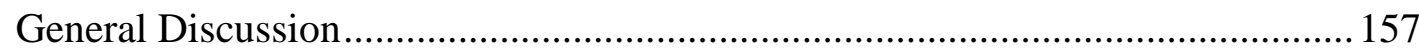

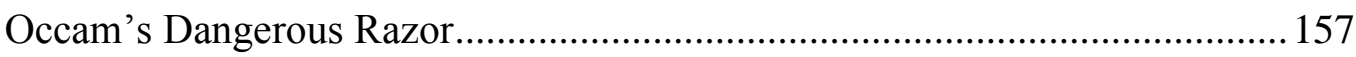

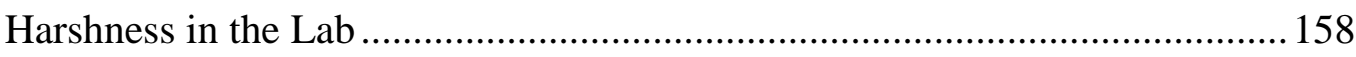

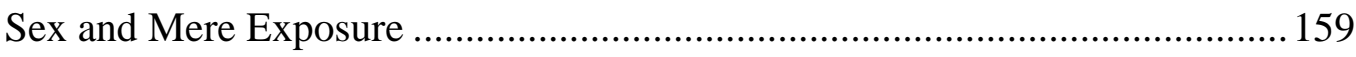

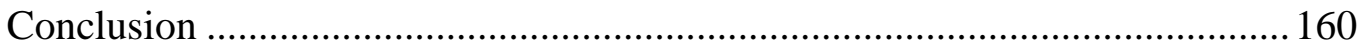

Chapter 6: An Increase in Male Short-Term Mating Preference Accompanies a Rise in

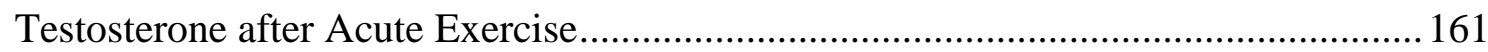

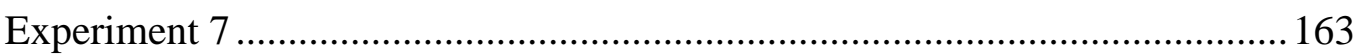

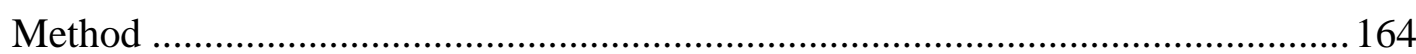

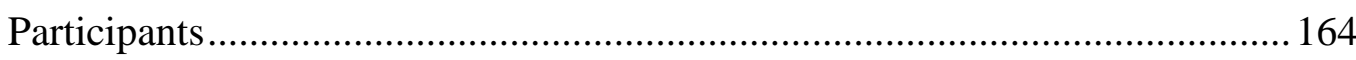

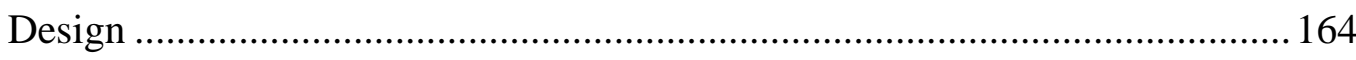

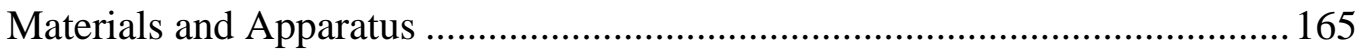

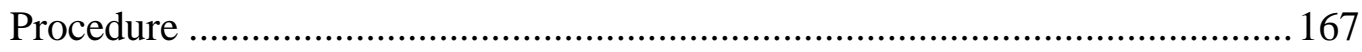

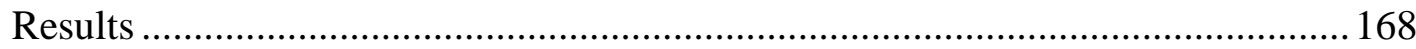

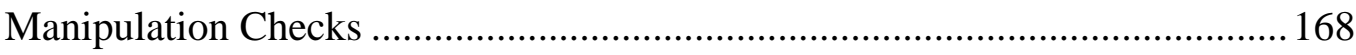

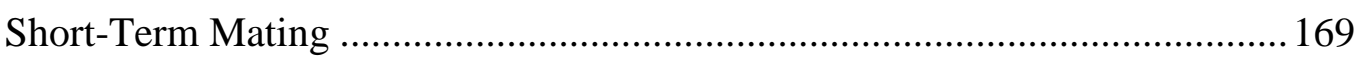

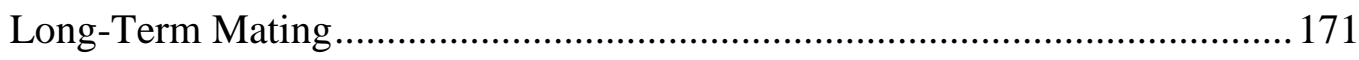

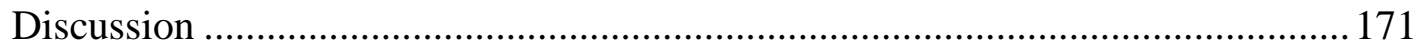

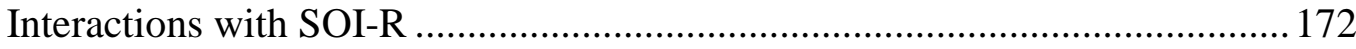

Potential for Replication with Females ....................................................... 173

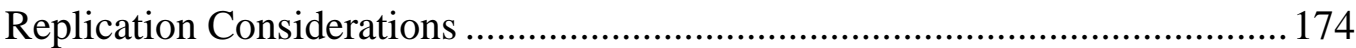

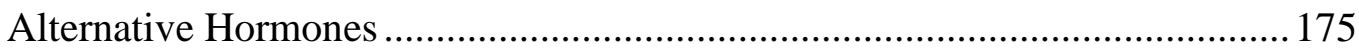

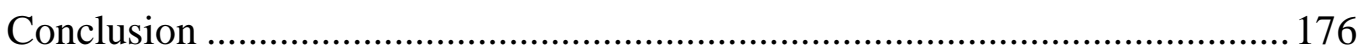

Chapter 7: To the Victor, the Reproductive Spoils .................................................. 177

Evidence for Within-Lifetime Change .......................................................... 178

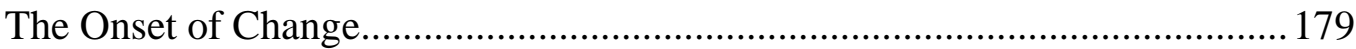

Victory, Defeat and Sexual Strategies ........................................................ 181

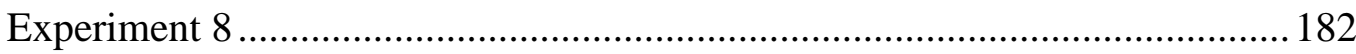

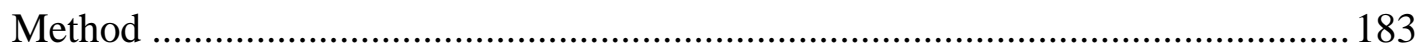

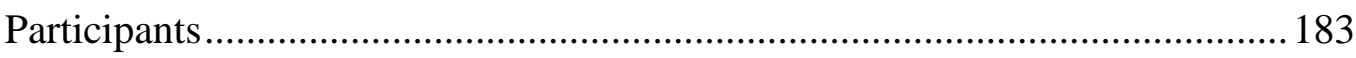

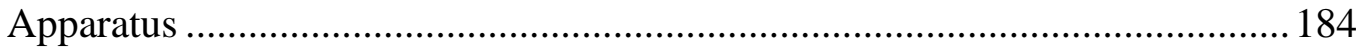

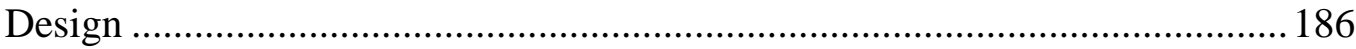

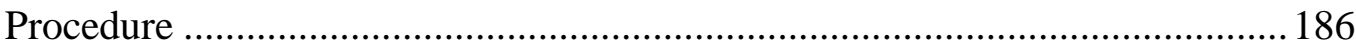


Results

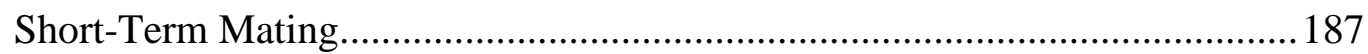

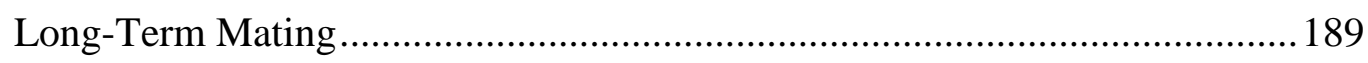

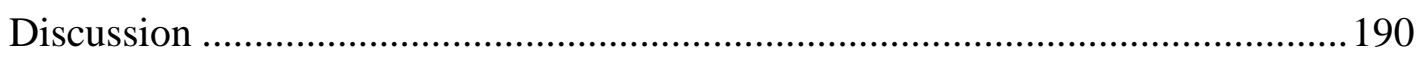

Victory, Testosterone, and Male Mating Strategies...................................... 191

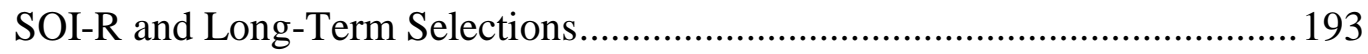

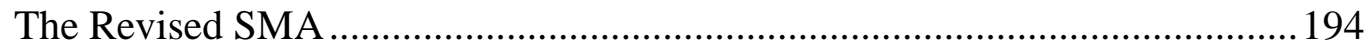

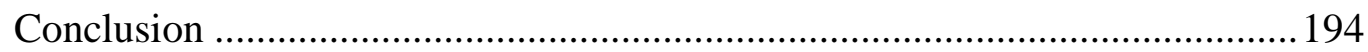

Chapter 8: One Man Too Many - Male-Biased Sex-Ratio Cues May Lead to Long-Term

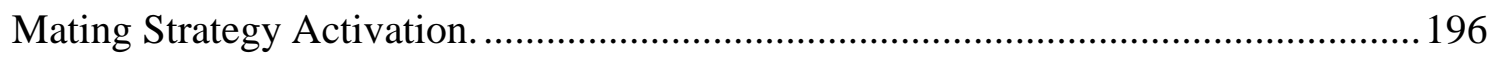

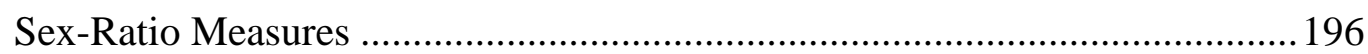

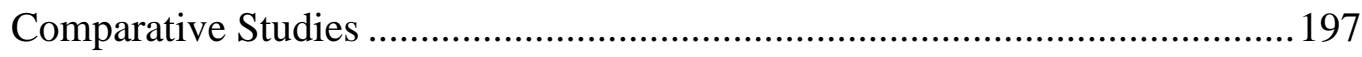

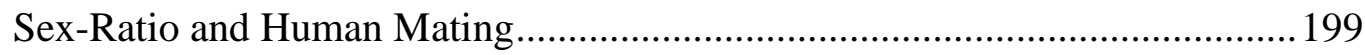

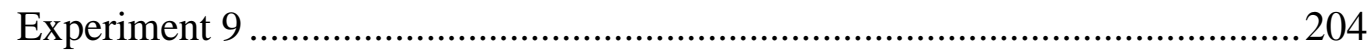

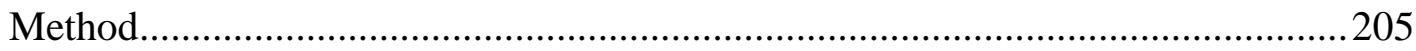

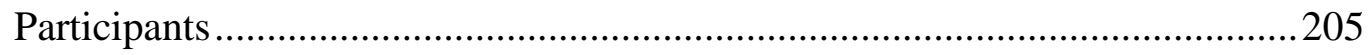

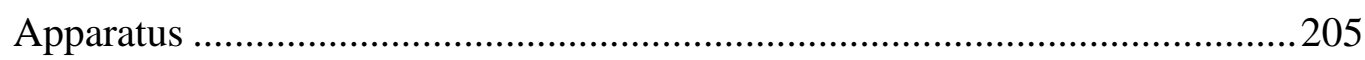

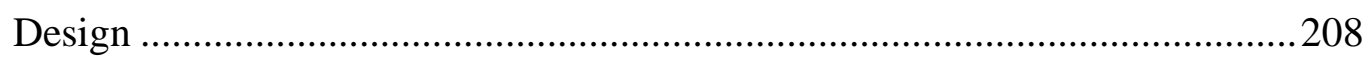

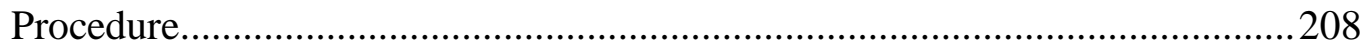

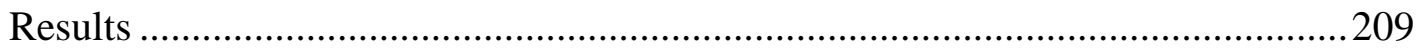

Post-Hoc Analysis Changes .......................................................................209

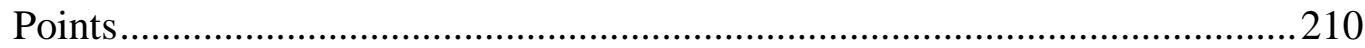

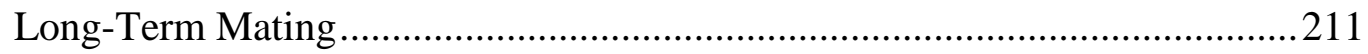

Short-Term Mating............................................................................... 212

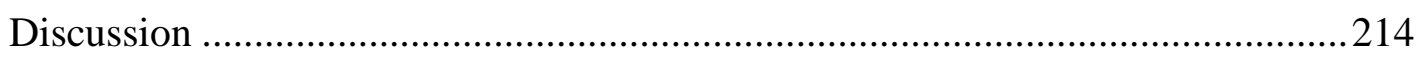

Lack of Female-Biased Bias .....................................................................2215

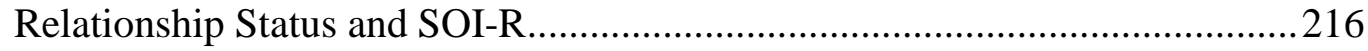

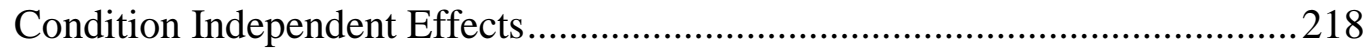

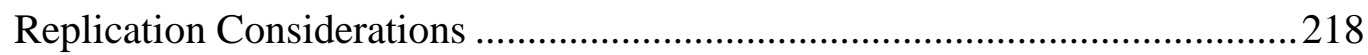

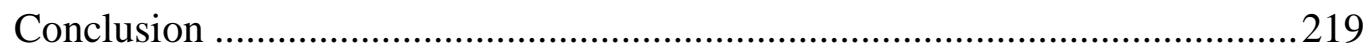

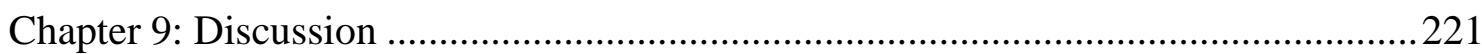

Section I: Summary of Findings and Integration with Current Evolutionary

Theory

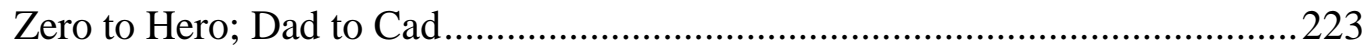




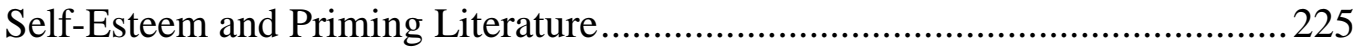

The Polygyny Problem..................................................................................... 227

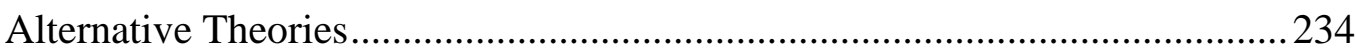

Section II: Methodological Considerations, Future Directions, and Conclusion ... 237

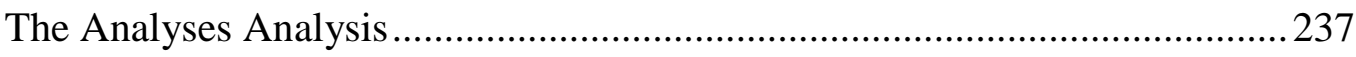

Non-Heterosexual Sexual Orientations and Mating Strategies........................ 245

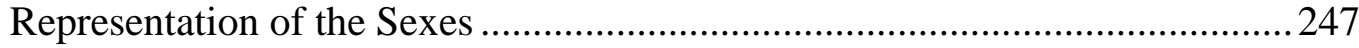

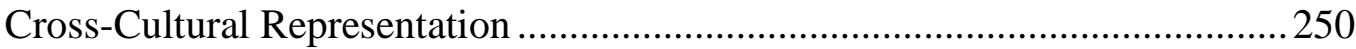

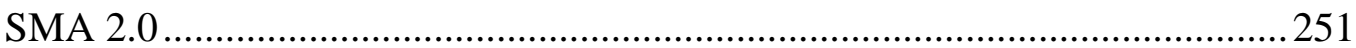

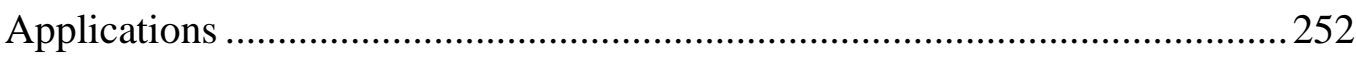

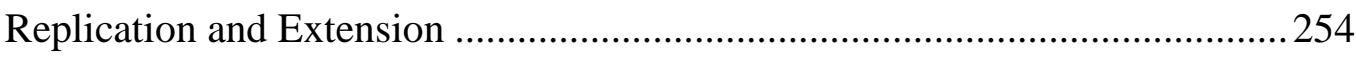

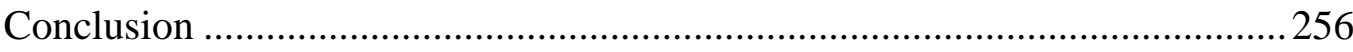

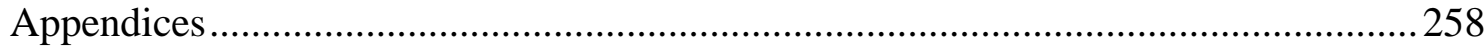

Appendix A: Socio-sexual Orientation Inventory …......................................... 258

Appendix B: The Socio-sexual Orientation Inventory - Revised ......................... 260

Appendix C: Instructions Given to Participants during the SMA task ................. 263

Appendix D: Instructions Given to Participants during the Attractiveness Rating

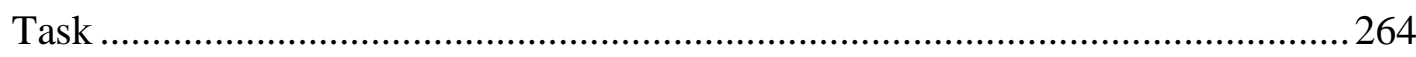

Appendix E: The Standard Demographic Questionnaire ......................................265

Appendix F: Consent Form Used for Experiment 0 in Chapter 2 ........................ 268

Appendix G: Debrief Form Used for Experiment 0 in Chapter 2 ........................ 270

Appendix H: Screenshots of the Mix\&Match Website Used to Administer False

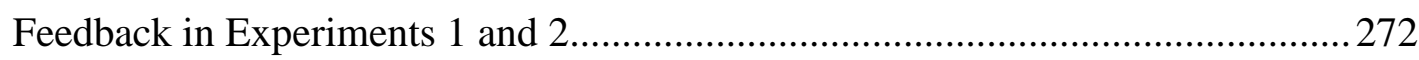

Appendix I: Shortened Versions of the Launay-Slade Hallucination Scale (LSHS-R)

and the Positive Affect Negative Affect Schedule (PANAS) ...............................2 280

Appendix J: Consent, Debrief, and Post-debrief Questionnaires Used During

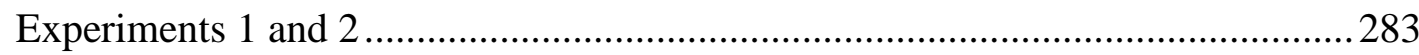

Appendix K: Bogus Market Research Forms used in Experiments 1 and 2 .......... 295

Appendix L: Consent and Debrief Forms for Experiments 3 and 4.....................299

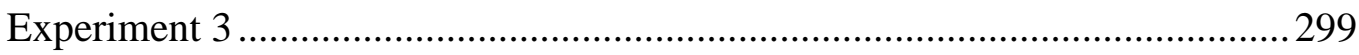

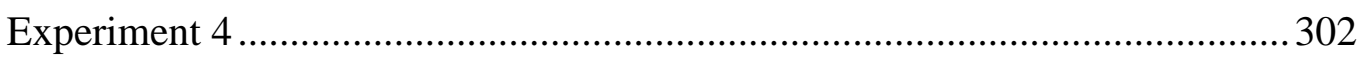

Appendix M: Screenshots of the Videos Used in Experiment 3 .......................... 305

Appendix N: Examples of the Slideshow Images Used in Experiment 4 ...............307

Appendix O: Consent and Debrief Forms for Experiments 5, 6 and 8 ...................309 
Experiment 5 309

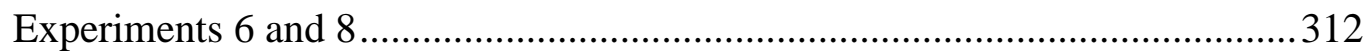

Appendix P: Examples of the Slideshow Images Used in Experiment 5...............316

Appendix Q: Wording and Screenshots of the Revised SMA Task....................... 318

Appendix R: Creativity Tasks Used in Experiment 6 ........................................ 321

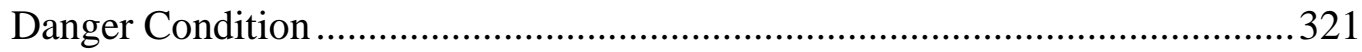

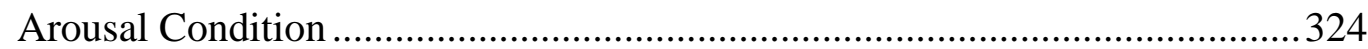

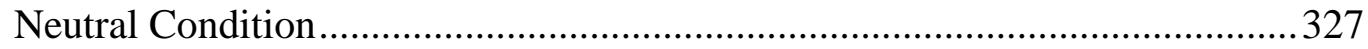

Appendix S: The Health Assessment Questionnaire Used in Experiment 7..........329

Appendix T: Consent and Debrief Forms for Experiment 7 ...............................330

Appendix U: Creativity Tasks Used in Experiment 8 ..........................................334

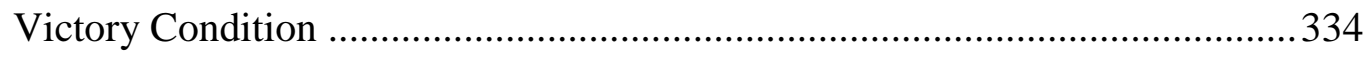

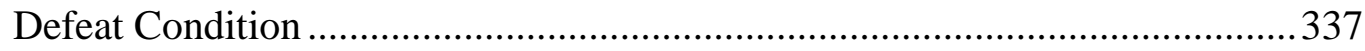

Appendix V: Consent and Debrief Forms for Experiment 9...............................340

Appendix W: Instructions Given to Participants during the Guessing Game Task344

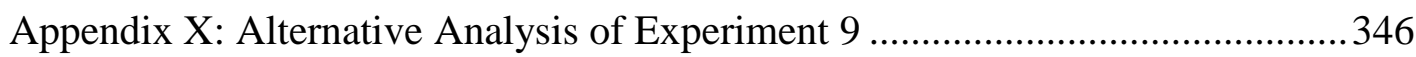

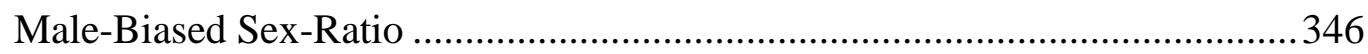

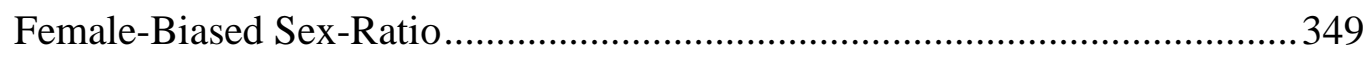

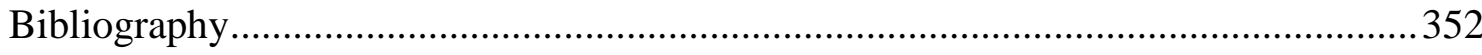




\section{Acknowledgements}

This research was conducted, part-time, over six years. During that time, I have received support and guidance from a large number of individuals, a small selection of which I recognise here. In terms of personal growth, I would like to show appreciation to following individuals for accepting me into the ivory tower of academia and constantly challenging my concepts and ideas: Ceri Carlisle, Louise Cleobury, Shakiela Davies, Darren Edwards, Gary Freegard, Katy Jones, Michail Kozlov, Rory Kumpata, Rob Lowe, Carlo Lucignoli, Louise McHugh, Anita Munnelly, Jordan Randell, Gemma Reynolds, Ian Russell, Heidi Seage, J James Tomash, Kathrin Weidacker, Christoph Weidemann, Claire Williams, and Hayley Young. At the same time, I would like to thank those who have kept me grounded throughout this time; those who have reminded me that humbleness, common sense, and compromise are useful skills in all walks of life: Joanne Ahmun, Elizabeth Allaway, Gareth Allsopp, Abdul Choudhary, Christina Gentles, Margaret Healan, Robin Lawson-Duckett (and family), Alan Llewellyn, Louise Lock, Joanne Middleton, Graham Perkins, Joanne Redmond, David Sharp, Ian Stocks, Khloe Stocks, Sonny Stocks, Benjamin Thomas, Roxanne Williams, and many others.

In terms of the development of the thesis, I would like to thank the various individuals who shared with me their resources, wisdom, patience, and social networks. These include Paul Brain, Neil Carter, Blair Crewther, Mark Johansen, Liam Kilduff, Michelle Lee, and Phil Reed. Over 1,400 participants were experimentally tested in this thesis. This sheer number of volunteers would have been near impossible for one researcher to recruit and test alone, so I would like to thank the following undergraduate students for their help with data collection: Emily Billington, Lisa Davies, Daniella DiCaro, Katheryn Egleton, Alexandra Fish, Leanne Francis, Sera Griffiths, Bryony Hawkins, Simon Johns, Anthony Jones, Bethan Jones, Louis Marson, Bethan Morgan, and Lucy Taylor.

Thank you to my parents, Angela and David, who, in two very different but equally important ways, supported me throughout this thesis. It is because of your love and support that I have been able to research an area I enjoy with the freedom to do so at my own pace. Finally, I would like to thank my mentor, supervisor, and psychological partner in crime Steve Stewart-Williams. Thank you for taking a chance on a self-funded bachelor graduate as your first $\mathrm{PhD}$ student. Your patience, wisdom, keen insight, and flexibility have helped me develop as an academic, and as a person, more than I ever could have alone. 


\section{List of Tables}

Table 2.1. The distribution of participants by SMA change category for the attractive condition

Table 2.2. Ordinal regression models predicting change to relationship responses in the attractive condition

Table 2.3. The distribution of participants by SMA change category for the unattractive condition.

Table 3.1. Change to short- and long-term relationship choices following negative attractiveness feedback.

Table 3.2. An ordinal logistic regression model predicting ST change category using self-reported attractiveness and relationship status

Table 3.3. Change to short- and long-term relationship choices following positive attractiveness feedback.

Table 3.4. An ordinal logistic regression model predicting LT change category using self-reported attractiveness .....

Table 4.1. The number of participants who increased, stayed the same, or decreased in their number of ST choices following exposure to either neutral or parental stimuli

Table 4.2. The number of participants who increased, stayed the same, or decreased in their number of LT choices following exposure to either neutral or parental stimuli

Table 4.3. The results of an ordinal regression to predict LT choice change using (a) condition alone and $(b)$ an interaction between condition and relationship status

Table 4.4. The number of participants who increased, stayed the same, or decreased in their number of ST choices following exposure to either a neutral or wealth stimuli

Table 4.5. The results of an ordinal regression to predict ST choice change using (a) just condition and $(b)$ an interaction between condition, relationship status and sex

Table 4.6. The results of two separate ordinal regression models, one for each sex, using condition and relationship status as predictors. 
Table 4.7. The number of participants who increased, stayed the same, or decreased in their number of LT choices following exposure to either neutral or wealth stimuli

Table 4.8. Two ordinal regression models predicting LT category. The first (a) used only condition and approached significance. The second (b) used an SOI-R by condition interaction

Table 5.1. The number of participants who increased, stayed the same, or decreased in their number of ST choices following exposure to either neutral or danger stimuli.

Table 5.2. The results of an ordinal regression to predict ST choice change using a) sex, b) an interaction between sex and condition, and c) an interaction between sex and SOI-R 143

Table 5.3. The number of participants who increased, stayed the same, or decreased in their number of LT choices following exposure to either neutral or danger stimuli.

Table 5.4. The results of an ordinal regression to predict LT choice change using condition

Table 5.5. The number of participants who increased, stayed the same, or decreased in their number of ST choices following exposure to either danger, neutral, or arousal stimuli 153

Table 5.6. The number of participants who increased or decreased in their number of ST choices following exposure to either danger or neutral stimuli

Table 5.7. The results of a binary logistic regression to predict ST choice change using (a) sex, (b) an interaction between sex and condition, and (c) an interaction between sex and SOI-R. 154

Table 5.8. The number of participants who increased, stayed the same, or decreased in their LT choices following exposure to either danger, neutral, or arousal stimuli 155

Table 5.9. The number of participants who increased or decreased in their LT choices following exposure to either danger or neutral stimuli 155

Table 5.10. The results of a binary logistic regression to predict LT choice change using condition 156

Table 6.1. The number of participants who increased, stayed the same, or decreased in their number of ST choices following the exercise intervention 169 
Table 6.2. The results of an ordinal regression to predict ST choice change using percentage change in testosterone following an exercise intervention.

Table 6.3. The number of participants who increased, stayed the same, or decreased in their number of LT choices following the exercise intervention

Table 7.1. The number of participants who increased, stayed the same, or decreased in their ST choices following the creativity task.

Table 7.2. The results of an ordinal regression to predict ST change category using a sex by condition interaction

Table 7.3. The number of participants who increased, stayed the same, or decreased in their LT choices following the creativity task

Table 7.4. The results of an ordinal regression to predict LT choice change using sex, SOI-R and condition

Table 8.1. The number of participants who increased, stayed the same, or decreased in their number of LT choices following exposure to either a male-or female-biased sex-ratio task

Table 8.2. The results of an ordinal regression to predict LT category change using condition, relationship status, and SOI-R

Table 8.3. The number of participants who increased, stayed the same, or decreased in their number of ST choices following exposure to either a male-or female-biased sex-ratio task

Table 8.4. The results of an ordinal regression to predict ST category change using condition, the number of points earned during the guessing game, self-perceived attractiveness and SOI-R

Table 9.1. A summary table showing the SMA experiments conducted within this thesis.

Table X.1. The number of participants who increased, stayed the same, or decreased in their number of LT choices following exposure to a male-biased sex-ratio task .....346

Table X.2. The results of an ordinal regression to predict change in LT choice using SOI-R, age, relationship status, points scored, and sex

Table X.3. The number of participants who increased, stayed the same, or decreased in their number of ST choices following exposure to a male-biased sex-ratio task .....348

Table X.4. The results of an ordinal regression to predict change in ST choice using points scored. 
Table X.5. The number of participants who increased, stayed the same, or decreased in their number of LT choices following exposure to a female-biased sex-ratio task

Table X.6. The number of participants who increased, stayed the same, or decreased in their number of ST choices following exposure to a female-biased sex-ratio task 350 


\section{List of Figures}

Figure 1.1. A graphical representation of the fitness benefits of following two mating strategies (a) and (b) given hypothetical variable $\mathrm{X}$.

Figure 2.1. The frequency of long- and short-term relationship choices during the first completion of the SMA task

Figure 2.2. The frequency of difference scores between the first and second performance of the SMA task for long-term (LT) and short-term (ST) relationships respectively

Figure 2.3. The median relationship choices in the first SMA measurement of the pilot study

Figure 3.1. Three examples of false feedback bar charts shown to the participants ......79

Figure 3.2. The histograms which were presented to participants on the "Completed" page after receiving their feedback through the Mix\&Match website 80

Figure 5.1. The hypothetical relationship between environmental harshness and the effectiveness of short- and long-term mating strategies

Figure 5.2. A modified version of Figure 5.1 to include hypothetical sex differences in the effectiveness of long- and short-term mating as environmental harshness (danger) varies

Figure 7.1. The images used to convey victory and defeat to the participants in the creativity tasks used in Experiment 8.

Figure 9.1a. The impact of evolutionarily relevant environmental input on mating strategies

Figure 9.1b. A hypothetical model of short-term mating strategy flexibility as inspired by M. R. Gross (1991)

Figure 9.2a. A hypothetical model showing the probability of a man entering into one of three different mating arrangements based upon his mating strategy..... 232

Figure $9.2 b$. A revised version of Figure $9.1 \mathrm{~b}$ whereby mating strategy is presumed to vary on a spectrum rather than conditionally

Figure 9.3. A demonstration of the effect of recoding the LT difference scores using three ordinal categories

Figure H.1. The sign-up page of the Mix\&Match website

Figure H.2. The log-in page of the Mix\&Match website which allowed individuals to access the "My Page" hub of the experiment.... 
Figure H.3. The "My Page" section of the Mix\&Match website. This page acted as a hub to guide participants through the experiment ................................................ 274

Figure H.4. Two of the steps from the "My Page" hub ........................................... 275

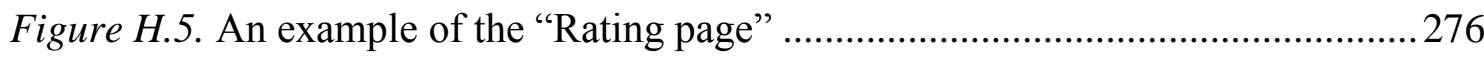

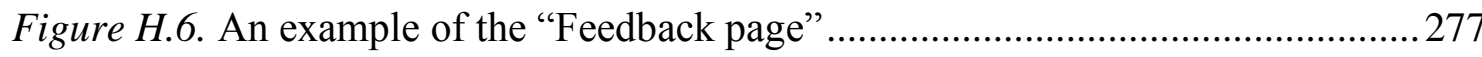

Figure H.7. An example of the "Completed page".....................................................2 278

Figure H.8. Two additional pages from the Mix\&Match website.............................2279

Figure M.1. Screenshots of the video used as parental stimuli during Experiment 3 .. 305

Figure M.2. Screenshots of the video used as neutral stimuli during Experiment 3 ....306

Figure N.1. Eight example images shown during the wealth slideshow in the experimental condition 307

Figure N.2. Eight example images shown during the neutral slideshow in the control condition 308

Figure P.1. Eight example images shown during the danger slideshow in the experimental condition

Figure P.2. Eight example images shown during the neutral slideshow in the control condition

Figure Q.1. Two screenshots of the revised SMA task 319

Figure W.1. Example of the Guessing Game task interface 344

Figure W.2. Positive (left) and negative (right) feedback signs used during the Guessing Game .345 


\section{Chapter 1: Literature Review and Introduction}

This combined introduction and literature review is divided into four sections. The first two sections cover the main evolutionary biological principles which are used within the thesis. These principles apply to humans as well as the animal kingdom in general. Section I introduces sexual selection and parental investment theory, while Section II outlines mating strategies and their implementation within the animal kingdom. The final two sections deal specifically with humans and their mating behaviour. Section III uses evolutionary psychological, anthropological, and paleoanthropological evidence to understand the typical mating behaviour of Homo sapiens. This understanding provides a foundation upon which individual variation in mating behaviour can be discussed. Section IV addresses this variation through the discussion of human mating strategies. This section also outlines the gap in the evolutionary psychological literature which the thesis attempts to fill, and presents some of the factors likely to affect mating strategy choice in humans within an experimental context. Collectively, these sections lead to the justification for the overall working hypothesis of this thesis: Human mating strategies are activated conditionally and change in historically adaptive ways in response to evolutionarily relevant stimuli.

\section{Section I: Mating Behaviour as Explained by Sexual Selection}

Mating behaviour in the animal kingdom is both complex and varied. When bed bugs (of the Cimex genus) mate, the male often uses his penis to break through the hard shell of the female rather than using her reproductive tract (Forsyth, 2001). Male spotted bower birds (Chlamydera maculata) spend hours building vibrant bowers to attract females using materials such as glass, bones, and colourful flowers (Borgia, 1995). After ejaculating, male red-eyed tree frogs (Agalychnis callidryas) will stay mounted to the female for hours at a time (Pyburn, 1970), while male angler fish of the Lophiiformes order fuse their flesh and nervous system with their partners in order to reproduce (Munk, 2000).

Even though there is large diversity in mating behaviour, science has been able to explain a great proportion of this using some relatively simple but powerful theories. At the centre of these theories is that of natural selection (Darwin, 1859). Although originally conceived in the absence of genetics, natural selection can be thought of as the filtering process whereby genes which are better suited to the environment proceed to form a greater proportion of genes in subsequent generations (Dawkins, 1976, 1994; G. C. Williams, 1966). This simple theory can account for the existence of all manner of 
survival enhancing adaptations including long teeth for hunting prey, keen eyesight for predator avoidance, and poisonous defences for protection. It is not immediately obvious, however, how such a theory accounts for attributes which appear to afford no survivability benefit at all. Why, for example, do birds of paradise have brightly coloured plumage when drab feathers would more likely go unnoticed by a predator? Why do the sexual organs of some insects form elaborate tunnels when the somatic resources required to create these could be better spent developing a thicker exoskeleton? Why do some mammals give gifts of food in exchange for sexual access, when extra calories could mean the difference between life and death? The answer is that evolution is not just about survival of the fittest, but also reproduction of the fittest, and this was revealed by Darwin in his subsequent work on a specific type of natural selection dubbed sexual selection (Darwin, 1871; Trivers, 1972).

Rather than variance in survivability, the focus of sexual selection theory is variance in reproduction. Classically, sexual selection distinguishes between the attraction of members of the opposite sex and competition among members of the same sex for mates. Those who are able to attract a large number of mates (to sire a greater quantity of offspring), or high quality mates (to sire offspring of better quality), are more likely to increase the number of their genes present in future generations. Likewise, those better able to fend off same-sex rivals for such mates are likely to increase their own fitness relative to their competitors. These two relatively simple processes are exceptionally powerful in accounting for non-survival adaptations. For example, bowerbirds build elaborate bowers, and birds of paradise have bright colourful feathers, as these are attractive to females. By attracting more mating partners these males are likely to increase the number of offspring they sire. In contrast, male frogs stay attached to their partners, and deer battle one another (Clutton-Brock, Albon, \& Harvey, 1980), to restrict the access of their competitors to females. This then increases their chance of paternity relative to these rival males. The power of sexual selection to change behaviour and anatomy can be demonstrated using two example species: southern elephant seals and marvellous spatuletails.

Southern elephant seals (Mirounga leonina) mate in large harems and intense male-male competition decides who will mate with the females. Dominant males, who oversee the harems, can account for almost $90 \%$ of the copulations within a given mating season, and these fertilisations strongly predict $\left(R^{2}=0.83-0.99\right)$ genetic paternity (Fabiani, Galimberti, Sanvito, \& Hoelzel, 2004). As the ratio of male-to- 
female elephant seals is roughly equal (0.89; Arnbom, Fedak, \& Rothery, 1994), many males find themselves without mates irrespective of their ability to survive. In the sample studied by Fabiani et al., 72\% of males failed to obtain a single copulation. This intense competition for mates leads to a selection pressure on males to become larger and more aggressive over the generations. Present day males can be up to seven times heavier, and 73\% longer, than females (Bryden, 1972; Lindenfors, Tullberg, \& Biuw, 2002) and are so aggressive that they will often kill pups while fighting with other males. In contrast, marvellous spatuletails (Loddigesia mirabilis) pursue a mating system categorized, not by male competition, but by female choice. Like male-male competition, female choice can have profound anatomical consequences on males over evolutionary time. The males of this species have exceptionally long tail feathers which are three to four times their body length (Zusi \& Gill, 2009). These have evolved because females have a preference for males with particularly long tails which in turn creates a selection pressure for such tails. This pressure is strong enough to override the survival handicap a long tail brings to flying ability and camouflage.

Often sexual selection creates anatomical changes which are extreme in nature, such as the $3.7 \mathrm{~m}$ wide antlers of the extinct Irish elk (Megaloceros giganteus), the 1.5 $\mathrm{m}$ tall feathers of the peacock, or the body-weight sexual dimorphism of gorillas in which males grow up to 2.5 times larger than females (R. J. Smith \& Cheverud, 2002). Such changes occur because, when a certain attribute becomes the focus of sexual selection, it can become progressively enhanced over many generations via a process known as runaway selection (R. A. Fisher, 1915, 1930). Using the example of spatuletails above, an initial sensory bias among females for long-tailed males would have led said males to start out-reproducing their shorter-tailed competitors.

Subsequently, the young from these partnerships would have inherited both the paternal genes for long tails and the maternal genes for long tail preference. As a consequence, the average male tail size in each generation would have increased as well as the strength of the tail preference in females. Such a runaway process can continue until the survivability costs of possessing the sexually selected trait outweigh the reproductive benefits it brings. The fact that this "upper limit" still allows for individuals with grossly elaborate, and sometimes debilitating, traits is testament to the power of sexual selection.

Runaway selection assumes that the initial trait under selection, such as plumage colour or tail length, is arbitrary. For example, long-tails in bird species may be selected 
purely due to an incidental bias within the female visual attention system. There are alternative theories which aim to explain the same effect. One of these is the good gene hypothesis, which states that attractive traits which are sexually selected for are typically linked to an organism's underlying fitness (Byers \& Waits, 2006; Hamilton \& Zuk, 1982; Møller \& Alatalo, 1999). In this view, a female preference for a sexually selected trait could start as a random mutation affecting the preference for a random trait. This random trait could be related to superior fitness (e.g. energetic singing), but could also indicate poor fitness (e.g. poor foraging ability or body asymmetry), or show no relation to fitness at all. If the preference is linked to a fitness related trait positively, the females who hold this preference would then choose higher quality partners compared to other females, and would pass on this preference to future generations. Other preferences not linked to a trait indicative of high fitness would be likely to die out within a few generations.

\section{Males Compete, Females Choose}

Until this point, the examples used within this section imply that it is generally only the males of a given species which engage in competition and display while the females simply choose from a selection of willing males. This males-compete/femaleschoose (MCFC; Stewart-Williams \& Thomas, 2013b) model holds true for a large number of species, but there are some very important exceptions. The first exception can be seen in the very few species which show reversed sex-roles. For example, in Mormon crickets (Anabrus simplex), and some pipefish (Syngnathus typhle), it is the males which brood the fertilized eggs until they hatch (Gwynne, 1981). ${ }^{1}$ Among these species it is the females who compete for mates and the males who are choosy. In other sex-role reversed species such as the tidewater goby (Eucyclogobius neberryi), sexual selection can also lead to female ornamentation designed to appeal to male preferences (Swenson, 1997, as cited in Eens \& Pinxten, 2000). The second exception can be seen among the large number of animals, mainly insects and fish, which utilise external fertilisation. In these species the female's eggs are first laid before being fertilized by the males. Here, sexual selection is still active, selecting for males who are able to

\footnotetext{
${ }^{1}$ The traditional exemplars of sex-role reversed animals are the seahorses (Hippocampus). Much like pipefish, it is male seahorses which brood fertilized eggs until they hatch. However, a recent review calls into doubt the sex-role reversed nature of seahorse mating behaviour (e.g. competitiveness and choosiness) due to a lack of discrepancy in operational sex ratio causing females to still be the "sex in demand" (Eens \& Pinxten, 2000).
} 
produce and deliver the most sperm to the eggs in a process known as sperm competition (Levitan, 1998).

The final exception involves species which are characterised by mutual mate choice (MMC). In these species there is a distinctly reduced discrepancy between the sexes in terms of ornaments, body size, competition for opposite sex partners, and choosiness. This reduced sexual dimorphism covers a vast array of animal groups and includes emperor penguins (Aptenodytes forsteri), lar gibbons (Hylobates lar) and black-backed jackals (Canis mesomelas). Among the birds (Aves), the majority of known species can be classed as socially (but not necessarily genetically) monogamous (Griffith, Owens, \& Thuman, 2002), and there is growing body of research evidence revealing MMC within these species (e.g. Amundsen, 2000; Holveck, Geberzahn, \& Riebel, 2011).

Initially these exceptions posed a problem for some of the early theories about the driving forces of sexual selection. The Bateman principle (Bateman, 1948) tried to explain sexual dimorphism in terms of the relative size of male and female gametes. In biology, females are defined as the sex which has the smaller number of gametes (eggs). Often, eggs are much larger and contain more nutrients than the gametes of the male (sperm). Simply because eggs are fewer in number, a mating market is created whereby eggs are in greater demand than sperm, and, as eggs are created in female bodies, females become in more demand among males than males are among females. This then places a pressure on males to try to obtain female mates, and a pressure on females to be picky about whom they mate with. This sex difference is further exaggerated by the fact that females have a lower potential reproductive rate than males. While females in some species have periods of infertility due to gestation (and sometimes lactation), the somatically cheap and quick production of sperm in males means that they hold the biological potential to impregnate a large number of females over a short space of time. The resulting selection pressure causes males to allocate more time and energy to mating effort by developing ornaments to attract females and/or by engaging in intrasexual competition. Females, in contrast, do not tend to engage in such behaviours but instead focus on discriminating between available males. While the Bateman principle linked sex differences in behaviour to differences in sex cells, it failed to explain why monogamous and sex-role reversed species show this same gamete differences, but not the accompanying sex differences in behaviour, armaments, or ornamentation. Over 20 years later, Trivers (1972) published a sub-theory of sexual 
selection, partly inspired by the Bateman principle, which shifted the focus from gamete size to parental investment.

According to Parental Investment Theory (PIT), it is not simply differences between sex cells which cause one sex to be higher in demand than the other. Rather, it is the relative levels of parental investment provided by each sex which is important. The sex which invests more in offspring will be in greater demand than the sex which does not within the mating market. The Bateman principle is somewhat encompassed by PIT because, at the level of sex cells, eggs are more costly to produce, and are fewer in number, than sperm. As such, combining gametes to produce offspring represents a greater initial investment by the female compared to the male. However, investment does not stop at gametes. In the majority of species, obligatory parental investment is larger among females, compared to males, due to a necessary gestation period. In placental mammals, this ranges from a month in the Siberian chipmunk (Eutamias sibiricus linetus) to almost two years in African elephants of the Loxodonta genus (Kawamichi \& Kawamichi, 1993; Wittemyer, Barner Rasmussen, \& Douglas-Hamilton, 2007). During this time, the female requires a higher amount of nutrients than normal and is at a higher risk of predation. In some species, even after giving birth, females have an extended period of lactation in which they are infertile and have to regularly share resources with their young. Thus, in the vast majority of species, the obligatory parental investment for females is much larger than that of males. ${ }^{2}$

In addition to the obligatory levels of parental investment (PI) within a species, there are also typical levels of parental investment which can extend beyond both birth and lactation. Even after weaning, the infants of many species are not yet self-sufficient and may require feeding and protection in order to ensure their optimal development. Due to this, there is a selection pressure on the parents of some species to continue investing in their young past that which could be considered the biological "minimum". As with obligatory PI, there can also be large sex differences in typical PI. Among internally fertilising species, males can easily desert the female post-copulation, leaving her as the only parent available to provide post-weaning infant care. Here, the discrepancy in both obligate and typical PI is large. However, in some cases the male pair-bonds with the female, staying with her long enough to also provide indirect parental care (e.g. by supporting her during pregnancy), or direct parental care (e.g.

\footnotetext{
${ }^{2}$ Even in species which show external fertilisation, such as most fish, eggs tend to be more costly to produce than sperm and so even here obligate PI can be seen as larger among females.
} 
feeding and protection), after the offspring are born. In this case, while the discrepancy in obligate PI is large, the difference in typical PI between the sexes is small. Thus, rather than relative gamete size, it is actually the discrepancy between typical PI that accounts for the usual pattern of sex differences found in the majority of species. ${ }^{3}$

Much like the Bateman principle, PIT can adequately explain the prevalence of MCFC behaviour in the animal kingdom. Among species where the obligate and typical PI of males is small, females have more to lose from indiscriminate mating. If a female were to mate with a low quality male, she may be left having to gestate and suckle offspring of inferior genetic quality. No such risk exists for the males of such species. However, unlike the Bateman principle, PIT can also explain the existence of sex-role reversed species and those which show MMC. In the former, the sex cells are consistent with an MCFC species as female eggs are larger and fewer in number than male sperm. Despite this, the typical PI of males is large as they engage in behaviours such as protecting and feeding their young, while the typical PI of females is small. As this sex difference in typical parental investment is reversed, it is then the males who have more to lose by indiscriminate mating. The males then become the choosier sex and are in demand by the females. In contrast, among MMC species, the level of typical PI is similar in both sexes. These species tend to engage in genetic or social monogamy, with both males and females contributing to offspring protection and survivability. Thus, both sexes have something to lose by mating indiscriminately. In such species, both sexes are choosy about their pair-bonded partner (sometimes leading to mutually selected ornamentation; Kekäläinen, Huuskonen, Tuomaala, \& Kortet, 2010; Kraaijeveld, Gregurke, Hall, Komdeur, \& Mulder, 2004; Kraaijeveld, Kraaijeveld-Smit, $\&$ Komdeur, 2007), and both participate in mate guarding behaviour.

\section{Within-Species Variation and Mating Strategies}

PIT explains the variation in typical sex differences between different species. However, there is also a great deal of mating behaviour variation within species. Male scorpion flies (Panorpidae) pursue reproduction in two ways, either by offering the female nutrients (an insect carcass or saliva ball) and mating with her as she eats, or by forced copulation (Thornhill, 1981); some female birds adjust the time and effort put into raising their offspring based on the genetic quality of their partner (Johnsen,

\footnotetext{
${ }^{3}$ The Operational Sex Ratio and the Potential Reproductive Rate of a species are alternative, related, ways of measuring and explaining sex differences (Clutton-Brock \& Vincent, 1991; Eens \& Pinxten, 2000). These are discussed in further detail in Chapter 8.
} 
Delhey, Schlicht, Peters, \& Kempenaers, 2005; Qvarnström, 1997); and a minority of male red-sided garter snakes (Thamnophis sirtalis parietalis) release female hormones to distract other male snakes from mating with the females (Forsyth, 2001, pp. 30-31). To account for this type of within-sex variance in sexual behaviour one needs to understand what mating strategies are and how they are implemented within nature. This is the focus of the next section.

\section{Section II: Mating Strategies in the Animal Kingdom}

A mating strategy is an 'integrated set of adaptations which guide mating effort' (Gangestad \& Simpson, 2000, p. 575). Thus, a given mating strategy would contain several related adaptations which, collectively, facilitate a larger mating goal. In its simplest form, a mating strategy (a male one in this example) might involve adaptations which facilitate copulation - such as the capacity to identify females, a bias to approach them once identified, and a bias toward mounting attempts when in range. Other mating strategies might include more complex behavioural adaptations such as the gift giving behaviour of scorpion flies mentioned earlier (Thornhill, 1981). Here, males either provide the carcass of another insect, or a ball of nutritious spit, to a female and then attempt to mount her while she consumes it. Such behaviour is once again likely to be due to a combination of several adaptations which help realise an overall goal. In this case, the male scorpion fly needs to be able to identify which resources are available, approach a female with said resources at the correct time, and wait until she accepts the gift before he attempts to mount her. These individual adaptions work together in order to produce a mating outcome.

Occasionally, multiple mating strategies can form in a species. This is because the success of a mating strategy is contingent on many external variables including the frequency of its use by same-sex competitors and the personal condition of the strategist (M. R. Gross, 1996). For example, in a hypothetical species, an isolated male, with an abundance of females to choose from, would have his fitness best enhanced by pursuing a mating strategy which entails high mating effort and harem polygyny. However, if this isolation was disrupted by introducing as many males as there are females, then the success of this strategy would no longer be guaranteed. If all males followed the same polygynous strategy, not all of them would be successful. Much like in the earlier example of southern elephant seals, there would be great variance in reproductive success with some males siring many offspring and others being left with none at all. For those in the latter camp, any mating behaviour which could improve fitness over 
and above that afforded by the primary strategy would likely to be selected for. For example, the primary mating strategy of the Woodhouse's toad (Bufo woodhousii) is to stay stationary and attract females through deep croaks. However, usually it is only the largest males with the loudest croaks who attract females, and thus the majority of copulations. Smaller males often employ a secondary strategy of satellite interception (C. J. Leary, Fox, Shepard, \& Garcia, 2005). Rather than trying to compete unsuccessfully with larger males, these males stay mobile and try to intercept females on the way to the alpha male.

While it sometimes appears as if animals which adopt secondary mating strategies are "making the best out of a bad situation", this is not strictly true. This issue shall be explored in depth later within this section. What is important is that the possession of several distinct mating strategies can sometimes benefit an organism's fitness by allowing them to mate via an alternative method.

\section{The Development of Mating Strategies}

Broadly speaking, an organism's mating strategy tends to develop in one of three ways. The first is when the males of a species show several mating strategies, but each of which are highly canalized by the presence of certain genes. That is, assuming a normal developmental environment, which mating strategy an organism pursues can be predicted with great accuracy simply by observing their DNA. Such species are few in number and tend to be polymorphic with males or females appearing as one of multiple distinct versions or 'morphs'. The marine isopod Paracerceis sculpta is one such species. The females have one morph, while the males have three: $\alpha, \beta$, and $\gamma$. The most frequent male morph $(81 \%)$ is the $\alpha$-male, which is larger than the female, has elongated posterior appendages for fending off other males, and presides over a harem of females who live in sponges. The second most common male is the $\gamma$-male (15\%) who is tiny compared to the other morphs and looks like the young of the species only with highly developed testes. These males try to sneak copulations from within harems without detection by the $\alpha$-male. Finally, the $\beta$-male (4\%) employs a similar tactic to the $\gamma$-male, but rather than mimicking an infant of the species he instead mimics females. He is roughly the same size as a female, if not smaller, and has no exaggerated posterior appendages for combat. Shuster and Wade (2003) have demonstrated that the male morphs exploit one another's weaknesses in a zero sum manner similar to "rockpaper-scissors" game, so that, on average, all morphs receive the same average fitness. 
Thus, the three strategies have become evolutionarily stable (Dawkins, 1976) in their current frequencies. Evidence of genetically maintained alternative reproductive strategies has been found in other species including lizards (Thompson, Moore, \& Moore, 1993) and birds (Tuttle, 2003).

The second way an organism can develop a mating strategy is through a longterm developmental process. Here, the organism starts with the capacity for many different types of strategy and is sent down a specific, and often irreversible, developmental "path" depending on environmental input. Insects provide the most obvious examples of this type of development. In the dung beetle (Onthophagus taurus), males show one of two morphs, horned (an ornament associated with malemale competition) and non-horned, and the expression of each is dependent on the quality and quantity of food the beetle receives while in its larval form (Moczek, 1998; Moczek \& Nijhout, 2002). Likewise, it has long been known that queen bees are derived from the same larvae as worker bees, but that the administration of royal jelly sets off a developmental chain of events which produces this different morph (Stephen J. Simpson, Sword, \& Lo, 2011). This capacity to display morphs (or distinct phenotypes) within a single organism is called polyphenism. Polyphenism is not restricted to insects, nor does it only occur in the larval/early development stages of animals. For example, experimental evidence has shown that the onset of sexual maturity and the mating behaviour of guppies can be influenced by social feedback and predator prevalence (Rodd, Reznick, \& Sokolowski, 1997) and clown fish (Amphiprion percula) are one of many fish which change their sex from female to male in response to the death of a dominant male (Buston, 2004). More commonly, mating strategy development is influenced by an organism's relative status compared to their conspecifics (see the status-dependent selection model of M. R. Gross, 1996). The Woodhouse's toad (Bufo woodhousii), introduced in the previous section, provides a good example. There are two distinct phenotypes among males; one is large and dominant, while the other is small, subordinate, and often referred to as a 'satellite male'. Unlike some species, satellite males are not just younger versions of soon-to-be dominants that grow larger with age. It is perfectly possible to find dominant and satellite males which are of the same age. The determining factor for which morph a toad will develop into surrounds the depletion of somatic resources during early aggressive intrasexual encounters. Males who are unsuccessful, and use up more resources, tend to stay smaller in size and adopt the indirect satellite strategy (C. J. 
Leary et al., 2005). Garter snakes (Thamnophis sirtalis parietalis) develop their mating strategies in a similar way (Shine, Langkilde, Wall, \& Mason, 2005).

The final way in which mating strategies are implemented involves behavioural plasticity. Rather than having distinct phenotypes or morphotypes, behavioural plasticity allows organisms to participate in a diverse range of mating strategies without the need for specific anatomical adaptations. The eastern gray squirrel (Sciurus carolinensis) is a good example. There is only one male morph of this species, yet two distinct types of mating behaviour are displayed. Dominant males actively pursue females while subordinate satellite males intercept females opportunistically. While dominant males are larger and more aggressive, this is not to the extent that males would be considered different phenotypes or morphs and although dominant males are almost always older, both young and old males have the capacity for both strategies. Even within a single breeding season some males will show both dominant and subordinate behaviour depending on context (Koprowski, 1993). There are many species capable of behavioural mating strategy plasticity. Male scorpion flies (Panorpidae) will change to more successful tactics when larger males are removed (Thornhill, 1981); long-tailed widow birds (Euplectes progne) will engage in more mating effort and intra-sexual competition when their tails (a sexually selected ornament) are artificially enhanced (Andersson, 1982); and female zebra finches (Taeniopygia guttata) will change the type of partners they court after their feathers are clipped, spending less time with attractive males that may desert them post copulation (Burley \& Foster, 2006).

Mammals generally do not have distinct within-sex morphotypes and yet show within-lifetime flexibility in mating behaviour. Thus, it is behavioural plasticity which is the most likely way conditional mating strategies are realised in mammals, including Homo sapiens. For example, African striped mice (Lemniscomys zebra) switch between group and solitary living actively in response to the amount of reproductive competition present (Carsten Schradin, König, \& Pillay, 2010). Likewise some of the great apes have fluid status hierarchies which can change on a yearly basis; a male chimpanzee's (Pan troglodytes) status in the hierarchy affects whether he attempts to mate through dominance displays or by sneaking copulations (de Waal, 2007).

\section{Identifying Strategies}

There is a potential criticism surrounding the use of mating strategies as a way of explaining variation in mating behaviour within a species. It is easy to see that 
distinct mating strategies exist among animals with distinct morphs, as there may be behaviours which can only be performed by one morph (such as fighting with horns in the dung beetle; Moczek, 1998) which make the differences between the strategies clear. However, among animals in which the deployment of strategies is behavioural and conditional (see below), one could argue that the identification of such strategies is more ambiguous and thus prone to error. Take a hypothetical species whereby males typically engage in polygyny. In one case, male $X$ attracts a total of five females which he defends in a territory. In another case, male $Y$ attracts only one female, and defends her. With such information, it would be unwise to assume that, while $X$ is following a mating strategy characterised by harem polygyny, $Y$ is following a separate strategy characterised by a socially monogamous pair-bond. It could just as likely be the case that both males are following the same strategy, but that they have just experienced different outcomes.

There are precautions which can be taken in order to prevent such a misattribution. For example, one could adopt the approach taken by some evolutionary theorists when identifying adaptations in general. Specifically, the default position when examining a behaviour or physical attribute should be that it does not constitute an adaptation but rather an exaptation or a by-product (Gould \& Lewontin, 1979; Gould \& Vrba, 1982; G. C. Williams, 1966). This position is then held until there is good evidence to the contrary. In a similar manner, behavioural outcomes or attributes which are not consistent with the typical mating behaviour of a species should not automatically be assumed to constitute an evolved alternative mating strategy without a good deal of support. Such support could be the identification of behaviours which are domain specific to the candidate mating strategy, or evidence that the fitness pay-off of the strategy appears to be evolutionarily stable in comparison to the alternatives (Dawkins, 1976; M. R. Gross, 1996; Shuster \& Wade, 2003).

When it comes to the study of mating strategies in humans, support can also be obtained by examining the thoughts and feelings of men and women. Rather than having to infer the presence of a type of mating strategy through behavioural analysis, it is possible to see if an individual's reported desires and beliefs match a hypothesized mating strategy. A man could be asked, for example, about his desire for multiple sex partners in order to gauge whether he actively pursues multiple mates (a short-term mating strategy) or is committed to the development of one pair-bond (a long-term mating strategy). His attitudes could also be recorded to see if they are concordant with 
his reported desires. Such evidence brings its own weaknesses; humans can sometimes deceive others as well as themselves. However, the ability to ask such questions is arguably more useful than relying on the analysis of behavioural outcomes alone. Evidence for distinct short- and long-term mating strategies in humans through such methods is discussed later in this chapter within Section IV.

\section{Conditional Mating Strategies}

When multiple mating strategies exist in a species, and the implementation of each is dependent on some environmental context or input, it is often argued that species possesses mating strategies which are 'conditional' in nature. Most conditional mating strategy models involve some sort of switchpoint (M. R. Gross, 1996; Tomkins \& Hazel, 2007). Two (or sometimes more) mating strategies co-exist in a species and which one is currently activated is determined by whether or not a certain personal or environmental factor exceeds a particular value. For example, in a hypothetical species where males show the mating strategies of harem dominance and copulation sneaking, whether a male follows one strategy or another might depend on their body mass. Here, males may adopt a sneaking strategy until they exceeded a certain body mass (e.g. $1 \mathrm{~kg}$ ) at which point they would switch and attempt to secure a harem. This switchpoint represents an intercept between the average fitness outcome of each strategy (Shuster, 2010; Shuster \& Wade, 2003). Before such a point, the fitness afforded by one strategy outweighs the other, while after the switchpoint this relationship is reversed. This switchpoint may not be the same for every individual of a species as variance between individuals, occasionally due to genetic differences, can be found (Tomkins \& Hazel, 2007).

Current thinking within the evolutionary psychological literature is that humans show two distinct mating strategies (short- and long-term; Buss \& Schmitt, 1993) and that these are implemented conditionally much like in other animal species. The seminal paper on the issue of implementation was published by Gangestad and Simpson (2000) in Behavioral and Brain Sciences. In the paper, some of the biological principles introduced in this section were applied to human reproductive behaviour (e.g. page 576) to form Strategic Pluralism Theory. This theory is discussed in further detail within Section IV.

If it is true that humans are conditional maters, then two questions remain to be answered. First, what are the factors which affect the fitness outcomes of each strategy, and what values of such factors are required for humans to "switch" strategies? Second, 
how readily do humans switch mating strategies? Is it the case that a small cue is enough to lead to a change of strategy, or is prolonged exposure to a relevant factor needed before a change in mating strategy occurs? Such questions are explored within this thesis. However, in order to do this, a good understanding of the typical mating behaviour of Homo sapiens, including their mating strategies and their mating flexibility, is needed. This is the focus of the next two sections of the introduction.

\section{Section III: Typical Human Mating Behaviour}

While animal nature is fascinating, this thesis relates to one specific type of animal: a rather hairless, neotenous, and upright standing ape called Home sapiens sapiens. Ultimately, the working hypothesis of this thesis surrounds the within-lifetime flexibility of human mating strategies. However, it is important to have a full and proper understanding of typical human reproductive behaviour before examining this area. This is because the specific environmental factors which can affect the fitness pay-offs of each mating strategy within a species can often depend on their primary mating system. For example, as resource giving is a key part of the primary mating strategy of the male scorpion fly (Thornhill, 1981), one might expect that manipulating the availability of such resources would affect their mating behaviour. That is, resource availability may represent the key factor which affects the fitness afforded by the scorpion fly's different mating strategies (Section IV). However, this logic would not hold for male eastern gray squirrels, whose mating strategy choice appears to be dependent on the strength of their competitors and not resource availability (Koprowski, 1993).

In this section, the typical mating behaviour of humans is established using several lines of evidence. These include information about human ancestors from the paleoanthropological fossil record, modern cross-cultural evidence about levels of typical parental investment in both sexes, and an examination of sexual dimorphism among extant humans from the anthropological and psychological literature.

\section{The Evolution of Homo}

As the fossil record grows, the picture of human evolution becomes ever clearer. The common ancestor shared by humans and their closest related extant genus, Pan (common chimpanzees and bonobos), is thought to have existed around seven million years ago (MYA; Soares et al., 2009). The split away from this ancestor led to the emergence of increasingly bipedal apes under a subtribe known as Hominina. Hominins include the Australopithecus genus, which appeared between 4.5 and 2.5 MYA, the Paranthropus genus, which appeared between 2.6 and 1 MYA, and the Homo genus 
which can be traced back as far as 2.4 MYA (Jones, Martin, \& Pilbeam, 1992). The precise taxonomic status of these hominin genera is still an openly debated issue. For example, for years some paleoanthropologists considered Paranthropus bosei to be part of the Australopithicus genus, although this issue now seems to be somewhat resolved (Rotman, 2005). Thus, while the description here is designed to give a brief overview as to how the hominins changed over history using current information, the picture may well change in the future.

Members of the Australopithecus genus consisted of slender apes adapted more for savannah habitats than forest dwelling. They were bipedal, an adaptation hypothesized to be beneficial for several reasons (for a recent review, see Niemitz, 2010), and likely had a diet based around soft fruit, nuts, and seeds (Teaford \& Ungar, 2000). Available fossil evidence suggests that they were sexually dimorphic, with males being 30-40\% taller and 35-55\% heavier than females (McHenry \& Coffing, 2000), and that they had a cranial capacity of around $400-500 \mathrm{cc}$ - similar to that of a modern chimpanzee (Jones et al., 1992). Paranthropus shared several traits in common with Australopithecus. They were also bipedal, had a similar brain size (Jones et al., 1992), and also appeared to be sexually dimorphic, with males being 10-20\% taller and 2545\% heavier than females (McHenry, 1994; McHenry \& Coffing, 2000). The main difference between the two genera was that Paranthropus appeared more "robust". They had flat foreheads, thick jaws and cheek bones, and large teeth. These adaptations were likely due to a dietary encompassing vegetation which required heavy grinding to process (Cerling et al., 2011). Finally, the Homo genus, of which modern day humans belong, was marked by a larger cranial capacity than both Paranthropus and Australopithecus. Homo species had neotenous facial features and showed a reduction in weight and height dimorphism between the sexes. The precision grip of this genus allowed for tool making, perhaps leading to a more varied diet by allowing tough plant matter to be cut and scavenged carcasses to be stripped (Braun et al., 2010; McHenry \& Coffing, 2000). Between 2 and 2.5 MYA ago all three genera shared a habitat which makes taxonomy and establishing lineage difficult. However, recent evidence suggests that both the Homo and Paranthropus genera diverged separately from an Australopithecus ancestor. That is, humans did not descend from Paranthropus (B. Wood, 2010).

The path between the first Homo (habilis) and the most recent (sapiens) is marked by a clear increase in brain capacity and tool use. There is also a general trend 
showing an initial reduction in sexual dimorphism which is then maintained. The fossil record is far from clear; estimates of height and weight dimorphism are given for some species and not others. However, compared to the Australopithecus and Paranthropus genera, all members of the Homo lineage show a reduction in either height or weight sexual dimorphism. While there are more candidates for separate Homo species being discovered regularly (P. Brown et al., 2004), for brevity only five species are discussed here. These are the species for which there is the most available fossil evidence.

Homo habilis existed between 2.5 and 1.5 MYA and was of a comparable height to members of the Paranthropus and Australopithecus genera at around $1.3 \mathrm{~m}$. However, unlike other hominins of the time, habilis (sensu stricto) had a much larger cranial capacity of around 600-800 cc - a 50\% increase. While the levels of sexual dimorphism in height are unknown for this species, there appears to be a reduction in weight dimorphism with males being 16\% heavier than females (Jones et al., 1992; McHenry \& Coffing, 2000). Homo ergaster/erectus (2.0 to 0.3 MYA) had a cranial capacity of around 750-1250 cc, and also showed an increase in average height to 1.6$1.8 \mathrm{~m}$ (Crow, 2002; Jones et al., 1992; McHenry \& Coffing, 2000). It is likely that the sex difference in height decreased for this species, while the difference in weight remained similar to that of habilis, with males being $18-21 \%$ heavier than females (Dixson, 2009, p. 6; McHenry \& Coffing, 2000). The next chronological species, Homo heidelbergensis (0.7-0.2 MYA), showed a further increase in brain size. Here, cranial capacity was around $1200-1325 \mathrm{cc}$, which was almost three times larger than that of the Australopithecines (Conroy et al., 2000). Heidelbergensis stood at around $1.6 \mathrm{~m}$ tall and males were only $7 \%$ taller than females (Carretero et al., 2012). No clear data for weight differences between males and females are available for this species. Finally, at around 0.2 MYA, two versions of Homo emerge, Homo neanderthalensis and anatomically modern Homo sapiens. These species had a cranial capacity ranging from 1200 to 1700 cc and were $1.6 \mathrm{~m}$ and $1.8 \mathrm{~m}$ tall respectively (Crow, 2002; Jones et al., 1992). Sexual dimorphism in terms of height remained low for these species at 7-13\% (Carretero et al., 2012) and body weight dimorphism is estimated to have been around 16-17\% (Froehle \& Churchill, 2009; McHenry \& Coffing, 2000).

The exact evolutionary history of the Homo lineage is subject to large debate. For example, for a time evidence suggested very little overlap between the existence of Homo erectus and habilis. Then, in 2007, a fossil find in Kenya suggested that the two species may have lived side by side for almost 500,000 years (Spoor et al., 2007). The 
numbers of hominin fossils available are so few in number that the landscape of human evolution can change dramatically with a single finding. The possible exception to this being Homo neanderthalensis of which surviving DNA is available (Eriksson \& Manica, 2012; Green et al., 2010). However, in terms of this thesis, what is important is the general pattern of anatomical changes to hominin anatomy over time. This pattern can provide some insight as to the selection pressures which early humans faced. The transition from Australopithecus to Homo sapiens, regardless of the particular ancestral path, was marked by a reduction in sexual dimorphism and an increase in cranial capacity. There are several theories which could individually account for this increase in brain size, such as an arms race in the social ability to outwit others, the onset of language, or even climatic changes (D. Bailey \& Geary, 2009; Dunbar, 1998; G. Miller, 2000a). However, none of these satisfactorily account for the concurrent reduction in height and weight dimorphism found between the sexes. To explain this, one must consider the consequences of having a large brain as well as the benefits.

\section{The Large Problem with a Large Brain}

Large brains mean large heads, both in adults and in infants. By the time Homo became an established genus, their ancestors had been adapting to bipedal movement for at least one million years (Lovejoy, Suwa, Spurlock, Asfaw, \& White, 2009), and, as a consequence, their pelvises had become shorter and broader with a more narrow opening. This, in and of itself, would have made childbirth a more difficult and dangerous process (Wittman \& Wall, 2007). This problem would have been exacerbated by an increase in infant cranial mass as Homo brains became larger. To solve this problem, Homo evolved to give birth pre-maturely. Whereas most mammalian offspring are born developed enough to be able to walk within weeks, days, or even minutes, humans are technically born 12 months prematurely, compared to our closest primate cousins, and remain in a helpless and totally dependent state for years (Bluestone, 2005; Garwicz, Christensson, \& Psouni, 2009; Martin, 1990). It is this helplessness which may explain the reduction in sexual dimorphism found within the Homo lineage.

In mammalian and bird species which display sexual dimorphism, only one parent, typically the female, remains after fertilisation to ensure offspring survival. In such species, apart from delivering genes, males essentially provide no care for their offspring. However, this pattern can change. If, over time, offspring survival rate is particularly low under single-parent investment, then there can be pressure for other individuals to provide care to an infant in order to enhance its chances of survival and 
proper development. Human babies, born with a need for constant nurture and attention, may have placed pressure both on fathers and the female's wider social network to provide care. It follows that, if sexual dimorphism is caused by discrepancies in typical levels of parental investment (see Section I), then a selection pressure for parental care, due to offspring being born under-developed, could have led to a reduction in sexual dimorphism within the Homo lineage. Specifically, the big-brain/short-gestation tradeoff would have led to a selection pressure towards social monogamy and bi-parental care. This would have subsequently reduced the amount reproductive variance between the sexes and thus reduced the levels of sexual dimorphism over time. A reduction, however, is relative, and although the fossil record suggests the presence of this pressure towards social monogamy, other lines of evidence need to be explored in order to understand where humans lie on the spectrum of mating behaviour.

\section{Typical Levels of Parental Investment in Humans}

As mentioned in Section I, Parental Investment Theory (PIT) emphasizes the role of typical levels of parental investment in the development of a species' mating system (Trivers, 1972). In humans, parental investment is typically high in both sexes. In hunter gatherer societies (see below), infants suckle for up to three years, and do not reach sexual maturity and calorific independence for a further 15-16 years (Kaplan, Hill, Lancaster, \& Hurtado, 2000; Robson \& Wood, 2008). During these years, they need protection, nutrients, and stimulation. Even though the typical parental investment provided by women is higher than that provided by men (simply because men have the option to abandon women post-fertilisation), many men still provide support during pregnancy and for many years beyond.

Hunter-gatherer societies are often used as a model of ancestral behaviour as their living conditions are a good approximation of those experienced by humans in the EEA, before the invention of agriculture. ${ }^{4}$ In such societies, fathers tend to provide high levels of both direct and indirect paternal care (Hewlett, 1991; Hewlett \& Macfarlan, 2010; Marlowe, 2000). Direct care involves close contact with a child, including sleeping in the same room as them, carrying them, or staying in close proximity to them

\footnotetext{
${ }^{4}$ EEA stands for the Environment of Evolutionary Adaptiveness (Cosmides \& Tooby, 1992b). Here, the environment does not refer to a single period in time or space. Instead it relates to a specific period for each adaptation in which environmental circumstances presented a problem which was persistent enough for the adaptation to evolve. For example, the EEA for bipedal movement in hominins would be in a completely different time and space compared to the EEA for the development of lactose persistence (a very recent adaptation by comparison; Holden \& Mace, 1997).
} 
during waking hours. For example, Aka men of the Central African Republic carry their child between $11 \%$ and $22 \%$ of the time up until the age of 18 months (Gettler, 2010). Indirect care, in contrast, includes the provisioning of calories, the transference of social status and knowledge, and providing protection/defence. In the Hadza of Tanzania, for example, males contribute almost half of the calories in a child's diet and even more when their partner's ability to forage is handicapped by pregnancy and lactation (Marlowe, 2003).

Bi-parental care most commonly occurs in the context of a long-term pair-bond. Even among the Hadza, who are known to have a high divorce rate, marriages have a $60.7 \%$ chance of lasting more than five years and a $32.4 \%$ chance of lasting more than 15 years. This is more than enough time for the father to have a positive contribution to a child's development (Blurton-Jones, Marlowe, Hakwes, \& O'Connell, 2000). The importance of paternal input is highlighted by the fact that many hunter-gatherer societies show a "father effect" on mortality; children are more likely to die if their father is absent through divorce or death (Blurton-Jones et al., 2000). This is the extreme outcome and around two-thirds of societies show no father effect on mortality at all (Blurton-Jones et al., 2000; Sear \& Mace, 2008). However, this does not mean that there is no negative impact of paternal absence. Any reduction in parental care can mean a potential reduction in fitness, and, if the father is unable to provide this, other friends and family members often offer support in the form of alloparental care.

There are some theories which state that the reason high levels of paternal care are found among men is not because this care improves the development of offspring (and thus fitness) but because it improves their sexual access. That is, men care for infants as a form of courtship display (Hawkes, 1991). If this were the case then stepfathers would be expected to provide the same amount of care to their biological offspring as their non-biological offspring. Yet, this effect is not found among the Hadza as evidenced by greater resource diversion towards biological offspring (Marlowe, 1999b).

There is, of course, a great deal of variation between men in how much they invest in their young and by what means, and this can be partially accounted for by cultural differences. Among the Kipsigi, for example, men hardly interact with their offspring until they reach the age of four and even then they do not feed or sooth them. However, even these "distant" fathers provide some form of indirect care such as intervening when their young are in danger, providing economic support, and 
disciplining children when necessary (Muller, Marlowe, Bugumba, \& Ellison, 2009). Even this level of investment vastly exceeds that offered by the males of almost all mammalian species, $95 \%$ of which provide no parental investment of any kind and are often completely indifferent to related or unrelated juveniles (Geary, 2000). If one were to create a scale of parental investment, with 'no investment' as the left anchor and 'typical maternal investment' as the right anchor, and then plot the typical level of investment provided by the males of each mammalian species, men would be one of the outliers. They would be closer to the right hand side of the scale than the vast majority of other males.

\section{A Committed Mind}

Our universal emotions and mate preferences also tell us something about our typical mating behaviour. In sexually dimorphic species, it is generally the females who are picky while males are relatively indiscriminate. Yet, this pattern is not found in humans. Both men and women are picky about their sexual partners and both sexes engage in behaviour designed to attract partners. This pattern tends to transcend culture. Similar to how male bower birds show off to females by creating elaborate bowers, some men strive to impress women by increasing their social status (Buss, 2003a). However, unlike the drab female bowerbird, which makes little effort to attract or maintain male attention, women spend tens of thousands of pounds during their lifetime on haircuts, make-up, diet schemes, jewellery, and attractive clothing, in order to attract men (Durante, Griskevicius, Hill, Perilloux, \& Li, 2011). Such behaviours are also echoed in non-Western cultures including the marital dresses of the Herero or the beaded necklaces and bracelets of the Tsimane (Hendrickson, 1994; Rucas, Gurven, Kaplan, \& Winking, 2010). In terms of pickiness, both men and women show high standards in their mate preferences. For example, both sexes typically desire intelligence, a sense of humour, an interesting personality, and romance in their longterm partners (Buss \& Barnes, 1986; Buss \& Shackelford, 2008; Li, Bailey, Kenrick, \& Linsenmeier, 2002). Although there are some robust differences between the sexes, these are often overshadowed when considering the presence of mutual of traits in both sexes. For example, the sex difference in preference for physical attractiveness has been replicated many times (Buss, 1989; Chang, Wang, Shackelford, \& Buss, 2011) and in Buss' classic study it was ranked for importance by men as 4.04 on average out of 13 traits. In women this was a lower 6.26 rank and this constituted the largest sex difference in the paper $(d=0.92)$. However, while there is indeed a difference, the larger 
finding is that humans in general are a species in which both sexes prefer physically attractive partners (Stewart-Williams \& Thomas, 2013a).

Two of the strongest human emotions also reveal our long-term nature: romantic love and jealously. Love is ubiquitous. Once considered a Western convention, the Standard Cross-Cultural Sample (Murdock \& White, 2006) revealed the presence of love in an overwhelming number of cultures (89\%; Jankowiak \& Fischer, 1992). Love is different from lust in as much as it involves an emotional bond and willingness to commit to an individual for a long period of time. Love is a pair-bonding mechanism and it functions to keep attention and resources focused on a particular partner. ${ }^{5}$ Feelings of love are enduring, lasting months or years. Most importantly, love occurs in both sexes, which is in line with MMC. If humans were an MCFC species, one may expect that only one sex (perhaps the one in lower demand) would be besotted with the other, or that love would not exist at all. This is not the case; love is the most important thing to both sexes in long-term partners (Buss et al., 1990).

The emotion of jealousy also appears to be a human universal. While it can lead to devastating consequences, jealousy may serve as a relationship maintenance mechanism within a long-term relationship (Buss, 2006a). Some research has focused on sex differences in jealousy, concluding that men seem to be more affected by a partner's sexual infidelity, rather than emotional infidelity, when compared to women (Buss, Larsen, Westen, \& Semmelroth, 1992; S. L. Miller \& Maner, 2009). In reality, these differences only reveal themselves when tightly controlled forced-choice paradigms are used. That is, when participants are asked to choose which behaviour would upset them the most, emotional or sexual infidelity, a larger proportion of men than women choose sexual infidelity as the most upsetting. The reason such paradigms are needed to tease out an effect is because both types of jealousy are very upsetting to both sexes (Stewart-Williams \& Thomas, 2013a). When these constraints are taken away, both men and women report both types of infidelity disturbing, with the sex difference either disappearing or showing a mixture of effect sizes which are large by psychological standards but small by biological standards $(d<1.0$; DeSteno, Bartlett, Braverman, \& Salovey, 2002; Pietrzak, Laird, Stevens, \& Thompson, 2002). In fact, a recent replication using over 60,000 participants found that the effect size was small-tomedium in size, $r=0.18$ (Frederick \& Fales, 2014). In terms of understanding typical

\footnotetext{
${ }^{5}$ Feelings of love have even been associated with specific brain regions, providing further evidence that it may be an evolved mechanism rather than a cultural invention (Aron et al., 2005).
} 
human reproductive behaviour, the fact that both men and women are prone to general jealousy is more informative than discussing the differences between the sexes.

Furthermore, although women do seem to be biased in favour of choosing emotional infidelity as more upsetting ( 20-80 split), men typically choose a more balanced ( 60 40) split between the two. ${ }^{6}$ This is poor evidence that men find sexual infidelity more upsetting than emotional infidelity. Rather, the specific split between the two types of jealousy in men suggests that one type of infidelity is just as upsetting as the other, even in a tightly controlled forced-choice context.

Finally, there are other psychological biases found within humans which suggest that our typical mating arrangement is that of a monogamous pair-bond. One such bias is the derogation of alternatives effect (Eastwick, 2013; Karremans, Dotsch, \& Corneille, 2011; J. A. Simpson, Gangestad, \& Lerma, 1990). This effect occurs when humans enter a pair-bond, and causes both men and women to show cognitive biases against attractive other sex suitors. For example, a recent study by Karremans et al. (2011) demonstrated that memory for attractive faces can be affected by relationship status. Despite there being no difference in how single and committed individuals perceived the initial attractiveness of faces, individuals who were in relationships later recalled attractive faces as being less attractive than they had originally rated them to be. The derogation of alternatives effect is described in more detail within Chapter 3.

\section{Sexual Dimorphism}

Humans are not exceptionally sexually dimorphic. In gorillas (Gorilla gorilla), males can be twice the weight of females, and males tend to be much more aggressive in nature, even from an early age (Leigh, 1992; Meder, 1990; Taylor, 1997). In contrast, men and women are of a similar height, share similar emotions and desires, and typically invest a lot in offspring. That being said, humans are also not exceptionally monomorphic; robust sex differences, both of a physiological and psychological nature, do exist. The purpose of this sub-section is to acknowledge these differences, while still recognising the fact that, comparatively, these are somewhat weak compared to other species.

In The Evolution of Homo section, it was established that, as the Homo line evolved, sexual dimorphism decreased. This was likely due to a selection pressure for

\footnotetext{
${ }^{6}$ The aforementioned large-scale replication of this original study found a more balanced split of 35-65 for women and 54-46 for men (Frederick \& Fales, 2014). Furthermore, there is some evidence that in small-scale, natural-fertility societies, sexual infidelity is found to be the most disturbing for both sexes (Scelza, 2014).
} 
additional childcare created by infants being born underdeveloped, which was then realised through an increase in paternal, as well as wider alloparental, care. However, such pressure may have also met some resistance. As previously discussed, obligate parental investment is generally much smaller for males than females. Although the discrepancy between men and women in the amount of typical investment they provide is much lower than for most mammals, the potential still exists for men to reproduce without heavy investment. Historically, men would have been restricted in the number of offspring they could have sired by the potential number of willing and fertile women available. Men who were able to successfully take advantage of an opportunity to mate with someone other than their primary partner could have experienced a large increase in their fitness at very little cost. For example, a man with two children by his primary partner could have, in theory, increased his reproductive output by $50 \%$ through one brief liaison with a different female. Indeed, examinations of the modern human genome suggest a long history of effective polygyny among Homo sapiens, although to what extent is still open to debate (Hammer, Mendez, Cox, Woerner, \& Wall, 2008).

Thus, there would have been a historic trade-off between the pressure on males to become parental, and the pressure on males to seek opportunistic mating due to a large potential fitness pay-off. This trade-off is akin to the earlier example of the male marvellous spatuletail, which possesses a tail which is the result of a trade-off between two selection pressures: one to grow progressively longer tails in order to attract females, and another to reduce tail size in order to enhance survivability. At a certain point, the fitness benefit $(X)$ of an increase in tail length would not be beneficial considering the reduction in fitness $(Y)$ experienced by the male because of the tails' impact on his survivability $(X+Y<0)$. Tail length would then reach an imposed maximum length at this balanced point. Similarly, in men, it may well be the case that, at a certain point, the fitness benefit of increased paternal care $(X)$ would not be beneficial considering the reduction in fitness $(Y)$ experienced by the man from not pursuing opportunistic mating. This would then lead to the maintenance of some shortterm or polygynous mating, thus causing sexual dimorphism to be sustained at a reasonably low level.

There are a variety of average sex differences in human behaviour which supports this idea. Compared to women, men typically want a greater number sexual partners in their lifetime, are more willing to consent to sex with a stranger or someone they have not known very long, and are more likely to have sexual desires involving 
strangers or multiple partners (Buss \& Schmitt, 1993; Ellis \& Symons, 1990). Men are more aggressive than women (Archer, 2004), are more likely to strive for status (Huberman, Loch, \& ÖNçüler, 2004; M. Wilson \& Daly, 1985), and engage in greater risk taking behaviour (Byrnes, Miller, \& Schafer, 1999; Daly \& Wilson, 2001; Pawlowski, Atwal, \& Dunbar, 2008). Physiologically, men are typically taller than women, and have greater muscle mass (Lassek \& Gaulin, 2009), denser bones (Naganathan \& Sambrook, 2003), and higher levels of testosterone (van Anders, Hamilton, \& Watson, 2007). In the majority of cultures (but not all), if there are differences in reproductive variance between the sexes it tends to be men who have the higher variance (Betzig, 2012; G. R. Brown, Laland, \& Mulder, 2009) and, if the local mating system permits, it is generally men who take multiple spouses rather than the women (Betzig, 2012; Marlowe, 2000). ${ }^{7}$ Generally, relationship infidelity is more likely to come from men than women (Betzig, 1989), although this pattern has reduced over time (Tsapela, Fisher, \& Aron, 2010).

It is important not to interpret these differences incorrectly by polarising the sexes. Every sex difference in a psychological, anatomical, or physiological attribute, if shared by both men and women, is one based on the means of distributions which overlap. Consider, for example, possibly one of the largest sex differences in humans: lean body-mass. Men are heavier than women on average, but by no means is the lightest man heavier than the heaviest woman. Statistically, the effect of sex on lean body weight is strong, $(d=2.06 \text { by one estimate; Lassek \& Gaulin, 2009 })^{8}$, but even with an effect size this large there is an overlap of around 18\% (Cohen's $U_{1}$ ) between the distributions (J. Cohen, 1988). When this is compared to highly sexually dimorphic and polygynous species, such as gorillas and elephant seals, much less of an overlap is found. Body weight sex differences in western lowland gorillas (Gorilla gorilla gorilla) have an effect size of over $d=4.4$, meaning that there is an overlap of only $1 \%$ between the male and female distributions (Leigh, 1992). In southern elephants seals (Mirounga leonina) this is even greater; an effect size of almost $d=5.2$ with an overlap of less than 0.5\% (Galimberti, Sanvito, Braschi, \& Boitani, 2007; Hindell, Slip, \& Burton, 1994). In more socially monogamous species, such as lars gibbons (Hylobates lar), this effect size

\footnotetext{
${ }^{7}$ There are some known cases, such as in Tibet, where examples of polyandry can be found. Typically this tends to be where two brothers share a wife out of necessity. Such arrangements often dissolve when economic circumstances become more favourable (L. Barrett, Dunbar, \& Lycett, 2002).

${ }^{8}$ Lean weight is a better choice in humans given that modern calorie-rich diets cause great variation in body size. In fact, the effect size when fat is included is a considerably weaker $d=0.42$ (Ogden, Fryar, Carroll, \& Flegal, 2004).
} 
is much smaller at around $d=1.0(d=0.8$ for carpenteri and $d=1.2$ for some entelloides) with an overlap between the male and female distributions of around $45 \%$ to $60 \%$ (Geissmann, 1993). The closer proximity of humans to a typically monomorphic species, rather than a highly polygynous one, suggests that Homo sapiens are not a highly polygynous species.

Rather than just physical differences, it would be beneficial to be able to compare sex differences in behaviour among sexually dimorphic species to that of humans. If, for example, the effect size of a given measure of aggression in northern elephant seals (Mirounga augustirostris) was $d=5.0$, while a similar measure in humans revealed an effect size of $d=1.0$, then this would tell us something about the relative level of psychological sex differences between the two species. However, this is hard to achieve for two reasons. First, among highly sexually dimorphic species, there is often such a marked difference in behaviour between the sexes that only one sex is considered during research on a sexually dimorphic trait (e.g. Christenson \& Le Boeuf, 1978; Leboeuf, 1972, in the case of elephant seals). Second, among less dimorphic animals, where sex differences are sometimes measured, the results are often presented in such a way that effect sizes are either hard to produce or hard to compare against those generated in humans studies. For example, in a group of chimpanzees, Kahlenberg and Wrangham (2010) found that male chimps were more likely than females to use sticks as play objects. For every 1000 observation hours the median number of times a stick was used as a weapon was 0.09 for males and 0.00 for females. While this sounds like a small difference, it produced a large Mann Whitney $U$ score $(U=597)$ and was highly significant with only 49 subjects. However, there is no way to translate $U$ to a comparable effect size such as $d$ or $r$. Similarly, in capuchin monkeys (Sapajus libidinosus) probes are often used to search for insects or lizards, and male monkeys account for $97 \%$ of probe use (Falótico \& Ottoni, 2014). Again, while this is likely to represent a large sex difference, it is not presented in a manner which allows for effect sizes to be generated and compared.

What is clear is that, among humans, even the largest psychological sex difference is small compared to physical differences such as height and weight. The desire component of the socio-sexual orientation inventory revised (Penke \& Asendorpf, 2008) shows one of the largest and most robust psychological sex differences ever 
found, with an effect size of $d=0.89 .{ }^{9}$ Yet, if one were to pick a woman and a man at random from the population, one would find that the woman had a higher desire for uncommitted sex than the man $29 \%$ of the time. As will be discussed later, women, as well as men, desire and engage in short-term opportunistic mating. Such behaviour can provide reproductive benefits to both sexes.

Effect sizes for other psychological sex differences are smaller still. A study of the abilities of men and women to mentally rotate 3D objects and remember object locations, using over 250,000 participants from 40 countries, found that men typically scored higher in the former task while women typically scored higher in the latter (Silverman, Choi, \& Peters, 2007). Though robust, these differences were of a moderate effect size with $d=0.48$ found for 3D rotation and $d=0.31$ found for location memory. Sex differences in aggression provide another example. These typically vary by both aggression type and measurement type. According to a meta-analysis by Archer (2004), the largest case (physical aggression as reported by peers) showed an effect size of $d=$ 0.84 , while the smallest (self-reported feelings of anger) had an effect size of only $d=$ 0.01. Likewise, the sex difference in sensation seeking and risk taking appears to be around $d=0.40$ (Cross, Copping, \& Campbell, 2011). Together, such small-to-medium effects, especially when compared to differences in observable physical attributes such as height, appear to suggest only moderate differences in behaviour between the sexes. Certainly, these are likely to be dwarfed by some of the differences seen elsewhere in nature among sexually dimorphic and polygynous species. To an alien observer (Stewart-Williams, in prep.), male and female Homo sapiens may seem as similar to one another as male and female gibbons or penguins appear to us.

\section{A Note about Miss-Match}

There exist many modern and historical accounts of human behaviour which, prima facie, provide evidence for evolutionarily relevant sex differences. Yet, when these accounts are examined in further detail, they are found to sometimes exaggerate such differences. Often this is because they occur in evolutionarily novel environments which are markedly different from the ancestral ones in which humans evolved. For example, one of the findings from the evolutionary psychological literature is that men tend to have greater reproductive variance than women. This is due to the biological

\footnotetext{
${ }^{9}$ This was calculated from our own sample of 1230 participants $(46.6 \%$ male). A similarly high value $(d$ $=0.74$ ) was found using a modified version of the original SOI in a study of over 200,000 participants (Lippa, 2009).
} 
restrictions unique to women which reduce the number of offspring they can produce. Men, in contrast, have very few restrictions and could potentially impregnate a large number of women within a short space of time. Over long periods of time this sex difference would have led to evolved differences in the mating psychology of men and women, such as men typically showing greater interest in sexual variety (Ellis \& Symons, 1990; Symons, 1979). Historical anecdotes of ruthless tyrants and kings siring thousands of children (Betzig, 2012) appear to support the idea that there is a sex difference in reproductive variance, and this could cause laymen to believe that the difference is very large. However, such prolific behaviour is likely the product of male mating psychology interacting with a set of evolutionarily novel circumstances. Specifically, kings and despots were able to mate with so many women because they lived in very large societies, made possible by the development of agricultural practices, with high levels of social stratification. This increase in power and population allowed the male preference for sexual variety to be realised in an extreme way for a small number of individuals.

In contrast, for the majority of human evolutionary history, the opportunities for men to gain sexual access to a large number of women would have been modest. Ancestral human groups would have had a population of around 150 members (Dunbar, 1993 ) and contained many infertile (e.g. postmenopausal) or related women. As a consequence, even the most influential and attractive man would have found it hard to gain sexual access to many fertile women. Instead, his primary mating arrangement would likely have been social monogamy or mild polygyny, with only occasional instances of short-term sexual access. Such a lack of reproductive variance is evident in extant groups of hunter-gatherers which show markedly reduced sex differences compared to pastoralists or agriculturalists (Betzig, 2012; G. R. Brown et al., 2009). ${ }^{10}$

The previous example demonstrates how the difference in reproductive variance between men and women can be overestimated when examining behaviour within evolutionarily recent societies. There are, however, other biases which can lead to sex differences being underestimated. The influence of religion, for example, can often create cultural conditions markedly dissimilar to ancestral ones. Most Judaeo-Christian religions have strict guidelines about monogamy and life-long pair-bonds. Over $17 \%$ of

\footnotetext{
${ }^{10}$ Even when pastoral and agricultural societies are considered, the level of male reproductive variance is still much smaller than that implied by the historical accounts of prolific leaders. For example, a sample of 93 polygynous societies from the SCCS revealed that, in $36 \%$ of cultures, no man had more than three wives, and in $78 \%$ of cultures, no man had more than ten (Low, 1988).
} 
the world's population describe themselves as Roman Catholic, a religion that generally forbids divorce (although annulments are allowed in some cases) and sex outside of marriage. Among a group of Catholics who subscribed to their doctrine faithfully, the difference between male and female reproductive variance would be very close to zero. This of course is not the case. Even in Ireland, whose population is predominantly Roman Catholic, divorce rates among Catholics continue to grow with $3.6 \%$ of the population reporting their marital status as 'divorced' as of 2011 (Central Statistics Office, 2012). Furthermore, the church allows widows to remarry, introducing another source of variance into the population. Nonetheless, such institutions may well reduce reproductive variance below the levels typically experienced by ancestral humans.

In societies more representative of ancestral conditions, marriage is much more of a relaxed concept. Individuals have several serial marriages throughout their life time, and these are relatively easy to enter into and annul (e.g. Kramer \& Greaves, 2007; Marlowe, 2002). For example, 40\% of Hadza and !Kung marriages end before their fifth year anniversary (Blurton-Jones et al., 2000). Thus, a marriage within these societies appears to be similar to a long-term relationship within modern western cultures, and so the sex difference in reproductive variance is likely to be larger among these cultures compared to ones in which life-long monogamy is forced. There are, of course, other cultural forces which lead to more reproductive variance than that found in pre-industrial societies. In the middle-east, especially in strongly Islamic countries, polygyny is considered both lawful and socially acceptable. Thus, there is a need to be especially cautious about the evidence used when trying to understand the size of evolutionarily relevant sex differences, especially when it pertains to an extant culture or a point in recent history, both of which may contain elements markedly different from our ancestral past.

\section{The Overall Picture}

Homo sapiens are not a grossly sexually dimorphic species, but neither are they a species in which males and females are truly monomorphic. Instead, humans lie somewhere in the middle of the spectrum, perhaps closer to the monomorphic anchor than the dimorphic one. This low level of sexual dimorphism does not appear to be due to a promiscuous past like that found in chimpanzees (promiscuous species are not highly dimorphic; Heske \& Ostfeld, 1990), as there existed clear selection pressures for bi-parental care, and modern humans show psychological adaptations to overcome commitment-related problems (e.g. the emotions of love and jealousy). At the same 
time, it is clear that some short-term mating behaviour was, and still is, part of human reproduction. For example, there exists strong evidence that short-term mating occurs cross-culturally (Buss \& Schmitt, 1993; Schmitt, 2005b) and that low levels of sperm competition exist in men (R. R. Baker \& Bellis, 1995; Shackelford, Pound, \& Goetz, 2005). A combination of evidence suggests that humans evolved as a socially monogamous hominin, typically engaging in long-term pair-bonds while providing biparental care to offspring. Occasionally, men and women would have engaged in extrapair mating and some would have been part of a polygynous relationship. Such behaviours would have led to the maintenance of a modest difference in reproductive variance between the sexes, and thus a moderate level of sexual dimorphism across a number of physical and psychological traits.

Now that the typical mating behaviour of humans has been established, the next step is to outline and understand the variance in mating behaviour which exists between individuals. According to evolutionary psychological theory, a large proportion of such variance can be accounted for by an individual's mating strategy. In the final section of this introduction, the types of mating strategy which are employed by men and women are discussed. This discussion includes the evolutionary costs and benefits of each strategy, as well as the types of personal and environmental factors which are likely to affect their effectiveness and therefore initiate strategy change.

\section{Section IV: Human Mating Strategies and Their Potential Flexibility}

There exists great variance in mating behaviour among humans, even when differences between the sexes are taken into account. Some men and women choose to engage in strict life-long monogamy with one individual, while others choose to only have brief sexual flings or one night stands. A great deal of this variation can be accounted for by mating strategies. In this final section, the specific mating strategies of humans are explored. The section starts with an outline of the evolutionary theory behind both strategy development and implementation. Following this, a summative section is presented which explores the types of evolutionarily relevant factors hypothesised to affect the pursuit of mating strategies. These factors ultimately became the focus of the experimental chapters of the thesis. Finally, a cross-section of current evidence is explored, from different areas within psychology, which provided some initial support for the idea that mating strategies are flexible in line with the working hypothesis.

\section{Human Mating Strategies}


Evolutionary psychology distinguishes between short-term and long-term mating strategies in humans (Buss \& Schmitt, 1993; Gangestad \& Simpson, 2000; Workman \& Reader, 2004). Long-term strategies guide mating effort towards relationships marked by love, commitment, and fidelity, which last for an extended period of time. Short-term strategies guide mating effort towards more temporary liaisons, low in commitment, with a focus on sex. Immediate evidence that humans hold the capacity for both types of strategies can be found in research which shows that participants often change the way they act when presented with different relationship contexts. That is, if participants are asked to make relationship choices within a shortterm context, their answers will sometimes differ markedly to those given when they are presented with a long-term context (Buss \& Schmitt, 1993; Confer, Perilloux, \& Buss, 2010; Lehr \& Geher, 2006; Scheib, 2001; Schmalt, 2006; Stewart, Stinnett, \& Rosenfeld, 2000). For example, both sexes find good looks a lot more important in a short-term partner than a long-term one (Li \& Kenrick, 2006), and women rate frugal behaviour in a short-term partner as much less desirable than in a long-term one (Buss $\&$ Schmitt, 1993). Likewise, women are more averse to potential partners who resemble themselves within a short-term mating context compared to a long-term one (DeBruine, 2005; Regan, Levin, Sprecher, Christopher, \& Gate, 2000), and attractive men show a preference for femininity in the faces of short-term, but not long-term, partners (Burriss, Welling, \& Puts, 2011).

According to Strategic Pluralism Theory (SPT; Gangestad \& Simpson, 2000) the variance found among humans in their mating behaviour can be attributed to the conditional deployment of these two strategies. For example, consider a man with a reliable history of strict monogamy who suddenly commits a promiscuous act of onetime adultery. This behaviour, according to SPT, could be explained by the differential activation of the two mating strategies. Specifically, the man in question would have initially pursued a long-term mating strategy, but at some point he would have temporarily switched to a short-term one. Why might these two types of distinct mating strategies exist in humans? In Section II, the fitness benefits of pursuing alternative mating strategies were explained. In brief, alternative mating strategies can evolve within a species if they provide a better reproductive outcome to a proportion of individuals than they would experience by following the primary strategy. A similar view can be applied to humans, and is done so in one of the key theories of mate preferences in psychology: Sexual Strategies Theory (SST; Buss \& Schmitt, 1993). 
SST outlines, and provides evidence for, the distinct long- and short-term mating strategies of both men and women, and summarises the costs and benefits of each. In terms of long-term mating, the ancestors of both sexes would have had access to several direct and indirect fitness enhancing benefits through the maintenance of a pair-bond. These benefits include enhanced offspring survival, the division of labour, the potential to increase social status, and access to a wider social network. According to the theory, sex specific benefits of such a strategy would have also existed. For men, having a longterm partner would have led to regular sexual access and increased paternity certainty, and their level of commitment would have allowed them to attract a high quality partner. For women, a long-term partner would have been able to provide protection for both her and her child, support her during pregnancy, and increase her access to economic resources.

Adopting a long-term mating strategy would have posed its own set of adaptive problems for human ancestors. For a woman, adopting such a strategy would have left her in a vulnerable position if her chosen partner was not as committed to the relationship as she was and deserted her after copulation. Likewise, for a man who decided to forego other mating opportunities to mate exclusively with one woman, choosing a partner of low reproductive value, or who would likely commit acts of infidelity, would have been detrimental to his fitness. Over time, human ancestors who were faced with these problems evolved adaptations to cope with them. For example, women tend to be more cautious than men when it comes to consenting to sex, often requiring some time to pass and for commitment to be displayed (Buss \& Schmitt, 1993; R. D. Clark \& Hatfield, 1989). This could function as a "test" of a potential partner's level of commitment in order to avoid abandonment, or may function to simply allow her more time to fully assess his fitness. Women also prefer older men as partners, typically two to three years older, likely due to their higher social status (Buss, 1989). To avoid choosing a long-term partner with low reproductive value, men typically show stronger preferences than women for attributes in a partner which signal youthfulness (such as having high energy and neotenous features), fertility (such as a low waist-to-hip ratio, Schmalt, 2006), and fidelity (Buss \& Schmitt, 1993). These are examples of adaptations to sex-specific problems, although many of the adaptive problems of mating would have been shared by both sexes. For example, both men and women would have been faced with the challenge of identifying a partner who would be devoted, kind, and possess good parenting skills. Such challenges appear to have been 
overcome with mutual psychological adaptations such as jealousy (perhaps as a method for securing commitment; Buss \& Haselton, 2005; Buss et al., 1992) and high standards when assessing an individual's suitability as a long-term partner (Stewart et al., 2000).

The pursuit of a short-term mating strategy provides its own set of reproductive benefits and adaptive problems. For ancestral men, short-term mating could have led to a large fitness boost. That is, for the relatively small cost of courtship and sperm, a man could have impregnated a woman and, providing the child survived, passed on his genes to the next generation without further investment. Pursuing short-term relationships may have also provided men with a way of assessing their mate value in preparation for long-term relationships later on. If a man finds that attractive women are willing to have a short-term relationship with him, then he may learn that he can attract an even more desirable woman for a long-term partnership. (This idea is adapted from Greiling and Buss (2000) where it is presented as a benefit of short-term mating in women. However, there is no reason to assume that this would not apply equally useful to both sexes.)

For women, the benefits of short-term mating are more complex. There are several theories as to why short-term mating would have been beneficial to ancestral women. These include having sex with men for protection from other men, for access to resources (such as food), to potentially "lure" a man of higher genetic quality into a long-term relationship via a short-term one (men lower their standards when it comes to short-term partners), or just to obtain high quality genes for their offspring (Buss \& Schmitt, 1993; Greiling \& Buss, 2000; Meston \& Buss, 2009; Regan \& Dreyer, 1999). These theories are not necessarily in direct competition with one another and short-term mating strategies in women may well have evolved for several reasons.

As with long-term strategies, pursuing a short-term strategy creates another set of adaptive problems to overcome. For example, an ancestral man who sought shortterm mating, but was unable to gauge the likelihood that a woman would grant him sexual access, would have risked wasting his time and reproductive effort pursuing a relationship unsuccessfully. Likewise, a woman who was unable to identify men who would be capable of protecting her from others, may have ended up exchanging sex for very little benefit, risking pregnancy in the process. Again, according to SST, human ancestors adapted to deal with these problems, and this can be seen in the desires and behaviours of modern men and women. When it comes to short-term relationships, men are less likely to be phased by rumours of promiscuity in a potential partner, are displeased by notions of commitment, and are sensitive to cues indicating ease of sexual 
access (Buss \& Schmitt, 1993). Women, in contrast, show typically stronger preferences for facial masculinity (Little, Jones, Penton-Voak, Burt, \& Perrett, 2002) and signals of resource generosity in their partners, compared to men, within a short-term mating context (Buss \& Schmitt, 1993).

In contrast to long-term strategies, there appears to be less of an overlap between the sexes in terms of mutual short-term mating adaptations. However, the similarities which do exist can be underemphasised in the evolutionary psychological literature. For example, in the summative article of Greiling and Buss (2000), the benefits of shortterm mating for women are broken down into various hypotheses, including the mate switching, mate skill-acquisition, and mate manipulation hypothesis (Greiling \& Buss, 2000). However, it would be unwise to assume that men do not also engage in such behaviours. Men sometimes have affairs with women they eventually date and use affairs as a revenge tactic to maintain the interest of their current partner. As previously mentioned, short-term mating may also allow men to understand their mate value.

Although, as a species, humans are typically socially monogamous, or mildly polygynous, there appear to be some cases in which it would benefit both men and women to pursue short-term relationships rather than long-term ones. If there was pressure on our ancestors to remain flexible in their mating strategy, as postulated by SPT (Gangestad \& Simpson, 2000), then this may go some way as to explaining the modern day individual differences found in human mating behaviour.

\section{The Gap in the Literature}

This thesis addresses a specific gap in the evolutionary psychological literature. According to the most recent theories, humans may retain some mating strategy flexibility throughout their life. However, to date, this is an area which has not been examined directly. According to Buss (2002):

Further research is needed on the context-sensitive nature of human mating strategies. Precisely which circumstances might cause a person to shift from a long-term mating strategy to a short-term mating strategy and vice-versa? Which circumstances might trigger an extramarital affair, or conversely, cause someone to forgo an alluring sexual opportunity? (p. 57)

and Gangestad and Simpson (2000) report that 'Conditional strategies are a central topic in behavioral ecology ... However, the possible role of conditional strategies in human mating has received relatively little attention' (p. 578). At time of writing, Strategic Pluralism Theory has been cited more than 660 times according to Scopus, and David Buss' work is even more influential. Yet, while some experiments have demonstrated 
that factors related to a particular strategy (such as mate preferences) can be manipulated (see Chapter 2), no study to date has demonstrated a within-subject change in mating strategy. That is, no researcher has taken an individual, measured their relative preference for, or willingness to engage in, short-term and long-term mating, and then observed if brief interventions can change this. The purpose of this thesis is to fill this gap in the literature. In order to accomplish this, a general working hypothesis is tested: Human mating strategies are activated conditionally and change in historically adaptive ways in response to evolutionarily relevant stimuli.

In this thesis, the working hypothesis is tested using brief experimental interventions on young adult men and women. Such an approach could be criticised for downplaying the role of early development in mating strategies. Indeed, there are some popular models (see below) which stress a period, early in life, in which several environmental factors can affect the development of an individual's mating strategy. However, an experimental format was chosen for two important reasons. First, by using experiments, a larger number of studies could be run in succession. This allowed for an array of different evolutionarily relevant mating factors to be examined. Second, experiments showing within-subject changes in inclination towards long- or short-term mating appear to be non-existent. In contrast, there appears to be a good deal of research on the effect of early life events on mating strategies in the evolutionary and developmental psychological literature, some of which are discussed in this section.

\section{Factors Influencing Strategy Choice in Humans}

In Section II, the mating strategies of several non-human animals were introduced, along with the idea that organisms which pursue mating strategies conditionally possess "switchpoints". That is, there are certain points in which the fitness benefits of one strategy become outweighed by another. Such a point is likely dependent or personal, social, and environmental factors related to the particular animals mating strategy. For example, among scorpion flies (Panorpidae), the point at which a male is expected to switch from the strategy of using a food offering to attract a female, to the strategy of pursuing forced copulation, may depend on the local availability of resources and their number of competitors (Thornhill, 1981). For the African striped mouse (Rhabdomys pumilio), population density seems to be the key factor. A territory guarding strategy is followed by males when the population is highly dense, while a roaming strategy is followed when the population density is low (C. Schradin \& Lindholm, 2011). This relationship is expressed in Figure 1.1 below. Here, 
the red line represents one strategy (a) and the blue line another (b). The fitness pay-off of each strategy changes given the level of $X . X$ is a variable likely to affect the success of each mating strategy, such as resource availability and competitor presence in the scorpion fly, or population density in the African striped mouse. It may well be that $X$ is simple, and relates to a single variable, or that it is more complex and multivariate. The switchpoint (c) represents the point where the average fitness pay-off of one strategy is identical to the other. To the left of this point, (a) should be the preferred strategy, while (b) should be preferred strategy to the right of this point.

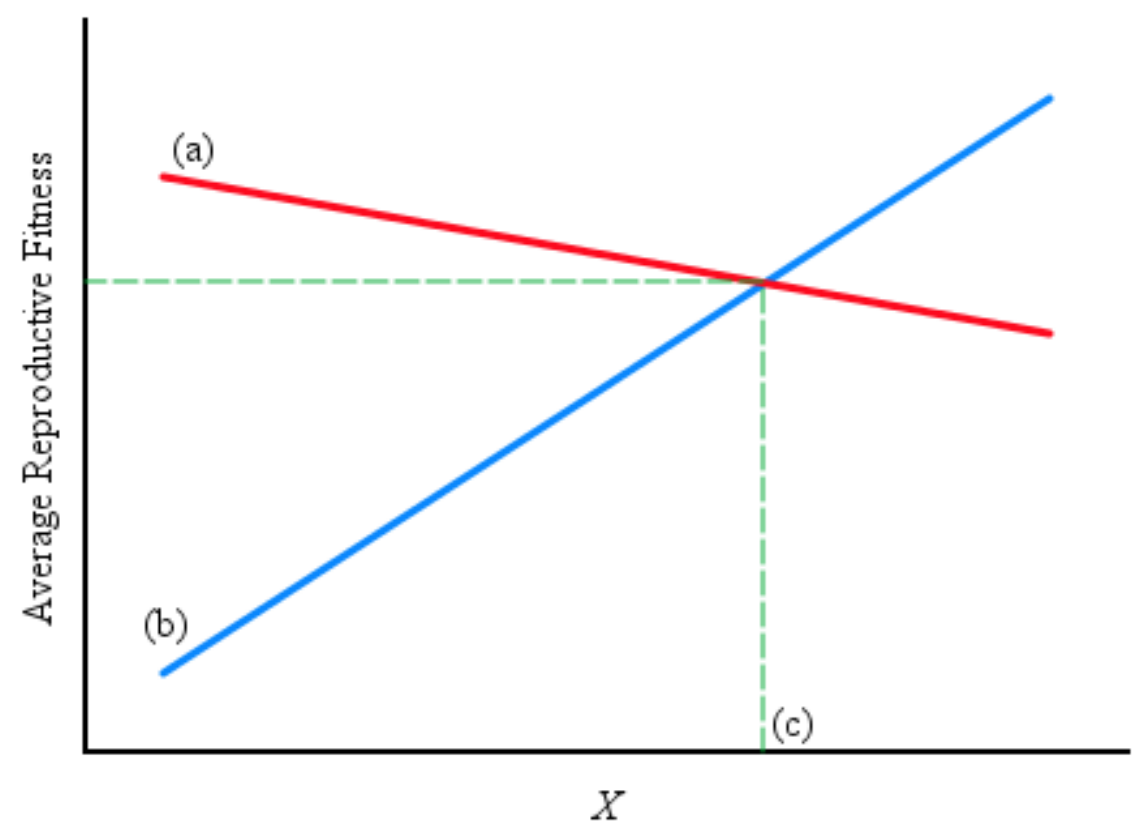

Figure 1.1. A graphical representation of the fitness benefits of following two mating strategies (a) and (b) given hypothetical variable X. A "switchpoint" (c) occurs between two mating strategies at a certain level of $X$. This representation was inspired by M. R. Gross (1996, pp. 95, Box 93), whose paper is one of the key influences behind Strategic Pluralism Theory (Gangestad \& Simpson, 2000).

If men and women have two distinct mating strategies, then, in accordance with SPT, we may expect there to also be a variable $X$ for humans, as well as a strategy switchpoint. Specifically, there should be evolutionarily relevant mating variables, either of a personal, social, or environmental nature, which affect the fitness pay-off of each strategy. We would then expect men and women to follow the strategy which 
provides the largest pay-off, switching from a long- to a short-term one (and vice versa) if necessary. The identification of such variables is crucial to this thesis as, in order to examine whether humans show behavioural plasticity in mating strategies, one must choose relevant variables to manipulate within an experimental setting.

There are several factors, likely to have been relevant in the ancestral environment, which would have affected the relative benefits of each mating strategy for men and women. The next few sub-sections provide a brief overview of the types of variables which are examined in this thesis, as well as how they might be expected to affect mating behaviour.

Environmental harshness. For the purpose of this sub-section, environmental harshness refers to a collection of factors which negatively affect population mortality and yet are outside the control of an individual or their social circle. Examples include drought, floods, predator prevalence, and food availability. Harsh, unpredictable environments tend to foster short-term mating behaviour in multiple animal species (Ridley, 2003), while predictable environments, with low predation threat and abundant resources, tend to foster long-term mating behaviour (Chisholm et al., 1993; Quinlan, 2007). In these latter environments mortality is low, life-span is long, and species tend to focus their reproductive efforts on offspring quality rather than quantity. Such an effect of environment also appears to be present in modern humans. In communities where resources are scarce, or the environment is particularly harsh, humans show a tendency to engage in behaviours associated with short-term mating. These include increased inter-sexual competition and aggression (E. M. Hill, Ross, \& Low, 1997; Kruger, 2010), earlier onset of reproduction (Allison \& Hyde, 2011; Dickins, Johns, \& Chimpan, 2012), riskier sexual behaviour (Rosenbaum, Zenilman, Rose, Wingood, \& DiClemente, 2012), and higher reproductive output (U.S. Census Bureau, 2011).

A great deal of the current research within evolutionary psychology on the effect of such environments on mating behaviour focuses on evolutionary-developmental models that begin in early life. Specifically, such models suggest that "harshness cues" in childhood lead to the development of risky short-term sexual behaviour in adults. For example, the early-stress model of Belsky, Steinberg, and Draper (1991), which differentiates between Type I (short-term) and Type II (long-term) reproductive strategies, states that an individual's strategy choice in adulthood is due to a culmination of several early stage psychosocial factors (such as father absence and poor upbringing) ultimately caused by a harsh environment. This model fits well with traditional life 
history theory (Chisholm et al., 1993; Ridley, 2003) which puts reproductive effort on a continuum between having a large number of offspring with an uncertain reproductive future and having fewer offspring of higher quality who are more likely to survive. In this view, the large variance seen in human mating behaviour is due to environmental input, including the parent-infant relationship, which then sends an individual down a specific developmental path leading to either a long- or short-term mating strategy.

The idea of a "critical period" in which a dangerous environment affects future mating behaviour is a debated one. For example, if mating strategies were entirely due to harshness cues during development (or other mating related cues for that matter), then one would expect there to be very little heritability of such strategies. That is, the relationship would be expected to persist once the mating strategy of the subject's genetic parents, or relatives, was controlled for. There is mixed evidence for this, with some studies claiming a strong heritable component (Pettay, Kruuk, Jokela, \& Lummaa, 2005; Rowe, 2000, 2002) and others claiming a weak or negligible one (Belsky, Houts, \& Fearon, 2010; Hoier, 2003; Quinlan, 2003). Then, there are scholars who believe that, while the model is correct, it only accounts for the presence of short-term mating among the small proportion of individuals who are developmentally challenged (Schmitt, 2005a). That is, not all of those who follow a ST strategy do so due to adaptive reasons. While the discussion of such models may seem like an aside, should they prove to be accurate, they have some real consequences for the working hypothesis of this thesis. If an individual's future mating strategy is crystallised during a developmental point in their past, then it may well be the case that variation in strategy choice is not due to within-lifetime mating flexibility. This is discussed in further detail within Chapter 9.

In a different direction to these evolutionary-developmental models, this thesis tests the effect of harshness cues on the mating behaviour of men and women in early adulthood. Specifically, Chapter 5 focuses on the factor of danger cues, with the idea that, if humans are flexible breeders, then such cues should affect mating strategies in ways predicted by life history theory and the models generated from it (e.g. Chisholm et al., 1993; Quinlan, 2007).

Partner availability. Another factor which can affect the effectiveness of a mating strategy is partner availability through variation in local sex-ratio. If, for example, there is an abundance of females available to a small number of males, then a given strategy (e.g. harem guarding) may well be very effective. However, if there is an abundance of competitor males in a particular area, and very few females, an alternative 
strategy (e.g. sneaking copulations via female mimicry) may be the only way in which some males can reproduce.

The idea that local mating behaviour can be dependent on the availability of each sex is well established in both ecology and evolutionary psychology (Puts, 2010; Shuster \& Wade, 2003). For example, among the Hadza, in communities marked by a skewed sex-ratio in the favour of women, men spend less time gathering resources for their young and more time engaging in mating effort (Marlowe, 1999a). Likewise, there is a strong negative correlation between sex-ratio and national sociosexuality scores; nations with more women than men show more relaxed attitudes towards uncommitted $\operatorname{sex}(r=-0.45 ;$ Schmitt, 2005b). The effect of the perceptions of sex-ratio is the subject of one of the experiments in this thesis found in Chapter 8.

Status and attractiveness. An individual's attractiveness and social standing relative to their peers can have a large impact on the type of mating strategies which they are able to pursue successfully. Among women, high status is seen as a desirable trait in a partner (Li et al., 2002), and so men of status find themselves able to attract higher quality wives and have enhanced lifetime reproductive output (Bereczkei \& Csanaky, 1996; Fieder \& Huber, 2007; Hopcroft, 2006) ${ }^{11}$. Likewise, men who are physically attractive (an attribute especially important to women in a short-term mate; Li \& Kenrick, 2006), report having a higher number of sexual partners (Bogaert \& Fisher, 1995; Rhodes, Simmons, \& Peters, 2005) and have a greater likelihood of being chosen as an extra-marital partner (Gangestad \& Thornhill, 1997). Thus, it appears as if highly attractive men, and indeed men of high status, may be in a better position to successfully pursue short-term mating.

Similar effects can be found among women. Physical attractiveness is highly desired by men in both long- and short-term contexts (Li \& Kenrick, 2006), and so physically attractive women are in a better position to attract more desirable high status partners (Udry \& Eckland, 1984). This is reflected in their high standards for a longterm partner (Buss \& Shackelford, 2008). Thus it appears as if highly attractive women may be in a better position to successfully pursue long-term mating.

\footnotetext{
${ }^{11}$ While the relationship between status and fitness is normally clear in traditional societies, it is harder to demonstrate in modern day urban cultures. This is because the relationship between social status and fertility has changed over time and no longer reflects the association present in our ancestral past. For example, the number of children born into highly educated high-status families is now low due to a selfimposed reduction in reproduction (Goodman, Koupil, \& Lawson, 2012). While not reflected in partner number, men of high status do tend to have sex more often (Hopcroft, 2006).
} 
Two of the experiments in this thesis, found in Chapters 3 and 7, attempt to influence an individual's perceived attractiveness and status relative to their peers. Specifically, the experiments in Chapter 3 use false feedback to convince participants that they are either more or less attractive than their peers, while the experiment in Chapter 7 uses cues of victory or defeat to increase or decrease a participants perceived levels of dominance. If humans are sensitive to their status relative to their peers, then we would expect those who are given positive attractiveness feedback, or cues that they are dominant, to switch to a mating strategy usually reserved for highly attractive and influential individuals within a group. A related experiment, found in Chapter 6, focuses on testosterone (T) levels and mating strategies. Circulating $\mathrm{T}$ levels are found to change following victory and defeat cues (Carré, Campbell, Lozoya, Goetz, \& Welker, In Press; Carré \& Putnam, 2010). Therefore, an increase in circulating T may well have similar effects as an artificial increase in status.

Strategy benefits. The final two variables explored in this thesis involve presenting participants with cues related to the benefits produced by the two mating strategies to see how they respond. In humans, and especially in women, there are many additional benefits associated with long- and short-term mating strategies which lead to enhanced fitness, both directly and indirectly. As explored earlier in this section, one such benefit is that of resource acquisition by women from men (Buss \& Schmitt, 1993; Greiling \& Buss, 2000; Meston \& Buss, 2009). It may well be the case that, if mating strategies are deployed conditionally, the presence of a potential strategy-linked benefit may lead to activation of such a strategy. Thus, women may be expected to shift towards a short-term mating strategy when they receive signals that resources are available to be acquired. This is tested in Chapter 4, where participants are exposed to cues of wealth. Similar cues have been found to increase behaviours related to shortterm mating (such as impulsivity) among women in earlier experiments (M. Wilson \& Daly, 2004).

The same chapter contains an experiment which cues one of the benefits of a long-term mating strategy: bi-parental care of infants. Within the context of a pair-bond, bi-parental care can be beneficial for several reasons (Section III) including offspring survival (Sear \& Mace, 2008) and enhanced development (e.g. Hewlett \& CavalliSforza, 1986). Thus, it may be the case that infant stimuli would lead to the activation of a long-term mating strategy. In other studies, the presence of stimuli related to infants 
has been shown to decrease the circulating testosterone of humans in some cases (see next sub-section). $\mathrm{T}$, in turn, is related to short-term mating (Chapter 6).

\section{Existing Support for the Working Hypothesis}

There are several lines of evidence which suggest that humans change their mating behaviour, throughout their lifetime, in response to input from the environment. Although not all of these relate specifically to short-term and long-term mating strategies, they provide some initial, tentative, support for the working hypothesis.

First, men and women are known to respond differently when asked about preferences across different mating contexts. In this type of task, participants are asked to rate mating characteristics, or approach a mating scenario, first within a short-term context and then within a long-term context (or vice versa). The two contexts are then compared. For example, in a long-term mating context, men and women rate kindness as a highly desirable characteristic in a partner, but this desirability almost halves within a short-term context (Li \& Kenrick, 2006). On average, men find promiscuity much more off-putting in long-term contexts compared to short-term ones, while women place more of a premium on a partners financial prospects when considering them for a long-term relationship (Buss \& Schmitt, 1993). Other examples of such behaviour can be found in the Human Mating Strategies sub-section earlier in the chapter.

Second, the 'pairing game' of Ellis and Kelley (1999) reveals potential shortterm flexibility in an individual's perception of their worth. In the game, several participants are given a random number on their forehead so that the owner is unaware of their own value but can see that of others. The objective for each participant is to then try to form a partnership with another participant, while also attempting to obtain a partner with the highest number possible. Needless to say, participants who have low numbers on their forehead find that they are often rejected when approaching those with high numbers. In contrast, those with high numbers find themselves approached by a large number of individuals who wish to form a partnership with them. After a while, participants form an idea of their approximate value and partner off assortatively (Cronbach's $\alpha \sim 0.80$ ).

A similar effect is found among studies from the sociometer literature. A sociometer (M. R. Leary, Tambor, Terdal, \& Downs, 1995) is a proposed mechanism whereby an individual's self-esteem functions as an indicator of their degree of social inclusion and approval. This indicator is then continuously affected by (or calibrated to) social feedback and experiences. An individual can have several sociometers for 
different social contexts, such as work or friendship (Kirkpatrick \& Ellis, 2004). Kavanagh, Robins, and Ellis (2010) demonstrated the domain specific nature of a "mating" sociometer. Single participants were given what appeared to be positive or negative written feedback about their dateability from three individuals who were average to above average in attractiveness. When these participants were subsequently asked to view high, medium, and low attractiveness dating profiles, those who had received positive feedback were more likely to see themselves as a good fit with high attractiveness potential partners compared to low attractiveness ones. Changes in selfesteem due to feedback almost completely mediated this effect, yet no such pattern was found when participants rated their compatibility with high or low attractiveness samesex models in the context of friendship.

Third, there is evidence that hormonal changes occur in humans and that these are connected with changes in mating behaviour. In men, lower levels of testosterone (T) have been associated with long-term mating strategies in both traditional (Muller et al., 2009) and industrialised (P. B. Gray et al., 2004) societies. Recently, this research has grown to include longitudinal studies which provide compelling evidence that it is not just low $\mathrm{T}$ men who are more likely to become fathers, but that $\mathrm{T}$ levels actually drop as a consequence of fatherhood (Gettler, McDade, Feranil, \& Kuzawa, 2011). Even exposing expectant fathers to parental stimuli (e.g. noises of crying infants or the smell of a new born) can cause a drop in circulating T levels (Storey, Walsh, Quinton, \& Wynne-Edwards, 2000). T has often been seen as a determinant of mating and parental effort allocation in the animal kingdom (Beehner, Bergman, Cheney, Seyfarth, \& Whitten, 2006; Stoehr \& Hill, 2000; Veiga, Salvador, Merino, \& Puerta, 1998) and high activation levels in men are associated with more intra-sexual competition (Carré, Putnam, \& McCormick, 2009; Slatcher, Mehta, \& Josephs, 2011), risk taking behaviour (Ronay \& Hippel, 2010), and polygynous tendencies (McIntyre et al., 2006; M. Peters, Simmons, $\&$ Rhodes, 2008). ${ }^{12}$ There is also evidence that the menstrual cycle can influence mate preferences in women. Women who are in the fertile phase of their cycle are more likely to show a preference for physical attractiveness $(d=0.40$ to 0.80$)$ and masculinity $(d=0.30)$ in men (Gangestad, Thornhill, \& Garver-Apgar, 2010; Provost, Troje, \& Quinsey, 2008; Thornhill \& Gangestad, 2008).

\footnotetext{
${ }^{12}$ It is interesting to note that, for several of these effects, it is not simply testosterone which counts; participants have to also be high in dominance for the effects to reveal themselves.
} 
Fourth, while models such as the early-stress model (Belsky et al., 1991) suggest a critical period whereby certain life events (such as father absence) send an individual down a developmental "path", such changes do not appear to be necessarily permanent or irreversible. As Chisholm et al. (1993) observe on page 10, 'The scientifically and politically essential point is that both theory and evidence suggest that change is possible and intervention can be effective.' Specifically, there are some individuals with insecure parental relationships, or who come from an environmentally harsh background, which can still develop a Type I (long-term) mating style later in life after being exposed to securely attached relationships with loving and supporting partners.

Fifth, there is a growing body of evidence which suggests that mate preferences can be biased in evolutionarily relevant ways. These findings tend to be centred on forced-choice preference tasks where participants choose a preferred partner from pairs of photographs that have had their masculinity or femininity manipulated. For example, when women are primed with "danger cues" (Little, Cohen, Jones, \& Belsky, 2007), indicating an environment where short-term mating may be more beneficial than longterm mating, they are more likely to pick the most masculine face of each pair. Masculinity is considered an indicator of good genes, and obtaining these for offspring is one hypothesised benefit of short-term mating in women (Buss \& Schmitt, 1993; Provost, Kormos, Kosakoski, \& Quinsey, 2006; Scheib, Gangestad, \& Thornhill, 1999). There are several studies which similarly show that mate preferences can be biased, including those by Little et al. (2002), D. Bailey, Durante, and Geary (2011), and Welling et al. (2007). However, such studies do not directly assess changes to preferences for long-term over short-term relationships and vice versa. The demonstration of a change in an attribute (such as desire for physical attractiveness or masculinity) which forms a small part of a particular mating strategy does not necessarily mean a strategy change has occurred.

Finally, there are some miscellaneous pieces of evidence which hint that mating behaviour can change in adulthood following certain experiences. Holmberg (1950) wrote of his involvement with a man from the Sirionó hunter-gatherers who had lost his wife, and his social standing, due to his poor hunting ability. After being taught how to use a shotgun, which artificially enhanced his hunting skills, the man ended up with a boost of both social status and sexual access within his group (see Chapter 7). Likewise, it is not uncommon for individuals who have experienced negative events in relationships to have these affect their approach to subsequent relationships. Someone 
who has suffered infidelity from a previous partner may be more sensitive to signs of cheating behaviour in subsequent relationships than they would have previously been (Burchell \& Ward, 2011). Within an experimental context, the perceived attractiveness of man by women can be influenced by the type of car they drive, or apartment they live in - factors likely to positively change within an individual's lifetime (Dunn \& Hill, 2014; Dunn \& Searle, 2010). Attractiveness, as previously discussed, has an impact on the types of mating strategies an individual can successfully pursue. Furthermore, data from social psychology reveals that, following a divorce, the attributes people choose in their second spouse can be markedly different from their first (Gelissen, 2004).

\section{Conclusion}

Humans are thought to possess two rather distinct mating strategies, one longterm and one short-term in nature. Recent evolutionary psychological theory, as informed by research on mating strategies in other animal species, suggests that these are employed conditionally depending on environmental circumstances. While there are a few lines of evidence which suggest that humans may well be capable of changing their mating strategy throughout their lifetime, this has yet to be formally demonstrated in a controlled experimental setting. In this final section, some factors were introduced which are thought to affect the effectiveness of each mating strategy in humans. These factors form the variables of interest within the experimental chapters of this thesis. Before presenting the first of these, however, the next chapter is devoted to the primary dependent measure of mating strategies which was developed for use in this thesis: the SMA task. 
Chapter 2: Snog, Marry, Avoid (SMA) - A Novel Tool for Measuring Mating Strategies

In order to test the overall working hypothesis, an appropriate method for measuring mating strategy was required. The experiments conducted in this thesis all followed a similar format. A baseline measure of a participant's tendency towards longand short-term mating was recorded, then an evolutionarily relevant cue or manipulation was introduced, and finally a second measure of mating strategy was taken. The effects of the manipulation were then established by analysing changes in mating strategies measurement between these two time points.

Such a measure needed to have two important attributes. First, it needed to be able to distinguish between a participant's inclination towards long- and short-term mating as separate constructs in acknowledgement of Sexual Strategies Theory and Strategic Pluralism Theory (Buss \& Schmitt, 1993; Gangestad \& Simpson, 2000). Second, it needed to be sensitive to change over a narrow temporal resolution given the design of the experiments. This chapter discusses some of the current measures of mating strategy available within the psychological literature, and explores why these were found unsuitable for use as a primary dependent variable in this thesis. These measures fall into two broad categories: pen and paper questionnaires and computerbased behavioural tasks. Following this discussion, a novel paradigm (the SMA task) is introduced which was designed to overcome some of the limitations of these existing measures. Data from a pilot study which used the SMA as a dependent variable is then introduced as well as the statistical techniques best suited to analyse the data it produces.

\section{Questionnaire Measures}

One of the most frequently utilised measures of mating strategy within the evolutionary psychological literature record a participant's sociosexuality (SO). The term sociosexuality was originally coined by the Kinsey lab to describe individual differences in willingness to engage in sex outside of a committed relationship (Kinsey, Pomeroy, \& Martin, 1948). The socio-sexual orientation inventory (SOI; Appendix A) was developed in the late 1980s and published in 1991 (J. A. Simpson \& Gangestad, 1991). Since its inception, the paper introducing the SOI has been cited over 800 times, and the measure itself has been used in some prominent high impact studies testing thousands of participants from dozens of nations (Lippa, 2009; Schmitt, 2005b). The original SOI questionnaire consisted of seven items used to measure an individual's 
previous sexual experience and their attitude towards uncommitted sex. This measure was found to be high in both construct validity and test-retest reliability during pilot studies (J. A. Simpson \& Gangestad, 1989, 1991). There have been some notable research findings from studies utilising the SOI. For example, males with higher (i.e. unrestricted) SO scores were found to be more likely to derogate competitors to impress women, mediating a relationship between competitiveness and fluctuating asymmetry (J. A. Simpson, Gangestad, Christensen, \& Leck, 1999). In another example, high SO individuals were found to be identifiable by high levels of facial attractiveness (in the case of women) and facial masculinity (in the case of men), with women showing a tendency to avoid the latter as mating partners (Boothroyd, Jones, Burt, DeBruine, \& Perrett, 2008). In an international study, the prevalence of pathogens in a local area correlated positively with SOI (Schaller \& Murray, 2008) in a manner consistent with life history theory (see Chapter 5). Also consistent with life history theory was the finding that students with parents who divorced when they were children showed reduced helping attitudes, increased Machiavellianism, and, importantly, higher SOI scores (Barber, 1998).

In 2007, a modified version of the SOI, the Socio-sexual Orientation Inventory Revised (SOI-R; Appendix B) was developed by Penke and Asendorpf (2008) and published in the Journal of Personality and Social Psychology. Improvements to the original measure included: a) the inclusion of three distinct sub-components of sociosexuality (behaviour, attitude, and desire); b) the introduction of a closed-ended scale for the behavioural questions to address the skewed nature of responses when open-ended questions were used; and c) greater validity and reliability checks. Due to these improvements, the SOI-R has been favoured over its predecessor in recent evolutionary psychological research (Lewis, Easton, Goetz, \& Buss, 2012; McDonald, Donnellan, \& Navarrete, 2012; Swami, Miller, Furnham, Penke, \& Tovée, 2008).

Although a measure of SO was a good candidate for the main dependent variable for the experiments, there were two concerns which led to it being judged as unsuitable. First, SO measures have very good test-retest reliability $(r=0.94 ;$ J. A. Simpson \& Gangestad, 1991). While this is usually a desirable trait in a questionnaire, a closer look at the SOI reveals that the source of this reliability may be due to the specific nature of the questions asked. For example, some of the questions are based on past, concrete, behaviours (e.g. 'With how many different partners have you had sex on one and only one occasion?'). Likewise, as a brief nine item pen and paper measure, 
questions on the SOI-R may be more likely to be influenced by the memory of previous responses. When these points were considered, along with the experimental nature of the intended studies, whereby repeated measures were likely to be taken within the same experimental session, the SOI and SOI-R appeared to lack the sensitivity needed in order to detect change in mating strategy over short durations.

Second, sociosexuality is a continuous variable in which low scorers are interpreted as following a long-term mating strategy, while high scorers are interpreted as following a short-term mating strategy. For example in A. P. Clark (2006, p. 1322), the difference between high and low SOI scorers is described dichotomously: 'high SOI scores indicate that the respondent is pursuing short-term mating... and low SOI scores indicate that the respondent is pursuing long-term mating.' In Boothroyd et al. (2008, p. 211), low SOI scorers are referred to as "restricted' individuals (i.e., people who prefer long-term relationships)', and high SOI scorers as ' unrestricted' individuals (i.e., people who are open to short-term relationships)'. Finally, in a study co-authored by David Buss, one of the researchers behind SST (Buss \& Schmitt, 1993), the relationship between SO and mating strategy is described as follows: 'High scores on the sociosexuality inventory (SOI) indicate pursuit of a short-term mating strategy; low scores indicate pursuit of a long-term mating strategy' (Haselton, Buss, Oubaid, \& Angleitner, 2005, p. 13). However, when the specific questions of the SO measures are explored, it is clear that they relate solely to the presence of short-term mating behaviour and attitudes. Does the presence of short-term mating inclination imply an absence of long-term mating desire? It appears to be assumed so. However, while the two types of strategy may necessarily trade-off (Buss \& Schmitt, 1993), how and to what extent seems to be a neglected area in the psychological literature, at least as far as measurement design is concerned. Unless the SO measures are modified, with questions capturing propensity towards long-term mating (such as 'I am the type of person who enjoys falling in love') this question may remain unanswered. ${ }^{13}$ Ultimately the SOI and the SOI-R were deemed unsuitable as dependent measures for the experiments in this thesis due to the fact that they were unable to measure a participant's desire for both short- and long-term relationships successfully.

\footnotetext{
${ }^{13}$ Jackson and Kirkpatrick (2007) have attempted to do this with a revised questionnaire. While the original SOI paper (J. A. Simpson \& Gangestad, 1991) did show some negative correlations between selfreported long-term behaviours and SOI, the revised questionnaire makes a strong case for short- and longterm strategies being statistically independent constructs. This does not, however, overcome the problems that a pen and paper measure of mating strategy presents when multiple recordings are taken within a short time frame.
} 
A second potential measure of mating strategy considered was self-perceived mating success (SPMS; Landolt, Lalumière, \& Quinsey, 1995). The SPMS consists of 8 items related to interactions with members of the opposite sex with which participants rated their agreement. These included 'Members of the opposite sex notice me' and 'I receive sexual invitations from members of the opposite sex'. Although valid and used in several notable studies (A. P. Clark, 2006; Goodwin et al., 2012; Lalumière \& Quinsey, 1996; Massar, Buunk, \& Rempt, 2012), this measure shares similar limitations as the sociosexuality ones. Namely, it is a pen and paper measure based mainly on past experience. Likewise, an individual's SPMS score may not allow for the prediction of a participant's mating strategy. For example, would a participant who considered their mate value to be high show a greater desire for short- rather than long-term mating? Perhaps if they were male but not female. Perhaps if they were young but not old. Perhaps if they were high in status but not low. In fact, Landolt et al. (1995) asked participants to make a forced choice about whether they would like to engage in shortor long-term relationships with a series of individuals (an idea returned to shortly). They found that the SPMS was only predictive of short-term mating preferences, and that this was found only in men - a result which has since been replicated (A. P. Clark, 2006).

Finally, there are two questionnaires in circulation which focus on the r-K selection spectrum. (K- and r-selection are discussed in further detail within Chapter 5). These are the Mini-K by Figueredo et al. (2006) and the High-K Strategy Scale (HKSS) by Giosan (2006). Unlike SO measures, which are solely based around sexual behaviour, the mini-K and HKSS also include questions about the individual's environment. The Mini-K for example, has 20 items, responded to using a -3 to +3 agreement scale, including 'I am often in social contact with my blood relatives', 'I often make plans in advance', and 'While growing up, I had a close and warm relationship with my biological father'. Likewise, the HKSS has 26 items answered using a 5-point likert scale including 'If something bad happened to me, I'd have many friends ready to help me', 'The neighbourhood where I live is safe', and 'If I wanted to, it would be easy for me to find and go on a new date'. Although these two measures have been employed in an array of evolutionary and developmental psychological studies (e.g. Giosan, 2013; Jonason, Koenig, \& Tost, 2010; McDonald et al., 2012), they too fell short in terms of suitability for experimental research. Much like the SO and SPMS questionnaires, some of the questions in the life history measures are anchored to past experiences and behaviours, although the larger battery of questions 
could make it hard for participants to respond during the second measurement using the memory of their previous answers alone. Finally, although the r-K spectrum is useful in terms of describing the typical mating system at a species level, these measures again neglect the conditional nature of human mating.

\section{Behavioural Measures}

After finding the available questionnaires unsuitable as dependent measures, behavioural measures were considered for their suitability. There are several tasks which, either modified or in their current form, could act as a mating strategies measure. One study by Confer et al. (2010) employed a quick experimental procedure whereby participants were shown two boxes presented vertically on a screen. One covered the head of a model while the other covered the body. Under both short- and long-term contexts, participants were asked which box they would like to uncover in order to assess if the person was a suitable dating partner. It was found that while women did not differ between conditions in which boxes they chose, men chose to reveal the body of potential dates more often than their heads in the short-term context. The authors suggest that this is because one of the adaptive problems historically faced by men pursuing a short-term strategy was that of identifying women who were currently fertile, and that this is more closely related to attributes about the body (such as waist-to-hip ratio) rather than the face (Confer et al., 2010, p. 349). This interpretation is in line with Sexual Strategies Theory (Buss \& Schmitt, 1993). Although this research primarily revealed a sex difference, such a paradigm could have potentially been modified to detect differences between individuals within the sexes as well as between them.

Another forced-choice style task comes from the various studies of the Little lab (Little, Burt, Penton-Voak, \& Perrett, 2001; Little, Cohen, et al., 2007; Little, DeBruine, \& Jones, 2011; Little et al., 2002). This task has participants pick the most attractive face out of a series of opposite sex pairs. In each pair the pictures are identical apart from the fact that they have had their masculinity altered by a computer program so that one appears more masculine than the other. Female participants who follow a short-term strategy tend to choose the most masculine face of each pair, while males following a short-term strategy tend to choose the more feminine face of each pair.

While these two paradigms (or modified versions thereof) could have overcome the problems of pen and paper measures, they too were considered not entirely suitable for the current research. First, they are tightly controlled and choices are forced. For example, there is no opportunity for a participant to say 'I find neither of these 
individuals attractive' when considering "masculine" vs. "very masculine" faces. As a result of this, small findings may become overly inflated. ${ }^{14}$ Second, they do not measure mating strategy directly but instead measure related behaviours which are hypothesised to vary with mating strategy (see Chapter 1; Section IV). Rather than seeing if participants show a preference for short-term relationships rather than long-term ones, they investigate a trait which is associated with a particular strategy (such as masculinity preference) and assume that changes to this trait reflect a strategy change. It was ultimately decided that these forced-choice paradigms were not suitable for research into mating strategy flexibility.

Aside from forced choice paradigms, there are also measures available which rely on a participant's reaction to stimuli to infer their attitudes and beliefs. Dwell time has been favoured by the Quinsey lab to uncover the true desires of men in non-clinical and clinical (e.g. sexually deviant) samples (Krupp, 2008; Quinsey, Ketsetzis, Earls, \& Karamanoukian, 1996). These measures involve giving participants control of how long an image is displayed on a screen as part of a slideshow (by holding down a button) before moving onto the next one. The length of time an individual spends looking at a photograph (their 'dwell time') is thought to be indicative of their preference towards it. A second paradigm, the Implicit Association Test (IAT; Greenwald, McGhee, \& Schwartz, 1998), involves participants responding to words or images on a screen with "negative" or "positive" keys. After a training period, participants are told to give a specific answer. For example, they are told to hit the negative key when they see pictures of black men or women and then, later on in the experiment, are told to use the positive key when responding to the same images. Faster response times indicate greater congruency between the target and the meaning of the key pressed. So in this instance, faster responding when asked to use the negative key in response to pictures of black people, compared to the positive key, may indicate that the person has an underlying racial bias against black individuals.

The difficulty with using these paradigms surrounds the development of appropriate stimuli. In Quinsey-like experiments, two sets of visual cues would be required which independently represented short- and long-term mating, and this task seemed unfeasible. For example, an image of sexy lingerie does not fit neatly into the

\footnotetext{
${ }^{14}$ In the Confer et al. (2010) study for example, a medium effect size of $\varphi=0.27$ was found ( $\varphi$ is an approximation of $r)$. Similarly, a medium effect size is found $\left(\eta^{2}{ }_{p}=0.07\right)$ in Little et al. (2011). This suggests that effects found within this thesis may too be medium or small-to-medium in size.
} 
category of short-term mating given that married women may also wear them. This could have potentially been overcome by increasing the complexity of the images used to include several short-term related attributes. However, given that the primary measure involves dwell time, this complexity may have added unnecessary noise to the data. ${ }^{15}$ With an IAT style task, words can be used instead of pictures. However, a list of target words, unique to each strategy, would have been required. Although there are clear differences in which attributes are desirable in long- and short-term partners (Buss \& Schmitt, 1993), this difference is a matter of degree. Most attributes which are desirable in one mating context are also desirable in the other, although to a greater or lesser extent. For example, being physically attractive may be more important in a short-term context, but it is also important in a long-term one (Li \& Kenrick, 2006). Given these points, these two types of measures were also deemed unsuitable to record mating strategy in the experiments.

Given the lack of suitable measures available, a novel task was created in order to measure mating strategy. The task was designed with the four following criteria in mind: a) it needed to address the potentially independent nature of short-term and longterm mating strategies; b) it needed to experimentally suitable by being able to be repeatedly administered within short spaces of time while maintaining the capacity to detect changes due to bias/manipulation; c) it needed to reflect changes in inclination towards short- and long-term relationships, rather than their associated factors (e.g. preferences for facial symmetry); and d) it needed to allow participants to "opt-out" of mating decisions, thus reducing the inflated effect size caused by forced choice paradigms.

\section{An Alternative Measure - The SMA Task}

In 2008, the BBC launched a television program called 'Snog Marry Avoid?' In the show, individuals with unconventional fashion styles are invited to take part in a "make-under". To prove to these individuals that their current appearance is not as attractive as they believe it to be, POD (the Personal Overhaul Device), one of the hosts of the show, reveals to the make-under candidate a video reel of the reactions of opposite sex members of the public to a photo of them. The public are asked a simple question: 'Would you snog, marry, or avoid this person?' Generally, before the make-

\footnotetext{
${ }^{15}$ A pilot study, not included in this thesis, was conducted to see if individuals of high and low sociosexuality differed in how long they dwelled on pictures of infants, but the data were exceptionally noisy and did not yield any significant results.
} 
under 'avoid' is the main choice, with the occasional 'snog', much to the surprise of the candidate. Following their make-under, a second reel is shown where most members of the public would 'snog' or 'marry' the candidate, who then promises to re-evaluate their lifestyle. It was this show which inspired a novel task for measuring mating strategies in this thesis. When asking the question 'Snog, marry, or avoid?' what is really being asked is the question: 'Is this individual worth pursuing as a short- or long-term partner, or should they be avoided entirely?

The SMA task begins with the participant being shown an array of opposite sex suitors or models. One by one the participant is asked which type of relationship they would prefer to have with each model. The choices are: a) a short-term fling; b) a longterm thing; or c) not interested. As participants who were already in a committed relationship may have approached the task with their current relationship status in mind, participants were given a scenario in which to imagine. Before making their relationship choices, participants were encouraged to a) imagine that they were single and open to a new relationship, and b) imagine that the models were known to them, having met them once or twice through friends. This second addition to the scenario was used to account for the fact that generally men and women show some reluctance to consider a relationship with a total stranger (Buss \& Schmitt, 1993, p. 211; R. D. Clark \& Hatfield, 1989; Hald \& Høgh-Olesen, 2010).

The SMA task produces three numbers; the number of long-term choices made (LT), the number of short-term choices made (ST), and the number of models not chosen for either type of relationship (NI). The first two figures are taken as an indication of the participant's disposition towards long- and short-term mating strategies respectively. This paradigm is somewhat similar to that used by Landolt et al. (1995). However, instead of forcing participants to choose between long-term and short-term relationships for each model, they were given an opt-out option of 'not interested'. Thus, it was more likely that the ST or LT selections were actually due to participant's desire rather than them being forced to choose an option.

To allow for validation of this paradigm, a pilot study was conducted in which the SMA task was used to measure relationship preferences before and after exposure to attractive and unattractive pictures of the opposite sex. It was expected that the introduction of these new stimuli would cause participants to re-evaluate the models they had previously seen. This is broadly known as a contrast effect, and is a very simple aspect of psychology found in many sub-disciplines including the visual 
(Gibson, 1933; Suzuki \& Cavanagh, 1998) and behavioural sciences (Bower, 1961). In the specific case of relationship research, showing images of unattractive and attractive same-sex individuals can increase or decrease participant self-esteem respectively (Thornton \& Moore, 1993), and a similar study has shown that women exposed to images of same-sex peers with "supermodel" physiques show increased physique anxiety and body dissatisfaction (Thornton \& Maurice, 1997).

\section{Experiment 0}

The aim of Experiment 0 was to test if the SMA was able to detect subtle changes in mating preference. If this were the case then support for a hypothesis based on the well-established contrast effect should have been found. Hypothesis 0 stated that exposure to attractive individuals would cause participants to lower their willingness to engage in relationships with average attractiveness individuals. In contrast, an increase in willingness to engage in relationships would follow exposure to unattractive individuals. Two predictions were developed to test the hypothesis. Prediction 1 was that, following exposure to attractive individuals, participants would choose fewer average attractiveness models for ST and LT relationships on the SMA task compared to baseline. Prediction 2 was that the opposite pattern of results would be found after participants were exposed to unattractive individuals.

\section{Method}

\section{Participants}

Seventy-eight participants (40 men and 38 women) were recruited from Swansea University to participate in the pilot study. The majority of participants were heterosexual $(n=74)$, with four participants describing themselves as bisexual. Half of the participants were in a committed relationship $(n=39)$, while the others were either single or in an uncommitted relationship. The average age of the participants was 20.79 $(S D=2.06)$ and they were all childless. The average SOI-R was $46.67(S D=10.35)$ for men and $29.70(S D=13.18)$ for women, and this sex difference was significant, $t(71)=$ 6.105, $p<0.01, d=1.45$. Participants considered themselves to be about average in attractiveness $(M=5.36, S D=1.41)$.

\section{Materials}

SMA task. The SMA task was coded using Visual Basic 6. The program was designed to take images from a designated stimuli folder, randomize their order, and display them one at a time in the centre of the screen. Under each picture were three buttons which the participant used to respond. These were ordered horizontally, and 
marked as 'short-term fling', 'nothing at all' and 'long-term thing' in that order. The display timing (starting from a blank screen) for each trial was as follows: 1) the image to be rated appeared in the centre of the screen; 2) after a two second pause the three response buttons appeared underneath the image; 3) after a response was given, both the image and the buttons disappeared; 4) a pause of $500 \mathrm{~ms}$ occurred before the next trial began. This process continued until all images in the stimuli folder had been displayed.

Instruction pages preceded the task in order to explain its format to the participants, define the different relationship choices, and encourage them to respond as if they were in a particular social context. The social context was as follows; participants were asked to imagine that: a) they were single; b) they were open to a new relationship; and c) the individual they were rating was known to them and not a stranger. This was to control for the potentially confounding variables of relationship status and reluctance to engage in a relationship with an unknown individual (e.g. Hald \& Høgh-Olesen, 2010). The task timeline and specific wording of the instructions can be found in Appendix C. Unless otherwise specified the SMA task always showed the participants opposite-sex models.

Attractiveness bias task. This task was completed between the two performances of the SMA and exposed participants to an array of either attractive or unattractive individuals. The task followed the same pattern as the SMA in terms of function and timing. However, instead of buttons being displayed, participants were shown a semantic differential scale between one and ten, and were instructed to rate each individual for their physical attractiveness. Instructions which preceded this rating task can be found in Appendix D. Participants viewed 30 attractive or unattractive opposite-sex individuals in total, and their responses were recorded.

Questionnaires and forms. A demographic questionnaire was included (Appendix E) which collected basic information from the participant including age, sex, relationship status, and self-perceived attractiveness. A copy of the SOI-R (Penke \& Asendorpf, 2008) was also included with this demographic form. The consent and debrief form used in this experiment can be found in Appendices F and G respectively.

Stimuli. Models for this study were gathered from the website hotornot.com. This website allows users to upload pictures of themselves for the public to view and rate for attractiveness (using a scale from one to ten). The users then receive an average attractiveness rating for their picture, as well as number of people who contributed to this figure. After rating a picture, members of the public are able to see this same 
information in a summary panel, presumably to allow them to compare their rating behaviour to the average. The users of the hotornot.com have willingly consented to have their picture viewed and rated by members of the public, so the use of their photographs within this study was considered ethical by the departmental ethics committee. In order to extract information about each picture from the hotornot website a rating of five was selected for each picture viewed by the experimenter.

Female models. A total of 194 images were taken from hotornot.com. Each picture was rated by at least 250 members of the public. To qualify for inclusion in the stimuli set, the pictures had to: a) be unedited (e.g. no enhancement or grey-scale effects); b) contain no indicators of country of origin (e.g. plug sockets on a wall, country flags, or car license plates); c) show the model's face clear from objects (e.g. sun glasses, costumes); and d) not be overly revealing (e.g. wearing bikinis or topless images). The average attractiveness of these images was $8.36(S D=1.23)$ out of 10 . From this pool of images, the 30 with the highest rating were used to create an "attractive" set, the 30 with the lowest rating were used to create an "unattractive" set, and a random sample of 50 images from the middle of the distribution were used in the SMA task. The average attractiveness rating for the images (derived from hotornot.com) in each set was $9.73(S D=0.13), 6.28(S D=0.74)$, and $8.57(S D=0.46)$ respectively. As there was no overlap between the distributions (e.g. all members of the high attractiveness set had a higher score than every member of the average attractiveness set), no tests of differences between the groups were performed.

Male models. A similar process was used to gather male images. The average attractiveness rating of the pool of 188 images was $8.92(S D=0.62)$. From these, three sets of models were formed. The average attractiveness ratings were: $9.72(S D=0.14)$ for the attractive set, $7.99(S D=0.30)$ for the unattractive set, and $8.99(S D=0.23)$ for the set from the middle of the distribution.

\section{Procedure}

Participants were recruited through local advertising within the university to participate in a study of 'attractiveness and relationship choice'. Participants started by reading the combined information sheet and consent form (Appendix F) and signing it. They were then given a copy of the demographic and SOI-R forms, which they completed in private. Once these were completed, the forms were sealed into a plain brown envelope with a random participant code written on the outside. This participant code was used in order to link the results of the SMA task with the participant's 
questionnaires in an anonymous manner. The same code was entered into the SMA task interface, and was stored in each participant's individual results file. Following the questionnaires, participants completed the SMA task twice, with exposure to either attractive or unattractive models in between. The programs were automated so that they passed from one to the other without experimenter intervention, and so the participant was left to complete the task in private. Upon completion, each participant was given a full verbal and written debriefing (Appendix G). The whole experiment took approximately 20 minutes to complete.

\section{Results}

\section{SMA Data and Chosen Analysis}

The most commonly selected relationship was 'not interested' $(M d n=31)$, followed by 'short-term fling' $(M d n=11)$, and then 'long-term thing' $(M d n=4.5)$. There was a significant difference between men and women in their number of shortterm relationship choices (Men: $M d n=13.5$, Women: $M d n=7 ; Z=-3.61, p<0.01$ ) but not their long-term relationship choices (Men: $M d n=4$, Women: $M d n=5.5 ; Z=-$ $0.517, p=0.61)$. Most participants, irrespective of sex, were both picky and selected a mixture of short- and long-term relationships.

The data produced for the SMA task did not appear suitable for parametric analysis. Figure 2.1 shows the number of models chosen during the first performance of the task for both long- and short-term relationships respectively. These distributions did not meet the assumptions of normality and this could not be rectified using statistical transformations (e.g. Log 10). Furthermore, the residuals from subtracting baseline ST and LT choices from those found post-manipulation were also not parametric in distribution (see Figure 2.2), showing marked kurtosis. While this could be addressed for differences in ST selection scores using kurtosis transformations, this was not possible for the LT differences. Thus, while it would have been desirable to use parametric tests to analyse the data, non-parametric analyses were used instead. 

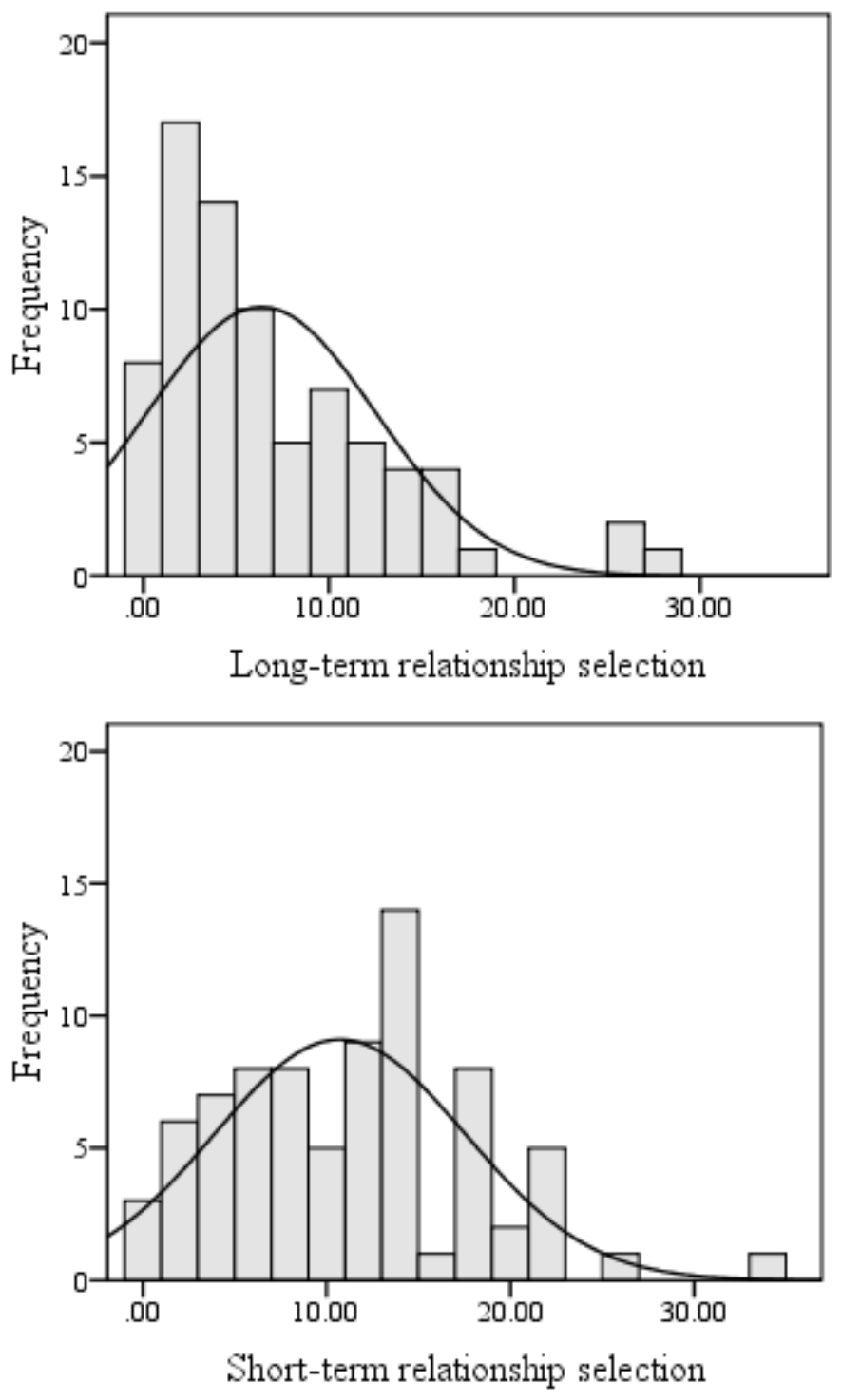

Figure 2.1. The frequency of long- and short-term relationship choices during the first completion of the SMA task. The distribution was positively skewed and showed a floor effect. There was, however, a good amount of variance in the data. 

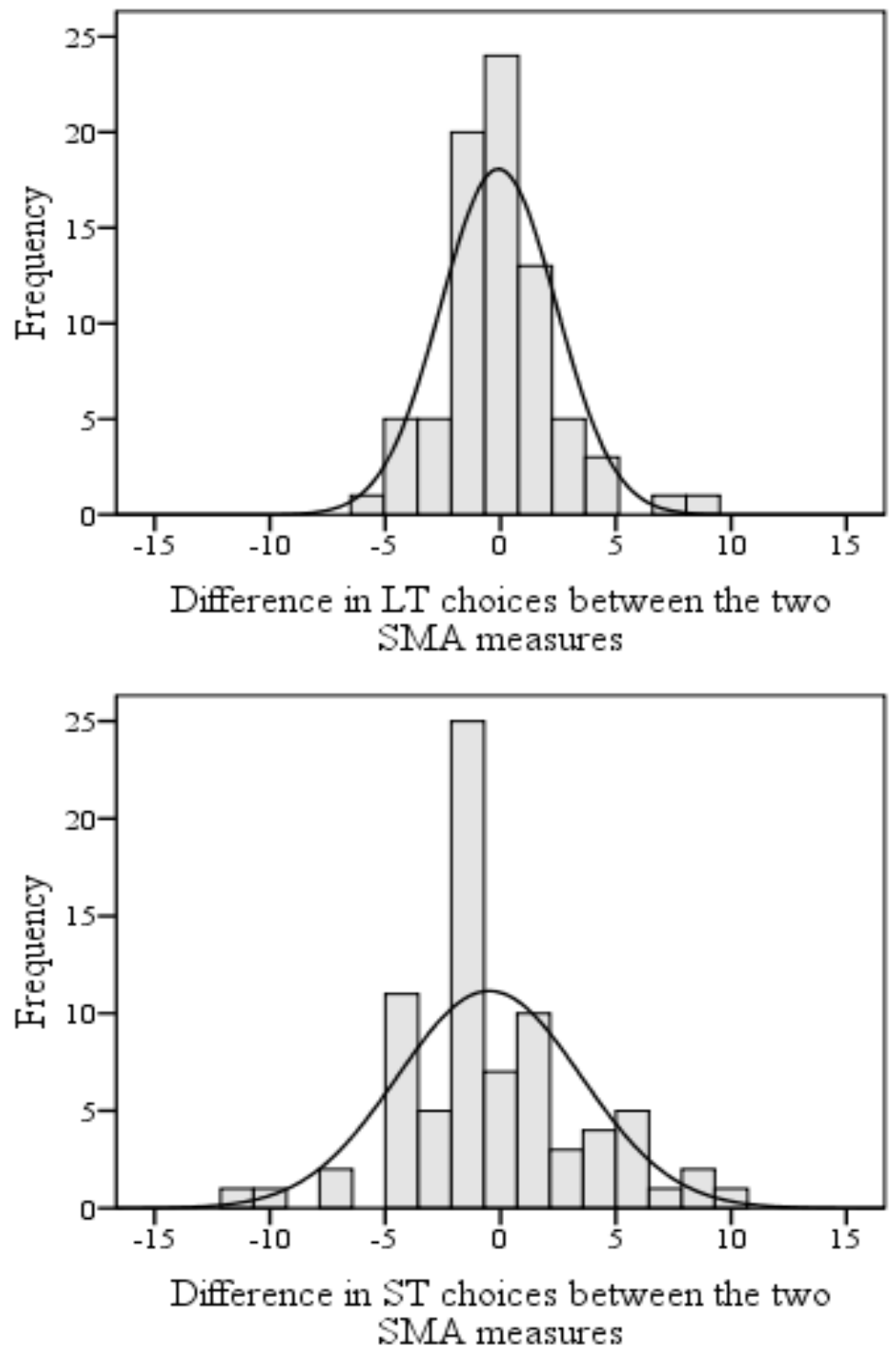

Figure 2.2. The frequency of difference scores between the first and second performance of the SMA task for long-term (LT) and short-term (ST) relationships respectively. Participants were shown a slideshow of either unattractive or attractive faces between performances. The differences in LT choices showed a marked degree of kurtosis which could not be rectified using data transformations.

Non-parametric analysis. There were several options available for nonparametric analysis of the data. For example, a simple Wilcoxon signed-rank $(W)$ test could have acted as a substitute for a parametric $t$-test. However, if $W$ had been used as a primary test, then it would have required a separate test to be conducted for each variable of interest, and doing so would have inflated the chances of making a Type I error. While a Bonferroni correction could have been applied, it was decided that an 
alternative analysis, which could provide the same answers but with fewer tests, was preferable. This was especially the case given the number of factors which could have had an impact on the results, such as reproductive value (Pawłowski \& Dunbar, 1999), sex (Buss \& Schmitt, 1993), perceived attractiveness (Little et al., 2001; Singh, 2004), and social status (Snyder, Kirkpatrick, \& Barrett, 2008). To be able to account for these influences simultaneously, it was decided that a non-parametric equivalent to regression would be used.

Binary logistic regression was entertained as one option. Whereas with linear regression variables are used to try to predict an interval or ratio outcome variable (e.g. income $=2000+\mathrm{IQ} * 200+\varepsilon$ ), binary regression uses variables to predict a simple yes or no answer. Thus $\beta$-values reflect an increased (or decreased) probability of appearing in the "yes" category rather than the "no" category. This is used quite often in the epidemiological literature (e.g. Bardwell et al., 2006; Freeman, Nkomo, Kafaar, \& Kelly, 2008; Muhlhauser et al., 1996). For example, smoking, red meat consumption, and sunbed use may all produce significantly positive $\beta$ 's indicating that they increase the chance that a participant will fall into the yes category for "develops cancer before age 40 " compared to the no category. The theory behind binary logistic regression is quite complex, and involves logarithmic transformations of probabilities to allow $\beta$ weights to be expressed using positive and negative values which are outside of the range of 0 and 1 (Field, 2009). Further complicating the matter is the fact that $\beta$-values are interpreted as odds. So whereas the interpretation of a standard linear regression may be written as "for every one point increase in IQ, predicted income grows by $£ 200$ ", the interpretation of a binary logistic regression is written in a manner such as "for every one point increase in $X$, the odds of appearing in the yes category are increased by $150 \%$ (or $2.5: 1$ )".

The data produced by the SMA task could have been made suitable for binary logistic regression by creating a dummy variable. This variable would have been coded based on whether ST or LT selections increased following the manipulation. For example, those who increased in LT selections would have been coded as one and those who decreased would have been coded as zero. This would have also allowed for the inclusion of covariates during analysis. However, as can be seen in Figure 2.2, there are several individuals who did not change their responses at all. If a binary logistic regression was employed, these participants would either need to be ignored or placed in one of the two outcome categories. For this reason, a binary logistic regression was 
deemed unsuitable. Instead, an ordinal logistic regression, which can support dummy variables with more than two outcomes, was chosen.

With ordinal logistic regression, the prediction shifts from a yes or no categorical one (as with binary regression), to one which decides between several categories which are in an ordered fashion but without necessarily equal intervals between each (Kleinbaum, Klein, \& Pryor, 2010, pp. 463-488). For example, if there were three categories (A, B, and C), conceptually what an ordinal regression does is conduct two binary logistic regressions using variables to first predict the chances of appearing in either A or B, and then to predict the chances of appearing in B or C. These models are then averaged to produce one which will predict the chances of a subject appearing in higher categories, based on the variables entered, no matter where the groups are split (i.e. A compared to $\mathrm{B}+\mathrm{C}$ will be the same as $\mathrm{A}+\mathrm{B}$ compared to $\mathrm{C}$ ). To validate this model a 'Test of Parallel Lines' is performed which ensures that the individual A-B and B-C models are not significantly different from one another. To use an applied example, a dataset could have three categories of contraception use among teenagers: "always", “occasionally" and "never". If a positive $\beta$-value for weekly alcohol use was found, of which the odds ratio indicated an increase of $200 \%$, this would mean that compared to the always group, high alcohol consumption leads to a $200 \%$ increase in appearing in the either the occasionally or never contraception groups. Likewise, it would also mean a 200\% increase of appearing in the never group compared to the always or occasionally groups, depending on where the comparison is made.

When applied to the SMA data, an ordinal regression would require the use of a three category dummy variable with -1 being assigned to those who chose fewer models, 0 for those who stayed the same and +1 who selected a greater number of models. A positive $\beta$-value would then indicate that participants who show the presence of a particular significant variable (dichotomous in this example) would have a higher chance of being in the +1 or 0 groups compared to -1 . In contrast, a negative $\beta$ would indicate a higher chance of being in -1 or 0 groups compared to +1 . It would be just as correct to move the 0 group to the other side, so that a positive $\beta$ indicates higher chances of being in the +1 group compared to 0 or -1 . However, the former interpretation is more conservative.

As ordinal regression appeared to fit well with the type of data produced by the repeat performance of the SMA task, and given that it would not neglect participants 
who showed no change, it was chosen as the primary method for analysing the effects of condition and covariates on mating strategy change. As a precursor to the ordinal regression, a simple chi-squared test on the dummy variable was also used to test for a simple main effect. This test compared the distribution of change categories from the experimental condition to either a control group or that which would be expected by chance. For example, if the experimental distribution was $-1=16,0=5$, and $+1=9$ (16/5/9), for LT selections in an experimental group, and 10/5/15 for a control group, then this would indicate that the experimental condition increased the chances of participants appearing in the -1 group. Likewise, the same distribution tested against chance $12.5 / 5 / 12.5$ would also yield a similar result. ${ }^{16}$

To accompany the two significance tests, two measures of effect size were used. For chi-squared analysis with more than three categories, Cramér's $V$ can be used as an approximate measure of $r$ (Cramér, 1999), and for ordinal regression models an approximation of $R^{2}$ is given in the form of Nagelkerke $R^{2}$.

One-tailed tests. All the hypotheses used within the individual experiments of this thesis were one-way. That is, they specified an anticipated direction of effect. In this chapter, for example, participants were predicted to increase their selection of LT and ST models following exposure to a set of attractive models. In contrast, a two-way hypothesis would simply predict that a change would occur following this exposure without the mention of a direction. It is conventional within statistics to use a one-tailed test during the analysis if a one-tailed hypothesis is used (Field, 2009). This is because during a two-tailed test the $\alpha$-value (usually 0.05 ) is split between the top and bottom ends of the distribution. Thus, the critical value of the test must usually be larger in order for statistical significance to be reached, but the test retains the ability to find a significant result in either direction. One-tailed tests, in contrast, do not split the $\alpha$ value. Instead they apply it to one end of the distribution. This makes the critical value required for significance lower, but means that if an effect is found in the non-predicted direction it must be disregarded.

Unless otherwise stated, the use of one- and two-tailed tests within this thesis are handled in the following manner: In all chi-squared tests where an experimental condition distribution (see below) is compared to a control one (or one which would be

\footnotetext{
${ }^{16}$ A split of $12.5 / 5 / 12.5$ was chosen as a comparison here rather than $10 / 10 / 10$ to make the test more conservative and place greater emphasis on the decrease or increase in relationship choices. Using this method, high or low numbers of non-changers would not contribute to the result.
} 
expected by chance), one-tailed $p$-values are presented. This is also the case in ordinal models when they reflect the experimental hypothesis precisely. In cases when an ordinal logistic regression model is generated which contains additional control variables, the effects of which are not stated in the experimental hypothesis, the results of a two-tailed test are presented.

\section{Influence of Attractive Individuals}

The set of attractive individuals was rated, on average, as $5.81(S D=1.01)$ out of ten for attractiveness by the participants. Applying chi-squared and ordinal regression to the data, it was found that exposing this set to the participants had an influence on their SMA ratings. Following exposure, participants were more likely to appear in the LT- and ST- categories (see Table 2.1) compared to that expected by chance.

Table 2.1

The distribution of participants by SMA change category for the attractive condition. The first table shows changes to ST responses, the second shows changes to LT responses

\begin{tabular}{|c|c|c|c|}
\hline \multicolumn{4}{|c|}{ Short-term change } \\
\hline & - & $=$ & + \\
\hline Actual & 30 & 2 & 8 \\
\hline Predicted & 19 & 2 & 19 \\
\hline$\chi^{2}(2)$ & \multicolumn{2}{|c|}{$12.737 * *$} & \\
\hline Cramér's $V$ & \multicolumn{2}{|l|}{0.28} & \\
\hline \multicolumn{4}{|c|}{ Long-term change } \\
\hline & - & $=$ & + \\
\hline Actual & 23 & 11 & 6 \\
\hline Predicted & 14.5 & 11 & 14.5 \\
\hline$\chi^{2}(2)$ & \multicolumn{2}{|c|}{$9.966 * *$} & \\
\hline Cramér's $V$ & \multicolumn{2}{|l|}{0.25} & \\
\hline
\end{tabular}

Note: Participants are categorised into the "." group if they showed a decrease, the "=" group if they stayed the same, and the "+" group if they increased in their model choices. A chi-squared test against a predicted distribution was then used to detect an overall change. ${ }^{\dagger} p<0.10$, ${ }^{*} p<0.05, * * p<0.01$.

When the variables of participant age, sex, SOI-R, self-perceived attractiveness, and relationship status were used in an ordinal regression, two significant models 
emerged (see Table 2.2). The first, related to LT change, saw a positive effect of selfperceived attractiveness. For every one point increase in self-perceived attractiveness the participant reported, the chances of them appearing in the LT= or LT+ group increased by $56 \%$. Conversely, individuals lower in attractiveness were more likely to be affected by the exposure to the attractive models than their attractive counterparts. In terms of ST change, there was a significant model produced using participant age as a covariate. As their age increased, the chances of a participant appearing in the ST= or $\mathrm{ST}+$ group increased by $53 \%$ for each year. Conversely, younger individuals were more likely to be affected by exposure to the models than their older counterparts.

Table 2.2

Ordinal regression models predicting change to relationship responses in the attractive condition. The first model predicts short-term response change using participant age, and the second predicts long-term response change using self-reported attractiveness

\begin{tabular}{|c|c|c|}
\hline \multicolumn{3}{|c|}{ Model ST } \\
\hline & $\beta$ & OR \\
\hline Age & $0.427 *$ & 1.53 \\
\hline Model & \multicolumn{2}{|c|}{$\chi^{2}(1)=4.495^{*}$} \\
\hline Nagelkerke $\mathrm{R}^{2}$ & \multicolumn{2}{|l|}{0.14} \\
\hline Accuracy & \multicolumn{2}{|c|}{$77.5 \%(+2.5 \%)$} \\
\hline Parallel lines & \multicolumn{2}{|c|}{$\chi^{2}(1)=0.429$} \\
\hline \multicolumn{3}{|c|}{ Model LT } \\
\hline \multirow[b]{2}{*}{ Attractiveness } & $\beta$ & OR \\
\hline & $0.455^{\dagger}$ & 1.56 \\
\hline Model & \multicolumn{2}{|c|}{$\chi^{2}(1)=4.238^{*}$} \\
\hline Nagelkerke $\mathrm{R}^{2}$ & \multicolumn{2}{|l|}{0.12} \\
\hline Accuracy & \multicolumn{2}{|c|}{$60.0 \%(+2.5 \%)$} \\
\hline Parallel lines & \multicolumn{2}{|c|}{$\chi^{2}(1)=0.020$} \\
\hline
\end{tabular}

${ }^{\dagger} p<0.10, * p<0.05, * * p<0.01$. OR = Odds ratio.

Accuracy. In some parametric analyses, the "base" model tends to be the mean. Other models are then tested to see if they can account for more of the variance in the data than this base. The base model equivalent in logistic regression assumes that all participants fall into the category with the highest $n$. So, for example, in the ST change 
table (Table 2.1), as 30 individuals were in the ST-group, the base model would assume that all participants were in that group, which would be correct $75 \%$ of the time. The fact that the new model improves on this predictability by $2.5 \%$ (Table 2.2 ) means that the model only leads to a small increase in accuracy.

\section{Influence of Unattractive Individuals}

The set of unattractive individuals was rated, on average, as $2.70(S D=0.90)$ out of ten for attractiveness by the participants, which was significantly different to the attractive set, $t(76)=14.25, p<0.01, d=3.27$. Following exposure to the unattractive set of individuals, the sample showed a tendency to increase in both LT and ST ratings. However, this was non-significant in the case of ST differences and only marginally significant ( $p=0.10$ ) in the case of LT differences (Table 2.3). Ordinal regression models testing for covariates were non-significant in both cases.

Table 2.3

The distribution of participants by SMA change category for the unattractive condition. The first table shows changes to ST responses, the second shows changes to LT responses

\begin{tabular}{|c|c|c|c|}
\hline \multicolumn{4}{|c|}{ Short-term change } \\
\hline & - & $=$ & + \\
\hline Actual & 15 & 5 & 18 \\
\hline Predicted & 16.5 & 5 & 16.5 \\
\hline$\chi^{2}(2)$ & 0.273 & & \\
\hline Cramér's $V$ & 0.06 & & \\
\hline \multicolumn{4}{|c|}{ Long-term change } \\
\hline & - & $=$ & + \\
\hline Actual & 8 & 13 & 17 \\
\hline Predicted & 12.5 & 13 & 12.5 \\
\hline$\chi^{2}(2)$ & $3.240^{\dagger}$ & & \\
\hline Cramér's $V$ & 0.20 & & \\
\hline
\end{tabular}

\section{SMA and Demographic Variables}

As secondary analysis, Spearman's rank correlations were performed between the number of ST and LT selections during the first performance of the SMA task and variables from the demographic questionnaire. In terms of short-term relationships, 
there was a strong correlation between the SOI-R and the number of ST selections, $r_{\mathrm{S}}(73)=0.48, p<0.01$, indicating that high SO individuals tended to pick more ST relationships. There was also a strong negative correlation between ST and sex, $r_{\mathrm{s}}(78)=$ $-0.41, p<0.01$, with males typically choosing more models for a short-term relationship than females. Finally, ratings of the attractive and unattractive models positively correlated with ST choices. This comparison implies that generally those who are more favourable in their rating behaviour are more likely to choose ST relationships during the task, $r_{\mathrm{s}}(78)=0.44, p<0.01$.

In terms of LT selection, a relationship between SOI-R and LT selection was absent, $r_{\mathrm{s}}(73)=-0.04, p=0.71$. However, there was a strong negative correlation with self-perceived attractiveness, $r_{\mathrm{s}}(78)=-0.36, p=0.01$, indicating that individuals were more likely to consider the models suitable for LT relationships if their self-perceived attractiveness was low. When considering the number of "not interested" (NI) choices, which acts as a rough measure of pickiness, a positive correlation with participant selfreported attractiveness was found, $r_{\mathrm{s}}(78)=0.24, p<0.05$, with more attractive individuals typically picking the NI option more frequently. In an expected mirror image of the ST findings, NI responses decreased as SOI-R increased, $r_{\mathrm{s}}(73)=-0.30, p$ $<0.01$.

A final piece of secondary analysis surrounded the interpretation of sociosexuality. As discussed in the introduction to this chapter, individuals who are high in $\mathrm{SO}$ are often referred to as primarily short-term maters. However, when the behaviour of high and low SO individuals was considered in terms of their median SMA responses, even high SO individuals were found to select long-term relationships some of the time. In Figure 2.3, the media LT, ST, and NI responses are displayed for twenty participants; the ten with the highest SO and ten with the lowest SO (with equal sex representation). Both groups selected models for short- and long-term relationships. Furthermore, while there was a significant difference between the groups in terms of ST selection $(U=81.5, p<0.05)$, there existed no difference in terms of LT selection $(U=$ $44.5, p=0.684)$. 


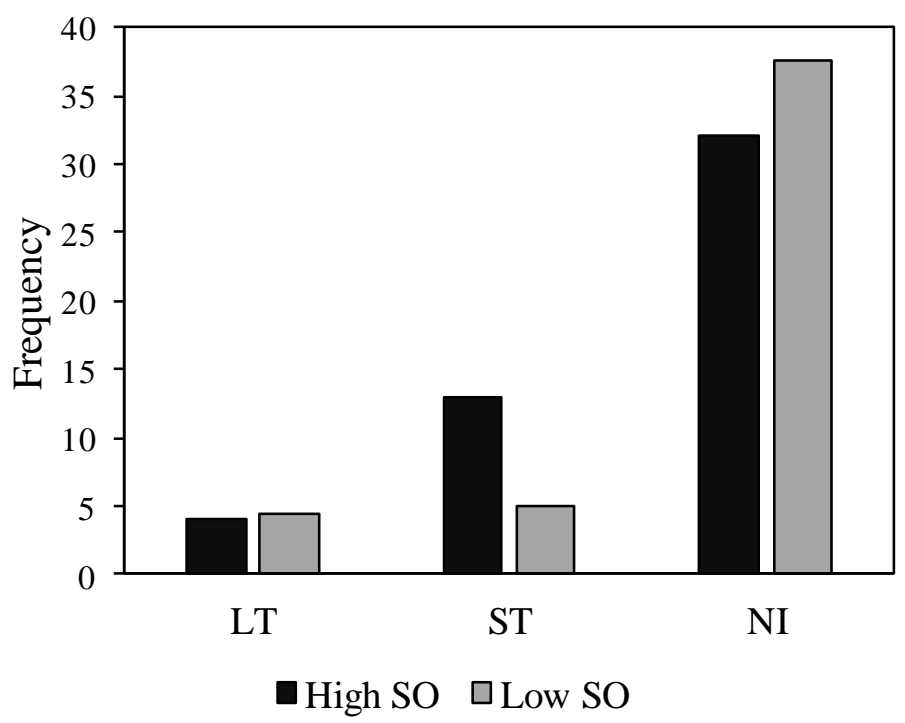

Figure 2.3. The median relationship choices in the first SMA measurement of the pilot study. The High SO group was formed from the five males and five females with the highest SOI-R scores. Similarly, the Low SO group was formed from the five males and five females with the lowest SOI-R scores.

\section{Discussion}

\section{A Contrast Effect}

This pilot experiment aimed to demonstrate that the SMA task is sensitive to changes in relationship preferences following a brief intervention. A simple contrast effect (Thornton \& Maurice, 1997; Thornton \& Moore, 1993) was chosen to demonstrate this whereby the SMA was completed by participants twice with a set of attractive or unattractive models shown in between measurements.

Overall, moderate support was found for Hypothesis 0. Following the presentation of attractive individuals, the willingness of the participants to engage in ST and LT relationships with an average attractiveness sample appeared to decrease, confirming Prediction 1. This was revealed using basic chi-squared analysis. While the opposite effect was expected following the display of unattractive individuals, changes to ST selection were non-significant and changes to LT selection were only marginally significant, although in both cases the results were in the anticipated direction. Thus Prediction 2 was only partially correct. Together the results indicates that, while the participants were willing to change their minds about their mating decisions after being presented with more desirable alternatives, less desirable alternatives had less of an impact on their previous decisions. This may have been due to the average models 
contrasting more with the attractive models than the unattractive ones. That is, the unattractive models may not have been unattractive enough to elicit an effect. This discrepancy between the conditions may have something to do with the way in which the pictures were collected. Ratings from hotornot.com were used to separate the models into groups, and ratings from this site may not be reliable given that users with poor scores have the option to remove their pictures (e.g. the picture with the lowest rating in the unattractive set was 3.2 followed by 5.1).

Another weakness of this study is that the attractiveness ratings provided by the participants during the task significantly departed from those collected from hotornot. For example, while the unattractive set of models had an average hotornot rating of $M=$ 7.99 ( $S D$ unknown), this was rated as $M=2.70(S D=0.44)$ by participants during the experiment. Precisely why the hotornot rating distribution is so skewed is unknown but it could relate to factors such as the age of those providing the ratings or the social context in which the rating task is performed. To rectify this problem, in the other experiments of this thesis hotornot was used as an image source only; attractiveness ratings were obtained from separate judges from the same population as the participant base.

Criticisms aside, the pilot study revealed that individuals can "change their mind" about potential mates quite readily following exposure to different stimuli. Furthermore, the ordinal logistic regression revealed a very small effect of two covariates of category change; age and self-perceived attractiveness. While these models were poor in terms of improvements to predictability, they did allow the opportunity to introduce the type of analysis used throughout the remainder of the thesis, and demonstrate that this analysis is indeed sensitive to covariates.

\section{SMA as a Dependent Variable}

Several points of interest emerged from the pilot study which related to the appropriateness of the SMA task as a dependent measure. First, participants were open to all three types of relationship category. Eighty-six per cent $(n=67)$ of individuals showed a preference for a mixture of both long- and short-term relationships during the first exposure to the task, which we would expect to find if humans were mixed strategists or could implement mating strategies conditionally (Gangestad \& Simpson, 2000).

Second, the frequency of choices within each category makes sense in light of what is known about human mating. Overall, participants of both sexes were highly 
selective and choose NI for most of the average attractiveness models. The second most selected relationship was short-term, and there was a sex difference with men selecting this option more than women. Finally, long-term relationships were chosen less frequently, reserved for a small handful of desirable individuals. There was no difference in LT choice between men and women, which makes sense given that humans are picky maters who seek the most desirable partner possible. Most individuals were not considered a viable dating partner, and even among those who were, only a small selection were considered for a long-term relationship. Such results should be unsurprising given the generally socially monogamous nature of our species (StewartWilliams \& Thomas, 2013b). Even individuals high in SO, who are seen to be "primarily short-term maters", picked not interested often and still showed a preference for pair-bonding (see Figure 2.3).

Third, the relationship choices from the SMA correlated with other variables in logical ways. Namely, ST correlated strongly with SOI-R, while LT correlated negatively with self-perceived attractiveness. Selectivity (as approximated through NI responses) also correlated positively with self-perceived attractiveness. These correlations also support a criticism of sociosexuality measures made at the start of the chapter, as the SOI-R correlated strongly with ST choice but not with LT choice. If low SO scores were indicative of the pursuit of a LT strategy then we would expect a strong negative correlation. This was not the case.

Fourth, although the spread of the data was non-parametric, a wide range of LT and ST selections were present. When designing questionnaire items using likert scales, one must take care to create questions which produce a diverse range of answers, or else there will be very little variance to explain. In a similar way, if there was no variance between individuals on LT and ST selection, it may compromise the ability of the statistical tests to detect changes. However, the data distributions (Figure 2.1) reveal a good range of responses. Although there was a floor effect found in initial relationship selections, LT was chosen at least once by the majority of participants. Only eight participants (10\%) deemed all models unsuitable for a long-term relationship and, discounting these, LT responses varied from 1 to 28 with a median of 5.5. Likewise, only three participants (4\%) found all models unsuitable for a short-term relationship. Excluding these, the number of ST responses ranged from 1 to 34 with a median of 11. Together, it appears that, while participants were pickier for LT relationships than ST 
ones, there was a sufficient amount of variance between individuals across the sample and that the paradigm was able to detect these individual differences.

\section{Conclusion}

The purpose of this chapter was to present the results of a pilot study using a dependent measure of mating strategies developed specifically for the research in this thesis. The SMA task was designed to: a) measure the independent constructs of LT and ST mating inclinations, while b) being sensitive enough to detect changes to participant behaviour following the administration of a manipulation. The second of these attributes was verified in this pilot study through the use of a contrast effect manipulation. The first attribute was tested in the experiments featured within subsequent chapters of this thesis. It is one thing to change relationship preferences by showing participants more desirable potential partners. It is another to illicit such an effect through, for example, cuing that the environment contains defenceless neonates (Chapter 4) or that one's attractiveness relative to other group members has shifted. It is this latter manipulation which is the focus of the next chapter. 
Chapter 3: Relative Attractiveness and Mating Strategies in Men - An Investigation Using False Feedback

An individual's physical attractiveness relative to his or her peers has the potential to vastly affect their mating opportunities. A simple classroom activity can illustrate this point. Ellis and Kelley (1999) designed a 'pairing game' whereby each individual is assigned a unique number which is placed on their forehead. This way each participant in the group can see everyone's number apart from their own. The goal of the game is for each participant to try to form a partnership with another member of the group. Incentives are given which are linked to partner number, so that those who partner with a high numbered individual receives a large reward, while those who partner with a low numbered individual receives a little reward. Typically, those with a low number find their attempts to partner-up met with rejection, while those with a midrange number find that high numbered suitors are not receptive to them but that they receive offers from lower numbered contemporaries. The participants with the highest numbers, however, find little rejection and are able to choose freely from the multiple offers they receive. Eventually, all the participants find a partner and this tends to be someone who has a similar number. Occasionally an individual may be paired with someone with a higher number than their own, but very rarely would a "1" and " 200 " mutually agree to be partners. In fact, the correlation tends to be around $r=0.70$ (Eastwick \& Buck, 2014). This simple pairing game reveals that humans are very good at learning the relative value of their attributes among their peers within a brief period of time and can adjust their behaviour accordingly.

In this sense, humans seem to be good "intuitive economists" and evidence for this can be found for this across several domains (L. Barrett et al., 2002; Rubin, 2003). The domain of mating is no exception. Much like in the pairing game, real life mate choices reveal that, although people would like the most desirable partner possible, they often end up mating assortatively with a partner who is similar to them. Research has revealed that men and women tend to settle down with a partner of similar physical attractiveness, intelligence, sense of humour, and educational level (Barelds \& BareldsDijkstra, 2010; Buss, 1985; Huber \& Fieder, 2011; Little, Burt, \& Perrett, 2006).

Despite humans showing a tendency to mate assortatively, this is still just a tendency and there are additional factors which can cause discrepancies in attributes between partners. For example, as attractiveness is a multifaceted construct there can 
still be a great deal of variance between an individual's various qualities. A lack of physical attractiveness may be coupled with high status or wealth, for instance.

Likewise, an individual's mating strategy may affect the quality of partners which they partner with. It is not simply the case that every individual desires a long-term relationship and so pairs off with someone who is as attractive as they are. Alternative mating strategies can be used to manipulate the system and gain access to a higher quantity, or quality of partners. Although a " 100 " man may well be able to attract a "98102 " woman for a committed long-term relationship, for instance, he may actually increase his reproductive fitness further by instead having several casual relationships with partners in the "70" range. In terms of female mating strategies (Greiling \& Buss, 2000), a "70" woman could use short-term mating to gain access to more desirable partner than the "68-72" men she could attract if she required strong evidence of commitment from her partner. Therefore, one's position among peers not only affects the type of partners which can be attracted, but also the type of mating strategies which are likely to be pursued successfully (Gangestad \& Simpson, 2000).

In this chapter, the influence of a man's perceived attractiveness on their mating strategy is examined. Two experiments using false feedback were conducted to accomplish this. In this introduction, a review of the current comparative and psychological literature related to attractiveness and mating strategies is presented as well as the strong one-way hypotheses which were developed based on this review.

\section{Comparative Studies}

Experiments on non-human animals reveal that the manipulation of relative attractiveness can indeed influence mating strategy choice. Some of these studies involve enhancing or crippling a sexually selected ornament which is an indicator of physical attractiveness. Others involve removing competitors from the mating market to increase the status of subordinates. In bluethroats (Luscinia svecica), Johnsen and Lifjeld (1995) showed that lowering a male's attractiveness by blackening their colourful throat patch, caused them to allocate more time to guarding their current partners than pursuing extra-pair copulations. In a similar study, when badge size was increased in male collared flycatchers (Ficedula albicollis) the number of their territories increased, particularly among older males (Qvarnström, 1997). A final avian example involves the common fowl (Gallus gallus). D. R. Wilson, Nelson, and Evans (2009) demonstrated that male fowls are very sensitive to changes in their relative 
attractiveness compared to others. Removal of attractive competitors for just 40 minutes was enough for males to engage in increased courtship behaviour.

It is not just birds which are sensitive to changes in the mating market. For example, in Arctic charr (Salvelinus alpinus) levels of sperm production are inversely related to a male's position in the status hierarchy. High status males spend more somatic resources on lekking behaviour and attracting females, while low status males produce larger quantities of sperm, as an alternative status-dependent strategy, to take advantage of the externally fertilizing nature of the species. Shifts in the sperm production of individual charr can be produced by changing their place in the social hierarchy. Low status males who are placed among males of an even lower status will decrease in the amount of sperm they produce (Rudolfsen, Figenschou, Folstad, Tveiten, \& Figenschou, 2006). Similarly, when the status hierarchy of deer is disrupted by removing the most successful males, subordinate males will increase their rate of intra-sexual competition to try to enhance their mating success (Apollonio, FestaBianchet, \& Mari, 1989). Likewise, intermediate-sized male sailfin mollies (Poecilia latipinna) will switch between the strategies of their small and large phenotypes depending on the size of other males present (Travis \& Woodward, 1989), while applying black nail polish to the eyes of horseshoe crabs (Limulus polyphemus) can lead them to switch to secondary "satellite" mating tactics (a strategy usually followed by males whose eyesight is weaker, or grip well worn; Duffy, Penn, Botton, Brockmann, \& Loveland, 2006).

Such experiments are not always successful. In Hector and Raleigh (1992) the removal of an alpha male vervet monkey (Chlorocebus pygerythrus) did not cause another to adopt his position. That is, none of the subordinate males showed an increase in dominance or aggressive behaviour, although they did seem to take advantage of the circumstance by approaching high ranking females more readily. Of course, this could have been due to the temporary nature of the removal; other studies have shown that following the death of a primate alpha male, the adoption of higher status by subordinates is almost inevitable (de Waal, 2007; Ren, Liang, Zhang, Li, \& Grueter, 2007). Most of the aforementioned animal studies use males-compete-females-choose (MCFC) species (Stewart-Williams \& Thomas, 2013b; Trivers, 1972) which is why the manipulations tend to have focused on male mating behaviour. However, there are examples of similar mating plasticity in females of socially monogamous species as 
well (Burley, 1986; Burley \& Foster, 2006; Limbourg, Mateman, Andersson, \& Lessells, 2004).

It appears that there is strong cross-species evidence that enhancing the relative physical attractiveness of an animal, or increasing its status by removing competitors, can cause it to adopt a more dominant or risky mating strategy. If humans are similar in this regard, then men and women who suddenly find themselves more attractive than their peers may switch to a mating strategy which, when pursued successfully, can lead to greatly enhanced fitness (e.g. a short-term strategy in men). Likewise, individuals who find themselves lower in status may also switch to a mating strategy which is more likely to maximise their fitness (e.g. a long-term strategy in men), rather than to persist with a strategy they are unable to pursue successfully. The evolutionary explanation for this behaviour would be that, in the ancestral environment, those who would have been sensitive to such changes in their relative attractiveness within the mating market, and maximised their fitness by choosing the appropriate strategy, would have been selected for over those who were unable to show such flexibility.

In humans, social status and physical attractiveness can be mutually exclusive. Whereas the most successful peacock within a given area is also likely to be the most physically attractive, it may not be the case that the highest status human within a group is necessarily the most physically attractive. They could have a dominant personality, wealth, and expert knowledge, and yet not be considered universally handsome. Other determinants of status will be discussed in later chapters which investigate the role of testosterone levels (Chapter 6) and wealth (Chapter 4). The experiments in this chapter focus specifically on physical attractiveness (or perception thereof) as the attribute under investigation.

\section{Psychological Studies}

While experiments which manipulate animal mating strategies by changing their relative attractiveness are plentiful, comparable human studies are few in number. Nonetheless, there are some studies which give us reason to believe that such manipulations are likely to affect mating strategies. For example, Surbey and Brice (2007) gave participants false feedback about their desirability as a date. They were told that they scored 92 out of 100 based on a personal characteristics questionnaire they had completed ten days prior. Change to mating behaviour was then assessed using a modified version of the original SOI (J. A. Simpson \& Gangestad, 1991). Responses to several vignettes were also used as a separate measure of mating preferences. The four 
vignettes were profiles of potential opposite sex partners who varied by attractiveness and the minimum time they were willing to wait before having sexual intercourse. Following the false feedback, an increase in willingness to date members of the shortterm sexual vignettes increased. Both sexes were tested in this experiment and men showed a greater increase in willingness than women. SO also increased, however, this was a small effect and was non-significant. This may well have been due to the modified nature of the SOI which did not include any desirability questions and had not undergone any form of validation.

In another example, D. Bailey et al. (2011) asked men to choose between two pictures of women in a forced choice paradigm. In each instance, both women were of similar attractiveness, however, only one was ovulating when their photograph was taken (although participants were not aware of this). The authors were able to demonstrate that, at baseline, men were more likely to choose women who were ovulating (around $55 \%$ of the time) unless the attractiveness of the models relative to the participant was high. In this case men were then more likely to choose the nonovulating woman of each pair (again roughly $55 \%$ of the time). The men then had their perceived mate value increased by being given positive false feedback about their desirability as a date using a bogus personal characteristics questionnaire. When the initial task was repeated, high attractiveness non-ovulating women were no longer picked over their ovulating counterparts; they were chosen equally. These results imply that a) men are sensitive to fertility in women, particularly so in those who are of a similar mate value; and b) increasing a man's perceived mate value can lead to a shift in what types of women are perceived to be of a similar mate value. Although exactly why participants would select, above chance levels, non-ovulating highly attractive women in the first instance is not clear from an evolutionary psychological perspective, the results imply that positive feedback administered to male participants about their mate value, can lead to changes in mate selection criteria.

Finally, there is evidence that self-esteem can be manipulated experimentally in the sociometer literature (M. R. Leary et al., 1995). Self-esteem is associated with a host of mating related factors including social status (Cheng, Tracy, \& Henrich, 2010), perceived mate value (Penke \& Denissen, 2008), and male sociosexuality (A. P. Clark, 2006). Thus, if a participant's self-esteem can be influenced by feedback about mate quality or low social status within a group then this could be a positive indication that mating strategy choice may also be affected by such feedback. In one such study 
participants played a virtual game of tossing a ball or Frisbee between members of a group comprised of several supposedly real players. Social exclusion was then manipulated by the computer which varied how often the participant was passed the object. The self-esteem of participants who underwent social exclusion during this task tended to decrease (E. F. Gross, 2009; K. D. Williams, Cheung, \& Choi, 2000). In another example (Pass, Lindenberg, \& Park, 2010), false feedback was given to participants about their potential quality as a mate as judged by a bogus 'Social and Mate Value Inventory'. In one condition, participants were told that 'there is a low probability that you will have good and fulfilling relationships and a high probability of frequent rejection by possible mates' and that even if they were currently in a relationship that 'this will change, and the older you get the more likely it becomes that you will end up without a partner later in life' (p. 233). This manipulation led to a decrease in self-esteem compared to a control condition. Furthermore, this effect was not found in a third experimental condition where 'mates' was replaced with 'friends'.

Despite sociometer experiments covering multiple domains, including friendship, kin, and mating bonds (Kavanagh et al., 2010; Kirkpatrick \& Ellis, 2004), the literature has not specifically investigated the effect of self-esteem change on mating behaviour with regards to the distinct long- and short-term mating strategies postulated by SST (Buss \& Schmitt, 1993).

\section{False Feedback Experiments in Psychology}

A common feature of the experiments mentioned above is the presence of false feedback. In animal studies desirable males are simply removed from or introduced into a group, or the physical attributes of individual group members are temporarily modified. Changes to the mating behaviour of members of the group are then recorded (Gage \& Barnard, 1996; Perrill, Gerhardt, \& Daniel, 1982; Qvarnström, 1997; Thornhill, 1981). In humans, such manipulations would be difficult to implement within a laboratory environment. Instead it is often ethically easier to temporarily manipulate a participant's perception using a false feedback paradigm.

False feedback paradigms are quite common in social sciences. One example comes from the domain of sexual psychology. Bach, Brown, and Barlow (1999) demonstrated that false feedback about sexual arousal could lead to real physiological changes. Male participants were shown three erotic films while having the circumference of their erection measured using a strain gauge. After the second film, either no feedback was given (control), or the experimenter asked the participants if 
they were 'finding it difficult to become aroused' (p. 84) and presented them with a fake graph indicating that their responses were below average. After being given negative feedback about their arousal, genital blood flow decreased during the presentation of the third film. Furthermore this effect was increased among those who had low self-efficacy in penile performance prior to the third video being shown. Similar studies with females show a change to subjective reports of arousal but not necessarily accompanying physiological changes (McCall \& Meston, 2007; Woll \& McFall, 1979).

Non-sexual false physiological feedback has also been found to change perceptions. A common manipulation involves giving participants false feedback about their heart rate and then seeing if this alters their perception of various stimuli (Hirschman, 1975; Holmes \& Frost, 1976; Makkar \& Grisham, 2013). In one recent study participants had their brain activity measured using an fMRI while they received false heart rate feedback indicating they were aroused (M. A. Gray, Harrison, Wiens, \& Critchley, 2007). Consistent with previous research, when participants were shown neutral faces, this feedback led them to increase their perceived emotional intensity of the models. However, the additional fMRI analysis also revealed that this feedback caused specific activation in brain areas related to emotional processing, including the amygdala, which could account for the shift in perception.

In social psychology, false feedback has been shown to affect group perceptions. W. Wood and Karten (1986) showed the effect of false feedback in discussion groups of equal sex. When individuals, who were unknown to each other, interacted in groups of four, a sex difference emerged. Males were more "task active" and rated themselves as higher in competence, while females engaged in more pro-social behaviour. If, however, each participant was given false feedback indicating they were "high" or "low" in competence compared to other members, this social dynamic changed: those given high feedback rated themselves as more competent and became more task-active, while those receiving low feedback were more pro-social and rated their own competence as lower. The power of this effect was so strong that the normal sex difference disappeared.

False feedback is a powerful research tool. It can be used to make people blush (Drummond, 2001), increase their perception of pain (Levine, Krass, \& Padawer, 1993; van den Hout, Vlaeyen, Peters, Engelhard, \& Van den Hout, 2000), and disrupt cooperative tasks (Monterosso, Ainslie, Pamela Toppi Mullen, \& Gault, 2002). With the potential for such strong effects on behaviour, great ethical care must be taken with such 
manipulations. In one Scottish study, the false suggestion to participants that they had become sick as a child by eating egg salad led to a real aversion to the food at a four month follow-up session (Geraerts et al., 2008).

\section{Experiment 1}

Experiment 1 used a bogus dating / social networking website in order to administer false attractiveness feedback to participants, across two experimental sessions, within a laboratory setting. Due to the time consuming nature of the paradigm, and the fact that a small-to-medium effect size was expected (see Chapter 2), it was decided that only men would be examined in order to increase experimental power. Combining findings from the sociobiological literature, evolutionary theory, and previous psychological studies, two experimental hypotheses were formed about how attractiveness feedback would affect men. Hypothesis 1 stated that positive attractiveness feedback would lead to short-term mating strategy activation in men, while Hypothesis 2 stated that negative attractiveness feedback would lead to long-term mating strategy activation in men. Two predictions were made to test the hypotheses. Prediction 1 was that, following positive feedback, men would increase in their selfreported SOI-R score. Prediction 2 was the reverse; following negative feedback the self-reported SOI-R score of men would decrease. A measure of sociosexuality (SOI-R) was used as the primary dependent measure as Experiment 1 was conducted before the development of the SMA task (Chapter 2).

\section{Method}

\section{Participants}

In total, 40 male participants were tested and seven of these were excluded from the analysis (see results section). The following demographic details reflect the remaining 33 participants. All participants were undergraduate $(n=29)$ or postgraduate $(n=4)$ students from Swansea University who participated for payment or course credit. Further details on recruitment can be found in the procedure section. The mean participant age was $19.97(S D=1.6)$ and only 6\% $(n=2)$ of the participants were nonCaucasian (both Black). The participants were childless and there was only one nonheterosexual participant. This male described himself as bisexual and so was included in the analysis. Two thirds of the participants were either single or in uncommitted relationships while the remainder $(n=11)$ were in a committed relationship lasting longer than 3 months. The average sociosexuality (SOI-R) score was 40.15 ( $S D=$ 11.58). 


\section{Materials}

The dating website. For the purpose of the experiment, a website called "Mix\&Match" (M\&M) was created. This website was accessible to the public and emulated the features of a social networking website with a large number of active users. In reality, all content was controlled by the experimenter. The apparent purpose of the website was to allow users to sign up, rate other members for attractiveness, and receive feedback about their own attractiveness. The true purpose of the website was to provide a unique way to administer false feedback to participants. The website was created using php and MySQL databases and was hosted on a web server. All elements of the website are described in detail below. Images of all major pages of the website can be found in Appendix H. The purposes of some aspects of the website (such as the "progress bar") are clarified in the following procedure section.

Sign-up and log-in systems. A "Sign-up" page was created to allow participants to register for an account. The page featured a form containing the following items: username, password, gender, age bracket, and city. A response was required for each item in order for the form to process. On successful completion of the form an account was created for the user in the MySQL database. They were then logged-in and sent to a personalised "My Page" hub. A "Log-in" page was created to facilitate participants returning to the study in the second session, this required a valid username and password from the database.

My page. This page acted as the main hub of the experiment. It gave participants access to different pages depending on their progress through the study. The top of the page always contained welcome text along with the participant's chosen username. Four "steps" were presented to the participant throughout the study. Step one asked participants to upload a photograph. The text 'Please select an image to upload' was accompanied by a form allowing an image to be uploaded to the web server. Step two gave participants access to the rating system (see below). Step three displayed a "progress bar" titled 'gathering feedback' that gradually filled up as the next experimental session approached. This was to add to the believability of the study should a participant access his account between experimental sessions. Finally, step four gave participants access to the feedback system.

Rating system. The purpose of the "Rating" page was to lead participants to believe they were rating 30 pictures of female users of the website. Pictures of women (also referred to as models) were displayed sequentially, with an age bracket (always 
18-25) and local city accompanying each. A semantic differential scale was presented below each picture which participants used to rate the physical attractiveness of each model between one and ten. The participant's rating for each of the models was not recorded. A counter informing participants as to how many pictures they had left to rate appeared above the model. After rating the 30 pictures, participants were redirected to the "My Page" hub and step three was made available.

Feedback system. The purpose of the "Feedback" page was to administer the experimental manipulation in the form of 50 pieces of false-feedback. Each feedback page consisted of a model on the centre-left of the page. As with the rating page, this was accompanied by an 18-25 age bracket as well as a location. To the right of the model appeared a bar chart titled 'The ratings' with a Y-axis labelled from zero to ten. The X-axis had two labels: 'Other guys' and 'You'. Once the page was fully loaded, a bar appeared above both labels, growing from zero to a specified value (see below) over the space of three seconds. The value of each bar appeared above it in text after it had finished growing. The values which these two bars took could be modified to give the impression that the participants were attractive or unattractive relative to their peers. An example of the bar chart can be found in Figure 3.1 below. A two second delay was then included before a link appeared allowing participants to move on to the next piece of feedback. This meant that each feedback piece was administered over five seconds. After viewing all 50 feedback pages, participants were redirected to the "Completed" page.

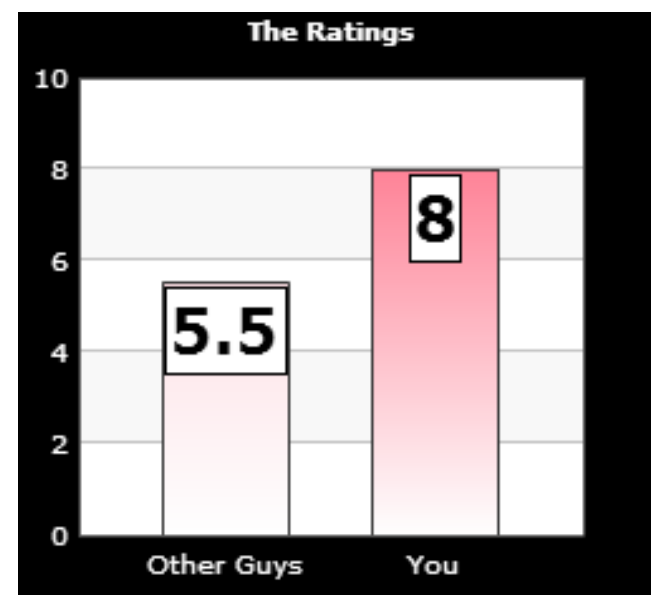



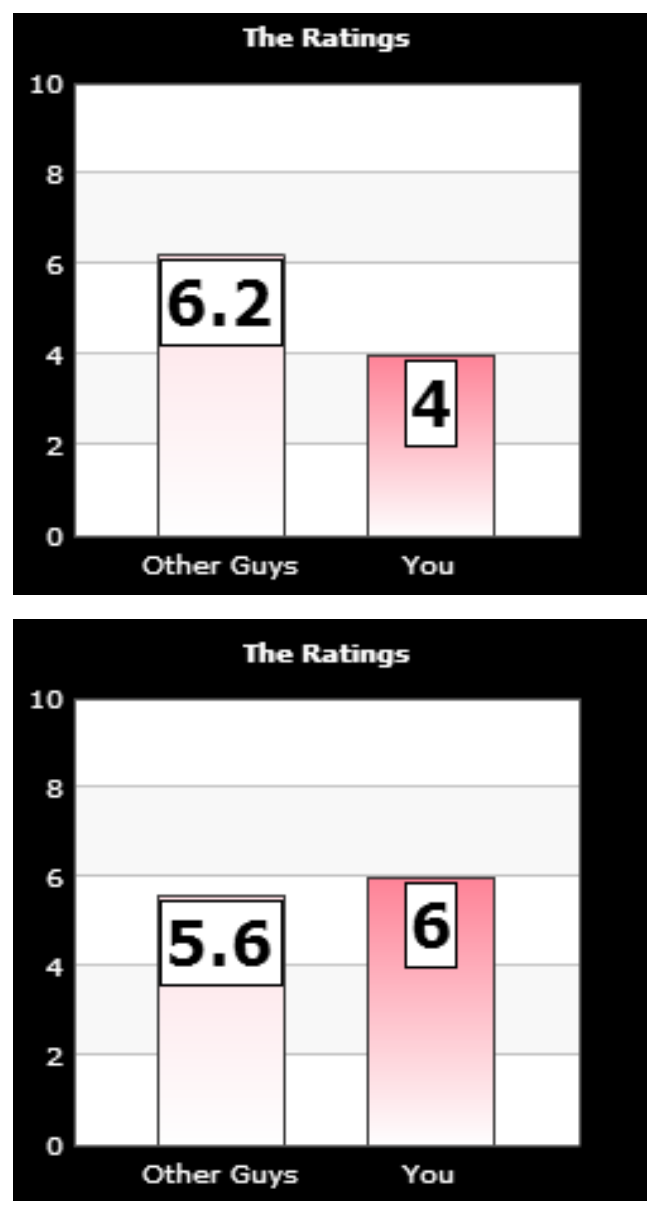

Figure 3.1. Three examples of false feedback bar charts shown to the participants. The top bar chart shows positive feedback, the middle bar chart shows negative feedback, and the bottom bar chart shows neutral feedback.

Completed page. To increase the intensity of the feedback, a "Completed" page was included which gave a bogus summary of the participant's feedback. A negative and positive version of this summary page was created. In the negative version participants were told that their average rating was 3.8 while the average rating for other men was 6.6. These figures were 7.8 and 4.6 respectively in the positive condition. A histogram (Figure 3.2) showing the participant's position in relation to other males was also shown. The end of this page signified that the study had completed and encouraged the participants to contact the experimenter. 


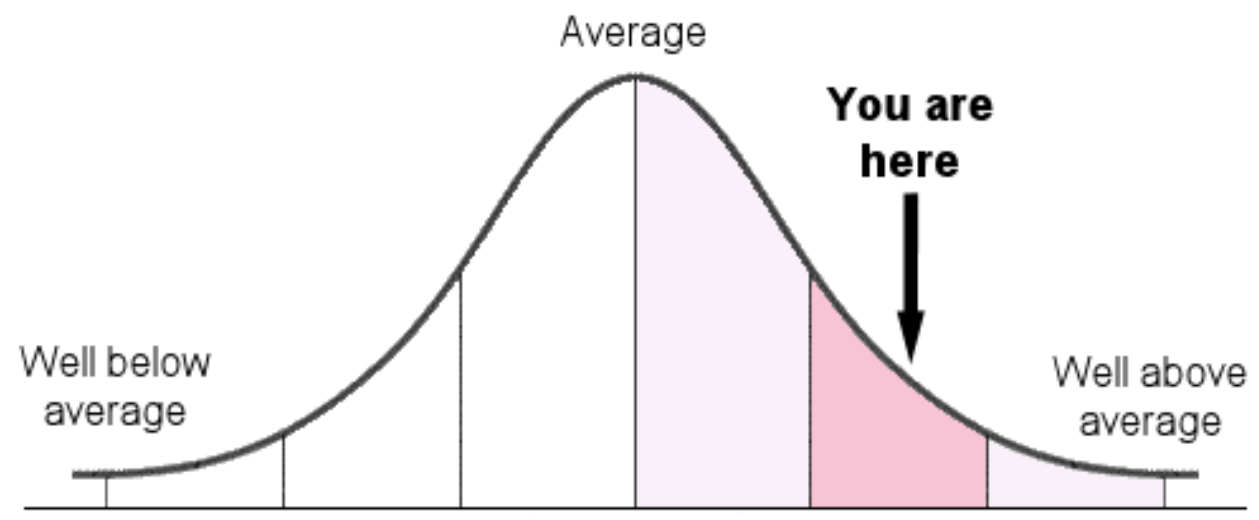

Attractiveness

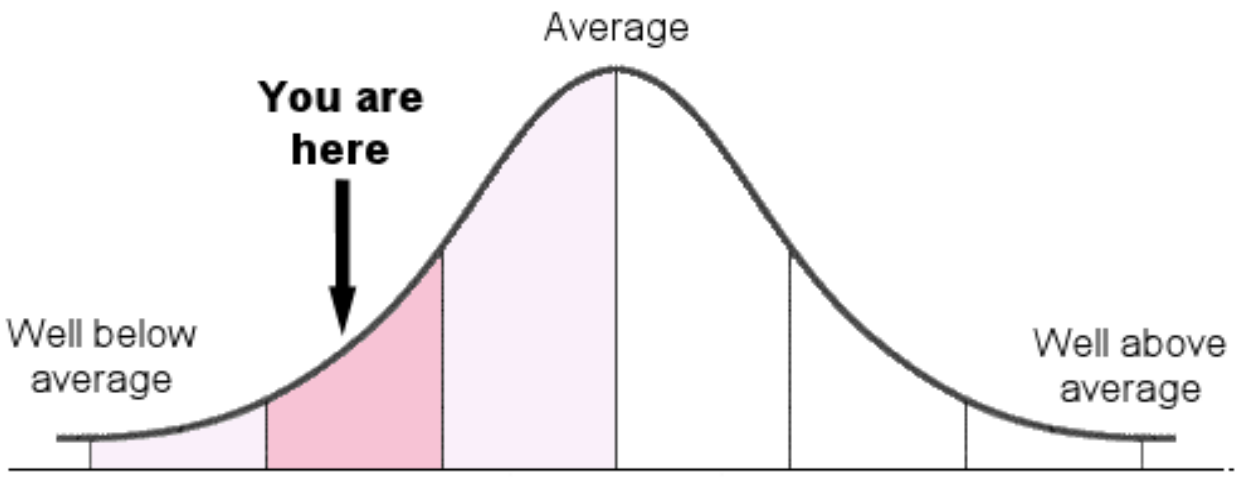

Attractiveness

Figure 3.2. The histograms which were presented to participants on the "Completed" page after receiving their feedback through the Mix\&Match website. The top histogram was positive and indicated that participants were more attractive than average. The bottom histogram was negative and indicated that participants were less attractive than average.

Online presence and restriction. The $M \& M$ website ran on a web server and as such was accessible to the public. To restrict access to parts of the study a "beta password" was used by the experimenter. The password was required to submit the form on the "Sign-up" page and to access the "Feedback" page towards the end of the experiment. The inclusion of this password meant that while members of the public could see the website they could not sign-up to an account. It also allowed participants to sign into their account from home, but meant they were unable to continue the experiment without the experimenter present. At home, participants would simply see a progress bar which indicated that feedback about their photograph was slowly being 
gathered as the next experimental session approached, or a full bar indicating that feedback was ready.

Banner ads and about section. To add to the realism of the M\&M paradigm, horizontal banner advertisements were added to the bottom of every page. There were seven banner ads in total which were picked at random for display. The banner ads had no link attached to them and were for neutral products such as web hosting and camping equipment. The website also featured an "About" page. This page included information about the bogus function of the website (i.e. to rate others and to be rated) along with a message saying that the website planned to launch next year following beta testing. Again, this was to add to the realism of the website and was visible to any site visitor.

Remove me. There was a "Remove me" link featured on the "My Page" hub. The purpose of which was to allow participants to log-in and remotely remove themselves from the study. This could be done from any PC with internet access and was included specifically for participants who may have changed their mind between study sessions and no longer wanted their image to be posted online. The website was set up in such a way that the experimenter was notified if anyone withdrew, allowing participants to be contacted and debriefed. No participant requested to be removed in either Experiment 1 or 2.

\section{Stimuli.}

Rated pictures. For this experiment 30 pictures of women were rated by the participants on the "Rating" page. The pictures were taken from the public domain website hotornot.com where they were independently rated for attractiveness by members of the public. The average attractiveness of the pictures was $7.94(S D=1.49)$, and each picture's rating was formed from at least 1000 votes. To be included in the study, pictures had to: a) be unedited (e.g. no enhancement or grey-scale effects); b) contain no indicators of country of origin (e.g. plug sockets on a wall, country flags, or car license plates); c) show the model's face clear from objects (e.g. sunglasses and costumes); and d) not be revealing (e.g. featuring bikinis or topless images). The images were sourced from the age 18-25 bracket of hotornot.com, which is why this information was included alongside each picture when presented to the participant. The name of a local city was also displayed with each photograph to give the impression that the models were from a university within Wales. The presentation of each city was randomised, although the probability of each appearing was weighted by its population 
(Bangor, 2.5\%; Cardiff, 45.6\%; Newport, 19.7\%; St. David's, 0.2\%; Swansea, 32.0\%; Wikipedia, 2012).

Feedback pictures. Fifty pictures of women were used on the "Feedback" page. These were also taken from hotornot.com. The average attractiveness of the set was $8.44(S D=0.96)$ and each picture's rating was formed from at least 1000 votes. The pictures were content filtered using the same criteria from the previous section. None of the pictures used during the feedback task were used during the rating task and vice versa.

Each picture was presented with a bar chart containing two bars ranging from one to ten in height. The first bar was labelled 'Other guys' and the second was 'You'. These values were presented to the participant as if they had come from the model in the picture they were currently viewing. The value for these bars was generated on page load by php and Adobe ${ }^{\circledR}$ Flash $^{\circledR}$ coding. The "Other guys" bar was intended to give the participant an idea of the average rating the model gave to other men. This value was formed by adding a random integer between 5 and 7 with a random decimal between one and nine (giving a range of 5.1 to 7.9 ).

In contrast the "You" bar was included to give the participant false feedback about their individual attractiveness, or relative attractiveness when contrasted with the "Other guys" bar. In the negative feedback condition, the participants received a "You" bar score which was lower than the "Other guys" bar 95\% of the time. This was always a whole number and was created by randomly subtracting two or three from the "Other guys" bar while ignoring any decimal point (e.g. 7.2 would become either 5.0 or 4.0). This resulted is a decrease of between 2.1 and 3.9 in comparison to the "Other guys" bar. The remaining 5\% of the time, the "You" bar score was calculated by randomly adding zero or one to the value of the "Other guys" bar, again with the decimal ignored (e.g. 7.2 would become 7.0 or 8.0). This resulted in a change ranging from a 0.9 decrease to a 0.9 increase in comparison to the "Other guys" bar.

In the positive feedback condition, the parameters were designed to try to mirror those of the negative condition. The "Other guys" value was formed by removing a random decimal between one and nine from a random integer between 4 and 6 (giving a range of 3.1 to 5.9). In $95 \%$ of the cases two or three points were added to this value (while ignoring the integer) to form the "You" bar (e.g. 4.2 would become 6.0 or 7.0). In the remaining $5 \%$ of cases zero or one was subtracted instead, leading to a slight 
decrease or increase (e.g. 4.2 would become 4.0 or 3.0). An example of this feedback can be found in Figure 3.1 above.

\section{Measures and equipment.}

Questionnaires. The standard demographic questionnaire (Appendix E) was used in Experiment 1. This was an older version of the form which did not have questions about the menstrual cycle, self-perceived attractiveness, and socio-economic status. The Socio-sexual Orientation Inventory - Revised (SOI-R; Penke \& Asendorpf, 2008) was also included and acted as the main dependent variable (Appendix B). Two bogus questionnaires, unrelated to the research hypothesis, were also used. These were shortened versions (first ten and 15 items respectively) of the Launay-Slade Hallucination Scale (LSHS-R; Launay \& Slade, 1981), a measure of predisposition to hallucination-like experiences, and the Positive Affect Negative Affect Schedule (PANAS; Watson, Clark, \& Tellegen, 1988), which measures the intensity of an individual's positive and negative mood states. A copy of these shortened measures can be found in Appendix I. These bogus questionnaires were included to try to mask the true dependent measure of the study. The responses to these questionnaires were not coded or analysed. Finally, a post-debrief questionnaire (Appendix J) was created to assess whether or not participants believed the feedback was real during the study. Participants were asked to indicate how much they believed they were completing real market research before and after the feedback was administered, as well as whether they still believed the feedback was real following debrief. This final question was included to check if the debrief was successful.

Consent and debriefforms. There were two consent forms and two debrief forms for this experiment. One of the consent forms was real and the other was bogus. The purpose of the bogus consent form is described below in the procedure sub-section. Of the two debrief forms, one was sent to the participant if they withdrew from the study remotely, while the other was given to the participant if they successfully completed the study. The former debrief form did not need to be used. Copies of these forms can be found in Appendix $\mathrm{J}$.

Market research forms. Two bogus market research forms (Appendix K) were created which were used in different phases of the experiment to add to the believability of the dating website. The forms asked whether participants liked the website, would recommend it to a friend, and would use it in the future. 
Equipment. The false feedback paradigm was hosted on a web server, thus allowing access via any PC with an internet connection. A JavaScript-enabled browser, and Adobe ${ }^{\circledR}$ Flash $^{\circledR}$ Player were required for the feedback elements of the website. In this experiment participants used a laptop running Mozilla ${ }^{\circledR}$ Firefox ${ }^{\circledR}$ version 3.5 on Windows ${ }^{\circledR}$ XP Home Edition with the desktop wallpaper displaying the M\&M logo. To add to the believability of the study, business cards were printed with the M\&M logo and website address on them. Also printed was the contact email address of the experimenter as well as designated spaces for participants to write their chosen username and password. This gave participants all the information they needed to allow them to access the site from home between tests sessions.

\section{Design}

This was a mixed-model design. Participants completed the SOI-R (dependent variable) at two time points to form the within-subjects variable of time. Between these two measures, participants were randomly assigned to receive either positive or negative attractiveness feedback. This functioned as the between-subjects variable of condition (independent variable). The data was analysed using a repeated measures ANCOVA to allow for the inclusion of covariates.

\section{Procedure}

Participants were recruited via a University-wide email and through the participant panel of the psychology department. The study was advertised as a market research study for 18-25 year old males which involved uploading a photograph online, having it rated, and receiving feedback:

Members of the psychology department are currently looking for volunteers to participate in some market research on a new social networking and dating website. This will involve viewing and rating pictures of other individuals, as well as having a photograph of yourself uploaded online to be rated by others. It was also stressed that participants would be asked questions about their sexual attitudes and beliefs. Participants were offered $£ 5$ or four course credits for their participation. Applicants for the study were signed up via email to two test sessions set one week apart, and were ask to bring a profile photograph on a USB stick. The photograph criteria given to participants were the same as that used to select images of models (e.g. unedited).

Session one. In the first test session participants were seated in the laboratory and the experimenter discussed with them both the selection criteria and the estimated time the experiment would take to complete (40 minutes over two sessions). If 
participants confirmed they understood this, and were still willing to participate, a consent form was signed and retained by the experimenter. The photograph which the participant brought for use in the study was then checked for suitability by the experimenter. In all cases the photographs were found to meet the study criteria.

Participants were told that they would be participating in two small studies. The first was a market research study which involved testing a new social networking system. The second was a questionnaire study. Participants were told that the second study was being undertaken on behalf of several members of the psychology department, and was combined with the "market research" study as it was short in nature and also required two study sessions. In reality, this explanation was simply a cover story to make the dependent variable, the SOI-R, seem unrelated to the market research.

The details of the first study were then explained to the participants. The experimenter opened a web browser and navigated to the hotornot website (hotornot.com) to show users the rating interface. At the time, the hotornot website allowed any member of the public to anonymously view the photographs of random members and rate them for attractiveness. The member would then see an overall average score as judged by the online community. Participants were told that they would be testing a similar system only, instead of feedback about their photograph being anonymous, they would be able to see specific feedback from individual users. The experimenter then brought up the M\&M homepage, and clicked the 'sign-up' button.

Participants were asked to complete the sign-up form, which included choosing a username and password. The experimenter suggested that the participant use their Swansea University student number as a password. Once participants reached the 'city' field of the form, they were stopped and warned that they may encounter someone they knew when using the system and, if they did, to tell the experimenter. Participants were told this to add to the believability of the paradigm. In reality, all pictures were taken from a website with predominantly U.S. users. As such, the chances of a participant seeing someone they knew were negligible. Once the participant had completed the sign-up form, the beta-password was entered by the experimenter and an account was created. The experimenter wrote the participant's username and password on an M\&M business card. The card was then given to the participant. 
After participants signed up to an account they were directed to the "My Page" hub which prompted them to upload a profile photograph. This was completed with assistance from the experimenter. Following this the "Rating" page was accessible. The experimenter informed the participants that in order for them to receive feedback about their attractiveness, they would need to rate some other individuals who were also taking part in the market research study. Participants engaged in the rating task in private with the experimenter sitting outside the room.

Once participants notified the experimenter that they had finished the rating task, they were issued with the first set of bogus market research forms to complete. Participants then logged out of their M\&M account. The experimenter reiterated to the participants that, over the coming week, their picture would be viewed online by other users in the market research study. In case participants changed their mind over the course of the week, the experimenter explained how they could log-in between sessions and remove themselves from the study remotely.

The experimenter then introduced the details of the "second study" using the cover story that the questionnaires were newly developed and needed provisional testing. Participants were warned that some of the questionnaires contained items of a sexual nature and were asked if they wanted to proceed. If participants agreed, they were asked to sign the bogus consent form which was retained by the experimenter. The purpose of this was to convince the participants that the questionnaires constituted a separate study. The participants were then issued with the demographic form, SOI-R, PANAS, and L-S in a random order as well as an envelope marked with their participant number. The inclusion of the envelope was to preserve confidentiality; once the participant had completed the questionnaires they were asked to seal them inside the envelope and write their signature over the seal. Due to the sensitive nature of the SOI$\mathrm{R}$, the experimenter left the participant alone to complete the questionnaires.

Session one was concluded by confirming a time slot for the second session. Participants were given the opportunity to ask any questions, thanked, and excused from the lab. Each participant's data envelope and consent forms were then stored in a locked filing cabinet. If a participant logged into their account between test sessions they would see two things on their "My page" hub: a) a link which would allow them to remove themselves from the study; and b) a progress bar marked 'gathering feedback' which filled relative to the time left before the next experimental session. 
Session two. Prior to the arrival of the participant, the website settings were checked to make sure that "My page" would give access to the feedback system when the participant logged in from the lab computer. Once the participant arrived they were welcomed, reminded of the format of session one, and talked through the format of session two. Participants were then asked to log into the M\&M system. If they had forgotten their username and password, the experimenter excused himself from the room to 'phone the site administrators'. (In reality the username and password was recovered from the website database using a different computer.) After the participant logged-in, the experimenter explained that they had 50 pieces of feedback to view. The feedback system was outlined to the participant who was also told that, due to the personal nature of the feedback, the experimenter would leave the room while they viewed it. Once the participant understood the feedback process, the beta password was entered by the experimenter to grant access to the "Feedback" page.

After the participant had viewed all of the feedback, they were shown the summary page. At the bottom of this page, participants were encouraged to call for the experimenter. The experimenter re-entered the lab and gave the participant the second bogus market research form to complete. Following this, the participants were thanked for their participation in the market research and reminded that they needed to complete a few more questionnaires for the "second study" before leaving.

For the second part of the second study participants were told that they, once again, were going to complete some questionnaires. They were told that some of the questions might be similar to those shown in the previous session, however, slightly different versions were being tested to establish validity. In reality the questionnaires were identical. The experimenter also reminded the participants of the sexual nature of some of the questions. All participants were willing to proceed.

The experimenter left a version of the SOI-R, PANAS, and L-S (this time with the number two written in the top corner of each form), in a random order, with the participant and asked to be called once they had completed them. The experimenter excused themselves from the room to allow the questionnaires to be completed in private. Upon returning, the experimenter showed the participant their sealed brown envelope from session one. This was opened in front of them, and its contents, as well as the newly completed questionnaires, placed into a fresh envelope. This was to reassure the participant of the anonymity of their responses. 
The second session was completed with a full verbal debrief making it clear to the participant that: a) the feedback they received was fictitious; b) their photograph was not viewed by any other individual; and c) the "second study" was actually a dependent measure. Participants were also given the printed debrief form. Following debrief, participants were asked if they were happy to have their data used and if they would be willing to complete the post-debrief questionnaire. All participants agreed to have their data used and to fill in the additional form. The post-debrief questionnaire was sealed away with the participant's data in the envelope upon completion. This envelope was then marked with a single letter to signify the feedback type ( $\mathrm{P}$ for positive and $\mathrm{N}$ for negative) and a participant number.

Finally, all participants were issued their course credit or cash payment and were asked not to reveal the true nature of the experiment to other potential participants. The participant was shown out of the lab and the experimenter then deleted their account and profile picture from the web server.

\section{Results}

Seven participants were excluded from the analysis. Three of these participants were homosexual, and four indicated that they did not believe that the feedback they received was real. (They answered one to three for the third question of the postdemographic form.) This led to a final sample of 33 men.

\section{SOI-R Change}

The average SOI-R score was $40.15(S D=11.58)$ with no difference between negative and positive feedback conditions, $t(31)=0.454, p=0.45$. The SOI-R variable was normally distributed while its individual subcomponents were not (KolmogorovSmirnov and Shapiro-Wilk tests both $p<0.05)$. As such, while parametric analysis was used for the SOI-R, non-parametric analysis was used for its subcomponents. Due to there being no neutral feedback condition, the positive and negative conditions were analysed independently with differences from baseline tested for significance.

Negative feedback. A repeated measures ANCOVA revealed no significant change to SOI-R over time, $F(1,17)=2.086, p=0.92 .{ }^{17}$ Several covariates were entered into the analysis including age, relationship status, and education, as well as selfreported enjoyment of the M\&M system and whether the participants felt their feedback was accurate (questions taken from the bogus market research forms). None of these

\footnotetext{
${ }^{17}$ The p-value of the two-tailed test was 0.17 with an effect in the incorrect direction. Thus, this value was halved and subtracted from one to give a one-tailed p-value.
} 
were significant covariates $(p>0.24)$. Three behavioural sub-components of SOI-R were examined using Wilcoxon signed-rank tests. Changes to the behaviour and desire components were non-significant ( $p s>0.72$ ). A change in the SOI-R attitude component was non-significant in terms of the one-tailed hypothesis $(Z=-1.832, p=$ 0.97). However, this would have been an increase significant to the $p=0.07$ level had a two-tailed test been used.

Positive feedback. A repeated measures ANCOVA revealed no significant change to SOI-R over time, $F(1,14)=0.105, p=0.62$. The same covariates were entered as in the analysis of the negative condition. Once again none of these were significant $(p>0.16)$. Wilcoxon signed-rank tests revealed no significant change to the SOI-R subcomponents following positive feedback ( $p s>0.84)$.

\section{Post-debrief information}

Information from the post-debrief questionnaire was used to assess how convincing the paradigm was. On a scale of one to nine, participants generally reported that, at the end of session one, they felt the $M \& M$ website was real $(M=8.21, S D=$ 0.99 , Range $=5-9$ ), and that their photograph was actually online and being rated by others $(M=8.39, S D=0.86$, Range $=6-9)$. Participants who indicated that they did not believe the feedback was real (those who selected one to three) were specifically excluded from the analysis. Of the remaining participants, reported believability was high $(M=7.27, S D=1.46$, Range $=4-9)$, as was reported truthfulness when responding to the SOI-R $(M=8.51, S D=0.75$, Range $=6-9)$. The debriefing proved successful as continued believability was low $(M=1.63, S D=1.43$, Range $=1-6)$. Participants also appeared to have enjoyed participating in the study as reported enjoyment was high $(M$ $=7.93, S D=0.83$, Range $=6-9$ ).

Only one of the above measures differed by condition. Participants in the negative condition reported being slightly less truthful on the SOI-R than the positive group, $t(31)=-2.079, p=0.05$. However, the average for the negative group was still $8.28(S D=0.89)$, and so this difference was considered unlikely to have any meaningful effect on the results.

\section{Discussion}

Experiment 1 failed to find support for either Hypothesis 1 or 2, as neither Prediction 1 nor Prediction 2 were correct. In fact, in the negative feedback condition there was an increase in the attitude component of the SOI-R which would have 
approached significance had a two-way hypothesis has been used. This would have been in direct contrast of Prediction 2

As discussed in Chapter 2, there are several issues with measures of socio-sexual orientation which suggest they may not be the best measure of mating strategies within an experimental context. These include the measure being linked to past behaviours and the small number of questions which makes it easier to remember previous responses. To address these concerns, the experiment was repeated using the newly developed SMA task to produce dependent variables.

\section{Experiment 2}

Experiment 2 was a replication of Experiment 1 with some minor changes and an increased sample size. Once again, an all-male sample was used. The experimental hypotheses remained the same, but different predictions were made to take into account the new measure. Prediction 3 stated that, following negative attractiveness feedback, men would choose fewer models for a ST, and a greater number of models for a LT relationship on the SMA task. Prediction 4 stated that, following positive attractiveness feedback, men would choose a greater number of models for a ST, and a fewer number of models for a LT relationship on the SMA task.

\section{Method}

\section{Participants}

Eighty male participants were tested in the second phase of the experiment and 12 of these were excluded from the analysis (see results section). The following demographic details reflect the remaining 68 participants whose data were analysed. Participants were undergraduate $(n=62)$ or postgraduate $(n=6)$ students at Swansea University who participated for payment or course credit. The mean participant age was $20.82(S D=1.68)$, and 93\% $(n=63)$ described themselves as Caucasian, while the remaining $7 \%$ consisted of participants who were Black $(n=2)$, Middle Eastern $(n=1)$, South Asian ( $n=1)$, and "Other" ( $n=1$; the participant did not elaborate on their ethnicity). The participants were childless and there were only two non-heterosexual men. These two men described themselves as bisexual and so were included in the analysis. Just under half of the participants were single or in an uncommitted relationship ( $n=33)$, while the remainder $(n=35)$ were in a committed relationship lasting longer than three months. The participants were typically from a middle socioeconomic background, averaging $3.32(S D=0.72)$ on a one to five scale ranging from upper to lower. Perceived attractiveness relative to peers was rated as 5.9 ( $S D=$ 
1.27) on average using a one to nine scale and the average SOI-R score was $40.46(S D=$ 11.99).

\section{Materials}

The paradigm remained largely unaffected apart from the changes listed in the following sub-sections.

Mix\&Match website. There was only one change made to the M\&M paradigm. On the "Rating" and "Feedback" pages instead of the locations which appeared next to each model being selected from one of five Welsh cities, this was expanded to eight cities which contained a major University. These included Exeter, Coventry, and Bath. Once again, the chance of each of these cities being displayed was weighted by their population size (Wikipedia, 2012).

SMA stimuli. The 50 pictures used in the SMA task were drawn from a pool of 339 images gathered from the hotornot.com website. None of these were used in Experiment 1 and for added reliability they were independently rated for physical attractiveness on a scale between one and nine by ten male and ten female judges (age $M=21.75, S D=2.12$ ). The pictures were gathered using the same criteria used in Experiment 1. The subsample of 50 images used for the task had an average attractiveness of $5.01(S D=1.57)$. This was markedly lower than the ratings given for the pictures in the first experiment. However, ratings on hotornot.com were negatively skewed, perhaps because individuals with low scores tended to remove their pictures from the site. A rating of five was about average for the total pool of photographs taken from the site when independently rated.

M\&M Stimuli. Thirty pictures were chosen for participants to rate during the first session. These were selected from the large sample of photographs mentioned above. The average attractiveness was $5.01(S D=1.60)$. The fifty pictures chosen to be presented with feedback during the second session had an average attractiveness of 5.01 $(S D=1.48)$. There was no repetition of models between the SMA, rating and feedback tasks.

Measures and Forms. In order to account for the extra time needed to complete the SMA task, the LSHS-R and PANAS were removed from the second experiment. Two questions were also added to the demographic form used in Experiment 1 (Appendix E). One measured self-perceived attractiveness and the other measured socio-economic status. These two questions remained in the demographic form for all subsequent experiments. Finally, the bogus consent form was modified to reflect the 
fact that participants would be completing the SMA as part of the "second experiment" rather than just questionnaire measures (Appendix $\mathbf{J}$ ).

\section{Design}

The experimental design remained the same, only the dependent variables were now derived from performance on the SMA task (ST and LT choices). Primary analyses used non-parametric chi-squared tests to explore main effects and ordinal logistic regression to allow for the analysis of covariates.

\section{Procedure}

The only change to the procedure surrounded the bogus "second" study which was now primarily a computer task rather than a questionnaire one. Participants were told that the SMA measure was a new task designed by the psychology department to measure relationship preferences that needed to be tested for validation. During the second performance of the SMA task participants were warned that they may recall some of the models featured in the task from the previous session, but that the majority would be different. In reality they were shown the exact same set of models. However, participants were given this warning so that they would be less likely to raise a concern with the experimenter during the measurement should they have recalled models from the previous session.

\section{Results}

The data from 12 participants were excluded as they indicated that they did not believe that the feedback they received was real (they answered one to three on the third question of the post-demographic form). This led to an analysed sample of 68 men.

\section{SMA Change}

For this experiment, there was no neutral condition to use as a control. As such, the negative and positive conditions were analysed independently by testing change to relationship choice category against that which would be expected by chance using chisquared analyses (see Chapter 2). Ordinal regression was then used to identify any related demographic factors. To facilitate these analyses two dummy variables were coded. The first was called STChange, where participants were given a 1 if they increased in their number of ST choices between sessions, a 0 if they did not change, and a -1 if they decreased. The same method was used to create LTChange from changes to LT ratings. Two ordinal models were then generated using an ordinal logistic regression to try to predict these two variables using a combination of covariates. 
Negative feedback. Table 3.1 displays the main effect chi-squared analysis conducted for the negative feedback condition. Following this feedback, participants were more likely to increase in the number of individuals chosen for a short-term relationship. Thus, this relationship was not significant in terms of the one-tailed hypothesis, $\chi^{2}(2)=4.800, p=0.95$, although this would have approached significance had a two-tailed hypothesis been used $(p=0.09)$. In terms of long-term relationship selection a general decrease was found, which again was not significant in terms of the one-tailed hypothesis, $\chi^{2}(2)=5.452, p=0.97$, but would have approached significance had a two-tailed hypothesis been used $(p=0.054)$.

Table 3.1

Change to short- and long-term relationship choices following negative attractiveness feedback. The change has been split across three ordinal categories. These distributions were then tested against that which would be expected by chance using chi-squared analysis

\begin{tabular}{|c|c|c|c|}
\hline \multicolumn{4}{|c|}{ Short-term change } \\
\hline & - & $=$ & + \\
\hline Actual & 9 & 4 & 21 \\
\hline Predicted & 15 & 4 & 15 \\
\hline$\chi^{2}(2)$ & 4.800 & & \\
\hline Cramér's $V$ & 0.19 & & \\
\hline \multicolumn{4}{|c|}{ Long-term change } \\
\hline & - & $=$ & + \\
\hline Actual & 21 & 5 & 8 \\
\hline Predicted & 14.5 & 11 & 14.5 \\
\hline$\chi^{2}(2)$ & 5.828 & & \\
\hline Cramér's $V$ & 0.21 & & \\
\hline
\end{tabular}

Ordinal logistic regression analyses were used to explore any potential interaction of demographic variables with the negative feedback. Several variables were used in the analysis as potential covariates. These included age, relationship status, perceived attractiveness, SES, and SOI-R. From the bogus market research forms a 
measure of self-reported enjoyment and feedback accuracy (whether the participant agreed the positive or negative feedback reflected their attractiveness) were included. Two significant predictors emerged in relation to ST change: relationship status and attractiveness (Table 3.2). Compared to single individuals, those in a relationship were 15 times more likely to belong to the $\mathrm{ST}=$ or ST+ categories, Wald $\chi^{2}(1)=8.222, p<$ 0.01 . When it came to the participant's self-reported attractiveness, the chance of appearing in the ST= or ST+ group decreased by $150 \%$ for every one point decrease in attractiveness, Wald $\chi^{2}(1)=4.786, p=0.03$. Overall the model was significant and accounted for a large percentage of variance in the data. No significant model was found for LT change.

Table 3.2

An ordinal logistic regression model predicting ST change category using self-reported attractiveness and relationship status

\begin{tabular}{lcc}
\hline \multicolumn{3}{c}{ Model ST } \\
\hline & $\beta$ & OR \\
\cline { 2 - 3 } Relat: Committed & $2.710 * *$ & 15.03 \\
Relat: Single & - & - \\
SPA & $-0.922 *$ & 0.4 \\
\hline Model & $\chi^{2}(2)=13.395^{*}$ \\
Nagelkerke $R^{2}$ & 0.39 \\
Accuracy & $76.5 \%(+23.8 \%)$ \\
Parallel lines & $\chi^{2}(2)=1.176$ \\
\hline
\end{tabular}

Note: Variables were coded as such: Relat: Committed (1), Relat: Single (0). Relat = relationship status. $\mathrm{SPA}=$ self-perceived attractiveness. $\mathrm{OR}=$ odds ratio. $^{\dagger} p<0.10,{ }^{*} p<0.05, * * p<0.01$.

Positive feedback. Table 3.3 displays the SMA category changes following positive attractiveness feedback. There was no overall effect of the feedback on either ST or LT choices ( $p$ s > 0.57).

Table 3.3

Change to short- and long-term relationship choices following positive attractiveness feedback. The change has been split across three ordinal categories. These distributions 
were then tested against that which would be expected by chance using chi-squared analysis

\begin{tabular}{|c|c|c|c|}
\hline \multicolumn{4}{|c|}{ Short-term change } \\
\hline & - & $=$ & + \\
\hline Actual & 13 & 5 & 16 \\
\hline Predicted & 14.5 & 5 & 14.5 \\
\hline$\chi^{2}(2)$ & 0.310 & & \\
\hline Cramér's $V$ & 0.05 & & \\
\hline \multicolumn{4}{|c|}{ Long-term change } \\
\hline & - & $=$ & + \\
\hline Actual & 12 & 8 & 14 \\
\hline Predicted & 13 & 8 & 13 \\
\hline$\chi^{2}(2)$ & 0.154 & & \\
\hline Cramér's $V$ & 0.03 & & \\
\hline
\end{tabular}

Ordinal regression analyses revealed no significant effect of the covariates on ST category. However, a significant model was revealed on LT relationship category (Table 3.4). Specifically, a participant's chances of appearing in the LT $=$ or LT + group dropped by $67.8 \%$ for every one point decrease in self-perceived attractiveness, Wald $\chi^{2}(1)=3.986, p=0.05$.

Table 3.4

An ordinal logistic regression model predicting LT change category using self-reported attractiveness

\begin{tabular}{|c|c|c|}
\hline \multicolumn{3}{|c|}{ Model LT } \\
\hline & $\beta$ & $\mathrm{OR}$ \\
\hline SPA & $-0.518 *$ & 0.60 \\
\hline Model & \multicolumn{2}{|c|}{$\chi^{2}(1)=4.315^{*}$} \\
\hline Nagelkerke $R^{2}$ & \multicolumn{2}{|l|}{0.13} \\
\hline Accuracy & \multicolumn{2}{|c|}{$47.1 \%(+14.3 \%)$} \\
\hline Parallel lines & \multicolumn{2}{|c|}{$\chi^{2}(1)=0.11$} \\
\hline
\end{tabular}

Note: $\mathrm{SPA}=$ self-perceived attractiveness. $\mathrm{OR}=$ odds ratio. ${ }^{\dagger} p<0.10, * p<0.05, * * p<0.01$. 
Post-debrief information. On a scale of one to nine, participants typically reported that, at the end of session one, they felt the $M \& M$ website was real $(M=7.49$, $S D=1.71$, Range $=2-9$ ), and that their photograph was actually online and being rated by others $(M=7.71, S D=1.33$, Range $=4-9)$. Participants who indicated that they did not believe the feedback was real (those who selected one to three) were specifically excluded from the analysis. Of the remaining participants, average reported believability was high $(M=7.03, S D=1.66$, Range $=4-9)$, as was self-reported truthfulness when responding to the SOI-R $(M=8.26, S D=1.13$, Range $=3-9)$. Between subjects $t$-tests were used to see if the samples from Experiments 1 and 2 differed on these variables. These tests revealed a reduction in how convinced participants were at the end of session one that the website was real, $t(102)=1.877, p=0.06, d=0.37$, and that their photograph was online and being rated, $t(102)=2.526, p=0.01, d=0.50$. However, no difference was found between the groups chosen for the analyses in terms of whether they thought the feedback in session two was real, $t(102)=0.846, p=0.40$.

\section{Discussion}

No clear support was found for Hypotheses 1 and 2 in Experiment 2. In the negative feedback condition there was no overall effect of the false feedback on the ST and LT category distributions in line with Prediction 3. On the contrary, rather than showing a bias towards increasing LT choices and decreasing ST choices, the opposite pattern was found in both cases. These changes would have approached statistical significance had a two-tailed test been used. When ordinal regression models were employed to test for the influence of covariates, relationship status was found to have a large effect, with individuals in committed relationships showing a large increase in ST choices following the negative feedback. There was also an influence of perceived attractiveness, with less attractive individuals being more likely to increase their number of ST choices. There was no significant predictive model found for the LT choice categories.

In the positive feedback condition, there was no overall effect of feedback on category choice for either the LT or ST change distributions. This was contrary to Prediction 4. A significant ordinal regression model was found for the LT category distribution which revealed that those who were low in attractiveness were more likely to select models for a LT relationship after the positive feedback.

\section{General Discussion}


Overall, the results of these two experiments failed to support the evolutionarybased hypotheses developed at the start of this chapter. In fact, the results appeared to be contradictory in the case of negative attractiveness feedback as men showed an increase in ST interest and a decrease in LT interest. These contradictory results do not necessarily undermine the overall working hypothesis of the thesis. Rather, they support the idea that human mating decisions can be flexible, but perhaps not in a manner which could be regarded as adaptive from an evolutionary psychological perspective. That said, literature exists from the areas of personal relationships and social psychology which could shed some light on the results found here. These are discussed in the following section, and are followed by methodological considerations and implications for the thesis as a whole.

\section{The Other Way Around?}

Considering the derogation of alternatives literature, one could conclude that the converse extreme is more likely: Men evolved to pursue long-term pair-bonding, only opting for short-term sexual relationships if they failed in this strategy (Eastwick, 2013, p. 186).

In response to a recent publication on the extent of human sex differences (Stewart-Williams \& Thomas, 2013b), Eastwick draws attention to several relevant areas of the close relationship literature. This branch of psychology often produces research which is supportive of evolutionary theory, integrates traditional attachment theory (Salter Ainsworth \& Bowlby, 1991), and has great methodological rigor. However, as Eastwick discusses, such literature is often times not combined with evolutionary psychology and acts almost as a separate entity. One relevant finding from the area is an effect called the derogation of alternatives (R. S. Miller, 1997; Rusbult \& Buunk, 1993) in which individuals in close pair-bonded relationships experience a change in psychology leading them to show disinterest in alternative partners. The view here, at least according to Eastwick, is that rather than long-term mating being a case of men "making the best out of a bad situation" because they cannot pursue short-term relationships, the opposite is actually the case. That is, a long-term pair-bond is actually the optimal human mating strategy and short-term relationships are a consequence of an individual becoming "stuck" in the initial phase of a relationship due to their inability to form or maintain a long-lasting relationship. This view is somewhat at odds with Sexual Strategies Theory and Strategic Pluralism Theory (Buss \& Schmitt, 1993; Gangestad \& Simpson, 2000), however, Eastwick does acknowledge that maintaining sequences of short-term mating can be adaptive. On page 188 for example he states 'Rather, 
attractive men's short-term mating successes are accrued during periods of time when they are in the initial stages of relationships and not pair-bonded'. Thus, this view of long-term (later-stage) relationships being developed via short-term (initial-stage) ones could be reconciled with evolutionary theory, as opposed to alternatives such as Attachment Fertility Theory which deny any evolutionarily adaptive benefit to shortterm mating (L. C. Miller, Pedersen, \& Putcha-Bhagavatula, 2005; Stewart-Williams \& Thomas, 2013b).

In light of the derogation of alternatives effect, one interpretation of the results could be that the negative attractiveness feedback led to a decreased interest in a longterm relationship by undermining the participant's confidence in their ability to maintain one. This may well explain why such a large effect of relationship status was observed in the ST ordinal model of the negative feedback condition. Men within relationships were much more likely to belong in the ST= and ST+ categories following negative feedback compared to single men. If negative feedback tells men about their ability to maintain a pair-bond, then this may have been more relevant to those already within a relationship. This interpretation is speculative but could be tested by revising the paradigm to include measures of self-esteem (see below) or a measure of mating self-efficacy (such as the SPMS introduced in Chapter 2) that was adapted to be sensitive to changes following an experimental intervention. In the absence of these measures, and the fact that the stated hypotheses were one-tailed and well-grounded in the evolutionary literature, the conclusion from these experiments is that the hypotheses were not supported and that the results cannot be explained satisfactorily from a SST perspective.

There are two other explanations for the pattern of results which use the theories from the sociological and social psychological literature. First, according to the overcompensation thesis (Willer, Rogalin, Conlon, \& Wojnowicz, 2013), men who have their masculinity threatened are subsequently more likely to temporarily "rebound" and show hyper masculine traits such as supporting war and wanting to climb status hierarchies - an effect not found in women. In this sense the results found here could be due to an initial "rebound" reaction by the participants in response to being told they are unattractive, or more specifically that they lack the attractiveness to pursue a short-term strategy. Thus, rather than becoming more long-term orientated, men temporarily showed a greater desire for short-term relationships. It is unlikely that this effect would persist in the face of consistent feedback; seeking an inappropriate strategy, despite 
continuous environmental signals that it would be unproductive compared to alternatives, is unlikely to be an evolutionarily stable behavioural tactic. As such, mating strategy cues which directly challenge an individual's perception of their own attributes may produce different results if the participant was exposed to them over several test sessions. One weakness of using the overcompensation thesis to explain the results is that it cannot account for the predictive effect of relationship status that was found within the ordinal regression model.

Second, some sociometer studies show that unexpected rejection can lead to feelings of aggression among participants (for example in the amount of hot sauce given to a partner; Wesselmann, Butler, Williams, \& Pickett, 2010). Although our participants indicated enjoyment at participating in the experiment at the post-debrief stage, if frustration or anger was developed in the negative condition due to rejection then that may well have influenced responses on the SMA task. Such an effect could also be potentially overcome in a similar manner to the overcompensation effect by administering the feedback over several sessions to remove any temporary effects. Such long-term administration, however, may be hard to implement ethically.

\section{Perceived Attractiveness}

In the ordinal models of Experiment 2, there was a small effect of participant self-reported attractiveness. In the ST model (Table 3.2), males who were more attractive were less likely to appear in the ST= or ST+ categories. While the explanation of the main effect is not clear (see previous section), it appears as if this was partially counteracted by participant attractiveness. This may have been because attractive individuals were less impacted by the negative feedback compared to unattractive individuals due to their increased self-confidence. As for the LT model (Table 3.4), attractive individuals were found to be less likely to appear in the LT+ categories. This could potentially be interpreted as support for part of Prediction 4 among a sub-group of attractive individuals. This is because even if there was no increase in ST interest a decrease in LT interest would lead to a more ST orientated mating strategy overall. However, given that there was no overall main effect, and that the negative condition produced inconclusive results, this interpretation should be approached cautiously.

\section{Methodological Considerations}

There were three main methodological considerations for this study. First, when considering the literature on sociometer theory and the derogation of alternatives effect, it became apparent that self-esteem plays an important role in an individual's perception 
of their mate worth. As such, a measure of self-esteem such as the Rosenberg selfesteem scale (Rosenberg, 1965), or implicit measures such as the initial preference task (Dijksterhuis, 2004), would have been beneficial as it would have allowed: a) the effect of the false feedback on participants to be clarified; and b) the interaction between selfesteem changes and mating strategies to be investigated.

Second, compared to sister experiments related to ours (e.g. D. Bailey et al., 2011; Surbey \& Brice, 2007), this experiment was far more intricate and required a deeper level of deception. Part of the reason for this was due to confidence placed in these previous studies: the M\&M paradigm was designed to enhance the effects found in these studies by making the feedback more salient. However, this had the adverse effect of reducing the overall sample size. Although evolutionary psychology has led to some of the largest effect sizes in the individual differences domain (Lippa, 2009; Schmitt, 2005b; Stewart-Williams \& Thomas, 2013b), the effect of some experimental manipulations still remains quite small (e.g. Shan et al., 2012). There is always an argument to be made to increase the participant base for such research, especially when effects are small-to-medium in similar studies (Little, Cohen, et al., 2007; Shan et al., 2012). According to $G^{*}$ Power (Erdfelder, Faul, \& Buchner, 1996), for example, a total sample of 264 people would be needed to detect a small effect size of $\left(d_{z}=0.20\right)$ using a simple within-subjects $t$-test (assuming 90\% power and an $\alpha$-value of 0.05 ).

Third, despite having a good rationale for testing only men, including the clarity of predictions for the male sex based on previous research and the availability of resources for the experiment, the exclusion of women from the participant base may have reduced the likelihood of changes in mating behaviour being detected. This is because the mating behaviour of women has been shown to be more flexible (Baumeister, 2000), although this is not always the case, especially within a short-term context (Penke \& Denissen, 2008).

\section{Implications for the Thesis}

This chapter presented results which not only do not support the overall working hypothesis but provide contradictory evidence of a specific prediction based upon it. What implication does this have for the direction of the thesis? One of the most interesting aspects of the theory of natural selection is how several, very distinct, lines of evidence have been used to support the overall theory. This is present even in The Origin of Species where Darwin presents the reader with evidence from different areas of naturalism which are pieced together to build an unquestionable body of evidence 
(Darwin, 1859). From seeds and fossils, to geology and embryos, definitive proof of natural selection did not come from a single source but from a culmination of observations. Thus, it is not always advantageous to concentrate on one particular domain or methodology with a series of studies built upon each other in a linear fashion. Due to the lack of success across two experiments in administering false feedback to participants, which has the potential to lead to some unintended effects (as described above), it was decided to pursue different methods of manipulation in order to examine human mating flexibility. In the upcoming chapters, low-level "prime-like" stimuli, biochemical changes, and a more subtle form of false feedback are used to try to initiate an evolutionarily relevant mating strategy change.

\section{Conclusion}

Although the large amount of comparative work led to a clear prediction for the effect of attractiveness feedback in men. This does not necessarily mean that this would be the factor most likely to influence male mating strategies. In one sociometer based study in the European Journal of Social Psychology, Pass et al. (2010) found that men's self-esteem was more likely to be influenced by feedback regarding their social status rather than their physical attractiveness, with the reverse being true for women. Perhaps this indicates that the M\&M paradigm would be more likely to produce evolutionarily consistent results in women. Why might social status be a more important cue to men than physical attractiveness? In other, less socially intelligent animals, such as those mentioned at the start of this chapter, status may be entirely determined by physical attractiveness. ${ }^{18}$ Factors such as dominance, prestige, and wealth are also linked with social status in humans with physical attractiveness only representing part of the puzzle. Furthermore, men may be more sensitive to changes in their status as it is a transient property more likely to change over time, compared to physical attractiveness which is more static. In the next chapter, this idea is explored further by using simple wealthrelated cues to manipulate mating strategy in a mixed-sex sample, as well the use of cues from the domain of parental care.

\footnotetext{
${ }^{18}$ That being said, even gallus gallus females are not fazed by manipulated sexually selected ornaments indicating that their choice is determined by multiple behavioural and physical factors (Zuk, Ligon, \& Thornhill, 1992).
} 
Chapter 4: Parental and Wealth Stimuli Influence Human Mating Strategies. (Are Significant Others Significant?)

In Chapter 3, false feedback was used in an attempt to change the participants' perception of their attractiveness relative to other men. This manipulation led to results inconsistent with the working hypothesis, which may have been accounted for by temporary "reactive" effects, such as overcompensation, following negative feedback (Willer et al., 2013). In this chapter, a different type of manipulation is explored. Instead of providing participants with information about their own attributes, two different cues were used to passively signal information about the environment to the participant. The first was that of parental care, which is associated with long-term mating. The second was that of wealth, which is arguably more closely associated with short-term mating (e.g. short-term mating can aid resource acquisition in women; Greiling \& Buss, 2000).

In Experiment 3, participants were shown visual and auditory cues related to parental care in the form of "how-to" videos, while a simple visual slideshow of wealth cues was used in Experiment 4. Unlike the previous experiments, these also included a neutral condition as a control. A neutral condition is important as there could be an artefact of the experimental methodology which causes a change in SMA responses between sessions. For example, participants could select fewer individuals for long- or short-term relationships during their second exposure to the task simply because they are restless or not concentrating, rather than due to the experimental manipulation itself. Without a neutral condition as a reference point, such an effect may be mistakenly attributed to the manipulation rather than other extraneous factors.

The introduction to this chapter begins with a general overview of the literature surrounding mating strategies and parental care, followed by a similar review of the literature related to mating strategies and wealth. As with Chapter 3, this literature is used to develop specific one-way hypotheses as to how parental and wealth stimuli should affect mating strategies in both men and women. As the experiments in this chapter used both men and women as participants, sex differences are also acknowledged in these hypotheses. Finally, this introduction also discusses similar paradigms which have been used to show that brief exposure to overt cues can lead to psychological changes.

\section{Parental Care and Mating Strategies}


Humans are one of the $5 \%$ of mammalian species in which both males and females provide parental care (Geary, 2000). As discussed in Chapter 1, current thinking among paleoanthropologists attributes the selection pressure for bi-parental care (and other types of alloparental care) to the trade-off between the narrowing of the pelvic girdle and the increase in infant brain size, leading to children being born underdeveloped and completely dependent for several years (Martin, 1990; Wittman \& Wall, 2007). This would have led to the gradual reduction of sexual dimorphism in hominids over the past four million years (McHenry \& Coffing, 2000), indicating that altricial young had a very real impact on the typical mating arrangement of our ancestors over evolutionary time. Although as a species extant humans show great flexibility in their mating behaviour, the most common relationship arrangement in human societies is still that of social monogamy (Stewart-Williams \& Thomas, 2013b).

Within one individual's lifetime, a host of psychological changes accompany the transition into parenthood. For example, compared to non-parents, those who enter parenthood show an optimistic bias towards paternity certainty in men (Bressan \& Dal Pos, 2012), increased sensitivity to potential threats from strangers (Eibach \& Mock, 2011; Fessler, Holbrook, Pollack, \& Hahn-Holbrook, 2014), more efficient neurological facial processing of emotions in infants (Proverbio, Brignone, Matarazzo, Del Zotto, \& Zani, 2006), and increased activation in the area of the brain responsible for jointattention (Abraham et al., 2014).

Parents and non-parents also show differences in their levels of sex hormones such as testosterone (T). T, which is discussed in further detail in Chapter 6, is a potential mediator between environmental input and mating strategy change. It is positively associated with an increase in mating effort and risk taking behaviour, especially in males (Book, Starzyk, \& Quinsey, 2001; M. Peters et al., 2008; S. J. Stanton, Liening, \& Schultheiss, 2011), and so can be thought of as a potential proxy for short-term mating behaviour. ${ }^{19}$ Importantly, this association is bi-directional: lower T levels are associated with a reduction in these traits and a greater focus on parental care. Several studies have found that as men enter a relationship, become married, and have children, their T levels drop at each successive stage (Alvergne, Faurie, \& Raymond, 2009; P. B. Gray, Jeffrey-Yang, \& Pope, 2006; P. B. Gray, Kahlenberg, Barrett, Lipson, $\&$ Ellison, 2002) and the same is true for women (Kuzawa, Gettler, Huang, \& McDade,

\footnotetext{
${ }^{19}$ Interestingly, sociosexuality and T correlations are often weak or non-existent (Edelstein, Chopik, \& Kean, 2011; van Anders et al., 2007).
} 
2010). There are, of course, some additional factors which moderate this relationship (Gettler, McDade, Agustin, Feranil, \& Kuzawa, 2013; McIntyre et al., 2006).

The fact that these changes occur at all, calls into question their possible evolutionary function. Why, for example, are all humans not born "parent ready" with a sufficiently low T level to match $?^{20}$ Reflecting on Sexual Strategies Theory (Buss \& Schmitt, 1993; Greiling \& Buss, 2000), it is clear that while there are key benefits to short-term mating for both men and women, that such strategies are not always compatible with the successful rearing of young in the way long-term strategies are. The Hadza provide a good example. Among men, those who are from villages with a high number of available women spend more time engaging in mating effort and less time caring for their young (Marlowe, 1999a). The same population also show a "Cinderella effect", whereby fathers provide less care for their step-children than their biological ones (Marlowe, 1999b). This effect is known to be just as, or even more, prominent among casual partners, often referred to as "mothers' boyfriends" (Damashek, Nelson, \& Bonner, 2013; Margolin, 1992; Starling, Holden, \& Jenny, 1995). Both these effects indicate that the pursuit of a short-term mating strategy by a caregiver could hinder the development of children. This may not always be the case. As discussed in Chapter 5, there are some situations, such as times of great environmental harshness, whereby both types of mating strategies have a negligible impact on offspring survivability or quality of development. However, the general point is that there are clear benefits of both longand short-term strategies for both sexes, but the relative benefits of each can change during the transition into parenthood.

If the different types of mating strategy have different implications for child rearing, then it makes sense for men and women to be able to conditionally switch from a short-term strategy to a long-term strategy depending on which is more likely to enhance fitness (Gangestad \& Simpson, 2000). To revisit the evolutionary rationale, an individual who follows one type of mating strategy may not be as reproductively successful as an individual who is able to pursue a risky short-term strategy when single with no dependants and then switch to a long-term strategy when fitness would be best served by looking after dependants. The use of the word "dependants" rather than "offspring" in the previous sentence emphasizes an important point; such a strategy may

${ }^{20}$ This issue is further discussed in Chapter 6 when reviewing the "challenge hypothesis". 
also benefit individuals who have young family members by enhancing indirect fitness (Hamilton, 1964).

There are a few experimental studies which use parental cues to initiate changes, mainly hormonal in nature, in men and women. For example, in an experiment by Storey et al. (2000), men were exposed to a combination of parental cues including nursing a baby doll (or new born baby if they had recently become a father), smelling a blanket which had been in contact with a new born, listening to the sound of baby cries, and watching a video about successful breastfeeding. Together these elements formed one large "super cue" of parental care and men who felt engaged with the stimuli showed a significant drop in T levels, and a significant increase in prolactin levels, 30 minutes after exposure to the cue (p.88). Interestingly, this effect applied both to new fathers, who were regularly exposed to parental cues, and expectant ones. The fact that this effect existed in the latter group suggests that the hormones, and perhaps mating strategies, of non-parents may also be affected by such stimuli. In other studies, the effects of the individual elements of the "super cue" have been examined, with child interaction and exposure to infant odour being independently associated with a decrease in T (Prudom et al., 2008; Storey, Noseworthy, Delahunty, Halfyard, \& McKay, 2011; Ziegler, Peterson, Sosa, \& Barnard, 2011) ${ }^{21}$. Crying, however, is more of a complex association with some contexts leading to an increase in circulating $\mathrm{T}$ (Fleming, Corter, Stallings, \& Steiner, 2002).

Once again, the current state of the literature is on the fringe of the issue having shown changes to factors related to mating strategies following parental cues, but not mating strategies directly. From an evolutionary perspective, we would expect men and women to be sensitive to cues of vulnerable infants and respond to this by switching towards a more long-term orientated mating strategy. This is because, historically, individuals who were not reactive to such cues could have potentially put their offspring at risk by pursuing a mating strategy less focused on parental care.

\section{Wealth and Mating Strategies}

Each week in the UK, 32 million individuals (almost half the population) buy a ticket for the National Lottery, each hoping to win a grand prize and become a millionaire (Woollaston, 2013). The benefits of wealth are fairly obvious. With wealth

\footnotetext{
${ }^{21}$ The relationship between $\mathrm{T}$ levels and infant odour was demonstrated using samples of male marmosets. While research has been conducted into the response of women to such cues (Lundström et al., 2013), the precise reaction of men to the smell of new born infants remains unreported.
} 
comes early retirement, not having to worry about paying bills, and the opportunity to experience things which no one else can. It also means longevity, with access to higher quality food and healthcare. More importantly, with regard to this thesis, it also brings benefits in terms of successful mating and parenting effort. For parenting effort, not only can wealth enhance one's longevity, it can also help one's immediate family, providing a substantial boost to inclusive fitness (L. Barrett et al., 2002, pp. 126-136). For mating effort, with wealth comes higher social status, and with higher social status comes greater bargaining power in the mating market. Consider the earlier cited "pairing game" example in Chapter 2 (Ellis \& Kelley, 1999). Here, participants are asked to choose a partner based on one attribute; a number on their forehead. Thus a "70" woman may normally only consider a man for a relationship who has a number similar to or greater than her own. However, humans are multifaceted, and one's overall attractiveness as a partner could be linked to several attributes. Thus, if a man were lacking in physical attractiveness, he may still be considered of high mate value overall due to other attributes he possesses such as wealth or social status. A man who would normally be considered a " 60 " based on just his physical appearance, could be revaluated as an " 80 " if he was wealthy, due to the inclusive fitness boost such resources could bring to the hypothetical " 70 " woman.

Wealth as a determinant of social status was likely unknown in the Pleistocene. Only after the onset of agriculture in the Neolitic era 10,000 years ago were humans able to accumulate the large quantities of food which set the foundation for wealth building (Mazoyer \& Roudart, 2006). In Experiment 4, wealth is used as a "modern" cue to social status, and other evolutionary psychological experiments have used such cues effectively to manipulate mating behaviour (Little, DeBruine, \& Jones, 2013; M. Wilson \& Daly, 2004). In order to develop a prediction as to how such cues might affect mating behaviour in men and women, it is necessary to review the other, more evolutionarily stable, determinants of social status and their effects on mating success. In hunter-gatherer societies, which are marked by low social stratification, variance in social status still exists and so does variance in mating success as a consequence. Here social status is realised through factors such as social prestige, dominance, and hunting ability (Henrich \& Gil-White, 2001; E. A. Smith, 2004; E. A. Smith, Bird, \& Bird, 2003). For example, among the Tsimané of Bolivia, men who are high in dominance or prestige have increased reproductive success. This is realised through several different 
means, including marrying earlier, having younger wives, and having a greater number of affairs (von Rueden, Gurven, \& Kaplan, 2011).

Such variance is in part driven by female preferences. Certainly women in industrial (Li \& Kenrick, 2006) and pastoral (M. B. Mulder, 1990) societies show a preference for social status in men, and while there is less conclusive evidence for this preference among hunter-gatherers, the pattern is in the right direction. For example, the reproductive effects of being a good hunter (one determinant of prestige) are quite clear (Henrich \& Gil-White, 2001; E. A. Smith, 2004) and among the Hadza, a partner's intelligence and ability to provide resources is of more importance to women than men (Marlowe, 2004). ${ }^{22}$ Why are there differences between the sexes in terms of preferences for social status? As discussed in Chapter 1, whereas both sexes can increase their inclusive fitness through social status in terms of improving offspring quality, men are in the unique position of being able to increase their total offspring number to a greater extent than women and at a lower biological cost (Trivers, 1972). Indeed, the total number of offspring sired correlates more strongly with social status in men than in women (Pérusse, 1993). As such, while women may be expected to seek high status partners to maximise their fitness, men may do so by seeking to improve their social status.

Whether through inheritance (L. Barrett et al., 2002), learned skills and expertise, or simply through changes in social alliances (de Waal, 2007), social status is an attribute which often varies throughout a single lifetime. Much like with parental cues, the relative benefits of long- and short-term strategies can be affected by one's status and so remaining sensitive to cues of wealth, and adjusting mating strategies in line with this, could potentially increase reproductive success. Indeed at the end of Chapter 3, one sociometer study was presented which found that men may well be sensitive to such cues, responding more to feedback about their wealth than physical attractiveness (Pass et al., 2010). From an evolutionary perspective, a man who simply pursued a long-term strategy irrespective of his social status would be at a disadvantage in terms of lifetime reproductive fitness compared to a man who could remain flexible in his mating behaviour and take advantage of high status to pursue short-term mating. Likewise, a woman who simply followed a long-term mating strategy would not be as

22 This difference did not reach statistical significance, however, this is likely due to small sample size. The desire of partner provisioning ability was clearly more important to women than men. 
successful as her peers who were able to remain flexible enough to pursue short-term mating to gain access to better genes or resources.

\section{Overt Stimuli Exposure and Behavioural Change}

The experiments in this chapter, and several which follow, use overt stimuli in an attempt to affect behaviour. That is, the participants were consciously aware of the type of stimuli they were observing and that this may have had an effect on their behaviour, but were not necessarily aware of what the intended effects of these stimuli were. This is in contrast to some priming tasks such as the scrambled word task which expose participants to a set of test words covertly (e.g. Shariff \& Norenzayan, 2007). Experiments using overt stimuli are not uncommon, and the social, evolutionary, and developmental psychological literature are full of examples of such studies. For example, Posavac, Posavac, and Posavac (1998) found that exposing undergraduate women that were dissatisfied with their body to pictures of glamour models had a negative impact on their body-esteem. This was compared to both a control group who viewed images of cars, and a "realistic" attractiveness condition where the models shown were average in attractiveness. Although not formally tested, there also appeared to be a mirrored effect for women who were satisfied with their body who showed an increase to body-esteem following model exposure. This study is important as it implies that pre-existing individual differences can affect how stimuli are responded to - a point which is discussed towards the end of this chapter.

In another study related to body image, young girls were exposed to a picture story book about a girl called Mira who went shopping (Dittmar, Halliwell, \& Ive, 2006). The book used images of Barbie dolls to represent Mira in one condition, Emme dolls (a doll of more realistic body proportions) in another, or no doll at all (just images illustrating the story). Following exposure to the Barbie images, girls aged 5-7 showed significantly more body dissatisfaction (as measured using various emoticons in lieu of a likert scale) compared to the Emme and control groups. Interestingly, the Emme and control groups did not differ from one another in their impact on body dissatisfaction.

Two final examples come from the evolutionary psychological literature. In the first, Little et al. (2011) found that by exposing participants to images of tape worms (vs. caterpillars), bodily fluid stained cloths (vs. a blue liquid stain), and an overcrowded train (vs. an empty carriage), they could induce a within-subjects change in preference for facial dimorphism and symmetry. Facial dimorphism was sex specific with men finding feminine faces appealing, and women finding masculine faces 
appealing, within the high-pathogen condition. The typical effect size for these effects was around $\eta_{\mathrm{p}}{ }^{2}=0.05$. In the second, Little et al. (2013) found that women who were shown cues of male-male competition and wealth showed an increase in their preference for masculine faces. Such a preference is associated with short-term mating in women as a form of good gene acquisition (Provost et al., 2006; Provost et al., 2008). This study is of particular interest as the wealth stimuli used were very similar to that used in Experiment 4 (e.g. a slideshow of expensive watches, high end cars, and luxury food) and the results support the one-tailed hypothesis which was formed for that experiment (see below). The effect size for the wealth cues in this study was $\eta_{\mathrm{p}}{ }^{2}=0.09$, although a forced-choice paradigm was used.

The experiments in this chapter followed the precedent set by these previous studies, and others, by using the overt presentation of parental and wealth stimuli as the experimental manipulation. However, there are some inevitable methodological concerns with such stimuli which are addressed in further detail in the general discussion of this thesis (Chapter 9).

\section{Expected Sex Differences}

Experiments 3 and 4 were the first experiments in this thesis to test evolutionarily relevant stimuli in a mixed-sex sample of participants. As such, the independent effects of parental and wealth cues on the sexes were considered and taken into account within the experimental hypotheses.

In terms of the parental stimuli, women have higher typical and obligate levels of parental investment than men (Trivers, 1972). While this discrepancy is lower among humans than in many other species, with men typically contributing to the rearing and protection of young, there is still a robust difference between the sexes in terms of typical parental investment (Marlowe, 2000). Due to this it was predicted that there would be a difference in how men and women responded to the parental stimuli. Specifically, women were predicted to be more sensitive to the cues than men.

Differences in parental investment also led to very different problems for the sexes in terms of short-term mating. While men faced problems such as sexual access, women faced problems of resource extraction (Buss \& Schmitt, 1993; Greiling \& Buss, 2000). As discussed in the previous section, women may be expected to be sensitive to cues of available resources which could potentially enhance their inclusive fitness (i.e. wealth). Men, in contrast, did not face the same adaptive problem of resource acquisition as women did (Buss, 2006b; Buss \& Schmitt, 1993), but instead were more 
likely to increase their fitness by actively seeking increased status. As such, men may not be expected to respond to wealth cues in the same way as women.

\section{Experiment 3}

Experiment 3 exposed participants to parental cues. Specifically, "how-to" videos about parental care were presented as well as footage of infants. Hypothesis 3 stated that parental cues would lead to long-term mating strategy activation in both men and women, and this activation would be more prominent among women. Three predictions were made to test the hypothesis. Prediction 1 was that, following exposure to parental cues, the number of LT selections participants would make on the SMA task would increase. Prediction 2 was that, following the same cues, a decrease in ST selection would be found among the participants. Prediction 3 related to differences in behaviour between the sexes. Specifically, that the effects stated in Predictions 2 and 3 would be more prominent among women than men.

\section{Method}

\section{Participants}

One-hundred and sixty volunteers participated in the experiment for either payment or course credit. Recruitment came from the psychology participant pool and local advertising within the university. The following demographic details relate to the 150 participants which were included in the analysis (ten were excluded - see results section). Seventy-five of the participants were male (50\%). Men were slightly older than women on average, with an average age of $21.01(S D=1.57)$ for men and $20.36(S D=$ 1.62 ) for women, $t(148)=2.503, p=0.01, d=0.41$. The sample was primarily Caucasian (95.3\%) with the other participants divided between Black $(n=2)$, East Asian $(n=2)$, and South-East Asian $(n=1)$ ethnicities. The remaining 2 participants described themselves as being of a 'mixed' ethnicity. Just under two-thirds $(n=92)$ of the participants were in a committed relationship (including marriage) while $38.6 \%$ ( $n=$ 58) were either single or in an uncommitted relationship. Participants were typically of a middle to lower-middle SES $(M=3.24, S D=0.70)$ with no significant difference found between the sexes, $t(148)=1.355, p=0.18$. Men considered themselves more attractive than women on average, with the average self-report rating being $5.76(S D=$ $1.43)$ for men and $4.76(S D=1.16)$ for women, $t(148)=4.699, p<0.01, d=0.77$. The average SOI-R score was $38.37(S D=12.51)$ for men and $28.16(S D=10.58)$ for women. This difference was also significant, $t(148)=5.400, p<0.01, d=0.88$. Finally, 
the sample was entirely heterosexual and childless with the exception of two participants who had one child each.

\section{Materials}

Forms. The SOI-R and standard demographic questionnaire were used in this study (Appendices B \& E). For the purpose of this experiment, two additional questions were added to the demographic questionnaire. These questions were used to gauge the participant's desire to have children and whether or not they would consider themselves to be good potential mothers or fathers. For both questions participants were asked to rate their agreement with a statement using a nine point semantic differential scale with the anchors of 'not at all' (one) and 'very much so' (nine). For the first question participants responded to the statement 'I like the idea of having children', and for the second question they responded to the statement 'I think I would make a great mother/father'. The consent and debrief forms used for this experiment can be found in Appendix L.

SMA models. The SMA task was used in this experiment and was identical to that specified in Chapter 3 with the exception that different models were used. An initial pool of 169 images of men was selected from the 18-25 age category of hotornot.com. These were then rated for attractiveness on a one to ten scale ranging from 'not at all attractive' to 'very attractive' by 20 judges. The judges had an average age of 21.05 (SD $=1.39$ ) and half were men. The average attractiveness for the male photographs was $4.17(S D=1.03)$ and from these a selection of 50 from the middle of the distribution were taken. The average attractiveness of this sub-set was $4.07(S D=0.23)$. Similarly for women, the same judges rated an initial set of 175 pictures and these had an average attractiveness of $4.90(S D=1.00)$. From these a sub-set was formed from the middle of the distribution which had an average attractiveness of $4.09(S D=0.38)$.

Parental stimuli. For the parental stimuli, the researchers gathered a collection of 20 videos, all around two to three minutes in length, which featured infants. These ranged from videos of toddlers falling over when attempting to walk to "how-to" videos instructing parents on how to bathe their babies. These videos were chosen to try to elicit a paternal response in the participants. From this collection of videos, three were chosen by the research group (see acknowledgements section) to be stimuli in the study. A description of each video can be found below. Screenshots from each of the videos can be found in Appendix M. 
Video one. The first video was produced by the "Baby Health Guru" and was titled 'Soothing your baby'. The video was presented by a male paediatrician who was sitting at a desk and talking directly to the viewer. The doctor spoke in second-person tense throughout, as if the viewer was the parent of a new-born child: 'As a parent, your job is to decode this message as quickly as possible - to stop the sobs. Luckily you'll soon be able to anticipate your babies' needs.' As the doctor spoke, the video alternated between clips of him at his desk looking at the viewer, and clips of crying babies being soothed by their parents. These clips included both male and female parents. The video lasted for 2 minutes 16 seconds.

Video two. The second video was produced as a "fun" segment by ITN (a British news programme). It was a short compilation clip showing the "five types" of baby laugh. These included "Gurgling" and "Hysteria". In each of the clips, an infant was being filmed from a first-person perspective. The person behind the camera made funny noises or tickling movements towards the child in order to make them laugh. Lighthearted music was being played in the background. There was a mixture of male and female cameramen (as recognised by speech directed at the child) and the video lasted 1 minute 42 seconds.

Video three. The final video was another created by the Baby Health Guru. The video was 2 minutes and 4 seconds long, and this time featured a female paediatrician sat behind a desk. The video was entitled 'Bathing your baby'. It followed a very similar format to video one as it alternated between clips of the female doctor talking directly to the parent (referring again to the child as 'your baby' throughout) and clips/stills of babies being bathed. Compared to video one, video three was more cautious in tone. It instructed parents to take care when washing their child and informed them of the potential consequences of carelessness: 'Infants can drown in as little as one inch of water, so keep a firm hand on your baby at all times.'

These three videos clips were combined together into one large video file. This file was 6 minutes 2 seconds long and presented the videos in the order described above. In the remainder of this chapter this is referred to as the "parental video" or the parental stimuli.

Neutral stimuli. Three videos were also chosen for the neutral condition. Originally 18 potential videos were collected, and content ranged from 'How to use chopsticks' to 'How to slice a pineapple'. These videos were chosen to try to match the level of movement and viewer engagement of the parental videos only without cuing 
parental care. From the ten videos, three were again chosen by the research group. Below are descriptions of these videos. Stills from these videos can also be found in Appendix M.

Video four. The first video was created by "TheBestSauces.com" and was an instructional video entitled 'How to cut a pineapple'. The video featured a male chef, teaching the viewer how to efficiently slice a pineapple into rings. The chef used second person tense much like videos one and three (e.g. 'What you want to look for is'). The video was edited down to 2 minutes 16 seconds and contained information that may have been novel to the participants, such as how to tell if a pineapple is ripe and the need to hang it upside down for a while before cutting it.

Video five. The second video of the neutral stimuli was a compilation video by YouTube user "SeriousFailDude". It was a "home-video" style compilation clip showing individuals failing at various tasks with some light rock music playing in the background. For example, one clip showed a women being flung from a bucking bronco while another showed a man in a superhero costume attempting to back-flip off a wall and missing. The video was 1 minute 24 seconds long.

Video six. The final video was again a how-to style tutorial, this time about growing orchids. The segment was presented by an enthusiastic female presenter who guided the viewer through the unique challenges of growing the flower. While the presentation did not use second person tense throughout, there were parallels with video three in terms of stern warnings to the viewer: 'And they make roots, and the roots need air. So if those roots don't get air they will die. So they need really good drainage.' Much like video four, several points were discussed which were likely to have been novel to the viewer, including that fact that orchids can be sunburnt, and that they will not grow out of traditional soil. The video was 2 minutes 32 seconds long.

In a similar manner to the parental video, a "neutral video" file was created. This was 6 minutes 12 seconds long and played the videos in the order presented here. This video is also described as the neutral stimuli in this chapter.

Hardware. The experiment was completed on a 19" Toshiba ${ }^{\circledR}$ laptop running Windows ${ }^{\circledR}$ XP on a 1024 by 768 resolution screen. Participants wore headphones during the video segment of the experiment.

\section{Design}

The experiment was a 2 by 2 mixed-model design with the within-subjects factor of time and the between-subjects factor of condition. Participants were randomly 
assigned to be exposed to either parental or neutral stimuli as part of the independent variable of condition. The LT and ST measures from the SMA task formed the dependent variables. Analysis utilised chi-squared tests for the main effect of condition as well as ordinal logistic regression to examine the effect of covariates as measured by the demographic and SOI-R questionnaires.

\section{Procedure}

Participants were recruited by the researchers to participate in a study about 'social perception and relationship choices'. When arriving at the laboratory, participants were first issued with a consent form. Upon giving consent, the format of the SMA task (described as a "relationship choice task") was discussed with the participant. They were told that they were about to see pictures of 50 potential oppositesex suitors of which they had to make relationship choices. Participants were then advised to read the instructions on the screen by the experimenter who then left the room so that the task could be completed in private.

After the task was completed the experimenter was called back into the room. Participants were then given a set of headphones to wear and were shown either the neutral or parental video in full screen mode. The volume of the video was set to default $(50 \%)$ on Windows ${ }^{\circledR}$ Media Player. As with the SMA task, the video was also watched in private.

Following the video, participants were asked to complete the SMA one more time. They were specifically told that they were being tested twice to 'make sure we have a full a proper idea of your relationship preferences'. They were also told that 'you may have seen some of the individuals that you have rated previously, but this is normal.' As with Chapter 3, the purpose of this latter statement was to reduce the likelihood that participants would interrupt the experiment should they recognise individuals from the previous performance of the SMA. Once again, the participants were left to complete the task in private. Upon completion of the second SMA measure, participants were thanked, given a full verbal and written debrief, and any questions they had were answered. Payment was then issued and participants were asked not to discuss the nature of the experiment with anyone who could be a potential future participant. Finally, the participant was shown out of the lab by the experimenter.

\section{Results}

Ten participants were excluded from the analysis. Seven were homosexual while three selected 'Not interested' for all models in the SMA during its first performance. 
The number of participants in each group was balanced with 37 of each sex appearing in the parental condition and 38 of each sex in the neutral condition. On average, the sample indicated that they quite liked the idea of having children $(M=6.82, S D=2.19)$ and were quite sure that they would be a good potential mother or father $(M=6.90, S D$ $=1.50)$. While there was no difference between men and women with regards to their perceived potential as a good parent, $t(148)=-0.599, p=0.55$, there was a difference between the sexes in how much they liked having the idea of children. Men reported $6.31(S D=2.30)$ on average while women reported a mean of $7.33(S D=1.96)$. This effect was statistically significant and of a medium size, $t(148)=-2.936, p<0.01, d=$ 0.48. A median of four models were chosen for a long-term relationships during the SMA task, and 11 were chosen for short-term relationships.

\section{Short-Term Mating}

In the parental condition, $44(59.5 \%)$ of the participants decreased their number of short-term choices (ST-) after viewing the parental stimuli. For $9(12.1 \%)$ of the participants there was no change (ST=) and $21(28.4 \%)$ increased their number of choices $(\mathrm{ST}+)$. When this distribution was compared to that produced by the neutral stimuli, it appeared as if the parental stimuli led to a greater number of participants appearing in the ST- category (see Table 4.1). The difference between these two distributions was marginally significant using a one-tailed chi-squared test, $\chi^{2}(2)=$ $3.562, p=0.08$.

Table 4.1

The number of participants who increased, stayed the same, or decreased in their number of ST choices following exposure to either neutral or parental stimuli. The difference between the two distributions approached statistical significance using a chisquared test

\begin{tabular}{lccc}
\hline \multicolumn{4}{c}{ Short-term change } \\
\hline & - & $=$ & + \\
\hline Parental & 44 & 9 & 21 \\
Neutral & 38 & 15 & 23 \\
\hline$\chi^{2}(2)$ & $3.562^{\dagger}$ & & \\
Cramér's $V$ & 0.11 & & \\
\hline
\end{tabular}

$$
{ }^{\dagger} p<0.10, * p<0.05, * * p<0.01 .
$$


When an ordinal regression analysis was conducted, a significant model could not be formed using condition to predict ST change group, $\chi^{2}(1)=0.795, p=0.186$. When the covariates from the demographic and SOI-R forms were introduced into the analysis using a backwards stepwise method, a significant model still could not be produced.

\section{Long-Term Mating}

In the parental condition, 24 participants (32.4\%) decreased their number of long-term choices (LT-), 16 (21.6\%) stayed the same (LT=), and 34 (45.9\%) increased $(\mathrm{LT}+)$. Compared to the distribution found in the neutral condition, it appeared as if the parental condition increased the likelihood that participants would appear in the LT+ category (see Table 4.2). This pattern was significant using a chi-squared test, $\chi^{2}(2)=$ $17.490, p<0.01$.

\section{Table 4.2}

The number of participants who increased, stayed the same, or decreased in their number of LT choices following exposure to either neutral or parental stimuli. The two distributions are significantly different from one another using a chi-squared test

\begin{tabular}{|c|c|c|c|}
\hline \multicolumn{4}{|c|}{ Long-term change } \\
\hline & - & $=$ & + \\
\hline Parental & 24 & 16 & 34 \\
\hline Neutral & 32 & 25 & 19 \\
\hline$\chi^{2}(2)$ & \multicolumn{3}{|c|}{$17.490 * *$} \\
\hline Cramér's $V$ & \multicolumn{3}{|l|}{0.24} \\
\hline
\end{tabular}

$$
{ }^{\dagger} p<0.10, * p<0.05, * * p<0.01
$$

When an ordinal regression was conducted to try to predict LT change group using experimental condition a significant model emerged. Participants exposed to parental stimuli were $95 \%$ more likely to belong to the LT+ group than their neutral stimuli counterparts (Table 4.3a). Furthermore, when a backwards stepwise ordinal regression was conducted using covariates, a more accurate model was produced using a condition by relationship status interaction (Table $4.3 \mathrm{~b}$ ). When this interaction was deconstructed (Table 4.3c) it became apparent that there was no difference between the conditions for those who were single. However, for those who were in a committed 
relationship, exposure to parental stimuli increased the chances of appearing in the LT+ group by $191 \%$ compared to the neutral condition.

Table 4.3

The results of an ordinal regression to predict LT choice change using (a) condition alone and (b) an interaction between condition and relationship status. A final regression (c) was performed to break down the interaction

\begin{tabular}{|c|c|c|}
\hline \multicolumn{3}{|c|}{ (a) Model LT } \\
\hline & $\beta$ & OR \\
\hline Condition & $0.668 *$ & 1.95 \\
\hline Model & \multicolumn{2}{|c|}{$\chi^{2}(1)=4.834^{*}$} \\
\hline Nagelkerke $R^{2}$ & \multicolumn{2}{|l|}{0.036} \\
\hline Accuracy & \multicolumn{2}{|c|}{$44 \%(+6.7 \%)$} \\
\hline Parallel lines & \multicolumn{2}{|c|}{$\chi^{2}(1)=2.582$} \\
\hline \multicolumn{3}{|c|}{ (b) Model LT } \\
\hline & $\beta$ & OR \\
\hline Condition $*$ Relat & $1.062 * *$ & 2.89 \\
\hline Model & \multicolumn{2}{|c|}{$\chi^{2}(1)=9.955$} \\
\hline Nagelkerke $R^{2}$ & \multicolumn{2}{|l|}{0.072} \\
\hline Accuracy & \multicolumn{2}{|c|}{$46.7 \%(+9.4 \%)$} \\
\hline Parallel lines & \multicolumn{2}{|c|}{$\chi^{2}(1)=0.623$} \\
\hline
\end{tabular}

\begin{tabular}{lcc}
\hline \multicolumn{3}{c}{ (c) Model LT } \\
\hline & \multicolumn{1}{c}{$\beta$} & OR \\
\cline { 2 - 3 } Single * Condition & 0.031 & - \\
Taken * Condition & $1.069 * *$ & 2.91 \\
\hline Model & $\chi^{2}(2)=9.961 * *$ \\
Nagelkerke $R^{2}$ & 0.072 \\
Accuracy & $46.7 \%(+9.4 \%)$ \\
Parallel lines & $\chi^{2}(2)=2.045$ \\
\hline
\end{tabular}

Note: Variables were coded as such: condition - neutral (0), parental (1); relationship status - single (0), in a relationship (1). Relat $=$ relationship status. Taken $=$ those who are currently in a committed relationship. $\mathrm{OR}=$ odds ratio. ${ }^{\dagger} p<0.10, * p<0.05, * * p<0.01$.

\section{Relationship Differences}

As relationship status was identified as a predictive variable within the longterm ordinal models, differences between committed and uncommitted participants on the mating and parenthood related variables from the questionnaires were explored. Specifically, the two parental demographic questions and SOI-R.

The first parental question asked participants whether they agreed with the statement that they would be a great mother or father. Single individuals rated themselves on average as $6.55(S D=1.57)$ for this question, while committed individuals rated themselves as $7.12(S D=1.41)$ on average. This was a statistically 
significant difference, $t(148)=2.296, p=0.02, d=0.38$. A similar pattern was found for the other parental question. While single individuals typically liked the idea of having children in the future $(M=6.24, S D=2.36)$, committed individuals showed a greater average preference $(M=7.18, S D=2.02)$. Once again, this difference was significant, $t(148)=2.613, p=0.01, d=0.43$. Finally, there was a significant difference in sociosexuality between the two groups. As committed individuals would, by the nature of their relationship, be less likely to have multiple partners over the previous 12 months, only the desire and attitude components of the SOI-R were used. Here, single individuals had an average score of $29.40(S D=10.00)$, while committed individuals had an average of $23.20(S D=9.20)$. This effect was of a medium to large size, $t(148)=$ 3.907, $p<0.01, d=0.65)$.

\section{Discussion}

In this experiment, the presence of parental cues was a significant positive predictor of LT change category; participants in the parental condition were more likely to increase their LT selections than those in the neutral condition. Thus, Prediction 1 was confirmed. Furthermore, the inclusion of relationship type as a covariate in the ordinal analysis led to an improved model. Among those in a committed relationship, the chances of appearing in the LT+ group after being shown parental stimuli was greater than those who were single or dating. Prediction 2 was somewhat correct, as there was an effect of condition on ST category change which approached significance. This was a negative effect, with participants in the parental condition being marginally less likely to appear in the ST+ category than those in the neutral condition. However, all subsequent ordinal models were non-significant. As there was no difference between the sexes for the effects of parental stimuli on ST or LT selections, Prediction 3 was found to be incorrect. In summary, partial support was found for Hypothesis 3. The evidence suggests that parental stimuli or cues may lead to a shift in relationship preference towards the long-term and that this might be most pronounced in individuals already within a close pair-bond.

While the fact that sex added no predictive power to the models did not support part of Hypothesis 3, this lack of effect does support an important finding within the evolutionary psychological literature. Specifically, while there are moderate to large sex differences in domains related to short-term mating (Buss \& Schmitt, 1993; Li \& Kenrick, 2006) such differences are less pronounced within the long-term domain (Buss et al., 1990). Again, this is to be expected if a selection pressure towards parental care 
was shared by the sexes in the ancestral environment. This is not to say that sex differences in long-term preferences do not exist, but that often times they are small. Consider the cross cultural study by Buss et al. (1990) for example. When men and women were asked to rate the importance of 18 characteristics in a marriage partner, the top four attributes were identical for men and women; love, dependability, emotional stability, and pleasing disposition. Among these variables there were still some sex differences. Dependability, for example, had a large effect size of $d=1.00$, while pleasing disposition had a smaller effect size of $d=0.27$. Thus it may well be the case that a sample size of 150 did not provide enough power to detect an effect. For example, in the LT model, the odds ratio of the non-significant $\operatorname{sex} \beta$ within the parental condition was 1.24 . According to $G^{*}$ Power (Erdfelder et al., 1996), with only a sample size of 74 participants, this model had a power of only $23 \%$ with a one-tailed hypothesis. For a power of $95 \%$, the sample size would need to have consisted of 947 participants. These results are explored further in the general discussion towards the end of this chapter.

\section{Experiment 4}

In this experiment, participants were exposed to a static slideshow of wealth cues. These included images of costly jewellery, holiday homes, and expensive cars. This slideshow was viewed between two measures of the SMA task. Using the literature from the introduction to this chapter, a one-tailed hypothesis was formed. Hypothesis 4 stated that wealth cues would lead to short-term mating strategy activation in both men and women, and this activation would be more prominent among women. Three predictions were made to test the hypothesis. Prediction 1 was that, following exposure to wealth cues, the number of ST selections participants would make on the SMA task would increase. Prediction 2 was that, following the same cues, a decrease in LT selections would be found among the participants. Prediction 3 was that these two effects would be more prominent among women than men.

\section{Method}

\section{Participants}

One-hundred and sixty volunteers participated in the study for either payment or course credit. Participants were recruited either from the psychology participant pool or via local advertising within the university. The following demographic details relate to the 151 participants which were included in the analysis (nine were excluded - see results section). Seventy-five of the participants were male (49.7\%) and all participants were heterosexual. The average participant age was $20.93(S D=1.72)$, and there was no 
significant difference between the sexes $t(149)=1.134, p=0.26$. The sample was primarily Caucasian (93.4\%) with the other participants divided between Black $(n=4)$, East Asian $(n=3)$, and Middle Eastern $(n=1)$ ethnicities. The remaining three participants described their ethnicity as 'mixed'. Over half of the participants $(n=84)$ were in a committed relationship (including marriage) while $44.4 \%(n=67)$ were either single or in an uncommitted relationship. Participants were typically from a middle SES background $(M=3.06, S D=0.66)$ and no significant difference was found between the sexes, $t(148)=1.355, p=0.17$. Men considered themselves more attractive than women, with men rating themselves on average as $5.74(S D=1.25)$ and women rating themselves on average as $4.97(S D=1.35), t(148)=3.625, p<0.01, d=0.60$. Ten of the participants had one child, and four had two children. The average SOI-R score for men was $43.47(S D=12.82)$, while women had an average score of $29.76(S D=11.23)$. This was a significant difference, $t(144)=6.880, p<0.01, d=1.15$.

\section{Apparatus}

Forms. The forms used in this experiment were identical to those used in Experiment 3, although the two parental questions were not included in the demographic form. Modified versions of the consent and debrief form, which reflected the specifics of Experiment 4, can be found in Appendix L.

SMA stimuli. A pool of 169 pictures of men were selected from the hotornot.com website and rated for attractiveness by 20 judges. The average age of the judges was $20.60(S D=0.82)$, and ten of them were male. Attractiveness was rated on a ten point scale ranging from 'not at all attractive' to 'very attractive'. The average attractiveness for the male photographs was $4.40(S D=0.83)$ and from these a selection of 50 from the middle of the distribution were used in the SMA task. The average attractiveness of this sub-set was $4.68(S D=1.50)$. Similarly for women, the same judges rated 202 pictures as having an average attractiveness of $5.19(S D=1.03)$. A sub-set of pictures was formed from the middle of the distribution. The average attractiveness of this sub-set was $4.68(S D=1.47)$.

Wealth stimuli. For this experiment, the manipulation came in the form of a slideshow viewed by the participants. The slideshow ran in full screen and used 75 pictures which were presented in a random order. Each picture stayed on the screen for three seconds before fading to reveal another. The whole slideshow lasted 3 minutes 45 seconds. The pictures shown as part of the "wealth slideshow" came from an initial sample of 166 images gathered by the researchers from various online websites. The 
images fell into four broad categories: a) mansions (53\%); b) expensive cars (25\%); c) jewellery/watches (12\%); and d) money/gold (10\%). These images were then rated by the same 20 judges who rated the SMA models. The judges were asked to observe each picture and rate it for how wealthy they would feel if they owned the item in the picture using a one to ten semantic differential scale labelled from 'not at all' to 'very much so'. These pictures were presented to the judges at the same time as the neutral stimuli (see below) which was also rated using the same criteria. Average ratings for each picture were then calculated and the 75 pictures with the highest average score $(M=9.00, S D=$ 1.43) were used in the wealth slideshow.

Neutral stimuli. A similar process was used to create the "neutral slideshow". An original set of 174 photos were gathered by the researchers using the broad categories of: a) household objects (50\%); b) inexpensive food (24\%); c) plants (21\%); and d) small pets (5\%). After being rated by the judges for wealth, the 75 pictures with the lowest average score $(M=1.87, S D=0.99)$ were chosen for inclusion in the neutral slideshow. Examples of the images used in both slideshows can be found in Appendix $\mathrm{N}$. As with the previous experiment, the neutral and wealth slideshows shall also be referred to as the neutral and wealth stimuli.

\section{Design}

The design remained the same as Experiment 3, however, the independent variable of condition now had the levels of neutral or wealth.

\section{Procedure}

The procedure remained identical to Experiment 3 aside from the following three changes: 1) the study was advertised as an investigation of 'relationship preferences and memory'; 2) Participants were shown either a neutral or wealth slideshow during the manipulation phase; and 3) when introduced to the slideshow, participants were told that their memory of the items would be tested at the end of the experiment. This was to ensure that participants attended to the stimuli; in reality no such test occurred.

\section{Results}

Nine homosexual participants were excluded from the analysis. The number of participants in each group was balanced with 38 men and 39 women present in the wealth condition and 37 of each sex present in the neutral condition. The sample chose an average (median) of two models for a long-term relationship, and 12 for a short-term relationship when first completing the SMA task. 


\section{Short-Term Mating}

In the wealth condition, $39(50.6 \%)$ of the participants decreased in their shortterm choices (ST-) following the prime. For 13 participants (16.9\%) there was no change ( $\mathrm{ST}=$ ) and for $25(32.5 \%)$ there was an increase in the number of choices (ST+). When this distribution was compared to that produced by the neutral stimuli, it appeared as if the wealth stimuli led to a larger number of participants appearing in the ST+ category (see Table 4.4). The difference between these two distributions was found to be significant using a chi-squared test, $\chi^{2}(2)=9.498, p<0.01$.

Table 4.4

The number of participants who increased, stayed the same, or decreased in their number of ST choices following exposure to either a neutral or wealth stimuli. The two distributions are significantly different from one another using a chi-squared test

\begin{tabular}{|c|c|c|c|}
\hline \multicolumn{4}{|c|}{ Short-term change } \\
\hline & - & $=$ & + \\
\hline Wealth & 39 & 13 & 25 \\
\hline Neutral & 47 & 13 & 14 \\
\hline$\chi^{2}(2)$ & 9.49 & & \\
\hline Cramér's $V$ & 0.18 & & \\
\hline
\end{tabular}

$$
{ }^{\dagger} p<0.10, * p<0.05, * * p<0.01
$$

When an ordinal regression analysis was performed to predict ST change group using condition alone, a significant model was produced, $\chi^{2}(1)=3.392, p=0.04$, but this failed to yield any predictive improvement over the base model (Table 4.5a). When a backwards stepwise analysis was conducted using additional variables from the demographic forms, a significant model emerged indicating the presence of a 3-way interaction between condition, sex, and relationship status (Table 4.5b).

Table 4.5

The results of an ordinal regression to predict ST choice change using (a) just condition and $(b)$ an interaction between condition, relationship status and sex

(a) Model ST

$\beta \quad$ OR

(c) Model ST

$\begin{array}{r}\beta \\ \hline\end{array}$




\begin{tabular}{|c|c|c|c|}
\hline Condition & $0.587 *$ & Cond $*$ Sex $*$ Relat & $0.686^{* *}$ \\
\hline Model & $\chi^{2}(1)=3.392 *$ & Model & $\chi^{2}(1)=10.710 * *$ \\
\hline Nagelkerke $R^{2}$ & 0.03 & Nagelkerke $R^{2}$ & 0.08 \\
\hline Accuracy & $57 \%(+0 \%)$ & Accuracy & $59.6 \%(+2.7 \%)$ \\
\hline Parallel lines & $\chi^{2}(1)=0.439$ & Parallel lines & $\chi^{2}(1)=0.312$ \\
\hline
\end{tabular}

Note: Variables were coded as such: condition - neutral (0), wealth (1); sex - male (0), female (1); relationship status - single $(0)$, in a relationship (1). Relat $=$ relationship status. Cond $=$ condition. $\mathrm{OR}=$ odds ratio. ${ }^{\dagger} p<0.10, * p<0.05, * * p<0.01$.

To break down the three-way interaction, a separate analysis was conducted for each sex. In males, there was no significant interaction between condition and relationship status (Table 4.6a). However, the same model was significant among female participants (Table 4.6b). In this model, no difference was found between the conditions among women who were single or dating. However, there was a large effect of condition among those who were in a committed relationship. Specifically, there was a $205 \%$ increase in likelihood of appearing in the ST+ group if these women were shown wealth stimuli compared to neutral stimuli.

Table 4.6

The results of two separate ordinal regression models, one for each sex, using condition and relationship status as predictors. The male model (a) was non-significant, however, the female model $(b)$ showed an effect of experimental condition among those participants who were in a relationship

\begin{tabular}{|c|c|c|c|c|c|}
\hline \multicolumn{3}{|c|}{ (a) Model ST Males } & \multicolumn{3}{|c|}{ (b) Model ST Females } \\
\hline \multirow{3}{*}{$\begin{array}{l}\text { Single * } \\
\text { Condition } \\
\text { Taken * } \\
\text { Condition }\end{array}$} & $\beta$ & OR & \multirow{3}{*}{$\begin{array}{l}\text { Single * } \\
\text { Condition } \\
\text { Taken } * \\
\text { Condition }\end{array}$} & $\beta$ & OR \\
\hline & 0.493 & - & & -0.698 & - \\
\hline & 0.956 & - & & $1.115^{*}$ & 3.05 \\
\hline Model & \multicolumn{2}{|c|}{$\chi^{2}(2)=3.144$} & Model & \multicolumn{2}{|c|}{$\chi^{2}(2)=8.502 *$} \\
\hline Nagelkerke $R^{2}$ & \multicolumn{2}{|l|}{0.05} & Nagelkerke $R^{2}$ & \multicolumn{2}{|l|}{0.12} \\
\hline Accuracy & \multicolumn{2}{|c|}{$61.3 \%(+0.0 \%)$} & Accuracy & \multicolumn{2}{|c|}{$57.9 \%(+5.3 \%)$} \\
\hline Parallel lines & \multicolumn{2}{|c|}{$\chi^{2}(2)=0.970$} & Parallel lines & \multicolumn{2}{|c|}{$\chi^{2}(2)=1.329$} \\
\hline
\end{tabular}

Note: Condition was coded as neutral $=0$ and wealth $=1 ; \mathrm{OR}=$ odds ratio. Taken $=$ in a committed relationship. ${ }^{\dagger} p<0.10, * p<0.05, * * p<0.01$. 


\section{Long-Term Mating}

In the wealth condition, 28 participants $(36.4 \%)$ decreased in their number of long-term choices (LT-), 35 (45.4\%) stayed the same (LT=), and 14 (18.2\%) showed an increase $(\mathrm{LT}+)$. Compared to the distribution found in the neutral condition, it appeared as if the wealth stimuli led participants to decrease in their LT interest (see Table 4.7). This pattern was confirmed as significant using a chi-squared test to compare the two distributions, $\chi^{2}(2)=6.125, p=0.02$.

Table 4.7

The number of participants who increased, stayed the same, or decreased in their number of LT choices following exposure to either neutral or wealth stimuli. The two distributions are significantly differently from each other when a chi-squared test is used

\begin{tabular}{lccc}
\hline \multicolumn{4}{c}{ Long-term change } \\
\hline & - & $=$ & + \\
\hline Wealth & 28 & 35 & 14 \\
Neutral & 18 & 39 & 17 \\
\hline$\chi^{2}(2)$ & $6.125^{*}$ & & \\
Cramér's $V$ & 0.14 & & \\
\hline
\end{tabular}

$$
{ }^{\dagger} p<0.10, * p<0.05, * * p<0.01
$$

When an ordinal regression analysis was used to predict LT category by condition alone, the model approached significance (Table 4.8a). However, when covariates were included using a backwards stepwise analysis, a model containing a significant condition by SOI-R interaction emerged (Table $4.8 \mathrm{~b}$ ). This model revealed that the main effect was driven by high SOI-R individuals. Among high SOI-R individuals, those who viewed wealth stimuli were $146 \%$ more likely to appear in the $\mathrm{ST}=$ or ST- categories compared to those who viewed neutral stimuli. The resulting model showed a decrease in prediction accuracy (-2.7\%) compared to baseline. This was due to the fact that the model had no predictive power for low SO individuals. When LT category was predicted for only high SO participants, the model showed a slight improvement of $0.4 \%$. 
Table 4.8

Two ordinal regression models predicting LT category. The first (a) used only condition and approached significance. The second (b) used an SOI-R by condition interaction. This latter model reveals that the main effect appears to be driven by participants with unrestricted sociosexuality

(a) Model LT

\begin{tabular}{|c|c|c|}
\hline & $\beta$ & OR \\
\hline Condition & $-0.461^{\dagger}$ & 0.63 \\
\hline Model & \multicolumn{2}{|c|}{$\chi^{2}(1)=2.238^{\dagger}$} \\
\hline Nagelkerke $R^{2}$ & \multicolumn{2}{|l|}{0.02} \\
\hline Accuracy & \multicolumn{2}{|c|}{$49.0 \%(+0.0 \%)$} \\
\hline Parallel lines & \multicolumn{2}{|c|}{$\chi^{2}(1)=0.527$} \\
\hline
\end{tabular}

(b) Model LT

\begin{tabular}{lcc}
\hline & \multicolumn{1}{c}{$\beta$} & OR \\
\cline { 2 - 3 } HSO * Condition & -0.901 & 0.41 \\
LSO * Condition & 0.038 & - \\
\hline Model & \multicolumn{2}{l}{$\chi^{2}(2)=6.425^{*}$}
\end{tabular}

Nagelkerke $R^{2} \quad 0.05$

Accuracy $\quad 46.6 \%(-2.3 \%)$

Parallel lines $\quad \chi^{2}(2)=3.281$

Note: Condition was coded as neutral $=0$ and wealth $=1 ; \mathrm{OR}=$ odds ratio. $\mathrm{SO}=$ sociosexuality. High and low SO were determined by a median split of the SOI-R variable within $\operatorname{sex}{ }^{\dagger} p<0.10, * p<0.05$, ** $p<$ 0.01 .

\section{Relationship Differences}

As with Experiment 3, relationship status was a significant predictor in one of the ordinal logistic regression models. Specifically, women who were in relationships were more likely to appear in the ST= or ST+ categories following wealth stimuli compared to single women. In the following discussion, these results are interpreted partially in terms of the relative sociosexuality of these two groups. While single women had an SOI-R of 26.7 ( $S D=9.8)$, those in committed relationships had an average score of $21.1(S D=8.1)$. This difference was both significant and of a medium effect size, $t(72)=2.682, p<0.01, d=0.62$. Here, the behavioural subcomponent of the SOI-R was excluded.

\section{Discussion}

In terms of short-term mating behaviour, following exposure to a wealth slideshow, participants were more likely to choose a greater number of models for a short-term relationship compared to a control group. This was initially confirmed with chi-squared analysis, and further supported by an ordinal regression model. Thus, Prediction 1 was found to be correct. Further analysis using ordinal regression revealed 
that this effect was mainly being driven by females who were in committed relationships. In terms of LT relationships, those participants who were exposed to wealth stimuli were more likely to decrease in their number of LT choices , confirming Prediction 2. Subsequent regression models revealed that this effect was primarily driven by high SO individuals. Prediction 3 was partially correct. In the case of shortterm mating, women appeared to be more affected by wealth cues than men. No such sex difference occurred in LT behaviour. In summary, good support was found for Hypothesis 4, although the effects partially depended on interactions with sex, relationship status, and sociosexuality.

\section{General Discussion}

Overall, the two experiments in this chapter found evidence for mating strategy plasticity following both parental and wealth stimuli. In both cases, this evidence provided support for the specific one-way hypotheses which were developed from the evolutionary psychological literature. Thus, this chapter supported the overall working hypothesis by demonstrating a shift in mating strategies in a potentially evolutionarily adaptive direction. In the following sections, the variables which were found to interact with the experimental conditions are discussed, and potential improvements to the research explored.

\section{Mating Strategy Plasticity and the Pair Bond}

In Experiment 3, both sexes were found to increase their selection of long-term relationships after being presented with parental stimuli. When ordinal regression analysis was employed, it was clear that this effect was being driven by individuals who were already in a committed relationship. What differences existed between these two subgroups which may have caused them to react to the stimuli differently? Compared to single individuals, those who were in committed relationships were more likely to consider themselves good parental material and like the idea of having children. Committed participants were also more likely to have a restricted (lower) sociosexuality, even when the behavioural component of the SOI-R was excluded. It appears then, that individuals who may already be pursuing a long-term mating strategy are more likely to be affected by parental cues. In comparison, those who were single may not have responded to the stimuli due to their greater disposition towards shortterm relationships and/or the fact that the stimuli were not relevant to them. This parallels a previous finding by Storey et al. (2000) that male T levels decreased when attending to parental stimuli but only when the participants felt engaged in the task. It 
may be the case that to be affected by a cue which signals that a long-term strategy would enhance fitness, one must already be further along the "path" to parenthood.

Evidence that effects can be restricted to a particular sub-group depending on stimuli relevance has been found across several psychological domains. One example can be found in the body dissatisfaction study by Posavac et al. (1998) which was cited earlier in this chapter. Another example from the social psychological literature reveals that individual differences in social value orientation (SVO) can affect ones susceptibility to priming. SVO measures identify whether an individual is more pro-self or pro-social, as well as how consistent this orientation is. For example, in a moneysharing task, one may show consistent pro-social behaviour by choosing the most equitable option in all cases, or inconsistent pro-social behaviour by choosing equitable options in only some cases. Smeesters, Yzerbyt, Corneille, and Warlop (2009) found that by using a priming task which exposed participants to religious words such as 'father', 'holy' and 'forgiveness' those with an inconsistent SVO could be made to perform more pro-socially on a dictator task.

In a study more relevant to this chapter, the dot probe task has been used to demonstrate that an individual's particular mating strategy can bias their attention towards certain stimuli. Maner, Gailliot, Rouby, and Miller (2007) found that, by exposing participants to a sexual arousal or mate-search prime, their attention became fixated upon attractive opposite-sex images. However, this effect was driven by individuals who were high in SO only. Likewise, individuals who were high in intrasexual vigilance (i.e. those who were jealous or concerned about potential partner infidelity) paid more attention to attractive same-sex targets when administered a jealousy prime. No such pattern was found among low vigilance individuals.

Effects which were driven by sub-groups were also found in this chapter. For example, in Experiment 4 it was pair-bonded women who showed an increase in ST preference following exposure to the wealth stimuli. This difference between single and committed women may be accounted for by the relative benefits of engaging in shortterm relationships for individuals who have a regular partner compared to those who do not. For a woman who is in a committed relationship, the costs of cheating on a partner can be great if caught, but there may be benefits if they are not (Greiling \& Buss, 2000; Meston \& Buss, 2009). They could acquire the affection, resources, and even the genes of a higher status male, while having the "safety net" of a committed relationship. The wealth stimuli could represent a cue to pair-bonded women that the pursuit of a short- 
term relationship may be beneficial. A study by Little et al. (2002) supports this idea. Here, the facial masculinity preference of women was found to be a function of their relationship status. Those already in a relationship showed a higher average preference for masculine faces than those who were single. Masculinity is seen as an indicator of good genes, which are in turn one of the hypothesized benefits of female extra-pair mating (Greiling \& Buss, 2000; Provost et al., 2006; Provost et al., 2008). In contrast, single individuals may already be following a relatively short-term strategy, and so the cue may not have changed their behaviour. Indeed, in Experiment 4 there was a difference in sociosexuality between the two different relationship groups of women with single women scoring higher.

\section{Sex Differences and Socio-Sexuality}

In Experiment 3, women were hypothesized to be more affected by parental stimuli than men. This hypothesis was informed by the fact that there exists a difference between the sexes in terms of minimum and typical parental investment (Geary, 2000; Marlowe, 2000; Trivers, 1972). However, the results revealed no such difference. In hindsight this makes sense in light of what is known about human mating. Ancestrally, the problems which both sexes had to overcome when pursuing a long-term relationship would have been very similar (Buss \& Schmitt, 1993). Both sexes would have faced the challenge of finding a partner who was loving, kind, faithful, and would have been a good parent. Thus, both men and women would have evolved similar adaptive behaviours to cope with these historically persistent challenges. It is not surprising then that if a parental cue biases mating strategies towards the long-term in one sex that it also does so in the other. The fact that no difference was apparent between the sexes may be indicative of the shared selection pressure faced by both sexes historically.

In contrast, the problems faced when pursuing a short-term mating strategy would have been fairly unique to each sex (Buss \& Schmitt, 1993; Greiling \& Buss, 2000; Stewart et al., 2000). For example, while men needed to overcome the problems of sexual access and assessing fertility, women had to identify men who were likely to share resources in exchange for mating and those who were of high genetic quality. As such we would expect the sexes to respond differently towards stimuli associated with short-term mating. In Experiment 4 wealth cues were found to have a general positive effect on the number of ST choices made by the participants, and further analysis revealed that this effect was driven mainly by women in relationships. One interpretation of this finding could be that the wealth cues signalled one of the potential 
benefits of ST mating in women (the acquisition of resources) and so shifted preferences in a way consistent with a short-term mating strategy. In contrast, the extraction of resources from women is not thought to have been a persistent problem for men in the EEA and so the stimuli did not signal the benefits of short-term mating to men (Buss, 2006b; Buss \& Schmitt, 1993). What sort of stimuli might produce a ST effect larger among men than women? Using the same logic, a cue that indicates greater sexual access to women might cause such an effect. One such cue, sex-ratio, is explored in Chapter 8.

Experiment 4 is not the first study to show that men and women respond differently to wealth cues. One study by M. Wilson and Daly (2004) also found a sex difference using a "discounting the future" task. This type of task involves making several choices between receiving a small amount of money immediately or larger amounts of money in the future. For example, participants might be asked if they would rather have $£ 5$ now or $£ 10$ in one week. The frequency in which participants choose the immediate reward provides an index as to how "impulsive" they are. Wilson and Daly's study found that, when presented with pictures of highly attractive opposite-sex individuals, only men increased in how much they discounted the future. However, when the stimuli presented were fast sports cars, women alone were the ones to increase. While discounting the future is not a direct measure of mating strategy, it does represent impulsivity, one of the behaviours related to short-term mating (Schmitt \& Shackelford, 2008, p. 247). The study compliments the findings of Experiment 4, adding confidence to the conclusion that cues to wealth increase ST mating inclination in women. Other examples of sex differences in response to stimuli include disgust cues (Curtis, Aunger, \& Rabie, 2004) and visual sexual stimuli (Hamann, Herman, Nolan, \& Wallen, 2004; Rupp \& Wallen, 2008).

One final effect which needs interpretation relates to socio-sexuality in Experiment 4. Here, following wealth stimuli, participants were less likely to choose models for LT relationships. However, further analysis revealed that this effect was driven by high SO individuals. Why was this the case? In Experiment 3, participants who were within a committed relationship (and had lower SO) were more likely to be effected by a "long-term" (parental) cue. This was attributed to the fact that committed individuals were further down the "path to parenthood" and so the parental cues were of greater relevance to this subgroup. Such interpretation could also be used to explain why high SO individuals decreased their LT choices following a "short-term" (wealth) 
cue; the stimuli were more relevant to this group as they were already closer down the "path to promiscuity".

\section{Replication Considerations}

One methodological concern of Experiment 4 surrounds the ambiguity of the wealth prime. Unlike the parental stimuli in which the presenters engaged with the participants, or the danger stimuli where interpretations are limited (see next chapter), there are different ways in which participants could interpret the slideshow. They could, for example, imagine how they would feel if they owned the items displayed to them. Likewise, the viewing of the images could cue the discrepancy between their current wealth and their desired wealth. The former interpretation would actually lead to the hypothesis that males would increase in ST but not females, while the second would imply the reverse. As such, an improved version of this experiment would give participants explicit instructions on what to focus on when viewing the slideshow. The disadvantage of this is that the prime would be made less subtle, leading participants to possibly guess the purpose of the experiment. An alternative suggestion would be to reduce ambiguity by showing models accompanying the stimuli. For example, instead of showing an expensive watch on its own, showing it in the presence of a model to which the item "belongs". Such stimuli have been used in other experiments such as Dunn and Searle (2010) and Dunn and Hill (2014) which manipulated the attractiveness of a male model by changing the car they were sitting in or the apartment they were living in (also see Marshall Townsend \& Levy, 1990).

The inclusion of additional factors could aid the interpretation of the effects. For example, in Experiment 3, a measure of intention to conceive (e.g. 'When do you plan on having your first/next child?') could help confirm the idea that the effect of parental stimuli on pair-bonded individuals was due to the stimuli being more relevant to those more likely to have a child in the near future. With regard to Experiment 4, although socio-economic status was assessed as a potential covariate, a closer examination of the participant's desire for resources could provide a useful moderator variable. For example, it might be the case that women in relationships who are disappointed with their combined household income are more likely to be affected by wealth cues than those who are content.

\section{Conclusion}

This chapter potentially contains the first two experiments which show that evolutionarily related stimuli can bias an individual's mating strategy in potentially 
adaptive ways. While other studies have approached this subject by looking at the effect of stimuli on an individual's preference towards factors associated with a particular mating strategy, the results here suggest that such primes can influence an individual's disposition towards long- and short-term relationships. The results of these experiments also draw attention to the fact that the effect of mating-related cues can vary by personal characteristics. That is, the conditional nature of mating strategies may depend on an interaction between the factor at hand (e.g. cues of resource availability) and the circumstances of the participant (e.g. if they are currently pair-bonded).

It appears as if basic visual stimuli are a good tool for testing the effect of evolutionarily related stimuli on mating behaviour. In Chapter 5, the use of this methodology is continued by exposing participants to danger cues in Experiment 5, and using an alternative "perspective taking" task in Experiment 6. 


\section{Chapter 5: Danger, Charles Darwin, Danger!}

Environmental harshness leading to early mortality is arguably the most historically persistent naturally selective pressure. There is not an extant species which is not at the mercy of local pathogens, resource availability, or intra/inter-species violence. While this pressure can lead to the evolution of intricate weapons and methods of defence, it also has consequences for the effectiveness of different reproductive strategies, creating a trade-off between offspring quality and quantity. For example, a female who decides to forego mating for several years until she finds a high quality male, in order to secure the best genes possible for her offspring, is likely to have her genetic lineage cut short if the environment leads to early mortality and thus a small reproductive window. Similarly, a female who mates quickly and indiscriminately when her potential reproductive lifespan is long may end up having offspring of a lower potential fitness than if she had been more discriminate when choosing a mate.

In accordance with life history theory (Chisholm et al., 1993; Kraus, Thomson, Kunkele, \& Trillmich, 2005; Reznick, Bryant, \& Bashey, 2002), a general pattern is observed in nature whereby high mortality risk environments are populated by species with increased reproductive output and decreased somatic growth (traditionally referred to in ecology as a species under r-selection). Such organisms include most insects, fish, and amphibians. In contrast, organisms with fewer predators and/or a relatively stable environment tend to grow larger, live longer, and reproduce at a slower rate (under what is referred to as K-selection). Such organisms include some mammals, lizards, and birds. When the harshness of the environment changes, species tend to evolve in line with this pattern. The extinct Dodo of Mauritius (Raphus cucullatus) is a popular example. Due to the absence of a natural predator, the Dodo evolved from a small bird of flight which reproduced quickly to a large flightless bird which reproduced slowly (with a clutch size of one; Livezey, 1993). Similar effects have been demonstrated under more controlled pseudo-naturalistic conditions using species such as wood frogs (Relyea, 2002). There are some noticeable exceptions to this trend, such as the island dwarfism experienced by very large mammals (e.g. elephants) which have previously evolved their large size as a specific defence against predators. Much like the Dodo's capacity for flight, such adaptations become lost in environments without natural predators. 
Within an individual's lifetime, changes to environmental harshness can lead to shifts in reproductive behaviour towards or away from each end of the life history spectrum. ${ }^{23}$ For example, when exposed to cues of predators (crushed eggs and tadpoles) or pathogens (seeds containing water mould) tadpoles have been shown to hatch faster, sacrificing a longer growth span for earlier reproductive capacity (Anderson \& Brown, 2009). In plankton, exposure to fish kairomones (a communication chemical similar to pheromones) leads to an increase in clutch size and reduced size at sexual maturity (Reede, 1995), and a large number of birds ranging from hermit thrushes (Catharus guttatus) to house wrens (Troglodytes aedon) engage in greater parental care and invest more resources (i.e. larger egg mass) in their clutches when local predators are removed (Fontaine \& Martin, 2006).

There are also cases in which temporary indicators of harshness (i.e. scarcity of food or predator presence) do not lead an organism to markedly change their current reproductive strategy, but simply adapt it to the temporary circumstances. For example, when grey-sided voles (Clethrionomys rufocanus) are exposed to the scent of a predator in the breeding environment, instead of increasing their rate of reproduction they instead temporarily cease reproduction (repressed fertility; Fuelling \& Halle, 2004) and in Siberian jays (Perisoreus infaustus) predator cues lead to increased parental investment in a reduced clutch size and the choice of more protective nesting sites (Eggers, Griesser, Nystrand, \& Ekman, 2006). Thus, it appears that how a particular species adapts its reproductive behaviour to environmental harshness can vary depending on their mating system (e.g. levels of parental/bi-parental care) and the longevity of the cue.

\section{Life History and Human Mating}

Compared to other mammals, the human growth process is marked by an extended infertile period for both sexes and a relatively large inter-birth interval for women (L. Barrett et al., 2002). While this does show variation across populations (Pennington, 2001, p. 182), the species-level pattern points to an ancestral environment which was safe enough for reproduction to be prolonged with more resources dedicated to somatic growth. That is not to say that the EEA was not hazardous. For example, paleoanthropological evidence from the Pleistocene suggests that, despite living in

\footnotetext{
${ }^{23}$ As previously mentioned, environmental harshness encompasses several factors including pathogen prevalence, violence from conspecifics, predation threat, and environment related mortality risk (e.g. flooding and drought). As a starting point, the experiments in this chapter focused on just a subset of these factors.
} 
communities, archaic humans were often killed by large predators (Hart \& Sussman, 2009; Prokop \& Fancovicova, 2010) as well as members of their own species (Thorpe, 2003). Among modern day hunter-gatherers and horticulturists such as the !Kung and the Tiwi, whose way of life is more representative of early Homo sapiens, intra-species violence can account for a large percentage of fatalities (Bowles, 2009; K. Hill, Hurtado, \& Walker, 2007; Wrangham, Wilson, \& Muller, 2006). ${ }^{24}$ Furthermore the prevalence of fears towards snake and spider-like stimuli which transcend culture (and the actual threat posed by local wildlife; Kasturiratne et al., 2008; Ohman \& Mineka, 2001) indicate that these hazards may have proved a persistent threat to our ancestors.

How might within lifetime variances in environmental danger or harshness influence human mating behaviour? Given life-history theory and its application to other species, one prediction might be that increased environmental harshness would lead to a shift from long-term pair-bonds producing a smaller number of children to short-term mating from a younger age involving earlier and more frequent pregnancies. Certainly there is some evidence which suggests this. In areas categorised by greater social stratification and increased homicide rates, there are higher levels of teen pregnancy (Kearney \& Levine, 2011; Pickett, Mookherjee, \& Wilkinson, 2005) which may in part be due to lower rates of contraceptive use (and casual sex) among women who are heavily dependent on resources from their partner (Rosenbaum et al., 2012). Likewise, there is growing support for the idea that women (and in some cases men) who undergo stressful family environments when young, enter puberty earlier, get married sooner, and tend to display sexual behaviours consistent with an unrestricted socio-sexual orientation (Bereczkei \& Csanaky, 2001; Griskevicius, Tybur, Delton, \& Robertson, 2011). Furthermore, there are some promising models which have attempted to formalise this relationship (Belsky, Schlomer, \& Ellis, 2012; Brumbach, Figueredo, \& Ellis, 2009). ${ }^{25}$ Finally, there appears to be a link between pathogen prevalence and short-term mating behaviour. In one correlational study pathogen rates were found to be linked with an increase in "good gene" interest in potential partners across a large number of cultures (Gangestad \& Buss, 1993) and in men the experimental

\footnotetext{
${ }^{24}$ There is of course great fluctuation in the amount of deaths by person-on-person violence (Pinker, 2011, p. 49, Fig. 2-2). The Aka pygmies, for example, are categorised by high paternal care and very low levels of person-on-person violence (Hewlett, 1991).

${ }^{25}$ As discussed in Chapter 1, whether this effect is driven more by shared environmental influences or genetic influences is still open issue within the developmental and evolutionary psychological literature.
} 
administration of disease cues led to an increase in the number of sexual partners desired in the future (Murray, Jones, \& Schaller, 2013).

So far the evidence presented indicates that following a danger cue both sexes may increase in their short-term mating inclination and perhaps decrease in their longterm inclination as they shift along the life-history spectrum. However, a contrasting view emerges when the typical levels of parental care in humans is considered. As discussed in both Chapters 1 and 4, human children require almost constant attention for several years after birth, and to cope with this our species has evolved various forms of extra-maternal care, including paternal care in the context of a pair-bond. However, in a peaceful environment where resources are plentiful, such care may contribute less to a reduction of offspring fatality. Thus, parental care may become more important as mortality risk increases, and various lines of research also support this (Geary, 2000; Little, Cohen, et al., 2007; Schmitt, 2005b). Certainly this is the case in other species where parental care is prominent, such as the Siberian jay (Perisoreus infaustus) and the Kentish plover (Charadrius alexandrinus; Al Rashidi, Kosztolányi, Shobrak, Küpper, \& Székely, 2011). As such, an alternative prediction might be that following exposure to danger cues men and women would both increase in LT and perhaps decrease in ST selection. Thus, there is a contradiction within the literature. Some evidence suggests danger cues would lead to a shift towards a more long-term strategy, while other evidence suggests this shift would be towards a more short-term strategy.

\section{Quinlan's Model - a Reconciliation}

Rather than pitting these two predictions against each other using two competing one-way hypotheses, or by using a two-way hypothesis, there exists a model produced by Quinlan (2007) which somewhat reconciles the two views. The model proposes that the effectiveness of maternal care on offspring mortality is contingent on environmental harshness (specifically pathogen stress) and follows a quadratic curve (red line, Figure 5.1). That is, initially maternal care increase survivability of offspring as environmental harshness increases. However, beyond a certain point its effects are negligible, such as if the chance of parental mortality is high. Applied to humans, such a model would predict that moderate and temporary cues of threat would lead to an increase in parental care (i.e. a long-term mating strategy), while chronic and persistent cues would lead to a decrease in parental care and a shift towards mating effort (i.e. a short-term mating strategy). There are some very noticeable cases in which experience with extremely harsh environments, such as warzones or those in which food is scarce, have produced 
an increase in promiscuous behaviour among men and women (Muhwezi et al., 2011; Weiser et al., 2007), and stressful family environments have been mentioned previously.

In applying Quinlan's model to the current research, two important assumptions are made. First, while the model was initially developed around pathogen stress, it is assumed that there is no reason why the same principles should not apply to other factors causing variance in mortality such as the threat of predation or resource scarcity. Second, while originally applied to maternal care exclusively, the model is assumed to apply to paternal care as well in species where paternal investment is high. If this extension of Quinlan's model is correct, then the effect of danger cues on participant mating preferences within an experimental context is likely to vary by the intensity of the stimuli. Specifically, temporary and moderately intense danger cues should lead to an increase in long-term mating interest in both sexes, and highly intense persistent stimuli should lead to an increase in short-term mating interest in both sexes. This relationship is expressed in Figure 5.1, where a second line has also been added as a hypothetical change to short-term mating behaviour in light of sexual strategies theory (Buss \& Schmitt, 1993).

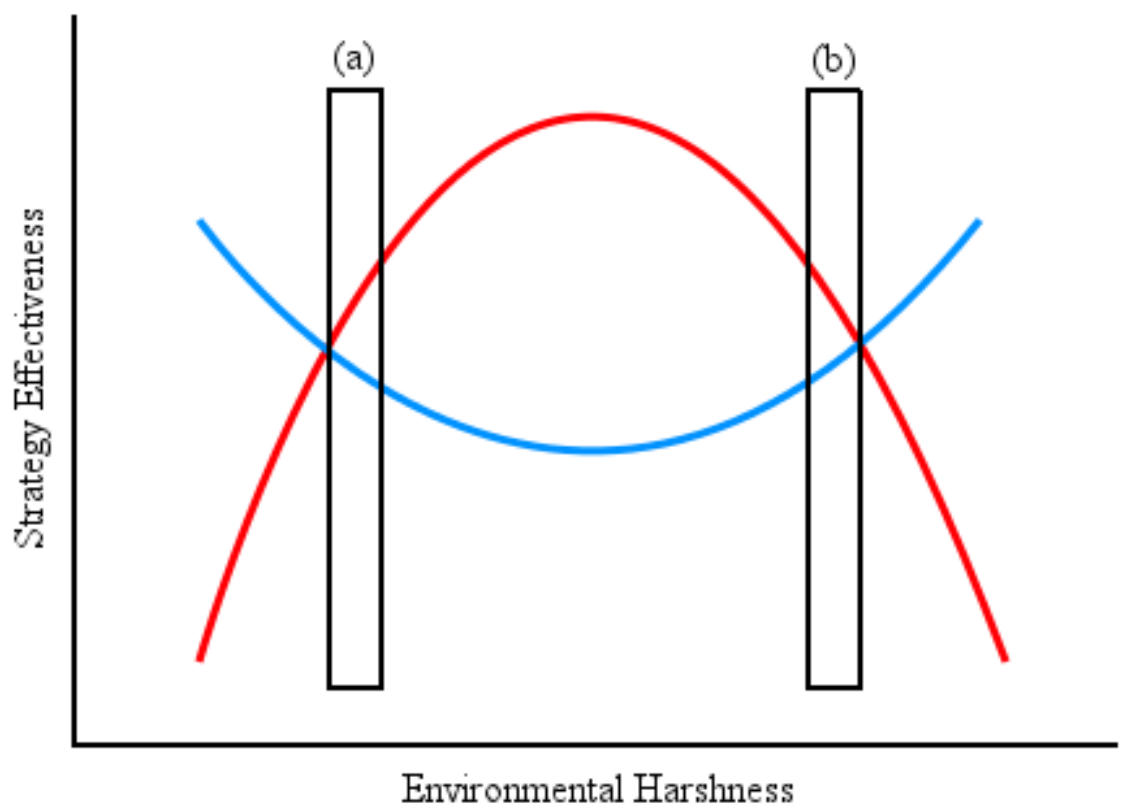

Figure 5.1. The hypothetical relationship between environmental harshness and the effectiveness of short- and long-term mating strategies. The red line represents longterm mating and is adapted from the quadratic curve of the influence of maternal care on offspring mortality as proposed by Quinlan (2007). The blue line represents short- 
term mating and is inspired by sexual strategies theory (Buss \& Schmitt, 1993). Crosssection (a) represents the outcome of moderate/temporary danger cues on the benefits of each mating strategy, while (b) represents the outcome of intense/persistent danger cues.

\section{Danger Cues and Sex Differences}

In many species, the implementation of an alternative mating strategy is simply a different way of reaching the same goal. Consider the Coho salmon (Oncorhynchus kisutch), where mating strategies are developed via two pathways. The first is phenotypical (large males are either "hooknose" or small "jack" morphs) which is an irreversible change decided at an early stage of development. The second is behavioural (the used of "fight" or "sneak" strategies) which can change with circumstance, but is more likely to be performed by one phenotype than the other. Irrespective of the phenotype or particular behavioural strategy, the goal of each salmon is clearly and directly the fertilisation of eggs. There is no additional social benefit of being a jack or a hooknose, or a sneaker rather than a fighter (M. R. Gross, 1991). However, in humans and other social animals, alternative mating strategies can often lead to additional social benefits which, while they can enhance fitness, do so in a more complex and indirect way. For example, Greiling and Buss (2000) highlight the many social and reproductive benefits of short-term strategies in women which are not generally conveyed to men through the pursuit of short-term mating. For example, one of the theories behind the prevalence of short-term mating strategies in females highlights the protection benefits afforded by "special male friends" who are given sexual access (Buss \& Schmitt, 1993; Smuts, 1992). ${ }^{26}$ Sex is not just exchanged for protection; females of several species also consent to sex in exchange for other resources such as food (Gomes \& Boesch, 2009; Thornhill, 1981). As such, short-term mating in and of itself can be a method of overcoming obstacles generated by harsh environments, but primarily for one sex. It follows then that it might be the case that cues signalling environmental danger or threat may cause an increase in short-term relationship willingness in females alone in order to extract fitness enhancing resources from males. In contrast to women, rather than decreasing, short-term mating is likely to be relatively unaffected by environmental harshness in men given that, although the payoff of such a strategy does vary, the cost

\footnotetext{
${ }^{26}$ While such theories are primarily centered on protection by males from other males of the same species, such protection likely extends to other threats such as those from predators (see RodriguezMunoz, Bretman, \& Tregenza, 2011 for a recent example with insects).
} 
of pursuing it as part of a mixed strategy (Gangestad \& Simpson, 2000) is relatively low (see Figure 5.2).

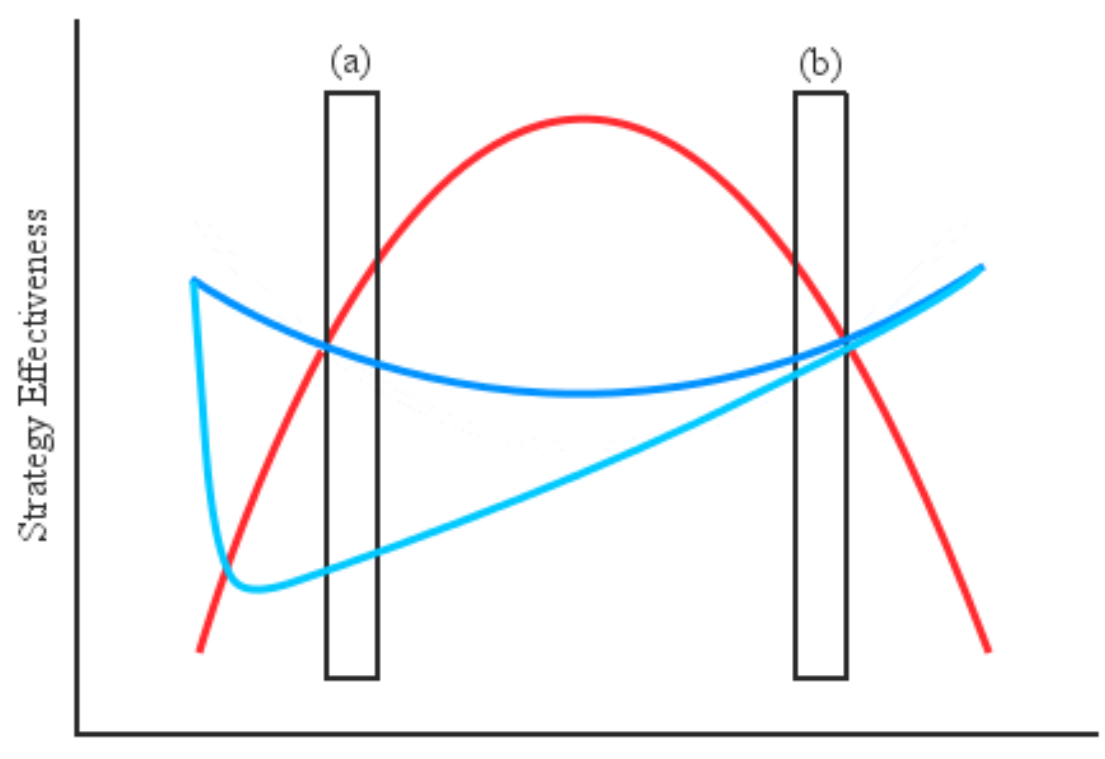

Environmental Harshness

Figure 5.2. A modified version of Figure 5.1 to include hypothetical sex differences in the effectiveness of long- and short-term mating as environmental harshness (danger) varies. Here, the blue line represents short-term mating in men and is slightly shallower than in Figure 5.1 due to the little cost which ST mating accrues when not pursued exclusively. The light blue line represents short-term mating in women and results in a sharper initial drop and a more progressive increase due to the added social benefits which short-term mating can accrue.

\section{An Integrated Prediction}

Given the information available, it might be expected that exposure to moderate and temporary cues of environmental harshness would lead to an increase in long-term mating interest for both sexes, while short-term interest would increase in females alone. In contrast, following extreme and persistent cues of threat, both men and women would be expected to shift towards short-mating and away from long-term mating, as shown in Figure 5.2 (a) and (b). It is highly unlikely that appropriate stimuli could be produced that would indicate the mortality risk of an extreme environment within the 
laboratory while easily passing ethical scrutiny. Thus, it is the former idea which is reflected in the experimental hypothesis used in this chapter.

\section{Experiment 5}

To test the prediction made above, Experiment 5 exposed participants to a slideshow consisting of images of dangers relevant to the ancestral environment, namely those of spiders and snakes (Ohman \& Mineka, 2001), aggressive/predatory animals (Prokop \& Fancovicova, 2010; Treves \& Naughton-Treves, 1999), and violent out-group members (Navarrete et al., 2009). This slideshow was of the same format used in Experiment 4 in the previous chapter. Hypothesis 5 stated that moderate danger cues would lead to long-term mating strategy activation in both men and women, as well as short-term mating strategy activation in women alone. Two predictions were developed to test this Hypothesis. Prediction 1 was that, following a slideshow of moderate danger cues, both sexes would choose a greater number of LT models on the SMA task compared to a neutral group. Prediction 2 was that, following the same cues, only women would show an increase in ST selection on the SMA task compared to a neutral group.

\section{Method}

\section{Participants}

One-hundred and twenty volunteers were recruited for the study using the psychology participant pool and through local advertising within the university. Participants were given either course credit or payment in return for completing the study. During analysis, four participants were excluded as they identified themselves as being homosexual. The following demographic details refer to the 116 remaining participants. Roughly half of the participants were female $(50.9 \% ; n=59)$ and, apart from three bisexuals, all participants described themselves as heterosexual. The sample was almost exclusively Caucasian ( $n=111 ; 95.7 \%)$ with two participants identifying themselves as Black, two as South Asian and one as South-East Asian. None of the participants had children and just over half were not in a committed relationship $(n=$ $59 ; 50.9 \%)$.

As Hypothesis 5 stated different effects for men and women, sex differences were explored for age, socio-economic status (SES), SOI-R, and self-reported attractiveness. The sample had an average age of $20.89(S D=1.30)$, with no statistical difference found between the sexes, $t(114)=0.770, p=0.44$. There was also no sex difference in average socio-economic status which was reported as $3.05(S D=0.60)$, 
$t(113)=0.331, p=0.74$. There was, however, a sex difference in the average selfreported attractiveness with $5.68(S D=1.21)$ reported by men and $5.14(S D=1.31)$ reported by women, $t(113)=2.312, p=0.02 d=0.43$. Likewise, a difference on the SOI-R was found with men having a score of $41.51(S D=13.77)$ and women having a score of $32.75(S D=9.22)$ on average, $t(113)=4.031, p<0.01, d=0.76$. All of these demographic variables were entered into the ordinal regression models below using a backwards stepwise method, and were discarded if found non-significant.

\section{Apparatus}

Forms. The SOI-R and standard demographic questionnaire were used in this experiment (Appendices B \& E). The only modification was the inclusion of four questions aimed to measure the participant's perceived fear towards the stimuli used in the experiment. Specifically, participants were asked 'How much do $X$ scare/frighten you?' with $X$ being replaced by 'spiders', 'snakes', 'large crowds', and 'large and aggressive animals'. Fear was recorded using a semantic differential scale between one ('not at all') and nine ('very much so'). Consent and debrief forms used for this experiment can be found in Appendix O.

SMA Models. The SMA task was used as the dependent measure for this experiment. One-hundred and seventy-three pictures of men were selected from the hotornot.com website and rated for attractiveness by 15 judges. Unless otherwise stated, all stimuli judges used in this thesis were different for each experiment. The judges had an average age of $20.47(S D=0.64)$ and ten were female. Together, these judges considered the sample of male photographs to be, on average, slightly unattractive $(M=$ $3.86 ; S D=1.04)$. From this large pool, a random selection of 50 photographs from the middle-to-high range of the distribution was taken. The average attractiveness of this sub-set was $4.50(S D=0.32)$ which is consistent with other male stimuli used in previous experiments. For the female photographs, 184 images were also gathered from hotornot.com to form an initial sample. These photographs were rated for attractiveness by another 15 judges (male $n=10$ ) whom had an average age of $20.33(S D=0.72)$. The female pictures were rated more favourably than males on average $(M=4.50, S D=$ 1.63 ) and this difference was statistically significant, $t(355)=4.33, p<0.01, d=0.46$. A sub-sample of 50 female images was chosen from the middle of the distribution. This sample had an average attractiveness of $4.49(S D=0.32)$ which was not significantly different from the male stimuli, $t(98)=0.08, p=0.93$. 
Danger and Neutral stimuli. To form the danger stimuli, 140 images were gathered from public domain websites. Each image contained either a snake, spider, aggressive animal (e.g. attacking bears, wolves, and sharks), or riot (e.g. masked rioters throwing projectiles and starting fires). Each category represented $25 \%$ of the image set. All of these images were then rated by 20 judges (female $n=10$; age $M=20.40, S D=$ 0.68 ) for how "threatening" they were on a nine point scale ranging from 'not at all' to 'very much so'. On average, these images were seen as relatively threatening $(M=6.44$, $S D=0.72$ ). The 75 images with the highest rating were used as the final stimuli set. This set contained 32 pictures of aggressive animals (42.7\%), 18 of spiders (24\%), 17 of riots $(22.7 \%)$, and eight of snakes $(10.7 \%)$. The average threat rating for these stimuli was $6.98(S D=2.06)$.

A similar process was used to form the neutral stimuli set. One hundred and fifty images featuring an equal split of non-threatening animals (e.g. giraffes and puppies), household objects (e.g. pillows and pencils), and landscape photographs were gathered. The same judges rated this stimuli set as very non-threatening on average $(M=1.30, S D$ $=0.27$ ) and the 75 images rated lowest for threat were chosen for the neutral stimuli set. These consisted of 66 pictures of household objects (88\%), six images of landscapes $(8 \%)$, and three images of animals (4\%). On average these pictures had a threat rating of $1.15(S D=0.43)$.

Two slideshows were created, one for the neutral stimuli and one for the danger stimuli. The process was identical to that used for the wealth stimuli discussed in Chapter 4. Each slideshow lasted 3 minutes and 45 seconds and displayed each image in a random order for three seconds. Examples of the neutral and danger stimuli can be found in Appendix P.

Hardware. The experiment was completed on a 19" Toshiba ${ }^{\circledR}$ laptop running Windows ${ }^{\circledR} \mathrm{XP}$ on a 1024 by 768 resolution screen.

\section{Design}

The experiment had a 2 by 2 mixed-model design with the within-subjects factor of time and the between-subjects factor of condition. The SMA task formed the dependent variable and exposure to danger or neutral stimuli formed the independent variable. Analysis utilised chi-squared to test for main effects, as well as ordinal regression to test for interaction effects using variables featured in the demographic and SOI-R questionnaires.

\section{Procedure}


The study procedure was identical to that used in Experiments 3 and 4 aside from the three following key differences. First, due to the nature of the stimuli used, participants were given forewarning in the information sheet and consent form (Appendix O) that the stimuli they were going to view contained images of snakes, spiders, and aggressive mammals, and that they should inform the experimenter if they felt uncomfortable viewing these types of images. Second, this warning was also foreshadowed by the inclusion criteria during recruitment advertising. The study was advertised as an investigation into 'social perception and relationship choices' and featured the inclusion criteria of 'being comfortable answering questions of a sexual nature and viewing images of spiders, snakes and aggressive animals'. Third, much like the earlier wealth cue study (Experiment 4), during the introduction of the slideshow participants were told that their memory of the items would be tested at the end of the experiment. No such test occurred.

\section{Results}

The number of participants in each group was reasonably balanced with 27 men and 30 women appearing in the danger condition and 30 men and 29 women in the neutral condition. There was a large sex difference in responses to the fear questions of the demographic form with women scoring higher on average in every case. For spiders, men reported an average of $3.32(S D=2.21)$ while women reported and average of 5.44 $(S D=2.60), t(113)=4.698, p<0.01, d=0.88$. For fear of snakes these figures were $2.73(S D=1.72)$ and $4.81(S D=2.32)$ respectively, $t(113)=5.448, p<0.01, d=1.03$. The weakest of the differences surrounded the fear of crowds. Here men reported 2.23 $(S D=1.54)$ on average, and women reported $3.54(S D=2.14), t(113)=3.749, p<$ $0.01, d=0.71$. Finally, men rated their fear of aggressive animals as $3.09(S D=1.61)$ on average, while women rated their fear as $4.95(S D=2.05), t(113)=5.398, p<0.01$, $d=1.01$.

At first performance of the SMA task, a median of one model was chosen for a long-term relationship and 12 models were chosen for a short-term relationship. This low number of LT selections is addressed in the results section.

\section{Short-Term Mating}

Following the presentation of the danger stimuli, 25 participants (42.4\%) selected fewer models for short-term relationships (ST-), nine (15.2\%) chose the same amount ( $\mathrm{ST}=$ ), and $25(42.4 \%)$ chose greater. Compared to the neutral stimuli (Table $5.1)$ it appeared as if participants in the danger condition were more likely to belong in 
the ST+ group. However, the difference between the two distributions was not significant, $\chi^{2}(2)=2.546, p=0.28$. Here, a two-tailed test was used as the effect of sex (which was specified as part of the one-way hypothesis) was not included.

Table 5.1

The number of participants who increased, stayed the same, or decreased in their number of ST choices following exposure to either neutral or danger stimuli. The two distributions are not significantly different from one another using a chi-squared test

\begin{tabular}{lccc}
\hline \multicolumn{4}{c}{ Short-term change } \\
\hline & - & $=$ & + \\
\hline Danger & 25 & 9 & 25 \\
Neutral & 30 & 7 & 20 \\
\hline$\chi^{2}(2)$ & 2.546 & & \\
Cramér's $V$ & 0.11 & & \\
\hline
\end{tabular}

${ }^{\dagger} p<0.10, * p<0.05, * * p<0.01$

No significant ordinal model could be produced using just a Conditon by Sex interaction and so variables from the demographic form and SOI-R were also included in the analysis to try to predict ST category change. Using a backwards stepwise method, a significant model emerged using the variables of sex, condition, and SOI-R (Table 5.2). According to the model, women were $229 \%$ more likely to appear in the $\mathrm{ST}+$ group following the presentation of danger stimuli compared to the neutral stimuli. There was also an interaction between sex and SOI-R, with men in both conditions being more likely to appear in ST+ if they had a high SOI-R score. Specifically, for every one point increase in SOI-R, men were $4 \%$ more likely to appear in ST+. This model depended on the attribute of sex being controlled for, as there was a very large effect of women selecting less ST partners during second exposure to the SMA task regardless of experimental condition (see Table 5.2).

Table 5.2

The results of an ordinal regression to predict ST choice change using a) sex, $b$ ) an interaction between sex and condition, and c) an interaction between sex and SOI-R

\section{Model ST}




\begin{tabular}{lcc} 
& \multicolumn{1}{c}{$\beta$} & OR \\
\cline { 2 - 3 } Sex & $-3.870 * *$ & 47.9 \\
Male * Condition & -0.433 & - \\
Female * Condition & $1.190^{*}$ & 3.29 \\
Male * SOI-R & $0.043^{*}$ & 1.04 \\
Female * SOI-R & -0.037 & - \\
\hline Model & $\chi^{2}(5)=13.911^{*}$ \\
Nagelkerke $R^{2}$ & 0.13 \\
Accuracy & $60 \%(+26.6 \%)$ \\
Parallel lines & $\chi^{2}(5)=3.862$ \\
\hline
\end{tabular}

Note: Variables were coded as such: Condition - neutral (0), danger (1); Sex - male (1), female (2). OR = odds ratio. SOI-R $=$ Socio-sexual Orientation Inventory Revised. ${ }^{\dagger} p<0.10,{ }^{*} p<0.05, * * p<0.01$

\section{Long-Term Mating}

In the danger condition, 12 participants $(21.1 \%)$ decreased in their long-term selection (LT-), 17 (29.8\%) stayed the same (LT=), and 28 (49.1\%) increased (LT+). When compared to the distribution found following the neutral condition (Table 5.3), it appeared as if exposure to the danger stimuli led participants to select more models for a long-term relationship. This was confirmed with a chi-squared test which showed that the two distributions were significantly different from one another, $\chi^{2}(2)=31.594, p<$ 0.01 .

Table 5.3

The number of participants who increased, stayed the same, or decreased in their number of LT choices following exposure to either neutral or danger stimuli. The two distributions are significantly different from one another using a chi-squared test

\begin{tabular}{lccc}
\hline \multicolumn{4}{c}{ Long-term change } \\
\hline & - & $=$ & + \\
\hline Danger & 12 & 17 & 28 \\
Neutral & 12 & 35 & 12 \\
\hline$\chi^{2}(2)$ & $31.594^{* *}$ \\
Cramér's $V$ & 0.37 \\
\hline
\end{tabular}

$$
{ }^{\dagger} p<0.10, * p<0.05, * * p<0.01
$$


In this instance, a significant ordinal regression model was produced using just the variable of condition (Table 5.4). An additional backwards stepwise ordinal regression was conducted using several variables including condition, sex, age, fear of the stimuli, SOI-R, SES and relationship status. However, this failed to produce any other model.

Table 5.4

The results of an ordinal regression to predict LT choice change using condition

\begin{tabular}{lll}
\hline \multicolumn{3}{c}{ Model LT } \\
\hline \multirow{2}{*}{ Condition } & \multicolumn{1}{c}{$\beta$} & OR \\
\cline { 2 - 3 } Model & $\chi^{2}(1)=5.172^{*}$ & 2.32 \\
Nagelkerke $R^{2}$ & 0.05 \\
Accuracy & $54.3 \%(+25.3 \%)$ \\
Parallel lines & $\chi^{2}(1)=7.731 * *$ \\
\hline
\end{tabular}

Note: Variables were coded as such: Condition - neutral (0), danger (1). OR $=$ odds ratio. ${ }^{\dagger} p<0.10,{ }^{*} p$ $<0.05, * * p<0.01$.

The test of parallel lines for this model was significant. This tests the assumption that the model predicts change between lower categories (e.g. LT+ to LT=) and higher categories (e.g. LT= to LT-) equally. If this assumption is not met then an ordinal regression is not an appropriate test. As such, the analysis was repeated using a multinomial logistic regression, which assumes no ordinal relationship between the DV categories. This revealed a significant overall model when using the variable of condition, $\chi^{2}(2)=12.910, p<0.01$. However, condition was only a significant predictor when contrasting $\mathrm{LT}=$ with $\mathrm{LT}+(\beta=-1.569, p<0.01)$ and not LT- and LT $=(\beta=-$ $0.722, p=0.925)$. This difference was likely due to the number of participants in the LT- category being equal for both conditions $(n=12)$, which may be due to the low median number of models chosen in the SMA task. Thus, the correct interpretation of the effect is that after receiving danger stimuli, participants were $480 \%$ times more likely to appear in the LT+ category than the LT= category.

\section{Discussion}

A test of the overall effect of the danger cues on participant mating behaviour revealed that, compared to participants in the neutral condition, short-term selection 
remained the same while long-term selection tended to increase. When these results were further explored using non-parametric regression analysis, a significant model was found for both short- and long-term mating change.

In the short-term model, irrespective of condition, women picked fewer models post-stimuli than men, and men of a higher SOI-R were more likely to pick a greater number of models post-stimuli. Once these effects were controlled for, a significant sex by condition interaction emerged suggesting that women in the danger condition were more likely to select a greater number of short-term models post-stimuli compared to those in the neutral condition. In the long-term model, only an effect of condition was found, confirming the results of the chi-squared analysis. As there was no difference between the neutral and danger distributions in the number of participants appearing in the LT- category, the model was restricted to predicting the difference between LT $=$ and LT+ categories only.

Thus, support was found for both the predictions made based on Hypothesis 5, with the caveat that non-condition related differences in responding behaviour were controlled for in the short-term ordinal model. In Experiment 6, the same hypothesis was maintained with the intention of replicating these results using a different type of danger cue, and an amended version of the SMA task. In addition to neutral and danger conditions, an "arousal" condition was introduced. This was included to test the assumption that the results were due to the dangerous aspect of the cues used rather than general arousal.

\section{Creativity Tasks as a Manipulation}

Rather than a slideshow, Experiment 6 used a novel manipulation which was also used in Chapter 7. This manipulation involved participants engaging in a "creativity" task in which they were asked to imagine themselves in different scenarios. They then had to write about their hypothetical thoughts, feelings, and potential actions in response to the scenario. This task was inspired by the 'Peacock, Picasso and Parental Investment' paper by Griskevicius, Cialdini, and Kenrick (2006). Here, participants had to imagine and write about either a short-term mating scenario, a long-term mating scenario, or a control scenario. In the short-term scenario, participants imagined they met a desirable individual during the last day of an exotic holiday, spent the day together, had a romantic meal, and finished by 'kissing on the beach' (implying a one night stand-like situation with a lack of commitment). In the long-term scenario, the participants imagined themselves meeting a potential romantic interest on campus, 
spending the day together, having a romantic candle lit dinner, and then sharing a 'sweet kiss goodnight'. The participant was told that they were considering the romantic interest's potential as a long-term partner in this scenario which concluded with the participant anticipating their first official date with the person. Finally, the control condition had participants visualise themselves walking down a street and asked them to discuss the most favourable weather conditions for admiring the buildings. In Griskevicius et al. (2006), these scenarios were the manipulation, with various measures of creativity employed to assess how the scenarios affected the creative performance of the participants. In general, the authors found that, after priming relationships, creative ability increased in men regardless of mating context, but that the effect was only apparent in women following high-commitment long-term primes.

\section{Experiment 6}

In Experiment 6 scenario-based manipulations were used, similar to those found in the Griskevicius et al. (2006) study. However, instead of seeing if mating context influenced another variable (e.g. creativity), the scenarios themselves were used to prime the variable of interest (in this case danger). Changes to mating preferences were then measured using a modified SMA task. These modifications were designed to produce parametric data and are discussed in full detail within the following method section. Experiment 6 used the same hypothesis as Experiment 5. The predictions also stayed the same as the revised SMA task also produced a value for ST and LT relationship choices.

\section{Method}

\section{Participants}

Two hundred and eighty-eight participants were recruited for the study in return for course credit or cash payment. Recruitment came from local advertising within the university or through the psychology department's participant pool. Two participants were excluded as their sexuality was unknown (they stated 'other' on the demographic form but did not elaborate as to what their sexual preference was). Just over half of the remaining 286 participants were male $(50.7 \% ; n=145)$ and all but three bisexual participants described themselves as heterosexual. The sample was predominantly Caucasian ( $n=268 ; 93.7 \%$ ) with ten participants identifying themselves as Black, one as South Asian, two as South-East Asian and two as 'other'. Only two participants had children (one each) and the majority of the participants were single $(55.9 \% ; n=160)$. 
For this sample, there were sex differences in age, SES, self-perceived attractiveness, and SOI-R. Men had an average of $21.30(S D=1.49)$ while women had an average of $20.65(S D=1.36), t(284)=3.851, p<0.01, d=0.46$. Men reported themselves to be of a slightly higher socio-economic background on average $(M=3.38$, $S D=0.82)$ compared to women $(M=3.15, S D=0.77), t(284)=2.447, p=0.02, d=$ 0.29 , and also typically reported higher self-perceived attractiveness, with women reporting $5.18(S D=1.50)$ and men reporting $5.61(S D=1.53), t(284)=2.430, p=$ $0.02, d=0.29$. Finally, consistent with previous experiments, the sample of men had a higher average SOI-R score than women. For men, this was $47.98(S D=13.59)$, and for women this was $34.74(S D=12.39), t(284)=8.599, p<0.01, d=1.02$. To account for these differences, sex, as well as the other variables were included in all ordinal regression models as potential covariates, but were ultimately excluded if they were non-significant.

\section{Apparatus}

Forms. In this experiment, the demographic form no longer contained questions about the participant's fear of snakes or spiders as the danger stimuli no longer included these animals (see below). As the neutral group from this experiment was also used in a subsequent experiment, two questions were also included about victory (see Experiment 8 in Chapter 7). Aside from these changes, the SOI-R and demographic forms were identical to those used in Experiment 5. The consent and debrief forms unique to this experiment can be found in Appendix $\mathrm{O}$.

Changes to the SMA paradigm. In the SMA task, participants are shown 50 models and are asked to choose one of three hypothetical relationships they would like to have with each individual, either a 'long-term thing', a 'short-term fling' or 'nothing at all'. There are two advantages of using this measure. First, it limits the task to 50 responses. This makes measurement quick, limits the exposure of the participant to the models, and reduces the chances of experimental fatigue. Second, the relationship choices participants make are "traded-off" against one another as the same individual cannot be picked for more than one relationship type. This adds confidence that each relationship choice a participant makes is a reflection of their true desires. If participants were able to choose both long- and short-term relationships for a particular model it would not be clear which type of relationship was the primary preference and which the participant may "settle for". For this replication, however, some experimental changes 
were made to the SMA task in order to try to produce data which met parametric assumptions and therefore would allow for more powerful statistical analysis.

To accomplish this goal, three key changes were made to the task. First, participants were asked whether they would pursue a long-term and short-term relationship with each model separately. That is, they were initially given the definition of a ST relationship and then made a 'yes' or 'no' decision for each of the models indicating whether they would want to pursue that relationship type. The process was then repeated for LT relationships. This presentation order was counterbalanced so that half the time an LT relationship was presented first. Second, the wording of the scenario in which participants imagined themselves to be under was altered. Participants were still told to imagine themselves as single and open to a new relationship and to imagine that they had met the model once or twice through friends. However, this time participants were told that 'after a while they ask you if you would be interested in a relationship with them. They make it clear that they are looking for a short-term [or long-term] relationship.' This scenario was then followed by the standard definitions of a short- and long-term relationship as specified in Chapter 2. Finally, as the task was doubled in length, a progress bar and a mandatory 30 second break was introduced between relationship types in order to reduce experiment fatigue. These changes can be seen in Appendix Q.

SMA models. A set of male and female photographs was constructed for use in this experiment as well as the experiment in Chapter 7. Twenty one judges were used to rate the gathered photographs (male $n=11$ ) and they had an average age of 20.48 (SD = 1.17). For the male pictures, an original pool of 116 images were gathered from hotornot.com which had an average attractiveness rating of $4.53(S D=0.97)$. From these, a sub-set of 50 pictures were used in the SMA task. These were taken from the middle-to-high end of the distribution and had an average attractiveness rating of 5.42 $(S D=0.66)$. The same process was conducted for the female photographs. One hundred and twenty-two were initially gathered $(M=5.14, S D=1.02)$ and a sub-set of 50 were chosen from the middle of the distribution so that the average attractiveness matched that of the male photographs $(M=5.41, S D=0.65), t(98)=0.08, p=0.94$.

Danger, arousal, and neutral stimuli. Inspired by Griskevicius et al. (2006), three written tasks were created to act as experimental manipulations. The first task was designed to include evolutionarily relevant danger cues, the second was designed to illicit general arousal in the participants, and the third was designed to act as a non- 
arousing control task. All three of the tasks followed a similar format. The task sheet began by informing participants that they were going to be asked to read two different imaginary scenarios, in which they were involved, and needed to write down what they imagine their 'actions and feelings [would be], describing them in as much detail as possible'. They were also specifically told that their creativity was being measured, and that they should take 30 seconds to visualise each scenario before writing down their response in as much detail as possible (a minimum word count of 150 words was suggested). Each written task had two scenarios which were responded to in the same way. The exact wording of all stimuli, as well as their accompanying pictures can be found in Appendix R.

Danger stimuli. In scenario one, participants were shown two pictures of aggressive dogs which looked as if they were preparing to attack. The participants were asked to imagine a scenario in which a pack of dogs had started attacking people in a street on which the participant was walking. They were then informed that the pack of dogs was approaching them and that it was highly likely that they would be attacked next. Participants were then asked to write down how they would feel emotionally about the situation and to describe how they would attempt to resolve it. For scenario two, participants were shown a picture of three hooded youths next to a park fence with bicycles at dusk. The participants were asked to imagine that they came across the gang in the dark, and had crossed over the road to avoid them. The gang noticed the participant and started to threaten and chase after them. Again participants were asked to describe how they would feel emotionally and how they would try to cope with the situation.

Arousal stimuli. The first situation in the arousal stimuli had participants imagine that they had just won a $£ 500$ Amazon voucher which they could spend on anything they wanted to on the amazon.co.uk website. Participants were asked to describe how they felt emotionally about the situation and to list the items they would purchase with the voucher and why. This scenario was accompanied by a picture collage of various products available on Amazon (including a camera, a watch, and a guitar) as well as the company logo. In the second situation, participants were told that they had to plan the perfect "night in" for themselves and their best (same-sex) friend. The scenario was accompanied by a picture collage of some popcorn, a pizza, and some DVDs used to represent a night in. Again, participants were asked to write down how 
they felt emotionally about the situation and to describe what this perfect night in would entail.

Neutral stimuli. The two neutral situations involved participants describing everyday routines in detail. In the first situation, participants were asked to imagine travelling from their home to the nearest post box and to describe the sounds and sights they would experience during the journey (as inspired by; Griskevicius et al., 2006). An image was displayed of a typical street containing student housing from the South Wales area. The second situation asked participants to describe their morning routine in detail and was presented alongside a collage of images including an alarm clock and bowl of cereal.

\section{Design}

The experiment followed a 2 by 3 mixed-model design with the within-subjects factor of time and the between-subjects factor of condition. The revised SMA task provided the dependent variables of ST and LT choice and exposure to danger, arousal, or neutral stimuli formed the independent variable. The analysis involved using the ordinal logistic regression models from Experiment 5 to try to predict mating strategy change in a new data set. The intention was also to assess whether any significant models were still quantitatively similar when the neutral condition was replaced by the arousal condition. This was to ensure that the effect of the danger stimuli was not purely due to its arousing nature.

\section{Procedure}

The study procedure was identical to that used in Experiment 5 aside from the two key differences. First, the forewarning participants were given about the nature of the stimuli used now referred only to aggressive animals. Second, instead of being exposed to a slideshow manipulation, participants were given one of the three experimental stimuli depending on their randomly assigned condition. They were allocated ten minutes to complete the creativity task on a lined sheet of paper. This was completed in private and these sheets of paper were then sealed in each participant's anonymous envelope with the demographic form and SOI-R.

\section{Results}

Participants were equally split between the conditions and both sexes were represented almost equally in each condition. In the neutral condition, there were 94 participants (male $n=47$ ), in the danger condition there were 95 participants (male $n=$ 48), and in the arousal condition there were 97 participants (male $n=50$ ). 
In this sample, men reported an average of $3.00(S D=1.90)$ for their fear of crowds, while women reported and average of $4.04(S D=2.04), t(284)=4.443, p<$ $0.01, d=0.53$. However, there was no sex difference in the fear of aggressive animals, $t(284)=0.284, p=0.81$, which had a mean rating of $5.02(S D=5.86)$. As with the analysis of Experiment 5, these factors, as well as sex were included as potential covariates in the ordinal analysis.

At first performance of the revised SMA task, a median number of 15.5 models were chosen for a long-term relationship and 18.5 models for a short-term relationship. This was a much more balanced selection than seen in previous experiments using the original SMA. During the creativity tasks, participants were encouraged to write a statement of around 150 words for each of the scenarios. The average number of words written for the first scenario was $71.89(S D=27.70)$ and $70.02(S D=28.86)$ for the second. While no qualitative analysis was performed on the statements, word count was included as a covariate in the regression models as those who answered the questions more thoroughly could potentially be more affected by the manipulations than those who answered superficially. However, previous research has shown that the time it takes to complete such tasks, as well as numbers of words a participant produces, tend to be non-significant covariates (Griskevicius et al., 2006).

\section{Short-Term Mating}

Following the presentation of the danger stimuli, 51 participants $(53.7 \%)$ selected fewer models for short-term relationships (ST-), five (5.3\%) chose the same number ( $\mathrm{ST}=)$, and $39(41 \%)$ chose greater $(\mathrm{ST}+)$. When compared to both the neutral and arousal conditions (Table 5.5) it appeared as if participants in the danger condition were more likely to appear in the ST-group. A chi-squared analysis revealed that the distribution in the danger condition was significantly different from both the neutral, $\chi^{2}(2)=10.492, p<0.01$, and arousal, $\chi^{2}(2)=7.078, p=0.03$, conditions. However, this significant difference may have been driven by the discrepancy between the number of participants in the ST= category between the neutral $(n=16)$ and danger $(n=5)$ conditions. Typically, the number of participants in the ST= category for the experimental and control conditions are both larger (around 7 to 16) and fairly balanced. Here however, there was a difference of 11 participants between the ST= categories which could have increased the chi-squared value. To this end, the analysis was repeated while excluding the ST= category (Table 5.6) at which point the significant 
difference between the neutral and danger distributions disappeared, $\chi^{2}(1)=1.044, p=$ 0.307 .

Table 5.5

The number of participants who increased, stayed the same, or decreased in their number of ST choices following exposure to either danger, neutral, or arousal stimuli. The danger distribution was significantly different from both the neutral and arousal distributions using chi-squared test

\begin{tabular}{|c|c|c|c|}
\hline \multicolumn{4}{|c|}{ Short-term change } \\
\hline & - & $=$ & + \\
\hline Danger (D) & 51 & 5 & 39 \\
\hline Neutral (N) & 40 & 16 & 38 \\
\hline Arousal (A) & 40 & 4 & 53 \\
\hline$\chi^{2}(2)-D: N$ & $10.492 * *$ & & \\
\hline Cramér's $V$ & 0.17 & & \\
\hline$\chi^{2}(2)-D: A$ & $7.078^{*}$ & & \\
\hline Cramér's $V$ & 0.14 & & \\
\hline
\end{tabular}

Table 5.6

The number of participants who increased or decreased in their number of ST choices following exposure to either danger or neutral stimuli. There is no significant difference between the distributions

\begin{tabular}{|c|c|c|}
\hline \multicolumn{3}{|c|}{ Short-term change } \\
\hline & - & + \\
\hline Danger & 51 & 39 \\
\hline Neutral & 40 & 38 \\
\hline$\chi^{2}(2)$ & 1.044 & \\
\hline Cramér's $V$ & 0.06 & \\
\hline
\end{tabular}

A binary logistic regression (which uses only two DV categories, see Chapter 2) was used in an attempt to replicate the ordinal logistic regression model from Experiment 5 using data from this experiment (Table 5.7). The resulting model was not 
significant. Furthermore, no significant model could be produced using a combination of condition and the variables included in the demographic form (including the number of words each participant wrote during the creativity task). This was also the case when the neutral condition was replaced with the arousal condition in the analysis and ordinal regression used, $\chi^{2}(3)=4.547, p=0.208$.

\section{Table 5.7}

The results of a binary logistic regression to predict ST choice change using (a) sex, (b) an interaction between sex and condition, and (c) an interaction between sex and SOI- $R$

\begin{tabular}{lcc}
\hline \multicolumn{3}{c}{ Model ST } \\
\hline & \multicolumn{1}{c}{$\beta$} & $\operatorname{Exp}(\beta)$ \\
\cline { 2 - 3 } Sex & 0.391 & - \\
Sex * Condition & -0.179 & - \\
Sex * SOI-R & 0.004 & - \\
\hline Model & $\chi^{2}(3)=2.276$ \\
Nagelkerke $R^{2}$ & 0.02 \\
Accuracy & $57.7 \%(+6.4 \%)$ \\
\hline
\end{tabular}

Note: Variables were coded as such: Condition - neutral (0), danger (1); sex - male (1), female (2). SOI$\mathrm{R}=$ Socio-sexual Orientation Inventory Revised. ${ }^{\dagger} p<0.10, * p<0.05, * * p<0.01$.

\section{Long-Term Mating}

In the danger condition, 48 participants $(50.5 \%)$ decreased in their long-term choices (LT-), one (1.1\%) stayed the same (LT=) and $46(48.4 \%)$ increased (LT+). When compared to the distribution found following the neutral and arousal conditions (Table 5.8), it appeared as if exposure to danger stimuli led participants to select fewer models for a long-term relationship. Once again, a chi-squared test showed that the distribution of the danger condition was significantly different from both the neutral, $\chi^{2}(2)=30.89, p<0.01$, and arousal conditions, $\chi^{2}(2)=17.48, p<0.01$. However, as with change to ST mating choices, there was a large difference between the number of participants in the LT= category in the danger condition $(n=1)$ compared to the neutral condition $(n=22)$. A repeat of the analysis excluding the $\mathrm{LT}=$ category revealed that there was no significant difference between the distributions, $\chi^{2}(1)=3.415, p=0.97$ (see Table 5.9). However, had a two-tailed test been used this would have been marginally significant $(p=0.06)$. 
Table 5.8

The number of participants who increased, stayed the same, or decreased in their LT choices following exposure to either danger, neutral, or arousal stimuli. The danger distribution is significantly different from both the neutral and arousal distributions using chi-squared test

\begin{tabular}{|c|c|c|c|}
\hline \multicolumn{4}{|c|}{ Long-term change } \\
\hline & - & $=$ & + \\
\hline Danger (D) & 48 & 1 & 46 \\
\hline Neutral (N) & 30 & 22 & 42 \\
\hline Arousal (A) & 34 & 13 & 50 \\
\hline$\chi^{2}(2)-D: N$ & \multicolumn{3}{|c|}{$30.889 * *$} \\
\hline Cramér's $V$ & \multicolumn{3}{|l|}{0.29} \\
\hline$\chi^{2}(2)-D: A$ & \multicolumn{3}{|c|}{$17.481 * *$} \\
\hline Cramér's $V$ & \multicolumn{3}{|l|}{0.21} \\
\hline
\end{tabular}

${ }^{\dagger} p<0.10, * p<0.05, * * p<0.01$.

Table 5.9

The number of participants who increased or decreased in their LT choices following exposure to either danger or neutral stimuli. There is no significant difference between the distributions

\begin{tabular}{lcc}
\hline \multicolumn{3}{c}{ Long-term change } \\
\hline & - & + \\
\hline Danger & 48 & 46 \\
Neutral & 30 & 42 \\
\hline$\chi^{2}(2)$ & 3.415 & \\
Cramér's $V$ & 0.10 & \\
\hline
\end{tabular}

${ }^{\dagger} p<0.10, * p<0.05, * * p<0.01$

The variables forming the significant LT ordinal regression from Experiment 5 were employed in a binary logistic regression to try to predict the variance in LT category change in this experiment. This model was again non-significant and no new model could be produced using condition and the variables from the demographic and 
SOI-R forms (Table 5.10). The model was also not significant if the neutral condition was replaced with the arousal condition, $\chi^{2}(1)=1.634, p=0.90$.

Table 5.10

The results of a binary logistic regression to predict LT choice change using condition

\begin{tabular}{|c|c|c|}
\hline \multicolumn{3}{|c|}{ Model LT } \\
\hline & $\beta$ & $\operatorname{Exp}(\beta)$ \\
\hline Condition & -0.379 & - \\
\hline Model & \multicolumn{2}{|c|}{$\chi^{2}(1)=1.449$} \\
\hline Nagelkerke $R^{2}$ & \multicolumn{2}{|c|}{0.01} \\
\hline Accuracy & \multicolumn{2}{|c|}{$54.2 \%(+2.3 \%)$} \\
\hline
\end{tabular}

Note: Variables were coded as such: Condition - neutral (0), danger (1). ${ }^{\dagger} p<0.10, * p<0.05, * * p<$ 0.01

\section{Discussion}

The results of Experiment 6 confirmed neither Predictions 1 nor 2 and so did not provide support for Hypothesis 5. When the significant models in Experiment 5 were applied to the data produced in Experiment 6, these no longer significantly accounted for variance in LT or ST change. Likewise, no new significant models using a different combination of variables could be generated. This lack of confirmatory results could be due to the initial results being a Type I error. However, another explanation could be that the creativity task manipulation was inadequate. The scenarios presented in the danger creativity task required participants to imagine situations to which they may have not been exposed and previous research has shown such exposure can be important. For example, in the study by Griskevicius et al. (2011), it was only participants from a low socioeconomic background who responded to a mortality cue by increasing their preference for risk taking. In contrast, the slideshow used in Experiment 5 exposed participants to images of danger irrespective of their previous experience with the content. The danger creativity task also tended to polarise the results, with fewer individuals appearing in the ST= and LT= categories. Given the changes to the SMA task, and the fact that each model was viewed four times instead of two, one would predict that this figure would actually increase as participants are given a greater opportunity to remember the models and therefore their previous relationship choices. None of the additional measures included in the regression models could account for this polarising effect, including SES (a potential measure of participants' likelihood as 
having encountered harsh environments) and task engagement (the number of words written in the creativity stats).

If it is true that the danger stimuli did not yield an effect as they were not of relevance to the participants, then the stimuli used in Experiment 8 should be more likely to produce a significant effect. Experiment 8 was a sister experiment conducted in parallel with Experiment 6 which also utilised the same SMA modifications and creativity tasks. Here, the creativity tasks involved the participant drawing on past memories of competitive success or defeat as well as fictional scenarios. Indeed, significant differences in category distributions and significant ordinal regression models were found in this experiment (see Chapter 7).

\section{General Discussion}

In summary, although good support for Hypothesis 5 was found in Experiment 5, no such support was found in Experiment 6. For the reasons mentioned above, there are good reasons to think that this failure to replicate may have been due to a weakness in the stimuli used rather than a Type I error. As such, the remainder of this chapter discusses the experimental results with this interpretation in mind, although the need for replication to confirm the results of Experiment 5 is still needed. This marks the second time in which a more complex manipulation (in terms of the creativity task) has produced inconsistent or null results. The strength of low-level slideshow-type manipulations over more complex tasks in which engagement and interpretation can influence the results is discussed further in Chapter 9.

\section{Occam's Dangerous Razor}

In Experiment 5, moderate danger cues affected both long- and short-term relationship choices. While in animal studies, introducing an organism into a more dangerous environment can lead them to follow a more short-term or riskier mating strategy (i.e. Anderson \& Brown, 2009; Fontaine \& Martin, 2006; Reede, 1995), the long-term pair-bonding nature of our species, and the fact that our alternative, shortterm, strategies convey more complicated social benefits as well as reproductive ones (especially in the case of women; Greiling \& Buss, 2000; Meston \& Buss, 2009), means that the picture is not so simple. In the introduction to this chapter, life-history predictions (Chisholm et al., 1993; Reznick et al., 2002) were combined with the hypothesized benefits of short-term mating in women (Buss \& Schmitt, 1993; Greiling $\&$ Buss, 2000) into an integrated hypothetical model which was supported by the results of Experiment 5. However, one weakness of taking a strategic pluralistic approach 
(Gangestad \& Simpson, 2000) to these manipulation experiments is that in trying to justify increases and decreases in interest within the two separate mating domains, one may actually be ignoring a larger, simpler effect. For example, while the two perspectives presented may account for the data, a parsimonious alternative could be that the danger cues simply led to a general increase in mating interest, and that this was just realised across two mating domains in women but not men (Buss \& Shackelford, 2008). If this were the case then a general decrease in 'not interested' responses among men and women following the manipulation compared to the control group should be present. Interestingly, when the inverse of not interested responses for Experiment 5 (50 - NI responses; i.e. the number of relationships chosen) is examined, neither chi-squared analyses nor various ordinal regression models utilising condition are found to be significant. That is, there appear to be two distinct effects present in the data, one involving ST responses and one involving LT responses. Indeed, the approach of separating long- and short-term mating domains still continues to produce distinct results in other experiments involving cues of environmental harshness (Little, Cohen, et al., 2007).

The interpretation of the data could be supported by further teasing apart different types of environmental harshness cues to see their individual impact on mating strategy in conjunction with demographic variables. For example, in women, an increase in preferences for masculinity and symmetry are found following a pathogen prime but not following a resource scarcity prime (Lee \& Zietsch, 2011). While these independent cues may both lead to an increase in short-term mating (i.e. to acquire good genes to combat pathogens, and to extract resources from partners irrespective of their genetic quality), pathogen cues may interact with demographic variables such as perceived personal condition (Prokop \& Fancovicova, 2010), while resource scarcity may interact with SES (Griskevicius et al., 2011). Further research into sex differences in this area may be fruitful as the responses of men to threat cues appears to be an overlooked area (e.g. Scheib et al., 1999; Watkins, DeBruine, Little, Feinberg, \& Jones, 2012).

\section{Harshness in the Lab}

In the introduction to this chapter, the quadratic model of the relationship between environmental harshness and parental care put forward by Quinlan (2007) was considered and extended. This extension came in the form of some basic assumptions about the model, including the introduction of a hypothesized relationship between 
environmental harshness for short-term relationship propensity (Figure 5.1), and differential patterns for the sexes given what is known about human mating strategies (Buss \& Schmitt, 1993; Figure 5.2). This hypothetical model was then used to predict the effect of each sex of moderate danger cues (Figure 5.2a), and evidence was found for these predictions in Experiment 5. A prediction was also made as to how extreme or persistent cues of danger would influence mating strategies (Figure 5.2b) as informed by literature in line with life history theory (Chisholm et al., 1993). However, this prediction was not tested in this chapter due to the ethical constraints concerned with realising such an experiment. What might such an experiment look like? One option might be to expose participants to a prolonged source of emotive danger stimuli - for example, the viewing of horror movies over several experimental trials (Cools, Schotte, \& McNally, 1992; Zillmann, Weaver, Mundorf, \& Aust, 1986). Another option might be to create a natural quasi-experiment whereby individuals who, irrespective of input from the experimenter, voluntarily put themselves in a dangerous environment. Earlier, the impact of warfare on promiscuous sexual behaviour was discussed (Muhwezi et al., 2011; Weiser et al., 2007). As such, one possibility could be to test the mating preferences of soldiers before and after exposure to combat. Though these two examples are simply suggestions at this point, they prove that the predictions of the developed model are able to be tested and therefore falsified.

\section{Sex and Mere Exposure}

In the ST ordinal model of Experiment 5 sex was found to be a predictive variable with a negative effect. That is, compared to men, women chose fewer individuals for a short-term relationship during their second exposure to the SMA irrespective of condition. Indeed, in addition to one other, this effect needed to be controlled for in order for the sex by condition effect to become significant. What might account for such a reduction in ST choice at second exposure to the models? One reason may be that as women see men for a second time the mere exposure effect (Rhodes, Halberstadt, \& Brajkovich, 2001; Zajonc, 1968) leads them to re-evaluate their suitability as long-term partners. If this were the case then one would expect there to be an inverse correlation between ST change and LT change when condition was ignored. This is indeed the case. In men, the Spearman's' rho for change in ST and change in LT was non-significant, $r_{\mathrm{s}}(42)=-0.16, p=0.319$. However, among women this was both significant and strongly positive, $r_{\mathrm{s}}(48)=0.41, p<0.01$. Even though parametric analysis is typically inappropriate given the non-parametric nature of the data, a partial 
correlation taking condition into account was also conducted to give an indication as to whether this effect was present irrespective of the nature of the stimuli. This was once again not significant for men, $r_{a b . c}(39)=-0.06, p=0.72$, but was for women, $r_{a b . c}(45)=$ $0.42, p<0.01$. If the familiarity effect did affect the results, the question remains as to why such sex difference exists. One reason may be that men's increased inclination towards short-term mating (Buss \& Schmitt, 1993; Lippa, 2009; Symons, 1979) leads them to be less choosy when selecting models for short-term relationships. It would then follow that these models would be less likely to be re-evaluated as a potential longterm suitor upon subsequent viewings. Indeed, in every SMA experiment, men typically choose more models for ST relationships than women at first measurement $(U=607.5$, $p<0.01$, in the case of Experiment 5). Although the original idea of the SMA task was to minimise the exposure of models to the participants as much as possible to reduce the impact of memory on responses, the presence of this familiarity effect may well indicate that this assumption is adding unwanted variance to the data. That being said, this was the only ordinal model in the thesis in which the control of sex was required for an effect to be found.

\section{Conclusion}

In this chapter, further evidence was provided that mating desire can be biased by short experimental interventions. In terms of danger cues, Experiment 5 yielded results which supported a life history perspective via an extended version of Quinlan's (2007) model. These results were not replicated in Experiment 6, which utilised a creativity intervention and produced null findings. In the next chapter, the results of an experiment involving the manipulation of circulating testosterone are presented. 
Chapter 6: An Increase in Male Short-Term Mating Preference Accompanies a Rise in Testosterone after Acute Exercise

As one of the oldest groups of hormones, androgens are found in even the most primitive of vertebrates. Their function is closely linked to sexual development and behaviour, especially in males. During foetal growth, androgens lead to the masculinisation of the brain and sexual organs (organisational effects), and at puberty they influence sexual behaviour as well as the development of secondary sexual characteristics (activational effects). During adult life, circulating levels of androgens are associated with a cluster of male mating behaviours including increased sexual interest, aggression towards intrasexual competitors, and risk taking. The importance of these hormones in mating is revealed by non-human animal studies which manipulate the availability of the circulating androgen testosterone (T). In animal castration studies, the $\mathrm{T}$ producing gonads are removed, causing sexual function to become greatly or completely suppressed (Boothe, 1977; Dixson \& Herbert, 1977; Lincoln, Guinness, \& Short, 1972). These behaviours can be subsequently re-established through the use of $\mathrm{T}$ implantation (Dixson \& Herbert, 1977; Moore, 1988; Tiefer, 1970; Wada \& Gorbman, 1977).

Other experiments have artificially enhanced $\mathrm{T}$ among normally functioning males, with varying effects on their mating behaviour. When given T implants, male house finches (Carpodacus mexicanus) will spend less time nurturing young and more time away from the nest seeking mating opportunities (Stoehr \& Hill, 2000), red grouse cocks (Lagopus lagopus scotica) will increase their territories, pushing non-implanted males out, to enhance their reproductive success (Moss, Parr, \& Lambin, 1994), and male prairie voles (Microtus ochrogaster) will become more aggressive towards their same-sex peers (Gaines et al., 1985). Similarly, T-implanted lambs will vigorously form dominance hierarchies when introduced to unknown individuals (Ruiz-de-la-torre \& Manteca, 1999) and male sand lizards (Lacerta agilis) will roam more to increase their mating opportunities (Olsson, Wapstra, Madsen, \& Silverin, 2000). These are not isolated examples; a large number of studies have shown similar effects (for a review of these, as well as species which show an exception to this pattern, see Lynn, 2008). ${ }^{27}$ The

\footnotetext{
${ }^{27}$ Such mating behaviours are associated with various risks, and are normally carried out by individuals able to cope with the costs. Artificially enhancing T often increases these behaviours at the cost of personal condition (Dufty Jr, 1989; A. Peters, 2000).
} 
general pattern, however, is quite clear; androgens are required for adequate performance of male mating, and higher levels are associated with an increase in mating effort. Outside of experimental manipulation, $\mathrm{T}$ is also useful as a predictor of reproductive strategy. For example, future social rank among baboons (which affects their mating opportunities) can be predicted by androgen by-products collected from stool samples (Beehner et al., 2006).

Men show a similar link between testosterone and mating effort, although this does not appear to have been demonstrated experimentally. Different types of T markers, reflecting both present (i.e. circulating $\mathrm{T}$ in saliva) and historic (as measured using 2D:4D) levels, are associated with aggression (Archer, 1991; Book et al., 2001), sexual partner number (Bogaert \& Fisher, 1995; Hönekopp, Voracek, \& Manning, 2006; M. Peters et al., 2008), and risk taking behaviour (Ronay \& Hippel, 2010; Sapienza, Zingales, \& Maestripieri, 2009; S. J. Stanton et al., 2011). Generally, T levels tend to increase in men during puberty and decline from middle age onwards (Nahoul \& Roger, 1990). ${ }^{28}$

Relationship context also seems to be a key determinant of a man's circulating $\mathrm{T}$ level. Single men tend to have the highest levels, followed by those in a committed relationship, and lastly by those who are coupled and have children (Alvergne et al., 2009; P. B. Gray et al., 2006; P. B. Gray et al., 2002). This is not just a between-subject finding; longitudinal research has revealed that the $\mathrm{T}$ levels of men change with their relationship status (Gettler et al., 2013; Gettler, McDade, Feranil, et al., 2011), perhaps suggesting that $\mathrm{T}$ acts as part of a mate acquisition system. Indeed, if a man intends to pursue extra-pair copulations then his level of $\mathrm{T}$ will remain high, even if he is in a long-term relationship with or without children (McIntyre et al., 2006).

An interesting study by Muller et al. (2009) further reiterates the relationship between mating effort and testosterone in men. The study focused on two cultures in close proximity, the Datoga and the Hadza of Tanzania. The Datoga have a culture marked by extreme polygyny; fathers do not sleep with their wives and children (indicative of reduced paternal care) and competition for wives is fierce. In contrast, Hadza men provide greater parental care, and tend towards social monogamy with mild polygyny. In the former culture, T levels are consistently high, while in the latter they start high in the morning and then drop throughout the day.

\footnotetext{
${ }^{28}$ Though robust, the effect size of some of these associations can often be quite low, $r=0.10$ in the case of aggression, and $r=0.10$ to 0.20 for sexual partner number.
} 
The challenge hypothesis proposed by Wingfield, Hegner, Dufty Jr, and Ball (1990), can explain such T variation in men. The hypothesis states that circulating T levels are up- or down-regulated in males depending on their mating context, remaining at the fairly low levels required for successful breeding until certain contextual cues arise (e.g. those of intrasexual competition or opportunity for extra-pair copulations). At such time they approach a physiological maximum and trigger a cluster of male mating behaviours. The non-human animal studies introduced above which use T implantation cause an artificial up-regulation of $\mathrm{T}$ levels and hence explain the increase in mating effort. While originally used to predict behaviour in seasonal breeders such as birds, the challenge hypothesis has been equally successful in predicting heightened testosterone in species which mate all year round, including chimpanzees (Muller \& Wrangham, 2004), and may explain the inflated T levels found in men who are seeking further mating opportunities (Archer, 2006).

There appear to be several contextual cues which cause the T levels of men to become differentially regulated, including both social and non-social factors. Non-social factors include time of day (e.g. Muller et al., 2009), nutrition (Prasad, Mantzoros, Beck, Hess, \& Brewer, 1996), and acute exercise (Crewther, Keogh, Cronin, \& Cook, 2006; Cumming, Brunsting, Strich, Ries, \& Rebar, 1986; Gatti \& De Palo, 2011). Social influences include the anticipation of sexual contact (Anonymous, 1970; Ronay \& Hippel, 2010; van der Meij, Buunk, van de Sande, \& Salvador, 2008) ${ }^{29}$, the outcome of competition (Bernhardt, Dabbs Jr, Fielden, \& Lutter, 1998; Brondino et al., In Press; Carré et al., In Press), and the presentation of parental and emotional stimuli (Cook \& Crewther, 2012; van Anders, Tolman, \& Volling, 2012). Such factors can both up- and down-regulate T. When exposed to parental cues, for example, expectant fathers show a drop in salivary T (Storey et al., 2000). Likewise, T levels drop in men when they hear baby cries and are able to engage in nurturing behaviour (and rise if they are unable to; Fleming et al., 2002; van Anders et al., 2012).

\section{Experiment 7}

In Experiment 7, the effect of changes in circulating $\mathrm{T}$ on male mating behaviour was explored. As it is difficult to ethically increase $\mathrm{T}$ in men using implantation, this change was initiated using a harmless non-social manipulation. Specifically,

\footnotetext{
${ }^{29}$ The anonymous reference refers to a letter published in Nature by a researcher who meticulously weighed his beard shavings while researching on an island. He found an increase in beard growth rate (a testosterone linked trait) in the final few days of research before his journey home. The author concluded that this increase was due to his 'anticipated sexual activity'.
} 
participants engaged in short bouts of intense exercise to cause a rise in their circulating T. Changes in their mating desires were then assessed using the SMA task. As T is associated with increased mating effort, and the pursuit of extra-pair copulations, Hypothesis 6 stated that an increase in circulating T would lead to short-term mating strategy activation in men. To test this hypothesis, two predictions were made.

Prediction 1 was that, following an increase in circulating $\mathrm{T}$ from exercise, men would select a greater number of models for a ST relationship on the SMA task compared to baseline. Prediction 2 was that, following the same increase in $\mathrm{T}$, fewer models would be chosen for a LT relationship on the SMA task.

\section{Method}

\section{Participants}

Forty-seven men were gathered from across the Swansea University campus to participate in a study investigating 'exercise, relationships, and cognitive ability'. The advert specifically called for men aged 18-25 who were comfortable providing saliva samples and answering questions of a sexual nature. The need for participants to be healthy enough to engage in acute exercise was also stressed. Participants were offered either course credit or a cash payment of $£ 8$ for successful completion of the two experimental sessions.

The following demographic details relate to 46 of the participants as one was excluded during analysis (see results section). Half of the participants ( $n=23)$ were in a committed relationship and the other half were either single or in an uncommitted relationship. All participants were heterosexual and childless. The majority of the sample described themselves as Caucasian $(n=43 ; 93.3 \%)$, two described themselves as East Asian, and one described himself as being of 'mixed' ethnicity. Thirty-eight of the participants were current undergraduate students (82.6\%) and the eight other volunteers were postgraduate students. The average age of the sample was 20.96 ( $S D=$ $2.41)$ and the average BMI was $23.76(S D=3.75)$. Typically, participants regarded themselves as middle-low to middle socioeconomic status $(M=3.39, S D=0.77)$ and slightly above average in attractiveness relative to their peers $(M=6.04, S D=1.23)$. The mean SOI-R score for the sample was $41.26(S D=13.16)$, which was consistent with other men tested in this thesis.

\section{Design}

The experiment followed a 2 by 2 mixed-model design. The first independent variable was time (pre- and post-exercise). The second was the quasi-independent 
variable of $\mathrm{T}$ change. The dependent variables were the responses given during the standard SMA task. In addition to the primary analysis, change in handgrip strength was used to discern the success of the exercise manipulation (see below).

\section{Materials and Apparatus}

Questionnaires. The standard demographic form (Appendix E) and SOI-R (Appendix B) were used in this study. All questions about the menstrual cycle were omitted from the demographic form. The inclusion of a health questionnaire is customary in studies involving exercise to reduce the risk of participant overexertion. This questionnaire (Appendix S) was split into two sections: one assessing cardiac history (e.g. heart attack or use of a pacemaker), and another assessing cardiovascular risk factors and general health issues (e.g. high cholesterol level or chest discomfort with exertion). A positive response to a single statement in the first section, or more than one statement in the second, identifies a participant who may not be suitable for physical exercise unless medical advice is sought. Some participants indicated that they did not know their blood pressure and also smoked. Under these circumstances only participants were allowed to participate if they indicated that they engaged in regular intensive exercise. No participants had to be excluded on the basis of their health. Consent and debrief forms for this study can be found in Appendix T.

SMA. The original SMA task was used for this study, as opposed to the revised version used in Chapters 5 and 7. Only female models were used in this experiment due to the male-only sample. The set of 50 female models were taken from a larger set of photographs gathered from the hotornot.com website for earlier experiments. The models were independently rated as having an average attractiveness of 4.68 ( $S D=$ 1.47 ) by 20 judges (male $n=10$ ) using a semantic differential scale between one ('not at all attractive') and nine ('very attractive').

Handgrip and exercise task. Handgrip strength was measured using a Takei ${ }^{\odot}$ grip strength dynamometer (T.K.K. 5001, Grip-A) set to level six. Handgrip strength correlates with various measures of T (Fink, Thanzami, Seydel, \& Manning, 2006; Gallup, White, \& Gallup Jr, 2007; S. T. Page et al., 2005) and should increase after acute exercise. For each grip trial, participants squeezed the dynamometer as hard as they could over a period of three seconds. This process was repeated three times with a 30 second rest period between each attempt. The average grip strength was then used as the score for the trial. 
The exercise manipulation itself involved cycling on a Monark ${ }^{\circledR}$ cycle ergometer. This was a stationary exercise bike with a piece of nylon rope wrapped around the front wheel. Resistance could be varied by adding weights to the end of the rope which would then constrict the wheel. The ergometer was friction-loaded to $7.5 \%$ of the participant's body mass which is a standard resistance used in the sports science literature (J. S. Baker, Thomas, \& Davies, 2009; Volek et al., 2001; Wilson II, Snyder, \& Dorman, 2009). The exercise task itself began with a two minute warm up where participants peddled at a rate of approximately $60 \mathrm{rpm}$ against no resistance. Five sprint cycles were performed in total. Each cycle consisted of six seconds of maximal effort sprinting against the bodyweight-derived friction, followed by 54 seconds "rest" at 60 rpm against no resistance. Following the final sprint the participant kept their rest pace for a further two minutes to warm down. The 6/54 sprint-split was chosen to provide appropriate exertion for the participants who varied in their athletic ability and came from a mixture of academic backgrounds. In other experiments, which have used sports science students who are used to being tested under high intensity conditions, splits of 6/30, 10/30, and 10/50 have been used (Glaister, Stone, Stewart, Hughes, \& Moir, 2006; Rockwell, Rankin, \& Toderico, 2001).

Anthropomorphic measures. Body weight was measured using a set of electronic scales and height was measured using a Harpenden Stadiometer by Holtain Ltd. The latter was a counterbalanced height measurement tool accurate to the nearest millimetre. Participants were encouraged to empty their pockets and remove articles of clothing such as shoes and jackets prior to measurement.

Cortisol and testosterone collection. Saliva samples were gathered in order to assess change to cortisol (C) and T levels. Immunoassay kits for both hormones were obtained from Salimeterics ${ }^{\circledR}$. The testosterone kits had a sensitivity of $1 \mathrm{pg} / \mathrm{ml}$ and a serum-saliva correlation of $r=0.96$, while the cortisol kits had a sensitivity of $<0.003$ $\mathrm{ud} / \mathrm{dL}$ and a serum-saliva correlation of $r=0.91$. Saliva samples were stored in polypropylene vials which were frozen at a temperature below $-20^{\circ} \mathrm{c}$ within half an hour of collection. At least $1 \mathrm{ml}$ was gathered for each saliva sample via passive drooling. Participants were required to avoid eating or drinking anything other than water for two hours before the study. If participants chose to drink water during the study (due to the intense nature of the exercise) samples were taken at least one minute after consumption to avoid diluting the saliva. Cortisol was recorded during this experiment for use by other researchers and so is not analysed in any detail within this 
chapter. However, baseline $\mathrm{C}$ was included in the ordinal regression models as a potential covariate.

\section{Procedure}

The study was separated into two sessions and the participants were encouraged to wear exercise appropriate clothing to both. Session 1 was a familiarisation session in which participants were briefed about the study, filled out paperwork, and became familiar with the tasks. The session began with participants being welcomed to the laboratory and asked to read and sign the combined information sheet and consent form. At the same time they also completed the health questionnaire. The experimenter then proceeded to check that consent had been given by the participant and that they had passed the health check. The format of the study was then reiterated orally to the participant to ensure they had good knowledge of what was required. Once this was in order, the participant's height and weight were measured and $7.5 \%$ of their body weight was calculated. A questionnaire pack containing the demographic form, SOI-R, and a coded envelope was then given to the participant. The purpose of the envelope was to allow participants to seal away their questionnaires to make their responses anonymous. After the questionnaires were completed and sealed away, participants performed a practice trial of the handgrip and exercise task. The nature of the SMA task was explained to the participant in session 1 but was not practiced to minimise exposure to the models used within it. The SMA also contains no skill or timed elements so the benefit of initial practice was considered negligible. At the end of session 1, participants were reminded of their time slot for session 2 and given more information about the saliva gathering process. They were also free to ask any procedural questions of the experimenter. Occasionally questions were asked about the hypothesis of the study. When this occurred, the experimenter explained that full details about the experiment would be revealed during the final debrief at the end of session 2 .

Session 2 occurred typically two days after the first at the same time of day (range $=1-7$ days). The first task participants completed when entering the lab was the SMA task. They were told that they would view pictures of 50 members of the opposite sex and had to make relationship choices about them. Participants were then encouraged to read the on screen instructions carefully. The experimenter was not present while the participant completed the SMA task to preserve privacy. Upon task completion handgrip strength was measured and recorded on the front of the participant's sealed envelope from session 1. Next, the first saliva sample was collected. Prior to collection 
the experimenter confirmed that participants had not consumed anything other than water for two hours before the session. Participants were encouraged to provide at least $1 \mathrm{ml}$ of saliva via passive drool and this typically took five minutes to accomplish. The exercise task began immediately after the saliva sample was sealed. Ten minutes were given following the sprints (including the two minute cool down) for participants to rest. During this time they were allowed to drink water until one minute before saliva collection. At the end of the cool down period, a second saliva sample was taken. The SMA and handgrip tasks were then repeated. Before completing the SMA task a second time, participants were told that 'most of the models you will view the second time around will be different, but some from the previous sample may appear.' Participants were told this so that they would not be concerned if they remembered any of the models (potentially disrupting the experiment). In reality all the pictures presented were the same as those shown at the start of session 2 but were presented in a different order.

After all the tasks were completed, participants were fully debriefed and allowed to ask any questions of the experimenter. Course credit or payment was then issued, the participants were thanked, and they were asked not to discuss the hypothesis of the study with any other potential participants.

\section{Results}

One participant was excluded from the analyses as he selected 'not interested' to all of the images displayed leaving a total sample size of 46 . The median numbers of LT and ST choices during the first performance of the SMA task were six and 17.5 respectively. The average baseline salivary $\mathrm{T}$ and $\mathrm{C}$ levels for the participants were $194.82(S D=73.45)$ and $0.30(S D=0.19)$. Percentage change in $\mathrm{T}$ following the exercise task was calculated by dividing post-task $\mathrm{T}$ by pre-task $\mathrm{T}$ and multiplying this number by 100 . Using percentage as a measure of change is common among experiments which utilise T (Salvador, Simón, Suay, \& Llorens, 1987; Zilioli, Caldbick, $\&$ Watson, 2014). On average, participants increased in their salivary $\mathrm{T}$ by $9.41 \%(S D=$ $0.31 \%$ ) after the intervention. However, this variable did not meet parametric assumptions (e.g. S-W $=0.827, p<0.01$ ) and this could not be rectified by transforming the data. As such, percentage $\mathrm{T}$ change was ranked for inclusion in the ordinal regression analysis below.

\section{Manipulation Checks}

Out of the 46 participants, 28 participants showed an increase in T, 16 showed a decrease, and one showed no change. A one-sample Wilcoxon ranked sign test revealed 
only a marginally significant change in $\mathrm{T}$ following the intervention when tested against a hypothesized median of 100 (i.e. no change), $Z=1.412, p=0.08$, one-tailed. Unexpectedly, a significant drop in cortisol was found post exercise $(Z=-3.700, p<$ 0.01 , two-tailed) which was an effect in the opposite direction from that usually found following intense exercise (Volek et al., 2001).

While change in $\mathrm{T}$ failed to meet the traditional standards of statistical significance, there were other effects present within the data which suggested that the measure of $\mathrm{T}$ was valid and that the intervention was successful. First, consistent with previous research, those who described themselves as either single, dating, or in an uncommitted relationship had higher baseline $\mathrm{T}$ than those who were in a committed relationship or married $(U=133.5, p=0.01)$. Second, average handgrip strength significantly increased from $42.23(S D=8.28)$ to $43.39(S D=7.99)$ following exercise and this difference was significant, $t(45)=3.285, p<0.01, d=0.48$. This handgrip strength effect is what would be expected if the cycle sprints increased $\mathrm{T}$ in participants (Crewther, Thomas, Kilduff, Stewart-Williams, \& Cook, In submission). Thus, this supports the idea that the marginally significant change in $\mathrm{T}$ represented a robust yet small effect rather than a Type I error.

\section{Short-Term Mating}

Due to resource limitations there was no control group for this experiment. As such, the distribution of ST change categories was compared to that expected by chance (see Chapter 2). While it appeared as if the exercise intervention caused more participants to appear in the ST+ category than the ST- category, this distribution was not significantly different from that expected by chance, $\chi^{2}(2)=1.256, p=0.27$ (see Table 6.1).

Table 6.1

The number of participants who increased, stayed the same, or decreased in their number of ST choices following the exercise intervention. The distribution was not significantly different from that expected by chance when a one-way chi-squared test was used

\begin{tabular}{lccc}
\hline \multicolumn{3}{c}{ Short-term change } \\
\hline & - & $=$ & + \\
\hline Actual & 16 & 7 & 23 \\
Predicted & 19.5 & 7 & 19.5 \\
\hline
\end{tabular}




$$
\begin{array}{ll}
\chi^{2}(2) & 1.256 \\
\text { Cramér's } V & 0.12
\end{array}
$$

$$
{ }^{\dagger} p<0.10, * p<0.05, * * p<0.01 \text {. }
$$

A backwards stepwise ordinal regression was used in order to predict ST change category. This model included percentage change in $\mathrm{T}$ as well as the items from the questionnaires and anthropomorphic data (e.g. BMI, cycle load and handgrip strength). Percentage change in $\mathrm{T}$ had to be ranked as it did not meet parametric assumptions (see above). A significant model emerged using only the T change variable (Table 6.2). According to the model, for every one rank increase in percentage $\mathrm{T}$ change, participants were 5\% more likely to appear in the ST= or ST+ categories compared to the ST- category. No other variables improved the predictive power of the model.

\section{Table 6.2}

The results of an ordinal regression to predict ST choice change using percentage

\begin{tabular}{|c|c|c|}
\hline \multicolumn{3}{|c|}{ Model ST } \\
\hline & $\beta$ & OR \\
\hline Rank T\%C & $0.048 *$ & 1.05 \\
\hline Model & \multicolumn{2}{|c|}{$\chi^{2}(1)=4.541^{*}$} \\
\hline Nagelkerke $R^{2}$ & \multicolumn{2}{|l|}{0.11} \\
\hline Accuracy & \multicolumn{2}{|c|}{$53.3 \%(+3.3 \%)$} \\
\hline Parallel lines & \multicolumn{2}{|c|}{$\chi^{2}(1)=0.103$} \\
\hline
\end{tabular}
change in testosterone following an exercise intervention. The percentage change variable was rank transformed

Note: Rank $\mathrm{T} \% \mathrm{C}=$ ranked percentage change in $\mathrm{T}$ following the exercise intervention. $\mathrm{OR}=$ odds ratio. ${ }^{\dagger} p<0.10,{ }^{*} p<0.05, * * p<0.01$

It is worth noting that this significant model did not depend on the nonparametric transformation of the $\mathrm{T}$ change variable. The model remains qualitatively the same when several types of $\mathrm{T}$ variable are used. These include the simple difference between $\mathrm{T}$ after exercise and $\mathrm{T}$ at baseline, the $\log 10$ transformed version of this difference, and the non-ranked percentage change in T. In fact, the model presented above is the most conservative of these different possibilities. 


\section{Long-Term Mating}

Following the exercise task, more participants were present in the LT- category compared to the LT+ category (see Table 6.3). However, a chi-squared test revealed that this distribution was not significantly different from a balanced distribution.

Table 6.3

The number of participants who increased, stayed the same, or decreased in their number of LT choices following the exercise intervention. The distribution is not significantly different from that expected by chance when a one-way chi-squared test was used

\begin{tabular}{lccc}
\hline \multicolumn{4}{c}{ Long-term change } \\
\hline & - & $=$ & + \\
\hline Actual & 20 & 12 & 14 \\
Predicted & 17 & 12 & 17 \\
\hline$\chi^{2}(2)$ & 1.059 & & \\
Cramér's $V$ & 0.11 & & \\
\hline
\end{tabular}

$$
{ }^{\dagger} p<0.10, * p<0.05, * * p<0.01
$$

Ranked percentage $\mathrm{T}$ change was not a significant predictor of LT category change in an ordinal regression, $\chi^{2}(1)=0.039, p=0.84$. The addition of other covariates using a backwards stepwise method led to no model which could significantly predict the data. These variables included baseline $\mathrm{T}$ and $\mathrm{C}$, percentage change in $\mathrm{C}$, handgrip strength, and the items recorded using the demographic form.

\section{Discussion}

The results of the study indicate that an increase in men's short-term relationship interest post-exercise can be predicted by an increase in circulating testosterone. In contrast, $\mathrm{T}$ increase did not help account for changes in long-term mating preference. Thus, only Prediction 1 proved to be correct, and so only some support was found for Hypothesis 6.

The results imply that short-term mating behaviour is linked with circulating $\mathrm{T}$ in males. As T increases so does the willingness to engage in ST relationships and this change can be brought about by a brief experimental intervention. This is consistent with the evolutionary psychological literature which shows that $\mathrm{T}$ levels are higher in 
men engaging in mating effort (i.e. seeking to acquire a partner or extra-pair partner) compared to those engaging in parental care (Alvergne et al., 2009; P. B. Gray et al., 2006; P. B. Gray et al., 2002). However, LT behaviour was unaffected by the intervention. As discussed in Chapter 2, it appears as if LT and ST willingness are not necessarily polar opposites on a spectrum, but are two different facets of mating behaviour which are present in varying amounts and can trade-off against one another. Thus activation of a ST mating strategy may overshadow, but not reduce, interest in LT mating. In this sense, higher levels of $\mathrm{T}$ (those above the level required for basic reproductive function) could have independent effects on ST and LT relationship desire.

\section{Interactions with SOI-R}

One interesting finding from the literature on testosterone and human behaviour is the lack of correlation between sociosexuality and T. In almost all reported cases no statistically significant relationship is found between the two variables. This is true for both salivary T and 2D:4D (Charles \& Alexander, 2011; Edelstein et al., 2011; van Anders et al., 2007). Even in the rare cases where a positive correlation is found this tends to only be weakly positive. Consistent with previous research, the correlation between SOI-R and $\mathrm{T}$ for participants in this experiment was positive but not statistically significant, $r_{\mathrm{s}}(45)=0.135, p=0.19$, one-tailed. This relationship implies that someone who is high in circulating $\mathrm{T}$ is just as likely to have a high SO score, or a high desire to have sex in the absence of commitment, as they are a low one.

Furthermore this lack of effect does not appear to be due to the behavioural component of the SOI-R as removing this did not qualitatively change the nature of the correlation, $r_{\mathrm{s}}(45)=0.165, p=0.14$, one-tailed. It is not just measures of sociosexuality which are uncorrelated with baseline T, ST choice on the SMA task at first measure also showed a non-significant correlation, $r_{\mathrm{s}}(45)=0.07, p=0.32$, one-tailed.

Once again this is at odds with the extensive literature which associates $\mathrm{T}$ with mating effort. These primarily include animal studies, but also human studies which show that partner number, aggression, and impulsivity are all linked to T (e.g. Archer, 1991; Bogaert \& Fisher, 1995). One possible explanation for this lack of effect could be that baseline $\mathrm{T}$ is not the most effective predictor of short-term mating behaviour, but instead it is the reactivity of the $\mathrm{T}$ system which counts (for a recent example with $\mathrm{T}$ and facial masculinity in men, see Pound, Penton-Voak, \& Surridge, 2009). That is, organisms which respond to external stimuli with a sharp increase in T may be more likely to show behaviours indicative of mating effort. Indeed, the non-parametric 
correlation between T percentage change and SOI-R in this sample of men approached significance $r_{\mathrm{s}}(45)=0.227, p=0.07$. This suggests that either a) men who are high in $\mathrm{SO}$ are more likely to show large increases in $\mathrm{T}$ following acute exercise, or b) men who show high $\mathrm{T}$ responsiveness are more likely to have an unrestricted sociosexuality. Further study is required to tease apart the causal direction of this finding. However, either of these scenarios would help account for individual differences in the pursuit of short-term mating among men. Future research correlating mating behaviour and testosterone may benefit from recording $\mathrm{T}$ reactivity in response to a simple stimulus (such as exercise or a victory cue) rather than baseline $\mathrm{T}$ alone.

\section{Potential for Replication with Females}

For the purpose of this experiment a male only sample was used. This was for two reasons: 1) there was a clearer hypothesis surrounding this sex given previous research from behavioural ecology; and 2) resource limitations meant that the sample size for this experiment was restricted to around $n=50$. As a result it was decided that the sex of the sample would be kept homogenous in order to increase the power of the study.

Testosterone does affect female mating behaviour in non-human animals in a similar way to males. In the sex-role reversed spotted sandpiper (Actitis macularia) females will allocate less time to incubating and more to mating effort when implanted with T (Oring, Fivizzani, \& el Halawani, 1989). Likewise, implants into the pre-optic area of the all-female lizard species (Cnemidophorus uniparens) leads to male like pseudo-copulatory behaviour (Mayo \& Crews, 1987). Females of males-competefemales-choose species also show an increase in "male-like" behaviour with $\mathrm{T}$ manipulation. For example, in ovariectomised mice and rats, T implants can increase aggressive behaviour (Albert, Jonik, \& Walsh, 1990; Barkley \& Goldman, 1977). Likewise in female ruffs (Philomachus pugnax) with intact ovaries, T implants can cause a growth in body size and the appearance of male breeding plumage (Lank, Coupe, \& Wynne-Edwards, 1999). However, these effects appear to be less consequential for female reproductive behaviour compared to that of males. For example, in the dark-eyed juncos (Junco hyemalis), T implants appear to do nothing more than reduce female body size and slightly delay reproduction (Clotfelter et al., 2004) and while "switching off" the testosterone system does seem to be somewhat detrimental to female mating behaviour, increasing amounts of oestrogen and progesterone appear to counteract this (Barraclough \& Gorski, 1962; Carlson, 2002). 
Should this study be replicated with a female sample, it may well be the case that a similar association between $\mathrm{T}$ change and short-term mating desire would be found, but this might be to a lesser extent than among men. Much like men, higher $\mathrm{T}$ in women is associated with aggression (Dabbs \& Hargrove, 1997; Dabbs, Ruback, Frady, Hopper, \& Sgoutas, 1988), risk taking (S. J. Stanton et al., 2011), sexual function and wellbeing (Davis \& Tran, 2001; Shifren et al., 2000), and sexual arousal (Tuiten et al., 2000). Mothers, like fathers, also have lower T than their non-parental peers (Kuzawa et al., 2010). One association unique to women is the fact that those with higher salivary $\mathrm{T}$ show greater attraction to masculine faces (Welling et al., 2007).

There does appear to be a tendency in the evolutionary psychological literature to report the impact of $\mathrm{T}$ on behaviour primarily in men, compared to just women or both sexes simultaneously (e.g. Carré \& Putnam, 2010). This may be due to a publication bias. If, as predicted, $\mathrm{T}$ does have a greater impact on male mating behaviour than female mating behaviour, then the testing of female samples would be more likely to yield null results. Certainly, studies which do investigate the relationship between $\mathrm{T}$ and mating behaviours for both sexes, or for women alone, show a decreased association for women compared to men (Carré et al., In Press; Kuepper et al., 2010; Rejeski, Parker, Gagne, \& Koritnik, 1990; although see Sapienza et al., 2009). It may also be the consequence of a bias within evolutionary psychology to focus solely on males when measuring traits related to intrasexual competition (Stewart-Williams \& Thomas, 2013b).

\section{Replication Considerations}

In this experiment, a 6/54 sprint-rest cycle was used which was repeated for five rounds. This specific sprint-rest split was chosen to account for the potential lack of physical fitness among the sample. Normally, studies of this nature employ students from sports studies courses which are used to engaging in maximal effort physical tasks such as the Wingate test (a test of anaerobic power and capacity) over longer periods of time. There were clear differences between the participants in terms of their sprinting ability. For some, peddling against $7.5 \%$ bodyweight presented only a minor challenge; others seemed almost incapable of sprinting against the weight and instead coped with the challenge as best as they could. This undoubtedly added noise to the data and may explain why the overall change in T only approached statistical significance. This is not an unknown occurrence. Fahey, Rolph, Moungmee, Nagel, and Mortara (1976) found an 
increase in serum $\mathrm{T}$ following exercise with college age students but not high school students stating that:

[L]ack of motivation by the high school subjects may have resulted in less vigorous weight training sessions in these subjects than experienced by the college males. This may have resulted in an absence of the testosterone increase that appears to occur in response to maximal levels of exercise. (Fahey et al., 1976, p. 33)

Thus, a replication of the current study would benefit from either: a) standardising the fitness levels of participants; b) carefully calibrating the resistance given to the participants in order to match their potential power output; or c) using fat free body mass when calculating 7.5\% of body mass (J. S. Baker et al., 2009). A larger sample would both improve statistical power and allow the addition of a control group. In this study, resources were limited and so all participants were given the experimental condition. Differences in T change and SMA choices were then compared to those expected by chance. While this was advantageous in terms of exploitation of resources, this approach has some obvious weaknesses which are outlined at the start of Chapter 4. Other useful potential covariates include perceived effort as rated by the participant and the experimenter (Eston \& Williams, 1988; Scherr et al., 2013), as well as measures of generated power during the exercise task. The latter would allow for the exclusion of participants who were unable to perform the task correctly.

\section{Alternative Hormones}

Testosterone is not the only hormone implicated in male parental responsiveness, and so it is unlikely that the conditional nature of mating strategies is regulated entirely through a T-related mechanism. Prolactin, for example, has been shown to increase in response to infant cries in experienced fathers (Fleming et al., 2002). Likewise, lower rates of cortisol during infant-play are also associated with paternal experience (Gettler, McDade, Agustin, \& Kuzawa, 2011). Oxytocin, a hormone associated with sociality, pair-bonding, and childbirth may also be a fruitful avenue of research on mating strategy plasticity as it has been shown to change neurological functioning to parental stimuli in women (Riem et al., 2012) and parental responsiveness in men (Naber, van Ijzendoorn, Deschamps, van Engeland, \& Bakermans-Kranenburg, 2010). Obviously the experimental manipulation of these hormones in a similar lab study present a unique challenge, perhaps with the exception of oxytocin which can be administered via an intranasal spray (Graustella \& MacLeod, 2012). 


\section{Conclusion}

Hormones are only one piece of the mating strategy puzzle. Whereas sexual behaviour can be greatly repressed in animals after castration, humans have been found to enjoy sex even when the accompanying hormones are absent, perhaps revealing the strong social bonding function of sex in our species (Carlson, 2002). Nonetheless, this experiment shows that male short-term mating behaviour may well be linked to fluctuations in the levels of the male mating hormone. The complex association between $\mathrm{T}$ and mating behaviour is further discussed in the next chapter. There, victory cues, which are associated with an increase in T (Bernhardt et al., 1998; Brondino et al., In Press; Carré et al., In Press), led to an increase in ST selections among men. However, departing from the results presented here, an increase in LT selection was also found among both sexes. 
Chapter 7: To the Victor, the Reproductive Spoils

Status hierarchies exist in a multitude of species, from insects to Great Apes, and ones place in the hierarchy is usually linked with reproductive fitness. For example, among northern elephant seals (Mirounga angustirostris) typically less than a third of males copulate during the breeding season. Higher social rank is strongly correlated with mating likelihood ( $r_{\mathrm{s}} \sim 0.9$ among the top ten males) and some alpha males secure up to $88 \%$ of the site copulations depending on the number of females in oestrus (Fabiani et al., 2004; Le Boeuf, 1974). A recent meta-analysis has revealed that, across several species of macaque (Macaca), the social rank of males correlates strongly with the number of offspring sired ( $r_{\mathrm{s}} \sim 0.4$; Rodriguez-Llanes, Verbeke, \& Finlayson, 2009) and in the brown lemming (Lemmus trimucronatus) females are more receptive to dominant males than subordinate ones, spending more time with them and engaging in lordosis more frequently (Huck \& Banks, 1982).

Social status is also linked with reproductive success in females. Among female mountain goats (Oreamnos americanus), social rank is a key predictor of kid production even when age is controlled for (Côté \& Festa-Bianchet, 2001), and among red-winged blackbirds (Agelaius phoeniceus) subordinate females (as measured by late territory establishment) suffer from delayed nesting and egg reproduction, due to lack of paternal care (Cristol, 1995).

Humans too show an association between social status and mating success. While dominance is the key determinant of social status in other animal species, an additional pathway to high status is found among humans in the form of prestige (Henrich \& Gil-White, 2001). These two pathways often correlate with different aspects of mating success. For example, in the von Rueden et al. (2011) study of Tsimane men introduced in Chapter 4, dominant individuals generally had younger wives (with a long reproductive window) whereas high prestige was independently associated with earlier marriage and the earlier onset of parenthood. In traditional societies, one of the consistent indicators of prestige is hunting ability. The Meriam turtle hunters from Australia show a higher than average number of mates, live with younger partners, and have children sooner than their non-hunter counterparts. This is especially true of hunt leaders who seem to accrue the most reproductive benefits (E. A. Smith et al., 2003). Importantly, it is the act of successfully hunting turtles itself, rather than simply provisioning their meat, which leads to prestige. Those who collect turtles at the time of 
year when they come ashore to mate (by simply picking them up) do not appear to have the same reproductive success as hunters. Other hunter-gatherer societies which show an association between hunting prowess and reproductive success include the Hadza of Tanzania and the Aché of Paraguay (E. A. Smith, 2004).

After the invention of agriculture, prestige could be inherited via land ownership. Among the 18th and 19th century Krummhörn population in Germany, farmland owners, compared to small holders or non-land owners, received several reproductive benefits including a larger number of children born per family, more surviving offspring, and greater fitness over a 100 year period (calculated by their number of descendants weighted by their coefficient of relatedness; Voland, 1990).

Status hierarchies are far from stable entities. Success in intrasexual competition (Bernstein, 1969; Setchell \& Dixson, 2001), the forming of social alliances (de Waal, 2007; Surbeck, Mundry, \& Hohmann, 2011), and mortality (Fitzpatrick et al., 2008; Le Boeuf, 1974) can instantly change one's position in the hierarchy and improve access to a high quantity, and high quality, of mates. Factors unique to humans include potential improvements (or decrements) in abilities linked with prestige (Holmberg, 1950; E. A. Smith et al., 2003; von Rueden et al., 2011) and the inheritance of resources (L. Barrett et al., 2002; Voland, 1990).

Given the fluidity of status hierarchies, and their influence on reproductive success, natural selection may well have selected for those individuals better able to detect and adapt to lifetime changes in social status in order to maximise their reproductive output. More specifically, human ancestors who were able to adapt their behaviour in accordance with changes in the hierarchy would have been able to accrue more lifetime reproductive benefits by choosing the most efficient mating strategy. ${ }^{30}$ In Experiment 8 this sensitivity was tested by administering victory or defeat cues to participants. If mating strategies are flexible and affected by such cues then participants who receive victory cues may be expected to switch to a mating strategy which would usually be successful for high status individuals. Likewise, those who are subjected to defeat stimuli may be expected to switch to a mating strategy which would be more efficient for those lower in status.

\section{Evidence for Within-Lifetime Change}

\footnotetext{
${ }^{30}$ Indeed, some experimental evidence shows that modern humans are sensitive to unstable status hierarchies at the group level (Scheepers, 2009).
} 
There are several well documented cases of mating success being directly affected by a status change in nonhuman animals. For example, subordinate female marmosets (Callithrix jacchus) suppress their ovulation to favour the reproduction of the dominant female but will begin to ovulate again if she is removed ( $\mathrm{J}$. Barrett, Abbott, \& George, 1990). ${ }^{31}$ Among chimpanzees (Pan troglodytes), a male's place in the status hierarchy may change several times within his lifetime depending on his age and alliances with other group members (de Waal, 2007). Similarly, in elephant seals, great within-male variance exists between mating seasons in insemination success (Le Boeuf, 1974). One male 'RAT', for example, inseminated zero females in 1968, two in 1969, and then 50 in 1970 before falling back to one the following year.

Human examples of such change are limited and tend to be qualitative in nature. One such example is that of Knee, the brother in-law of a Sirionó chief, whose poor hunting ability had made him the target for insults by his peers and whose low status had cost him a wife. After an anthropologist surreptitiously gave him some game to pass off as his own, and taught him to use a shotgun, Knee's status increased and he gained several sexual partners (Holmberg, 1950, p. 53). Likewise, among the rural Malawi of southern Africa, young men often leave their villages before marriage in order to make money in urban areas only to be 'sought out by local women due to their increased social status' upon their return (Soldan, deGraft-Johnson, Bisika, \& Tsui, 2007, p. 35). When it comes to quantitative data, most evidence is associational in nature, such as the aforementioned example of the Merium turtle hunters (E. A. Smith et al., 2003), or among the Sereer of Senegal where wrestlers experience higher reproductive success (Llaurens, Raymond, \& Faurie, 2009). Thus, there is a gap in the literature for controlled experimental studies in humans which examine how status change affects mating behaviour.

\section{The Onset of Change}

Several physiological and psychological changes occur when an organism achieves dominant social status. As discussed in Chapter 6, testosterone (T) is linked with the outcome of competition and has been shown to be elevated in victors and lowered in losers. This 'winner effect' occurs across a variety of mammals including Great Apes and Old World monkeys (Beehner et al., 2006; Muller \& Wrangham, 2004;

\footnotetext{
${ }^{31}$ This repression appears to be self-imposed as subordinate females are subject to no more acts of aggression than dominant females. One theory is that this self-imposition is due to an adaptation against infanticide (Saltzman, Digby, \& Abbott, 2009).
} 
Rose, Bernstein, \& Gordon, 1975), other animals (Huhman, Moore, Ferris, Mougey, \& Meyerhoff, 1991; Oliveira, Silva, \& Canário, 2009; Oyegbile \& Marler, 2005), and most importantly humans (Bernhardt et al., 1998; Booth, Shelley, Mazur, Tharp, \& Kittok, 1989; Brondino et al., In Press; Carré \& Putnam, 2010; Maner, Miller, Schmidt, \& Eckel, 2008). Similarly, serotonin (5-hydroxytryptamine or 5-HT) metabolism is also affected by loss and defeat. Among vervet monkeys (Chlorocebus pygerythrus), for example, an artificial increase in 5-HT metabolism through various methods (e.g. diet or SSRIs) each lead to an increase in social behaviours such as approaching and grooming, and a decrease in "dominant" behaviours including vigilance and territorial locomotion (Raleigh, Brammer, McGuire, \& Yuwiler, 1985). Such an association is found in species as diverse as lizards and crickets (Dyakonova, Schürmann, \& Sakharov, 1999; Larson \& Summers, 2001).

Psychological changes also occur following victory and defeat. Most noticeably self-esteem is affected. Sociometer theory (M. R. Leary et al., 1995) as introduced in Chapter 1, postulates a self-esteem regulation mechanism, and would predict that cues indicating one is victorious would lead to enhanced self-esteem and positive feelings about oneself. Research suggests this to be the case. For example, participants who perform better at (rigged) tasks than their peers, report significantly higher levels of perceived self-worth irrespective of their initial level of self-esteem (J. D. Brown \& Dutton, 1995). Likewise in a study in which false feedback was used to manipulate the participants' perceived acceptance and dominance within a group (i.e. their leadership potential as expressed by peers), both factors lead to enhanced self-esteem, though in qualitatively different ways (M. R. Leary, Cottrell, \& Phillips, 2001).

Despite knowledge of these individual changes, it is not clear how victory cues might change a person's mating behaviour. Although T is linked with short-term mating (Bogaert \& Fisher, 1995; Hönekopp et al., 2006; M. Peters et al., 2008), this relationship can be affected by other factors such as an individual's sex (Mazur, Susman, \& Edelbrock, 1997) and their motivations (Edelstein et al., 2011; Suay et al., 1999). The effect of variance in 5-HT metabolism in humans has not been investigated in light of its effect on mating strategies (the area seems to be restricted to studies involving mood and aggression, perhaps due to 5-HT's association with depression; Quan-Bui et al., 1984; Sarai \& Kayano, 1968). Likewise, when adapted to the mating domain, sociometer research predicts a general increase in perceived mate value and therefore increased self-efficacy in attracting and maintaining high quality relationships 
following an increase in self-esteem (Kavanagh et al., 2010). However, this is meaningless in terms of predicting individual mating strategies unless it is understood what constitutes a "high quality" relationship and why.

\section{Victory, Defeat and Sexual Strategies}

Sexual strategies theory (SST; Buss \& Schmitt, 1993), in conjunction with strategic pluralism theory (Gangestad \& Simpson, 2000), allows for the development of informed predictions as to how cues to victory and loss may affect mating strategies. For example, individuals who are consistently victorious over their peers (and are thus high in status) may be expected to pursue a mating strategy that is normally only successful for a small percentage of individuals within the mating market.

Expected changes in men. In men, victory cues are likely to lead to an increase in short-term mating interest, as this is a quick and relatively inexpensive way to enhance fitness. This prediction fits well with what is known about $\mathrm{T}$ levels and competitive outcome (see Chapter 6) as well as the view of some evolutionary psychologists that an increase in male self-esteem (i.e. via a mating sociometer) should increase interest in mating effort among men (Penke, Todd, Lenton, \& Fasolo, 2008). Loss cues, in contrast, would be predicted to show a reverse pattern. Here, men should show less short-term interest following such cues as this strategy is less likely to be effectively pursued.

In terms of a long-term mating strategy we might expect to find, following an increase in status, no change in the desire for long-term relationships in men. This prediction is partially informed from the results found in Experiment 7. Those results revealed that, while an increase in $\mathrm{T}$ was associated with an increase in ST selection, no such pattern was found for LT selection on the SMA task. If men are receiving input from the environment that they are victorious and of high status, why should they maintain a long-term mating strategy? Among men, a long-term pair-bond with a high quality mate can lead to more surviving offspring of a higher quality, and this is irrespective of whether extra-pair partners are pursued. Historically, the benefits of mating within a pair-bond would have been so great that the desire for long-term mating would have become deeply engrained in the evolved psychology of men. Indeed, in modern humans, we find that even men who are high enough in status to boycott a longterm mating strategy in favour of the exclusive pursuit of short-term partners rarely do so. Some of the most powerful despots from recorded history have chosen high quality long-term partners despite access to a large number of mistresses (Masters, 1997; 
Stewart-Williams \& Thomas, 2013b). ${ }^{32}$ In this sense, an increase in status may not reduce the desire for long-term relationships.

Expected changes in women. In contrast to men, environmental input indicating that a woman is high in status should have a different effect on her short-term mating behaviour. Among women of low social status, the pursuit of a short-term mating strategy can be used to fulfil the inadequacies within their primary pair-bonded relationship (Greiling \& Buss, 2000; Symons, 1979), through access to resources and good genes (Gangestad \& Buss, 1993; Thornhill \& Gangestad, 1993). In contrast, high status women who are able to secure high-quality partners, whom satisfy their desires within a long-term relationship, would not benefit from the pursuit of short-term mating (Buss \& Schmitt, 1993; Symons, 1979). Thus the sexes would be expected to act differently in response to victory cues; with men shifting towards, and women away from, short-term mating. The reversed pattern would be expected following consistent cues of loss.

In terms of long-term mating, an increase in status should strengthen a woman's preference for long-term partners. This is because, as her status grows, her confidence in her ability to attract high quality partners, and maintain such a relationship, should increase in line with sociometer theory (M. R. Leary et al., 1995). The opposite pattern would then be found in the case of defeat cues. Here, women should show a decrease in their pursuit of long-term partners as they shift towards a short-term strategy in order to best maximise their fitness.

\section{Experiment 8}

In this experiment, participants were given a creativity task similar to that used in Experiment 6 of Chapter 5. This task was modified to act as either a neutral, victory, or defeat cue, which then formed the different experimental conditions. For the victory condition participants were asked to recall a time in which they were victorious within a particular domain, and also to imagine a fictional victorious scenario. In the defeat condition the task was the same but involved recalling and creating scenarios in which the participant was defeated. Changes to participant's mate selections were then measured using the revised SMA task, as introduced in Experiment 6.

\footnotetext{
${ }^{32}$ Masters (1997) refers to King Charles II who famously had 11 mistresses in addition to his wife. Did Charles love his wife? Several anecdotes suggest so. These include the time when Charles cleaned up Catherine of Braganza's vomit when she was sick, wept at her bedside when she became ill, and refused to divorce her at the request of his ministers when she was unable to produce a child.
} 
For this experiment, two experimental hypotheses were developed, one for each sex. Hypothesis 7 stated that victory cues would lead to short-term mating strategy activation in men as well as long-term mating strategy activation. Two predictions about the experimental outcome were then made to test this hypothesis. Prediction 1 was that men would choose a greater number of models for a ST relationship on the revised SMA task following victory feedback, and fewer following defeat feedback. Prediction 2 was that men would choose the same number of models for a LT relationship on the revised SMA task following either victory or defeat feedback.

Hypothesis 8 stated that victory cues would lead to long-term mating strategy activation in women. Once again, two predictions were made to test this hypothesis. Prediction 3 was that women would choose fewer models for a ST relationship on the revised SMA task following victory feedback, and a greater number following defeat feedback. Prediction 4 was that women would choose a greater number of models for a LT relationship on the revised SMA task following victory feedback, and fewer following defeat feedback.

\section{Method}

\section{Participants}

Two hundred and eighty-six participants were recruited for the study in return for course credit or cash payment. Of these participants, 94 had previously completed the neutral condition for Experiment 6 which also used a creativity task as its manipulation. To reduce data redundancy, these data from these participants were also used in this experiment to form the neutral condition.

Recruitment came from local advertising within the university or through the psychology department participant pool. One participant identified himself as homosexual and was excluded from the analysis. The remaining participants were all heterosexual apart from three who described themselves as bisexual. The participants were equally split by sex (male $n=143)$ and were mainly Caucasian $(n=268,94 \%)$ with 15 participants identifying themselves as a mixture of ethnicities including Black, East Asian, and Middle Eastern. Ethnicity information was missing for two individuals. All participants were childless and the majority were single $(n=159,55.8 \%)$.

As the experimental hypotheses stated different effects for men and women, some of the demographic variables were analysed for sex differences. On average, men $(M=21.03, S D=1.37)$ were slightly older than women $(M=20.46, S D=1.34), t(283)$ $=3.516, p<0.01, d=0.42$. The average self-perceived attractiveness score for men $(M$ 
$=6.04, S D=1.53)$ was higher than for women $(M=5.18, S D=1.33), t(283)=5.095, p$ $<0.01, d=0.61$, which is consistent with the other samples within this thesis. Finally, there was a sex difference in SOI-R score. This was in the expected direction with men scoring $48.59(S D=14.10)$, and women scoring $35.39(S D=13.41)$, on average, $t(283)$ $=8.091, p<0.01, d=0.96$. There was no difference between the sexes in terms of reported socio-economic status which had a mean score of $3.27(S D=0.80), t(282)=-$ $0.784, p=0.43$.

\section{Apparatus}

Forms. A demographic form and the SOI-R (Appendices B \& E) were used in this experiment. As with Experiment 6, four additional items were added to the standard demographic form. Two related to the participant's fear of aggressive animals and crowds. These are described in Chapter 5. The other two related to competitiveness. The first item read 'Compared to my peers I consider myself to be:' and was accompanied by a nine point scale with the anchors of 'not at all competitive' (one) and 'very competitive' (nine). The second item read 'In general, when competing one-on-one against others, I tend to be victorious' and participants also responded using a nine point scale for this item. There were three anchors for this scale ranging from 'rarely or never' (one), to 'half the time' (five) to 'frequently' (nine). All participants in Experiment 6 and Experiment 8 answered these two items as well as the "danger" items. This facilitated the sharing of the neutral condition between experiments by ensuring all participants answered the same questions. The consent and debrief forms used in this experiment were identical to those used for Experiment 6 which can be found in Appendix O.

SMA version and models. As with Experiment 6, this experiment utilised the revised version of the SMA task whereby participants gave 'yes' or 'no' responses to members of the opposite sex for short- and long-term relationship types separately. Full information about this revised paradigm can be found in Chapter 5 as well as in Appendix Q. As the neutral condition from Experiment 6 was also used in this experiment, the participants in the victory and loss conditions were required to view the same models during the SMA task. The male model set contained 50 images from hotornot.com which were independently rated as having an average attractiveness score of $5.42(S D=0.66)$ out of 10 . The 50 images of females were also gathered from hotornot.com and received an average rating of $5.41(S D=0.65)$ from the same judges. Further information on the selection process of these models can be found in Chapter 5. 
Victory and defeat stimuli. The stimuli used in this experiment were of a similar format as that used in Experiment 6. However, instead of focusing on danger and arousal cues, two creative writing tasks were designed to act as victory and defeat stimuli. These tasks can be found in their entirety in Appendix $U$ and the neutral task can be found in Appendix R.

Victory stimuli. In situation one, participants were asked to recall and write about a time in their life when they felt victorious. In order to facilitate recall, participants were given four example domains from which to choose. These were the domains of sport, education, work, and games. In situation two, this task was repeated, only this time participants were asked to imagine being victorious in an important domain of their life. Again, the four example domains were given to facilitate task response. Both scenarios were accompanied by a collage of pictures related to the specified domains (e.g. video game consoles and poker cards) as well as an image of a man punching the air in victory (see Figure 7.1).

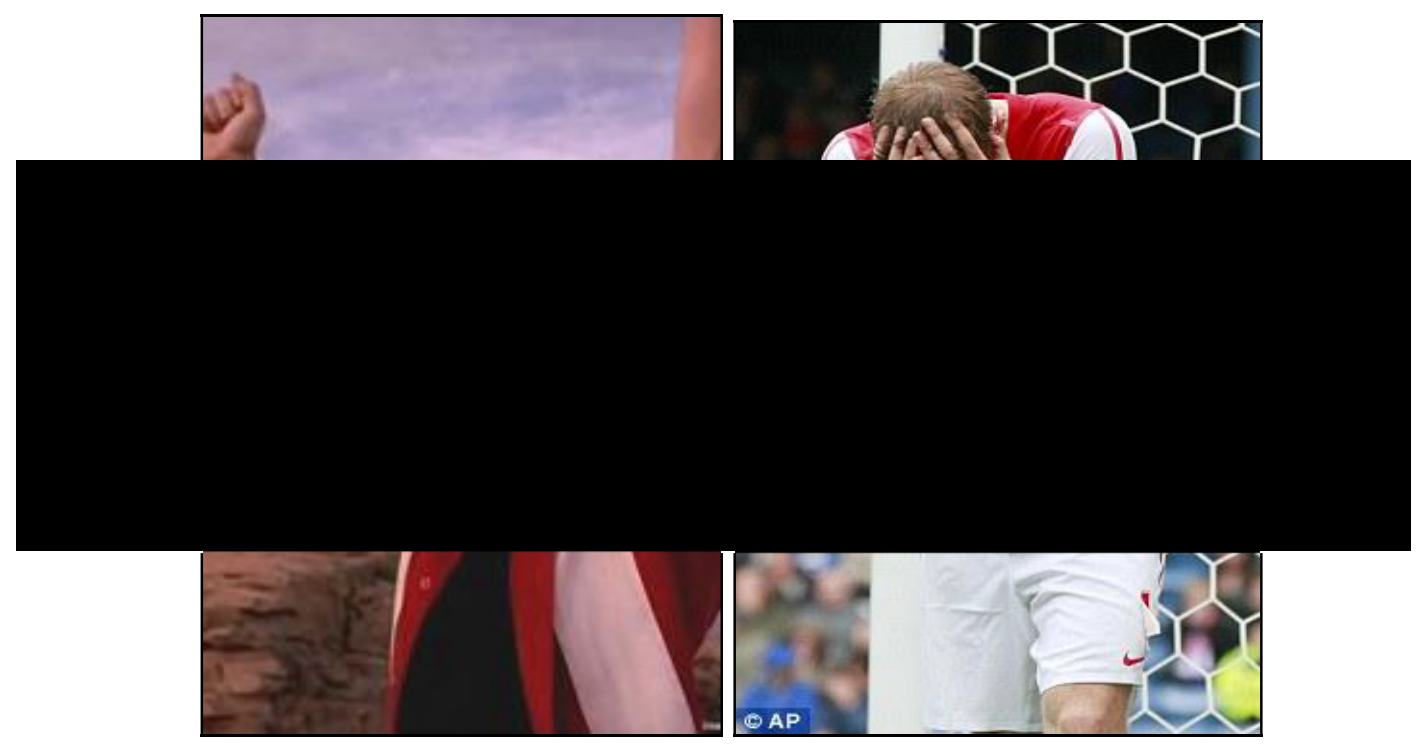

Figure 7.1. The images used to convey victory and defeat to the participants in the creativity tasks used in Experiment 8. These images were featured next to a collage of pictures related to sports, education, work, and gaming. Participants were encouraged to write about one of these example domains.

Defeat stimuli. The defeat situations followed the same format as the victory ones. In the first situation participants were asked to recall a time in which they felt most defeated within one of the four domains. Likewise, in the second situation they 
were asked to imagine being defeated by another person in a domain which was important to them. Accompanying both tasks was the same collection of images used in the victory examples above; however, the image of a man punching the air in victory was replaced by the image of a footballer who was covering his face in shame (see Figure 7.1).

Hardware. The experiment was run on several laptops to allow for portable testing in multiple laboratories. The minimum screen size of these laptops was 15.4" with a minimum screen resolution of 1280 by 800 . The operating system was always windows based, using a version of Windows ${ }^{\circledR} \mathrm{XP}$ or higher.

\section{Design}

The experiment was a 2 by 3 mixed-model design with the within-subjects factor of time and the between-subjects factor of condition. The revised SMA task produced the two dependent variables of ST and LT choice at times one and two, and exposure to either victory, defeat, or neutral stimuli formed the independent variable. The analysis involved using chi-squared analysis to test for main effects and ordinal logistic regression to test for an interaction between condition and several covariates.

\section{Procedure}

The study procedure was identical to that used in Experiment 6 aside from the fact that participants were only warned about being asked questions of a sexual nature, and not that the task involved aggressive animals. The nature of the victory and defeat tasks was not discussed with the participants before the experiment as they were considered unlikely to cause the participants any ethical distress.

\section{Results}

Ninety-six participants (female $n=48$ ) were included in the victory condition, and 95 (female $n=47$ ) participated in the defeat condition. The neutral condition consisted of 47 males and 47 females. Overall, participants indicated that they were fairly competitive. Men reported an average competitiveness rating of $6.61(S D=1.79)$ out of nine, while women reported an average rating of $5.68(S D=1.68)$. The difference between the two groups was significantly different, and showed a medium effect size, $t(283)=4.504, p<0.01, d=0.54$. Likewise, men reported being more victorious in competition, rating themselves as $6.26(S D=1.48)$ on average, compared to the 5.18 $(S D=1.40)$ rating which women reported. Again, this was a significant difference, and showed a large effect size, $t(283)=6.362, p<0.01, d=0.76$. 
During the first performance of the revised SMA task, participants chose a median number of 14 models for a long-term relationship and 18 for a short-term relationship. This number was similar to that found in Experiment 6, and a much more balanced ratio of LT to ST choices than found in previous experiments using the standard SMA task. The average number of words written for the first scenario across all conditions was $72.25(S D=39.12)$ and $62.66(S D=34.44)$ for the second scenario. The word count for both tasks was included as a covariate during analysis. No qualitative analyses of the responses were performed.

\section{Short-Term Mating}

The ST change category distributions following neutral, victory, and defeat conditions for all participants can be found in Table 7.1a below. When examining the distributions, it appeared as if there that there was no real difference between the two conditions. Indeed, when chi-squared analyses were used to test the differences between the distributions (defeat compared to victory, for example) all three were non-significant when using Bonferroni corrected alpha levels.

As an experimental hypothesis was created for each sex, the analysis was repeated separately for men and women (Table 7.1b and c). In men, it appeared that participants in the victory condition were more likely to appear in the ST+ category compared to both the neutral and defeat conditions. However, there did not appear to be a difference between these two latter groups. This pattern was confirmed with chisquared analysis. In women, it appeared that participants in the victory condition were more likely to appear in the ST- group compared to the neutral and defeat conditions. At the same time, it appeared that those in the defeat condition were more likely to appear in the ST+ group compared to the neutral and victory conditions. A chi-squared analysis revealed that these differences were only significant when comparing the victory and defeat groups.

\section{Table 7.1}

The number of participants who increased, stayed the same, or decreased in their ST choices following the creativity task. Three separate distributions are shown for the victory, defeat, and neutral conditions. There were no significant differences found between the distributions using chi-squared tests

(a) Short-term 


\begin{tabular}{lrcc} 
& - & $=$ & + \\
\hline Defeat & 38 & 15 & 42 \\
Neutral & 40 & 16 & 38 \\
Victory & 36 & 18 & 42 \\
\hline$\chi^{2}(2)-\mathrm{D}: \mathrm{N}$ & 0.567 & $\mathrm{CV}$ & 0.04 \\
$\chi^{2}(2)-\mathrm{V}: \mathrm{N}$ & 1.007 & $\mathrm{CV}$ & 0.05 \\
$\chi^{2}(2)-\mathrm{D}: \mathrm{V}$ & 0.688 & $\mathrm{CV}$ & 0.04 \\
\hline
\end{tabular}

\begin{tabular}{lccc}
\hline \multicolumn{3}{c}{ (b) Men } & \\
\hline & - & $=$ & + \\
\hline Defeat & 24 & 7 & 17 \\
Neutral & 22 & 10 & 16 \\
Victory & 15 & 11 & 22 \\
\hline$\chi^{2}(2)-\mathrm{D}: \mathrm{N}$ & 1.144 & $\mathrm{CV}$ & 0.08 \\
$\chi^{2}(2)-\mathrm{V}: \mathrm{N}$ & $4.577^{*}$ & $\mathrm{CV}$ & 0.15 \\
$\chi^{2}(2)-\mathrm{D}: \mathrm{V}$ & $7.991^{* *}$ & $\mathrm{CV}$ & 0.20 \\
\hline
\end{tabular}

\begin{tabular}{lccc}
\hline \multicolumn{4}{c}{ (c) Women } \\
\hline & - & $=$ & + \\
\hline Defeat & 14 & 8 & 25 \\
Neutral & 19 & 6 & 22 \\
Victory & 27 & 7 & 20 \\
\hline$\chi^{2}(2)-\mathrm{D}: \mathrm{N}$ & 2.392 & $\mathrm{CV}$ & 0.11 \\
$\chi^{2}(2)-\mathrm{V}: \mathrm{N}$ & 0.527 & $\mathrm{CV}$ & 0.05 \\
$\chi^{2}(2)-\mathrm{D}: \mathrm{V}$ & $7.749 * *$ & $\mathrm{CV}$ & 0.20 \\
\hline
\end{tabular}

Note: $\mathrm{CV}=$ Cramér's $V ; \mathrm{D}=$ Defeat condition; $\mathrm{V}=$ Victory condition; $\mathrm{N}=$ Neutral condition. ${ }^{\dagger} p<0.10, * p<0.05, * * p<0.01$.

A backwards stepwise ordinal regression analysis was conducted to try to predict variation in ST relationship choice change. The analysis included condition, SOI-R, the variables from the demographic form, and the number of words written during the creativity tasks. A model emerged using only the variables of sex and condition entered as an interaction term. This model approached significance, $\chi^{2}(2)=$ 4.270, $p=0.06$ (see Table 7.2). The model revealed a positive association between condition and ST category for men, and a negative association for women. Specifically as the experimental conditions progressed from defeat-to-neutral-to-victory (DNV), men's chances of appearing in the ST= or ST+ category increased by $35 \%$ for each step. In contrast this DNV progression was associated with an increase in women's chances of appearing in the ST= or ST- category by $30 \%$ for each step. This model could not be enhanced through the inclusion of additional variables.

\section{Table 7.2}

The results of an ordinal regression to predict ST change category using a sex by condition interaction. The beta for men is positive, while the beta for women is negative. These both approached significance when using an adjusted alpha for a one-tailed prediction 


\begin{tabular}{lll}
\hline \multicolumn{3}{c}{ Model ST } \\
\hline & \multicolumn{1}{c}{$\beta$} & OR \\
\cline { 2 - 3 } Male * Condition $^{*} 0.297^{\dagger}$ & 1.35 \\
Female * Condition $^{*}$ & $-0.265^{\dagger}$ & 0.76 \\
\hline Model & $\chi^{2}(2)=4.268^{\dagger}$ \\
Nagelkerke $R^{2}$ & 0.02 \\
Accuracy & $45.6 \%(+2.8 \%)$ \\
Parallel lines & $\chi^{2}(2)=1.308$ \\
\hline
\end{tabular}

Note: Variables were coded as such: Condition - defeat (-1), neutral (0), victory (1). OR $=$ odds ratio. ${ }^{\dagger} p$ $<0.10, * p<0.05, * * p<0.01$.

\section{Long-Term Mating}

The LT change categories following neutral, victory, and defeat stimuli can be found in Table 7.3 below. When compared to the neutral distribution, it appeared as if exposure to the victory stimuli led to a greater number of participants selecting more models for a long-term relationship following stimuli exposure, while the defeat stimuli led participants to select a fewer number of models. This was confirmed with three chisquared tests, all of which were significant. When a Bonferroni correction was applied (i.e. an $\alpha$-level of $0.05 / 3=0.017$ ), the differences all remained statistically significant.

\section{Table 7.3}

The number of participants who increased, stayed the same, or decreased in their LT choices following the creativity task. Three separate distributions are shown for the victory, defeat, and neutral conditions. All the distributions are significantly different from one another when using a chi-squared test

\begin{tabular}{lccc}
\hline \multicolumn{4}{c}{ Long-term change } \\
\hline & - & $=$ & + \\
\hline Defeat & 48 & 16 & 31 \\
Neutral & 30 & 22 & 42 \\
Victory & 25 & 15 & 56 \\
\hline$\chi^{2}(2)-\mathrm{D}: \mathrm{N}$ & $15.146^{* *}$ & $\mathrm{CV}$ & 0.20 \\
$\chi^{2}(2)-\mathrm{V}: \mathrm{N}$ & $7.525^{* *}$ & $\mathrm{CV}$ & 0.14 \\
$\chi^{2}(2)-\mathrm{D}: \mathrm{V}$ & $30.909^{* *}$ & $\mathrm{CV}$ & 0.28 \\
\hline
\end{tabular}

Note: $\mathrm{C} V=$ Cramér's $V ; \mathrm{D}=$ Defeat condition; $\mathrm{V}=$ Victory condition; $\mathrm{N}=$ Neutral condition. ${ }^{\dagger} p<0.10$, $* p<0.05, * * p<0.01$. 
When LT change category was predicted using the demographic variables in a backwards step-wise ordinal regression, a significant model emerged using a main effect of condition and an SOI-R * condition interaction. A within-sex median split was performed on SOI-R in order to further understand this interaction (Table 7.4). The model revealed a positive association between LT group and DNV progression. Specifically, as participants passed from the defeat to the neutral condition, or from neutral to the victory condition, the chances of them appearing in the LT+ category increased by $171 \%$ each time. As specific predictions were made for each sex, the ordinal regression model below displays the effects independently for men and women. Both were in the same direction and both were significant to below the $p=0.01$ level. In terms of SOI-R, individuals who were high in SO were more likely to show this positive association than their low SO counterparts.

\section{Table 7.4}

The results of an ordinal regression to predict LT choice change using sex, SOI-R and condition. SOI-R was median split into high and low groups within sex

\begin{tabular}{lcc}
\hline \multicolumn{3}{c}{ Model LT } \\
\hline & \multicolumn{1}{c}{$\beta$} & OR \\
\cline { 2 - 3 } Male * Condition & $1.065^{* *}$ & 2.9 \\
Female * Condition & $0.851^{* *}$ & 2.34 \\
High SO * Condition & $0.766^{* *}$ & 2.15 \\
Low SO * Condition & - & - \\
\hline Model & $\chi^{2}(3)=23.133^{* *}$ \\
Nagelkerke $R^{2}$ & 0.09 \\
Accuracy & $49.1 \%(+3.9 \%)$ \\
Parallel lines & $\chi^{2}(3)=0.889$ \\
\hline
\end{tabular}

Note: Variables were coded as such: condition - defeat (-1), neutral (0), victory (1). OR $=$ odds ratio. ${ }^{\dagger} p<$ $0.10, * p<0.05, * * p<0.01$.

\section{Discussion}

The results of Experiment 8 supported three out of the four predictions based upon the two experimental hypotheses. Prediction 1, which expected that victory cues would cause an increase, and defeat cues a decrease, in the number of models men 
chose for a ST relationship, proved to be correct. However, Prediction 2, which predicted no relationship between the experimental stimuli and LT relationship choices, did not. Here, men were found to choose more models for a LT relationship following victory cues and less following defeat cues. Thus, only partial support was found for Hypothesis 7.

Prediction 3 was correct as women showed a tendency to choose fewer models for a ST relationship following victory stimuli, and a greater number following defeat stimuli. Prediction 4, which expected an inverse relationship between victory and LT relationship choice, was also correct. Thus, good support was found for Hypothesis 8. Victory, Testosterone, and Male Mating Strategies

In the case of men, the number of models chosen for a short-term relationship was predicted to increase following a victory cue and decrease following a defeat cue. This prediction was built from a combination of two related findings: 1) several experiments have demonstrated that victory cues can cause an increase in testosterone (e.g. Bernhardt et al., 1998; Brondino et al., In Press; Carré \& Putnam, 2010); and 2) testosterone is associated with behaviours related to mating effort and a short-term mating strategy (Archer, 1991; Bogaert \& Fisher, 1995; M. Peters et al., 2008; Ronay \& Hippel, 2010; S. J. Stanton et al., 2011). Further evidence for this second finding was demonstrated in Experiment 7, where an increase in circulating $\mathrm{T}$ was found to be positively associated with short-term mating strategy change. Thus, the assumption was made that victory cues would lead to an increase in circulating T, which in turn would cause mating behaviour in men to become more short-term orientated.

While the changes to ST choices following victory cues found here appear to support this assumption, it is worth noting that actual $\mathrm{T}$ change was not measured in the experiment. As such there is still the chance that a change in circulating $\mathrm{T}$ did not occur and that the key underlying change affecting mate choices was a psychological one rather than a physiological one. Another complication surrounding the "victory $(+) \rightarrow$ testosterone $(+) \rightarrow \mathrm{ST}(+)$ ") assumption is the fact that the experimental results also revealed a positive effect of victory cues on long-term mate selection in men. This was not found in the standalone $\mathrm{T}$ experiment from the previous chapter, and so warrants explanation.

This different outcome may well be due to the additional complexity of the present experiment compared to the former. The exercise intervention used in Experiment 7 was non-social in nature and was designed specifically to increase $\mathrm{T}$ in 
isolation (or in as much isolation as possible). In contrast, the victory and defeat cues used in Experiment 8 required cognitive engagement and interpretation from the participants. This would have added an additional layer of complexity for two reasons. First, aside from T, when a human is victorious in competition, a host of other changes are likely to occur including changes to 5-HT metabolism, self-esteem levels, and circulatory cortisol (Bernhardt et al., 1998; Kudryavtseva, 2000; M. R. Leary et al., 1995; Raleigh et al., 1985; Wirth, Welsh, \& Schultheiss, 2006). Even levels of oxytocin have been associated with gloating behaviours in competitive contexts (Shamay-Tsoory et al., 2009). Second, when participants are engaged in a form of psychologically engaging task, experience, contextual, and motivational factors can also interact with biochemical changes leading to different behavioural outcomes. For example, as discussed in previous chapters, the T response of men to infant cries can be heavily influenced by whether or not they are allowed to comfort the infant, as well as their previous parental experience (Fleming et al., 2002; van Anders et al., 2012). Likewise, investigations of the 'mismatch effect' reveal how the performance of cognitive tasks can be affected by an interaction between the social labels given to participants and their underlying T levels (Josephs, Sellers, Newman, \& Mehta, 2006).

Unfortunately, the variables which were recorded as part of this study were not able to shed light on the proximate mechanisms driving the relationship choice changes in this experiment, despite introducing variables such as relationship status, selfperceived attractiveness, and competitiveness into the analysis. Future replications introducing biochemical variables such as circulating $\mathrm{T}$ and cortisol might help us better understand the effect. One may find, for example, that changes in $\mathrm{T}$ do actually account for changes in short-term relationship willingness, and that the change to long-term willingness is accounted for by a different biological or psychological change uniquely triggered by victory stimuli.

While the proximate reason for the increase in LT behaviour is unclear, the ultimate reason may be easier to understand. While it is tempting to consider the pursuit of a short-term mating strategy as the best way for men to maximise their fitness, it is important to remember that due to the dependent nature of human offspring, a pair-bond with a high quality partner would have historically proved a better way of ensuring low offspring mortality. Thus, as alluded to in the introduction to this chapter, even modern men of very high social status still pursue long-term relationships and benefit from this arrangement through the rearing of offspring with a high quality partner. This line of 
thought was originally used to form the prediction that a maintenance of LT partner selection following victory feedback would be found. However, if participants subsequently felt more capable of attracting and maintaining a high quality relationship following victory feedback then an increase in LT interest could also represent a shift in mating behaviour which could maximise fitness among men. The idea that both longand short-term mating strategies can be activated simultaneously by the same stimuli, though for different reasons, was introduced earlier in this thesis in Chapter 5. There, danger cues were predicted to increase both ST and LT selections in women due to the fitness benefits both types of strategies would afford in harsh environments.

\section{SOI-R and Long-Term Selections}

Why might individuals who are high in SO increase their LT selections following victory feedback (and lower them during defeat feedback) more than those low in $\mathrm{SO} ?^{33}$ One possibility is that high SO individuals have a different perception of commitment within a long-term relationship, seeing it as another method in which to obtain sexual partners and underestimating the level of commitment required in order to engage in sexual activity with a new partner. Supporting this interpretation is the fact that individuals who are high in SO have been shown to still engage in long-term relationships but to be less committed to them (Seal, Agostinelli, \& Hannett, 1994). Indeed in Chapter 2, high SO individuals still showed a preference for LT relationships and in some cases this was even higher than among low SO individuals.

The question then remains, why has SO not been a significant factor in all significant models produced throughout this thesis? This may be due to the revisions made to the SMA task used here. In the original SMA, participants generally expressed a desire for which type of relationship they would prefer by picking either a ST or LT relationship for each model. In the revised SMA task, the question asked is quite different; participants are explicitly told which relationship type is available and with whom and are simply asked if they would accept this offer or not. That is, rather than desires, it is willingness which is being measured using the revised SMA. Suffice to say, if high SO individuals are offered a long-term relationship with an individual or nothing at all, and their goal is sex without commitment, then they may favour the former choice as this could lead to sexual relations compared to the latter which guarantees nothing.

\footnotetext{
${ }^{33}$ Note: the significance of the ordinal model did not depend on the inclusion of SOI-R. When simply run with condition alone a significant model was produced. However, adding an SOI-R high/low split variable increased the Nagelkerke $R^{2}$ value from 0.06 to 0.09 , leading to a more powerful model.
} 
This is also reflected in the number of LT partners selected by high SO individuals during the first round of the revised SMA task. Here 16-17 individuals were chosen for a long-term relationship, compared to 2-4 using the original task (see Chapter 2).

\section{The Revised SMA}

In Chapter 5, changes were made to the SMA task to try to alter the type of data which it produced. Specifically, ST and LT decisions were separated in an attempt to generate output which was suitable for more powerful parametric analyses. The experiment in this chapter also used the same revised task. While participants ended up selecting a more balanced median of $14 \mathrm{LT}$ to $18 \mathrm{ST}$ relationships during the revised SMA, compared to the usual split of around 5 to 12 found using the regular SMA, the data produced still did not meet parametric assumptions.

In Experiment 6, the failure to repeat the effect of danger cues on mating behaviour was assumed to have been caused by faults with the creativity task used. However, there was also the possibility that this could have been due to changes to the SMA measure. Here, the revised SMA task was able to identify changes to mating strategies following a different cue. Thus, while parametric data was not produced, there is some evidence that the revised task is still a valid measure of mating strategy. Furthermore, in this experiment, the data produced did not seem to be subject to the "polarising effect" discussed in Chapter 5, whereby a very small number of participants showed no change to relationship selections after the second exposure to the revised SMA task. Taken together, the results from Experiment 8 seem to support the idea that the null results and abnormal distributions found in Experiment 6 may have been due to inadequacies in the stimuli used.

Despite not showing any obvious benefit over the original task, the revised SMA may still be of interest in further studies given the "willingness" rather than "desire" question posed to participants. This is further discussed in Chapter 9.

\section{Conclusion}

In Chapter 5, the results of the first danger cue experiment demonstrated that, while men and women can respond quite consistently to stimuli in terms of long-term mating behaviour, sex differences can emerge within short-term mating. Here, a similar result was found. While both men and women increased their receptivity to long-term relationships following cues of victory, there was a sex difference in their short-term receptivity which was in line with what sexual strategies theory would predict. 
Together, the results of Experiment 8 suggest that men and women may adjust their mating strategies in line with their social status in order to maximise their fitness. 
Chapter 8: One Man Too Many - Male-Biased Sex-Ratio Cues May Lead to Long-Term

Mating Strategy Activation.

This chapter focuses on sex-ratio as a potential contributing factor in mating strategy variation. Unlike personal factors which influence one's desirability in the mating market, such as physical attractiveness, intelligence, and kindness (Buss \& Barnes, 1986; Li et al., 2002), sex-ratio is a group level attribute which influences the availability of potential mates. Any change to the sex-ratio consequently alters the demand for, and the buying power of, individuals in the mating market. To give a simple analogy, in a busy city centre, one has many retailers from which to purchase a cup of coffee, and with this selection comes choosiness. One can shop around and make a purchase based on size, taste, and value for money; in response, the coffee retailers may produce eye catching adverts or run competitive offers to attract business. In contrast, on a remote university campus, one may have a cheap coffee vending machine as a sole source of caffeine. This latter scenario reduces one's choice, and with it one may find inflated coffee prices, reduced product quality, and little advertising. The same principles can be applied to the mating domain. The presence of many available males may lead to females to be highly selective over their choice of partner, and cause males to compete with one another for sexual access, or to adopt alternative mating strategies. However, if some event caused the population of fertile males to suddenly drop, then the pool of potential mates from which females could choose would be reduced. As a consequence, a reduction may be found in the choosiness of females and the level of competition found among males.

\section{Sex-Ratio Measures}

In a large number of animal species, from coyotes to crayfish, the sex-ratio is effectively male-biased. This is because, even when the absolute numbers of each sex are equal, females generally have periods of infertility due to biological restraints such as gestation. This principal holds for most species which fertilise internally. Thus, sexratio in the sociobiological literature is discussed in terms of the number of fertile males to fertile females (the operational sex-ratio; OSR) or the number of offspring each sex has the potential to produce over a period of time (their potential rate of reproduction; Clutton-Brock, 2007; Clutton-Brock \& Vincent, 1991; Emlen \& Oring, 1977). In economic terms, a male-skewed OSR creates a "buyer's market" for females, in which fertile females are in demand, and fertile males are in abundance. Over evolutionary 
time such an imbalance leads to an increase in sexual selection pressure on males through both intrasexual competition and female choice, which in turn can affect the typical levels of male aggression and the presence of male ornaments and armaments in a species (Clutton-Brock \& Vincent, 1991; Shuster \& Wade, 2003).

The OSR is typically male-biased in populations where the quantity of males and females are equal. Yet this bias can be further enhanced or diminished by the presence of mitigating factors which change the absolute number of each sex or their state of fertility. For example, riskier behaviour in many species can lead to higher mortality rates among males than females (Daly \& Wilson, 2001; Mathers, Sadana, Salomon, Murray, \& Lopez, 2001; M. Wilson \& Daly, 1985), some diseases, such as chlamydia, can suppress the reproductive capacity of one sex more than the other (Eggert-Kruse, Gerhard, Näher, Tilgen, \& Runnebaum, 1990), and in some species parents can bias the sex of their offspring (Burley, 1986; Saragusty et al., 2012; Trivers \& Willard, 1973). In mobile species, the availability of food and shelter can also create sex-ratio variance as it leads populations of females to be differentially distributed across space and time (Shuster \& Wade, 2003).

While the OSR is seen as an ideal measure of sex-ratio within sociobiological studies, for simplicity much of the human psychological literature uses the absolute number of men to women as a basic measure of sex-bias (although at least one study has tried to calculate human OSR using equations from the sociobiological literature; Marlowe \& Berbesque, 2012). This trend is continued here with sex-ratio, unless specifically stated otherwise, referring to differences in the absolute number of each sex without fertility status taken into consideration.

\section{Comparative Studies}

A great number of non-human animal studies were published towards the end of the $20^{\text {th }}$ century which examined the association between OSR and mating behaviour. Most of these appear to have been influenced by a classic 1977 paper published in Science by Emlen and Oring discussing the evolution of mating systems. For example, Schwagmeyer and Brown (1983) observed the male intra-sexual conflicts in a group of thirteen lined ground squirrels (Spermophilus tridecemlineatus) during the 1978 and 1979 breeding seasons. In 1978, the OSR was calculated as one female for every 2.5 males. However, the following year this balanced out to a more even 1:1.5. At the same time, there was a reduction in intra-sexual competitive behaviours (such as the disruption of mounting) among males between the two years. Similarly, a multi-year 
comparison of the intra-sexual competitiveness of adders (Vipera berus) by Madsen and Shine (1993) revealed that as the number of fertile males relative to females increased, so did the number of copulations which were preceded by male combat. When there was one female for 23 males, for example, combat occurred $100 \%$ of the time, compared to just $26.5 \%$ of the time when there were 14 females for 20 males. Across seven years' worth of data, male combat and OSR correlated strongly $(r=0.85)$. Amphibians too show such a pattern. In Woodhouse's toad (Bufo woodhousei) the number of croaks per minute emitted by males in order to attract a mate was found to be a function of male population density ( $r \sim 0.9$; Woodward, 1984).

More pertinent to this thesis are the experimental studies of animals which show that the artificial manipulation of sex-ratio can affect reproductive behaviour. Kvarnemo, Forsgren, and Magnhagen (1995) used sand gobies (Pomatoschistus minutus), a species with a roughly equal OSR, as their test animal. In one condition, three males were placed in a tank with six females, and in the second condition this ratio was reversed. Intrasexual and intersexual interactions between the gobies were then observed. In the male-biased condition, there were greater instances of male-on-male aggressive displays, and the same pattern was found among females in the femalebiased condition. Furthermore, while all males adopted a dominant strategy and built nests in the female-biased condition, only large males did so in the male-biased condition. Smaller males instead engaged in copulation sneaking. This conditional implementation of alternative mating strategies provides further example of the plasticity of mating strategies in non-human animals.

In a carefully controlled experiment, Steinmann and Priotto (2011) kept two groups of corn mice (Calomys musculinus) in separate enclosures. In one enclosure there were equal numbers of males and females, while in the other there was one female for every 2.8 males. Each enclosure contained several families which formed their own nests. To observe the amount of male-male aggression, researchers placed a small transparent chamber near one of the nests and then placed the "nest owner" male inside along with an "intruder" male from another part of the enclosure. Percentages of hostile and non-hostile interactions were then reported. Among the mice from the equal sexratio enclosure, only $20 \%$ of interactions were aggressive while $80 \%$ of the time the mice were either amicable towards, or simply ignored, each other. In contrast, among the mice from the skewed ratio enclosure, $60 \%$ of the interactions were aggressive, $40 \%$ were non-interactive, and none were amicable. 
A final example reveals sex-ratio manipulation does not solely affect intrasexual aggression but also intersexual choosiness. Using sex-role reversed pipefish (Syngnathus typhle), Berglund (1994) created an experimental set-up in which a lone male was placed in a tank adjacent to one containing a large female, a small female, and a mixed-sex group. This mixed-sex group was manipulated so that males were either in excess (two males, including the one to be tested, to four females) or in demand (four males to two females). After a 24-hour period, the test male was exposed to the small and large females and his mating behaviour was observed. In the male-biased condition, test males were found to mate with both females indiscriminately. However, in the female-biased condition, they selectively mated with the large female. These males also spent more time swimming in front of the large female prior to exposure when she was kept in the adjacent tank. The results indicate that a skewed female-sex-ratio leads male pipefish to become choosier, preferentially selecting the most fecund female.

\section{Sex-Ratio and Human Mating}

To understand how changes in the sex-ratio may influence human mating strategies, it is useful to consider the sexual strategies of both men and women and how they are predicted to change under extreme circumstances. If a member of each sex had unlimited access to willing members of the opposite sex, what sexual strategies might they follow? A man could choose to pursue a string of short-term partners, as an expression of his (typically) greater desire for sexual variety (Buss \& Schmitt, 1993; Stewart-Williams \& Thomas, 2013b; Symons, 1979). However, given the many psychological and biological indicators of man's disposition towards social monogamy (Chapter 1), it may well be that he would select one (or a few) high-quality long-term partners while taking advantage of occasional extra-pair mating opportunities.

Certainly, there are some good examples in history of kings and despots who take advantage of their access to a near limitless supply of lovers, while still taking wives (L. Barrett et al., 2002; Stewart-Williams \& Thomas, 2013b).

A woman, in contrast, may simply choose the highest quality partner possible for a long-term relationship and not feel the need to engage in extra-pair mating. This is because a large number of the hypothesized benefits of a short-term mating strategy in women surround the acquisition of resources from multiple casual partners in lieu of a single committed one (Greiling \& Buss, 2000). These include evolutionarily persistent benefits such as good genes and protection, as well as evolutionarily novel benefits such as access to money and self-esteem enhancements. If these benefits can be provided by 
one, high quality, long-term partner then the pursuit of extra-pair partners by women may not enhance her fitness. ${ }^{34}$ This dynamic is reflected in the average sex difference in the desire for a large number of desired lifetime sexual partners (Buss \& Schmitt, 1993, p. 211).

Such predictions are reversed in circumstances of low partner availability. For a man, extra-pair partnerships may be out of the question as it is generally not advantageous for women to engage in short-term mating if they can find a high-quality long-term partner easily. In such a case, he may opt to pour all his effort and resources into obtaining a single long-term partner. Thus it is expected that a change in the sexratio from female-biased to male-biased will correspond to an increase or maintenance of a long-term, and a reduction in short-term, mating strategy activation in men. For a woman, a small pool of men from which to choose may lead her to settle for a longterm relationship with a less-than-desirable man and then make up for inadequacies in her current partner through the additional benefits afforded by short-term mating (Greiling \& Buss, 2000; Symons, 1979, p. 232). Alternatively, she may engage in uncommitted relationships exclusively. As such, we would expect that, as the sex-ratio went from male-biased to female-biased, women would decrease or maintain their activation of a long-term strategy and increase their activation of a short-term strategy.

A few key studies have looked at the effect of varying levels of sex-ratio on sexual behaviour and desires in human societies. ${ }^{35}$ In Hadza hunter-gatherers, the amount of time men allocate to parental care and mating effort appears to be affected by the local sex-ratio. Marlowe (1999a) conducted over 30,000 hourly spot checks of father-child interactions across six Hadza villages and coded them for the presence of paternal care (e.g. talking to, playing with, and being in proximity of, their children). Also coded were indicators of mating opportunities, as reflected by number of fertile women, the number of single women, and the ratio of women-to-men in the camp. These three indicators, to varying degrees, were inversely related to the frequency of paternal care among men (with an average standardised $\beta$ value of around -0.5 ). One interpretation of these results is that the presence of mating opportunities, as reflected

\footnotetext{
${ }^{34}$ This of course assumes that she is satisfied with her relationship. As Symons (1979, p. 232) acknowledged, short-term extra-pair mating can be used to make up for missing attributes in a partner or as a way of facilitating mate-switching.

${ }^{35}$ It appears that the influence of sex-ratio on human mating behaviour has been considered for some time including one publication by Cox (1940), the title of which (Sex-Ratio and Marital Status Among Negroes) reflects its antiquity.
} 
by a higher female-to-male sex-ratio, leads to increased mating opportunities for men whom then reduce their level of parental care by pursuing those opportunities.

There have been some large scale population studies which have shown a link between sex-ratio and mating behaviour. This includes research by Pedersen (1991), which found that male-biased sex-ratios in the United States were associated with lower divorce rates and higher paternal care. A similar effect was found by Schmitt (2005b) in a large 48-nation study of sociosexuality. When the national levels of sociosexuality were correlated with the number of males-to-females in each country, a significant negative correlation emerged $\left(R^{2}=0.2\right)$ providing evidence that as sex-ratio becomes more female-biased, positive national attitudes and desires towards uncommitted sex, as well as behaviour, increase. Such effects would likely be larger if the OSR was used instead of the absolute numbers of people, as these ignore infertile individuals and the influence of the menstrual cycle. Nevertheless, these population studies suggest that as males become the sex in short supply, short-term mating becomes more prevalent, perhaps reflecting the fact that most men show a higher desire for sexual variety than women.

A final example of a correlational sex-ratio study examined the effect of sexratio in a pre-WWI United States population. Pollet and Nettle (2008) predicted that, as sex-ratios became more male-biased, women should have become more selective about their marriage partners. Therefore, being of high socioeconomic status (SES) would be a better predictor of marriage in male-biased societies than sex-balanced ones. Using census data of almost 22,000 men aged 15 to 50, regression analysis revealed that an individual's likelihood of marriage was predicted by a significant sex-ratio by SES interaction. The resulting model predicted that a 30 year old male had their chances of marriage increase from $56 \%$ to $60 \%$ if they were high in SES within a balanced sexratio population (1:1). However, a male-bias of just 1:1.1 was enough to change these figures to $24 \%$ and $46 \%$ respectively. Thus, not only did a male-biased population lower a man's chance of marriage, but in order to overcome this he needed to appeal to the desire of women for a marriage partner of high SES.

When an immediate change in behaviour is considered, there are a small number of laboratory experiments which show that sex-ratio cues can affect attributes related to mating strategy. Most of these use a between-subjects design. One multi-experiment paper by Griskevicius et al. (2012) showed the effects of sex-ratio manipulations on consumer behaviour. Their first experiment used a discounting-the-future measure 
inspired by the M. Wilson and Daly (2004) experiment mentioned in Chapter 4. The sex-ratio cue came in the form of a task whereby participants had to report how many models of each sex were present in a group photograph following brief exposure (one second). After answering, participants "checked their own answers" by subsequently viewing each picture again for 15 seconds. The process was repeated three times, and the participants were presented with either a male-biased, female-biased, or neutral condition. Analysis revealed that men in the male-biased condition showed a significant decrease in how long they were willing to wait for monetary rewards compared to the neutral and female-biased conditions (which were not significantly different from one another). No such difference between the groups was found among women.

In their second and third experiments, the authors used a fictional newspaper article as their manipulation. The article, which ostensibly came from a national newspaper, gave a fictional account of the demographics among students in the participant's local area. The article gave the impression that the sex-ratio was skewed in the direction of men in one condition and women in the other. Participants were asked to memorise the content of the article and then asked various questions postmanipulation as the dependent measure. For the second experiment, they were a) given a hypothetical wage and asked how much they would like to put into savings each month, and b) asked how much they would be comfortable borrowing on a credit card for luxuries each month. Results once again revealed a discounting the future effect in men but only in the male-biased condition. For the third experiment, participants were asked the appropriate values for courting gifts including a romantic meal, engagement ring, and Valentine's Day gift. This time an effect was found for both sexes. In the male-biased condition, both men and women assigned higher values to these gifts. The authors concluded that an increased male sex-ratio led to enhanced intra-sexual competition in men which changed their behaviour, and which was detectable by women who changed their expectations appropriately.

A separate study (of which Vladas Griskevicius was also a co-author) found that sex-ratio cues can affect career desire in women. After finding a negative correlation between high-paying careers and sex-ratio among women (i.e., U.S.A. states with fewer men also had more women in high paying job roles), Durante, Griskevicius, Simpson, Cantu, and Tybur (2012) predicted that career aspiration could be affected by sex-ratio cues. The experiment used the same type of pictorial sex-ratio cue described in the previous paper whereby participants had to guess how many members of each sex were 
in a photo after being briefly exposed to it. The experimenters found that when women were given a female-biased sex-ratio cue, they were more likely to favour their career over their family, as measured using a three-item questionnaire $\left(\eta_{p}^{2}=0.10\right)$, compared to neutral and male-biased conditions. In another experiment within the same paper, the authors showed that perceived mate-value also influences responses. Women who considered themselves low in mate value increased their career aspirations when presented with female-biased cues, while women who considered themselves high in mate value remained unchanged, presumably as the latter felt that they would not have a hard time attracting a high quality mate even among a group of competitors.

In a final example, Watkins, Jones, Little, DeBruine, and Feinberg (2012) found that sex-ratio cues could influence the selectivity of a female test sample in a study of facial symmetry. Participants were exposed to 20 pairs of photographs and asked to pick which one of each pair they considered the most attractive. Each pair consisted of two photos of the same model and these were identical apart from the fact that one had been altered to show enhanced facial symmetry. Half of the pairs featured men and half featured women. Following this task, participants were exposed to a slideshow of 30 more models, each presented for two seconds. In the female-biased sex-ratio condition, $83 \%$ of these models were women and $17 \%$ men. In the male-biased condition this was reversed. Finally, the original symmetry preference task was performed again. The female participants showed an average increase in preference for male symmetry in the male-biased condition, and female symmetry in the female-biased condition $\left(\eta^{2} \mathrm{p}=0.04-\right.$ 0.06). The authors interpret this as a bias towards choosing the most attractive partners (in the case of male-biased sex-ratio) and identifying competitors (in the case of femalebiased sex-ratio). The lack of men in the participant base of this study means that these results are not fully interpretable.

Alternative interpretations. From the literature presented one might predict that, as the sex-ratio becomes either male or female biased, both sexes would change their mating behaviour in order to maximise their fitness. However, there are two alternative interpretations of the same findings which could produce similar results. First, it may be that overall patterns in behaviour at the population-level (such as the association between SOI and sex ratio Schmitt, 2005b) may in fact be driven by a small number of individuals. These individuals could show marked changes in their mating behaviour in response to the local sex-ratio, which then has an influence on the mean of the population for a given measureable trait such as SOI. Second, it may be the case that 
the typical mating behaviour of the population, while appearing to be driven by strategy fluctuations in both sexes in response to sex-ratio, may in fact be the consequence of a strategy change primarily in one sex. For example, it could be the case that men are open to short-term mating but, when there are few women, end up engaging in a monogamous relationship out of necessity despite following a short-term mating strategy. If circumstances then changed, with men becoming fewer than women, then women may change their sexual strategy, causing them to be more open towards shortterm mating. Such a change would appear to be accompanied by a change in the mating behaviour of men, when, in reality, they would simply be pursuing the same initial strategy only with a different outcome.

If either of these interpretations is correct, then we would expect support to be found for them in the following experiment. Namely, if the first interpretation was true then we would expect only a small sub-section of men and women to change their mating behaviour following sex-ratio cues, perhaps identified by a demographic variable such as initial SOI or self-perceived attractiveness. If the second interpretation was true, then we would expect to find no change within the desire for ST or LT mating choices within one sex, and a marked change within the other. However, for the purpose of hypothesis development within this chapter, changes to the mating strategies of both sexes in reaction to cues of male- and female-biased sex-ratios were assumed.

\section{Experiment 9}

The purpose of this experiment was to see if exposing participants to sex-ratio cues could lead to measurable changes in their mating orientation as recorded using the SMA task. In contrast to some of the recent sex-ratio manipulation studies, both men and women were tested in this experiment and a within-subject design was used.

Participants were presented with an array of individuals and given bogus information about their relationship status. This information was designed to imply that one sex was in abundance within the local community while the other was in demand. While the underlying rationale for the direction of change in response to the sex-ratio cues is qualitatively different for each sex, the outcome was predicted to be quantitatively similar. Therefore the experimental hypotheses did not state a sex difference. Hypothesis 9 stated that male-biased sex-ratio cues would lead to long-term mating strategy activation in both men and women. Two predictions were formed to test Hypothesis 9. Prediction 1 was that, following a male-biased sex-ratio cue, both men and women would choose a greater number of partners for a LT relationship on the 
SMA task. Prediction 2 was that, following the same cues, both sexes would choose fewer partners for a ST relationship on the SMA task.

Hypothesis 10 stated that female-biased sex-ratio cues would lead to short-term mating strategy activation in both men and women. This second hypothesis was also tested using two predictions. Prediction 3 stated that, following a female-biased sexratio cue, both men and women would choose a greater number of partners for a ST relationship on the SMA task. Prediction 4 stated that, following the same cues, both sexes would choose fewer partners for a LT relationship on the SMA task.

\section{Method}

\section{Participants}

One-hundred and sixty volunteers (an equal split of men and women) participated in the study for either payment or course credit. An additional incentive was offered to encourage the participants to engage with the task. Participants were told that, at the end of the study, the individual who scored the most number of points on the task would receive a $£ 30$ Amazon gift voucher. In reality, as the "correct" answers on the manipulation task were fixed, the voucher was issued to a random participant at the end of the study. This was explained to the participants during their experimental debrief.

Recruitment came from local advertising within the university and through social contacts of the experimenter. Two homosexual participants were removed from the analysis and so the following demographic information refers to the 158 remaining subjects. The sample was primarily Caucasian (97.5\%) with the other participants divided between East Asian $(n=2)$, South Asian $(n=1)$, and mixed $(n=1)$ ethnicities. The majority of the sample was single or in an uncommitted relationship (58.2\%) and the remainder was in a committed relationship which had lasted longer than three months. The participants had an average age of $20.67(S D=1.74)$ and typically reported themselves as being average in attractiveness compared to their peers $(M=4.23, S D=$ 1.52). On average, participants considered themselves to be of a middle to upper-middle socioeconomic status $(M=2.62, S D=0.82)$. No sex differences were found for age, self-perceived attractiveness, or SES in this sample. However, an average sex difference was found for SOI-R, $t(155)=5.320, p<0.01, d=0.85$. Men had an average score of $37.63(S D=12.30)$, while women had an average score of $28.46(S D=9.00)$. All participants were heterosexual, and only one participant had a child.

\section{Apparatus}


Forms. As with previous chapters, a standard demographic form and copy of the SOI-R were included in this study (Appendices B and E). No additional changes were made to these forms for this particular experiment. Debrief and consent forms for the study can be found in Appendix V.

SMA models. The set of male and female models presented during the task were sourced from hotornot.com. For the male models, 145 initial images were gathered and rated for attractiveness on a scale spanning from one ('very unattractive') to ten ('very attractive') by 20 judges. The judges were an equal split of both sexes and their average age was $20.55(S D=1.28)$. The full set of male photos had an average attractiveness rating of $3.51(S D=0.69)$. From these, a sub-set of 50 models were chosen from the middle of the distribution which had an average attractiveness of 3.20 $(S D=0.21)$. For the female models, the same judges rated an initial set of 145 females. This set had an average attractiveness of $4.94(S D=0.68)$. A sub-set of 50 models were taken from the middle of the distribution. These models had an average attractiveness rating of $4.91(S D=0.25)$. There was a large difference in the attractiveness of the final male and female model sets. While the male photos ranged in attractiveness from 2.753.55 , female photos ranged from to 4.50-5.30. No statistical test was performed to test for a difference between these groups as their distributions did not overlap. The implications of this difference are addressed in the discussion.

The Guessing Game. A short task was created to administer the sex-ratio cue. The Guessing Game (GG) was introduced to the participants as a task used to measure their ability to guess the relationship status of other individuals based solely on their appearance. The task showed participants 100 pictures of models and asked them to guess the relationship status for each one. Participants were told whether they were right or wrong after each guess. The "correct" answers were designed to give the impression that one sex was in greater demand than the other. For example, in the male-biased condition, many men would be single while many women would be in committed relationships.

The GG started with some basic instructions (Appendix W) which introduced the task and informed the participants that the models used within the game were from the local area. This location information was given in order to make the sex-ratio cue relevant to the participant. Participants were told that the pictures were volunteered by students studying at Swansea University, Swansea Metropolitan University, and Cardiff University. The instructions then explained that performance on the task was measured 
in terms of points: A single point was awarded for every correct guess the participant made, while a point was subtracted for every incorrect guess. Finally, to cement the idea that the models were from the local area, participants were informed that they may come across someone known to them and to contact the experimenter if this occurred (a similar device was used in Chapter 3).

The task itself consisted of 100 rounds of guessing. Each round started with a blank grey screen. After a $1500 \mathrm{~ms}$ pause a model was shown in the middle of the screen with two buttons below them. The left button was labelled 'single' and the right was labelled 'spoken for'. When participants clicked one of the response buttons, the screen briefly cleared and was replaced by one of two on-screen messages. For a correct response a large green tick appeared in the middle of the screen along with the message ' +1 point'. For an incorrect response a large red cross appeared in the middle of the screen along with the message '-1 point'. Also accompanying the incorrect response message was an unpleasant buzzer sound (this was the same sound used for an incorrect answer in the television game show Family Fortunes). The message remained on the screen for $700 \mathrm{~ms}$ before disappearing. A blank screen was then shown to the participants for $1500 \mathrm{~ms}$ before the next round began.

The sex of the models alternated for the duration of the 100 rounds, and the correct answer for each round was decided by a condition dependent algorithm. In the male-biased condition, a guess that a male model was single yielded correct feedback $75 \%$ of the time, while a guess that a female model was single yielded correct feedback $25 \%$ of the time. Thus participants were given feedback that there was an abundance of men while women were in demand. In the female-biased condition this pattern was reversed: A guess of single when presented with a male model yielded correct feedback $25 \%$ of the time, while a guess that a female model was single yielded correct feedback $75 \%$ of the time.

Throughout the task, a counter was present in the top-left hand corner of the screen displaying the number of points the participant had earned. This counter was set to ' 0 ' points in the first round. Thus, the highest number of points which could be scored on the guessing game was +100 while the lowest number of points was -100 . The scoring system was designed in such a way that a purely random selection of relationship category by the participant would result in an average score of zero. The GG was sandwiched between two performances of the standard SMA task. 
The Guessing Game models. A separate set of models was gathered from hotornot.com for use in the GG, and these were rated for attractiveness by the same judges mentioned in the 'SMA models' section above. For males, a set of 185 images were rated as having an average attractiveness of $3.29(S D=0.67)$ and from this a subset of 50 images was taken from the middle of the distribution. These images had a final attractiveness rating of $3.21(S D=0.21)$. For the female models, 190 images were originally gathered and these had an average attractiveness of $4.15(S D=0.67)$. From these, a sub-set of 50 images were chosen from the middle of the distribution which had an attractiveness level of $4.91(S D=0.29)$.

Much like the SMA models, there was a difference between the attractiveness of the male and female stimuli, whose attractiveness distributions did not overlap. However, there was no within-sex difference in the attractiveness of the images used for the SMA and GG tasks. That is, for a female participant, the males they encountered in the GG task were of a similar attractiveness to the males they encountered in the SMA task, $t(98)=0.07, p=0.941$. The same applied to the female models viewed by male participants, $t(98)=0.07, p=0.944$. Thus, any changes found in the relationship choices participants made were unlikely to be due to the exposure of models which were of higher or lower attractiveness between the SMA measures. However, attractiveness differences between the male and female models during the GG could have implications for the results. This is discussed in detail towards the end of the chapter.

Hardware. The experiment was run on several laptops to allow for portable testing in multiple locations. The minimum screen size of these laptops was 15.4" with a minimum screen resolution of 1440 by 900 . The operating system was always Windows ${ }^{\circledR}$ based, using a version of Windows ${ }^{\circledR}$ XP or higher. Participants wore headphones during the GG in order to hear the negative feedback sound effect.

\section{Design}

The experiment was a 2 by 2 mixed-model design with the within-subjects factor of time and the between-subjects factor of condition. The SMA task provided the dependent variables and GG feedback (either male- or female-biased) formed the independent variable. The data were analysed using chi-squared analyses to test for main effects. Ordinal regression models were also used to predict change in mating behaviour, after the manipulation, using the items present in the demographic form and the SOI-R.

\section{Procedure}


The study was advertised as an investigation into 'relationship preferences and social cognition'. When the participant entered the lab they began by reading the combined information sheet and consent form. After they had provided consent they completed the demographic form and the SOI-R. These were subsequently sealed in an envelope with a random participant reference number written on the front. Before beginning the experiment, the general format of the study was confirmed with the participant to check their understanding.

The SMA and GG computer programs were both written in Visual Basic 6 and were linked together for the purpose of this experiment. So, without the need for experimenter intervention, the program would present the SMA task, the GG, and the SMA task again in that order. The experimenter set up the SMA-GG program by entering the participant's unique reference number into it, and selecting the experimental condition. This was done out of the view of the participant. The participant was then issued with headphones and allowed to complete the experimental tasks in private. Together, all three tasks took around 20 minutes to complete, and the participant notified the experimenter when they had finished. A full verbal and written debrief was then administered, and the participant was allowed to ask any questions to the experimenter they wished.

\section{Results}

\section{Post-Hoc Analysis Changes}

In Experiment 2, participants received either negative or positive feedback about their attractiveness via the Mix\&Match website. As there was no neutral condition present, the changes to ST and LT relationship choices were tested separately for each condition against that which would be expected by chance. There are two weaknesses to such an approach. First, an assumed equal distribution may not be a good substitution for a true neutral condition. For example, following negative feedback, if the ST-, ST=, and ST+ categories held the values of 10, 7, and 20 respectively, then the procedure to date would be to test this distribution against the expected values of 15, 7 and 15. This would have suggested that the participants had a tendency to increase in ST choice. However, it may actually be the case that a neutral condition would have produced quite a different split. If this distribution had been 9, 7, and 21, for example, then the conclusion drawn from the analysis would have been that the distributions did not differ from one another in a meaningful way. 
Second, the significant predictors within the ordinal models could be attributed either to the intervention or to general time related effects. For example, if age were a significant positive predictor in a ST model within the negative condition, then this could be interpreted as: a) older individuals having responded to the negative feedback differently than younger participants; or b) older individuals having responded to the SMA task differently at second exposure, compared to younger participants, for some non-condition related reason. Perhaps, for example, younger participants were more likely to become bored with the study and choose 'not interested' more readily during the second measure. This ambiguity is removed when a condition variable is present, as these latter variables become significant in the models irrespective of condition.

The approach described above was originally applied to the results from the current experiment; the female-biased and male-biased conditions were analysed separately. When this occurred, no significant main effects were found for either condition using chi-squared analyses. While no significant ordinal models emerged in the female-biased condition, both a LT, $\chi^{2}(6)=25.64, p<0.01$ and a ST, $\chi^{2}(1)=6.42, p$ $<0.05$ model emerged in the male-biased condition. The interpretation of these significant models was subject to the second issue raised above, as there was no neutral condition present. As a result, the decision was made to change the nature of the analysis post-hoc by using the female-biased condition (which yielded null results) as a comparison group. This would then allow for the identification of "general effect" variables (such as the example of age above) and demographic variables which legitimately interacted with condition. The full results of the original analysis can be found in Appendix X.

\section{Points}

The average number of points scored by the participants was $12.67(S D=$ 20.26). The points variable was normally distributed, $\mathrm{K}-\mathrm{S}(158)=0.07, p>0.05$; $\mathrm{S}$ $\mathrm{W}(158)=0.99, p>0.05$, and so was assessed using parametric analysis and included in the ordinal regression analyses (see below) as a continuous variable. A two-way between-subjects ANOVA was used to see if the number of points earned by the participants differed by their condition or sex. There was no significant main effect of sex, $F(1,154)=0.368, p=0.54$, and no significant interaction between condition and sex (this had the same $F$-value and significance level). However, there was a main effect of condition, $F(1,154)=48.988, p<0.01, \eta^{2} \mathrm{p}=0.24$, with participants scoring an average of 2.64 points $(S D=18.12)$ in the female-biased condition and $22.45(S D=$ 
17.31) in the male-biased condition. Further analysis revealed that while the former did not differ from a hypothesized distribution with a mean of zero, $t(77)=1.287, p=0.20$, the latter did, $t(79)=11.600, p<0.01, d=2.61$.

\section{Long-Term Mating}

After playing the male-biased version of the guessing game, 34 participants chose fewer models for a LT relationship (LT-), 21 chose the same number (LT=), and 25 chose a greater number (LT+). In comparison, following female-biased feedback, these figures were 37, 20, and 21 respectively. Thus, there did not appear to be a marked difference between the two conditions in how the participants were distributed across the LT categories. This was confirmed with a chi-squared test which revealed no significant difference between the distributions (Table 8.1).

\section{Table 8.1}

The number of participants who increased, stayed the same, or decreased in their number of LT choices following exposure to either a male- or female-biased sex-ratio task. The distributions are not significantly different from each other using a chisquared test

\begin{tabular}{lccc}
\hline \multicolumn{3}{c}{ Long-term change } \\
\hline & - & $=$ & + \\
\hline Male-biased & 34 & 21 & 25 \\
Female-biased & 37 & 20 & 21 \\
\hline$\chi^{2}(2)$ & 0.979 & & \\
Cramér's $V$ & 0.06 & & \\
\hline
\end{tabular}

$$
{ }^{\dagger} p<0.10, * p<0.05, * * p<0.01 .
$$

A backwards ordinal logistical regression was performed in order to predict LT change category. The same variables were used in this analysis as were entered into the short-term analysis above. A significant model emerged which also utilised four variables (Table 8.2).

Table 8.2

The results of an ordinal regression to predict LT category change using condition, relationship status, and SOI-R 


\begin{tabular}{lll}
\hline \multicolumn{3}{c}{ Model LT } \\
\hline \multirow{2}{*}{ Condition } & \multicolumn{1}{c}{$\beta$} & OR \\
\cline { 2 - 3 } Relat * Condition & $-0.459^{*}$ & 0.63 \\
SOI-R * Condition & $-0.023^{*}$ & 0.98 \\
\hline Model & $\chi^{2}(3)=8.392^{*}$ \\
Nagelkerke $R^{2}$ & 0.06 \\
Accuracy & $48.4 \%(+3.5 \%)$ \\
Parallel lines & $\chi^{2}(3)=0.655$ \\
\hline
\end{tabular}

Note: Relat. = relationship status. Variables were coded as such: Condition - female-biased (1), malebiased (2); Relat - single/uncommitted (0), committed/married (1). OR = odds ratio. ${ }^{\dagger} p<0.10,{ }^{*} p<$ $0.05, * * p<0.01$

A significant effect of condition was observed in the model. Compared to the female-biased condition, those who were subjected to the male-biased version of the Guessing Game were 236\% more likely to appear in the LT= and LT+ category compared to the LT- category. However, the predictive power of condition in this model depended on the inclusion of two interaction terms. The first was a relationship status * condition interaction and the second was SOI-R * condition interaction. When these variables were split in order to reveal the nature of the interactions, it was found that single participants in the male-biased condition were $194 \%$ more likely than participants in committed relationships to appear in the $\mathrm{LT}=$ or $\mathrm{LT}+$ categories $(\beta=-1.077, p=$ 0.04). No such effect was found among participants in the female-biased condition ( $\beta=$ $-0.169, p=0.72$ ). Likewise, in the male-biased condition only, SOI-R was a negative predictor. With each additional point scored on the SOI-R, the participants chance of appearing in the LT $=$ or LT- category increased by $5 \%(\beta=-0.047, p=0.02)$. No such effect was present in the female-biased condition $(\beta=-0.023, p=0.28)$. This expanded model is not shown here, as by splitting the categories, the $\chi^{2} \mathrm{df}$ inflates and causes of model to fall out of significance, $\chi^{2}(5)=8.945, p=0.11$.

\section{Short-Term Mating}

After playing the male-biased version of the guessing game, 42 participants chose fewer models for a ST relationship (ST-), nine chose the same number (ST=), and 29 chose a greater number of models (ST+). In comparison, following female-biased feedback, these figures were 39,12 , and 27 respectively. Thus, there did not appear to 
be a marked difference between the two conditions in how the participants were distributed across the ST categories. This was confirmed with a chi-squared test which revealed no significant difference between the distributions (Table 8.3).

Table 8.3

The number of participants who increased, stayed the same, or decreased in their number of ST choices following exposure to either a male-or female-biased sex-ratio task. The distributions are not significantly different from each other using a chisquared test

\begin{tabular}{lccc}
\hline \multicolumn{3}{c}{ Short-term change } \\
\hline & - & $=$ & + \\
\hline Male-biased & 42 & 9 & 29 \\
Female-biased & 39 & 12 & 27 \\
\hline$\chi^{2}(2)$ & 1.051 & & \\
Cramér's $V$ & 0.06 & \\
\hline
\end{tabular}

${ }^{\dagger} p<0.10, * p<0.05, * * p<0.01$

A backwards ordinal logistical regression was performed in order to predict ST change category. Included in the analysis were the factors of condition, points earned, sex, SOI-R, self-perceived attractiveness, age, relationship status, and socio-economic status. A significant model emerged from the analysis using four variables (Table 8.4).

Table 8.4

The results of an ordinal regression to predict ST category change using condition, the number of points earned during the guessing game, self-perceived attractiveness and SOI-R

\begin{tabular}{lcc}
\hline \multicolumn{3}{c}{ Model ST } \\
\hline & \multicolumn{1}{c}{$\beta$} & OR \\
\cline { 2 - 3 } SPA & $0.238^{*}$ & 1.27 \\
SOI-R & $0.035^{*}$ & 1.04 \\
MBGG * Points & $-0.022^{*}$ & 0.98 \\
FBGG * Points & -0.008 & - \\
\hline Model & $\chi^{2}(4)=17.681^{* *}$ \\
Nagelkerke $R^{2}$ & 0.12
\end{tabular}




\begin{tabular}{ll} 
Accuracy & $59.2 \%(+7.9 \%)$ \\
Parallel lines & $\chi^{2}(3)=3.749^{\mathrm{a}}$ \\
\hline
\end{tabular}

Note $:$ MBGG = male-biased guessing game condition. $\mathrm{FBGG}=$ female-biased guessing game condition. $\mathrm{SPA}=$ self-perceived attractiveness. $\mathrm{OR}=$ odds ratio. ${ }^{\dagger} p<0.10, * p<0.05, * * p<0.01 .{ }^{\mathrm{a}}$ The test of parallel lines here refers to the model where the condition * points interaction term was not broken down. The test could not be performed in SPSS for the $4 \mathrm{df}$ model.

A significant effect of condition * points was observed in the model. Table 8.2 shows the outcome of splitting the female-biased and male-biased condition to reveal the direction of the interaction. Within the male-biased condition, for every 10 additional points the participant earned, the likelihood of them appearing in the ST= or ST- group increased by $22 \%$. Thus, among participants in the male-biased condition who were able to score points effectively, ST selection appeared to decrease. Although in a similar direction, points were not found to be a significant predictor within the female-biased condition. In order for the condition * points interaction to be revealed, it was necessary to control for both participant self-perceived attractiveness and sociosexuality. Both of these variables were positive predictors. Participants were more likely to appear in the ST= or ST+ categories if they perceived themselves to be high in attractiveness or if they had an unrestricted sociosexuality. These effects were irrespective of condition.

\section{Discussion}

The results of Experiment 9 provided support for two out of the four predictions based upon the two hypotheses. Prediction 1 stated that, following male-biased feedback, an increase in LT selection would be found in both sexes. Indeed, when compared to the female-biased condition, participants in the male-biased had a greater chance of appearing in the LT= or LT+ categories. This effect was apparent once the relationship status and sociosexuality of the male-biased participants was controlled for. Prediction 2 stated that, following male-biased feedback, a decrease in ST selection would be found. The results also seem to confirm this prediction. Among the malebiased participants, those who scored highly on the GG task were more likely to appear in the ST= or ST-groups upon second exposure to the SMA task. No such effect was found among the female-biased group. This effect emerged after the self-perceived attractiveness and SOI-R of all participants was controlled for. Thus, support was found for Hypothesis 9. 
In contrast, no support was found for Predictions 3 or 4 . When the analysis was originally run as intended (Appendix X), no significant chi-squared tests or ordinal models could be found within the female-biased condition. This condition was then used as a post-hoc comparison group in order to clarify the effects of male-biased sexratio feedback. Thus, Hypothesis 10 was not supported.

\section{Lack of Female-Biased Bias}

The results of this experiment revealed no influence of female-biased sex-ratio on ST or LT choice variation. There are at least three potential reasons for this. The first is that a male-biased sex-ratio cue could have a greater influence on the mating behaviour of both sexes than a female-biased one. As discussed in the introduction, male-biased sex-ratios tend to be accompanied by more intense intra-sexual competition and violence among males. If this was also true ancestrally, it may well be that our mating psychology evolved to be more reactive to changes in one direction of sex-ratio change than the other. This explanation would also account for the lack of effect of female-biased ratio found in the previously mentioned Griskevicius et al. (2012) study. That being said, other studies have found that female-biased cues can influence female psychology (i.e. Durante et al., 2012), so other reasons for a lack of an effect in this experiment must be considered.

The second potential reason is that the natural sex-ratio of the sample was already skewed in the female direction and so the female-biased condition was actually a "neutral" condition from the perspective of the participants. When the demographic statistics of students at Swansea University are explored (City and County of Swansea, 2010), gender is found to be fairly equally split with 1.13 men for every one woman. ${ }^{36}$ However, this ratio is quite different when we consider students in the psychology department, a large proportion of which formed the participant base for this study. According to the academic registry, for the academic year 2011-2012, for every one man enrolled in a single or joint-honours psychology course at the University there were 3.7 women (women $n=407$, men $n=111$; Swansea University Registry Office, personal communication). Had this experiment been conducted using students from an academic area where the sex-ratio is reversed (e.g. computer science or engineering), it may well have been the female-biased cue which affected behaviour rather than the male-biased one. Of course, a student's academic community represents only one social

\footnotetext{
${ }^{36}$ Although, as previously mentioned in the study by Pollet and Nettle (2008), this small male bias can have a large influence on behaviour.
} 
sphere out of many to which they belong. To properly test this claim, a measurement of group salience would be required, perhaps adopted from the self-categorisation theory literature which focuses on the transient nature of group affiliation and its accompanying attitudes and beliefs (Turner, Hogg, Oakes, Reicher, \& Wetherell, 1987). Similarly, the analysis could be repeated using the alternative male-biased student populations mentioned previously. However, if it was true that the female-biased condition had no effect because it was "neutral" and reflected the status quo, then female-biased participants would have been expected to perform well on the GG task by scoring a lot of points. This was not the case, but may have been due to the relative attractiveness of the models used. This is addressed below.

The third and final reason is one of methodological criticism. When creating a set of models to be displayed to the participants, great care was taken in selecting models of the same attractiveness for the GG and SMA tasks. That is, the within-sex model sets were of similar attractiveness. Unfortunately, there was a large sex difference in the attractiveness of the models with all female models exceeding males in their attractiveness rating. This may have been partly due to basic sex differences in perceived attractiveness; in every set of pictures taken from the hotornot.com website throughout this thesis, women were rated as more attractive than men by independent judges. It was possible to minimise these differences in other studies by selecting an appropriate sub-set of images. However, this was not the case in this experiment. Subsequently, this may have affected how the GG was perceived. In the male-biased condition, participants would have received the "message" that average looking men were in abundance, while very attractive women were in short supply. Conversely, in the female-biased condition, this message would have been that average attractiveness men were in short-supply while very attractive women were in abundance. Out of the two scenarios, the former seems more realistic than the latter given that men of average attractiveness should find it harder to attract and maintain a partner compared to highly attractive women. This appears to be supported by the fact that there was a significant difference in the number of points scored by participants in the male-biased and femalebiased condition. Not only did participants in the female-biased condition score fewer points, their scoring distribution did not differ from a hypothesized distribution with a mean of zero. As such, the male-biased cue may have been more plausible and therefore more likely to influence behaviour.

\section{Relationship Status and SOI-R}


Within the LT model, an overall effect of condition was found when two interaction terms were included in the analysis. The first was an interaction between condition and relationship status; the second was an interaction between condition and SOI-R. Subsequent analysis revealed that both relationship status and SOI-R had a predictive effect within the male-biased condition only. The first predictor, relationship status, showed a negative association due to the way the variable was coded. This $\beta$ value can be interpreted as individuals who were single being more likely to appear in the $\mathrm{LT}=$ or $\mathrm{LT}+$ categories compared to those within a committed relationship. Thus, when considered in conjunction with the general condition effect, it appeared as if single participants were more likely to be affected by the male-biased cue. From an evolutionary perspective, it makes sense that individuals who are currently single, and who are perhaps seeking to acquire a partner, should be more affected by stimuli which reflect the relationship status of other individuals within the mating market. Human ancestors capable of such social reasoning would have had a clear advantage over their peers. For those already within a pair-bond, who are not searching for extra-pair partnerships, such information may only be useful for mate retention (Buss \& Shackelford, 1997) as opposed to mate acquisition. Supporting this idea is the previously discussed literature in Chapters 3 and 4 showing that humans are more likely to attend to cues which are relevant to their circumstances (e.g. Maner et al., 2007; Posavac et al., 1998) and that several changes in psychology occur when entering a pairbond (e.g. R. S. Miller, 1997; Rusbult \& Buunk, 1993). Such evidence includes the results of Experiment 3 within this thesis in which only participants within committed relationships appeared to increase their LT selections in response to parental stimuli.

The second variable of interest here was SOI-R. The SOI-R $\beta$ was negative which was in the opposite direction of the main condition effect. Thus, it appeared that the higher an individual's willingness to engage in sex without commitment, the less likely they were to be affected by the male-biased stimuli. Such an effect has been found in previous chapters, whereby an individual's pre-existing mating strategy appears to make a shift towards a certain relationship type more or less likely. For example, in Experiment 4, the effect of reduced LT interest following wealth stimuli appeared to have been driven almost entirely by high SO individuals. Here too, it appeared that individuals who were higher in SO were affected differentially by the experimental manipulation. However, as discussed in the next section, a general effect of SOI-R was found in the ST model, suggesting that an individual's sociosexuality 
affected how they responded to the second SMA task irrespective of experimental condition. As there is no explanation as to why a general effect would be found for one relationship type, while a condition dependent effect would be found in the other, the interpretation of the interaction of SOI-R with condition should be approached with caution.

\section{Condition Independent Effects}

In the ordinal model used to predict ST category change, two demographic covariates emerged. These variables were unrelated to condition, and were required in the model for the condition * points interaction to be significant. The first variable was self-perceived attractiveness while the second was SOI-R. Both of these were positive, indicating that attractive and socio-sexually unrestricted individuals were more likely to appear in the $\mathrm{ST}=$ or $\mathrm{ST}+$ categories during the second exposure of the SMA task.

Why might these individuals have responded differently to their low SO and low attractiveness counterparts? One suggestion could be that there is something about the Guessing Game task, shared by both conditions, which led to these effects. Stripped of the relationship status element, the GG gave participants additional exposure to members of the opposite sex, as well as some same sex models. For men, this meant exposure to more highly attractive potential partners and some average attractiveness competitors. In this case, those men who were high in SO may have responded to this scenario by acting as if they had good access to attractive women. The converse would then have been true of women, who may have responded to stimuli as if average attractiveness potential partners were abundant but that competitors were very attractive. In this case, those women who were high in SO could have been responding to this scenario by acting as if they had poor access to average attractiveness men.

Unfortunately, such reasoning does not explain fully the presence of selfperceived attractiveness as a covariate. Here, men would be expected to increase in their ST selections if they were highly attractive, but among women it would actually be low attractiveness individuals who would be expected to increase in ST in the presence of highly attractive competitors. While the "additional exposure" explanation is speculative, such thought does highlight an interesting avenue of future research using the SMA. If the exposure to attractive or unattractive individuals can have a contrast effect on participants (as in Experiment 0), could a similar effect be established by exposing participants to attractive or unattractive competitors?

\section{Replication Considerations}


When attempting to interpret the results of this experiment, it became apparent that a measure of perceived local sex-ratio would likely have improved the clarity of the results. Such a measure may be indispensable during further investigation of sex-ratio manipulations. To date only one experiment appears to have taken this measure (Durante et al., 2012) and this was only used as a manipulation check. If data were gathered about whether the participants viewed their environment as male- or femalebiased from the outset of the study, this could then be controlled for during analysis. This would also allow the prediction (made in the Lack of Female-Biased Bias section) that participants from an environment with a female-biased sex-ratio are more likely to be affected by male-biased cues, and vice versa, to be tested. Of course, this does create the added risk of having the variable of interest exposed to the participants.

While the GG looks to have been somewhat successful as a way of administering a sex-ratio cue, some participants still performed quite poorly. That some of the effects seemed dependent on participants earning points (or engaging with the task), and with $10 \%$ of the male-biased condition scoring $<=0$ and $25 \%$ scoring $<=10$ points, there is clearly room for improvement in this task. The methodology used by Griskevicius et al. (2012) and Durante et al. (2012) did not influence the design of this experiment as they were published after the data collection had begun. This was unfortunate as they contain two manipulations (a slide show memory task and a newspaper reading task) which have produced significant between-subject effects suggesting the successful bias of mating-related behaviour. ${ }^{37}$ As such these may have served as an ideal independent variable for this topic and could be utilised in the future with an SMA-style task to validate these results.

\section{Conclusion}

In this final experimental chapter, some evidence was found that a male-biased sex-ratio cue can lead to a shift away from a short-term mating strategy and towards a long-term one. In contrast, a shift in strategy could not be found following femalebiased cues. Most likely this was due to some of the experimental stimuli being inappropriate and the fact that sex-ratio of which the participants were regularly exposed was likely female-biased. However, such cues may well affect mating behaviour given previous evidence (e.g. Durante et al., 2012). In the final chapter of this

\footnotetext{
${ }^{37}$ A similar "newspaper" manipulation was considered for inclusion in this thesis in 2011. However, a pilot experiment, whereby participants read about individuals becoming more long-term or short-term in relationship mind-set due to the economic recession, failed to significantly affect the participants.
} 
thesis, the results from all of the experimental chapters are summarised, and support for the overall working hypothesis is addressed. 


\section{Chapter 9: Discussion}

This final chapter is divided into two main sections. Section I summarises the main findings of the thesis, introduces an extended evolutionary biological model of human mating flexibility, and discusses the implications of polygyny on mating strategy theory. Section II addresses the methodology and analysis used in the thesis, future directions of the research area, and the potential for application of the results. This second section also discusses how alternative methods of data analysis affect the results, as well as potential improvements which could be made to the SMA task.

\section{Section I: Summary of Findings and Integration with Current Evolutionary Theory}

In Chapter 1, the overall working hypothesis was developed: Human mating strategies are activated conditionally and change in historically adaptive ways in response to evolutionarily relevant stimuli. This hypothesis was tested in the subsequent experimental chapters by exposing participants to six cues thought to be ancestrally relevant to mating outcomes: personal attractiveness, the presence of children, social status, environmental harshness, sex-ratio, and competitive outcome. In addition, the association between mating strategies and circulating testosterone levels was explored. Within each chapter, comparative evidence from non-human animals was presented. In most cases this evidence revealed how changes to the variable of interest affected the reproductive success of a given species, and provided examples of how these animals responded to such changes by modifying their mating behaviour. If available, human studies from the psychological literature were also introduced. These were typically correlational studies or experiments which found changes to partner preferences. Together, this evidence was combined with sexual strategies theory (Buss \& Schmitt, 1993) and strategic pluralism theory (Gangestad \& Simpson, 2000) to develop subhypotheses specifying the expected change in human mating strategy following exposure to each cue.

A summary of all the experiments which used the SMA task can be found in Table 9.1 in Section II. Out of the ten evolutionary hypotheses tested with these experiments, support, or partial support, was found for seven. This support was the outcome of two different types of analyses. The first utilised simple chi-squared tests. Here, participants were added to one of three categories following their exposure to a cue depending on how their behaviour changed. For example, they were assigned to the category of LT $=$ if they did not change their responses, LT+ if they chose a greater 
number of models for a long-term relationship, or LT- if they chose fewer models. Chisquared tests were then used to see whether the distribution of these change categories differed from either a control group or what would be expected by chance. Of the seven significant chi-squared analysis, the average Cramér's $V$ score (an effect size measure equivalent to $r)$ was $0.24(S D=0.07)$. That is, the effects were small-to-medium in size, ranging from 0.14 to 0.37 (Cramér, 1999).

The chi-squared analyses were supplemented using non-parametric regression models. This allowed for the inclusion of demographic variables which could account for some of the variance in the data. For example, in the first danger experiment (Chapter 5), there was no overall main effect of the manipulation on the mating behaviour when all participants were considered together. However, when an ordinal regression was used, women were found to be more likely to appear in the ST+ category following the danger cue. In this case, the manipulation was successful for a subgroup instead of the sample as a whole and, rather than splitting cells several times using a series of chi-squared tests, regression was used to detect this. Of the seven hypotheses for which support was found, it was often the case that other demographic variables were needed in order for a significant model to become apparent or to improve the overall power of the model. These variables included sex, relationship status, SOI-R, and age. Overall the average effect size $\left(R^{2}\right.$ as approximated from Nagelkerke $\left.R^{2}\right)$ of the significant models was $0.12(S D=0.09)$. This was a medium effect size and ranged from small (0.05) to large (0.39).

The three strongest overall findings from the experiments were as follows. First, general support was found for the overall working hypothesis. When change to mating behaviour occurred following a manipulation, this tended to be in a direction consistent with the sub-hypothesis tested in the experiment. Had the working hypothesis been false, then the results would have either been predominantly null or significant but in directions inconsistent with evolutionary psychological theory. Second, the fact that some demographic variables were required in order for the experimental effects to become apparent revealed something about the complexity of human mating. The relative benefits of short- and long-term mating strategies do not apply to all individuals equally but can depend on factors such as their age or relationship status. Third, the fact that not all experiments caused a change among participants indicates that mating strategies are actually quite robust and, potentially, hard to influence. This is also evident by the small-to-medium effect size produced by the manipulations. 


\section{Zero to Hero; Dad to Cad}

If mating strategies can be affected by brief experimental manipulation, then one could claim that, in a similar way to other animals (e.g. Duffy et al., 2006; Johnsen \& Lifjeld, 1995), such manipulations could cause a low status individual to adopt a mating strategy usually pursued by those high in status. There are clearly some large lifechanging events which could have such an impact on mating strategy, such as the example of Knee the unsuccessful Sirionó hunter (Holmberg, 1950). However, these events are rare and more common day-to-day interactions are unlikely to have such an effect. If every successful flirtation attempt led men to more actively pursue extra-pair mating then we would expect persistent long-term relationships to be few and far between. Thus, while the experiments in this thesis show that mating behaviour can be biased in evolutionarily relevant directions, it is unlikely that such manipulations would cause concrete and enduring changes in mating behaviour. A graphical representation of this relationship can be found in Figure 9.1a which shows an individual on a point between high and low ST relationship desire. The red box represents the potential influence which large life changing events could exert on mating strategies. Such events might include a change in social status (L. Barrett et al., 2002; Soldan et al., 2007) or local sex-ratio (Marlowe, 1999a), which are implied by wider correlational and quasiexperimental evolutionary psychological research but are beyond the scope of this experimental thesis. In contrast, the green box represents the small amount of variance which can be caused by experimental manipulations of the type contained within this thesis.

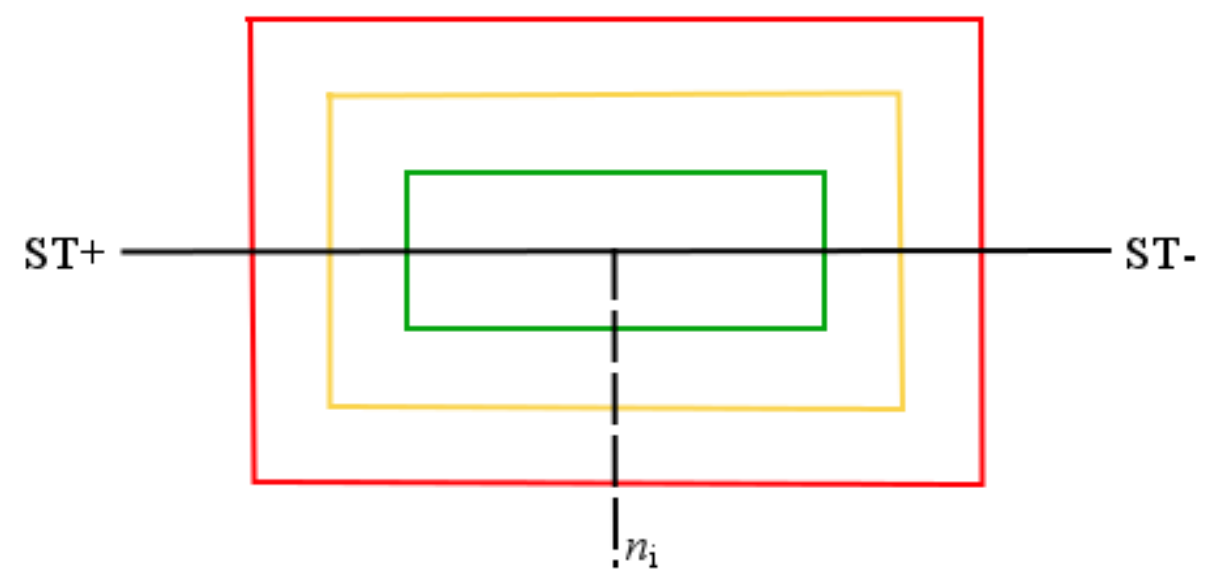

Figure 9.1a. The impact of evolutionarily relevant environmental input on mating strategies. The $\mathrm{x}$-axis represents a spectrum between high and low short-term (ST) mating preferences, but could be equally applied to long-term (LT) preferences. The 
green box represents the amount of variance which can be created by experimental bias (small effect) while the red box shows the influence of large life events (large effect). The yellow shows an intermediate (medium) effect.

How might one's initial place on this line be determined? It is unlikely that it would be entirely due to experience within the mating market. In Chapter 6, the mismatch effect was discussed. While initially found in other animals such as birds (Qvarnström, 1997; Rohwer \& Rohwer, 1978), this effect has also been found to exist in humans. When low T participants were placed into a position of subordination, they performed well, but if placed into a position of dominance they became stressed and performed badly (Josephs et al., 2006). This effect, tied to one of the key sexual hormones, suggests that there may be a somewhat rigid element to mating strategies. That is, for some individuals simply putting them into a more favourable mating position would not be enough to trigger a drastic change in their mating behaviour. What then, could create initial differences between individuals in their mating strategy? Most likely this would be due to an initial interaction between genes and environment during a critical stage of development (such as those introduced in Chapter 1). This concept is not farfetched. In Chapter 5 a model was introduced (M. R. Gross, 1991), which integrated an initial phonotypical difference, formed during early development via a gene-environment interaction, with life-long changes in behaviour which were partially restrained by the original phenotype. This is expressed in Figure 9.1b where two individuals of differing phenotypes are represented at different levels of ST relationship preference. If this application and extension of Matt Gross' model is correct, it is likely that the within-lifetime flexibility of one's mating strategy is somewhat anchored in place, and is then able to be shifted depending on the salience and intensity of mating related cues. 


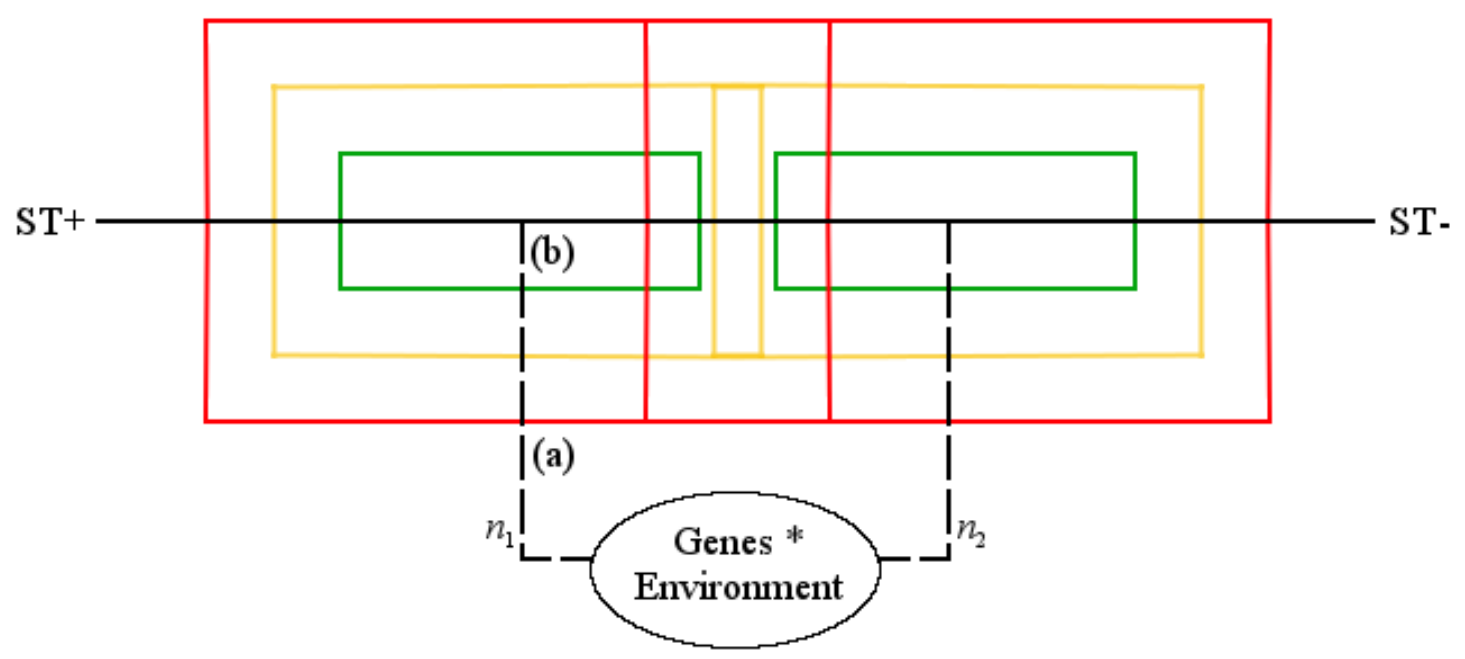

Figure 9.1b. A hypothetical model of short-term mating strategy flexibility as inspired by M. R. Gross (1991). An individual's genotype interacts with their early life experiences (a) leading to an initial preference for short-term mating (b). This preference continues to be open to influence depending on the life events encountered by the individual as discussed in Figure 9.1a.

\section{Self-Esteem and Priming Literature}

The cues used in this thesis broadly fell into one of two groups. The first group contained personal cues which told the participants something about themselves or their status in relation to others. These included the victory cues, which had participants visualise being victorious over their peers, and the sex-ratio cues, in which participants were told whether members of their sex were in high or low demand. The second group contained general "prime-like" cues. These cues signalled something about the environment in general but were not designed to affect the participant's perception of themselves in relation to others. These included the parental, wealth, and danger cues. ${ }^{38}$

It is important to differentiate between these two types of cue as the way in which they influence the psychology of the participants may well be different. In terms of personal cues, the pathway of change may involve a shift in one's perceived value as a mate, perhaps via a self-esteem mechanism. In the chapters which used such cues the results were interpreted in light of changes to self-esteem as presented in sociometer

\footnotetext{
${ }^{38}$ The testosterone manipulation may also belong in this category, although it was not a cue in the technical sense.
} 
theory (SMT; Kavanagh et al., 2010; M. R. Leary et al., 1995). ${ }^{39}$ This theory was also considered when interpreting results which were inconsistent with the sub-hypotheses (such as the results of Chapter 3). One significant issue with using SMT for interpretation is the lack of self-esteem measurement throughout this thesis. Rather than adopting a linear research path in which the design of one study influenced the design of subsequent ones, this thesis tested the working hypothesis across several evolutionary domains concurrently. Indeed, it was not until data collection had begun that the literature on SMT was discovered. Now that evidence has been found that mating strategies can indeed be affected by brief changes in environment, the next stage is to refine the knowledge of how such personal cues affect strategy choice by a) replicating the results to ensure that the findings are consistent, and b) confirming self-esteem as a mechanism of change. For example, the results of these experiments could be replicated while taking a pre- and post-measurement of self-esteem (e.g. Rosenberg, 1965). If the effects are due to changes to a sociometer, then the inclusion of a self-esteem difference score as a covariate should lead to the effect being diminished or removed entirely.

In contrast, external factors, such as parental cues, would not be expected to change self-esteem. Instead, priming may act as the mechanism of change. Priming involves presenting participants with a conscious or unconscious cue to see if this affects their subsequent behaviour. For example, Shariff and Norenzayan (2007) found that using a scrambled sentence task to prime 'god' as well as secular moral institutions (e.g. 'jury', 'civic' and 'police') led to an increase in pro-social sharing behaviour with a confederate $(d \sim 0.8-1.0)$. Priming co-operation using the same task can lead to faster baton exchange among amateurs within $400 \mathrm{~m}$ relay races (Greenlees, Figgins, \& Kearney, 2014), and sociability primes have even been found to increase non-verbal expressive behaviours (such as smiling or using hand gestures) among schizophrenic patients (Del-Monte et al., 2014). Priming paradigms have been used to investigate a wide range of research topics, from the influence of junk food adverts on healthy eating habits (Harris, Bargh, \& Brownell, 2009) to the priming of 'individualism' vs. 'collectivism' to explore cultural differences in self-identification (Oyserman \& Lee, 2008).

\footnotetext{
${ }^{39}$ In his response to Stewart-Williams and Thomas (2013b), Eastwick (2013) argues for the integration of the independently pursued bodies of relationship research within social and evolutionary psychology. Such an attempt has been made in this thesis through the employment of theories such as SMT.
} 
Priming works by temporarily activating mental representations along with their associated cognitions. Such representations then become more readily accessible for a short period and affect perceptions and behaviour (Bargh, 2006; Bargh \& Chartrand, 2000). In the first example above, by presenting participants with words such as 'jury' and 'police', a schema for moral institutions is primed. Such schema contains a whole framework of information about such institutions, including punishment for transgressions and the idea of a "higher body" observing one's behaviour. These activated concepts are then temporarily more accessible in memory and subsequently influence participant behaviour. Returning to the example, when the schema for moral institutions was activated, it was for a brief time more accessible to the participants, thus causing them to act more pro-socially during the money sharing task. This effect persisted even though the prime itself was absent during the final task. Such an interpretation could also be applied to the cues used in this thesis, such as the parental cues found in Chapter 4. In this case, the use of videos surrounding infants and infant care would have likely activated related concepts such as protection, kindness, conscientiousness, and, perhaps, pair-bonded parental roles. As such concepts are more related to long-term relationships than short-term ones, this would then lead participants to favour this relationship type during the second measure of the SMA task.

Although some classic prime experiments are currently under scrutiny due to reproducibility issues (see Chapter 3), there is still a large body evidence supporting the idea that priming can affect behaviour. The priming literature is vast, containing several paradigms for activating different schema. In this thesis, three types of prime were used: static image slideshows, informative videos, and hand written scenarios. Of the three cue types, it appears that low level visual cues seem to produce the cleanest results. However, other alternatives, such as the aforementioned scrambled sentence task, could be used to verify the effects.

\section{The Polygyny Problem}

Short- and long-term mating strategies have been approached in this thesis as if they were two conditionally chosen constructs pursued in an "on or off" binary fashion. However, not all human mating arrangements fall neatly into either of the two hypothesized strategies. This becomes apparent when we try to describe the mating behaviour of two hypothetical men - John and Gudo.

John is a 35 year old man from Great Britain who has been in a long-term relationship with his partner for several years. He claims to be happy in his 
relationship, showing love and affection for his partner. However, a year ago, John found himself tempted by the prospect of a casual liaison with a female coworker and ultimately decided to have uncommitted sex with her. After the event, John felt remorseful but decided not to tell his partner about his infidelity. John continues his long-term relationship to the present day.

How might John's behaviour be described using a mating strategies perspective? One interpretation of the event could be that originally John pursued a long-term mating strategy which guided him towards a monogamous mating arrangement. Then, temporarily, John pursued a short-term mating strategy which guided him towards promiscuous mating. According to strategic pluralism theory (Gangestad \& Simpson, 2000), the reason one strategy was temporarily pursued over the other may have been due to a change in an environmental factor which, in turn, affected the relative fitness benefits of each mating strategy. Thus, one strategy became more strongly activated than the other, causing it to have a stronger influence on John's behaviour. The triggering factor may have been something simple, such as a cue to increased sexual access among work colleagues. John may have, for example, recently had a job promotion which made him feel higher in status relative to his peers (von Rueden et al., 2011). ${ }^{40}$

While John's behaviour seems to be adequately explained by a "switching" of mating strategy, this may partially be due to the fact that the mating arrangements (or outcomes) he participated in compliment the two hypothesized human strategy types. That is, a promiscuous mating arrangement fits well with the psychological adaptations which form a short-term mating strategy, while a monogamous arrangement is a close fit with a long-term mating strategy. However, monogamy and promiscuity are not the only mating arrangements participated in by humans. According to H. E. Fisher (1989), the majority $(84 \%)$ of pre-industrial societies permit polygynous relationships whereby a man has several wives, and an average of $10 \%$ of men from these societies enter into such marriages (although there exists great variation between there societies). Likewise, in very rare cases, women can be found to take several husbands in a system of polyandry (L. Barrett et al., 2002). As polygyny is far more common among pre-

\footnotetext{
${ }^{40}$ In an initial pilot study, not included in this thesis, the SOI scores (J. A. Simpson \& Gangestad, 1991) of a sample of men and women were monitored over the space of two weeks, as well as any key life changes they experienced. The life changes of those who varied in their SO were then examined. Findings included one man who increased in his SO around the same time he received a job promotion, and one woman who decreased in her SO after she started trying to conceive (Thomas, 2010).
} 
industrial societies than polyandry, this sub-section focuses primarily on the former, though the arguments can presumably be applied to the latter.

Unlike John's situation, in which a person alternates between a monogamous and promiscuous mating arrangement, changes which involve polygyny or polyandry are more difficult to explain using a flexible mating strategy perspective. Consider, for example, the fictional example of Gudo:

Gudo is a 50 year old man from the Hadza tribe in Tanzania. He has been happily married to his wife for ten years and has four children with her. He is well respected in his village because of his hunting expertise. Due to this high social status, and the fact that the Hadza allow polygamy, Gudo is permitted to marry additional wives. Early into his first marriage Gudo showed no desire for a second wife, yet changed his mind about one year ago. During this time, he courted and eventually wed a woman younger than his first wife. While Gudo's first wife was not happy with the arrangement, she reluctantly accepted Gudo's choice and tolerated his new wife. Gudo is still married to both wives and claims to love both of them very much. He continues to be a devoted father and husband.

Could Gudo's change from a monogamous to a polygynous mating arrangement also be explained by a change in mating strategy? Much like John, Gudo's initial behaviour might well be interpreted as him pursuing a long-term strategy which led him towards a monogamous mating arrangement. However, it is less clear how Gudo's mating strategy changed in order to guide him towards a polygynous relationship.

One explanation could be that a polygynous mating arrangement is an outcome of a switch from a long-term to a short-term mating strategy. If this were the case then we would expect certain elements of men's psychology to be different under conditions of polygyny compared to monogamy. For example, we might expect men to show less love and a weaker emotional bond with their partners within a polygynous union compared to a monogamous one, or we might expect men to be less picky about the characteristics of their additional partners. Both behaviours would be indicative of a short-term mating strategy (Buss, 2002; Buss \& Schmitt, 1993). Unfortunately, there appears to be no comprehensive scientific review of differences in mate preferences between first and additional wives apparent within the evolutionary psychological literature. A search for the terms "polygyny" or "polygamy" on Scopus in the journals of Evolution and Human Behavior, Human Nature, and Evolutionary Psychology returns 85 results. None of these articles discuss mate preferences within the context of polygyny. (There are, however, some references which discuss the effect of polygynous marriage on the psychological wellbeing of women; M. Mulder, 1992.) 
What little evidence there is seems to suggest that a man's approach to finding an additional wife is more indicative of a long-term mating strategy than a short-term one. For example, one of the attributes that men find important in a long-term partner is that of youth as this signifies greater lifetime reproductive potential (Buss, 1989). In contrast, in the short-term sexual strategies of men, it is simply fecundity which is important in a temporary partner. Thus, if a man were pursuing a long-term strategy we would expect him to desire an additional wife who was young and with a large reproductive window. ${ }^{41}$ There is some evidence to this effect. In a study of over 21,000 individuals in polygynous societies, Bean and Mineau (1986) found that, irrespective of the age of the groom, wives tended to marry while in their early 20s. In a sample taken from 1820-1839, among monogamous couples the average age of a woman at marriage was $21.6(S D=4.7)$, while among polygamous unions the age of the first wife at marriage was $20.7(S D=4.6)$. The average ages of the second and additional wives at marriage were $21.3(S D=5.7)$ and $22.5(S D=6.4)$ respectively. This preference for youth is even clearer when the ages of men at marriage are considered. While the average age of men at their first marriage was $24.6(S D=5.2)$ and $26.6(S D=6.2)$, for monogamous and polygamous unions respectively, this grew to $33.8(S D=6.7)$ at the time of a man's marriage to his second wife, and $40.3(S D=9.2)$ when marrying his third.

One may argue, given the example cited in Buss $(1989$, p. 2$)$, that a woman in her early 20 s is actually at her most fecund, and therefore this age would be most desirable to a man following a short-term mating strategy rather than a long-term one (in which having a partner of around 13 would hold the largest reproductive potential). However, it is important to note that this study was about actual mating outcome rather than the desires of men, which are often compromised. For example, when the average desired age difference between a man and his wife is compared to the actual average age difference, a strong correlation is found $(r=0.71)$, yet more than $50 \%$ of the variance still remains unaccounted for (Buss, 1989, p. 9). More important is the fact that polygynous men typically choose second or third wives who are of a similar age to the first wife of monogamous men. If the motivation for obtaining a second or third wife was short-term in nature, then we might expect to find large differences here. Similarly,

\footnotetext{
${ }^{41}$ For an exception to this pattern, which demonstrated that Western men prefer younger women for short-term relationships see (Buunk, Dijkstra, Kenrick, \& Warntjes, 2001).
} 
if polygyny was the result of a man switching from a long-term to a short-term strategy we might expect the ages of these latter wives to be substantially older and more varied.

A second example comes from the fact that the motivations of men to marry additional wives often appears to be at odds with a male short-term strategy usually marked by sex in the absence of commitment and a desire for sexual variety (Buss, 2003a; Buss \& Schmitt, 1993; Penke \& Asendorpf, 2008). Such motivations include to build a larger family (Slonim-Nevo \& Al-Krenawi, 2006; Speizer, 1995) and divide labour (Dorjahn, 1958), but also include the pursuit of romantic love. In a qualitative study of the functioning of polygamous families in Israel, Slonim-Nevo and Al-Krenawi (2006) provided an example of one man whose motivation for taking an additional wife was purely driven by love: 'I was on a trip in Jordan, I saw her and as they say love is blind, I was like a teenager, I knew I would marry her' (p. 317). Likewise, there is some evidence that, among Mormon communities of the $19^{\text {th }}$ century, plural spouses showed deep emotional attachment to their husbands (Flake, 2009).

Although this is still a grossly under-researched area, some evidence suggests that polygyny may well be a mating arrangement which lies within the boundaries of a long-term mating strategy rather than a short-term one. If this is the case, then Gudo's change from a monogamous mating arrangement to a polygynous one cannot be adequately accounted for by hypothesizing a simple "switch" from a long- to a shortterm mating strategy. This is The Polygyny Problem inherent within the human mating strategies perspective.

One potential objection to this argument is that polygyny can be accounted for by strategy "switching" providing that polygyny represents a trade-off between strategy and environment. For instance, it could be that the only method available for men in a given community to satisfy their desire for sexual variety is through marriage, thus leading a short-term strategy to be realised through a mating arrangement less consistent with its goals, such as polygyny rather than promiscuity (see Identifying Strategies in Chapter 1). However, opportunities for casual sex are reported to be large in the majority of cultures featured in the SCCS, and are likely present at some level in the vast majority of all human cultures (Scelza, 2013). Such reasoning would also not explain why men from polygynous cultures choose additional wives who possess characteristics desirable in a long-term mate rather than a short-term one (such as youth mentioned above), or why men who are in the position to have casual sex with a large number of women (such as powerful despots) often choose to engage in polygynous 
marriages rather than having a single wife and several mistresses (Stewart-Williams \& Thomas, 2013b).

One way to account for The Polygyny Problem within evolutionary psychological theory is to disregard the concept of a "switch" between strategies. That is, men and women are not like Coho salmon (M. R. Gross, 1991), which pursue one of two distinct mating strategies at any given time, but instead have a mating strategy which shifts on a "spectrum" between a purely long-term and a purely short-term strategy. Under this view, different mating arrangements would be more common at different parts of the spectrum (see Figure 9.2a below). Such an interpretation would also fit in well with the model of mating presented earlier in this Chapter (Figure 9.2b).

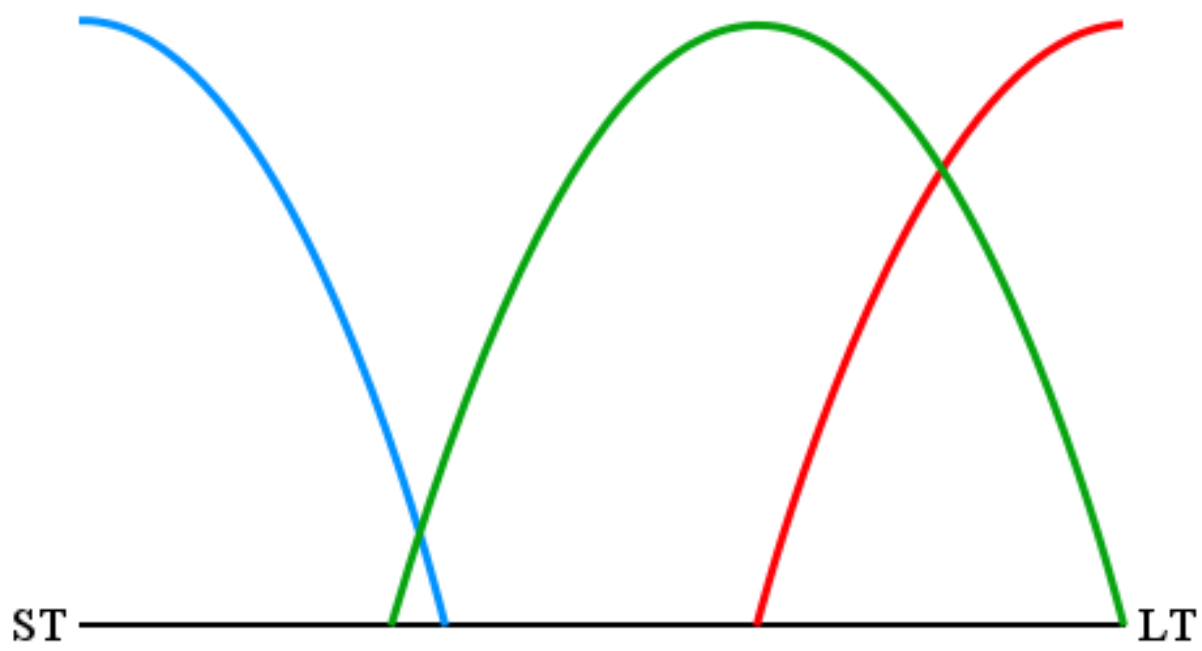

Figure 9.2a. A hypothetical model showing the probability of a man entering into one of three different mating arrangements based upon his mating strategy. When pursuing a strategy which is primarily long-term in nature, monogamy (red) is the most likely mating arrangement. If the strategy is primarily short-term then promiscuity (blue) is more likely. An intermediate strategy leads to an increased chance of entering a polygynous mating arrangement (green). 


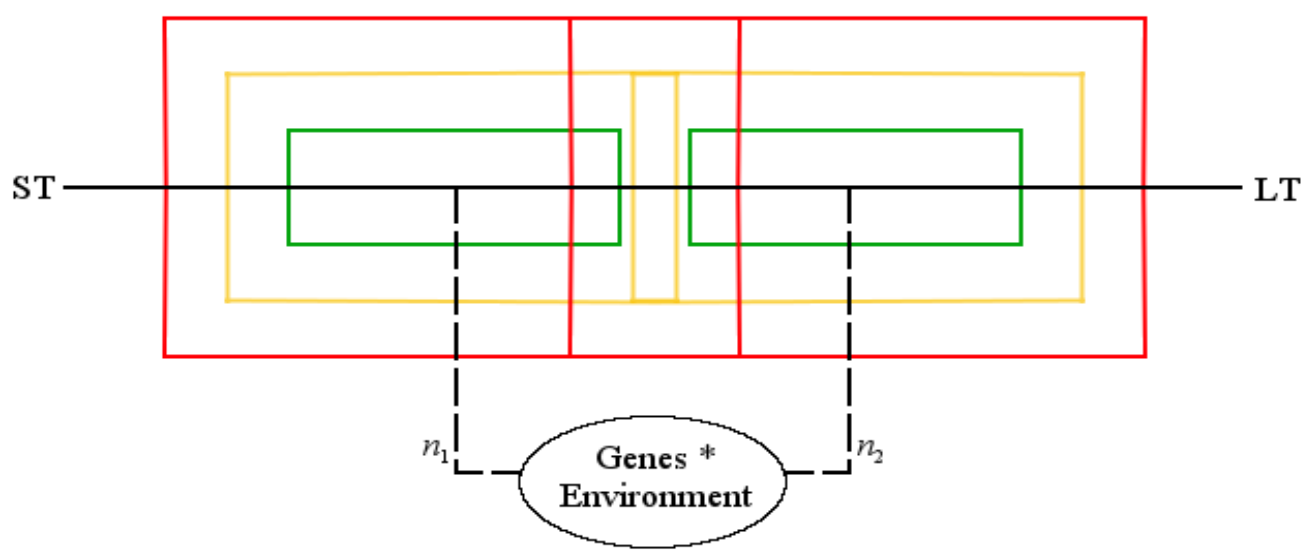

Figure 9.2b. A revised version of Figure $9.1 \mathrm{~b}$ whereby mating strategy is presumed to vary on a spectrum rather than conditionally.

If true, then such a concept has some serious implications for the evolutionary psychological literature. For example, strategic pluralism uses a status-dependent "switching" model from the evolutionary biological literature as the hypothesized mechanism behind mating strategy change (Gangestad \& Simpson, 2000; M. R. Gross, 1996). Likewise, studies which try to demonstrate a "dual-nature" of human mating by revealing changes to partner preferences under different mating contexts may be painting a false picture of how human mating strategies are implemented (e.g. Confer et al., 2010; DeBruine, 2005; Li \& Kenrick, 2006). Nonetheless, the relationship between polygynous mating arrangements and mating strategies appears to be an overlooked area, although researchers have been aware of the complications polygyny introduces into research for some time. For example, according to Buss (2003b), after approaching a Nigerian researcher to gather the mate preferences of men, he found that his 'Nigerian colleague wished to know whether [Buss] sought mate preferences for a man's first wife, second wife, or third wife.' (p. 221). This caused the research team to use a modified version of their mate preference questionnaire for the $8 \%$ of cultures studied which permitted legal polygyny.

Polygyny and the SMA. What implication does polygyny have for the results in this thesis? The underlying assumption of each of the experimental sub-hypotheses was that as an individual changes from a short- to a long-term mating strategy, their interest in short-term mating would increase and their interest in long-term mating would decrease (and vice versa). Thus, when it came to the specific predictions as to how participants would change their SMA responses, LT and ST choices were assumed to be 
inversely related. That is, a decrease in the number of LT choices was predicted to be accompanied by an increase in ST choices. Ultimately, however, there were only two occasions within this thesis (in Experiment 4 and among women in Experiment 8) in which such a pattern was clearly found.

Can this low occurrence be explained? One possibility is that some of the assumptions made when developing the SMA task were incorrect as they did not take into account polygynous tendencies. Within the SMA, a shift in mating strategy towards the long-term is assumed to result in more models being chosen for a long-term relationship. Similarly, a shift in mating strategy towards the short-term is assumed to result in more models being chosen for a short-term relationship. Yet, when we consider the case of polygyny, where a man chooses several partners and has a committed relationship with each, a flaw in the task is revealed. If the engagement in polygyny marks a step closer to the short-term end of the mating strategy spectrum (Figure 9.2a), this may not be accurately captured by the SMA task. This is because the pursuit of multiple long-term partners by a participant would actually increase their number of LT responses rather than decrease them. Thus, much like the model presented by Quinlan (2007), it may be the case that there is a quadratic relationship between the degree to which a man's mating strategy is short-term and his interest in long-term relationships. That is, as a man transitions from a predominantly long-term to a predominantly shortterm strategy, his interest in long-term partnerships may actually increase in line with a polygynous mating arrangement, before decreasing in line with a promiscuous arrangement.

Such an explanation is tentative, but it might explain why, among men, an increase in both LT and ST interest could be produced by a victory stimulus (Experiment 8). These results could be due to a combination of the behaviours of different subsets of men, with one developing a polygynous tendency following the cues and another developing a promiscuous tendency. Both of these findings could be explained by a shifting of mating strategy towards the short-term end of the mating strategies spectrum. Simple modifications to the SMA, such as asking which mating arrangement (i.e. monogamy, polygyny/polyandry, or promiscuity) would be preferred, could confirm this explanation as well as providing a tool of investigation for an underexplored element of human mating psychology.

\section{Alternative Theories}


Could the results of this thesis be explained without needing to invoke evolutionary psychological theories of mating strategies? It has already been outlined in this discussion that the potential proximate mechanisms of change behind the results could involve self-esteem and priming - two constructs with a long history within social and cognitive psychology which stand as theories of behaviour in and of themselves (Bargh, 2006; Bargh \& Chartrand, 2000; Kirkpatrick \& Ellis, 2004; M. R. Leary et al., 1995). However, there are some individual findings within the thesis which would be harder to explain using these theories. For example, the results of the testosterone study of Chapter 6 cannot be accounted for by priming. The effect could potentially be attributed to a change in self-esteem, but only if one assumes that those who showed large increases in circulating $\mathrm{T}$ were also those better able to perform during the exercise task, thus giving them a self-esteem boost. Even if this were proved to be the case, one would be left needing to explain why an increase in self-esteem specifically affected short-term mating desire and not long-term mating desire (see The Onset of Change in Chapter 7).

Even in cases where priming or sociometer theories can be used to explain the results, these still only provide a proximate rather than an ultimate explanation of the behavioural change, and sometimes this is incomplete. For example, in Experiment 5, both sexes were found to respond to danger cues by increasing the number of relationships they chose. While the overall "increase" effect could potentially be explained by priming, priming theory in and of itself is not enough to explain the existence of the effect. That is, one would still be left with no explanation as to why danger cues activate mating-related concepts even if priming was proven to be the mechanism behind this change. Furthermore, among men, the effect was restricted to LT relationships and priming theory would struggle to explain why such a sex difference exists. In contrast, a combination of sexual strategies theory and strategic pluralism theory can provide this answer irrespective of the proximate mechanism of change.

Are there other theories which could account for some of the results within this thesis? Prima facie there appear to be several. The results of wealth cues on behaviour could be explained as the product of a more general psychological phenomenon whereby a multitude of factors appear to make an individual more orientated towards short-term rewards. These include fast cars, gender threats, stress, and even exposure to urban environments (Lempert, Porcelli, Delgado, \& Tricomi, 2012; van der Wal, 
Schade, Krabbendam, \& van Vugt, 2013; Weaver, Vandello, \& Bosson, 2013; M. Wilson \& Daly, 2004). In this sense, the influence of wealth cues on mating could simply be a non-functional by-product of the influence of such cues on economic decision making. ${ }^{42}$ Likewise, it could be the case that victory cues (Chapter 7) simply put participants in a better mood, leading them to change the way they responded to the experimental task at second measurement (Erber, Wegner, \& Therriault, 1996; Fishbach \& Labroo, 2007; Forgas, 2007; Isbell, 2004; Storbeck \& Clore, 2005). As a final example, the increase in LT selections in Experiment 3 could stem purely from the fact that participants have come to learn that childrearing primarily exists within the context of a long-term relationship, and so their increase in LT responses at time two could be due to a form of demand characteristic bias (Orne \& Whitehouse, 2000; M. M. Page, 1974, 1981).

It appears then that the results found during this research could be explained either through the use of a sexual strategies framework, or through invoking individual, unrelated, effects as described above. While both explanations may be valid, we ultimately side with the former for several reasons. First, the use of a general theoretical framework rather than individual theories is more parsimonious. When faced with two equally predictive theories it is typically the simpler one, that which invokes fewer entities, which is more favourable. This is because simple theories can be generally be applied to a larger number of cases and are better open to testing and falsification (Popper, 1992). Second, the evolutionary psychological framework allowed for strong $a$ priori hypotheses to be generated, while alternative theories fit better as post-hoc explanations. For example, while mood change could be a valid post-hoc explanation for the effect of victory and defeat cues on mating behaviour, one might struggle to predict in precisely what direction such effect would take at the experimental prediction stage. This is similar to the difficulties faced when using sociometer theory to predict changes to mating strategy described in Chapter 7 (The Onset of Change). Third, there are sex differences in relation to some stimuli which can be accounted for by an evolutionary framework. In contrast, other theories based in social and cognitive domains would need to invoke supplemental theories to explain these sex differences. Finally, as already alluded to, alternative theories fail to explain ultimate causes of

\footnotetext{
42 That being said, the general effect of making more short-term economic decisions in response to stress may well serve its own evolutionarily adaptive purpose, directing an individual towards immediate rewards when the certainty of larger rewards in the future is questionable.
} 
behaviour; they are unable to explain why the individual effect forms part of our modern day psychology. For these reasons, we argue that an evolutionary psychological approach leads to a better, and more parsimonious, explanation of the results found.

\section{Section II: Methodological Considerations, Future Directions, and Conclusion} The Analyses Analysis

Data analysis was kept fairly consistent between the chapters. Initial chi-squared tests were performed to test for overall main effects followed by non-parametric ordinal (and in some cases multilogistic) regression analyses to test for the influence of covariates. To make the dependent variables more suitable for the analysis, ST and LT difference scores were recoded into a dummy variable based on whether participants increased, stayed the same, or decreased their choices following the manipulation (a three stage DV model). This was performed due to the skewed nature of the difference score (See Chapter 2) which meant that parametric analysis was unsuitable. However, two potential criticisms can be made of this approach. The first concerns the suitability of the data for use with an ordinal regression, while the second concerns the use of such analysis over robust parametric alternatives. These two criticisms are addressed in the following two sub-sections.

Use of ordinal regression. The primary method of analysis used throughout this thesis was ordinal logistic regression. This form of regression uses a combination of variables to predict one's likelihood of appearing in one of several ordered outcome categories. For this research, there were three such outcome categories and these reflected how participants changed in their response to the SMA task after an experimental intervention. Participants were coded as "-1" if they chose fewer models following the intervention, " +1 " if they chose a greater number, and " 0 " if they showed no change. This coding was performed separately for ST and LT selections.

One potential concern surrounding this coding procedure is whether or not this dummy dependent variable is more suitable for ordinal logistic regression compared to a multinomial logistic regression. A key assumption of an ordinal regression is that the outcome categories are related to one another in an ordered way without necessarily being separated by equal intervals (Norušis, 2011, p. 69). For example, if one wanted to use a model to predict whether a participant fell into a "high", "medium", or "low" category of smoking frequency then an ordinal analysis may be appropriate. In contrast, trying to predict whether a participant will choose a new car that is "red", "blue", or "green" in colour would not be suitable for such an analysis. There is no objective 
ordinal relationship between the categories; a red car is not more "colourful" than a blue car. In this case, a multinomial regression would be more appropriate. This type of regression assumes no ordered relationship between the categories and instead runs individual binary logistic regressions on every potential combination of the outcome variable (whether a variable can predict blue over red car choice, then red over green, and so on).

For two reasons it can be argued that the recoded SMA difference scores produced a DV which was suitable for an ordinal logistic regression. First, Figure 9.3 below contains one of the histograms displayed in the SMA Data and Chosen Analysis section from Chapter 2. This was used to demonstrate that the raw difference scores from the SMA task (LT in this case) formed a distribution with marked kurtosis that could not be made normal using data transformations. Below the histogram is another showing the result of the recoding process used in the thesis whereby the two tails of the distribution are "compacted" into two categories. Such coding does lead to a simplification of the data; however, the three remaining categories are clearly ordered in nature. Those who are coded as " 0 " or " +1 " have LT difference scores which are higher than those in the " -1 " category, and those who are coded as "+1" have LT difference scores which are higher than those in the " -1 " or the " 0 " categories. 

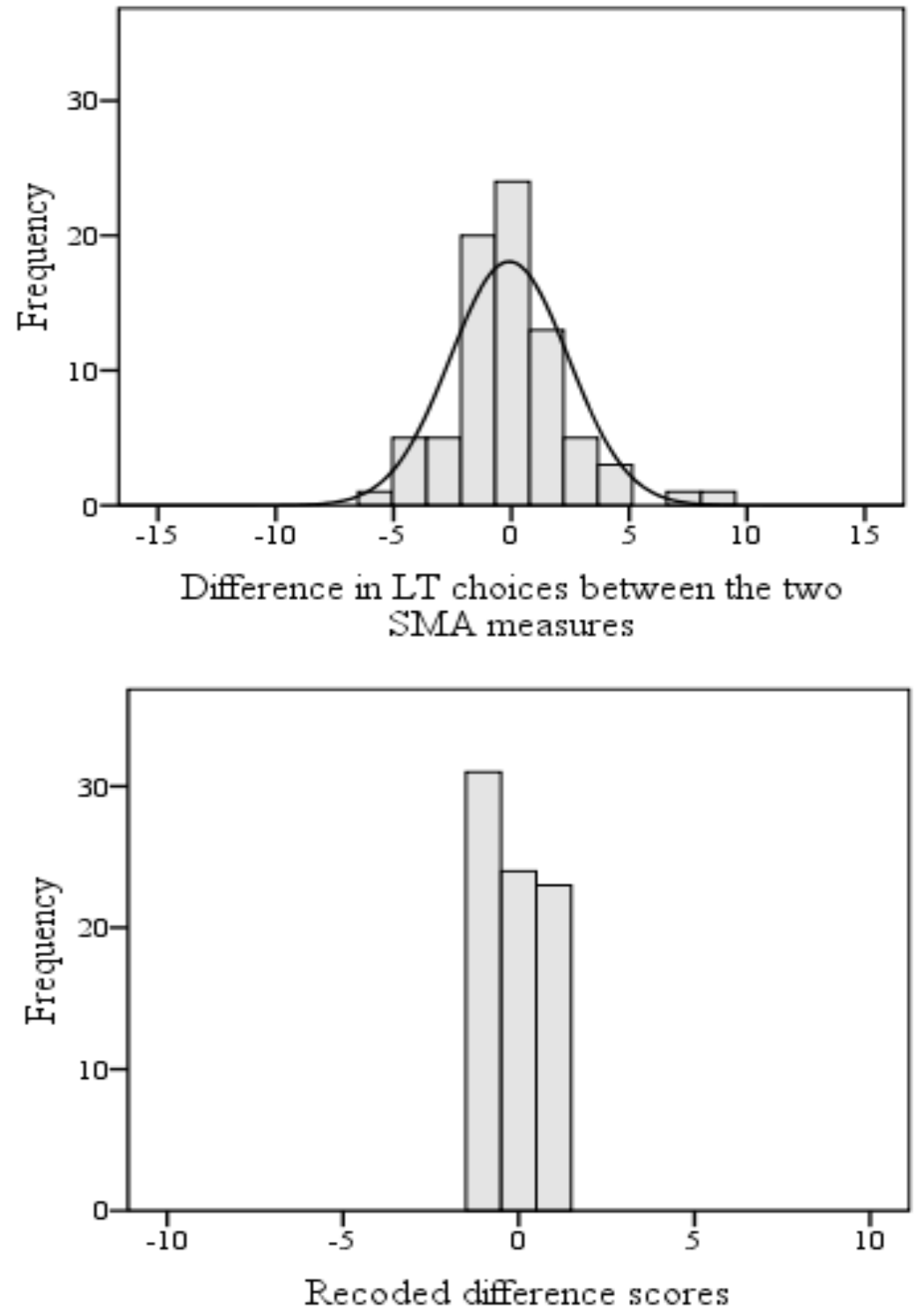

Figure 9.3. A demonstration of the effect of recoding the LT difference scores using three ordinal categories. The top figure shows the distribution of LT difference scores for participants in Experiment 0 . The bottom shows the outcome of the recoding process.

The second line of support for adopting ordinal analysis derives from the fact that, in all but one case (Experiment 5) where ordinal regression models were used, the test of parallel lines was non-significant. The test of parallel lines tests the null hypothesis that the ordinal model produced applies equally to the ordinal categories at different splits (see Long \& Freese, 2001, pp. 150-152; Norušis, 2011, pp. 69-89). Thus, if the recoded DV was formed from unordered categories then we would not expect this test to be consistently null. Yet this was the case for 21 out of 22 of models (95.5\%). In 
the one case in which this test was significant, a multinomial regression was employed in order to analyse the results.

A final note about the recoding of the data surrounds that of power. By fitting continuous data to a non-parametric mould, variance between individuals is inevitably lost. It follows that this recoding method may have led to an inflated chance of accepting the null hypothesis when in fact it should be rejected. That is, the recoding process leads to a higher risk that a true difference between experimental groups is masked as borderline cases become forced into categories (Irwin, 2001). Fortunately, further investigation and reanalysis of the data revealed that similar results were obtained with alternative statistical tests as to those obtained by the ordinal models (see below). The fact that these alternative analyses did not return novel significant results gives us confidence that Type II errors were not made, despite the increased likelihood that they would occur given the recoding method used.

Use of alternative tests. Some effects are strong enough, and some tests robust enough, that parametric analysis can sometimes be useful even if some of their statistical assumptions are broken (Heeren \& D'Agostino, 1987; Schmider, Ziegler, Danay, Beyer, \& Bühner, 2010). This, coupled with the fact that the use of nonparametric recoding leads to a reduction in power (see previous sub-section), it could be claimed that alternative analyses of the same data would return qualitatively different results to those presented in the experimental chapters. To address this criticism, the analyses for the experiments with significant results (Table 9.1) were repeated with both ordinal analyses using data recoded into five categories and parametric linear regression. For the five category models, participants were recoded as either -2 or +2 if their ST or LT change scores exceeded $+/$ - three. For the parametric models, untransformed difference scores were entered as the criterion variable. In the majority of cases the same models could be recreated and were found to be statistically significant at the $p<0.05$ level. In a few cases the model changed slightly with a variable becoming excluded. For example, in the ST sex-ratio model, SOI-R was excluded. In one case (the testosterone study) the significant finding became marginally significant (just short of the $p=0.05$ cut-off). However, for all practical purposes, the results were qualitatively the same. 
Table 9.1

A summary table showing the SMA experiments conducted within this thesis. Main effects (as established using the results of the non-parametric regression models. The final two columns show how the results change if alter Rows in grey represent non-supported hypotheses, yellow rows represent partially supported hypotheses, and gr hypotheses

\begin{tabular}{|c|c|c|c|c|c|}
\hline Manipulation & Hypothesis & ST/LT & Main effect & $\mathrm{NP}^{43}$ & Interpretation \\
\hline \multirow{2}{*}{$\begin{array}{l}\text { False } \\
\text { attractiveness } \\
\text { feedback on } \\
\text { men (negative) }\end{array}$} & \multirow{2}{*}{$\begin{array}{c}\mathrm{H} 1 \\
(\mathrm{Ch} 3: \mathrm{Ex} 2)\end{array}$} & ST & - & $\begin{array}{l}\text { Relat. status (+); } \\
\text { Attractiveness (-) }\end{array}$ & $\begin{array}{l}\text { Men who are in a relationship, or } \\
\text { low in attractiveness, are more } \\
\text { likely to increase their number of } \\
\text { ST selections following negative } \\
\text { feedback. }\end{array}$ \\
\hline & & LT & - & - & N/A \\
\hline \multirow{2}{*}{$\begin{array}{l}\text { False } \\
\text { attractiveness } \\
\text { feedback on } \\
\text { men (positive) }\end{array}$} & \multirow{2}{*}{$\begin{array}{c}\mathrm{H} 2 \\
(\mathrm{Ch} 3: \mathrm{Ex} 2)\end{array}$} & ST & - & - & - \\
\hline & & LT & - & Attractiveness (-) & $\begin{array}{l}\text { Men lower in attractiveness are } \\
\text { more likely to increase their } \\
\text { number of LT selections } \\
\text { following positive feedback. }\end{array}$ \\
\hline
\end{tabular}

43 "NP3" refers to an ordinal or multiple logistic regression where the SMA results were coded as -1 (a negative change), 0 (no change repeat of this analysis using two more ordinal categories: -2 (a negative change greater than three) and +2 (a positive change greater th analysis using a parametric linear regression on raw difference scores. $C V=$ Cramér's $V$. QS = Quantitatively Similar. 


\begin{tabular}{|c|c|c|c|c|c|}
\hline \multirow{2}{*}{ Parental cues } & \multirow{2}{*}{$\begin{array}{c}\mathrm{H} 3 \\
(\mathrm{Ch} 4: \mathrm{Ex} 3)\end{array}$} & \multicolumn{3}{|c|}{$\begin{array}{c}\text { Decrease }(\dagger) \\
C V=0.11\end{array}$} & \multirow{2}{*}{$\begin{array}{l}\text { A marginally significant decrease } \\
\text { in the number of ST selections is } \\
\text { found following negative } \\
\text { feedback in both sexes. } \\
\text { A general increase in number of } \\
\text { LT selections is found following } \\
\text { exposure to parental cues. The } \\
\text { effect is more prominent among } \\
\text { those who are in a relationship. }\end{array}$} \\
\hline & & LT & $\begin{array}{c}\text { Increase } \\
\mathrm{CV}=0.24\end{array}$ & $\begin{array}{l}\text { Condition * Relat. } \\
\text { status }(+)\end{array}$ & \\
\hline \multirow{2}{*}{ Wealth cues } & \multirow{2}{*}{$\begin{array}{c}\mathrm{H} 4 \\
(\mathrm{Ch} 4: \mathrm{Ex} 4)\end{array}$} & ST & $\begin{array}{c}\text { Increase } \\
\mathrm{C} V=0.18\end{array}$ & $\begin{array}{l}\text { Condition * Sex * } \\
\text { Relat. status (+) }\end{array}$ & $\begin{array}{l}\text { A general increase in number of } \\
\text { ST selections is found following } \\
\text { exposure to wealth cues. The } \\
\text { effect is more prominent among } \\
\text { women who are in a relationship. }\end{array}$ \\
\hline & & LT & $\begin{array}{l}\text { Decrease } \\
C V=0.14\end{array}$ & Condition $*$ SOI-R (-) & $\begin{array}{l}\text { A general decrease in number of } \\
\text { LT selections is found following } \\
\text { wealth cues. The effect is more } \\
\text { prominent among individuals who } \\
\text { are high in SO. }\end{array}$ \\
\hline \multirow{2}{*}{ Danger cues } & \multirow{2}{*}{$\begin{array}{c}\text { H5 } \\
\text { (Ch5:Ex5) }\end{array}$} & ST & - & $\begin{array}{l}\text { Sex }(-) ; \operatorname{Sex} * \\
\text { Condition }(+) \\
\text { Sex } * \text { SOI-R (-) }\end{array}$ & $\begin{array}{l}\text { An increase in ST selections is } \\
\text { found among women. The effect } \\
\text { is revealed only when SO and sex } \\
\text { are included in the analysis. }\end{array}$ \\
\hline & & LT & $\begin{array}{l}\text { Increase } \\
C V=0.37\end{array}$ & Condition (+) & $\begin{array}{l}\text { A general increase in number of } \\
\text { LT selections is found following } \\
\text { danger cues for both sexes. }\end{array}$ \\
\hline
\end{tabular}


Danger cues $\quad \begin{gathered}\text { H5 } \\ \text { (Ch5:Ex6) }\end{gathered}$

LT

ST

H6

Testosterone change in men
Ranked \% change in Testosterone (+)
A positive change in circulating $T$ can predict an increase in number of ST selections in men.

LT

ST $\quad$ Increase $\quad$ Condition $(+) \dagger$

Victory cues in men H7 (Ch7:Ex8)

$\begin{array}{ccc}\text { LT } & \text { Increase } & \text { Condition (+); } \\ & \mathrm{C} V=0.28 & \text { Condition * SO }\end{array}$
Condition * SOI-R (+)
A marginally significant increase in the number of ST choices is found following the presentation of victory cues in men.

A general increase in number of LT selections is found for both sexes. The effect is further enhanced among individual who are high in SO.

A marginally significant decrease in the number of ST choices is found following the presentation of victory cues in women. 


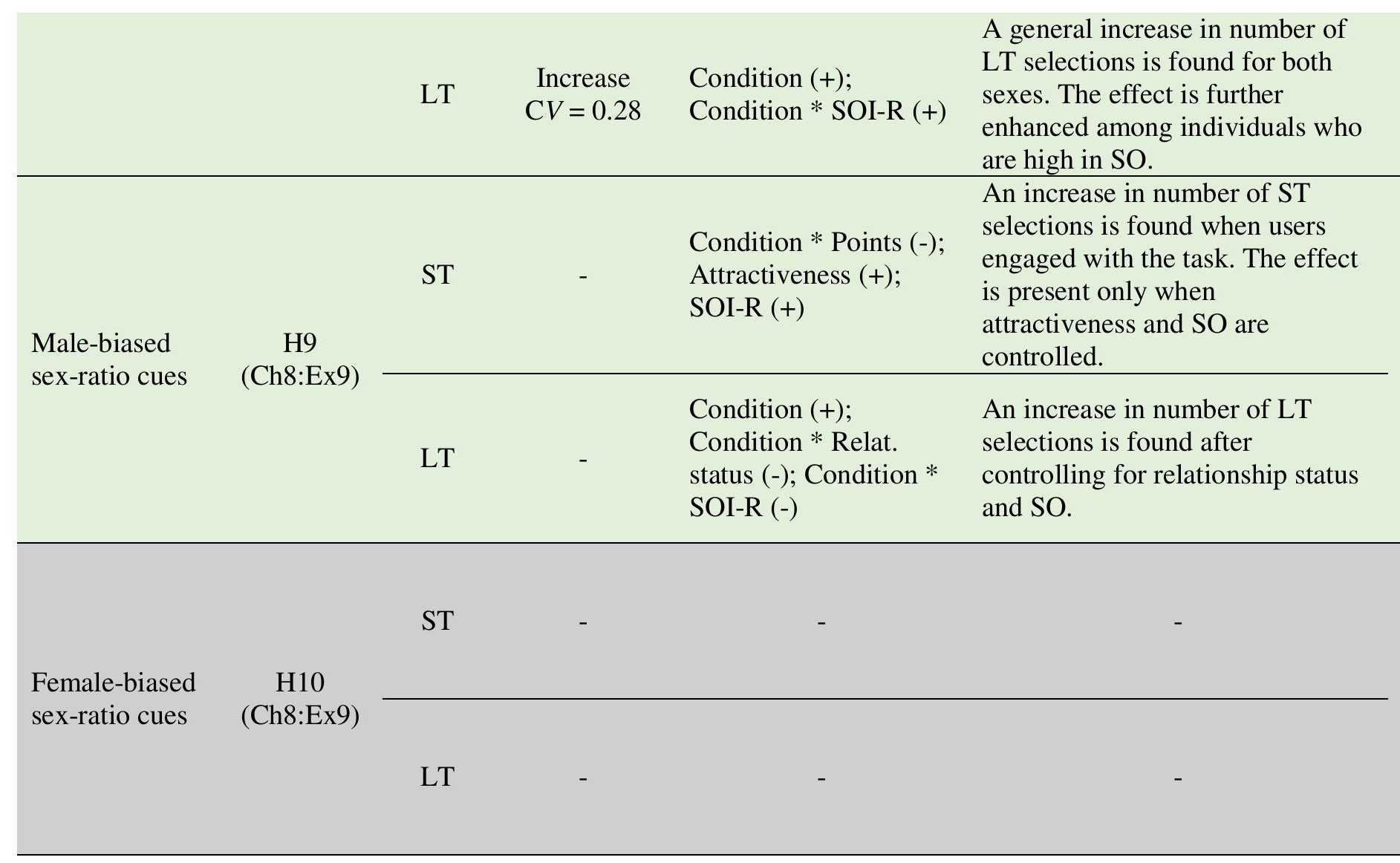




\section{Non-Heterosexual Sexual Orientations and Mating Strategies}

Homosexuality. While the study of human mating from an evolutionary perspective has predominantly focused on heterosexual desires and behaviours, there has been some notable research in the area of homosexual mate preferences. As Symons (1979) identified over a generation ago, homosexual men tend to show similar preferences as heterosexual men only with a same-sex target. That is, they show preferences for younger partners, a desire for sexual variety, and are more open to uncommitted sex. Homosexual women also show a similar pattern of behaviour as their heterosexual counterparts, preferring, on average, sex within the confines of a committed relationship with strong emotional bonds. Subsequent research has confirmed Symons' observations. For example, the age preferences of homosexual men appear to be similar to those of heterosexual men (Kenrick, Keefe, Bryan, Barr, \& Brown, 1995), and a large study of over 200,000 individuals from 52 nations found more variance between the sexes than between sexual orientations within each sex (Lippa, 2007a). Some interesting differences have also been found, such as homosexual and heterosexual men being affected differently by the administration of oxytocin (Thienel et al., 2014), as well as evidence for distinct long- and short- term sexual strategies among homosexuals (Regan, Medina, \& Joshi, 2001; Schmitt, 2007). While knowledge about the similarities and differences between homosexual and heterosexual mating behaviour has advanced, explaining homosexuality from an evolutionary perspective still remains open to debate. Theories range from homosexuality being a byproduct of a genotype which usually leads to increased mating success among heterosexuals (E. M. Miller, 2000; Zietsch et al., 2008), to the result of later birth order allowing the immune system of mothers to build up a "resistance" to Y-chromosome linked cells in the foetal brain (Blanchard, 2001; Cantor, Blanchard, Paterson, \& Bogaert, 2002).

What about homosexuality in relation to this thesis? Across the seven experimental chapters, 15 participants identified themselves as homosexual and were excluded from the analyses. To make the experiments as ethical as possible, participants were not asked for their sexual orientation in advance. A small number of individuals made their sexual orientation clear at the beginning of the experiments, and so were shown same-sex models during the SMA task, while others completed the task while viewing their non-preferred sex. As such, there is no accurate SMA dataset for homosexual participants and so mating strategy plasticity within this subgroup remains 
an open question. However, given the similarity of homosexual preferences to that of heterosexuals, it follows that homosexual mating plasticity may also be similar. In the same way that an increase in testosterone appears to be linked with short-term mating in heterosexual males (Chapter 7), one would predict this to also be true of homosexual males. Likewise, one may expect homosexual women to respond to moderate danger cues by increasing interest in both short- and long-term mating (Chapter 5).

Unfortunately, as the number of homosexual participants present within the experiments of this thesis was so small, these questions remain unanswered.

Bisexuality. In each sample of the population tested as part of this thesis, participants predominantly described themselves as heterosexual. There were, however, a small number of individuals in each experiment who described themselves either as homosexual or bisexual. Specifically there were 15 homosexual and 16 bisexual participants. During the analysis, the data of homosexual participants were excluded (see previous section) while the data of bisexual participants were included. The rationale behind the inclusion of the latter was that, unlike homosexuals, bisexuals participants by their very definition would have held some attraction to the sex they were exposed to during the SMA task. However, this inclusion also makes the assumption that the sexual strategies of bisexual and heterosexual individuals are homogenous. Unfortunately, information on mating strategies and preferences of bisexuals within the evolutionary psychological literature is sparse. Where sexuality is examined it is often the case that the research question focuses on differences between homosexual and heterosexual preferences and so bisexuals tend to be an excluded group (e.g. Gobrogge et al., 2007; Kenrick et al., 1995; Valentova, Stulp, Třebický, \& Havlíček, 2014). In the limited research which does exist, the sexual desires of bisexual men are found to be similar to that of their homo and heterosexual counterparts. Bisexual women, in contrast, show a moderate decrease in sexual restrictiveness compared to homosexual and heterosexual women, although this is still much more restricted than men of any sexuality (Schmitt, 2003, 2012). Outside of the evolutionary psychological domain, bisexual men and women have been shown to have mating preferences similar to their homosexual counterparts or somewhere between hetero and homosexuals (A. B. Cohen \& Tannenbaum, 2001; Lippa, 2007b; Teuscher \& Teuscher, 2007).

While the current state of (admittedly scarce) research appears to suggest that the sexual strategies of bisexual men and women are not too dissimilar to their 
heterosexual counterparts, should this prove to be to the contrary there would be very little implication for the results in this thesis. None of the reported effects depended on the inclusion of bisexual participants as they remained qualitatively similar when repeated using only heterosexuals. Should subsequent research confirm that sexuality has very little effect on sexual strategies, other than the target of sexual interest, there are still some improvements which could be implemented in further research using the SMA task to ensure participants have been shown models in line with their desires. For example, some prominent research on sexuality utilises the Kinsey scale (Kinsey et al., 1948) when obtaining demographics (J. M. Bailey, Dunne, \& Martin, 2000; Golombok \& Tasker, 1996; Li, Smith, Griskevicius, Cason, \& Bryan, 2010; Valentova et al., 2014). This item, rather than simply asking participants what sex they are attracted to, instead records sexuality on a scale, allowing participants to indicate, for instance, if they are primarily attracted to men but also somewhat attracted to women or if they are equally attracted to both sexes. In the same way as the data gathered from homosexual participants who are shown opposite sex models during the SMA is redundant, so too may be the data gathered from women who are shown men but have a bisexual nature more focused on women as their sex of interest.

\section{Representation of the Sexes}

While the majority of the experiments within the thesis used a mixed-sex sample, there were two chapters containing experiments conducted solely on men. These were Chapter 3 and Chapter 6 which tested the influence of a self-perceived attractiveness manipulation, and of changes in circulating $\mathrm{T}$ levels, on mating behaviour respectively. In this section, the reasons why these studies utilised an all-male sample, and the limitations of this approach, are discussed in further detail.

Mix\&Match. For the Mix\&Match experiments of Chapter 3, two main factors led to an all-male sample being tested. The first was theoretical in nature. The majority of studies within the thesis took inspiration from existing research within the behavioural ecology literature. In the specific case of the manipulation of attractivenesslinked sexually selected ornaments, the number of studies focusing on the male of the species is overwhelming (e.g. Apollonio et al., 1989; Duffy et al., 2006; Qvarnström, 1997; Travis \& Woodward, 1989; D. R. Wilson et al., 2009). In contrast, the number of similar experiments surrounding females is relatively few in number (e.g. Burley, 1986; Burley \& Foster, 2006; Limbourg et al., 2004). Thus, due to a stronger body of 
comparative evidence demonstrating that manipulation of attractiveness-related attributes affects mating strategy in males, men were chosen as the focus of Chapter 3.

The second factor was economic. Even though the literature review suggested that men may be more susceptible to an attractiveness manipulation, a sample of women within the experiment would have provided a useful comparison group. However, according to $\mathrm{G}^{*}$ Power (Erdfelder et al., 1996), in order to achieve $80 \%$ power with a predicted effect size $\left(\eta_{\mathrm{p}}{ }^{2}\right)$ of 0.03 and an $\alpha$-value of 0.05 , a sample of 66 participants would have been needed. This assumes the use of an all-male sample with two experimental conditions. Adding a group of women into the experiment would have increased this required sample size to 96 . While an additional 30 participants may not seem like a large number, the experiments were quite time consuming in nature. This meant that the research budget could only afford to cover the costs of a small number of participants. As such it was decided that the study would focus purely on the group which was predicted to change in response to the experimental manipulation, allowing for slightly more participants to be tested than required (in the case of Experiment 2) as a precaution in case the effect size was smaller than anticipated.

In hindsight, the choice to test only men was less than ideal. Although the comparative literature is full of examples of male mating strategies being manipulated by affecting their attractiveness, this is mainly reported for MCFC species (StewartWilliams \& Thomas, 2013b). Specifically, these males are from species where males compete for access to multiple females. These females, in turn, choose among several males who vary in the quality of their sexually selected ornaments or their place in a status/dominance hierarchy. However, as discussed in the introduction, humans are not an MCFC species, but are instead a species which show high levels of mutual mate choice (MMC). Thus, it is a fallacy to assume that manipulations which affect MCFC species will necessarily affect MMC species in the same way. Once the typical mating behaviour of humans is considered, it becomes apparent that, rather than expecting a change in the behaviour of just one sex in response to an attractiveness manipulation, it is more likely that both men and women would be affected by such cues. Furthermore, considering that men place a higher emphasis on physical attractiveness in their partner compared to women (Li et al., 2002; Meltzer, McNulty, Jackson, \& Karney, 2014; Stewart et al., 2000), and that physical attractiveness typically contributes more to a woman's self-esteem than a man's (Bale \& Archer, 2013; Shackelford, 2001; Wade, 
2000), we may even expect such a manipulation to affect women more than men. This point was initially raised in the conclusion of Chapter 3.

This thesis was conducted over seven years. During that time, and through careful thought and reflection on human sex differences, a subsequent piece of research (The Ape That Thought It Was a Peacock, Stewart-Williams \& Thomas, 2013b) was independently developed by the research team behind this thesis. The work identified that humans are collectively more representative of a MMC species than a MCFC one and demonstrated that evolutionary psychological researchers sometime use evolutionary theories based on these latter species and apply them too readily to Homo sapiens. This theoretical work commenced after the collection of data for Chapter 3 and it is now apparent that the rationale for Experiments 1 and 2 may well have been influenced by the very MCFC bias which we grew to critique several years later. Indeed, experiments conducted later in the thesis have experimental hypotheses which were clearly formed with a more MMC perception of human mating in mind.

The Mix\&Match paradigm was retired early into the research programme following the finding that basic visual cues provided a more cost effective method for administering evolutionarily relevant mating strategy cues to participants. That said, it may be that a revised version of the experiment (or one using a more 'basic' form of a self-perceived attractiveness manipulation), would provide fruitful results in the future when utilising a mixed-sex sample.

Testosterone. Much like Chapter 3, Chapter 6 also focused on an all-male sample. This was for similar reasons; limited resources (in this case the number of salivary assay kits) meant that we were able to test 46 participants for their pre- and post-exercise $T$ levels. $G^{*}$ Power calculates that an overall sample size of 41 is needed in order to provide $80 \%$ power with an $\alpha$-level of 0.05 (one-tailed) and an expected effect size of $d_{\mathrm{z}}=0.40$. This assumes the use of a simple pre-post design for one sample. Introducing a control condition would have required 25 more participants, and the recommended sample size would have increased to 96 if a separate group of women were tested. Again, these numbers could not be tested using the resources available and so a homogenous sample of men was used in a single experimental condition. A larger study utilising a formal control condition with a mixed-sex sample would provide a much clearer view of the effect of circulating $\mathrm{T}$ on short-term mating behaviour.

Unlike the hypotheses formed in Chapter 3, the choice of men as the sex of interest in Chapter 6 was not due to an underlying MMC bias. In contrast to the other 
experiments in the thesis, in which the predicted influence of a given experimental intervention on mating strategies was reliant on existing effects found in other species, there is a healthy amount of research about $\mathrm{T}$ changes in both men and women. This research suggest that women can experience a change in $\mathrm{T}$ in response to environmental cues such as victory, and that such changes can be as large (in relative terms) as those found in men (Bateup, Booth, Shirtcliff, \& Granger, 2002; Edwards, Wetzel, \& Wyner, 2006). However, the influence of such cues on female $\mathrm{T}$ is not as consistent as those found in men, often producing null results (Steven J. Stanton, Beehner, Saini, Kuhn, \& LaBar, 2009) or a very different pattern than that of men (Mazur et al., 1997; Rejeski et al., 1990). As such, there is good reason to believe that, if $\mathrm{T}$ is connected to short-term mating propensity, $\mathrm{T}$ interventions would have a more consistent impact on the behaviour of men than women. Ultimately, however, this remains an assumption untested by this research.

\section{Cross-Cultural Representation}

Evolutionary psychology investigates behaviour from the perspective that humans are organisms with evolved psychological mechanisms forged in response to the challenges of a relatively stable ancestral environment. It follows then, that psychological adaptations should, for the most part, transcend the boundaries of culture. There may well be some exceptions. Some evolved traits, such as the case of lactose persistence (Holden \& Mace, 1997), evolved relatively recently in human history and so are not represented equally across all ethnicities. However, psychological traits such as a preference for facial symmetry (Scheib et al., 1999), and specific attributes in a mate (Buss \& Schmitt, 1993), show good cross-cultural consistency, albeit with minor fluctuations in effect strength (but see Pound et al., 2014). For example, in all 37 cultures sampled, Buss (1989) found that men showed a greater than average preference for younger partners. Likewise, the preference for facial averageness and symmetry found among western samples (Thornhill \& Gangestad, 1999) has been replicated using Chinese and Japanese participants (Rhodes, Yoshikawa, et al., 2001). This latter psychological adaptation is even found among other animals (e.g. López, Muñoz, \& Martín, 2002; Waitt \& Little, 2006). Outside the realm of mating, phobias such as the fear of spiders and snakes (Ohman \& Mineka, 2001), cognitive biases (Ruffle \& Sosis, 2006), and emotion recognition/display (Ekman et al., 1987; Sauter, Eisner, Ekman, \& Scott, 2010), have also been replicated cross-culturally. 
Within this thesis, participant ethnicity was measured as part of the demographic form. This was included in every regression model and in no case did the variable contribute to variance in SMA change. Thus, there appeared to be no effect of ethnicity on a participant's capacity to change their mating strategy in response to environmental cues. While ethnicity was recorded, the culture in which the participant was socialised, unfortunately, was not. Thus, a participant who described themselves as 'South-East Asian' may well have been born in UK, but could have easily been socialised in another country. Therefore, evidence for cross-cultural consistency of mating strategy flexibility is required. Indeed, while we may expect mating flexibility to be present among other cultures, we may also expect slight differences between societies given that mating strategy flexibility, unlike some of the aforementioned domain-specific adaptions, appears to be more dependent on moderating variables (such as relationship status and age). For example among the Datoga (Muller et al., 2009), where men spend little time with their young, a sex difference may be found in how parental cues affect mate choices. In contrast, such a sex difference may not be found among the Ache of Paraguay which are categorised by high paternal, as well as maternal, investment (Hewlett, 1991). Likewise, communist (e.g. China or parts of eastern Europe), or egalitarian (e.g. Israeli Kibbutz or the Hadza; Marlowe, 1999a) cultures may be less affected by wealth cues.

\section{SMA 2.0}

One reoccurring issue with the SMA task is that participants tend to choose a small number of models for a relationship and this tends to make data from the task unsuitable for parametric analysis. At the same time, as mentioned in Chapter 2, this frequency of selection is interesting as it tells us something about the nature of human mating. Specifically, it tells us that humans are quite selective when considering potential partners and are often unwilling to entertain the idea of a relationship with someone based on their picture alone. This is true for both sexes and for individuals of varying sociosexuality. However, now that this is known to be the case, it may be of further interest to control for this behaviour and simply present participants with individuals who they are attracted to. This could be accomplished by offering participants an array of individuals and asking them to pick those which they consider attractive enough with which to have a relationship. Once a sample of 50 models was selected, the SMA task could then be conducted by asking participants to choose between short- and long-term relationships for each of these individuals. This would 
have the added benefit of producing a simple ratio of LT to ST selections for each SMA measure. Furthermore, images could be enhanced by including some basic information such as age and social status in a "profile" format. As it stands, men choose more models for relationships in the SMA than women. This may be because physical attractiveness ranks higher in men's reported desirable mate characteristics compared to women (Buss, 1989; Symons, 1979, p. 301). The inclusion of ambition (which shows the opposite pattern, in that it is more important to women; Buss, 1989) may help redress this balance.

Other possible modifications to the SMA task include an expansion in the number of relationship choices. Within the category of short-term mating, for example, there exists a spectrum of behaviour from an anonymous one night stand to a fling lasting weeks. Likewise, marriage or a prolonged informal relationship could both be considered long-term in nature. Some authors recognise a 'medium-term' relationship as one lasting between six months and three years (Muñoz, Khan, \& Cordwell, 2011). Such a term may apply to those models a participant would happy to date, but unlikely to consider suitable for marriage. Although SST has not been expanded to include this, such an additional category may allow for more intricate analysis of mating preferences and further develop our understanding as to how evolutionarily relevant cues affect mating strategies. It may be the case, for example, that some cues are enough to bias an individual's relationship choices from short-term towards medium-term, but not enough to lead them to select more long-term choices and vice versa. Alternatively, as suggested in The Polygyny Problem section, the SMA could be modified to capture propensity towards various mating arrangements (e.g. monogamy, promiscuity, and polygyny).

A final element which remained static throughout the thesis was the social context given to the participants during the SMA task. Namely, participants were told to act as if they were single and open to a new relationship. There exists the opportunity, however, to change this context to see if participants become more or less susceptible to cues. For example, if female participants were asked to imagine being in a relationship with a low-status male, would they react to wealth cues differently? If participants were asked to imagine they were unsatisfied with their relationship, would they be easier to bias in the direction of short-term mating? Such combinations are potentially limitless.

\section{Applications}


It is likely that the area of mating strategy plasticity has either few applications, or applications which are limited in scope. A simple thought experiment can illustrate this. Take, for example, a man who wanted to guide his behaviour towards being more long-term orientated. This thesis suggests that exposure to parental cues, danger cues, and perhaps other rival males, may provide a small boost to the number of female models he may select for a long-term relationship. Could this then be translated into a sort of "monogamy therapy"? Perhaps it could, but it would likely involve persistent exposure to a number of steady cues in a manner akin to behavioural conditioning. Even if this were the case, the results may not be promising; there are many men who are exposed to new born infants within their relationship who still pursue extra-pair mating. Even if this type of therapy could somehow be established, it is unclear whether it would be a better alternative to other behavioural management techniques, such as mindfulness interventions (Grossman, Niemann, Schmidt, \& Walach, 2004; KabatZinn, 2003; D. S. Wilson, Hayes, Biglan, \& Embry, 2014), which have recently been able to help participants increase their willpower in domains as diverse as dieting and ethical decision making (Ruedy \& Schweitzer, 2010; Tapper et al., 2009).

That said, the knowledge that our mating behaviour can be flexible, and that certain situations may make us vulnerable to strategy shifts, may well be useful. For example, women who are in relationships may be sensitive to cues of wealth in other men and this may lead to greater short-term mating interest (Chapter 4). Thus, women who find a sudden lack of resources in their relationship due to economic hardship, but who want to remain committed to their partner, could prepare themselves for the fact that they may, temporarily, find a short-term relationship with an affluent male desirable. Likewise in men, circulating testosterone $(\mathrm{T})$ increase was associated with increased ST selection (Chapter 6). There are many factors which can increase $\mathrm{T}$ in men and these can be both natural (such as increased anaerobic exercise; Ari et al., 2004) and unnatural (such as when using drugs for disorders such as low sperm count or anaemia; Heller, Rowlety, \& Heller, 1969; Hendler, Goffinet, Ross, Longnecker, \& Bakovic, 1974 ) in nature. A man who intends to stay committed to a partner may be able to use the information on mating strategy flexibility to prepare him for a potential change in his sexual desires. At the beginning of this thesis, David Buss was quoted as saying that deeper knowledge of our mating strategies 'gives us greater power to design our own mating destiny than any other humans have ever possessed' (Buss, 2003a, p. 96). 
However, in light of the small-to-medium effects of the manipulations in this thesis, the implication of the findings on one's 'mating destiny' appears to be minimal.

\section{Replication and Extension}

The structure of this thesis was unusual in as much as several evolutionary topics were tested using a small number of experiments. This method was chosen in order to cover a large amount of ground and hopefully gain a wider range of support for the overall working hypothesis. Though there are some obvious weaknesses of this approach, there is also a clear strength. Had only two or three topics been covered (e.g. physical attractiveness, sex-ratio, and danger cues), with each being replicated a few times after receiving tweaks in methodology, the overall conclusion drawn from the thesis as a whole may have been markedly different. For example, the Mix\&Match studies (Chapter 3) produced null results followed by inconsistent results, the danger cues studies (Chapter 5) produced consistent results followed by null results, and the sex-ratio study (Chapter 8) produced an effect in only one of the main conditions. Given these findings, support for the working hypothesis would have been weak. In order to compensate for the lack of depth in each topic, the power of each study was increased with a total of 1419 participants being tested throughout the thesis. With $70 \%$ of the one-way hypotheses supported, 10\% contradicted, and 20\% unsupported (null results), it is unlikely that all of the support for the working hypothesis were subject to Type I errors.

Despite the positive aspect of this approach, there have nonetheless been recent cases within social psychology of established effects failing to be reproduced (Abbott, 2013; Shanks et al., 2013; Yong, 2012), and a subsequent push towards consistent replication of effects. Thus, the future focus of any subsequent research on mating flexibility using the SMA should be on the reproduction of the effects found here, perhaps in conjunction with pre-existing findings from the psychological literature. For example, one could combine the several fluctuating asymmetry studies with an SMA measurement to see if previous manipulations simply cause a bias in favour of facial symmetry alone or are enough to produce a change in mating strategy as well.

Duration of effects. If evolutionarily relevant cues are indeed able to affect mating strategies, then one necessary follow up question is: for how long does such an effect last? Is it the case that a cue which leads to a strategy change must be persistently present in order for the change to remain, or that relatively brief exposure to a succinct cue will have a lasting effect on strategy change? To date no experimental studies on 
humans have addressed this issue, even among studies which only elicit changes to a property related to a given mating strategy. For example, none of the studies which produced a change to facial masculinity or symmetry preferences (e.g. Little et al., 2011; Little et al., 2013) assessed the duration of this effect.

The ecological literature may provide some idea of what we might find in such studies. The winner effect (Chapter 6) is the phenomenon that, among several organisms, the winners of intra-sexual competition tend to be more likely to win in future bouts. In one study using rats, winning a battle was associated with increased circulating testosterone, a reduction in freezing responses, and lower attack latency (Oyegbile \& Marler, 2005). Further instances of victory caused these attributes to become enhanced cumulatively (up to a maximum of four fights, as tested in the experiment). Importantly, the duration between each of these fights was set at two days. This means that, at least in rats, the winner effect must last at least two days. If this were not the case, then such an effect would not be expected to accumulate. By comparison, in non-mammals such as fish, the winner effect can be as short as one hour (Chase, Bartolomeo, \& Dugatkin, 1994). Although speculative, this might suggest that some of the evolutionarily relevant mating cues thought to affect $\mathrm{T}$ (such as parental or victory cues) may have effects in humans which last hours or days, rather than seconds or minutes.

Effects of the menstrual cycle. There is a growing body of research indicating that, although physically hidden, women experience oestrus (Thornhill \& Gangestad, 2008) and that this is accompanied by some interesting changes in partner preferences and social interaction. One classic example found that women at peak fertility wore more revealing clothing in nightclubs (Grammer, 1996 as cited in Buss, 2003a, p. 247). Another study found that female strippers who were at peak ovulation earned more tips (G. Miller, Tybur, \& Jordan, 2007), although it is unclear whether this was because men could "detect" their fertile status, if the women "performed" better, or both. Similarly, Geoffrey Miller is currently writing a book about how consumer behaviour is influenced by a woman's menstrual cycle stage (personal communication). This research area has recently produced some promising results (Pine \& Fletcher, 2011; Saad \& Stenstrom, 2012).

Perhaps most related to this thesis are the studies which show that women who are menstruating, or using oral contraceptives, show different mate preferences to those who are in the most fertile part of their cycle. Specifically, ovulating women consider 
men with more masculine (Penton-Voak \& Perrett, 2000) and symmetrical (Little, Jones, Burt, \& Perrett, 2007) faces to be more attractive than their non-ovulating counterparts do. It is hypothesized that this change in preference serves a function in directing women towards the acquisition of the best possible genes at the time in which they are most likely to become pregnant. These studies share similar methodological weaknesses as the aforementioned mating behaviour "biasing" studies (e.g. Griskevicius et al., 2011; Little et al., 2011; Watkins, Jones, et al., 2012) as they do not take measures of mating strategies. If it is true that women possess an adaptation guiding them towards good genes from high quality men when they are most fertile, then we might expect to find a change in their willingness to engage in short-term mating at the same time - a relationship type in which they are more likely to successfully pursue highly attractive men (but see Soler et al., 2014).

It follows then that, given a large enough sample, women who are in their fertile period may select more individuals for a short-term relationship on the SMA task, and/or score higher on measures of sociosexuality at this time, when compared to their non-fertile counterparts. The standard demographic form for each of the experiments in this thesis did in fact include information on the menstrual cycle, and so one next step might be to try to make sense of these data. If fruitful, other "medium-term" influences on mating behaviour could be investigated with similar sample sizes. For example, one could observe whether impact of naturally occurring stressors, such as varying levels of examination pressure on students throughout the academic year, affect mating behaviour.

\section{Conclusion}

As an organism, we have various psychological adaptations which ensure our survival. These include a taste for highly nutritious food, a fight-or-flight response when reacting to threats, and cognitive biases which steer us away from danger (Ohman \& Mineka, 2001). From an evolutionary psychological perspective, such adaptations form evolved psychological mechanisms which meet several stringent criteria including being efficient, complex, universal, and domain specific (Cosmides \& Tooby, 1992a; G. Miller, 2000b). In the same way as our mind has specialised tools for maximising survival, it may also have an equivalent suite of reproductive maximisation tools. For example, emotions such as jealousy seem to function to stop cheating within relationships (Buss \& Haselton, 2005; Buss et al., 1992), and specific preferences for each sex, such as the desire for an older partner typically found in women, may steer 
people towards more efficient mating outcomes (Buss, 1989; Buss \& Schmitt, 1993). Could there be a set of mating flexibility tools in our mental pocket knife; unique adaptations allowing individuals to shift their mating preferences in order to maximise their fitness? Previously, a large body of comparative literature, some initial human research, and some anecdotal evidence seemed to indicate that this was the case. This thesis furthers this support by providing some evidence that an individual can change the way they select potential mates depending on their exposure to evolutionarily relevant stimuli such as cues of increased danger, changed social status, vulnerable offspring, and a skewed sex-ratio. The experiments also produced some novel findings including the interest of high SO individuals in long-term relationships (Chapter 2) and the importance of several demographic variables in the prediction of mating strategy change. Initial support for the overall working hypothesis, coupled with the high potential for the topic to be expanded and further explored, suggests that the area of human mating flexibility may be a fruitful area for future research. 
Appendices

\section{Appendix A: Socio-sexual Orientation Inventory}

Survey about sexual thoughts, attitudes and experiences ${ }^{44}$

With

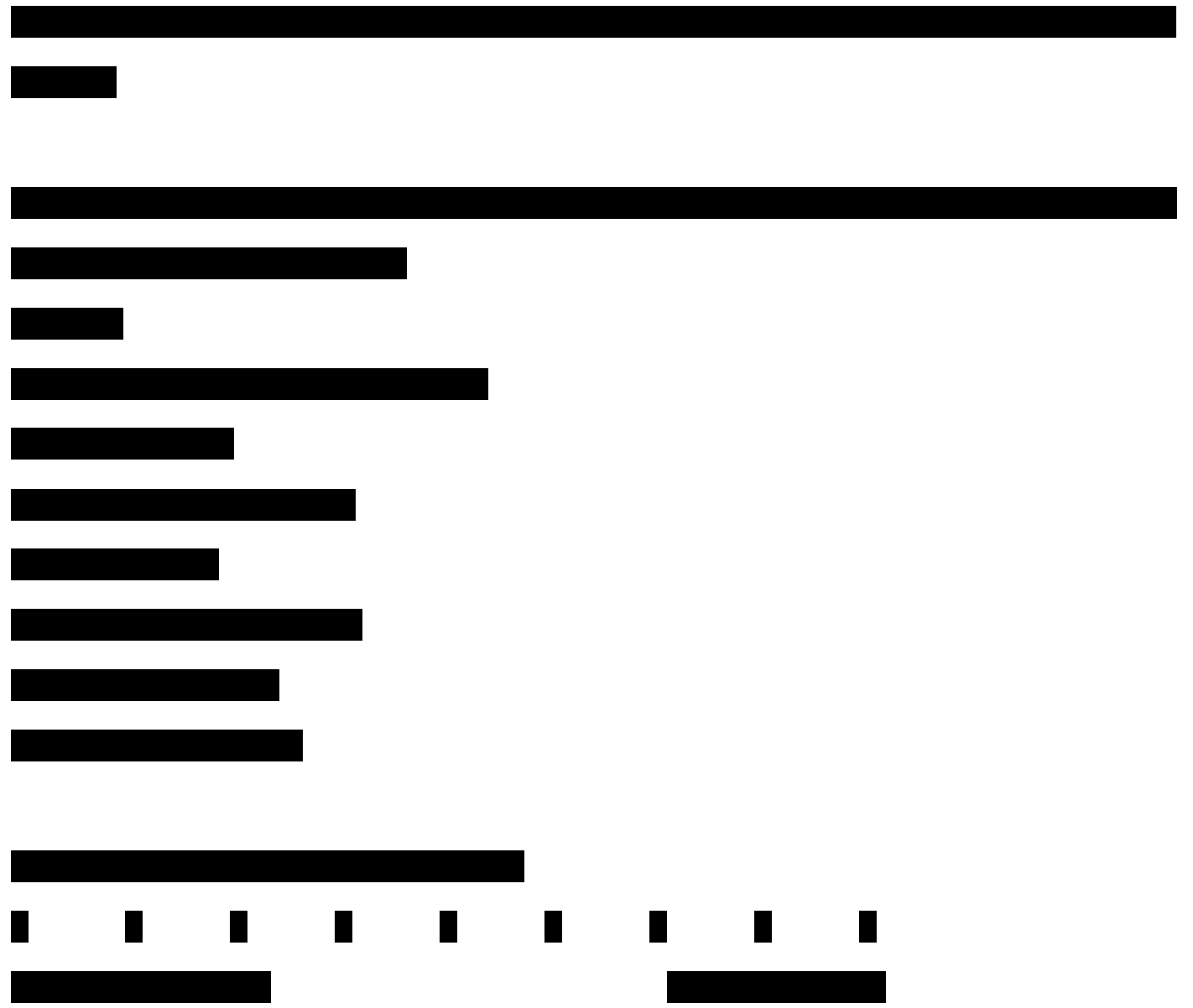

\footnotetext{
${ }^{44}$ Simpson, J. A., \& Gangestad, S. W. (1991). Individual-differences in sociosexuality - evidence for convergent and discriminant validity. Journal of Personality and Social Psychology, 60(6), 870-883. doi: $10.1037 / 0022-3514.60 .6 .870$
} 


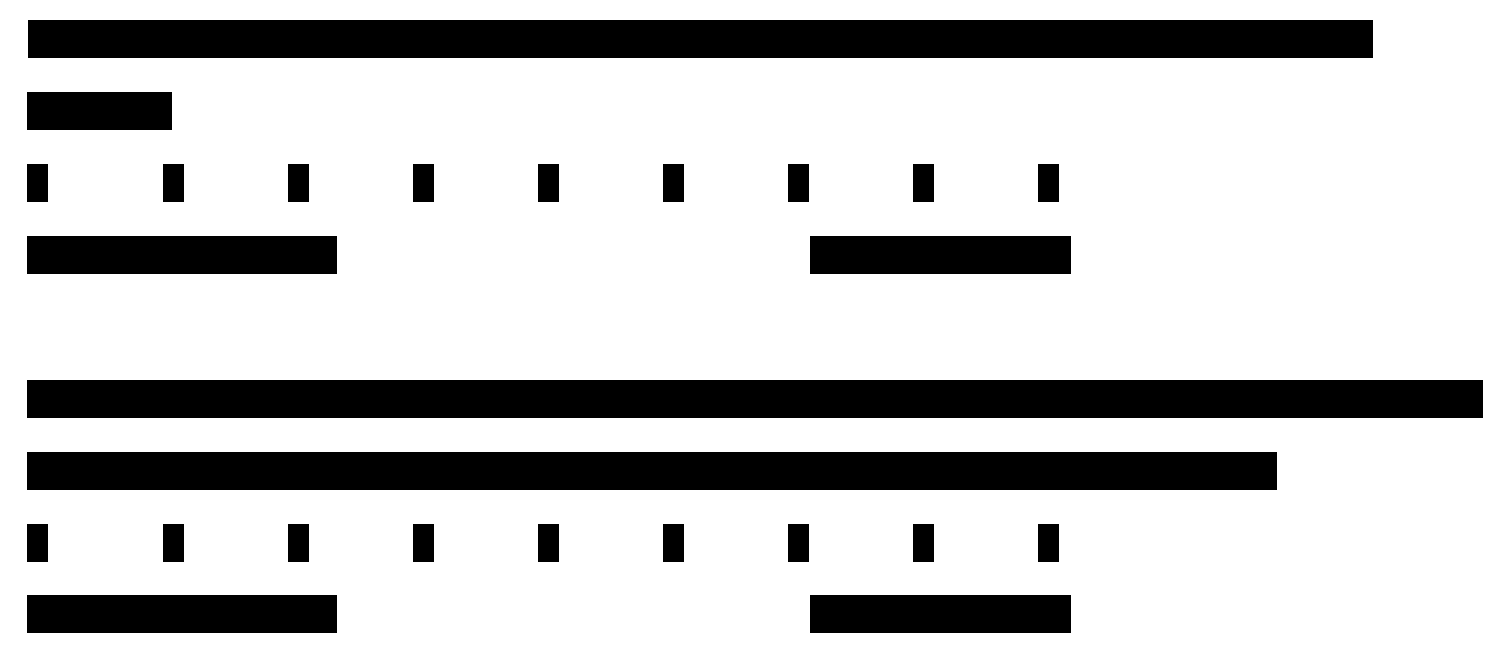




\section{Appendix B: The Socio-sexual Orientation Inventory - Revised}

Survey about sexual thoughts, attitudes and experiences ${ }^{45}$

Please respond honestly to the following questions:
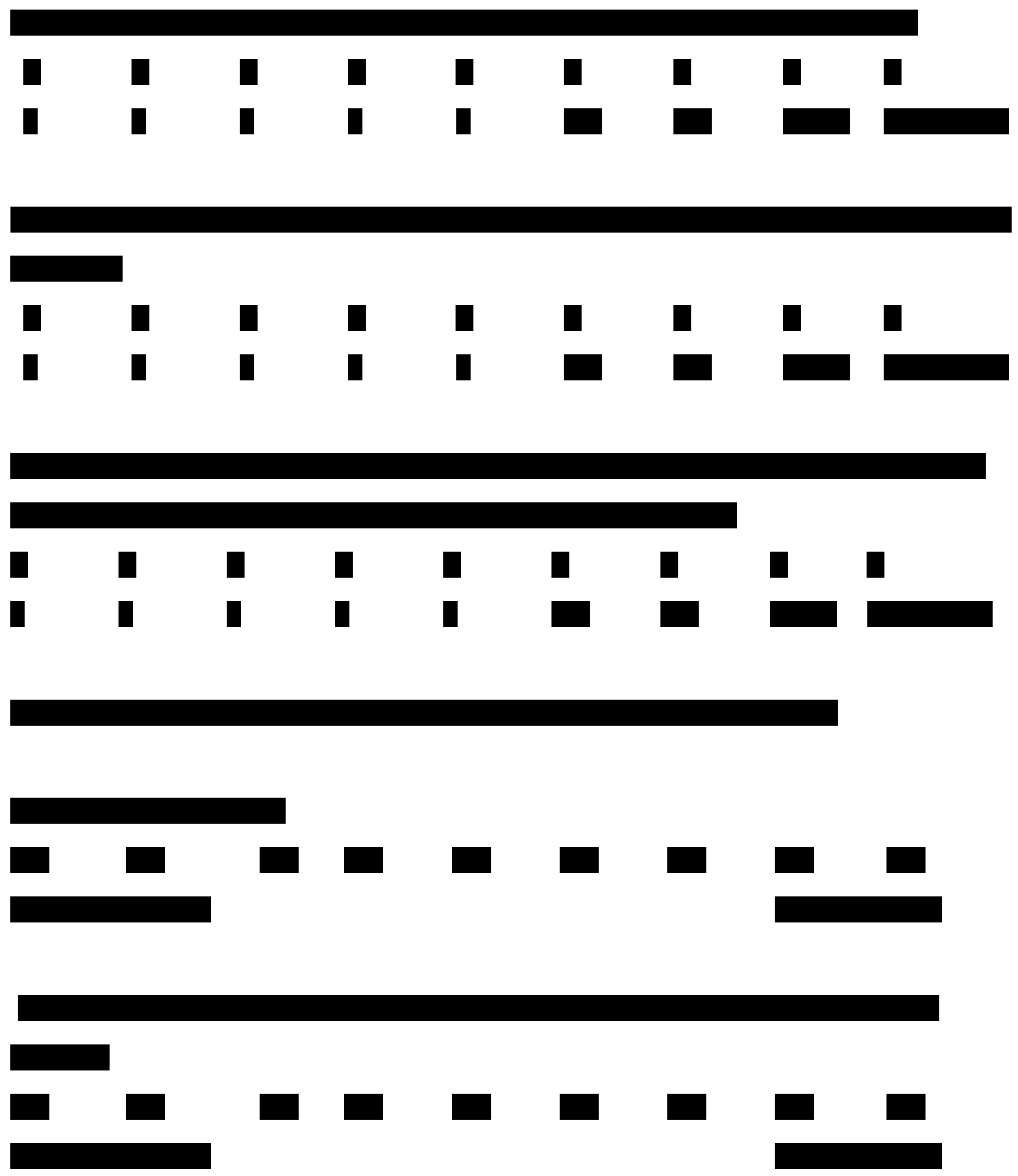

\footnotetext{
${ }^{45}$ Penke, L., \& Asendorpf, J. B. (2008). Beyond global sociosexual orientations: A more differentiated look at sociosexuality and its effects on courtship and romantic relationships. Journal of Personality and Social Psychology, 95(5), 1113-1135. doi: 10.1037/0022-3514.95.5.1113
} 


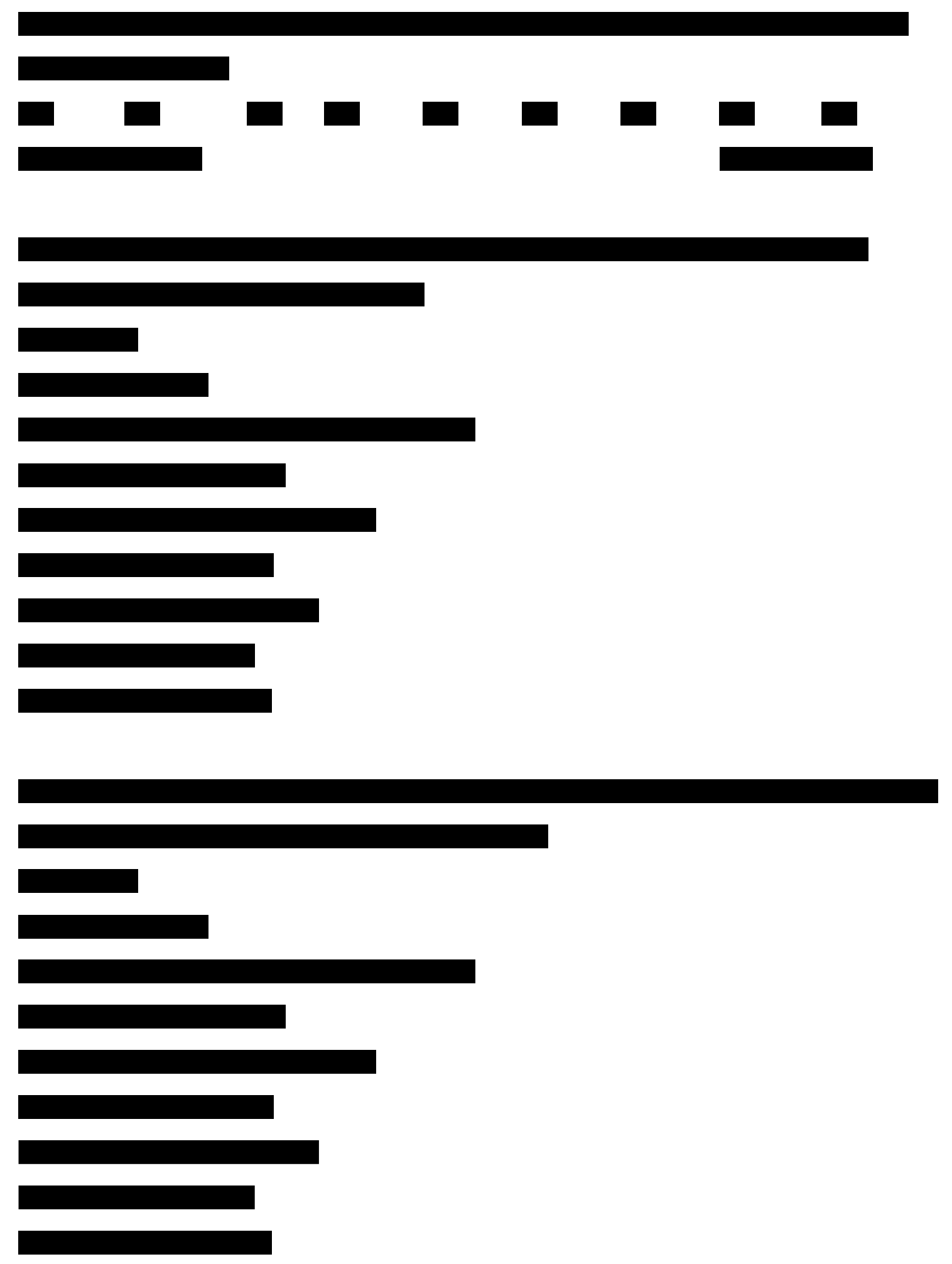


In everyday

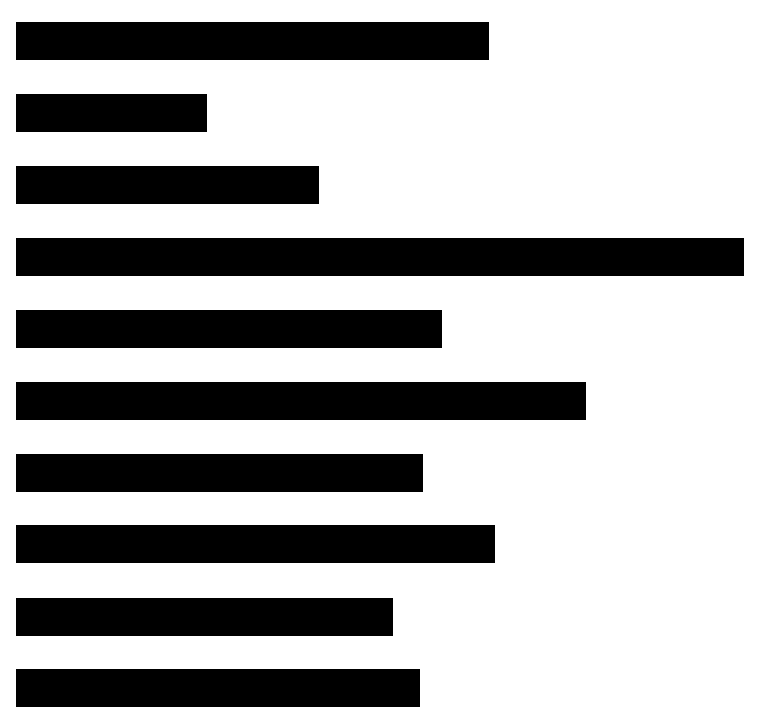




\section{Appendix C: Instructions Given to Participants during the SMA task}

For this study we are interested in how people rate others based on their attractiveness. Part of the study will ask you to indicate what type of relationship you'd prefer to have with a person based on their photo. Another part of the study involves [Description of the manipulation task].

To begin with we would like you to indicate what type of relationship you'd prefer to have with a person based on their photo. For the purpose of this study we would like you to imagine that you are single and open to starting a new relationship with someone. As you view each person imagine you have met them once or twice through friends. Your friends have made it clear to you that the person finds you attractive.

You will be shown photos individually and be able to select from three options. Please select the one you'd most prefer.

For a short-term sexual relationship with the person select: 'Short-term fling'. For a long-term committed relationship with the person select: 'Long-term thing'. Or, if you would not be interested in either of these, please pick: 'Not interested'.

[Manipulation slideshow shown here]

Thank you. You have now reached the final part of the study. Just like the first part we would like you to look at some people and indicate what type of relationship you'd prefer to have with them.

Remember, for the purpose of this study we would like you to imagine that you are single and open to starting a new relationship with someone. As you view each person, imagine you have met them once or twice through friends. Your friends have made it clear to you that the person finds you attractive.

Thank you! You have now completed the study. Please contact the experimenter who will debrief you. 


\section{Appendix D: Instructions Given to Participants during the Attractiveness Rating Task}

Another part of the study involves looking at pictures of people and rating their attractiveness on a scale of $1-10 .{ }^{46}$

We would now like to show you pictures of some more people. This time, instead of three buttons below each picture you will see a scale between 1 and 10 . We would like you to use this scale to rate the attractiveness of the people in these pictures.

Rating 10 on this scale means that the individual in the picture is highly attractive, while rating 1 means the individual is highly unattractive.

46 This appeared in the opening instructions of the SMA task, describing the general format of the study to the participants (See Appendix C, point 1). 


\section{Appendix E: The Standard Demographic Questionnaire}

Demographic Questionnaire

1. What is your age?

2. What is your sex? (Please tick).

Male

Female

3. What is your Sexual Orientation? (Please tick).

Heterosexual (primarily attracted to members of the other sex)

Homosexual (primarily attracted to members of the same sex)

Bisexual (attracted to both sexes)

Asexual (not attracted to either sex)

Other (Please specify)

4. What is your current relationship status? (Please tick all that apply).

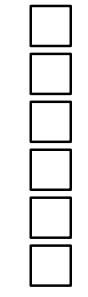

Married

Divorced/Separated

In a committed relationship with one person

In an uncommitted relationship

Not currently dating or romantically involved with anyone

Other (Please specify)

5. Do you have any children? (Please tick).

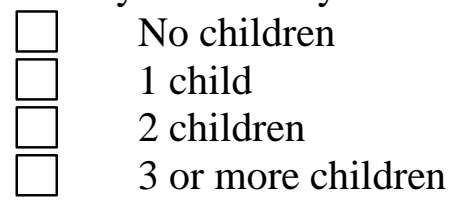

6. What is your ethnic/racial background? (Please tick all that apply).

Caucasian/White

Black

Native American

Middle Eastern/North African

South Asian - Indian, Pakistani, etc.

Southeast Asian - Thai, Cambodian, etc.

East Asian - Chinese, Japanese, Korean, etc.

Inuit/Arctic Peoples

Australian Aboriginal/ Aboriginal New Guineans

New Zealand Maori/Pacific Islander

Other (Please specify)

7. What is the highest level of education you have obtained? (Please tick).

Some High School or less 
Graduated High School

Some College/University

Completed Undergraduate Degree

Completed Postgraduate Degree

8. Compared to my peers I consider myself to be:

1 .

$2 \square$

$3 \square \quad 4 \square$

5 口

$6 \square$

$7 \square$
$8 \square \quad 9 \square$
Very Attractive

Much less attractive

9. Please indicate your social/economic status:

$\begin{array}{ccccc}1 \square & 2 \square & 3 \square & 4 \square & 5 \square \\ \text { Upper } & \text { Upper-Middle } & \text { Middle } & \text { Lower-Middle } & \text { Lower }\end{array}$

Please only answer the following questions if you are female. Otherwise this questionnaire is now complete.

10. Are you currently on any form of contraceptives which stops your menstrual cycle? (Please tick).

$\square \quad$ Yes

If you answered 'yes' to question 10 then do not continue, this questionnaire is now complete. Otherwise please continue.

11. On average, by how many days does the length of an entire cycle vary from month to month? (Please tick).

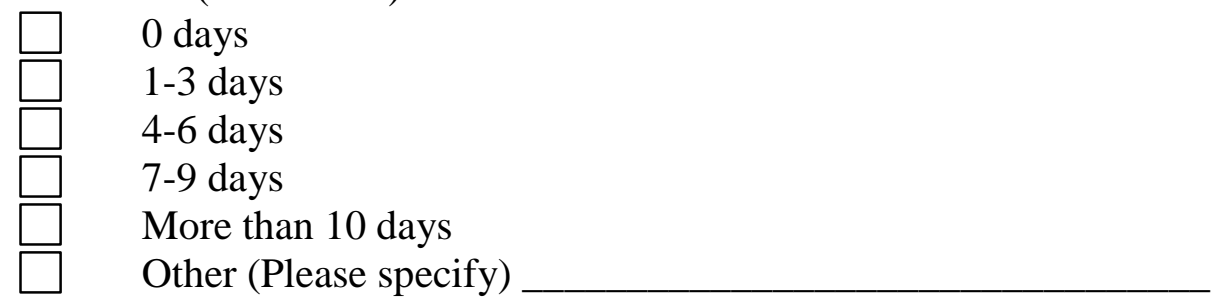

12. How long is your menstrual cycle? (Please tick).

Note: Count the first day you bleed as day one and the day before your next bleed as the last day.

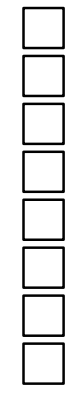

Less than 21 days

Between 22 and 26 days

Between 27 and 30 days

Between 31 and 35 days

Between 36 and 42 days

Over 43 days

N/A

Other (Please specify)

13. When is your next cycle due to start? (Please try to be as accurate as possible). In days time.

14. Do you experience regular cycles? (Please tick).

Yes 
No

Sometimes

Other (Please specify)

Thank you, this questionnaire is now complete. 


\section{Appendix F: Consent Form Used for Experiment 0 in Chapter 2}

\section{Consent form}

This study is about attractiveness and relationship choice. We will ask you to fill in a number of short questionnaires and then perform a computer based task.

The questionnaires involve questions of a sexual nature - specifically about your sexual experience, attitudes and beliefs.

The computer based task involves looking at pictures of the opposite sex and making a judgements as to what type of relationship you'd prefer to have with them. You'll also be asked to rate some individuals for how attractive they are.

The questionnaires and data gathered will not contain any information that can be traced back to you personally and will be added to a pool of data from other participants.

At the end of the study you will receive credits or payment (of a prearranged amount) for your participation. Participation in this study is not compulsory and you are free to withdraw at any time without penalty. This study is conducted in accordance with British Psychological Society ethical guidelines.

If you have any queries feel free to contact the experimenter (Andrew Thomas, Swansea University) at [EMAIL ADDRESS]. 
Please read each of the following statements and, if you agree with them, please sign below to begin the study.

I consent to participate in this study. I am satisfied with the instructions I have been given.

I have also been informed that any information kept after study termination will be anonymous and confidential in nature.

I have not been coerced in any way to participate in this study and I understand that I may terminate my participation in the study at any point should I wish. I am at least 18 years of age.

Data Protection: I agree to the University processing information that I have supplied. I understand that I am unable to withdraw my participation from the study following its termination, because my data will be anonymised and unidentifiable.

Full Name:

Signature:

Date: 


\section{Appendix G: Debrief Form Used for Experiment 0 in Chapter 2}

\section{Debrief form}

Thank you for your participation.

This study was designed to examine the factors which contribute to the types of relationships people engage in. Specifically, we are looking to see if the presentation of attractive or unattractive members of the opposite sex can change an individual's preference for a short or long-term relationship.

In order to do this we asked you to pick what type of relationships you'd like to engage in for a group of people before and after exposure to attractive or unattractive individuals.

Your participation in the study was greatly appreciated. We would further ask you not to mention your participation in this study to other students who may be asked to participate themselves.

If you have any further questions or queries regarding this experiment feel free to contact the researcher (Andrew Thomas: [EMAIL ADDRESS]) or research supervisor (Dr. Steve Stewart-Williams: [EMAIL ADDRESS]).

Finally, some references related to this research area are given below which may be of interest. 
References

Buss, D.M. (2003). The evolution of desire. New York: Basic Books.

Gangestad, S.W., \& Simpson, J.A. (2000). The evolution of human mating: trade-offs and strategic pluralism. Behavioral and Brain Sciences, 23(4), 573-587.

Schmitt, D.P. (2005). Sociosexuality from Argentina to Zimbabwe: A 48-nation study of sex, culture, and strategies of human mating. Behavioral and Brain Sciences, 28, 247-311.

Simpson, J.A., \& Gangestad, S.W. (1992). Sociosexuality and romantic partner choice. Journal of Personality, 60, 31-51. 


\section{Mix\&Match}

Log-in Signt-up About

\section{Welcome to Mix\&Match!}

To get started you need an account! Once you have registered you can upload a photo, rate other people and be rated yourself!

Right now, Mix\&Match is still in its testing phase. If you were lucky enough to be chosen as a tester then you can sign up below!

Note: you will need the beta password in order to sign-up.

Please choose a log-in name.

Please choose a password.

Please confirm this password.

What is your gender?

Male Female

Please select your age bracket.

- $\quad \boldsymbol{T}$

Which is the closest city to which you live?

Bangor $\checkmark$

What is the Beta password?

Submit

TESTING MODE

Copyright $\odot 2011$ Mix\&Match

Figure H.1. The sign-up page of the Mix\&Match website. In order for an account to be created, a password was required from the experimenter. 


\section{Mix\&Match}

Log-in Sign-up About

\section{Welcome to the Log-in page!}

Username:

testuser

Password:

(.........

Submit

TESTING MODE

Copyright $\odot 2011$ Mix\&Match

Figure H.2. The log-in page of the Mix\&Match website which allowed individuals to access the "My Page" hub of the experiment. 


\section{Mix\&Match}

Testing Page Remove Me Log-out About

\section{Welcome, testuser 2 .}

Thank you for offering to test a new feature of the Mix\&Match website.

The feature you'll be testing is our new rating system. This involves rating the photographs of other website users for attractivness and receiving feedback about your own.

Below you will be guided through the four simple steps of the testing process.

\begin{tabular}{|c|c|}
\hline \multirow{2}{*}{ Today } & $\begin{array}{c}\text { Step 1: Upload your photo } \\
\text { Please select an image to upload } \\
\text { Choose File }\end{array}$ \\
& \\
\cline { 2 - 2 } & No file chosen \\
\hline & Submit \\
\hline & Step 2: Rate other people \\
\hline Next session & Step 4: Youthering your results \\
\hline
\end{tabular}

Figure H.3. The "My Page" section of the Mix\&Match website. This page acted as a hub to guide participants through the experiment. 


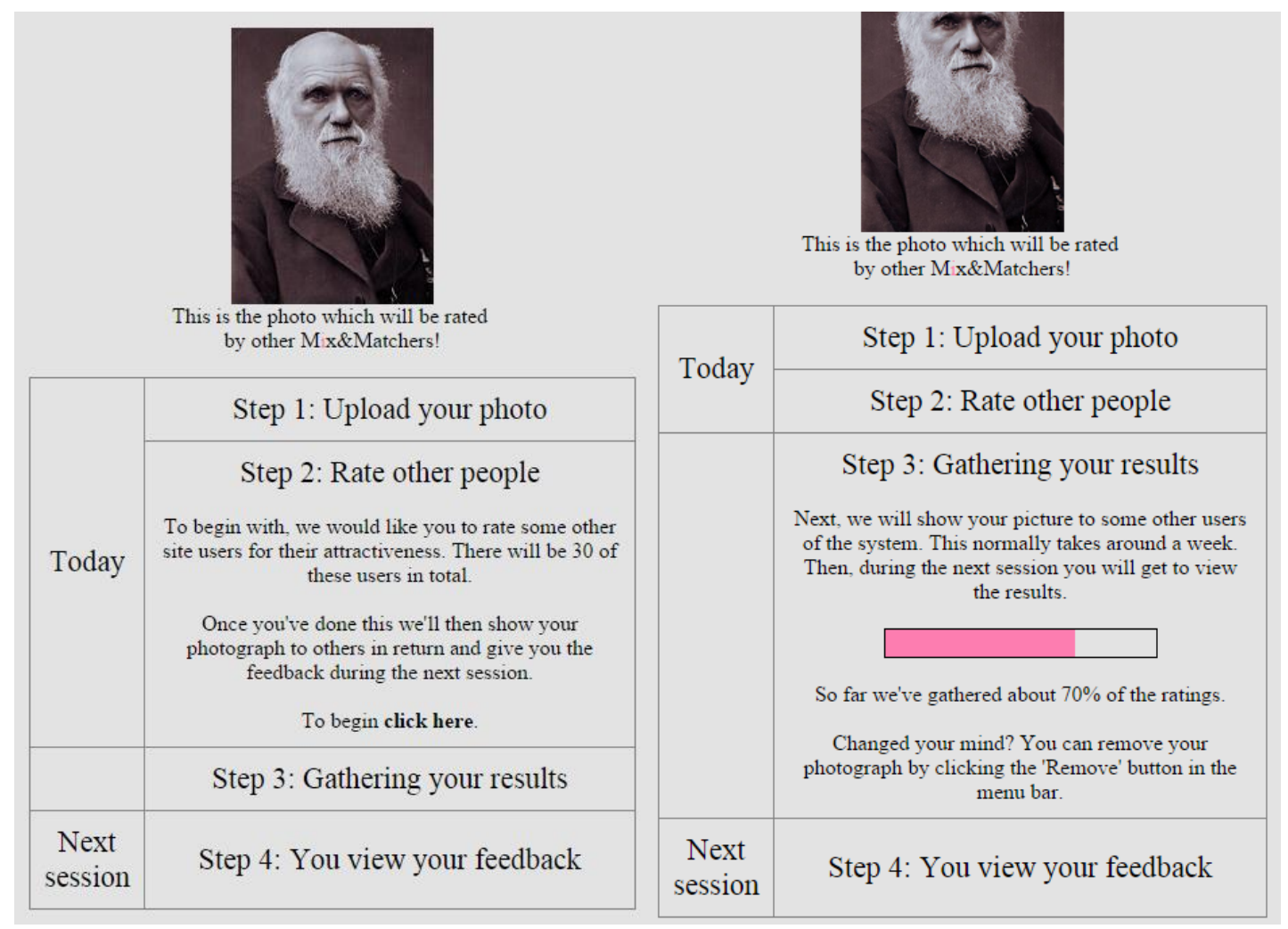

Figure H.4. Two of the steps from the "My Page" hub. The image on the left shows the participant's view after they have uploaded their photograph to be rated. The image on the right shows an example of the "progress bar". This would be seen by the participant if they logged into their account between test sessions. 


\section{Mix\&Match}

Testing Page Remove Me Log-out About

You have 10 more users to rate.

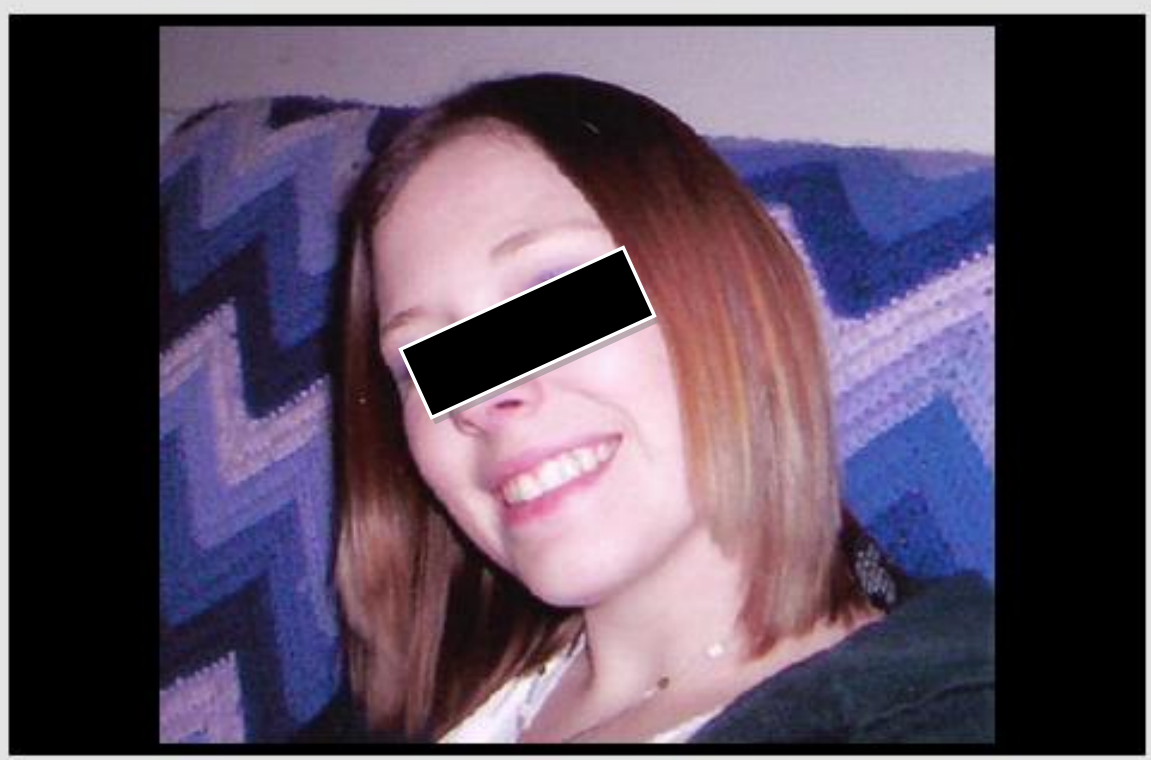

18-25 from Swansea

$\begin{array}{lllllllllll}1 & 2 & 3 & 4 & 5 & 6 & 7 & 8 & 9 & 10\end{array}$

Figure H.5. An example of the "Rating page". Participants rated 30 images for attractiveness on a scale of 1 to 10. Each image was of a woman aged 18-25 and the name of a city accompanied each image to add to its believability. 


\section{Mix\&Match}

Testing Page Remove Me Log-out About

You have 42 more piece(s) of feedback to view.

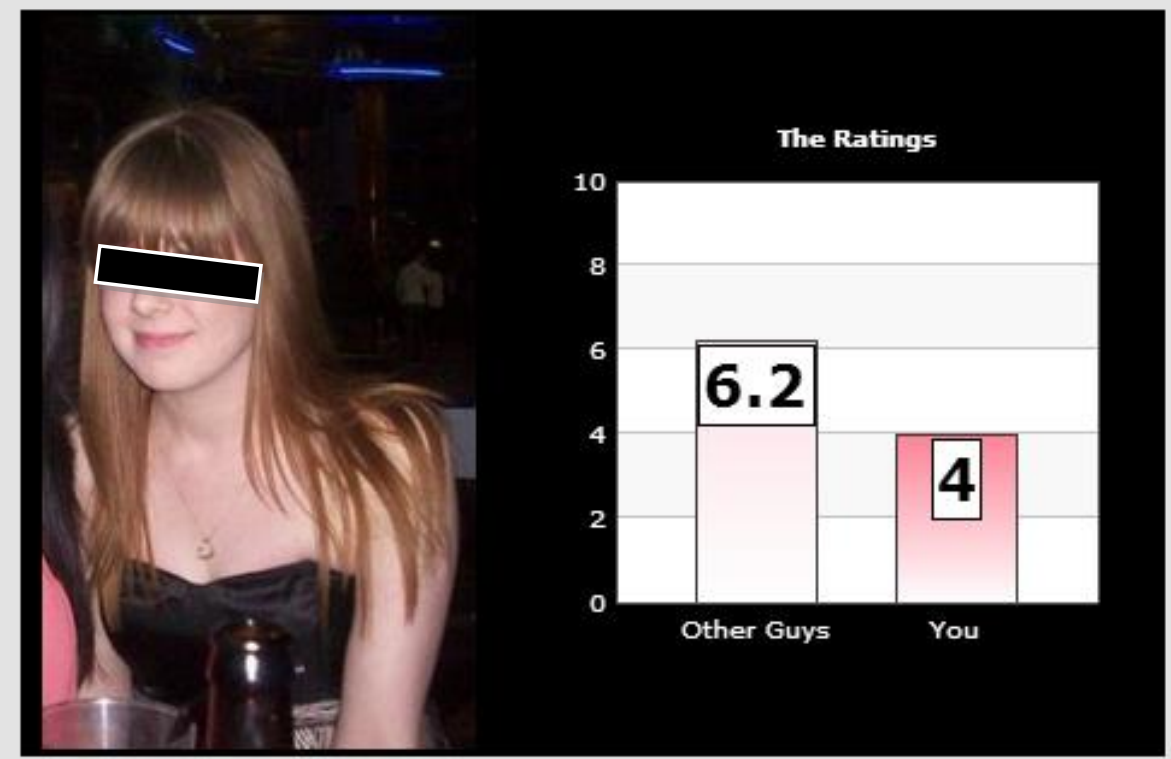

\section{8-25 from Exeter Next}

Figure H.6. An example of the "Feedback page". Participants saw 50 images of women along with an 18-25 age bracket and a location. They also saw a bar chart containing two bars which purportedly represented each woman's rating behaviour. The first bar showed the woman's average rating given to men; the second bar showed the rating of the participant. All reported ratings were bogus and generated by a computer algorithm. 


\section{Mix\&Match}

\section{Log-in Signt-up About}

Summary

Your average rating

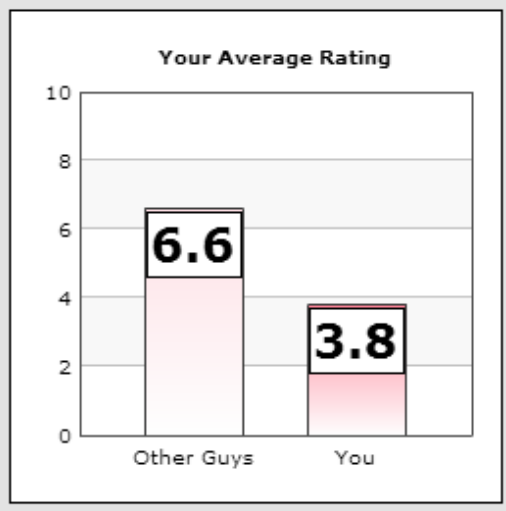

You scored an average rating of 3.8 overall. This was formed from a total of 50 ratings The average score of other males on Mix\&Match is 6.6 .

How you compare

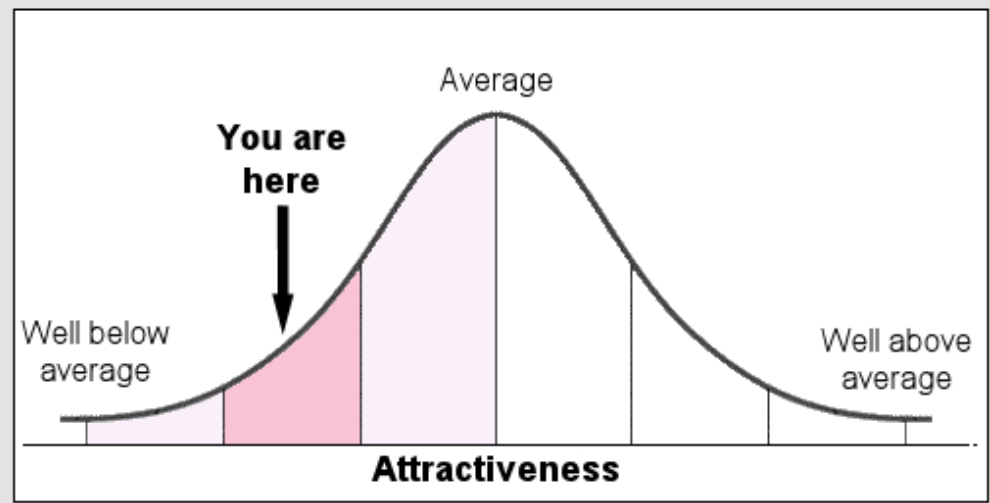

Your current score puts you as lower than average in attractiveness. Of the 629 males tested with Mix\&Match to date 540 (86\%) scored higher than you while $89(14 \%)$ scored lower.

Thank you for helping us to test one of our new site features. Please contact your advisor who will gather your feedback about the feature to help us develop it further.

Figure H.7. An example of the "Completed page". This page gave a fake summary to the participant indicating they were either high or low in attractiveness compared with other men. 


\section{Mix\&Match}

Testing Page Remove Me Log-out About

Main user page

Are you sure you would like to remove your account and stop Beta-testing?

Note: If you testing this website through our collegues at Swansea University, removing your account will withdraw you from the study.

Please remove my account

\section{Mix\&Match}

Mix $\& M$

\section{What is Mix\&Match?}

Mix\&Match is an exciting new website which will allow users to rate the profiles of others for attributes such as attractiveness - and be rated themselves!

However, unlike other rating websites, Mx\&Match will allow users to see their feedback broken down into individual ratings. This means that you'll actually get to see the users who rated you as well as what they selected.

Right now Mix\&Match is in a beta-testing phase only with participation being restricted to selected UK areas. Participation is by invitation only.

If you have any concerns you can contact us at mail@mix-match.org. However, please do not ask for a beta password, we are currently not issuing these through email.

Mix\&Match

Figure H.8. Two additional pages from the Mix\&Match website. The top image shows the page which allows users to remotely remove themselves from the study from outside the lab in between experimental sessions. The second was an "About" page which was viewable by the public. This latter page was present to increase believability. 


\section{Appendix I: Shortened Versions of the Launay-Slade Hallucination Scale (LSHS- R) and the Positive Affect Negative Affect Schedule (PANAS)}

$$
\mathbf{L}-\mathbf{S}^{47}
$$

Please read each sentence and circle the number describes you best. Answer all items even if unsure of your answer. When you have finished, check over each one to make sure you have answered them. Please answer thinking about your usual state during these last five years (excluding taking substances such as cannabis, alcohol, and ecstasy). All responses will be confidential.

\section{KEY:}

0: certainly does not apply to me

1: possibly does not apply to me

2: unsure

3: possibly applied to me

4: certainly applies to me

\# Item

Answer

1
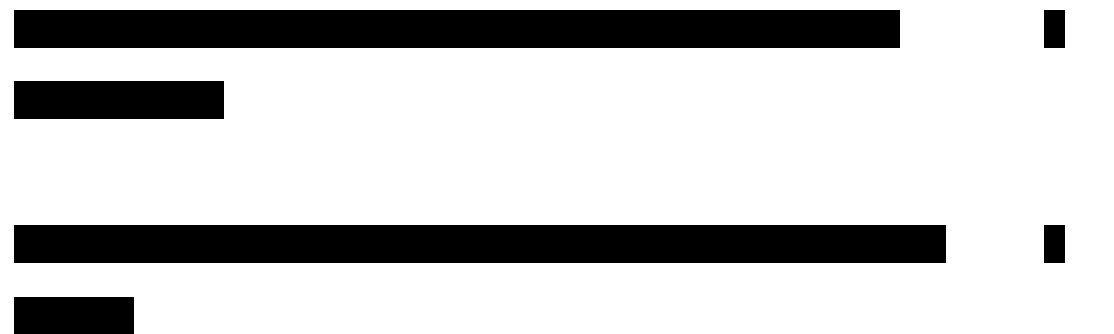

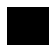
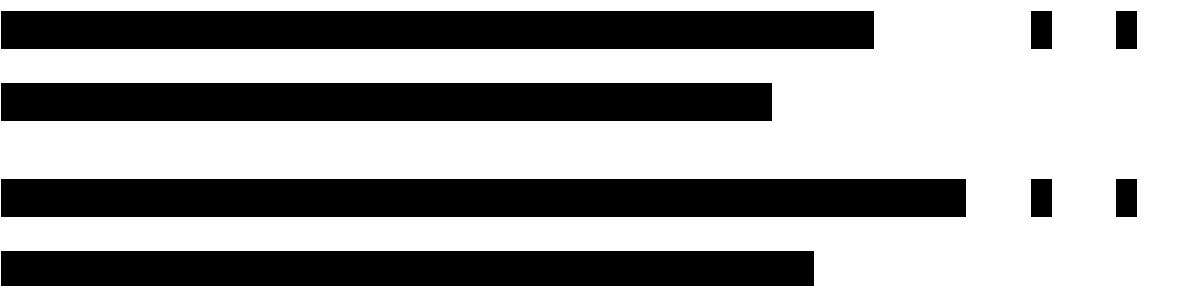

\footnotetext{
${ }^{47}$ Launay, G., \& Slade, P. (1981). The measurement of hallucinatory predisposition in male and female prisoners. Personality and Individual Differences, 2(3), 221-234. doi: 10.1016/0191-8869(81)90027-1
} 


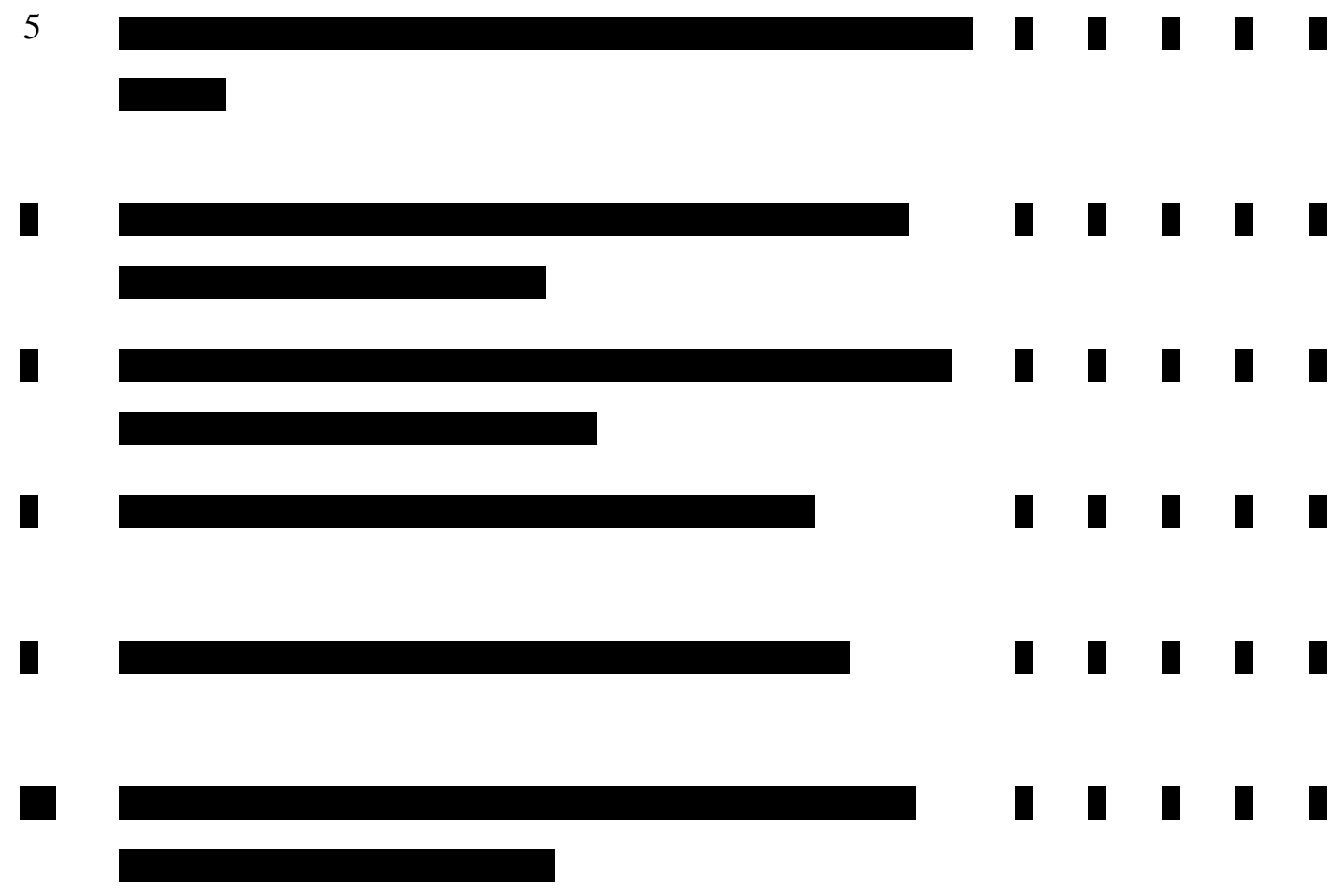



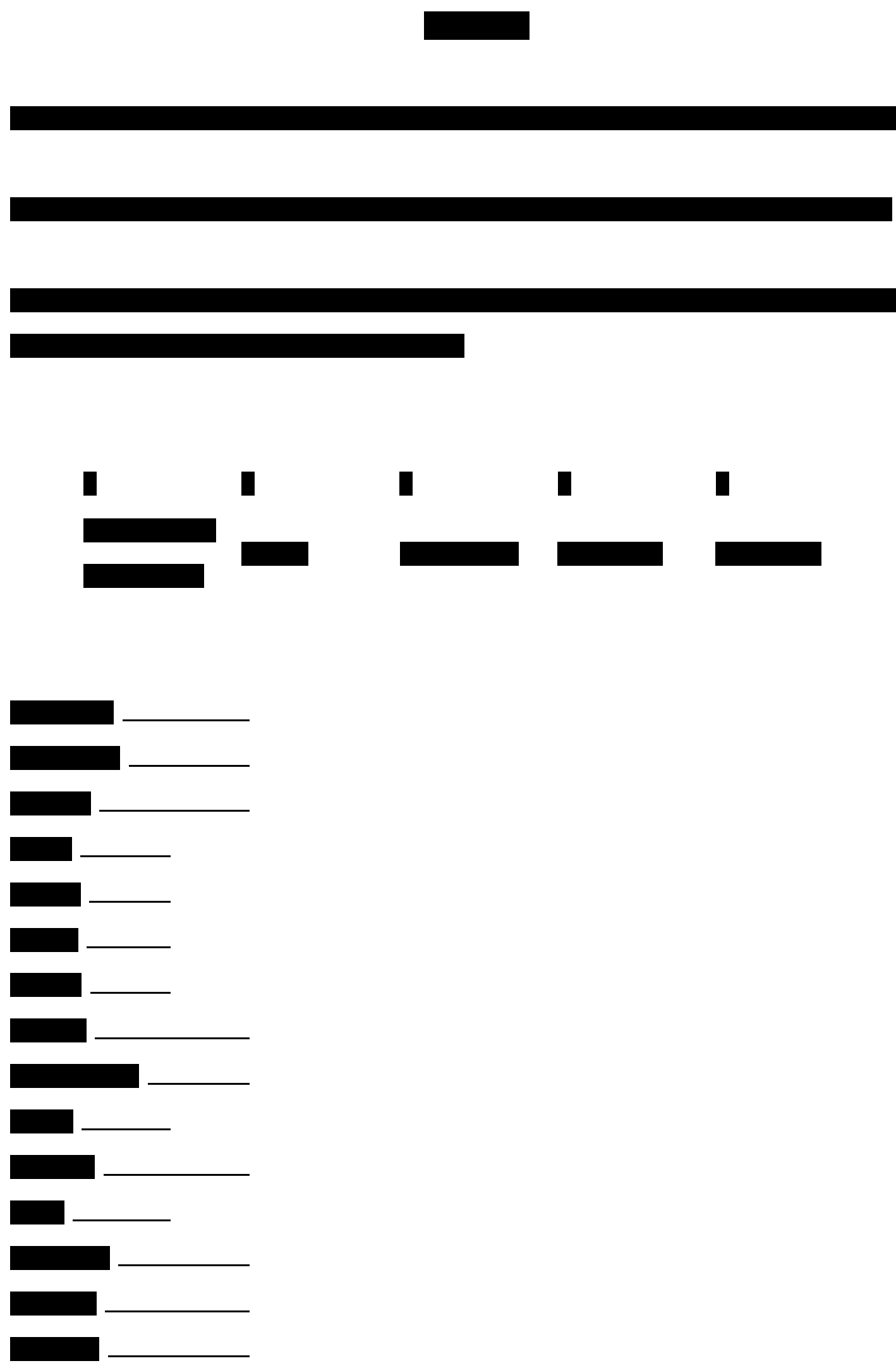

${ }^{48}$ Watson, D., Clark, L. A., \& Tellegen, A. (1988). Development and validation of brief measures of positive and negative affect: The PANAS scales. Journal of Personality and Social Psychology, 54(6), 1063-1070. doi: 10.1037/0022-3514.54.6.1063 


\section{Appendix J: Consent, Debrief, and Post-debrief Questionnaires Used During Experiments 1 and 2}

\section{Consent Form}

Members of the psychology department have been hired to get public feedback on some of the features of a new social website Mix \& Match that will launch in 2012 and would be grateful for your input. The research will be conducted over two twenty minute sessions.

The first session will involve viewing some online profiles and testing the websites "attractiveness rating" feature. You will then be required to create your own profile, which will be viewed and rated by the general public. The second session will then be conducted after one week. In this session you will be able to view the feedback that your profile has received.

You will be able to login to your online profile at any time between session 1 and 2 if you should wish to withdraw, your profile will then be immediately removed from the website. Participation in this research is not compulsory and you are free to withdraw at any time without penalty.

You will have the opportunity to give your feedback at the end of both sessions; feedback and resulting data will be kept anonymous.

You will receive 2 credits for your participation.

If you have any questions, or concerns please feel free to email the university contact for the market researcher Andrew Thomas ([EMAIL ADDRESS]), his assistant [NAME] ([EMAIL ADDRESS]), or contact the website owners directly: mail@mixmatch.org

Please read each of the following statements and, if you agree with them, please sign below to begin the study. 
I consent to participate in this research. I am happy that my picture will be viewed by the public online and I am satisfied with the instructions I have been given.

I have also been informed that any information kept after study termination will be anonymous and confidential in nature.

I have not been coerced in any way to participate and I understand that I may terminate my participation in the study at any point should I wish. I am at least 18 years of age. Data Protection: I agree to the University processing information that I have supplied. I understand that I am unable to withdraw my participation from the study following its termination, because my data will be anonymised and unidentifiable.

Full Name:

Signature: Date: 


\section{Consent Form ${ }^{49}$}

This study is a two part study designed to test the reliability of some questionnaires. That is, we are testing to see whether people score the same on these questionnaires over time.

We will ask you to fill in a number of short questionnaires this week, and then ask you to fill in the same questionnaires approximately one week later.

Some of the questionnaires will contain questions of a sexual nature.

The questionnaires will not be looked at until both are collected and have been anonymised. This means that when the study finishes your data will be made anonymous.

At the end of the study you will receive 2 credits for your participation. Participation in this study is not compulsory and you are free to withdraw at any time without penalty. This study is conducted in accordance with British Psychological Society ethical guidelines.

If you have any queries feel free to contact the experimenter [NAME] ([EMAIL ADDRESS]) or the research supervisor (Dr. Steve Stewart-Williams, Swansea University) at [EMAIL ADDRESS].

\footnotetext{
${ }^{49}$ Note. This second consent form was included to give the impression that the questionnaire measures constituted a separate study.
} 
Please read each of the following statements and, if you agree with them, please sign below to begin the study.

I consent to participate in this study. I am satisfied with the instructions I have been given.

I have also been informed that any information kept after study termination will be anonymous and confidential in nature.

I have not been coerced in any way to participate in this study and I understand that I may terminate my participation in the study at any point should I wish. I am at least 18 years of age.

Data Protection: I agree to the University processing information that I have supplied. I understand that I am unable to withdraw my participation from the study following its termination, because my data will be anonymised and unidentifiable.

Full Name:

Signature: Date: 


\section{Consent Form ${ }^{50}$}

This study is a two part study designed to test the reliability of a new psychological measurement. That is, we are testing to see whether people score the same on this measure over time.

We will ask you to fill in a number of short questionnaires and then perform a computer based task.

The questionnaires involve questions of a sexual nature - specifically about your sexual experience, attitudes and beliefs.

The computer based task involves looking at pictures of the opposite sex and making a judgements as to what type of relationship you'd prefer to have with them.

In approximately one week's time, we will ask you to repeat this task. There may be a small number of changes to its format when you perform it the second time.

The questionnaires and data gathered will not contain any information that can be traced back to you personally and will be added to a pool of data from other participants.

At the end of the study you will receive 2 credits for your participation. Participation in this study is not compulsory and you are free to withdraw at any time without penalty. This study is conducted in accordance with British Psychological Society ethical guidelines.

If you have any queries feel free to contact the experimenter [NAME] ([EMAIL ADDRESS]) or research supervisor (Dr. Steve Stewart-Williams, Swansea University) at [EMAIL ADDRESS].

\footnotetext{
${ }^{50}$ Note. This version second consent form was used in Experiment 2 where the SMA task was used as a dependent variable.
} 
Please read each of the following statements and, if you agree with them, please sign below to begin the study.

I consent to participate in this study. I am satisfied with the instructions I have been given.

I have also been informed that any information kept after study termination will be anonymous and confidential in nature.

I have not been coerced in any way to participate in this study and I understand that I may terminate my participation in the study at any point should I wish. I am at least 18 years of age.

Data Protection: I agree to the University processing information that I have supplied. I understand that I am unable to withdraw my participation from the study following its termination, because my data will be anonymised and unidentifiable.

Full Name:

Signature:

Date: 


\section{Debrief Form}

Thank you for taking part in our experiment.

The aim of the study was to examine the effect of attractiveness feedback on participant mating behaviour.

You were allocated to one of two conditions; the first group (condition 1) received 'positive feedback' that indicated they were above average in attractiveness. The second group (condition 2) received 'negative feedback', that they were below average in attractiveness. All feedback was, in fact, generated by a computer program and was not real; in addition, your photograph was not uploaded to an online forum.

It is hypothesised that participants in condition 1 will tend to alter their mating behaviour towards short-term relationships, whereas those in condition 2 will lean towards more long-term relationships. Due to the confidentiality and anonymity of all experimental data, it will not be possible to give individual feedback as to your results in this study.

Following this information, if you wish to withdraw your data, please inform the experimenter.

If you would like more information with regards to the background literature that the present study is based on, please see the following references:

Buss, D.M. (2003). The Evolution of Desire. New York: Basic Books.

Penke, L. \& Asendorpf, J.B. (2008). Beyond Global Sociosexual Orientations: A More Differentiated Look at Sociosexuality and Its Effects on Courtship and Romantic Relationships. Journal of Personality and Social Psychology, 95(5), 1113-1135. 
Alternatively, if you have any further questions regarding the research, or feedback about how the experiment was conducted, please contact either [NAME] ([EMAIL ADDRESS]) or Dr. Steve Stewart Williams ([EMAIL ADDRESS]; Study Supervisor). 


\section{Debrief Form (Email Opt-Out $)^{51}$}

Dear [PARTICIPANT NAME].

Thank you for informing us of your wish to opt-out of the Mix\&Match study.

This email contains some important information regarding the study.

This study was and experiment designed to examine the factors which contribute to the types of relationships people engage in. Specifically, we are looking to see if positive or negative feedback about physical attractiveness can change an individual's preference for a short or long-term relationship.

In order to test this it was necessary to lead you to believe that your picture was being shown to members of the public and rated for attractiveness.

However, this was not the case and your picture was not shown to the public. The Mix\&Match website is a research tool designed to look and act like a real website with an online community.

Following this email, the photo you submitted and "online profile" will be deleted. Your participation in the study up to this point is greatly appreciated. You will be credited for the first study session you attended accordingly.

We would further ask you not to mention your participation in this study to other students who may be asked to participate themselves.

If you have any further questions or queries regarding this experiment feel free to contact the researcher (Andrew Thomas: [EMAIL ADDRESS]) or research supervisor (Dr. Steve Stewart-Williams: [EMAIL ADDRESS]).

Below are some references related to this research area which may be of interest.

${ }^{51}$ Note. This debrief form was designed to be emailed to any participant who withdrew from Experiment 1 or Experiment 2 remotely. It did not need to be used. 
References

Buss, D.M. (2003). The evolution of desire. New York: Basic Books.

Gangestad, S.W., \& Simpson, J.A. (2000). The evolution of human mating: trade-offs and strategic pluralism. Behavioral and Brain Sciences, 23(4), 573-587.

Schmitt, D.P. (2005). Sociosexuality from Argentina to Zimbabwe: A 48-nation study of sex, culture, and strategies of human mating. Behavioral and Brain Sciences, 28, 247-311.

Simpson, J.A., \& Gangestad, S.W. (1992). Sociosexuality and romantic partner choice. Journal of Personality, 60, 31-51. 


\section{Post-Session Questionnaire}

We would appreciate it if you could answer a few more questions with regard to your experience within this experiment.

After session 1 of the experiment, how convinced were you that Mix\&Match was a real website?

$\begin{array}{lllllllllll}\text { Not convinced } & 1 & 2 & 3 & 4 & 5 & 6 & 7 & 8 & 9 & \text { Fully }\end{array}$

at all

convinced

After session 1 of the experiment, how convinced were you that your photo was being rated by real people?

$\begin{array}{lllllllllll}\text { Not convinced } & 1 & 2 & 3 & 4 & 5 & 6 & 7 & 8 & 9 & \text { Fully }\end{array}$

at all

convinced

During session 2 of the experiment, how convinced were you that the feedback you were given was real?

$\begin{array}{lllllllllll}\text { Not convinced } & 1 & 2 & 3 & 4 & 5 & 6 & 7 & 8 & 9 & \text { Fully }\end{array}$

at all

convinced

Now that you have completed the experiment, do you still believe the feedback you received was real?

$\begin{array}{lllllllllll}\text { Not at all } & 1 & 2 & 3 & 4 & 5 & 6 & 7 & 8 & 9 & \text { Very much so }\end{array}$

How truthfully did you answer the questionnaire given to you about your sexual 
thoughts, feelings and behaviours?

$\begin{array}{lllllllllll}\text { Not truthful } & 1 & 2 & 3 & 4 & 5 & 6 & 7 & 8 & 9 & \text { Very truthful }\end{array}$

at all

How enjoyable did you find the experiment to participate in?

$\begin{array}{lllllllllll}\text { Not enjoyable } & 1 & 2 & 3 & 4 & 5 & 6 & 7 & 8 & 9 & \text { Very enjoyable }\end{array}$ at all 


\section{Appendix K: Bogus Market Research Forms used in Experiments 1 and 2}

\section{Mix\&Match}

Beta-tester feedback form - Week 1

Thank you for helping us test Mix\&Match

We're interested in what you think of the website so far. Please answer the questions below and be as honest as you can.

General usage

On a scale of 1-9 how much did you enjoy rating others using Mix\&Match? (Please circle).

$\begin{array}{lllllllllll}\text { Not at all } & 1 & 2 & 3 & 4 & 5 & 6 & 7 & 8 & 9 & \text { A lot }\end{array}$

On a scale of 1-9 how likely would you be to use Mix\&Match when it launches late 2010? (Please circle).

$\begin{array}{lllllllllll}\text { Not likely } & 1 & 2 & 3 & 4 & 5 & 6 & 7 & 8 & 9 & \text { Definitely will }\end{array}$

On a scale of 1-9 how likely would you be to recommend Mix\&Match to a friend? (Please circle).

Not likely

$\begin{array}{llllllllll}1 & 2 & 3 & 4 & 5 & 6 & 7 & 8 & 9 & \text { Definitely will }\end{array}$

On a scale of 1-9 how unique an experience did you find Mix\&Match? (Please circle).

Not at all

$\begin{array}{llllllllll}1 & 2 & 3 & 4 & 5 & 6 & 7 & 8 & 9 & \text { A lot }\end{array}$


On a scale of 1-9 how excited are you about receiving your attractiveness feedback next week? (Please circle).

$\begin{array}{lllllllllll}\text { Not at all } & 1 & 2 & 3 & 4 & 5 & 6 & 7 & 8 & 9 & \text { A lot }\end{array}$

When using Mix\&Match did you recognise any individuals using the system?

$\square \quad$ Yes

$\square \quad$ No

If you answered 'yes' to question 6, how do you know that person?

Finally, do you have any other feedback you wish to send us regarding Mix\&Match?

We're all done. Thank you for your feedback! 


\section{Mix\&Match}

Beta-tester feedback form - Week 2

Thank you for helping us test Mix\&Match

Now that you have tried out some of the features of our website we would love to get your feedback. Please answer the questions below and be as honest as you can.

When did you complete your first session with us? : days ago

General usage

On a scale of 1-9 how much did you enjoy using Mix\&Match? (Please circle).

$\begin{array}{lllllllllll}\text { Not at all } & 1 & 2 & 3 & 4 & 5 & 6 & 7 & 8 & 9 & \text { A lot }\end{array}$

On a scale of 1-9 how likely would you be to use Mix\&Match when it launches late 2010? (Please circle).

$\begin{array}{lllllllllll}\text { Not likely } & 1 & 2 & 3 & 4 & 5 & 6 & 7 & 8 & 9 & \text { Definitely will }\end{array}$

On a scale of 1-9 how likely would you be to recommend Mix\&Match to a friend? (Please circle).

$\begin{array}{lllllllllll}\text { Not likely } & 1 & 2 & 3 & 4 & 5 & 6 & 7 & 8 & 9 & \text { Definitely will }\end{array}$

On a scale of 1-9 how unique an experience did you find Mix\&Match? (Please circle).

$\begin{array}{lllllllllll}\text { Not at all } & 1 & 2 & 3 & 4 & 5 & 6 & 7 & 8 & 9 & \text { A lot }\end{array}$

On a scale of 1-9 how accurate did you find the feedback your peers gave you? (Please circle). 
$\begin{array}{lllllllllll}\text { Not accurate } & 1 & 2 & 3 & 4 & 5 & 6 & 7 & 8 & 9 & \text { Very accurate }\end{array}$ at all

On a scale of 1-9 when receiving your feedback, how important was it for you that your raters were from the same region (Please circle).

$\begin{array}{lllllllllll}\text { Not important } & 1 & 2 & 3 & 4 & 5 & 6 & 7 & 8 & 9 & \text { Very important }\end{array}$ at all

On a scale of 1-9 when receiving your feedback, how important was it for you that your raters were from the same age (Please circle).

$\begin{array}{lllllllllll}\text { Not important } & 1 & 2 & 3 & 4 & 5 & 6 & 7 & 8 & 9 & \text { Very important }\end{array}$ at all

When using Mix\&Match did you recognise any individuals using the system?

$$
\text { Yes }
$$

No

If you answered 'yes' to question 8 , how do you know that person?

Finally, do you have any other feedback you wish to send us regarding Mix\&Match?

We're all done. Thank you for your feedback! 


\section{Appendix L: Consent and Debrief Forms for Experiments 3 and 4}

\section{Experiment 3}

\section{$\underline{\text { Participant Information Sheet }}$}

Please read the information on this sheet carefully

This study is about social perception and relationship choices. If you decide to take part you will be asked to complete a series of questionnaires, complete two computer tasks, and watch some person-centred videos.

If you are currently in a relationship, the computer task requires that you imagine you are single throughout when responding. Full instructions regarding what to do during the computer task will appear on the screen before the task commences.

Completion of the study should take no longer than 15 to 25 minutes.

This study is voluntary therefore you are free to withdraw your participation or your data from this investigation now or at any stage without penalty.

Please note that some questions are of a sexual nature and you may skip a question if you do not want to answer it. However, any questions you do answer will be entirely anonymous. If you agree to participate, the consent form that you will need to sign will be kept separately from the questionnaires in order to ensure your anonymity.

This study is conducted in accordance with the British Psychological Society, and has been approved by departmental ethics guidelines.

If you agree to take part in this experiment please read the consent form on the next page and then sign it if you agree with the statements. 


\section{$\underline{\text { Participant Consent Form }}$}

I consent to participate in this study. I am satisfied with the instructions I have been given so far and I have been reassured that any further information I request regarding the study will be supplied to me at the end of the experiment.

I have been informed that the data I provide will remain confidential and be password protected. I am free to ask any questions at any time before and during the study.

I understand that I will be required to answer questions of a sexual nature. I understand that I am free to withdraw my consent and terminate my participation at any time and without penalty.

I have not been coerced in any way to participate in this study and I understand that I may terminate my participation in the study at any point should I wish to do so. I am at least 18 years of age.

Data Protection: I agree to the University processing personal data that I have supplied. I agree to the processing of such data for any purposes connected with the Research Project as outlined to me.

Name: Signature:

Date: Student Number:

Researcher: [NAME] ([EMAIL ADDRESS])

Research Supervisor: Dr Steve Stewart-Williams ([EMAIL ADDRESS]) 


\section{$\underline{\text { Participant Debrief Form }}$}

Thank you for taking time to participate in the study, it is much appreciated.

This study is about relationship choices and is investigating whether parental cues, such as looking at a video of how to bathe your baby affects an individual's preference for long-term relationships over short-term ones. One group was show videos to do with parenting, while another was shown control videos.

It is important that you are aware that all results are anonymous and confidential and are strictly used for research purposes only. If, following this information, you wish to withdraw your data please let me know and I will do so. If you would like more information about the background literature on this study here are some references:

I hope you have enjoyed taking part in the study, and below is a small list of reading that is relevant to the topic if you are interested in knowing more about mating strategies.

If you have any further comments or queries, please email myself at [EMAIL ADDRESS] or Dr. Steve Stewart-Williams at [EMAIL ADDRESS]

\section{References}

Buss, D. M. (1994). The evolution of desire: Strategies of human mating. New York: Basic.

Buss, D. M., \& Schmitt, D. P. (1993). Sexual Strategies Theory: An evolutionary perspective on human mating. Psychological Review, 100(2), 204-232.

Gangestad, S. \& Simpson, J. (2000). The evolution of human mating; Trade-offs and strategic pluralism. Behavioural and Brain Sciences, 23(4), 587-644. 


\section{Experiment 4}

\section{Information about the study}

Please read the information on this sheet carefully

In this study you will be asked to complete some questionnaires, a computer based task, and a memory test. The questionnaires will contain some anonymous questions of a sexual nature.

For the computer based task, you'll be required to make some relationship choices. That is, we'll ask you to look at some pictures of individuals and decide what relationship type you'd prefer to have with them.

You may find the topic of investigation particularly sensitive. If you think you may be affected you are free to withdraw your participation or your data from this investigation now or at any stage without penalty.

For the memory test we'll ask you to view a slideshow of some pictures and then complete a questionnaire to test your memory.

This study is conducted in accordance with British Psychological Society, and departmental ethics guidelines. Your rights as a participant, including the right to withdraw at any point without penalty, are ensured. All results will be anonymous and it will be possible to identify individual participant's data.

If you agree to take part in this study then please read and sign the consent form on the next page. 


\section{$\underline{\text { Participant Consent Form }}$}

I consent to participate in this study. I am satisfied with the instructions I have been given so far and I have been reassured that any further information I request regarding the study will be supplied to me at the end of the experiment.

I have been informed that the data I provide will remain confidential and be password protected. I am free to ask any questions at any time before and during the study. I have been provided with a copy of this form and the participant information sheet.

I have not been coerced in any way to participate in this study and I understand that I may terminate my participation in the study at any point should I so wish. I am at least 18 years of age.

Data Protection: I agree to the University processing personal data that I have supplied. I agree to the processing of such data for any purposes connected with the Research Project as outlined to me.

Participant signature: Date:

Researcher(s)'s contact details: [NAME] ([EMAIL ADDRESS])

Supervisor contact details: Dr. Steve Stewart-Williams ([EMAIL ADDRESS]) 


\section{$\underline{\text { Participant Debrief Form }}$}

Thank you for taking time to participate in the study, it is much appreciated.

This study is about relationship choices and is investigating whether wealth cues, such as looking at images of expensive items, can change an individual's preference for longterm relationships over short-term ones. One group was shown a slideshow to do with wealth, while another was shown a control slideshow.

It is important that you are aware that all results are anonymous and confidential and are strictly used for research purposes only. If, following this information, you wish to withdraw your data please let me know and I will do so.:

I hope you have enjoyed taking part in the study, and below is a small list of reading that is relevant to the topic if you are interested in knowing more about mating strategies.

If you have any further comments or queries, please email me at [EMAIL ADDRESS] or Dr. Steve Stewart-Williams at [EMAIL ADDRESS].

\section{References}

Buss, D. M. (1994). The evolution of desire: Strategies of human mating. New York: Basic.

Buss, D. M., \& Schmitt, D. P. (1993). Sexual Strategies Theory: An evolutionary perspective on human mating. Psychological Review, 100(2), 204-232.

Gangestad, S. \& Simpson, J. (2000). The evolution of human mating; Trade-offs and strategic pluralism. Behavioural and Brain Sciences, 23(4), 587-644. 
Appendix M: Screenshots of the Videos Used in Experiment 3

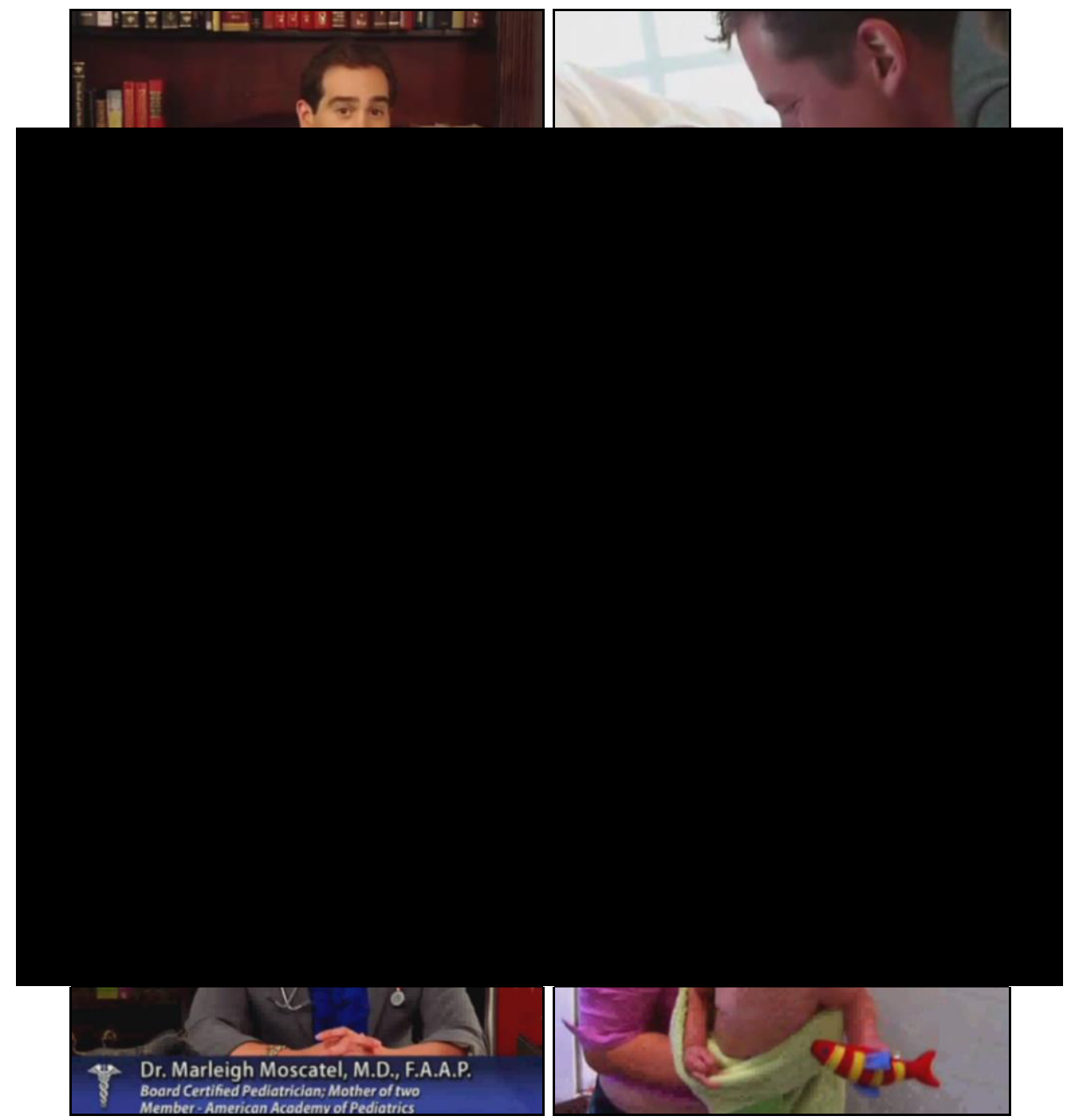

Figure M.1. Screenshots of the video used as parental stimuli during Experiment 3. 


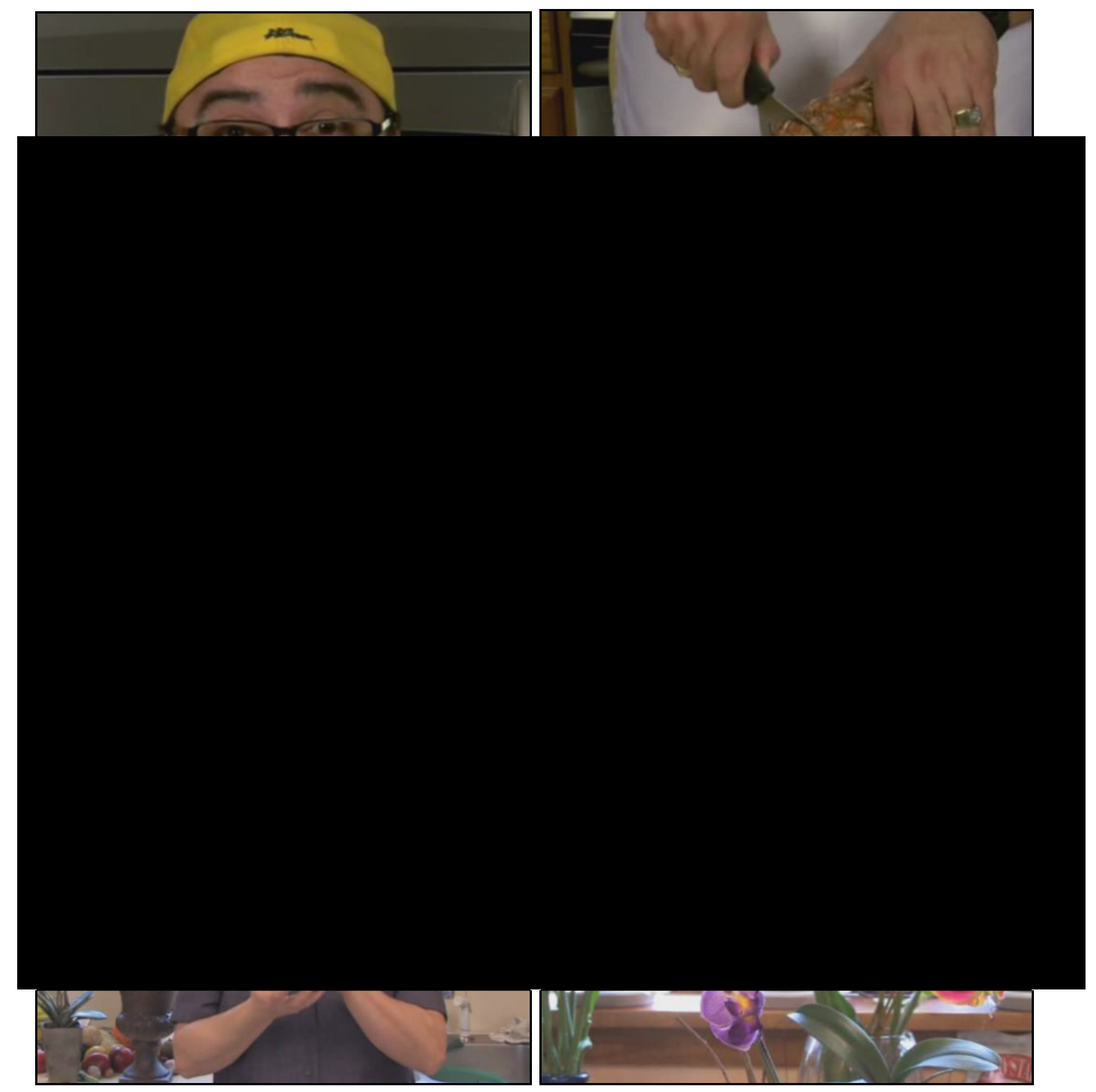

Figure M.2. Screenshots of the video used as neutral stimuli during Experiment 3. 
Appendix N: Examples of the Slideshow Images Used in Experiment 4

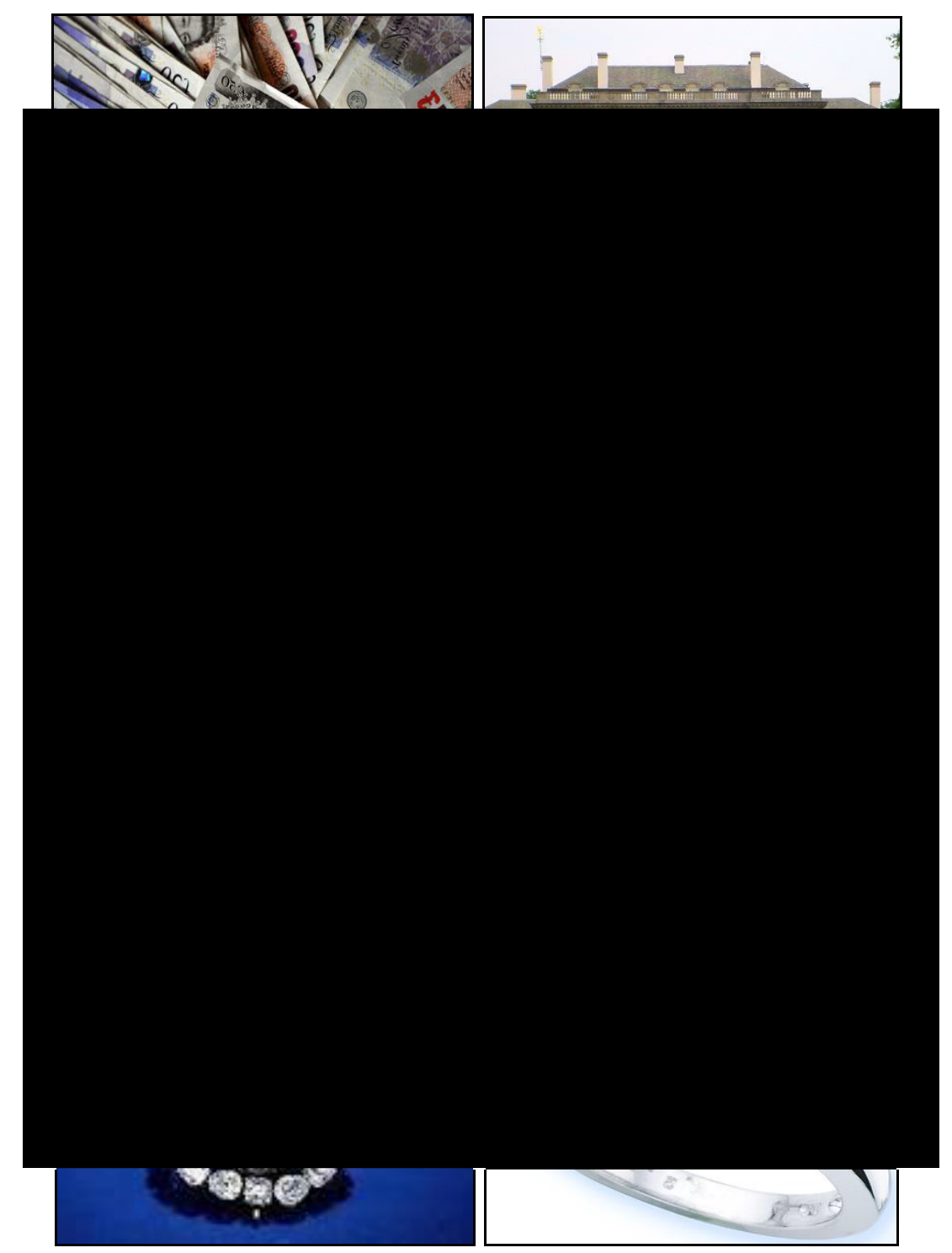

Figure N.1. Eight example images shown during the wealth slideshow in the experimental condition. 


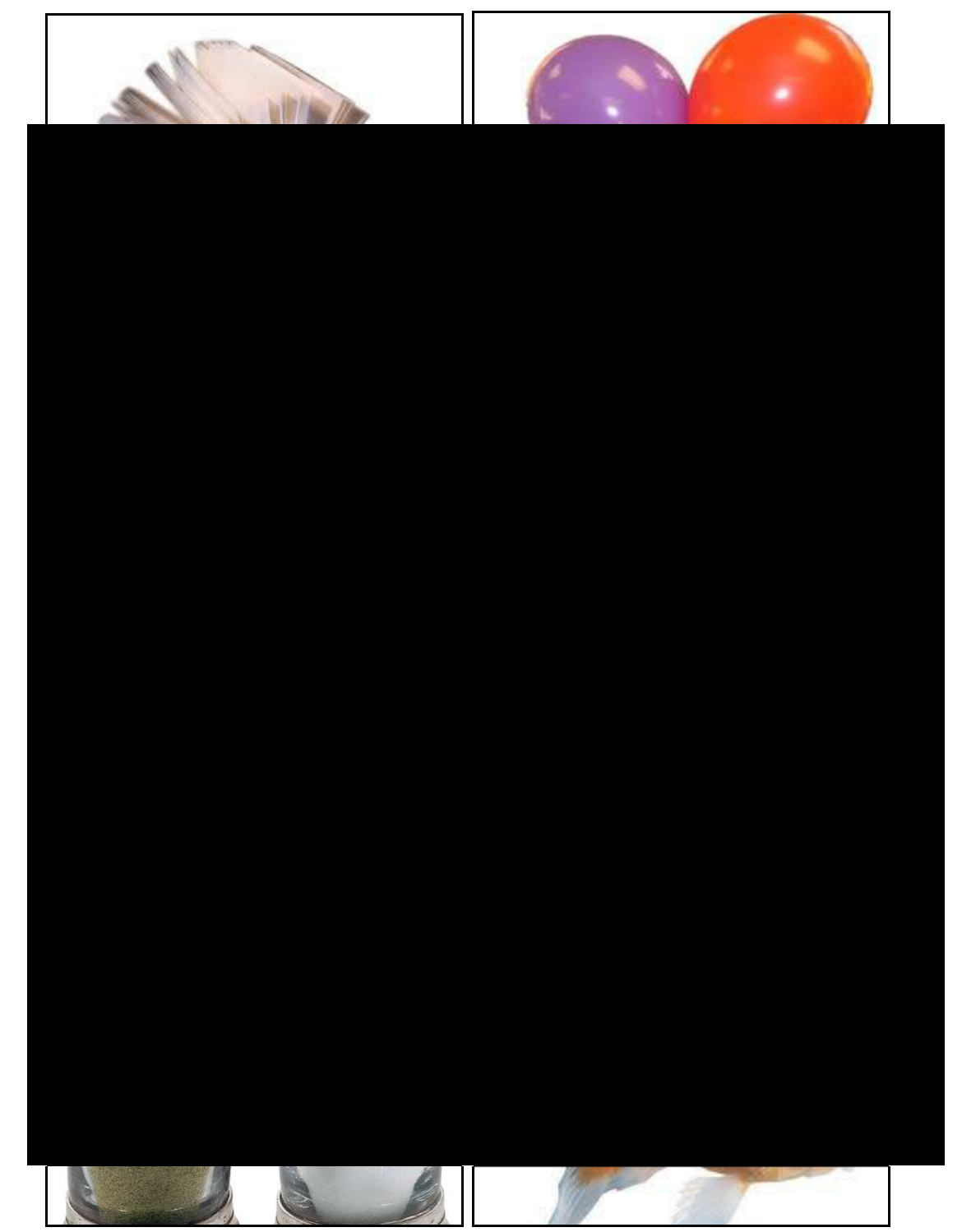

Figure N.2. Eight example images shown during the neutral slideshow in the control condition. 


\section{Appendix O: Consent and Debrief Forms for Experiments 5, 6 and 8}

\section{Experiment 5}

\section{Information about the study}

Please read the information on this sheet carefully

In this study you will be asked to complete some questionnaires, a computer based task, and a memory test. The questionnaires will contain some anonymous questions of a sexual nature.

For the computer based task, you'll be required to make some relationship choices. That is, we'll ask you to look at some pictures of individuals and decide what relationship type you'd prefer to have with them.

For the memory test we'll ask you to view a slideshow of some pictures and then complete a questionnaire to test your memory. This slideshow will contain images of snakes, spiders, crowds, and aggressive animals.

You may find the topic of investigation particularly sensitive. If you think you may be affected you are free to withdraw your participation or your data from this investigation now or at any stage without penalty.

This study is conducted in accordance with the British Psychological Society's ethical guidelines. Your rights as a participant, including the right to withdraw at any point without penalty, are ensured. All results will be anonymous and it will be possible to identify individual participant's data.

If you agree to take part in this study please read and sign the consent form on the next page. 


\section{$\underline{\text { Participant Consent Form }}$}

I consent to participate in this study. I am satisfied with the instructions I have been given so far and I have been reassured that any further information I request regarding the study will be supplied to me at the end of the experiment.

I have been informed that the data I provide will remain confidential and be password protected. I am free to ask any questions at any time before and during the study. I have been provided with a copy of this form and the participant information sheet.

I have not been coerced in any way to participate in this study and I understand that I may terminate my participation in the study at any point should I so wish. I am at least 18 years of age.

Data Protection: I agree to the University processing personal data that I have supplied. I agree to the processing of such data for any purposes connected with the Research Project as outlined to me.

Participant signature Date

Researcher(s)'s contact details: [NAME] ([EMAIL HERE ADDRESS])

Supervisor contact details: Dr. Steve Stewart-Williams ([EMAIL ADDRESS]) 


\section{$\underline{\text { Participant Debrief Form }}$}

Thank you for taking time to participate in the study, it is much appreciated.

This study is about relationship choices and is investigating whether danger cues, such as looking at images of spiders and snakes, affects an individual's preference for longterm relationships over short-term ones. One group was shown a slideshow to do with danger, while another was shown a control slideshow.

It is important that you are aware that all results are anonymous and confidential and are strictly used for research purposes only. If following this information you wish to withdraw your data please let me know and I will do so. If you would like more information about the background literature on this study here are some references:

I hope you have enjoyed taking part in the study, and below is a small list of reading that is relevant to the topic if you are interested in knowing more about mating strategies.

If you have any further comments or queries, please email me at [EMAIL ADDRESS] or Dr Steve Stewart-Williams at [EMAIL ADDRESS].

\section{References}

Buss, D. M. (1994). The evolution of desire: Strategies of human mating. New York: Basic.

Buss, D. M., \& Schmitt, D. P. (1993). Sexual Strategies Theory: An evolutionary perspective on human mating. Psychological Review, 100(2), 204-232.

Gangestad, S. \& Simpson, J. (2000). The evolution of human mating; Trade-offs and strategic pluralism. Behavioural and Brain Sciences, 23(4), 587-644. 


\section{Experiments 6 and 8}

\section{$\underline{\text { Consent Form }}$}

Relationship choice and creativity

What will you do in this research: At the start of the study we will ask you to complete two anonymous questionnaires designed for us to learn more about you. These questionnaires will ask you to indicate things such as your age and relationship status. There will also be some questions of a sexual nature. If you feel uncomfortable answering any particular question you may skip it.

We will then ask you to complete a relationship choice task. This short task takes about six minutes and involves looking at pictures of males/females and telling us which type of relationship you'd prefer with them. It will require you to imagine yourself as being single and open to starting a new relationship.

A creative measuring task will then begin which will require you to imagine a particular experience as vividly as you can and then discuss it in as much detail as possible.

To make sure we have an accurate view of your relationship preferences the relationship choice task will be administered a second time.

Time required: Participation will take between 20-25 minutes to complete.

Risks: Questions of a sexual nature will be asked but information will be kept strictly anonymous. The creativity task may ask you to imagine yourself in a dangerous or upsetting situation. Although we don't anticipate risk, should you feel uncomfortable you will be allowed to withdraw from the study at any time without penalty and discuss any concerns with the researcher.

Compensation: Compensation for the study varies. Please see the researcher for details.

Confidentiality: Your participation in this study will remain confidential, and your identity will not be stored with your data. Your responses will be assigned a participant number, and kept in a sealed envelope 
Participation and Withdrawal: Your participation in this study is completely voluntary, and you may withdraw at any time without penalty. You may withdraw by informing the researcher that you no longer wish to participate (no questions will be asked).

How to contact researchers: If you have questions or concerns about your participation or payment, or want to request a summary of research findings, please contact the researcher: [NAME] ([EMAIL ADDRESS])

For any other problems related to this study, you may also contact the staff member supervising this work Dr. Steve Stewart-Williams ([EMAIL ADDRESS]).

Agreement:

The nature and purpose of this research have been sufficiently explained and I agree to participate in this study. I understand that I am free to withdraw at any time without incurring any penalty.

Signature:

Date:

Name (print): 


\section{$\underline{\text { Participant Debrief Form }}$}

Thank you for participating in the present study concerning relationship choice and creativity. We are currently interested in the effects of various cues on an individual's relationship preferences. That is, we are looking to see if an individual's preference for long- and short-term relationships changes under certain conditions.

In this particular test, we used a creativity task to encourage you to think carefully about a theme that we feel may or may not influence relationship preferences.

At the end of this form are a few references related to this study that may be of interest to you. Should you have any questions about the research feel free to ask the researcher [NAME] now or contact them another time at: [EMAIL ADDRESS].

Alternatively, you can contact the supervisor of this project (Dr. Steve StewartWilliams) by email: [EMAIL ADDRESS].

As a reminder, all results we have gathered during this study are anonymous. They will be mixed together with those of other participants and will not be able to be personally traced back to you. Should you object to your data being used in the study, then please let the researcher know now as it will become impossible to do so once the data becomes combined with others.

Again, thank you for your participation in this study. If you know of any friends or acquaintances that are eligible to participate in this study, we request that you not discuss it with them until after they have had the opportunity to participate. Prior knowledge of questions asked during the study can invalidate the results. We greatly appreciate your cooperation. 
References

Buss, D. M., \& Schmitt, D. P. (1993). Sexual Strategies Theory: An evolutionary perspective on human mating. Psychological Review, 100(2), 204-232. doi: $10.1037 / 0033-295 x .100 .2 .204$

Buss, D. M. (1994). The evolution of desire: Strategies of human mating. New York: BasicBooks

Wilson, M., \& Daly, M. (2004). Do pretty women inspire men to discount the future? Proceedings of the Royal Society of London. Series B: Biological Sciences, 271(Suppl 4), S177-S179. doi: 10.1098/rsbl.2003.0134 
Appendix P: Examples of the Slideshow Images Used in Experiment 5

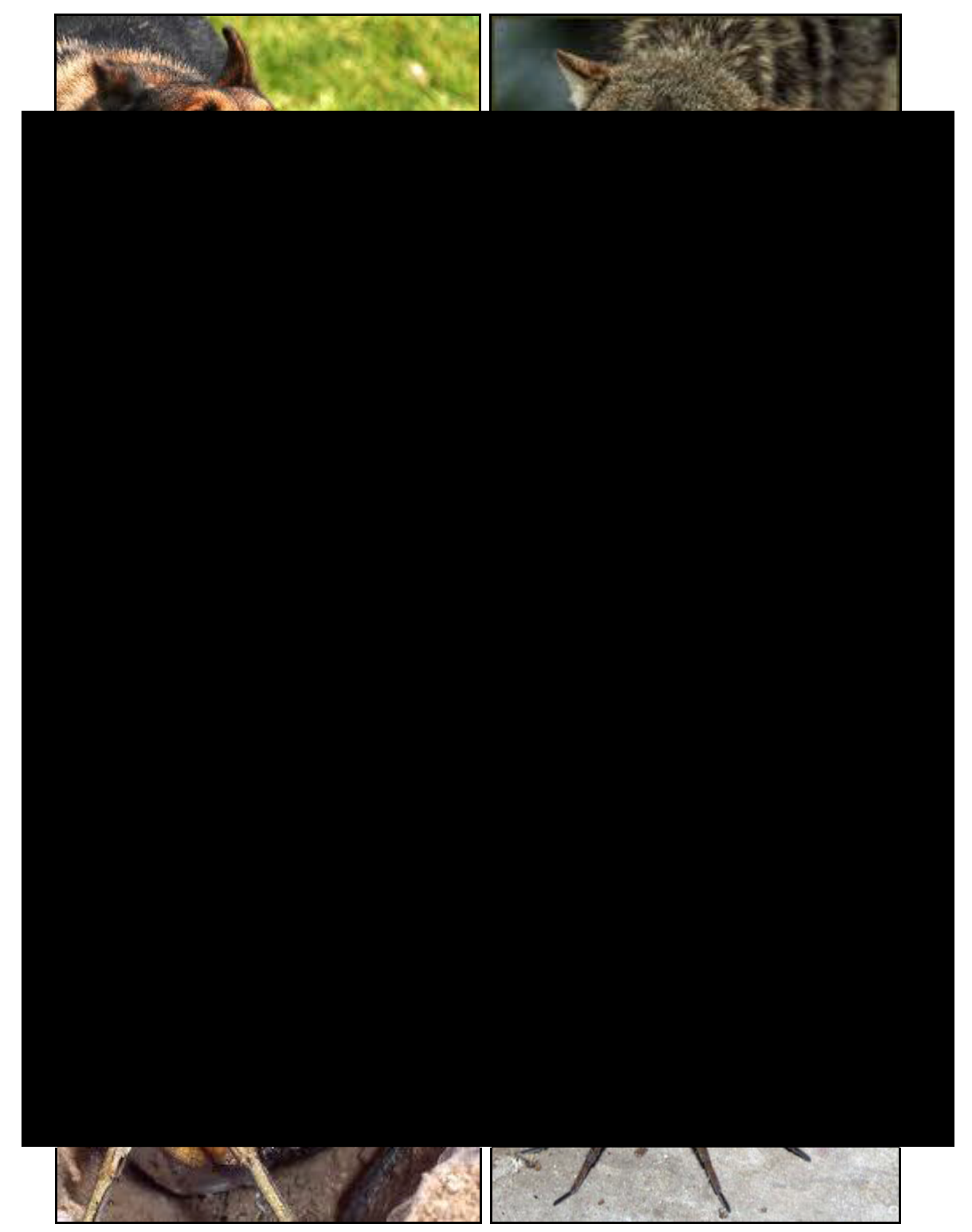

Figure P.1. Eight example images shown during the danger slideshow in the experimental condition. 


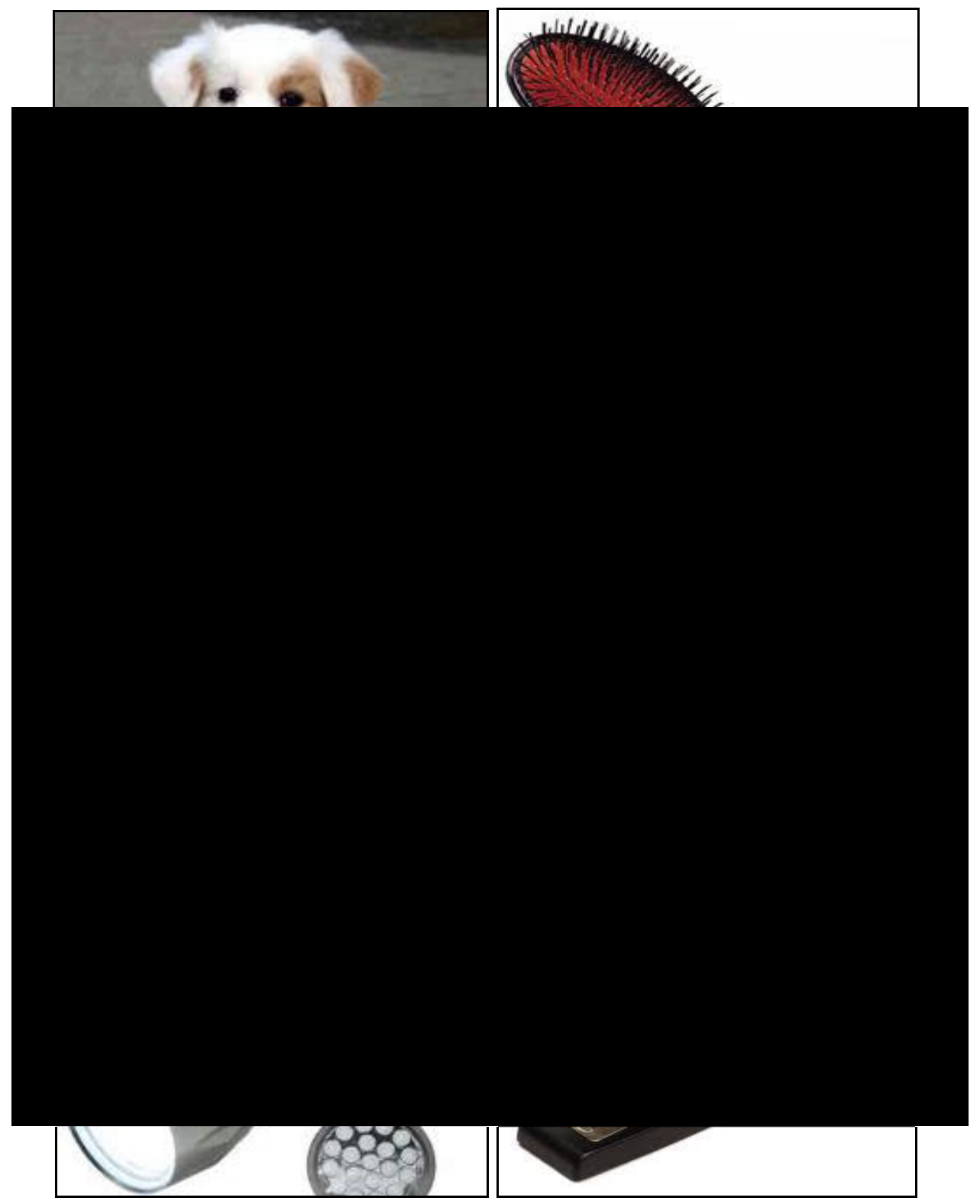

Figure P.2. Eight example images shown during the neutral slideshow in the control condition. 


\section{Appendix Q: Wording and Screenshots of the Revised SMA Task}

Welcome to the relationship choice task! During this task we will ask you to imagine yourself in a certain situation and make a judgment about how you'd behave.

For the purpose of this study we would like you to act as if you were single and open to starting a new relationship as we show you several pictures of people. As you view each person we would like you to imagine yourself in the following scenario: 'You have met them once or twice through friends and have gotten on well with them each time. They appear to have similar interests to you and you're comfortable hanging out with them.'

'After a while this person asks you if you be interested in a relationship with them. They make it clear that they are looking for a [relationship type]'.

[At random, one of the two following descriptions is shown to the participant.]

Long-term relationships are marked by love and commitment and are long in duration (months or years).

Short-term relationships are marked by brief affairs, one-night stands or temporary liaisons (days or weeks).

With this in mind, we would like you to tell us if you would be interested in the offer by selecting 'Yes' or 'No'. There is no right or wrong answer, so please go with your gut feeling.

[The participant then completes the ratings for the first relationship type.] 

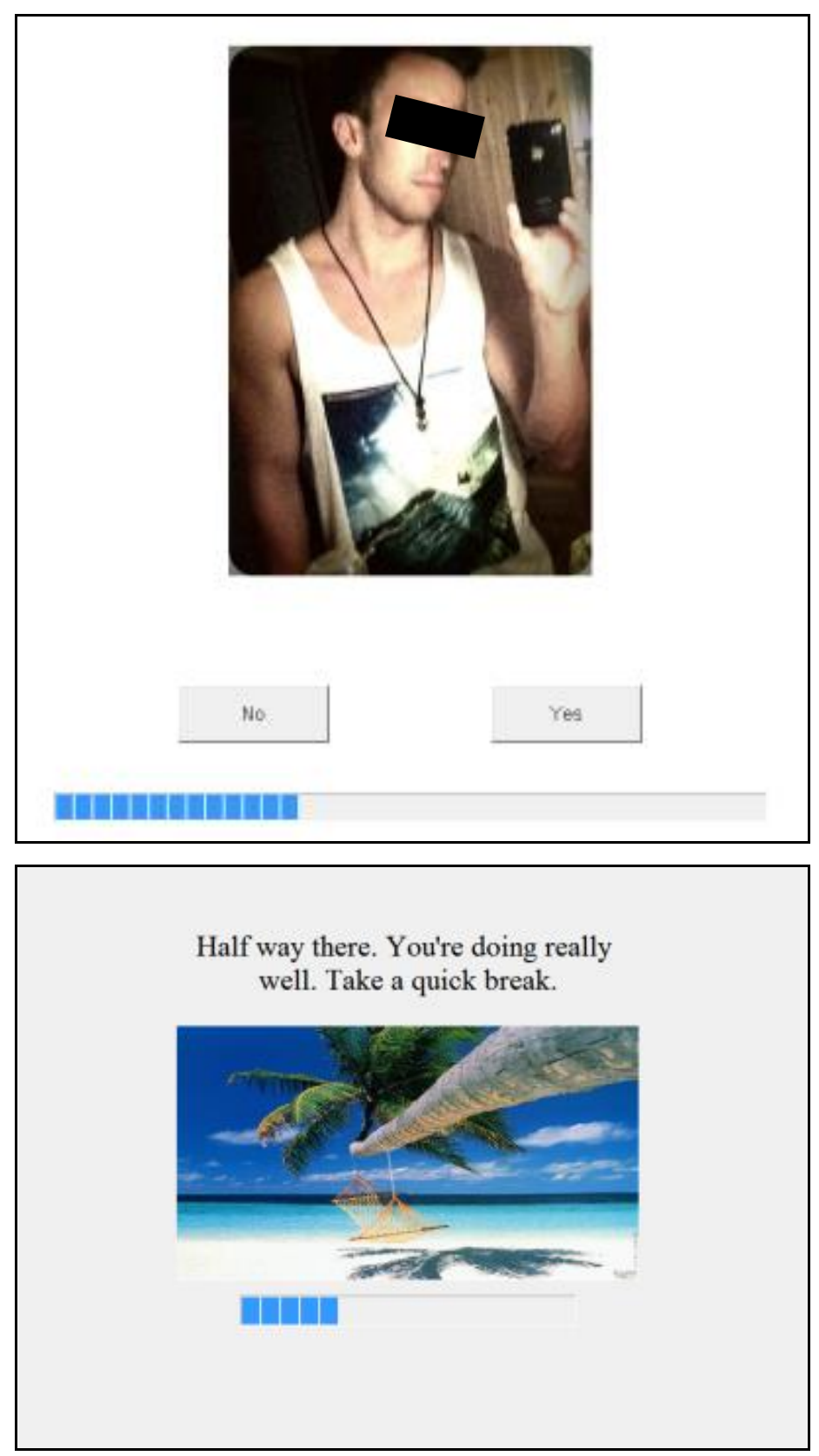

Figure Q.1. Two screenshots of the revised SMA task. The first (top) shows the 'yes' and 'no' rating system complete with a progress bar. The second (bottom) shows the page which was shown to participants before rating the same models for a different relationship type. This page stayed on the screen for 30 seconds. 
You've reached the second half of the relationship choice task. The second half is much like the first, although now we're interested in your responses to a different relationship type. Though the instructions on the next page seem familiar, it is important that you read them carefully as there are some important changes.

[\#2 is repeated.]

[\#3 is repeated with the different relationship type displayed.]

The task is now complete. Please contact the experimenter. 


\section{Appendix R: Creativity Tasks Used in Experiment 6}

\section{Danger Condition}

\section{Creativity Task}

Please read the following instructions carefully.

In this part of the study, we are going to ask you to imagine several different situations and describe how you would feel in each. We would like you to close your eyes and create each situation in your mind's eye, visualising it as vividly as you possibly can. You'll have 30 seconds to do this. Then, we would like you to write down your actions and feelings, describing them in as much detail as possible. This is a task designed to measure your creativity, so we would like you to answer in lots of detail.

\section{Situation \#1}

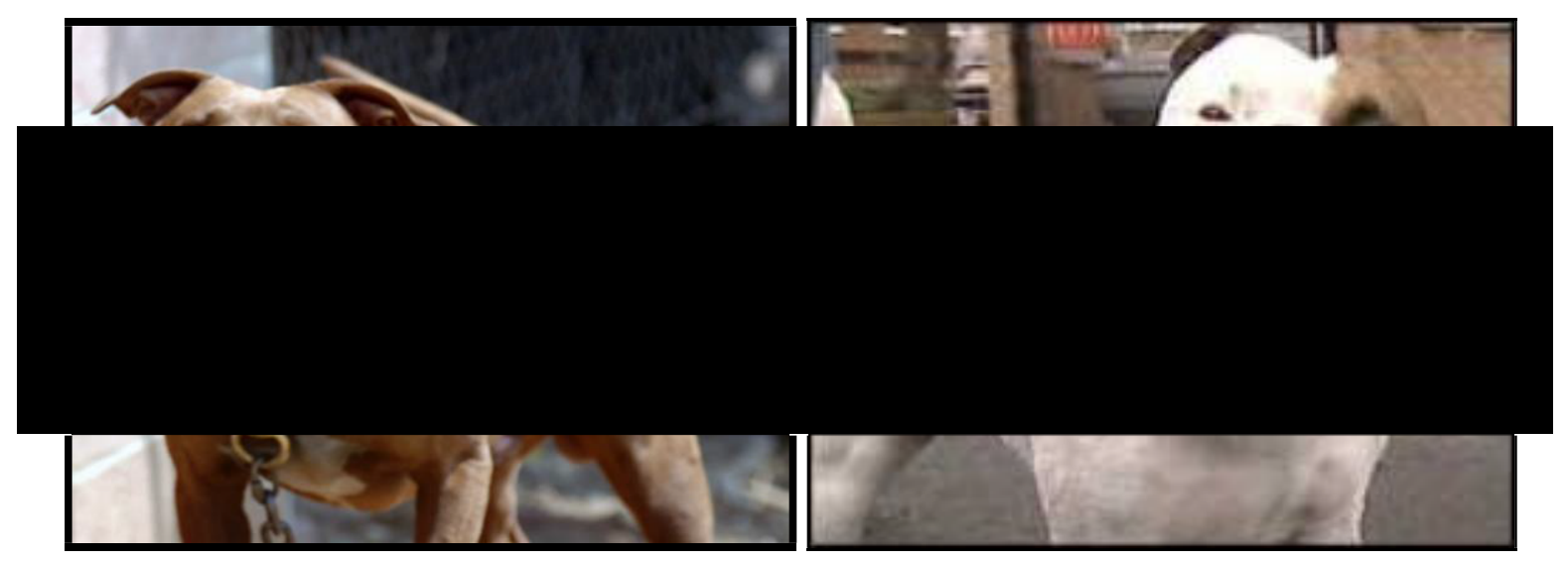

You are walking down a busy high street on your way to work. Suddenly you hear screams behind you and turn around to see several escaped pit-bull dogs have started attacking people. As you back away from the pack you find yourself caught with your back against the wall of a shop. You can't see any path to leave the area without passing a dog - some of which have started turning their attention to you. It is very likely you will come to some sort of harm - at the very least, you're going to get a nasty bite or two.

Please take 30 seconds to visualise this situation. 
In around 150 words, describe how you would feel emotionally about the situation, describe realistically how you would escape this situation and what you expect the outcome to be. 
Situation \#2

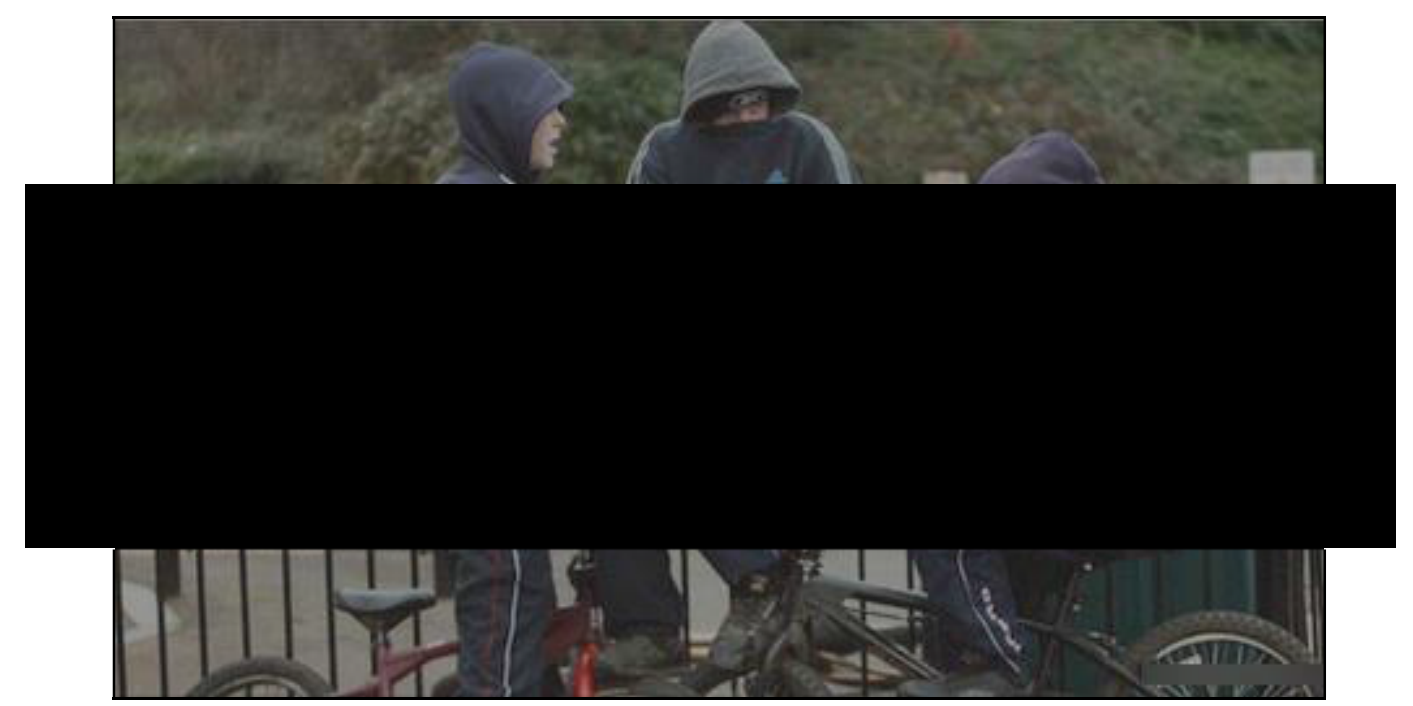

You find yourself walking home alone in the dark after a party. At one point in your journey you spot a gang of 6-8 youths huddled around a park bench in a notoriously rough area. You cross over the road to avoid passing the youths directly. As you pass them one of the youth shouts "Why'd you cross over? Afraid we'll cut ya?" Ignoring the gang, you start walking faster, only to hear footsteps behind you and another voice shout "Oi! We're talking to you". The gang is getting closer, and you're sure that if you can't resolve the situation it is very likely you will come to some sort of harm.

Please take 30 seconds to visualise this situation.

In around 150 words, describe how you would feel emotionally about the situation, describe realistically how you would escape this situation and what you expect the outcome to be. 


\section{Arousal Condition}

\section{Creativity Task}

Please read the following instructions carefully.

In this part of the study, we are going to ask you to imagine several different situations and describe how you would feel in each. We would like you to close your eyes and create each situation in your mind's eye, visualising it as vividly as you possibly can. You'll have 30 seconds to do this. Then, we would like you to write down your actions and feelings, describing them in as much detail as possible. This is a task designed to measure your creativity, so we would like you to answer in lots of detail.

\section{Situation \#1}

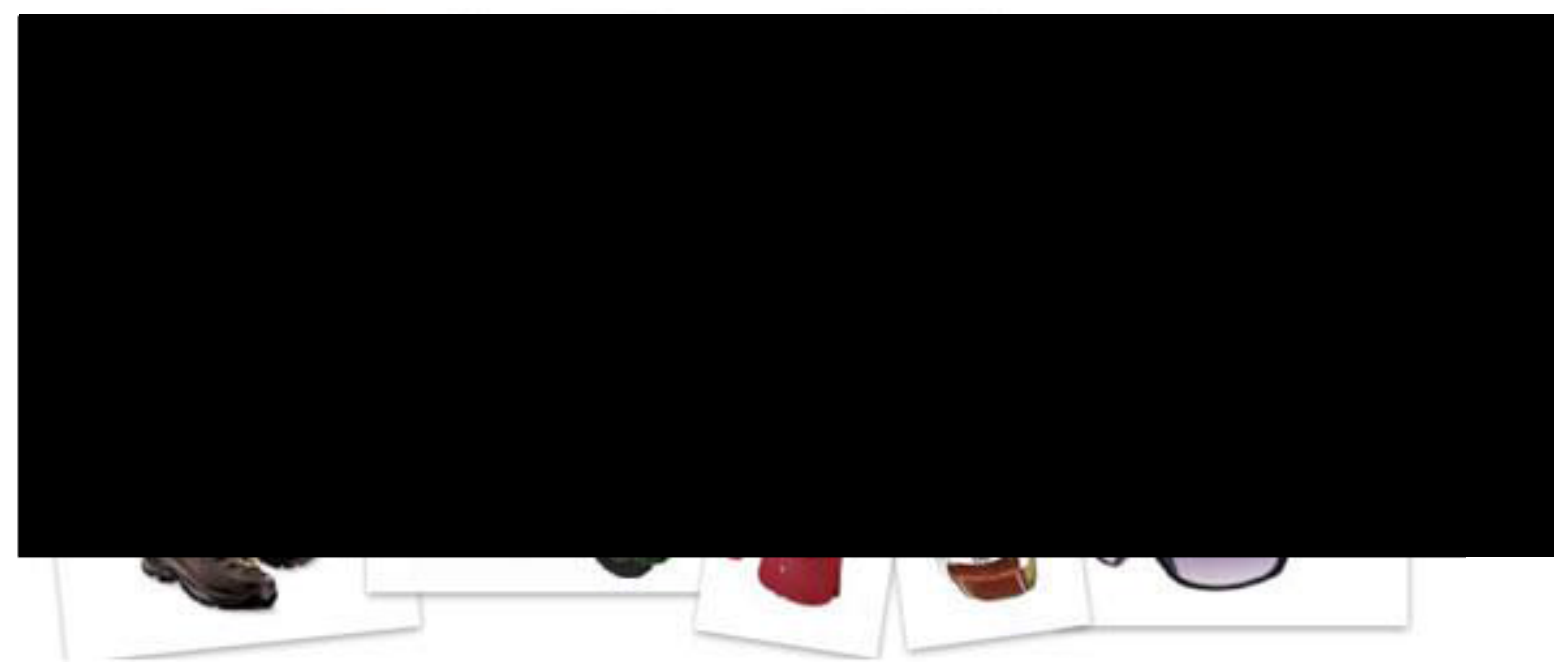

You open your email inbox and notice a new email from a researcher. Several months ago you participated in online study and were entered into a prize draw for an Amazon voucher, though you'd forgotten about this until now. The email states your name was drawn and that you have won an Amazon e-voucher to the value of $£ 500$. The email is definitely authentic: The voucher is attached and can be used immediately to buy anything you want.

Please take 30 seconds to visualise this situation. 
In around 150 words, describe how you would feel emotionally about the situation and give us a break down of how you would most likely use your voucher and why. 
Situation \#2

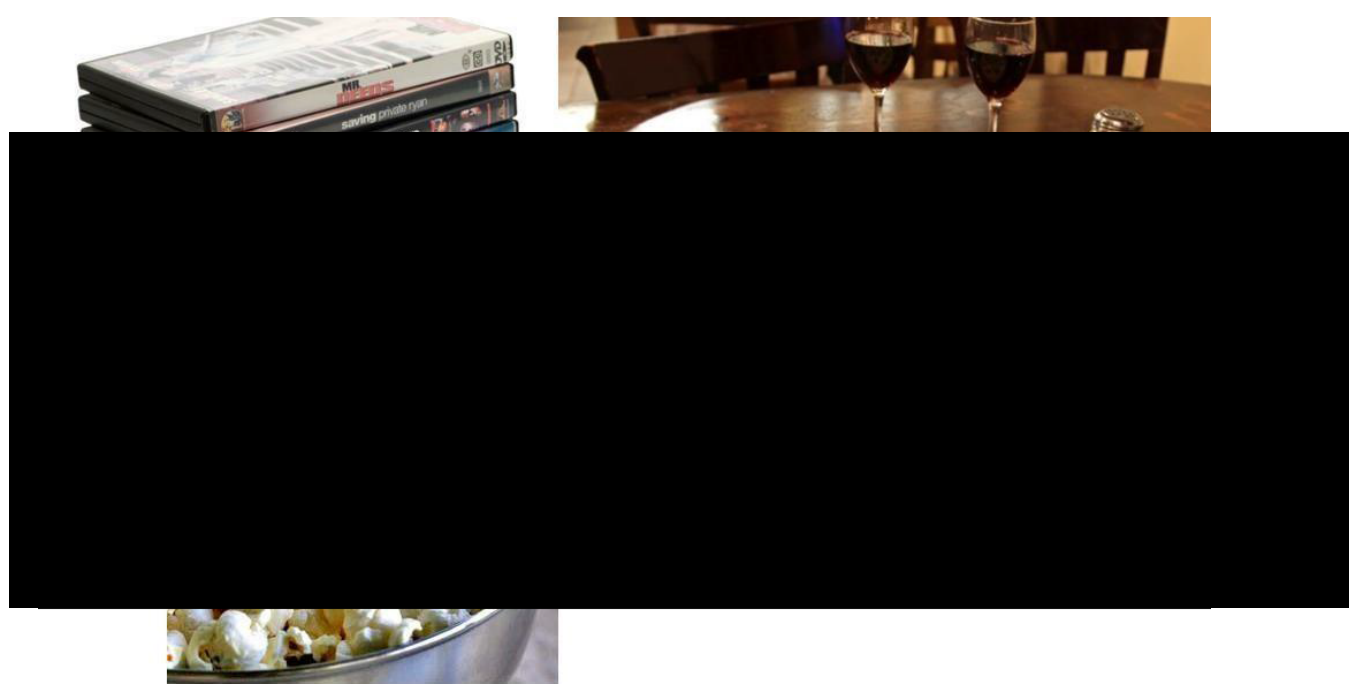

You get a text from your best same-sex friend who you haven't seen in a few months. It turns out you have a day off at the same time and you decide to hang out. Up until now you have taken it in turns to plan 'the perfect night in' and this time it's your turn. Your friend is pretty easy going and you know that whatever you decide to plan they will find just as fun as you will.

Please take 30 seconds to visualise this situation.

In around 150 words, describe how you would feel emotionally about the situation and describe your plan for the "perfect night in". 


\section{Neutral Condition}

\section{$\underline{\text { Creativity Task }}$}

Please read the following instructions carefully.

In this part of the study, we are going to ask you to imagine several different situations and describe how you would feel in each. We would like you to close your eyes and create each situation in your mind's eye, visualising it as vividly as you possibly can. You'll have 30 seconds to do this. Then, we would like you to write down your actions and feelings, describing them in as much detail as possible. This is a task designed to measure your creativity, so we would like you to answer in lots of detail.

\section{Situation \#1}

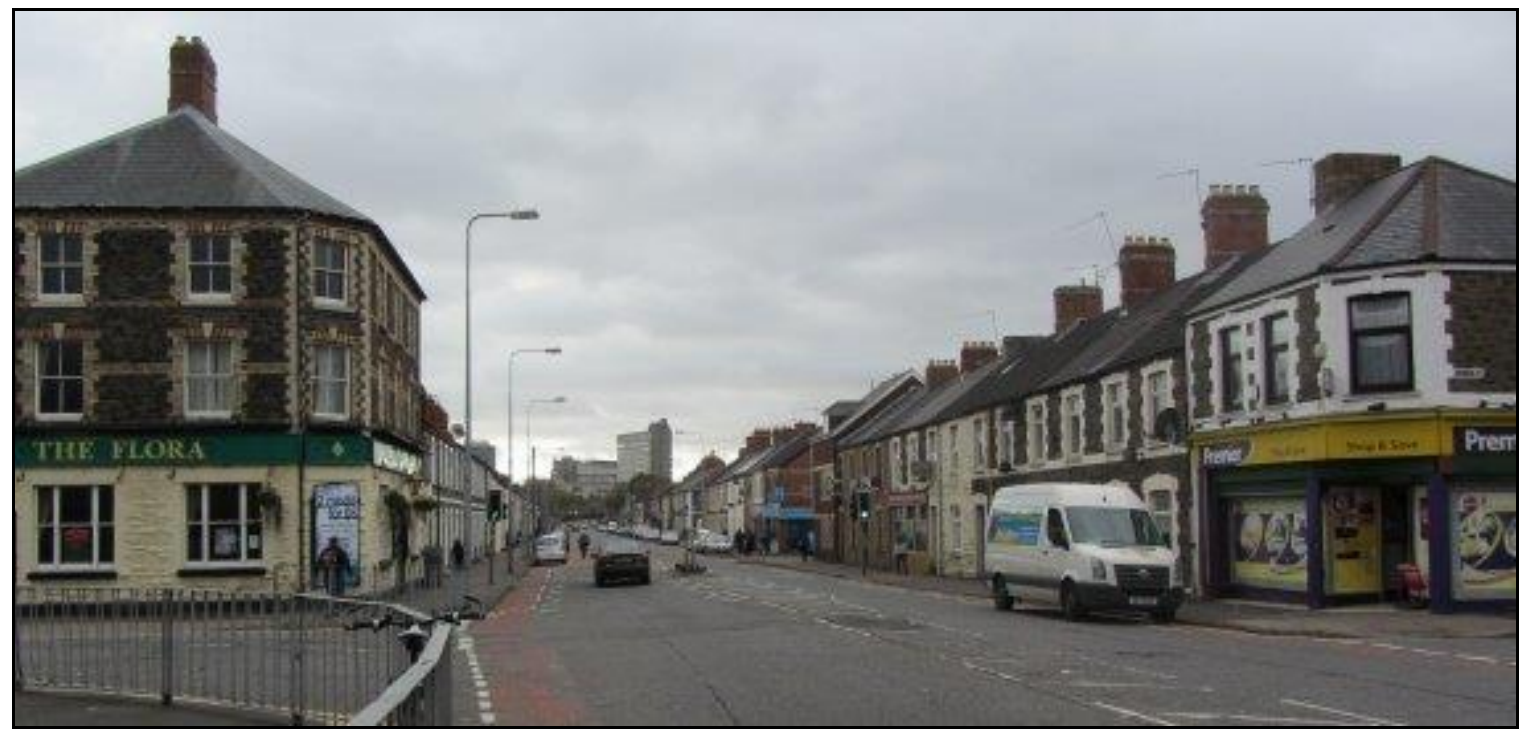

You are at home and need to mail a letter. You walk to the nearest post box on a fair autumn day (not too hot, not too cold) taking in all the sights as you go along.

Please take 30 seconds to visualise this situation.

In around 150 words, describe how you would feel emotionally about the situation and describe your surroundings during the journey; what would you see and experience? Situation \#2 


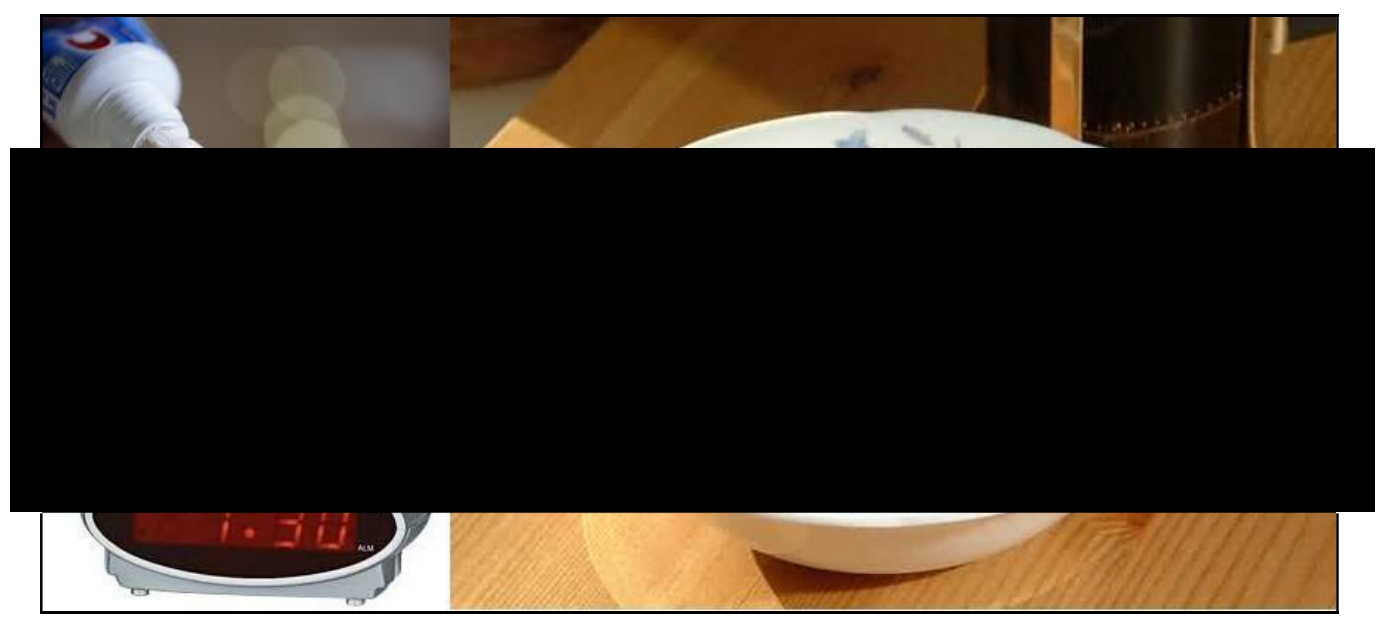

You wake up in the morning a few hours before you're due to be at work/school. You have plenty of time and can get ready at a leisurely pace.

Please take 30 seconds to visualise your morning routine.

In around 150 words, describe how you would feel emotionally about the situation and describe your typical morning routine in detail. 


\title{
Appendix S: The Health Assessment Questionnaire Used in Experiment 7
}

\author{
$\underline{\text { Health Questionnaire }}$
}

\section{APPENDIX C:}

AHA/ACSM Health/Fitness Facility Preparticipation Screening Questionnaire.

\section{Name \\ Address}

Phone number home work

Emergency contact name phone Date of birth

\section{History}

You have had:

$\square$ a heart attack

$\square$ heart surgery

$\square$ cardiac catheterization

$\square$ coronary angioplasty (PTCA)

Dpacemaker/implantable cardiac

defibrillator/rhythm disturbance

$\square$ heart valve disease

$\square$ heart failure

Dheart transplantation

$\square$ congenital heart disease

\section{Symptoms and other health issues:}

$\square$ You experience chest discomfort with exertion.

$\square$ You experience unreasonable breathlessness.

¿You experience dizziness, fainting, blackouts.

$\square$ You take heart medications.

$\square$ You take prescription medication(s).

$\square$ You have musculoskeletal problems.

$\square$ You have concerns about the safety of exercise.

$\square$ You are pregnant.

Cardiovascular risk factors

$\square$ You are a man older than 45 years.

$\square$ You are a woman older than 55 years or you have had a

hysterectomy or you are postmenopausal.

$\square$ You smoke.

$\square$ Your blood pressure is greater than $140 / 90$.

$\square$ You don't know your blood pressure.

$\square$ You take blood pressure medication.

$\square$ Your blood cholesterol level is $>240 \mathrm{mg} / \mathrm{dL}$.

$\square$ You don't know your cholesterol level.

$\square$ You have a blood relative who had a heart attack

before age 55 (father or brother) or age 65 (mother or sister).

$\square$ You are diabetic or take medicine to control your blood sugar

$\Xi$ You are physically inactive (i.e., you get less than 30 minutes

If you marked any of the statements in this section, consult you a health provider before engaging in exercise. You may need to use a facility with a medically qualified staff.

of physical activity on at least 3 days per week)

$\square$ You are more than 20 pounds overweight.

\section{$\square$ None of the above is true.}

You should be able to exercise safely without consulting your healthcare provider in almost any facility that meets your exercise programme needs.

AHAVACSM indicates American Heart Association/American College of Sports Medicine. 


\title{
Appendix T: Consent and Debrief Forms for Experiment 7
}

\author{
Information Sheet and Consent Form
}

This study is looking for the effect of exercise on task performance and relationship choices. It involves performing some simple tasks before and after some exercise.

The study is split into two sessions. An initial session where you will try out the exercise and some of the tasks to get used to them (today), and a main session where the actual experiment is conducted. The initial session takes about 30 minutes and the main session takes around 60 minutes. Upon successful completion, you will be paid $£ 8.00$ for your participation.

The tasks involved in the study include:

Bicycle Sprints - This is the exercise which will consist of you sprinting as fast as you can on an exercise bicycle for 6 seconds, and then cycling at a moderate pace for 54 seconds. This one minute pattern will be repeated 5 times. It is recommended that you wear suitable clothing for this during the main session. The experimenter will discuss this with you.

Cognitive Tasks - Two short measures of reaction time will be administered. One where you have to quickly name the colour of an item without making mistakes, and one where you have to place items in an order quickly.

A measure of handgrip strength

Relationship choice task - This task involves looking at pictures of the other sex and deciding whether (if you were single) you would prefer a no relationship, a long-term relationship or a short-term relationship with them.

During the course of the experiment we will:

Take some saliva samples before and after exercise (to make these accurate you must not eat or drink anything 2 hours prior to the main experiment).

Require you to complete a short health assessment form to make sure you are suitable for the study

Answer some questionnaires about yourself which contain anonymous questions about sex and aggression. These questionnaires will be kept confidential. 
Please read the following statements below and, if you agree with them, print and sign your name at the bottom of the sheet.

I consent to participate in this experiment. I am satisfied with the instructions I have been given thus far and I have been reassured that any further information I request regarding the experimental topic will be supplied to me at the end of the experimental procedure.

I have been informed that the data I provide will remain confidential and be password protected. I am free to ask any questions at any time before and during the experiment. I understand that I will be required to answer fairly personal questions, but will do so for the purpose of the experiment.

I have not been coerced in any way to participate in this study and I understand that I may terminate my participation in the experiment at any point should I wish to do so. I meet the age requirements of being no younger than 18 years of age and no older than 27 years of age.

Data Protection: I agree to the University processing personal data that I have supplied. I agree to the processing of such data for any purposes connected with the Research Project as outlined to me.

Participant Name

Participant Signature

Date 


\section{Debrief form}

Thank you for taking part in the study. We are currently interested in the effect of testosterone on behaviour. In this study, we had you engage in some tasks before and after exercise. Short bursts of vigorous exercise have been shown to increase testosterone and we were seeing whether this increase affected your performance on several tasks.

Two of these tasks tested your cognitive abilities, namely your performance in reaction time and sorting tasks. Another tested your hand-grip strength which is known to correlate with testosterone. Finally you engaged in a relationship choice task.

Testosterone is often associated with mate seeking behaviour and this task allowed us to measure your preferences for short- and long-term relationships.

All your data including the sexual and aggression questionnaires will be kept strictly confidential and anonymous as will the rest of the data we have gathered. If you would like to have your data removed from analysis then let us know now. Once you leave the laboratory we will combine your data with other individuals and it will be indistinguishable.

Thank you for your participation once again. If you have any questions feel free to ask now, or contact the experimenter (Andrew Thomas, [EMAIL ADDRESS]) or research supervisor (Dr. Steve Stewart-Williams, [EMAIL ADDRESS]) at a later date.

Below are some references related to the research hypotheses should you be interested.

\section{References}

Gangestad, S. W., \& Simpson, J. A. (2000). The evolution of human mating: Trade-offs and strategic pluralism. Behavioral and Brain Sciences, 23, 573-644. 
Storey, A. E., Walsh, C. J., Quinton, R., \& Wynne-Edwards, K. E. (2000). Hormonal correlates of paternal responsiveness in new and expectant fathers. Evolution and Human Behavior, 21, 79-95.

Thornton, I.M., Horowitz, T.S. (2004). The multi-item localization (MILO) task: Measuring the spatiotemporal context of vision for action. Perception and Psychophysics, 66(1), 38-50. 


\section{Appendix U: Creativity Tasks Used in Experiment 8}

\section{Victory Condition}

\section{Creativity Task}

Please read the following instructions carefully.

In this part of the study, we are going to ask you to imagine some different situations and describe how you would feel in each. We would like you to close your eyes and create each situation in your mind's eye, visualising it as vividly as you possibly can. You'll have 30 seconds to do this. Then, we would like you to write down your actions and feelings, describing them in as much detail as possible. This is a task designed to measure your creativity, so we would like you to answer in lots of detail.

\section{Situation \#1}

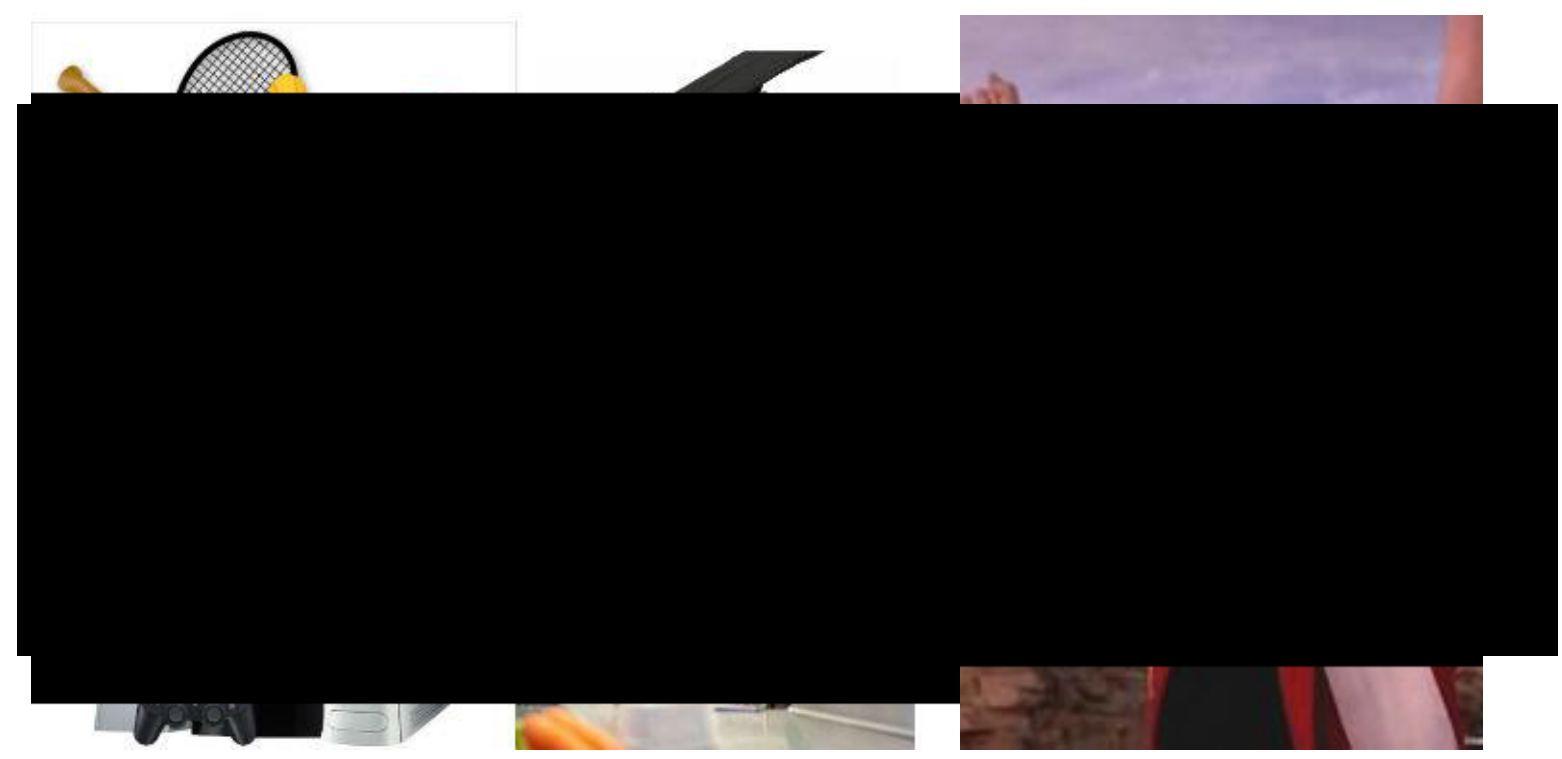

We would like you to remember the time in your life when you felt the most victorious.

Please choose one of the following settings:

Sports (e.g. beating a rival team or player)

Education (e.g. scoring higher than your friends on an exam; winning an academic prize) 
Work (e.g. receiving a promotion)

Games (e.g. beating someone at a video game or board game)

Really try to think of a scenario which really gave you a 'fist in the air' sense of victory.

Please take 30 seconds to remember/visualise this situation.

In around 150 words, describe the event in detail as well as how you felt emotionally about the situation. 
Situation \#2

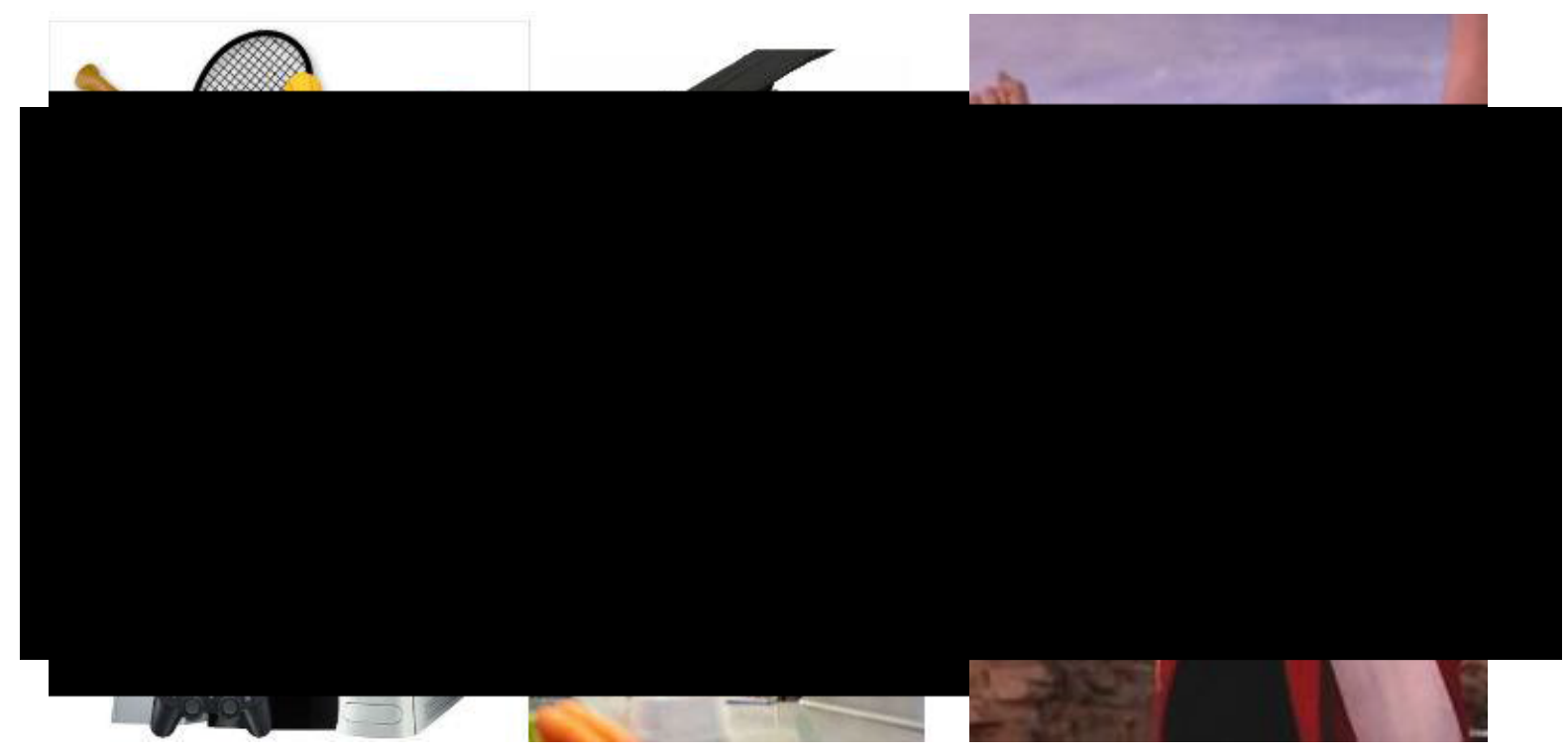

You've written about a real victory you've had in your life. Now we would like you to imagine yourself being victorious in an important area of your life. Again, please choose one of the following areas:

Sports (e.g. beating a rival team or player)

Education (e.g. scoring higher than your friends on an exam; winning an academic prize)

Work (e.g. receiving a promotion)

Games (e.g. beating someone at a video game or board game)

As before, really try to think of a scenario which would really give you a 'fist in the air' sense of victory.

Please take 30 seconds to visualise this situation.

In around 150 words, describe the event in detail as well as how you would feel emotionally about the situation. 


\section{Defeat Condition}

\section{$\underline{\text { Creativity Task }}$}

Please read the following instructions carefully.

In this part of the study, we are going to ask you to imagine some different situations and describe how you would feel in each. We would like you to close your eyes and create each situation in your mind's eye, visualising it as vividly as you possibly can. You'll have 30 seconds to do this. Then, we would like you to write down your actions and feelings, describing them in as much detail as possible. This is a task designed to measure your creativity, so we would like you to answer in lots of detail.

Situation \#1

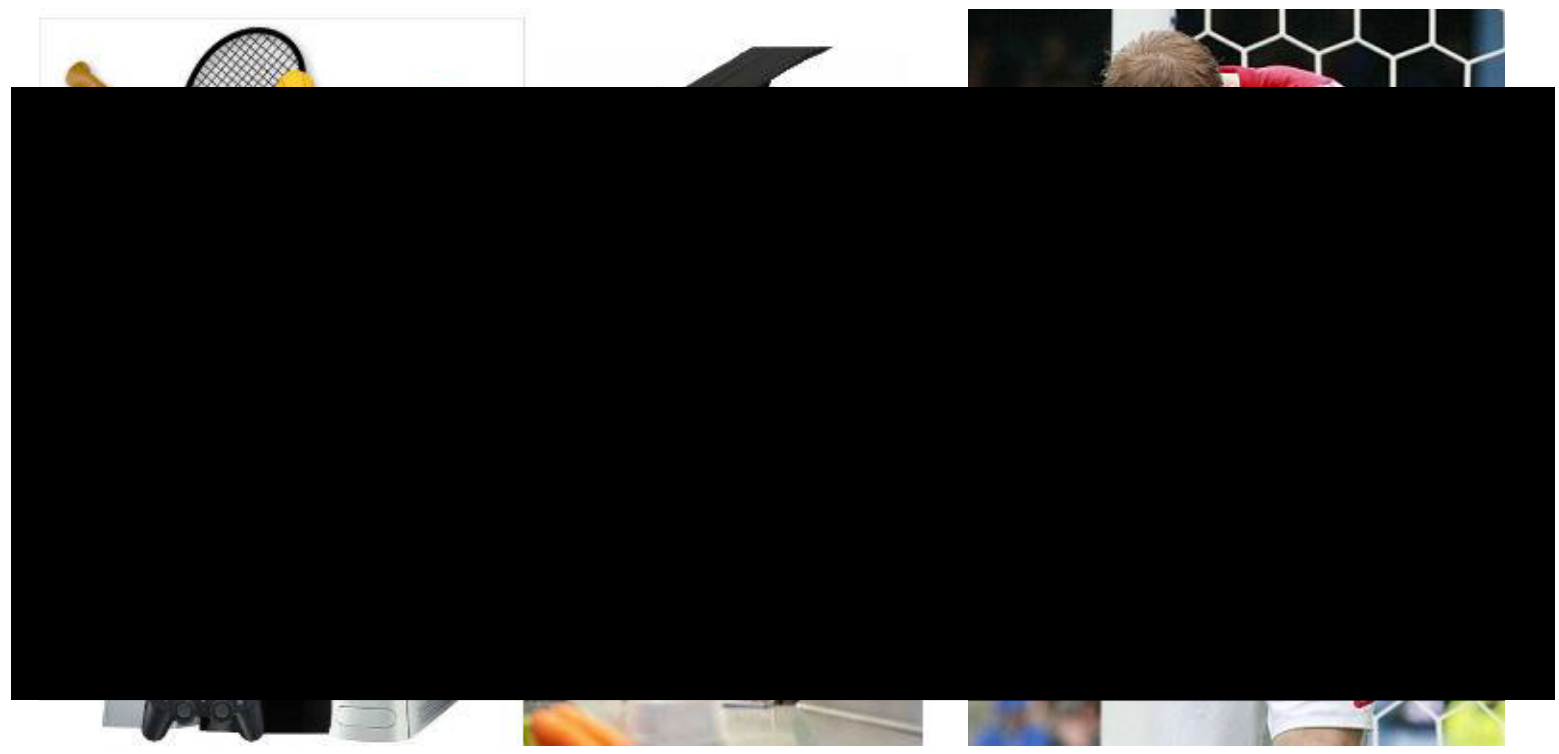

We would like you to remember the time in your life when you felt the most defeated by another person or persons within one of the following settings:

Sports (e.g. being beaten by a rival team or player)

Education (e.g. scoring lower than your friends on an exam; answering a question wrong in front of your class)

Work (e.g. being turned down for a promotion)

Games (e.g. losing to someone in a video game or board game) 
Try to think of a scenario which really gave you a 'hang your head' sense of defeat.

Please take 30 seconds to remember/visualise this situation.

In around 150 words, describe the event in detail as well as how you felt emotionally about the situation. 
Situation \#2

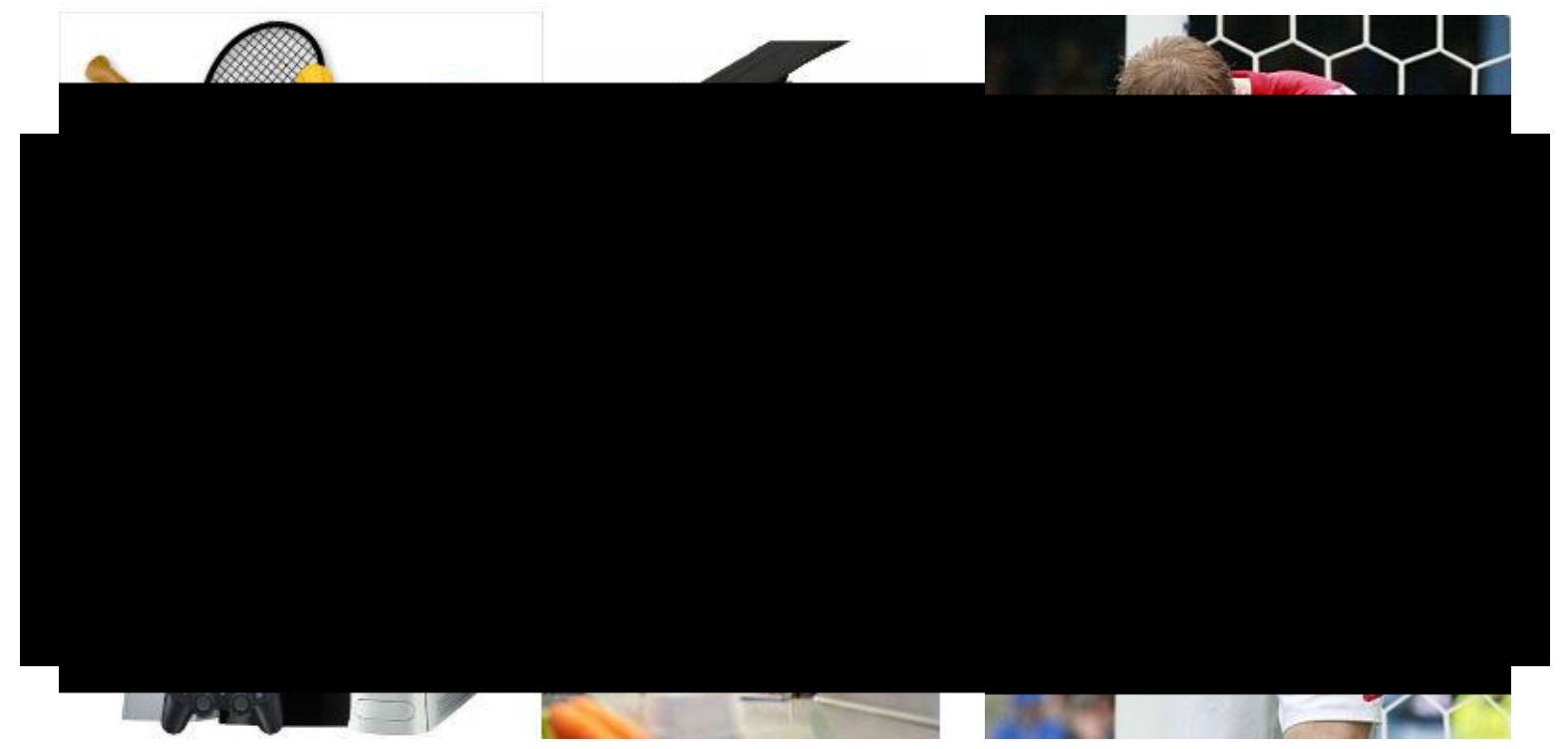

You've written about a real defeat you've had in your life. Now we would like you to imagine yourself being defeated by another person or persons in an important area of your life related to:

Sports (e.g. being beaten by a rival team or player)

Education (e.g. scoring lower than your friends on an exam; answering a question wrong in front of your class)

Work (e.g. being turned down for a promotion)

Games (e.g. losing to someone in a video game or board game)

Try to think of a scenario which would really give you a 'hang your head' sense of defeat.

Please take 30 seconds to visualise this situation.

In around 150 words, describe the event in detail as well as how you would feel emotionally about the situation. 


\section{Appendix V: Consent and Debrief Forms for Experiment 9}

\section{$\underline{\text { Participant Information Sheet and Consent Form }}$}

In the following study you will be required to complete some questionnaires and two types of computer tasks.

The questionnaires will ask you basic questions about yourself as well as some anonymous questions of a sexual nature.

The computer tasks involve: (a) looking at pictures of individuals and trying to guess their relationship status; and (b) looking at some different individuals and deciding which type of relationship you'd prefer to have with them. You will complete task (a) once and task (b) twice.

There will be a prize awarded for the best score obtained during task (a). Instructions will accompany each task, so please read these thoroughly.

All information you give during the study will remain confidential. All questionnaires will be sealed in an envelope immediately after they have been completed and all computer based information will be kept password protected. Information will only be used by the researcher once all other individuals have taken part in the study.

If at any point during the study you feel uncomfortable with the tasks you are required to complete, you have the right to withdraw at any time and your results will not be included in any part of this experiment.

Please read the following statements on the next page and sign if you agree with them. 
I consent to participate in this experiment. I am satisfied with the instructions I have been given thus far and I have been reassured that any further information I request regarding the experimental topic will be supplied to me at the end of the experimental procedure.

I have been informed that the data I provide will remain confidential and be password protected. I am free to ask any questions at any time before and during the experiment. I understand that my responses will be kept anonymous.

I have not been coerced in any way to participate in this study and I understand that I may terminate my participation in the experiment at any point should I wish to do so. I meet the age requirements of being no younger than 18 years of age and no older than 25 years of age.

Data Protection: I agree to the University processing personal data that I have supplied. I agree to the processing of such data for any purposes connected with the Research Project as outlined to me.

Participant signature

Date

Researcher(s)'s contact details: [NAME] ([EMAIL ADDRESS]) 


\section{Debrief form}

Thank you for your co-operation and completion of this experiment. The aim of the experiment was to examine the effects of sex-ratio within a population on relationship choices. More specifically, the experiment set out to assess what factors affect relationship choices.

The relationship statuses of the individuals shown within the 'guessing game' task were bogus and decided in advance by the experimenters.

Depending on the condition, some participants were led to believe males were mainly single while females were mainly in a relationship, whereas others were led to believe females were mainly single while males were mainly in a relationship. These conditions were set in place because we believe that the availability of the desired sex has an effect on one's own relationship choices.

If following this information you wish to withdraw your data, please let the experimenter know and this will be carried out.

As there was no real 'right' or 'wrong' answer in the guessing game, we will be issuing the $£ 30$ gift voucher to a random participant at the end of the study, rather than giving it to the highest scorer.

If you would like further information regarding the literature behind this topic please consult the following readings:

\section{References}

Buss, D.M. (1994). The Evolution of Desire: Strategies of Human Mating. New York: Basic Books.

Buss, D.M. \& Schmitt, D.P. (1993). Sexual strategies theory: An evolutionary perspective on human mating. Psychological Review, 100, 204-232. 
Gangestad, S.W. \& Simpson, J.A. (2000). The evolution of human mating: Trade offs and strategic pluralism. Behavioral and Brain Sciences, 23, 573-644.

Stone, E.A., Shackelford, T.K. \& Buss, D.M. (2007). Sex ratio and mate preferences: A cross cultural investigation. European Journal of Social Psychology, 37, 288-296. 


\section{Appendix W: Instructions Given to Participants during the Guessing Game Task}

The Guessing Game task was presented between two performances of the SMA task. For the specific wording of the SMA task, please see Appendix C.

Thank you! For the next part of the study, we are interested in whether people can guess someone's relationship status purely by their picture.

On the next screen we will show you several pictures of both men and women. These are students at Swansea, Swansea Met., and Cardiff University who have volunteered their pictures and relationship statuses for the study. As you are shown each picture, you will be asked to guess if the person is single or spoken for (in a relationship).

If you guess correctly you will be awarded a point; if you are wrong you will lose a point. At the end of the study, the participants with the highest number of points will receive a prize of $£ 30$ in Amazon gift vouchers. As these pictures were taken from students in the local area, you may spot someone that you know. If this happens, please make a note of the individual so that they can be discounted from your results. The experimenter will have issued you a piece of paper for this purpose.

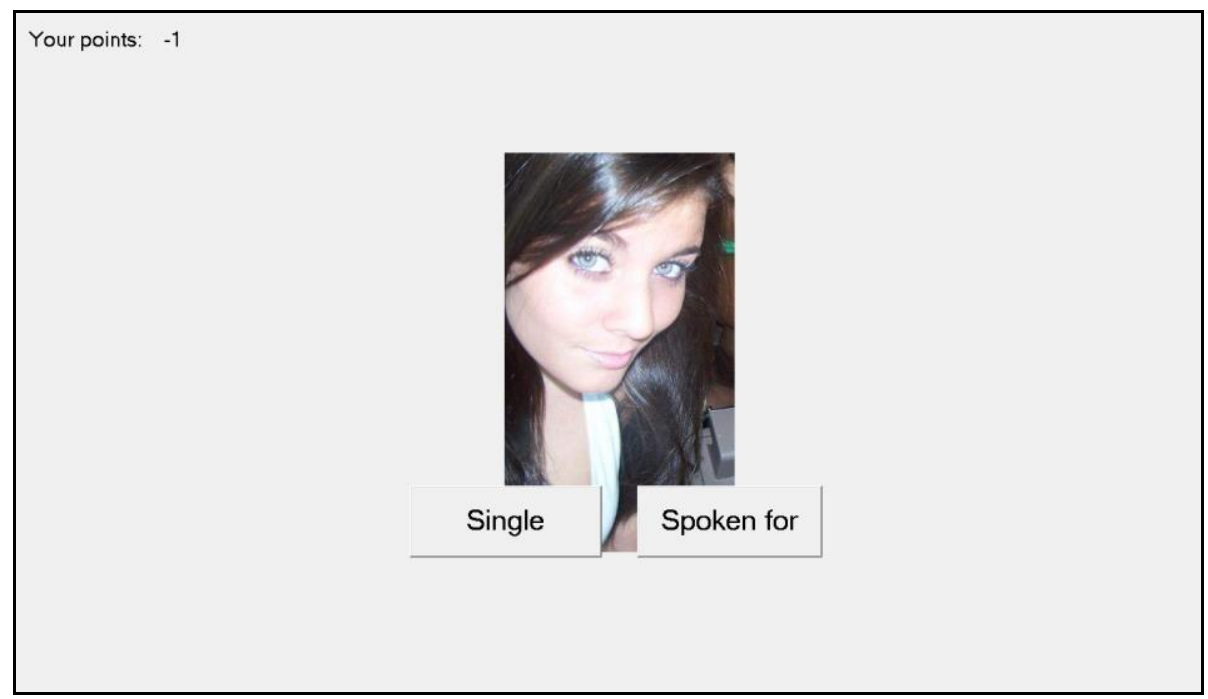

Figure W.1. Example of the Guessing Game task interface. The number of points earned are shown in the top left-hand corner of the screen. 


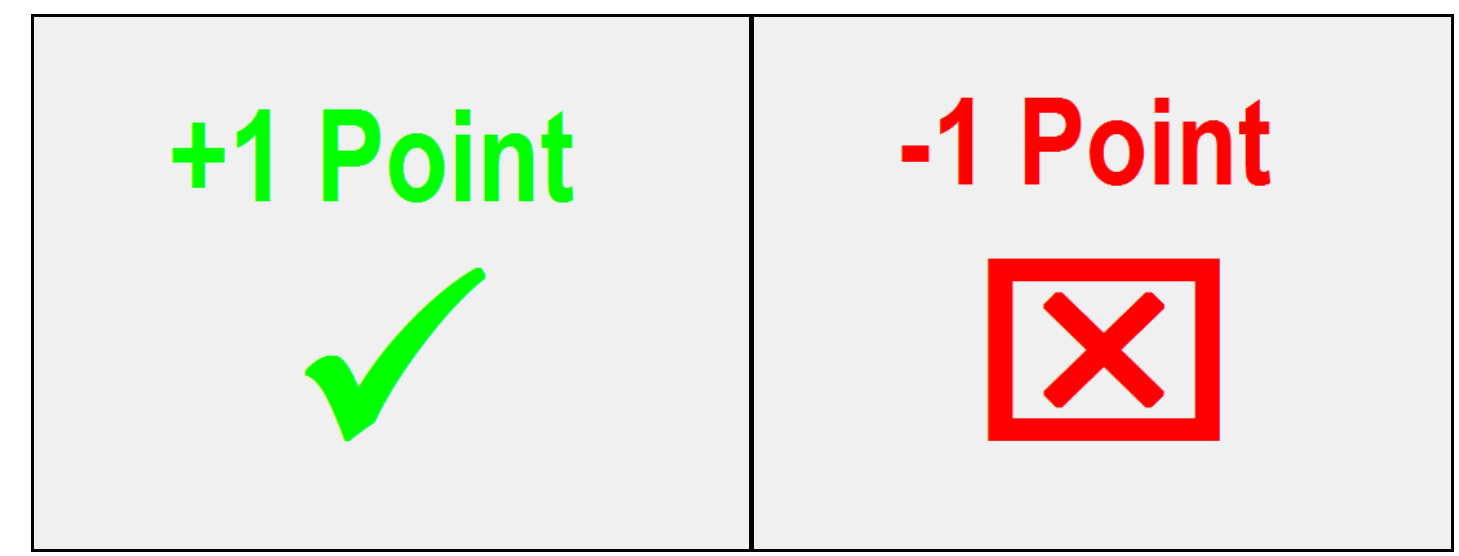

Figure W.2. Positive (left) and negative (right) feedback signs used during the Guessing Game. The signs were shown full screen, and the negative feedback was accompanied by an incorrect "game show buzzer" sound effect.

Thank you! You are now at the final part of the study. To make sure we have an accurate picture of your relationship preferences, we would like you to complete the relationship choice task a second time. The task is identical to last time and some of the people you are about to be shown may have appeared before. This is normal. 


\section{Appendix X: Alternative Analysis of Experiment 9}

\section{Male-Biased Sex-Ratio}

Points. The average number of points scored by participants in this condition were $22.45(S D=17.31)$. The points variable was normally distributed, $(\mathrm{K}-\mathrm{S}=0.08, p=$ $0.2 ; \mathrm{S}-\mathrm{W}=0.99, p=0.67$ ) and so was assessed using parametric analysis and included in the ordinal regression (see below) as a continuous variable. The average number of points earned by participants was significantly higher than zero using a one-sample $t$ test, $t(79)=11.600, p<0.01$, indicating that on average the participants made relationship guesses somewhat in line with the algorithm (zero would be expected if participants guessed randomly). There was no clear difference between the sexes in terms of task performance. Both men $(M=22.45, S D=18.51)$ and women $(M=22.45$, $S D=16.26)$ scored significantly more than zero, $\mathrm{t}(39)=7.67$ for men, and 8.73 for women, both $p$ s $<0.01$, and these scores were not significantly different from one another, $\mathrm{t}(78)<0.01, p>0.99$.

Long-term mating. After playing the guessing game, 34 participants decreased in their LT selection (LT-), 21 stayed the same (LT=), and 25 increased (LT+). Thus it appeared as if LT selection decreased following the task. However, a chi-squared analysis revealed that the distribution did not significantly deviate from that expected by chance (see Table X.1).

\section{Table X.1}

The number of participants who increased, stayed the same, or decreased in their number of LT choices following exposure to a male-biased sex-ratio task. The distributions are not significantly different from each other using a chi-squared test

\begin{tabular}{lccc}
\hline \multicolumn{4}{c}{ Long-term change } \\
\hline & - & $=$ & + \\
\hline Found & 34 & 21 & 25 \\
Predicted & 29.5 & 21 & 29.5 \\
\hline$\chi^{2}(2)$ & 1.373 & & \\
Cramér's $V$ & 0.07 & & \\
\hline
\end{tabular}

${ }^{\dagger} p<0.10, * p<0.05, * * p<0.01$ 
An ordinal regression analysis was performed in order to predict LT change after the male-biased sex-ratio feedback. The variables included in the analysis were gender, SOI-R, self-perceived attractiveness, age, points earned, relationship status, and socioeconomic status. After testing for main and two-way interaction effects among the variables, a significant model emerged (see Table X.2).

\section{Table X.2}

The results of an ordinal regression to predict change in LT choice using SOI-R, age, relationship status, points scored, and sex

\begin{tabular}{lcc}
\hline \multicolumn{3}{c}{ Model LT } \\
\hline \multirow{2}{*}{ SOI-R } & \multicolumn{1}{c}{$\beta$} & OR \\
\cline { 2 - 3 } Age & $-0.08 * *$ & 0.92 \\
Male * Relat & -1.016 & - \\
Female * Relat & $-1.728^{*}$ & 0.18 \\
Male * Points & $0.05 * *$ & 1.05 \\
Female * Points & 0.01 & - \\
\hline Model & $\chi^{2}(6)=25.64 * *$ \\
Nagelkerke $R^{2}$ & 0.31 & \\
Accuracy & $57.5 \%(+35.3 \%)$ \\
Parallel lines & $\chi^{2}(6)=0.819$ \\
\hline
\end{tabular}

Note $:$ Relat. $=$ relationship status. Variables were coded as such: Relat - single/uncommitted (0), committed/married (1). OR = odds ratio. ${ }^{\dagger} p<0.10, * p<0.05, * * p<0.01$.

There were several significant predictors in the model. Predictors in the positive direction included age and points scored, while SOI-R and relationship status were in a negative direction. Sex interacted with some of these. Starting with the non-interaction variables, age was found to be a positive predictor with each additional year of age leading to a $46 \%$ increase in likelihood of appearing in the LT+ category. SOI-R was found to be a negative predictor, with every one point increase leading to an $8 \%$ decrease in the likelihood of appearing in the LT+ category.

In terms of the interacting variables, it was found that being in a relationship decreased the likelihood of increasing in LT following the GG. However, this effect was restricted to women only. Such an effect makes more sense when interpreted inversely following the task single women were $463 \%$ more likely to appear in the LT+ group 
than their in-relationship counterparts. Finally, an effect restricted to men was also found. For each additional point which a male scored during the task, their likelihood of appearing in LT+ increased by $5.1 \%$.

Short-term mating. After playing the guessing game, 42 participants decreased in their number of ST selections (ST-), nine stayed the same (ST=), and 29 increased (ST+). Thus it appeared as if ST selection decreased following the task. However, chisquared analysis revealed that this distribution did not significantly differ from that expected due to chance (see Table X.3).

Table X.3

The number of participants who increased, stayed the same, or decreased in their number of ST choices following exposure to a male-biased sex-ratio task. The distribution is not significantly different from an expected balanced distribution using a chi-squared test

\begin{tabular}{lccc}
\hline \multicolumn{4}{c}{ Short-term change } \\
\hline & - & $=$ & + \\
\hline Found & 42 & 9 & 29 \\
Predicted & 35.5 & 9 & 35.5 \\
\hline$\chi^{2}(2)$ & 2.380 & & \\
Cramér's $V$ & 0.09 & \\
\hline
\end{tabular}

$$
{ }^{\dagger} p<0.10, * p<0.05, * * p<0.01 .
$$

The same analysis and variables used to predict LT category change were used here to predict short-term category change. Following the same procedure, a significant model emerged consisting of a single predictive variable: the number of points scored by the participants (see Table X.4).

Table X.4

The results of an ordinal regression to predict change in ST choice using points scored

\begin{tabular}{|c|c|c|}
\hline \multicolumn{3}{|c|}{ Model ST } \\
\hline & $\beta$ & OR \\
\hline Points & $-0.03 *$ & 0.97 \\
\hline Model & $\chi^{2}(1)=t$ & $0 * *$ \\
\hline
\end{tabular}

Nagelkerke $R^{2} \quad 0.09$ 


\begin{tabular}{ll} 
Accuracy & $53.8 \%(+2.4 \%)$ \\
Parallel lines & $\chi^{2}(1)=0.860$ \\
\hline
\end{tabular}

Note: $\mathrm{OR}=$ odds ratio. ${ }^{\dagger} p<0.10, * p<0.05, * * p<0.01$

Irrespective of the participant's sex or values of any of the other variable included in the analysis, the number of points scored was the sole predictor of ST category change. For every one point earned by the participant in the GG task, likelihood of appearing in the ST+ group decreased by $3 \%$.

\section{Female-Biased Sex-Ratio}

Points. As in the male-biased condition, this variable was normally distributed $(\mathrm{K}-\mathrm{S}=0.08, p=0.20 ; \mathrm{S}-\mathrm{W}=0.98, p=0.48)$ and so it was assessed using parametric analysis and treated as a continuous variable. The average number of points scored by participants in this condition was much lower than in the previous one. Participants on average scored only $2.64(S D=18.12)$ points and this was not significantly higher than 0 using a one-sample $t$-test, $t(77)=1.29, p=0.20$. There was no clear difference between the sexes in terms of task performance with men scoring an average of 4.36 points $(S D=17.34)$ and women scoring an average of $0.92(S D=18.93)$. These two averages were not significantly different from one another, $t(76)=0.836, p=0.41$. A between-subjects $t$-test confirmed that participants from the male-biased sex-ratio condition scored significantly more points than the female-biased condition, $t(156)=$ $7.028, p<0.01, d=1.13$.

Long-term mating. After playing the guessing game, 37 participants decreased in their number of LT selections (LT-), 20 stayed the same (LT=), and 21 increased (LT+). Thus it appeared as if LT selection decreased following the task. Chi-squared analysis was employed to see if this distribution deviated from an expected distribution in which LT- and LT+ were balanced (see Table X.5), however, this was nonsignificant.

Table X.5

The number of participants who increased, stayed the same, or decreased in their number of LT choices following exposure to a female-biased sex-ratio task. The distribution is not significantly different from an expected balanced distribution using a chi-squared test 


\begin{tabular}{lccc}
\hline \multicolumn{4}{c}{ Long-term change } \\
\hline & - & $=$ & + \\
\hline Found & 37 & 20 & 21 \\
Predicted & 29 & 20 & 29 \\
\hline$\chi^{2}(2)$ & 4.414 & & \\
Cramér's $V$ & 0.12 & & \\
\hline
\end{tabular}

$$
{ }^{\dagger} p<0.10, * p<0.05, * * p<0.01 \text {. }
$$

A backwards stepwise ordinal regression analysis was performed in order to predict LT change after the female-biased sex-ratio feedback. The factors included in the analysis were gender, SOI-R, self-perceived attractiveness, age, points earned, relationship status and socio-economic status. However, no significant model using the individual variables or interactions between them could be produced.

Short-term mating. After playing the guessing game, 39 participants decreased in their LT selection (ST-), 12 stayed the same (ST=), and 27 increased (ST+). Thus it appeared as if ST selection decreased following the task. A chi-squared analysis revealed that there was no significant difference between this distribution and that which would be expected by chance (see Table X.6).

\section{Table X.6}

The number of participants who increased, stayed the same, or decreased in their number of ST choices following exposure to a female-biased sex-ratio task. The distribution is not significantly different from an expected balanced distribution using a chi-squared test

\begin{tabular}{lccc}
\hline \multicolumn{4}{c}{ Short-term change } \\
\hline & - & $=$ & + \\
\hline Found & 39 & 12 & 27 \\
Predicted & 33 & 12 & 33 \\
\hline$\chi^{2}(2)$ & 2.183 & & \\
Cramér's $V$ & 0.08 & & \\
\hline
\end{tabular}

$$
{ }^{\dagger} p<0.10, * p<0.05, * * p<0.01 \text {. }
$$


An ordinal regression analysis was performed in order to predict ST change following the female-biased sex-ratio feedback. This used the same factors included in the LT model. No significant model could be produced. 


\section{Bibliography}

Abbott, A. (2013). Disputed results a fresh blow for social psychology. Nature, 497(7447), 16. doi: 10.1038/497016a

Abraham, E., Hendler, T., Shapira-Lichter, I., Kanat-Maymon, Y., Zagoory-Sharon, O., \& Feldman, R. (2014). Father's brain is sensitive to childcare experiences. Proceedings of the National Academy of Sciences, 111(27), 9792-9797. doi: 10.1073/pnas.1402569111

Al Rashidi, M., Kosztolányi, A., Shobrak, M., Küpper, C., \& Székely, T. (2011). Parental cooperation in an extreme hot environment: Natural behaviour and experimental evidence. Animal Behaviour, 82(2), 235-243. doi: 10.1016/j.anbehav.2011.04.019

Albert, D. J., Jonik, R. H., \& Walsh, M. L. (1990). Aggression by ovariectomized female rats with testosterone implants - competitive experience activates aggression toward unfamiliar females. Physiology \& Behavior, 47(4), 699-703. doi: 10.1016/0031-9384(90)90080-N

Allison, C. M., \& Hyde, J. S. (2011). Early menarche: Confluence of biological and contextual factors. Sex Roles, 1-10. doi: 10.1007/s11199-011-9993-5

Alvergne, A., Faurie, C., \& Raymond, M. (2009). Variation in testosterone levels and male reproductive effort: Insight from a polygynous human population. Horm Behav, 56(5), 491-497. doi: 10.1016/j.yhbeh.2009.07.013

Amundsen, T. (2000). Why are female birds ornamented? Trends in Ecology \& Evolution, 15(4), 149-155. doi: 10.1016/S0169-5347(99)01800-5

Anderson, A. L., \& Brown, W. D. (2009). Plasticity of hatching in green frogs (rana clamitans) to both egg and tadpole predators. Herpetologica, 65(2), 207-213. doi: 10.1655/08-016r1.1

Andersson, M. (1982). Female choice selects for extreme tail length in a widowbird. Nature, 299(5886), 818-820. doi: 10.1038/299818a0

Anonymous. (1970). Effects of sexual activity on beard growth in man. Nature, 226(5248), 869-870. doi: 10.1038/226869a0

Apollonio, M., Festa-Bianchet, M., \& Mari, F. (1989). Effects of removal of successful males in a fallow deer lek. Ethology, 83(4), 320-325. doi: 10.1111/j.14390310.1989.tb00539.x

Archer, J. (1991). The influence of testosterone on human-aggression. British Journal of Psychology, 82, 1-28. doi: 10.1111/j.2044-8295.1991.tb02379.x 
Archer, J. (2004). Sex differences in aggression in real-world settings: A meta-analytic review. Review of General Psychology, 8(4), 291-322. doi: 10.1037/10892680.8.4.291

Archer, J. (2006). Testosterone and human aggression: An evaluation of the challenge hypothesis. Neuroscience \& Biobehavioral Reviews, 30(3), 319-345. doi: 10.1016/j.neubiorev.2004.12.007

Ari, Z., Kutlu, N., Sami Uyanik, B., Taneli, F., Buyukyazi, G., \& Tavli, T. (2004). Serum testosterone, growth hormone, and insulin-like growth factor-1 levels, mental reaction time, and maximal aerobic exercise in sedentary and long-term physically trained elderly males. International Journal of Neuroscience, 114(5), 623-637. doi: 10.1080/00207450490430499

Arnbom, T., Fedak, M. A., \& Rothery, P. (1994). Offspring sex-ratio in relation to female size in Southern elephant seals, Mirounga-leonina. Behavioral Ecology and Sociobiology, 35(6), 373-378. doi: 10.1007/BF00165838

Aron, A., Fisher, H., Mashek, D. J., Strong, G., Li, H., \& Brown, L. L. (2005). Reward, motivation, and emotion systems associated with early-stage intense romantic love. Journal of Neurophysiology, 94(1), 327-337. doi: 10.1152/jn.00838.2004

Bach, A. K., Brown, T. A., \& Barlow, D. H. (1999). The effects of false negative feedback on efficacy expectancies and sexual arousal in sexually functional males. Behavior Therapy, 30(1), 79-95. doi: 10.1016/S0005-7894(99)80047-1

Bailey, D., Durante, K. M., \& Geary, D. C. (2011). Men's perception of women's attractiveness is calibrated to relative mate value and dominance of the women's partner. Evolution and Human Behavior, 32(2), 138-146. doi:

10.1016/j.evolhumbehav.2010.08.004

Bailey, D., \& Geary, D. (2009). Hominid brain evolution. Human Nature, 20(1), 67-79. doi: 10.1007/s12110-008-9054-0

Bailey, J. M., Dunne, M. P., \& Martin, N. G. (2000). Genetic and environmental influences on sexual orientation and its correlates in an Australian twin sample. Journal of Personality and Social Psychology, 78(3), 524-536. doi: $10.1037 / / 0022-3514.78 .3 .524$

Baker, J. S., Thomas, N. E., \& Davies, B. (2009). Physiological, biochemical and mechanical issues relating to resistive force selection during high-intensity cycle ergometer exercise. Journal of Exercise Science \& Fitness, 7(2), S51-S60. doi: $10.1016 / \mathrm{S} 1728-869 \mathrm{X}(09) 60023-\mathrm{X}$ 
Baker, R. R., \& Bellis, M. A. (1995). Human sperm competition: Copulation, masturbation, and infertility. London: Chapman and Hall.

Bale, C., \& Archer, J. (2013). Self-perceived attractiveness, romantic desirability and self-esteem: A mating sociometer perspective. Evolutionary Psychology, 11(1), 68-84. doi: 10.1177/147470491301100107

Barber, N. (1998). Sex differences in disposition towards kin, security of adult attachment, and sociosexuality as a function of parental divorce. Evolution and Human Behavior, 19(2), 125-132. doi: 10.1016/S1090-5138(98)00004-X

Bardwell, W. A., Natarajan, L., Dimsdale, J. E., Rock, C. L., Mortimer, J. E., Hollenbach, K., \& Pierce, J. P. (2006). Objective cancer-related variables are not associated with depressive symptoms in women treated for early-stage breast cancer. Journal of Clinical Oncology, 24(16), 2420-2427. doi:

10.1200/jco.2005.02.0081

Barelds, D. P. H., \& Barelds-Dijkstra, P. (2010). Humor in intimate relationships: Ties among sense of humor, similarity in humor and relationship quality. Humor, 23(4), 447-465. doi: 10.1515/HUMR.2010.021

Bargh, J. A. (2006). What have we been priming all these years? On the development, mechanisms, and ecology of nonconscious social behavior. European Journal of Social Psychology, 36(2), 147-168. doi: 10.1002/Ejsp.336

Bargh, J. A., \& Chartrand, T. L. (2000). Studying the mind in the middle: A practical guide to priming and automaticity research. In H. Reis \& C. Judd (Eds.), Handbook of research methods in social psychology (pp. 253-285). New York: Cambridge University Press.

Barkley, M. S., \& Goldman, B. D. (1977). Testosterone-induced aggression in adult female mice. Horm Behav, 9(1), 76-84. doi: 10.1016/0018-506x(77)90052-6

Barraclough, C. A., \& Gorski, R. A. (1962). Studies on mating behavior in the androgen-sterilized female rat in relation to the hypothalamic regulation of sexual behaviour. J Endocrinol, 25(2), 175-182. doi: 10.1677/joe.0.0250175

Barrett, J., Abbott, D. H., \& George, L. M. (1990). Extension of reproductive suppression by pheromonal cues in subordinate female marmoset monkeys, Callithrix jacchus. Journal of Reproduction and Fertility, 90(2), 411-418. doi: 10.1530/jrf.0.0900411

Barrett, L., Dunbar, R. I. M., \& Lycett, J. (2002). Human evolutionary psychology. Princeton, N.J.: Princeton University Press. 
Bateman, A. J. (1948). Intra-sexual selection in Drosophila. Heredity, 2(3), 349-368. doi: 10.1038/hdy.1948.21

Bateup, H. S., Booth, A., Shirtcliff, E. A., \& Granger, D. A. (2002). Testosterone, cortisol, and women's competition. Evolution and Human Behavior, 23(3), 181192. doi: 10.1016/S1090-5138(01)00100-3

Baumeister, R. F. (2000). Gender differences in erotic plasticity: The female sex drive as socially flexible and responsive. Psychol Bull, 126(3), 347-374. doi: 10.1037/0033-2909.126.3.347

Bean, L. L., \& Mineau, G. P. (1986). The polygyny-fertility hypothesis: A reevaluation. Population Studies, 40(1), 67-81. doi: 10.2307/2174280

Beehner, J. C., Bergman, T. J., Cheney, D. L., Seyfarth, R. M., \& Whitten, P. L. (2006). Testosterone predicts future dominance rank and mating activity among male chacma baboons. Behavioral Ecology and Sociobiology, 59(4), 469-479. doi: 10.1007/s00265-005-0071-2

Belsky, J., Houts, R. M., \& Fearon, R. M. P. (2010). Infant attachment security and the timing of puberty: Testing an evolutionary hypothesis. Psychological Science, 21(9), 1195-1201. doi: 10.1177/0956797610379867

Belsky, J., Schlomer, G. L., \& Ellis, B. J. (2012). Beyond cumulative risk: Distinguishing harshness and unpredictability as determinants of parenting and early life history strategy. Dev Psychol, 48(3), 662-673. doi: 10.1037/a0024454

Belsky, J., Steinberg, L., \& Draper, P. (1991). Childhood experience, interpersonal development, and reproductive strategy: And evolutionary theory of socialization. Child Dev, 62(4), 647-670. doi: 10.2307/1131169

Bereczkei, T., \& Csanaky, A. (1996). Mate choice, marital success, and reproduction in a modern society. Ethology and Sociobiology, 17(1), 17-35. doi: 10.1016/01623095(95)00104-2

Bereczkei, T., \& Csanaky, A. (2001). Stressful family environment, mortality, and child socialisation: Life-history strategies among adolescents and adults from unfavourable social circumstances. International Journal of Behavioral Development, 25(6), 501-508. doi: 10.1080/01650250042000573

Berglund, A. (1994). The operational sex-ratio influences choosiness in a pipefish. Behavioral Ecology, 5(3), 254-258. doi: 10.1093/beheco/5.3.254

Bernhardt, P. C., Dabbs Jr, J. M., Fielden, J. A., \& Lutter, C. D. (1998). Testosterone changes during vicarious experiences of winning and losing among fans at 
sporting events. Physiology \& Behavior, 65(1), 59-62. doi: 10.1016/S00319384(98)00147-4

Bernstein, I. S. (1969). Stability of the status hierarchy in a pigtail monkey group (macaca nemestrina). Animal Behaviour, 17, Part 3(0), 452-458. doi: 10.1016/0003-3472(69)90146-8

Betzig, L. (1989). Causes of conjugal dissolution: A cross-cultural study. Current Anthropology, 30(5), 654-676. doi: 10.1086/203798

Betzig, L. (2012). Means, variances, and ranges in reproductive success: Comparative evidence. Evolution and Human Behavior, 33(4), 309-317. doi: 10.1016/j.evolhumbehav.2011.10.008

Blanchard, R. (2001). Fraternal birth order and the maternal immune hypothesis of male homosexuality. Horm Behav, 40(2), 105-114. doi: 10.1006/hbeh.2001.1681

Bluestone, C. D. (2005). Humans are born too soon: Impact on pediatric otolaryngology. International Journal of Pediatric Otorhinolaryngology, 69(1), 1-8. doi: 10.1016/j.ijporl.2004.07.021

Blurton-Jones, N. G., Marlowe, F. G., Hakwes, K., \& O'Connell, J. F. (2000). Paternal investment and hunter-gatherer divorce rates. In L. Cronk, N. Chagnon \& W. Irons (Eds.), Adaptation and human behaviour: An anthropological perspective (pp. 69-90). New York: Aldine de Gruyter.

Bogaert, A. F., \& Fisher, W. A. (1995). Predictors of university men's number of sexual partners. The Journal of Sex Research, 32(2), 119-130. doi: 10.2307/3812964

Book, A. S., Starzyk, K. B., \& Quinsey, V. L. (2001). The relationship between testosterone and aggression: A meta-analysis. Aggression and Violent Behavior, 6(6), 579-599. doi: 10.1016/S1359-1789(00)00032-X

Booth, A., Shelley, G., Mazur, A., Tharp, G., \& Kittok, R. (1989). Testosterone, and winning and losing in human competition. Horm Behav, 23(4), 556-571. doi: $10.1016 / 0018-506 X(89) 90042-1$

Boothe, J. E. (1977). Sexual behaviour of neonatally castrated rats injected during infancy with oestrogen and dihydrotestosterone. Journal of Endocrinology, 72(2), 135-141. doi: 10.1677/joe.0.0720135

Boothroyd, L. G., Jones, B. C., Burt, D. M., DeBruine, L. M., \& Perrett, D. I. (2008). Facial correlates of sociosexuality. Evolution and Human Behavior, 29(3), 211218. doi: 10.1016/j.evolhumbehav.2007.12.009 
Borgia, G. (1995). Complex male display and female choice in the spotted bowerbird specialized functions for different bower decorations. Animal Behaviour, 49(5), 1291-1301. doi: 10.1006/anbe.1995.0161

Bower, G. H. (1961). A contrast effect in differential conditioning. Journal of Experimental Psychology, 62(2), 196-199. doi: 10.1037/h0048109

Bowles, S. (2009). Did warfare among ancestral hunter-gatherers affect the evolution of human social behaviors? Science, 324(5932), 1293-1298. doi:

$10.1126 /$ science. 1168112

Braun, D. R., Harris, J. W. K., Levin, N. E., McCoy, J. T., Herries, A. I. R., Bamford, M. K., . . Kibunjia, M. (2010). Early hominin diet included diverse terrestrial and aquatic animals 1.95 ma in East Turkana, Kenya. Proceedings of the National Academy of Sciences, 107(22), 10002-10007. doi:

10.1073/pnas.1002181107

Bressan, P., \& Dal Pos, S. (2012). Fathers see stronger family resemblances than nonfathers in unrelated children's faces. Archives of Sexual Behavior, 41(6), 1423 1430. doi: 10.1007/s10508-012-9983-X

Brondino, N., Lanati, N., Giudici, S., Arpesella, M., Roncarolo, F., \& Vandoni, M. (In Press). Testosterone level and its relationship with outcome of sporting activity. Journal of Men's Health(0). doi: 10.1016/j.jomh.2012.10.003

Brown, G. R., Laland, K. N., \& Mulder, M. B. (2009). Bateman's principles and human sex roles. Trends in Ecology and Evolution, 24(6), 297-304. doi: 10.1016/j.tree.2009.02.005

Brown, J. D., \& Dutton, K. A. (1995). The thrill of victory, the complexity of defeat self-esteem and peoples emotional-reactions to success and failure. Journal of Personality and Social Psychology, 68(4), 712-722. doi: 10.1037//00223514.68.4.712

Brown, P., Sutikna, T., Morwood, M. J., Soejono, R. P., Jatmiko, Wayhu Saptomo, E., \& Awe Due, R. (2004). A new small-bodied hominin from the late Pleistocene of flores, indonesia. Nature, 431(7012), 1055-1061. doi: 10.1038/nature02999

Brumbach, B. H., Figueredo, A. J., \& Ellis, B. J. (2009). Effects of harsh and unpredictable environments in adolescence on development of life history strategies. Human Nature, 20(1), 25-51. doi: 10.1007/s12110-009-9059-3 
Bryden, M. M. (1972). Body size and composition of elephant seals (Mirounga leonine): Absolute measurements and estimates from bone dimensions. Journal of Zoology, 167(3), 265-276. doi: 10.1111/j.1469-7998.1972.tb03110.x

Burchell, J. L., \& Ward, J. (2011). Sex drive, attachment style, relationship status and previous infidelity as predictors of sex differences in romantic jealousy.

Personality and Individual Differences, 51(5), 657-661. doi:

10.1016/j.paid.2011.06.002

Burley, N. T. (1986). Sex-ratio manipulation in color-banded populations of zebra finches. Evolution, 40(6), 1191-1206.

Burley, N. T., \& Foster, V. S. (2006). Variation in female choice of mates: Condition influences selectivity. Animal Behaviour, 72(3), 713-719. doi: 10.1016/j.anbehav.2006.01.017

Burriss, R. P., Welling, L. L. M., \& Puts, D. A. (2011). Men's attractiveness predicts their preference for female facial femininity when judging for short-term, but not long-term, partners. Personality and Individual Differences, 50(5), 542-546. doi: 10.1016/j.paid.2010.11.022

Buss, D. M. (1985). Human mate selection: Opposites are sometimes said to attract, but in fact we are likely to marry someone who is similar to us in almost every variable. American Scientist(73), 47-51.

Buss, D. M. (1989). Sex differences in human mate preferences: Evolutionary hypotheses tested in 37 cultures. Behavioral and Brain Sciences, 12(1), 1-49. doi: $10.1017 / \mathrm{s} 0140525 \times 00023992$

Buss, D. M. (2002). Human mating strategies. Samfundsøkonomen, 4, 47-58.

Buss, D. M. (2003a). The evolution of desire: Strategies of human mating. New York: Basic Books.

Buss, D. M. (2003b). Sexual strategies: A journey into controversy. Psychological Inquiry, 14(3/4), 219-226. doi: 10.2307/1449679

Buss, D. M. (2006a). The evolution of love. In R. J. Sternberg \& K. Weis (Eds.), The new psychology of love (pp. 65-86). New Haven, CT: Yale University Press.

Buss, D. M. (2006b). Strategies of human mating. Psychological Topics, 15(2), 239260.

Buss, D. M., Abbott, M., Angleitner, A., Asherian, A., Biaggio, A., Blanco-Villasenor, A., ... Yang, K.-S. (1990). International preferences in selecting mates: A study 
of 37 cultures. Journal of Cross-Cultural Psychology, 21(1), 5-47. doi: $10.1177 / 0022022190211001$

Buss, D. M., \& Barnes, M. (1986). Preferences in human mate selection. Journal of Personality and Social Psychology, 50(3), 559-570. doi: 10.1037/00223514.50.3.559

Buss, D. M., \& Haselton, M. (2005). The evolution of jealousy. Trends Cogn Sci, 9(11), 506-507; author reply 508-510. doi: 10.1016/j.tics.2005.09.006

Buss, D. M., Larsen, R. J., Westen, D., \& Semmelroth, J. (1992). Sex differences in jealousy: Evolution, physiology, and psychology. Psychological Science, 3(4), 251-255. doi: 10.1111/j.1467-9280.1992.tb00038.x

Buss, D. M., \& Schmitt, D. P. (1993). Sexual strategies theory: An evolutionary perspective on human mating. Psychological Review, 100(2), 204-232. doi: $10.1037 / 0033-295 x .100 .2 .204$

Buss, D. M., \& Shackelford, T. K. (1997). From vigilance to violence: Mate retention tactics in married couples. Journal of Personality and Social Psychology, 72(2), 346-361. doi: 10.1037/0022-3514.72.2.346

Buss, D. M., \& Shackelford, T. K. (2008). Attractive women want it all: Good genes, economic investment, parenting proclivities, and emotional commitment. Evolutionary Psychology, 6(1), 134-146.

Buston, P. M. (2004). Territory inheritance in clownfish. Proceedings of the Royal Society of London. Series B: Biological Sciences, 271(Suppl 4), S252-S254. doi: 10.1098/rsbl.2003.0156

Buunk, B. P., Dijkstra, P., Kenrick, D. T., \& Warntjes, A. (2001). Age preferences for mates as related to gender, own age, and involvement level. Evolution and Human Behavior, 22(4), 241-250. doi: 10.1016/S1090-5138(01)00065-4

Byers, J. A., \& Waits, L. (2006). Good genes sexual selection in nature. Proceedings of the National Academy of Sciences, 103(44), 16343-16345. doi: 10.1073/pnas.0608184103

Byrnes, J. P., Miller, D. C., \& Schafer, W. D. (1999). Gender differences in risk taking: A meta-analysis. Psychol Bull, 125(3), 367-383. doi: 10.1037/00332909.125.3.367

Cantor, J. M., Blanchard, R., Paterson, A. D., \& Bogaert, A. F. (2002). How many gay men owe their sexual orientation to fraternal birth order? Archives of Sexual Behavior, 31(1), 63-71. doi: 10.1023/a:1014031201935 
Carlson, N. R. (2002). Foundations of physiological psychology (5th ed.). Boston: Allyn and Bacon.

Carré, J. M., Campbell, J. A., Lozoya, E., Goetz, S. M. M., \& Welker, K. M. (In Press). Changes in testosterone mediate the effect of winning on subsequent aggressive behaviour. Psychoneuroendocrinology(0). doi: 10.1016/j.psyneuen.2013.03.008

Carré, J. M., \& Putnam, S. K. (2010). Watching a previous victory produces an increase in testosterone among elite hockey players. Psychoneuroendocrinology, 35(3), 475-479. doi: 10.1016/j.psyneuen.2009.09.011

Carré, J. M., Putnam, S. K., \& McCormick, C. M. (2009). Testosterone responses to competition predict future aggressive behaviour at a cost to reward in men. Psychoneuroendocrinology, 34(4), 561-570. doi: 10.1016/j.psyneuen.2008.10.018

Carretero, J.-M., Rodríguez, L., García-González, R., Arsuaga, J.-L., Gómez-Olivencia, A., Lorenzo, C., . . Quam, R. (2012). Stature estimation from complete long bones in the Middle Pleistocene humans from the Sima de los Huesos, Sierra de Atapuerca (Spain). Journal of Human Evolution, 62(2), 242-255. doi: 10.1016/j.jhevol.2011.11.004

Central Statistics Office. (2012). Profile 7: Religion, ethnicity and Irish travellers: Government of Ireland.

Cerling, T. E., Mbua, E., Kirera, F. M., Manthi, F. K., Grine, F. E., Leakey, M. G., . . Uno, K. T. (2011). Diet of Paranthropus boisei in the early Pleistocene of East Africa. Proceedings of the National Academy of Sciences. doi: 10.1073/pnas.1104627108

Chang, L., Wang, Y., Shackelford, T. K., \& Buss, D. M. (2011). Chinese mate preferences: Cultural evolution and continuity across a quarter of a century. Personality and Individual Differences, 50(5), 678-683. doi: 10.1016/j.paid.2010.12.016

Charles, N. E., \& Alexander, G. M. (2011). The association between 2D:4D ratios and sociosexuality: A failure to replicate. Archives of Sexual Behavior, 40(3), 587595. doi: 10.1007/s10508-010-9715-Z

Chase, I. D., Bartolomeo, C., \& Dugatkin, L. A. (1994). Aggressive interactions and inter-contest interval: How long do winners keep winning? Animal Behaviour, 48(2), 393-400. doi: 10.1006/anbe.1994.1253 
Cheng, J. T., Tracy, J. L., \& Henrich, J. (2010). Pride, personality, and the evolutionary foundations of human social status. Evolution and Human Behavior, 31(5), 334347. doi: 10.1016/j.evolhumbehav.2010.02.004

Chisholm, J. S., Ellison, P. T., Evans, J., Lee, P. C., Lieberman, L. S., Pavlik, Z., . . . Worthman, C. M. (1993). Death, hope, and sex: Life-history theory and the development of reproductive strategies [and comments and reply]. Current Anthropology, 34(1), 1-24. doi: 10.2307/2743728

Christenson, T. E., \& Le Boeuf, B. J. (1978). Aggression in the female northern elephant seal, Mirounga angustirostris. Behaviour, 64(1-2), 158-172. doi: $10.1163 / 156853978 \times 00495$

City and County of Swansea. (2010). Students in Swansea University Health, Social Care and Well-being Needs Assessment: City and County of Swansea.

Clark, A. P. (2006). Are the correlates of sociosexuality different for men and women? Personality and Individual Differences, 41(7), 1321-1327. doi: 10.1016/j.paid.2006.05.006

Clark, R. D., \& Hatfield, E. (1989). Gender differences in receptivity to sexual offers. Journal of Psychology \& Human Sexuality, 2(1), 39-55. doi: 10.1300/J056v02n01_04

Clotfelter, E. D., O'Neal, D. M., Gaudioso, J. M., Casto, J. M., Parker-Renga, I. M., Snajdr, E. A., . . Ketterson, E. D. (2004). Consequences of elevating plasma testosterone in females of a socially monogamous songbird: Evidence of constraints on male evolution? Horm Behav, 46(2), 171-178. doi: 10.1016/j.yhbeh.2004.03.003

Clutton-Brock, T. H. (2007). Sexual selection in males and females. Science, 318(5858), 1882-1885. doi: 10.1126/science.1133311

Clutton-Brock, T. H., Albon, S. D., \& Harvey, P. H. (1980). Antlers, body size and breeding group size in the cervidae. Nature, 285(5766), 565-567. doi: $10.1038 / 285565 \mathrm{a} 0$

Clutton-Brock, T. H., \& Vincent, A. C. (1991). Sexual selection and the potential reproductive rates of males and females. Nature, 351(6321), 58-60. doi: $10.1038 / 351058 \mathrm{a} 0$

Cohen, A. B., \& Tannenbaum, H. J. (2001). Lesbian and bisexual women's judgments of the attractiveness of different body types. Journal of Sex Research, 38(3), 226-232. doi: 10.1080/00224490109552091 
Cohen, J. (1988). Statistical power analysis for the behavioral sciences (2nd ed.).

Hillsdale, N.J.: L. Erlbaum Associates.

Confer, J. C., Perilloux, C., \& Buss, D. M. (2010). More than just a pretty face: Men's priority shifts toward bodily attractiveness in short-term versus long-term mating contexts. Evolution and Human Behavior, 31(5), 348-353. doi:

10.1016/j.evolhumbehav.2010.04.002

Conroy, G. C., Weber, G. W., Seidler, H., Recheis, W., Zur Nedden, D., \& Mariam, J. H. (2000). Endocranial capacity of the bodo cranium determined from threedimensional computed tomography. American Journal of Physical Anthropology, 113(1), 111-118. doi: 10.1002/10968644(200009)113:1<111::aid-ajpa10>3.0.co;2-X

Cook, C. J., \& Crewther, B. T. (2012). Changes in salivary testosterone concentrations and subsequent voluntary squat performance following the presentation of short video clips. Horm Behav, 61(1), 17-22. doi: 10.1016/j.yhbeh.2011.09.006

Cools, J., Schotte, D. E., \& McNally, R. J. (1992). Emotional arousal and overeating in restrained eaters. Journal of Abnormal Psychology, 101(2), 348-351. doi: $10.1037 / 0021-843 x .101 .2 .348$

Cosmides, L., \& Tooby, J. (1992a). Cognitive adaptations for social exchange. In J. H. Barkow, L. Cosmides \& J. Tooby (Eds.), The adapted mind : Evolutionary psychology and the generation of culture (pp. xii, 666 p.). New York: Oxford University Press.

Cosmides, L., \& Tooby, J. (1992b). The psychological foundations of culture. In J. H. Barkow, L. Cosmides \& J. Tooby (Eds.), The adapted mind : Evolutionary psychology and the generation of culture (pp. xii, 666 p.). New York: Oxford University Press.

Côté, S. D., \& Festa-Bianchet, M. (2001). Reproductive success in female mountain goats: The influence of age and social rank. Animal Behaviour, 62(1), 173-181. doi: 10.1006/anbe.2001.1719

Cox, O. C. (1940). Sex ratio and marital status among negroes. American Sociological Review, 5(6), 937-947. doi: 10.2307/2084528

Cramér, H. (1999). Mathematical methods of statistics. Princeton: Princeton University Press. 
Crewther, B. T., Keogh, J., Cronin, J., \& Cook, C. (2006). Possible stimuli for strength and power adaptation: Acute hormonal responses. Sports Med, 36(3), 215-238. doi: 10.2165/00007256-200636030-00004

Crewther, B. T., Thomas, A. G., Kilduff, L., Stewart-Williams, S., \& Cook, C. (In submission). Dual effect of testosterone and cortisol on hand-grip strength in healthy men.

Cristol, D. A. (1995). Early arrival, initiation of nesting, and social status: An experimental study of breeding female red-winged blackbirds. Behavioral Ecology, 6(1), 87-93. doi: 10.1093/beheco/6.1.87

Cross, C. P., Copping, L. T., \& Campbell, A. (2011). Sex differences in impulsivity: A meta-analysis. Psychological Bulletin, 137(1), 97-130. doi: 10.1037/a0021591

Crow, T. J. (2002). The speciation of modern Homo sapiens. Oxford ; New York: Oxford University Press.

Cumming, D. C., Brunsting, L. A., 3rd, Strich, G., Ries, A. L., \& Rebar, R. W. (1986). Reproductive hormone increases in response to acute exercise in men. Med Sci Sports Exerc, 18(4), 369-373. doi: 10.1249/00005768-198608000-00001

Curtis, V., Aunger, R., \& Rabie, T. (2004). Evidence that disgust evolved to protect from risk of disease. Proceedings of the Royal Society of London. Series B: Biological Sciences, 271(Suppl 4), S131-S133. doi: 10.1098/rsbl.2003.0144

Dabbs, J. M., \& Hargrove, M. F. (1997). Age, testosterone, and behavior among female prison inmates. Psychosomatic Medicine, 59(5), 477-480. doi: 10.1097/00006842-199709000-00003

Dabbs, J. M., Ruback, R. B., Frady, R. L., Hopper, C. H., \& Sgoutas, D. S. (1988). Saliva testosterone and criminal violence among women. Personality and Individual Differences, 9(2), 269-275. doi: 10.1016/0191-8869(88)90088-8

Daly, M., \& Wilson, M. (2001). Risk-taking, intersexual competition, and homicide. Nebraska Symposium on Motivation, 47, 1-36.

Damashek, A., Nelson, M. M., \& Bonner, B. L. (2013). Fatal child maltreatment: Characteristics of deaths from physical abuse versus neglect. Child Abuse \& Neglect, 37(10), 735-744. doi: 10.1016/j.chiabu.2013.04.014

Darwin, C. (1859). On the origin of species by means of natural selection. London,: J. Murray.

Darwin, C. (1871). The descent of man, and selection in relation to sex. London,: J. Murray. 
Davis, S. R., \& Tran, J. (2001). Testosterone influences libido and well being in women. Trends Endocrinol Metab, 12(1), 33-37. doi: 10.1016/S10432760(00)00333-7

Dawkins, R. (1976). The selfish gene. Oxford: Oxford University Press.

Dawkins, R. (1994). Blind watchmaker. New York: W.W. Norton.

de Waal, F. B. M. (2007). Chimpanzee politics : Power and sex among apes (25th anniversary ed.). Baltimore, Md.: Johns Hopkins University Press.

DeBruine, L. M. (2005). Trustworthy but not lust-worthy: Context-specific effects of facial resemblance. Proceedings of the Royal Society B: Biological Sciences, 272(1566), 919-922. doi: 10.1098/rspb.2004.3003

Del-Monte, J., Raffard, S., Capdevielle, D., Salesse, R. N., Schmidt, R. C., Varlet, M., . . Marin, L. (2014). Social priming increases nonverbal expressive behaviors in schizophrenia. PLoS ONE, 9(10), e109139. doi: 10.1371/journal.pone.0109139

DeSteno, D., Bartlett, M. Y., Braverman, J., \& Salovey, P. (2002). Sex differences in jealousy: Evolutionary mechanism or artifact of measurement? Journal of Personality and Social Psychology, 83(5), 1103-1116. doi: 10.1037/00223514.83.5.1103

Dickins, T. E., Johns, S. E., \& Chimpan, A. (2012). Teenage pregnancy in the United Kingdom: A behavioral ecological perspective. Journal of Social, Evolutionary, and Cultural Psychology, 6(3), 344-359. doi: 10.1037/h0099247

Dijksterhuis, A. (2004). I like myself but i don't know why: Enhancing implicit selfesteem by subliminal evaluative conditioning. Journal of Personality and Social Psychology, 86(2), 345-355. doi: 10.1037/0022-3514.86.2.345

Dittmar, H., Halliwell, E., \& Ive, S. (2006). Does Barbie make girls want to be thin? The effect of experimental exposure to images of dolls on the body image of 5to 8-year-old girls. Developmental Psychology, 42(2), 283-292. doi: $10.1037 / 0012-1649.42 .2 .283$

Dixson, A. F. (2009). Sexual selection and the origins of human mating systems (1st ed.). Oxford ; New York: Oxford University Press.

Dixson, A. F., \& Herbert, J. (1977). Gonadal hormones and sexual behavior in groups of adult talapoin monkeys (miopithecus talapoin). Horm Behav, 8(2), 141-154. doi: 10.1016/0018-506X(77)90031-9

Dorjahn, V. R. (1958). Fertility, polygyny and their interrelations in Temne society. American Anthropologist, 60(5), 838-860. doi: 10.1525/aa.1958.60.5.02a00050 
Drummond, P. D. (2001). The effect of true and false feedback on blushing in women. Personality and Individual Differences, 30(8), 1329-1343. doi: 10.1016/S01918869(00)00115-X

Duffy, E. E., Penn, D. J., Botton, M. L., Brockmann, H. J., \& Loveland, R. E. (2006). Eye and clasper damage influence male mating tactics in the horseshoe crab, Limulus polyphemus. Journal of Ethology, 24(1), 67-74. doi: 10.1007/s10164005-0163-5

Dufty Jr, A. M. (1989). Testosterone and survival: A cost of aggressiveness? Horm Behav, 23(2), 185-193. doi: 10.1016/0018-506X(89)90059-7

Dunbar, R. I. M. (1993). Coevolution of neocortical size, group size and language in humans. Behavioral and Brain Sciences, 16(04), 681-694. doi: $10.1017 / \mathrm{S} 0140525 \mathrm{X} 00032325$

Dunbar, R. I. M. (1998). The social brain hypothesis. Evolutionary Anthropology, 6(5), 178-190.

Dunn, M. J., \& Hill, A. (2014). Manipulated luxury-apartment ownership enhances opposite-sex attraction in females but not males. Journal of Evolutionary Psychology, 12(1), 1-17. doi: 10.1556/jep.12.2014.1.1

Dunn, M. J., \& Searle, R. (2010). Effect of manipulated prestige-car ownership on both sex attractiveness ratings. British Journal of Psychology, 101(1), 69-80. doi: $10.1348 / 000712609 \times 417319$

Durante, K. M., Griskevicius, V., Hill, S. E., Perilloux, C., \& Li, N. P. (2011). Ovulation, female competition, and product choice: Hormonal influences on consumer behavior. Journal of Consumer Research, 37(6), 921-934. doi: $10.1086 / 656575$

Durante, K. M., Griskevicius, V., Simpson, J. A., Cantu, S. M., \& Tybur, J. M. (2012). Sex ratio and women's career choice: Does a scarcity of men lead women to choose briefcase over baby? Journal of Personality and Social Psychology, 103(1), 121-134. doi: 10.1037/a0027949

Dyakonova, V. E., Schürmann, F. W., \& Sakharov, D. A. (1999). Effects of serotonergic and opioidergic drugs on escape behaviors and social status of male crickets. Naturwissenschaften, 86(9), 435-437. doi: 10.1007/s001140050647

Eastwick, P. W. (2013). The psychology of the pair-bond: Past and future contributions of close relationships research to evolutionary psychology. Psychological Inquiry, 24(3), 183-191. doi: 10.1080/1047840x.2013.816927 
Eastwick, P. W., \& Buck, A. A. (2014). Too much matching: A social relations model enhancement of the pairing game. Teaching of Psychology, 41(3), 246-250. doi: $10.1177 / 0098628314537980$

Edelstein, R. S., Chopik, W. J., \& Kean, E. L. (2011). Sociosexuality moderates the association between testosterone and relationship status in men and women. Horm Behav, 60(3), 248-255. doi: 10.1016/j.jyhbeh.2011.05.007

Edwards, D. A., Wetzel, K., \& Wyner, D. R. (2006). Intercollegiate soccer: Saliva cortisol and testosterone are elevated during competition, and testosterone is related to status and social connectedness with teammates. Physiology \& Behavior, 87(1), 135-143. doi: 10.1016/j.physbeh.2005.09.007

Eens, M., \& Pinxten, R. (2000). Sex-role reversal in vertebrates: Behavioural and endocrinological accounts. Behavioural Processes, 51(1-3), 135-147. doi: $10.1016 / \mathrm{s} 0376-6357(00) 00124-8$

Eggers, S., Griesser, M., Nystrand, M., \& Ekman, J. (2006). Predation risk induces changes in nest-site selection and clutch size in the Siberian jay. Proceedings of the Royal Society B-Biological Sciences, 273(1587), 701-706. doi: 10.1098/rspb.2005.3373

Eggert-Kruse, W., Gerhard, I., Näher, H., Tilgen, W., \& Runnebaum, B. (1990). Chlamydial infection - a female and/or male infertility factor? Fertility and sterility, 53(6), 1037-1043. doi: 10.1016/0020-7292(91)90635-I

Eibach, R. P., \& Mock, S. E. (2011). The vigilant parent: Parental role salience affects parents' risk perceptions, risk-aversion, and trust in strangers. Journal of Experimental Social Psychology, 47(3), 694-697. doi: 10.1016/j.jesp.2010.12.009

Ekman, P., Friesen, W. V., O'Sullivan, M., Chan, A., Diacoyanni-Tarlatzis, I., Heider, K., .. . Tzavaras, A. (1987). Universals and cultural differences in the judgments of facial expressions of emotion. Journal of Personality and Social Psychology, 53(4), 712-717. doi: 10.1037/0022-3514.53.4.712

Ellis, B. J., \& Kelley, H. H. (1999). The pairing game: A classroom demonstration of the matching phenomenon. Teaching of Psychology, 26, 118-121. doi: 10.1207/s15328023top2602_8

Ellis, B. J., \& Symons, D. (1990). Sex differences in sexual fantasy: An evolutionary psychological approach. The Journal of Sex Research, 27(4), 527-555. doi: $10.1080 / 00224499009551579$ 
Emlen, S. T., \& Oring, L. W. (1977). Ecology, sexual selection, and evolution of mating systems. Science, 197(4300), 215-223. doi: 10.1126/science.327542

Erber, R., Wegner, D. M., \& Therriault, N. (1996). On being cool and collected: Mood regulation in anticipation of social interaction. Journal of Personality and Social Psychology, 70(4), 757-766. doi: 10.1037/0022-3514.70.4.757

Erdfelder, E., Faul, F., \& Buchner, A. (1996). Gpower: A general power analysis program. Behavior Research Methods, Instruments, and Computers, 28(1), 1-11. doi: 10.3758/BF03203630

Eriksson, A., \& Manica, A. (2012). Effect of ancient population structure on the degree of polymorphism shared between modern human populations and ancient hominins. Proceedings of the National Academy of Sciences, 109(35), 1395613960. doi: 10.1073/pnas.1200567109

Eston, R. G., \& Williams, J. G. (1988). Reliability of ratings of perceived effort regulation of exercise intensity. British Journal of Sports Medicine, 22(4), 153155. doi: 10.1136/bjsm.22.4.153

Fabiani, A., Galimberti, F., Sanvito, S., \& Hoelzel, A. R. (2004). Extreme polygyny among southern elephant seals on Sea Lion Island, Falkland Islands. Behavioral Ecology, 15(6), 961-969. doi: 10.1093/beheco/arh112

Fahey, T. D., Rolph, R., Moungmee, P., Nagel, J., \& Mortara, S. (1976). Serum testosterone, body-composition, and strength of young-adults. Med Sci Sports Exerc, 8(1), 31-34. doi: 10.1249/00005768-197621000-00019

Falótico, T., \& Ottoni, E. B. (2014). Sexual bias in probe tool manufacture and use by wild bearded capuchin monkeys. Behavioural Processes, 108(0), 117-122. doi: 10.1016/j.beproc.2014.09.036

Fessler, D. M. T., Holbrook, C., Pollack, J. S., \& Hahn-Holbrook, J. (2014). Stranger danger: Parenthood increases the envisioned bodily formidability of menacing men. Evolution and Human Behavior, 35(2), 109-117. doi:

10.1016/j.evolhumbehav.2013.11.004

Fieder, M., \& Huber, S. (2007). The effects of sex and childlessness on the association between status and reproductive output in modern society. Evolution and Human Behavior, 28(6), 392-398. doi: 10.1016/j.evolhumbehav.2007.05.004

Field, A. P. (2009). Discovering statistics using SPSS: (and sex, drugs and rock ' $n$ ' roll) (3rd ed.). Los Angeles: SAGE Publications. 
Figueredo, A. J., Vásquez, G., Brumbach, B. H., Schneider, S. M. R., Sefcek, J. A., Tal, I. R., . . Jacobs, W. J. (2006). Consilience and life history theory: From genes to brain to reproductive strategy. Developmental Review, 26(2), 243-275. doi: 10.1016/j.dr.2006.02.002

Fink, B., Thanzami, V., Seydel, H., \& Manning, J. T. (2006). Digit ratio and hand-grip strength in german and mizos men: Cross-cultural evidence for an organizing effect of prenatal testosterone on strength. American Journal of Human Biology, 18(6), 776-782. doi: 10.1002/Ajhb.20549

Fishbach, A., \& Labroo, A. A. (2007). Be better or be merry: How mood affects selfcontrol. Journal of Personality and Social Psychology, 93(2), 158-173. doi: 10.1037/0022-3514.93.2.158

Fisher, H. E. (1989). Evolution of human serial pairbonding. American Journal of Physical Anthropology, 78(3), 331-354.

Fisher, R. A. (1915). The evolution of sexual preference. Eugenics Review, 7, 184-192.

Fisher, R. A. (1930). The genetical theory of natural selection. Oxford: Clarendon Press.

Fitzpatrick, J. L., Desjardins, J. K., Milligan, N., Stiver, K. A., Montgomerie, R., \& Balshine, S. (2008). Female-mediated causes and consequences of status change in a social fish. Proceedings of the Royal Society B: Biological Sciences, 275(1637), 929-936. doi: 10.1098/rspb.2007.1449

Flake, K. (2009). The emotional and priestly logic of plural marriage. Paper presented at the Arrington Annual Lecture.

Fleming, A. S., Corter, C., Stallings, J., \& Steiner, M. (2002). Testosterone and prolactin are associated with emotional responses to infant cries in new fathers. Horm Behav, 42(4), 399-413. doi: 10.1006/hbeh.2002.1840

Fontaine, J. J., \& Martin, T. E. (2006). Parent birds assess nest predation risk and adjust their reproductive strategies. Ecology Letters, 9(4), 428-434. doi:

10.1111/j.1461-0248.2006.00892.x

Forgas, J. P. (2007). When sad is better than happy: Negative affect can improve the quality and effectiveness of persuasive messages and social influence strategies. Journal of Experimental Social Psychology, 43(4), 513-528. doi: 10.1016/j.jesp.2006.05.006

Forsyth, A. (2001). A natural history of sex - the ecology and evolution of mating behavior: Firefly Books. 
Frederick, D. A., \& Fales, M. R. (2014). Upset over sexual versus emotional infidelity among gay, lesbian, bisexual, and heterosexual adults. Archives of Sexual Behavior, 1-17. doi: 10.1007/s10508-014-0409-9

Freeman, M., Nkomo, N., Kafaar, Z., \& Kelly, K. (2008). Mental disorder in people living with hiv/aids in south africa. South African Journal of Psychology, 38(3), 489-500. doi: 10.1177/008124630803800304

Froehle, A. W., \& Churchill, S. E. (2009). Energetic competition between neandertals and anatomically modern humans. PaleoAnthropology, 2009, 96-116.

Fuelling, O., \& Halle, S. (2004). Breeding suppression in free-ranging grey-sided voles under the influence of predator odour. Oecologia, 138(1), 151-159. doi: 10.1007/s00442-003-1417-y

Gage, A. R., \& Barnard, C. J. (1996). Male crickets increase sperm number in relation to competition and female size. Behavioral Ecology and Sociobiology, 38(5), 349-353. doi: 10.1007/s002650050251

Gaines, M. S., Fugate, C. L., Johnson, M. L., Johnson, D. C., Hisey, J. R., \& Quadagno, D. M. (1985). Manipulation of aggressive behavior in male prairie voles (Microtus ochrogaster) implanted with testosterone in Silastic tubing. Canadian Journal of Zoology, 63(11), 2525-2528. doi: 10.1139/z85-374

Galimberti, F., Sanvito, S., Braschi, C., \& Boitani, L. (2007). The cost of success: Reproductive effort in male southern elephant seals (Mirounga leonina). Behavioral Ecology and Sociobiology, 62(2), 159-171. doi: 10.1007/s00265007-0450-y

Gallup, A. C., White, D. D., \& Gallup Jr, G. G. (2007). Handgrip strength predicts sexual behavior, body morphology, and aggression in male college students. Evolution and Human Behavior, 28(6), 423-429. doi:

10.1016/j.evolhumbehav.2007.07.001

Gangestad, S. W., \& Buss, D. M. (1993). Pathogen prevalence and human mate preferences. Ethology and Sociobiology, 14(2), 89-96. doi: 10.1016/01623095(93)90009-7

Gangestad, S. W., \& Simpson, J. A. (2000). The evolution of human mating: Trade-offs and strategic pluralism. Behavioral and Brain Sciences, 23(04), 573-587. doi: 10.1017/S0140525X0000337X 
Gangestad, S. W., \& Thornhill, R. (1997). The evolutionary psychology of extrapair sex: The role of fluctuating asymmetry. Evolution and Human Behavior, 18(2), 69-88. doi: 10.1016/S1090-5138(97)00003-2

Gangestad, S. W., Thornhill, R., \& Garver-Apgar, C. E. (2010). Fertility in the cycle predicts women's interest in sexual opportunism. Evolution and Human Behavior, 31(6), 400-411. doi: 10.1016/j.evolhumbehav.2010.05.003

Garwicz, M., Christensson, M., \& Psouni, E. (2009). A unifying model for timing of walking onset in humans and other mammals. Proceedings of the National Academy of Sciences, 106(51), 21889-21893. doi: 10.1073/pnas.0905777106

Gatti, R., \& De Palo, E. F. (2011). An update: Salivary hormones and physical exercise. Scandinavian Journal of Medicine \& Science in Sports, 21(2), 157-169. doi: 10.1111/j.1600-0838.2010.01252.x

Geary, D. C. (2000). Evolution and proximate expression of human paternal investment. Psychol Bull, 126(1), 55-77. doi: 10.1037/0033-2909.126.1.55

Geissmann, T. (1993). Evolution of communication in gobbons (Hylobatidae). Ph.D., Zürich University.

Gelissen, J. (2004). Assortative mating after divorce: A test of two competing hypotheses using marginal models. Social Science Research, 33(3), 361-384. doi: 10.1016/j.ssresearch.2003.06.003

Geraerts, E., Bernstein, D. M., Merckelbach, H., Linders, C., Raymaekers, L., \& Loftus, E. F. (2008). Lasting false beliefs and their behavioral consequences. Psychological Science, 19(8), 749-753. doi: 10.1111/j.1467-9280.2008.02151.x

Gettler, L. T. (2010). Direct male care and hominin evolution: Why male-child interaction is more than a nice social idea. American Anthropologist, 112(1), 721. doi: 10.1111/j.1548-1433.2009.01193.x

Gettler, L. T., McDade, T. W., Agustin, S. S., Feranil, A. B., \& Kuzawa, C. W. (2013). Do testosterone declines during the transition to marriage and fatherhood relate to men's sexual behavior? Evidence from the Philippines. Horm Behav, 64(5), 755-763. doi: 10.1016/j.yhbeh.2013.08.019

Gettler, L. T., McDade, T. W., Agustin, S. S., \& Kuzawa, C. W. (2011). Short-term changes in fathers' hormones during father-child play: Impacts of paternal attitudes and experience. Horm Behav, 60(5), 599-606. doi: 10.1016/j.yhbeh.2011.08.009 
Gettler, L. T., McDade, T. W., Feranil, A. B., \& Kuzawa, C. W. (2011). Longitudinal evidence that fatherhood decreases testosterone in human males. Proceedings of the National Academy of Sciences, 108(39), 16194-16199. doi: 10.1073/pnas.1105403108

Gibson, J. J. (1933). Adaptation, after-effect and contrast in the perception of curved lines. Journal of Experimental Psychology, 16(1), 1-31. doi: 10.1037/h0074626

Giosan, C. (2006). High-K strategy scale: A measure of the High-K independent criterion of fitness. Evolutionary Psychology, 4, 394-405.

Giosan, C. (2013). 'Slow' reproductive strategy: A negative predictor of depressive symptomatology. Australian Journal of Psychology, 65(3), 156-162. doi: 10.1111/ajpy.12016

Glaister, M., Stone, M. H., Stewart, A. M., Hughes, M. G., \& Moir, G. L. (2006). Aerobic and anaerobic correlates of multiple sprint cycling performance. $J$ Strength Cond Res, 20(4), 792-798. doi: 10.1519/R-18705.1

Gobrogge, K. L., Perkins, P. S., Baker, J. H., Balcer, K. D., Breedlove, S. M., \& Klump, K. L. (2007). Homosexual mating preferences from an evolutionary perspective: Sexual selection theory revisited. Archives of Sexual Behavior, 36(5), 717-723. doi: 10.1007/s10508-007-9216-X

Golombok, S., \& Tasker, F. (1996). Do parents influence the sexual orientation of their children? Findings from a longitudinal study of lesbian families. Developmental Psychology, 32(1), 3-11. doi: 10.1037/0012-1649.32.1.3

Gomes, C. M., \& Boesch, C. (2009). Wild chimpanzees exchange meat for sex on a long-term basis. PLoS ONE, 4(4), e5116. doi: 10.1371/journal.pone.0005116

Goodman, A., Koupil, I., \& Lawson, D. W. (2012). Low fertility increases descendant socioeconomic position but reduces long-term fitness in a modern post-industrial society. Proc Biol Sci 279(1746), 4342-4351. doi: 10.1098/rspb.2012.1415

Goodwin, R., Marshall, T., Fülöp, M., Adonu, J., Spiewak, S., Neto, F., \& Hernandez Plaza, S. (2012). Mate value and self-esteem: Evidence from eight cultural groups. PLoS ONE, 7(4), e36106. doi: 10.1371/journal.pone.0036106

Gould, S. J., \& Lewontin, R. C. (1979). The spandrels of San Marco and the Panglossian paradigm: A critique of the adaptationist programme. Proc R Soc Lond B Biol Sci, 205(1161), 581-598. doi: 10.1098/rspb.1979.0086

Gould, S. J., \& Vrba, E. S. (1982). Exaptation; a missing term in the science of form. Paleobiology, 8(1), 4-15. 
Graustella, A. J., \& MacLeod, C. (2012). A critical review of the influence of oxytocin nasal spray on social cognition in humans: Evidence and future directions. Horm Behav, 61(3), 410-418. doi: 10.1016/j.yhbeh.2012.01.002

Gray, M. A., Harrison, N. A., Wiens, S., \& Critchley, H. D. (2007). Modulation of emotional appraisal by false physiological feedback during fmri. PLoS ONE, 2(6), e546. doi: 10.1371/journal.pone.0000546

Gray, P. B., Chapman, J. F., Burnham, T. C., McIntyre, M. H., Lipson, S. F., \& Ellison, P. T. (2004). Human male pair bonding and testosterone. Human Nature, 15(2), 119-131. doi: 10.1007/s12110-004-1016-6

Gray, P. B., Jeffrey-Yang, C.-F., \& Pope, H. G. (2006). Fathers have lower salivary testosterone levels than unmarried men and married non-fathers in Beijing, China. Proceedings of the Royal Society B: Biological Sciences, 273(1584), 333339. doi: 10.1098/rspb.2005.3311

Gray, P. B., Kahlenberg, S. M., Barrett, E. S., Lipson, S. F., \& Ellison, P. T. (2002). Marriage and fatherhood are associated with lower testosterone in males. Evolution and Human Behavior, 23(3), 193-201. doi: 10.1016/S10905138(01)00101-5

Green, R. E., Krause, J., Briggs, A. W., Maricic, T., Stenzel, U., Kircher, M., . . Paabo, S. (2010). A draft sequence of the neandertal genome. Science, 328(5979), 710722. doi: $10.1126 /$ science. 1188021

Greenlees, I., Figgins, S., \& Kearney, P. (2014). Can achievement goals be primed in competitive tasks? Journal of Human Kinetics, 40, 245-250. doi: 10.2478/hukin2014-0026

Greenwald, A. G., McGhee, D. E., \& Schwartz, J. L. (1998). Measuring individual differences in implicit cognition: The implicit association test. Journal of Personality and Social Psychology, 74(6), 1464-1480. doi: 10.1037/00223514.74.6.1464

Greiling, H., \& Buss, D. M. (2000). Women's sexual strategies: The hidden dimension of extra-pair mating. Personality and Individual Differences, 28(5), 929-963. doi: 10.1016/S0191-8869(99)00151-8

Griffith, S. C., Owens, I. P. F., \& Thuman, K. A. (2002). Extra pair paternity in birds: A review of interspecific variation and adaptive function. Molecular Ecology, 11(11), 2195-2212. doi: 10.1046/j.1365-294X.2002.01613.x 
Griskevicius, V., Cialdini, R. B., \& Kenrick, D. T. (2006). Peacocks, picasso, and parental investment: The effects of romantic motives on creativity. Journal of Personality and Social Psychology, 91(1), 63-76. doi: 10.1037/00223514.91.1.63

Griskevicius, V., Tybur, J. M., Ackerman, J. M., Delton, A. W., Robertson, T. E., \& White, A. E. (2012). The financial consequences of too many men: Sex ratio effects on saving, borrowing, and spending. Journal of Personality and Social Psychology, 102(1), 69-80. doi: 10.1037/a0024761

Griskevicius, V., Tybur, J. M., Delton, A. W., \& Robertson, T. E. (2011). The influence of mortality and socioeconomic status on risk and delayed rewards: A life history theory approach. Journal of Personality and Social Psychology, 100(6), 1015-1026. doi: 10.1037/A0022403

Gross, E. F. (2009). Logging on, bouncing back: An experimental investigation of online communication following social exclusion. Developmental Psychology, 45(6), 1787-1793. doi: 10.1037/A0016541

Gross, M. R. (1991). Salmon breeding-behavior and life-history evolution in changing environments. Ecology, 72(4), 1180-1186. doi: 10.2307/1941091

Gross, M. R. (1996). Alternative reproductive strategies and tactics: Diversity within sexes. Trends in Ecology \& Evolution, 11(2), 92-98. doi: 10.1016/01695347(96)81050-0

Grossman, P., Niemann, L., Schmidt, S., \& Walach, H. (2004). Mindfulness-based stress reduction and health benefits: A meta-analysis. Journal of Psychosomatic Research, 57(1), 35-43. doi: 10.1016/S0022-3999(03)00573-7

Gwynne, D. T. (1981). Sexual difference theory: Mormon crickets show role reversal in mate choice. Science, 213, 779-780.

Hald, G. M., \& Høgh-Olesen, H. (2010). Receptivity to sexual invitations from strangers of the opposite gender. Evolution and Human Behavior, 31(6), 453458. doi: 10.1016/j.evolhumbehav.2010.07.004

Hamann, S., Herman, R. A., Nolan, C. L., \& Wallen, K. (2004). Men and women differ in amygdala response to visual sexual stimuli. Nat Neurosci, 7(4), 411-416. doi: $10.1038 / \mathrm{nn} 1208$

Hamilton, W. D. (1964). The genetical evolution of social behaviour. I. J Theor Biol, 7(1), 1-16. 
Hamilton, W. D., \& Zuk, M. (1982). Heritable true fitness and bright birds: A role for parasites? Science, 218(4570), 384-387. doi: 10.1126/science.7123238

Hammer, M. F., Mendez, F. L., Cox, M. P., Woerner, A. E., \& Wall, J. D. (2008). Sexbiased evolutionary forces shape genomic patterns of human diversity. PLoS Genet, 4(9), e1000202. doi: 10.1371/journal.pgen.1000202

Harris, J. L., Bargh, J. A., \& Brownell, K. D. (2009). Priming effects of television food advertising on eating behavior. Health Psychology, 28(4), 404-413. doi: $10.1037 / \mathrm{a} 0014399$

Hart, D., \& Sussman, R. W. (2009). Man the hunted : Primates, predators, and human evolution (Expanded ed.). Boulder, CO: Westview Press.

Haselton, M. G., Buss, D. M., Oubaid, V., \& Angleitner, A. (2005). Sex, lies, and strategic interference: The psychology of deception between the sexes.

Personality and Social Psychology Bulletin, 31(1), 3-23. doi: $10.1177 / 0146167204271303$

Hawkes, K. (1991). Showing off. Tests of an hypothesis about men's foraging goals. Ethology and Sociobiology, 12(1), 29-54. doi: 10.1016/0162-3095(91)90011-E

Hector, A. K., \& Raleigh, M. J. (1992). The effects of temporary removal of the alpha male on the behavior of subordinate male vervet monkeys. American Journal of Primatology, 26(2), 77-87. doi: 10.1002/ajp.1350260202

Heeren, T., \& D'Agostino, R. (1987). Robustness of the two independent samples t-test when applied to ordinal scaled data. Statistics in Medicine, 6(1), 79-90. doi: $10.1002 / \operatorname{sim} .4780060110$

Heller, C. G., Rowlety, M. J., \& Heller, G. V. (1969). Clomiphene citrate: A correlation of its effect on sperm concentration and morphology, total gonadotropins, icsh, estrogen and testosterone excretion, and testicular cytology in normal men. The Journal of Clinical Endocrinology \& Metabolism, 29(5), 638-649. doi: 10.1210/jcem-29-5-638

Hendler, E. D., Goffinet, J. A., Ross, S., Longnecker, R. E., \& Bakovic, V. (1974). Controlled study of androgen therapy in anemia of patients on maintenance hemodialysis. New England Journal of Medicine, 291(20), 1046-1051. doi: 10.1056/NEJM197411142912002

Hendrickson, H. (1994). The 'long' dress and the construction of Herero identities in Southern africa. African Studies, 53(2), 25-54. doi:

$10.1080 / 00020189408707800$ 
Henrich, J., \& Gil-White, F. J. (2001). The evolution of prestige: Freely conferred deference as a mechanism for enhancing the benefits of cultural transmission. Evolution and Human Behavior, 22(3), 165-196. doi: 10.1016/S10905138(00)00071-4

Heske, E. J., \& Ostfeld, R. S. (1990). Sexual dimorphism in size, relative size of testes, and mating systems in North-American voles. Journal of Mammalogy, 71(4), 510-519.

Hewlett, B. S. (1991). Intimate fathers : The nature and context of aka pygmy paternal infant care. Ann Arbor: University of Michigan Press.

Hewlett, B. S., \& Cavalli-Sforza, L. L. (1986). Cultural transmission among aka pygmies. American Anthropologist, 88(4), 922-934. doi:

10.1525/aa.1986.88.4.02a00100

Hewlett, B. S., \& Macfarlan, S. J. (2010). Fathers' roles in hunter-gatherer and other small-scale cultures. In M. E. Lamb (Ed.), The role of the father in child development (Vol. 413-434). Hoboken, N.J.: Wiley.

Hill, E. M., Ross, L. T., \& Low, B. S. (1997). The role of future unpredictability in human risk-taking. Human Nature, 8(4), 287-325. doi: 10.1007/bf02913037

Hill, K., Hurtado, A. M., \& Walker, R. S. (2007). High adult mortality among hiwi hunter-gatherers: Implications for human evolution. Journal of Human Evolution, 52(4), 443-454. doi: 10.1016/j.jhevol.2006.11.003

Hindell, M. A., Slip, D. J., \& Burton, H. R. (1994). Body-mass loss of molting female Southern elephant seals, Mirounga-leonina, at macquarie island. Polar Biology, 14(4), 275-278.

Hirschman, R. (1975). Cross-modal effects of anticipatory bogus heart rate feedback in a negative emotional context. Journal of Personality and Social Psychology, 31(1), 13-19. doi: 10.1037/h0076170

Hoier, S. (2003). Father absence and age at menarche: A test of four evolutionary models. Human Nature, 14(3), 209-233. doi: 10.1007/s12110-003-1004-2

Holden, C., \& Mace, R. (1997). Phylogenetic analysis of the evolution of lactose digestion in adults. Human Biology, 69(5), 605-628.

Holmberg, A. R. (1950). Nomads of the long bow; the Siriono of eastern bolivia. Washington, DC: U.S. Government Printing Office. 
Holmes, D. S., \& Frost, R. O. (1976). Effect of false autonomic feedback on selfreported anxiety, pain perception, and pulse rate. Behavior Therapy, 7(3), 330334. doi: 10.1016/S0005-7894(76)80059-7

Holveck, M.-J., Geberzahn, N., \& Riebel, K. (2011). An experimental test of conditiondependent male and female mate choice in zebra finches. PLoS ONE, 6(8), e23974. doi: 10.1371/journal.pone.0023974

Hönekopp, J., Voracek, M., \& Manning, J. T. (2006). 2nd to 4th digit ratio (2D:4D) and number of sex partners: Evidence for effects of prenatal testosterone in men. Psychoneuroendocrinology, 31(1), 30-37. doi: 10.1016/j.psyneuen.2005.05.009

Hopcroft, R. L. (2006). Sex, status, and reproductive success in the contemporary United States. Evolution and Human Behavior, 27(2), 104-120. doi: 10.1016/j.evolhumbehav.2005.07.004

Huber, S., \& Fieder, M. (2011). Educational homogamy lowers the odds of reproductive failure. PLoS ONE, 6(7), e22330. doi: 10.1371/journal.pone.0022330

Huberman, B. A., Loch, C. H., \& ÖNçüler, A. (2004). Status as a valued resource. Social Psychology Quarterly, 67(1), 103-114. doi: 10.1177/019027250406700109

Huck, U. W., \& Banks, E. M. (1982). Male dominance status, female choice and mating success in the brown lemming, Lemmus trimucronatus. Animal Behaviour, 30(3), 665-675. doi: 10.1016/S0003-3472(82)80136-X

Huhman, K. L., Moore, T. O., Ferris, C. F., Mougey, E. H., \& Meyerhoff, J. L. (1991). Acute and repeated exposure to social conflict in male golden hamsters: Increases in plasma pomc-peptides and cortisol and decreases in plasma testosterone. Horm Behav, 25(2), 206-216. doi: 10.1016/0018-506X(91)90051-I

Irwin, J. (2001). Continuous and discrete variables. In D. Iacobucci (Ed.), Journal of consumer psychology's special issue on methodological and statistical concerns of the experimental behavioral researcher (Vol. 10, pp. 51-52). Mahwah, NJ: Lawrence Erlbaum Associates.

Isbell, L. M. (2004). Not all happy people are lazy or stupid: Evidence of systematic processing in happy moods. Journal of Experimental Social Psychology, 40(3), 341-349. doi: 10.1016/j.jesp.2003.06.003

Jackson, J. J., \& Kirkpatrick, L. A. (2007). The structure and measurement of human mating strategies: Toward a multidimensional model of sociosexuality. 
Evolution and Human Behavior, 28(6), 382-391. doi:

10.1016/j.evolhumbehav.2007.04.005

Jankowiak, W. R., \& Fischer, E. F. (1992). A cross-cultural perspective on romantic love. Ethnology, 31(2), 149-155.

Johnsen, A., Delhey, K., Schlicht, E., Peters, A., \& Kempenaers, B. (2005). Male sexual attractiveness and parental effort in blue tits: A test of the differential allocation hypothesis. Animal Behaviour, 70(4), 877-888. doi:

10.1016/j.anbehav.2005.01.005

Johnsen, A., \& Lifjeld, J. T. (1995). Unattractive males guard their mates more closely: An experiment with bluethroats (aves, turdidae: Luscinia s. Svecica). Ethology, 101(3), 200-212. doi: 10.1111/j.1439-0310.1995.tb00358.x

Jonason, P. K., Koenig, B. L., \& Tost, J. (2010). Living a fast life. Human Nature, 21(4), 428-442. doi: 10.1007/s12110-010-9102-4

Jones, S., Martin, R. D., \& Pilbeam, D. R. (1992). The cambridge encyclopedia of human evolution. Cambridge England ; New York, NY, USA: Cambridge University Press.

Josephs, R. A., Sellers, J. G., Newman, M. L., \& Mehta, P. H. (2006). The mismatch effect: When testosterone and status are at odds. Journal of Personality and Social Psychology, 90(6), 999-1013. doi: 10.1037/0022-3514.90.6.999

Kabat-Zinn, J. (2003). Mindfulness-based interventions in context: Past, present, and future. Clinical Psychology: Science and Practice, 10(2), 144-156. doi: 10.1093/clipsy.bpg016

Kahlenberg, S. M., \& Wrangham, R. W. (2010). Sex differences in chimpanzees' use of sticks as play objects resemble those of children. Current Biology, 20(24), R1067-R1068. doi: 10.1016/j.cub.2010.11.024

Kaplan, H., Hill, K., Lancaster, J., \& Hurtado, A. M. (2000). A theory of human life history evolution: Diet, intelligence, and longevity. Evolutionary Anthropology: Issues, News, and Reviews, 9(4), 156-185. doi: 10.1002/15206505(2000)9:4<156::aid-evan5>3.0.co;2-7

Karremans, J. C., Dotsch, R., \& Corneille, O. (2011). Romantic relationship status biases memory of faces of attractive opposite-sex others: Evidence from a reverse-correlation paradigm. Cognition, 121(3), 422-426.

Kasturiratne, A., Wickremasinghe, A. R., de Silva, N., Gunawardena, N. K., Pathmeswaran, A., Premaratna, R., . . . de Silva, H. J. (2008). The global burden 
of snakebite: A literature analysis and modelling based on regional estimates of envenoming and deaths. PLoS Med, 5(11), e218. doi:

10.1371/journal.pmed.0050218

Kavanagh, P. S., Robins, S. C., \& Ellis, B. J. (2010). The mating sociometer: A regulatory mechanism for mating aspirations. Journal of Personality and Social Psychology, 99(1), 120-132. doi: 10.1037/a0018188

Kawamichi, T., \& Kawamichi, M. (1993). Gestation period and litter size of Siberian chipmunk Eutamias sibiricus lineatus in hokkaido, northern japan. Journal of the Mammalogical Society of Japan, 18(2), 105-109.

Kearney, M. S., \& Levine, P. B. (2011). Income inequality and early non-marital childbearing: An economic exploration of the "culture of despair". National Bureau of Economic Research Working Paper Series, No. 17157. doi: $10.3386 / \mathrm{w} 17157$

Kekäläinen, J., Huuskonen, H., Tuomaala, M., \& Kortet, R. (2010). Both male and female sexual ornaments reflect offspring performance in a fish. Evolution, 64(11), 3149-3157. doi: 10.1111/j.1558-5646.2010.01084.x

Kenrick, D. T., Keefe, R. C., Bryan, A., Barr, A., \& Brown, S. (1995). Age preferences and mate choice among homosexuals and heterosexuals: A case for modular psychological mechanisms. Journal of Personality and Social Psychology, 69(6), 1166-1172. doi: 10.1037/0022-3514.69.6.1166

Kinsey, A. C., Pomeroy, W. B., \& Martin, C. E. (1948). Sexual behavior in the human male. Philadelphia, USA: W. B. Saunders Co.

Kirkpatrick, L. A., \& Ellis, B. J. (2004). An evolutionary-psychological approach to self-esteem: Multiple domains and multiple functions. In M. B. B. M. Hewstone (Ed.), Self and social identity (pp. 52-77). Malden: Blackwell Publishing.

Kleinbaum, D. G., Klein, M., \& Pryor, E. R. (2010). Logistic regression : A selflearning text (3rd ed.). New York: Springer.

Koprowski, J. L. (1993). Alternative reproductive tactics in male Eastern gray squirrels - making the best of a bad job. Behavioral Ecology, 4(2), 165-171.

Kraaijeveld, K., Gregurke, J., Hall, C., Komdeur, J., \& Mulder, R. A. (2004). Mutual ornamentation, sexual selection, and social dominance in the black swan. Behavioral Ecology, 15(3), 380-389. doi: 10.1093/beheco/arh023 
Kraaijeveld, K., Kraaijeveld-Smit, F. J. L., \& Komdeur, J. (2007). The evolution of mutual ornamentation. Animal Behaviour, 74(4), 657-677. doi: 10.1016/j.anbehav.2006.12.027

Kramer, K. L., \& Greaves, R. D. (2007). Changing patterns of infant mortality and maternal fertility among pumé foragers and horticulturalists. American Anthropologist, 109(4), 713-726. doi: 10.1525/aa.2007.109.4.713

Kraus, C., Thomson, D. L., Kunkele, J., \& Trillmich, F. (2005). Living slow and dying young? Life-history strategy and age-specific survival rates in a precocial small mammal. Journal of Animal Ecology, 74(1), 171-180. doi: 10.1111/j.13652656.2004.00910.x

Kruger, D. J. (2010). Socio-demographic factors intensifying male mating competition exacerbate male mortality rates. Evolutionary Psychology, 8(2), 194-204.

Krupp, D. B. (2008). Through evolution's eyes: Extracting mate preferences by linking visual attention to adaptive design. Archives of Sexual Behavior, 37(1), 57-63. doi: 10.1007/s10508-007-9273-1

Kudryavtseva, N. N. (2000). An experimental approach to the study of learned aggression. Aggressive Behavior, 26(3), 241-256. doi: 10.1002/(sici)10982337(2000)26:3<241::aid-ab4>3.0.co;2-j

Kuepper, Y., Alexander, N., Osinsky, R., Mueller, E., Schmitz, A., Netter, P., \& Hennig, J. (2010). Aggression-interactions of serotonin and testosterone in healthy men and women. Behavioural Brain Research, 206(1), 93-100. doi: 10.1016/j.bbr.2009.09.006

Kuzawa, C. W., Gettler, L. T., Huang, Y.-y., \& McDade, T. W. (2010). Mothers have lower testosterone than non-mothers: Evidence from the Philippines. Horm Behav, 57(4-5), 441-447. doi: 10.1016/j.yhbeh.2010.01.014

Kvarnemo, C., Forsgren, E., \& Magnhagen, C. (1995). Effects of sex ratio on intra- and inter-sexual behaviour in sand gobies. Animal Behaviour, 50(6), 1455-1461. doi: 10.1016/0003-3472(95)80002-6

Lalumière, M. L., \& Quinsey, V. L. (1996). Sexual deviance, antisociality, mating effort, and the use of sexually coercive behaviors. Personality and Individual Differences, 21(1), 33-48. doi: 10.1016/0191-8869(96)00059-1

Landolt, M. A., Lalumière, M. L., \& Quinsey, V. L. (1995). Sex differences in intra-sex variations in human mating tactics: An evolutionary approach. Ethology and Sociobiology, 16(1), 3-23. doi: 10.1016/0162-3095(94)00012-V 
Lank, D. B., Coupe, M., \& Wynne-Edwards, K. E. (1999). Testosterone-induced male traits in female ruffs (Philomachus pugnax): Autosomal inheritance and gender differentiation. Proceedings of the Royal Society of London. Series B: Biological Sciences, 266(1435), 2323-2330. doi: 10.1098/rspb.1999.0926

Larson, E. T., \& Summers, C. H. (2001). Serotonin reverses dominant social status. Behavioural Brain Research, 121(1-2), 95-102. doi: 10.1016/S01664328(00)00393-4

Lassek, W. D., \& Gaulin, S. J. C. (2009). Costs and benefits of fat-free muscle mass in men: Relationship to mating success, dietary requirements, and native immunity. Evolution and Human Behavior, 30(5), 322-328. doi:

10.1016/j.evolhumbehav.2009.04.002

Launay, G., \& Slade, P. (1981). The measurement of hallucinatory predisposition in male and female prisoners. Personality and Individual Differences, 2(3), 221234. doi: 10.1016/0191-8869(81)90027-1

Le Boeuf, B. J. (1974). Male-male competition and reproductive success in elephant seals. American Zoologist, 14(1), 163-176. doi: 10.1093/icb/14.1.163

Leary, C. J., Fox, D. J., Shepard, D. B., \& Garcia, A. M. (2005). Body size, age, growth and alternative mating tactics in toads: Satellite males are smaller but not younger than calling males. Animal Behaviour, 70(3), 663-671. doi: 10.1016/j.anbehav.2004.12.013

Leary, M. R., Cottrell, C. A., \& Phillips, M. (2001). Deconfounding the effects of dominance and social acceptance on self-esteem. Journal of Personality and Social Psychology, 81(5), 898-909. doi: 10.1037//0022-3514.81.5.898

Leary, M. R., Tambor, E. S., Terdal, S. K., \& Downs, D. L. (1995). Self-esteem as an interpersonal monitor: The sociometer hypothesis. Journal of Personality and Social Psychology, 68(3), 518-530. doi: 10.1037/0022-3514.68.3.518

Leboeuf, B. J. (1972). Sexual behavior in the Northern elephant seal Mirounga angustirostris. Behaviour, 41(1), 1-26.

Lee, A. J., \& Zietsch, B. P. (2011). Experimental evidence that women's mate preferences are directly influenced by cues of pathogen prevalence and resource scarcity. Biology Letters, 7(6), 892-895. doi: 10.1098/rsbl.2011.0454

Lehr, A. T., \& Geher, G. (2006). Differential effects of reciprocity and attitude similarity across long- versus short-term mating contexts. Journal of Social Psychology, 146(4), 423-439. doi: 10.3200/SOCP.146.4.423-439 
Leigh, S. R. (1992). Body weight in captive lowland gorillas. Gorilla Gazette, 6(3), 1-3.

Lempert, K. M., Porcelli, A. J., Delgado, M. R., \& Tricomi, E. (2012). Individual differences in delay discounting under acute stress: The role of trait perceived stress. Frontiers in Psychology, 3, 251. doi: 10.3389/fpsyg.2012.00251

Levine, F. M., Krass, S. M., \& Padawer, W. J. (1993). Failure hurts: The effects of stress due to difficult tasks and failure feedback on pain report. Pain, 54(3), 335340. doi: 10.1016/0304-3959(93)90034-M

Levitan, D. R. (1998). Sperm limitation, gamete competition, and sexual selection in external fertilizers. In T. R. Birkhead \& A. P. Moller (Eds.), Sperm competition and sexual selection (pp. 175-206). London: Academic Press.

Lewis, D. M. G., Easton, J. A., Goetz, C. D., \& Buss, D. M. (2012). Exploitative male mating strategies: Personality, mating orientation, and relationship status. Personality and Individual Differences, 52(2), 139-143. doi: 10.1016/j.paid.2011.09.017

Li, N. P., Bailey, J. M., Kenrick, D. T., \& Linsenmeier, J. A. W. (2002). The necessities and luxuries of mate preferences: Testing the tradeoffs. Journal of Personality and Social Psychology, 82(6), 947-955. doi: 10.1037/0022-3514.82.6.947

Li, N. P., \& Kenrick, D. T. (2006). Sex similarities and differences in preferences for short-term mates: What, whether, and why. Journal of Personality and Social Psychology, 90(3), 468-489. doi: 10.1037/0022-3514.90.3.468

Li, N. P., Smith, A. R., Griskevicius, V., Cason, M. J., \& Bryan, A. (2010). Intrasexual competition and eating restriction in heterosexual and homosexual individuals. Evolution and Human Behavior, 31(5), 365-372. doi: 10.1016/j.evolhumbehav.2010.05.004

Limbourg, T., Mateman, A. C., Andersson, S., \& Lessells, C. M. (2004). Female blue tits adjust parental effort to manipulated male UV attractiveness. Proc Biol Sci, 271(1551), 1903-1908. doi: 10.1098/rspb.2004.2825

Lincoln, G. A., Guinness, F., \& Short, R. V. (1972). The way in which testosterone controls the social and sexual behavior of the red deer stag (cervus elaphus). Horm Behav, 3(4), 375-396. doi: 10.1016/0018-506X(72)90027-X

Lindenfors, P., Tullberg, B., \& Biuw, M. (2002). Phylogenetic analyses of sexual selection and sexual size dimorphism in pinnipeds. Behavioral Ecology and Sociobiology, 52(3), 188-193. doi: 10.1007/s00265-002-0507-х 
Lippa, R. A. (2007a). The preferred traits of mates in a cross-national study of heterosexual and homosexual men and women: An examination of biological and cultural influences. Archives of Sexual Behavior, 36(2), 193-208. doi: $10.1007 / \mathrm{s} 10508-006-9151-2$

Lippa, R. A. (2007b). The relation between sex drive and sexual attraction to men and women: A cross-national study of heterosexual, bisexual, and homosexual men and women. Archives of Sexual Behavior, 36(2), 209-222. doi: 10.1007/s10508006-9146-z

Lippa, R. A. (2009). Sex differences in sex drive, sociosexuality, and height across 53 nations: Testing evolutionary and social structural theories. Archives of Sexual Behavior, 38(5), 631-651. doi: 10.1007/s10508-007-9242-8

Little, A. C., Burt, D. M., Penton-Voak, I. S., \& Perrett, D. I. (2001). Self-perceived attractiveness influences human female preferences for sexual dimorphism and symmetry in male faces. Proceedings of the Royal Society of London. Series B: Biological Sciences, 268(1462), 39-44. doi: 10.1098/rspb.2000.1327

Little, A. C., Burt, D. M., \& Perrett, D. I. (2006). Assortative mating for perceived facial personality traits. Personality and Individual Differences, 40(5), 973-984. doi: 10.1016/j.paid.2005.09.016

Little, A. C., Cohen, D. L., Jones, B. C., \& Belsky, J. (2007). Human preferences for facial masculinity change with relationship type and environmental harshness. Behavioral Ecology and Sociobiology, 61(6), 967-973. doi: 10.1007/s00265006-0325-7

Little, A. C., DeBruine, L. M., \& Jones, B. C. (2011). Exposure to visual cues of pathogen contagion changes preferences for masculinity and symmetry in opposite-sex faces. Proc Biol Sci, 278(1714), 2032-2039. doi: 10.1098/rspb.2010.1925

Little, A. C., DeBruine, L. M., \& Jones, B. C. (2013). Environment contingent preferences: Exposure to visual cues of direct male-male competition and wealth increase women's preferences for masculinity in male faces. Evolution and Human Behavior, 34(3), 193-200. doi:

10.1016/j.evolhumbehav.2012.11.008

Little, A. C., Jones, B. C., Burt, D. M., \& Perrett, D. I. (2007). Preferences for symmetry in faces change across the menstrual cycle. Biological Psychology, 76(3), 209-216. doi: 10.1016/j.biopsycho.2007.08.003 
Little, A. C., Jones, B. C., Penton-Voak, I. S., Burt, D. M., \& Perrett, D. I. (2002). Partnership status and the temporal context of relationships influence human female preferences for sexual dimorphism in male face shape. Proceedings of the Royal Society B-Biological Sciences, 269(1496), 1095-1100. doi:

10.1098/rspb.2002.1984

Livezey, B. C. (1993). An ecomorphological review of the dodo (Raphus cucullatus) and solitaire (pezophaps solitaria), flightless columbiformes of the mascarene islands. Journal of Zoology, 230(2), 247-292. doi: 10.1111/j.14697998.1993.tb02686.x

Llaurens, V., Raymond, M., \& Faurie, C. (2009). Ritual fights and male reproductive success in a human population. Journal of Evolutionary Biology, 22(9), 18541859. doi: 10.1111/j.1420-9101.2009.01793.x

Long, S. J., \& Freese, J. (2001). Regression models for categorical dependant variables using STATA. College Station, Texas: Stata Press.

López, P., Muñoz, A., \& Martín, J. (2002). Symmetry, male dominance and female mate preferences in the iberian rock lizard, Lacerta monticola. Behavioral Ecology and Sociobiology, 52(4), 342-347. doi: 10.1007/s00265-002-0514-y

Lovejoy, C. O., Suwa, G., Spurlock, L., Asfaw, B., \& White, T. D. (2009). The pelvis and femur of Ardipithecus ramidus: The emergence of upright walking. Science, 326(5949), 71, 71e71-71e76. doi: 10.1126/science.1175831

Low, B. S. (1988). Measures of polygyny in humans. Current Anthropology, 29(1), 189-194.

Lundström, J. N., Mathe, A., Schaal, B., Frasnelli, J., Nitzsche, K., Gerber, J., \& Hummel, T. (2013). Maternal status regulates cortical responses to the body odor of newborns. Frontiers in Psychology, 4. doi: 10.3389/fpsyg.2013.00597

Lynn, S. E. (2008). Behavioral insensitivity to testosterone: Why and how does testosterone alter paternal and aggressive behavior in some avian species but not others? General and Comparative Endocrinology, 157(3), 233-240. doi: 10.1016/j.ygcen.2008.05.009

Madsen, T., \& Shine, R. (1993). Temporal variability in sexual selection acting on reproductive tactics and body size in male snakes. American Naturalist, 141(1), 167-171. doi: 10.1086/285467 
Makkar, S. R., \& Grisham, J. R. (2013). Effects of false feedback on affect, cognition, behavior, and postevent processing: The mediating role of self-focused attention. Behavior Therapy, 44(1), 111-124. doi: 10.1016/j.beth.2012.07.005

Maner, J. K., Gailliot, M. T., Rouby, D. A., \& Miller, S. L. (2007). Can't take my eyes off you: Attentional adhesion to mates and rivals. Journal of Personality and Social Psychology, 93(3), 389-401. doi: 10.1037/0022-3514.93.3.389

Maner, J. K., Miller, S. L., Schmidt, N. B., \& Eckel, L. A. (2008). Submitting to defeat: Social anxiety, dominance threat, and decrements in testosterone. Psychol Sci, 19(8), 764-768. doi: 10.1111/j.1467-9280.2008.02154.x

Margolin, L. (1992). Child abuse by mothers' boyfriends: Why the overrepresentation? Child Abuse \& Neglect, 16(4), 541-551. doi: 10.1016/0145-2134(92)90070-8

Marlowe, F. W. (1999a). Male care and mating effort among Hadza foragers. Behavioral Ecology and Sociobiology, 46(1), 57-64. doi: $10.1007 / \mathrm{s} 002650050592$

Marlowe, F. W. (1999b). Showoffs or providers? The parenting effort of Hadza men. Evolution and Human Behavior, 20(6), 391-404. doi: 10.1016/S10905138(99)00021-5

Marlowe, F. W. (2000). Paternal investment and the human mating system. Behav Processes, 51(1-3), 45-61. doi: 10.1016/S0376-6357(00)00118-2

Marlowe, F. W. (2002). Why the Hadza are still hunter-gatherers. In S. Kent (Ed.), Ethnicity, hunter-gatherers, and the "other": Association or assimilation in africa (pp. 247-275). Washington D.C.: Smithsonian Institution Press.

Marlowe, F. W. (2003). A critical period for provisioning by Hadza men: Implications for pair bonding. Evolution and Human Behavior, 24(3), 217-229. doi: 10.1016/S1090-5138(03)00014-X

Marlowe, F. W. (2004). Mate preferences among Hadza hunter-gatherers. Human Nature, 15(4), 365-376. doi: 10.1007/s12110-004-1014-8

Marlowe, F. W., \& Berbesque, J. C. (2012). The human operational sex ratio: Effects of marriage, concealed ovulation, and menopause on mate competition. Journal of Human Evolution, 63(6), 834-842. doi: 10.1016/j.jhevol.2012.09.004

Marshall Townsend, J., \& Levy, G. D. (1990). Effects of potential partners' costume and physical attractiveness on sexuality and partner selection. Journal of Psychology, 124(4), 371. 
Martin, R. D. (1990). Primate origins and evolution: A phylogenetic reconstruction Princeton: Princeton University Press.

Massar, K., Buunk, A. P., \& Rempt, S. (2012). Age differences in women's tendency to gossip are mediated by their mate value. Personality and Individual Differences, 52(1), 106-109. doi: 10.1016/j.paid.2011.09.013

Masters, B. (1997). The mistresses of Charles II. London: Constable Publishers.

Mathers, C. D., Sadana, R., Salomon, J. A., Murray, C. J. L., \& Lopez, A. D. (2001). Healthy life expectancy in 191 countries, 1999. The Lancet, 357(9269), 16851691. doi: 10.1016/S0140-6736(00)04824-8

Mayo, M. L., \& Crews, D. (1987). Neural control of male-like pseudocopulatory behavior in the all-female lizard, Cnemidophorus uniparens: Effects of intracranial implantation of dihydrotestosterone. Horm Behav, 21(2), 181-192. doi: 10.1016/0018-506X(87)90043-2

Mazoyer, M., \& Roudart, L. (2006). A history of world agriculture : From the neolithic age to the current crisis. New York: Monthly Review Press.

Mazur, A., Susman, E. J., \& Edelbrock, S. (1997). Sex difference in testosterone response to a video game contest. Evolution and Human Behavior, 18(5), 317326. doi: 10.1016/S1090-5138(97)00013-5

McCall, K. M., \& Meston, C. M. (2007). The effects of false positive and false negative physiological feedback on sexual arousal: A comparison of women with or without sexual arousal disorder. Archives of Sexual Behavior, 36(4), 518-530. doi: 10.1007/s10508-006-9140-5

McDonald, M. M., Donnellan, M. B., \& Navarrete, C. D. (2012). A life history approach to understanding the dark triad. Personality and Individual Differences, 52(5), 601-605. doi: 10.1016/j.paid.2011.12.003

McHenry, H. M. (1994). Behavioral ecological implications of early hominid body-size. Journal of Human Evolution, 27(1-3), 77-87. doi: 10.1006/jhev.1994.1036

McHenry, H. M., \& Coffing, K. (2000). Australopithecus to Homo: Transformations in body and mind. Annual Review of Anthropology, 29, 125-146. doi: 10.1146/annurev.anthro.29.1.125

McIntyre, M., Gangestad, S. W., Gray, P. B., Chapman, J. F., Burnham, T. C., O'Rourke, M. T., \& Thornhill, R. (2006). Romantic involvement often reduces men's testosterone levels--but not always: The moderating role of extrapair 
sexual interest. Journal of Personality and Social Psychology, 91(4), 642-651. doi: 10.1037/0022-3514.91.4.642

Meder, A. (1990). Sex differences in the behaviour of immature captive lowland gorillas. Primates, 31(1), 51-63. doi: 10.1007/BF02381029

Meltzer, A. L., McNulty, J. K., Jackson, G. L., \& Karney, B. R. (2014). Sex differences in the implications of partner physical attractiveness for the trajectory of marital satisfaction. Journal of Personality and Social Psychology, 106(3), 418-428. doi: $10.1037 / \mathrm{a} 0034424$

Meston, C. M., \& Buss, D. M. (2009). Why women have sex : Understanding sexual motivations from adventure to revenge (and everything in between) (1st ed.). New York: Times Books.

Miller, E. M. (2000). Homosexuality, birth order, and evolution: Toward an equilibrium reproductive economics of homosexuality. Archives of Sexual Behavior, 29(1), 1-34. doi: 10.1023/a:1001836320541

Miller, G. (2000a). The mating mind : How sexual choice shaped the evolution of human nature (1st ed.). New York: Doubleday.

Miller, G. (2000b). Mental traits as fitness indicators: Expanding evolutionary psychology's adaptationism. Annals of the New York Academy of Sciences, 907(1), 62-74. doi: 10.1111/j.1749-6632.2000.tb06616.x

Miller, G., Tybur, J. M., \& Jordan, B. D. (2007). Ovulatory cycle effects on tip earnings by lap dancers: Economic evidence for human estrus? Evolution and Human Behavior, 28(6), 375-381. doi: 10.1016/j.evolhumbehav.2007.06.002

Miller, L. C., Pedersen, W. C., \& Putcha-Bhagavatula, A. (2005). Promiscuity in an evolved pair-bonding system: Mating within and outside the Pleistocene box. Behavioral and Brain Sciences, 28(2), 290-291. doi: $10.1017 / \mathrm{s} 0140525 \mathrm{x} 05370051$

Miller, R. S. (1997). Inattentive and contented: Relationship commitment and attention to alternatives. Journal of Personality and Social Psychology, 73(4), 758-766.

Miller, S. L., \& Maner, J. K. (2009). Sex differences in response to sexual versus emotional infidelity: The moderating role of individual differences. Personality and Individual Differences, 46(3), 287-291. doi: 10.1016/j.paid.2008.10.013

Moczek, A. P. (1998). Horn polyphenism in the beetle onthophagus taurus: Larval diet quality and plasticity in parental investment determine adult body size and male 
horn morphology. Behavioral Ecology, 9(6), 636-641. doi:

10.1093/beheco/9.6.636

Moczek, A. P., \& Nijhout, H. F. (2002). Developmental mechanisms of threshold evolution in a polyphenic beetle. Evolution \& Development, 4(4), 252-264. doi: 10.1046/j.1525-142X.2002.02014.X

Møller, A. P., \& Alatalo, R. V. (1999). Good-genes effects in sexual selection. Proceedings of the Royal Society B: Biological Sciences, 266(1414), 85-91. doi: 10.1098/rspb.1999.0607

Monterosso, J., Ainslie, G., Pamela Toppi Mullen, P. A. C., \& Gault, B. (2002). The fragility of cooperation: A false feedback study of a sequential iterated prisoner's dilemma. Journal of Economic Psychology, 23(4), 437-448. doi:

10.1016/S0167-4870(02)00095-8

Moore, M. C. (1988). Testosterone control of territorial behavior: Tonic-release implants fully restore seasonal and short-term aggressive responses in free-living castrated lizards. General and Comparative Endocrinology, 70(3), 450-459. doi: 10.1016/0016-6480(88)90121-9

Moss, R., Parr, R., \& Lambin, X. (1994). Effects of testosterone on breeding density, breeding success and survival of red grouse. Proceedings of the Royal Society of London. Series B: Biological Sciences, 258(1352), 175-180. doi:

10.1098/rspb.1994.0159

Muhlhauser, I., Bender, R., Bott, U., Jorgens, V., Grusser, M., Wagener, W., . . Berger, M. (1996). Cigarette smoking and progression of retinopathy and nephropathy in type 1 diabetes. Diabet Med, 13(6), 536-543. doi: 10.1002/(SICI)10969136(199606)13:6<536::AID-DIA110>3.0.CO;2-J

Muhwezi, W. W., Kinyanda, E., Mungherera, M., Onyango, P., Ngabirano, E., Muron, J., . . Kajungu, R. (2011). Vulnerability to high risk sexual behaviour (hrsb) following exposure to war trauma as seen in post-conflict communities in eastern uganda: A qualitative study. Confl Health, 5, 22. doi: 10.1186/1752$1505-5-22$

Mulder, M. (1992). Women's strategies in polygynous marriage. Human Nature, 3(1), 45-70. doi: 10.1007/bf02692266

Mulder, M. B. (1990). Kipsigis women's preferences for wealthy men: Evidence for female choice in mammals? Behavioral Ecology and Sociobiology, 27(4), 255264. doi: 10.1007/bf00164897 
Muller, M. N., Marlowe, F. W., Bugumba, R., \& Ellison, P. T. (2009). Testosterone and paternal care in east african foragers and pastoralists. Proc Biol Sci, 276(1655), 347-354. doi: 10.1098/rspb.2008.1028

Muller, M. N., \& Wrangham, R. W. (2004). Dominance, aggression and testosterone in wild chimpanzees: A test of the 'challenge hypothesis'. Animal Behaviour, 67(1), 113-123. doi: 10.1016/j.anbehav.2003.03.013

Munk, O. (2000). Histology of the fusion area between the parasitic male and the female in the deep-sea anglerfish Neoceratias spinifer pappenheim, 1914 (teleostei, ceratioidei). Acta Zoologica, 81(4), 315-324. doi: 10.1046/j.14636395.2000.00062.x

Muñoz, L. C., Khan, R., \& Cordwell, L. (2011). Sexually coercive tactics used by university students: A clear role for primary psychopathy. Journal of Personality Disorders, 25(1), 28-40. doi: 10.1521/pedi.2011.25.1.28

Murdock, G. P., \& White, D. R. (2006). Standard cross-cultural sample: On-line edition, from http://eclectic.ss.uci.edu/ drwhite/courses/

Murray, D. R., Jones, D. N., \& Schaller, M. (2013). Perceived threat of infectious disease and its implications for sexual attitudes. Personality and Individual Differences, 54(1), 103-108. doi: 10.1016/j.paid.2012.08.021

Naber, F., van Ijzendoorn, M. H., Deschamps, P., van Engeland, H., \& BakermansKranenburg, M. J. (2010). Intranasal oxytocin increases fathers' observed responsiveness during play with their children: A double-blind within-subject experiment. Psychoneuroendocrinology, 35(10), 1583-1586. doi: 10.1016/j.psyneuen.2010.04.007

Naganathan, V., \& Sambrook, P. (2003). Gender differences in volumetric bone density: A study of opposite-sex twins. Osteoporosis International, 14(7), 564569. doi: 10.1007/s00198-003-1422-3

Nahoul, K., \& Roger, M. (1990). Age-related decline of plasma bioavailable testosterone in adult men. Journal of Steroid Biochemistry, 35(2), 293-299. doi: $10.1016 / 0022-4731(90) 90287-3$

Navarrete, C. D., Olsson, A., Ho, A. K., Mendes, W. B., Thomsen, L., \& Sidanius, J. (2009). Fear extinction to an out-group face: The role of target gender. Psychological Science, 20(2), 155-158. doi: 10.1111/j.1467-9280.2009.02273.x 
Niemitz, C. (2010). The evolution of the upright posture and gait - a review and a new synthesis. Naturwissenschaften, 97(3), 241-263. doi: 10.1007/s00114-009-06373

Norušis, M. J. (2011). IBM SPSS statistics 19 advanced statistical procedures companion. Upper Saddle River: Prentice Hall.

Ogden, C. L., Fryar, C. D., Carroll, M. D., \& Flegal, K. M. (2004). Mean body weight, height, and body mass index, United States 1960-2002. Adv Data(347), 1-17.

Ohman, A., \& Mineka, S. (2001). Fears, phobias, and preparedness: Toward an evolved module of fear and fear learning. Psychological Review, 108(3), 483-522. doi: $10.1037 / / 0033-295 x .108 .3 .483$

Oliveira, R. F., Silva, A., \& Canário, A. V. M. (2009). Why do winners keep winning? Androgen mediation of winner but not loser effects in cichlid fish. Proceedings of the Royal Society B: Biological Sciences. doi: 10.1098/rspb.2009.0132

Olsson, M., Wapstra, E., Madsen, T., \& Silverin, B. (2000). Testosterone, ticks and travels: A test of the immunocompetence-handicap hypothesis in free-ranging male sand lizards. Proceedings of the Royal Society of London. Series B: Biological Sciences, 267(1459), 2339-2343. doi: 10.1098/rspb.2000.1289

Oring, L. W., Fivizzani, A. J., \& el Halawani, M. E. (1989). Testosterone-induced inhibition of incubation in the spotted sandpiper (actitis mecularia). Horm Behav, 23(3), 412-423. doi: 10.1016/0018-506X(89)90053-6

Orne, M. T., \& Whitehouse, W. G. (2000). Demand characteristics. In A. E. Kazdin (Ed.), Encyclopedia of psychology (pp. 469-470). Washington, DC: American Psychological Association and Oxford University Press.

Oyegbile, T. O., \& Marler, C. A. (2005). Winning fights elevates testosterone levels in california mice and enhances future ability to win fights. Horm Behav, 48(3), 259-267. doi: 10.1016/j.yhbeh.2005.04.007

Oyserman, D., \& Lee, S. W. S. (2008). Does culture influence what and how we think? Effects of priming individualism and collectivism. Psychol Bull, 134(2), 311342. doi: 10.1037/0033-2909.134.2.311

Page, M. M. (1974). Demand characteristics and the classical conditioning of attitudes experiment. Journal of Personality and Social Psychology, 30(4), 468-476. doi: $10.1037 / \mathrm{h} 0037036$ 
Page, M. M. (1981). Demand compliance in laboratory experiments. In J. T. Tedeschi (Ed.), Impression management theory and social psychological research (pp. 57-79). New York: Academic Press.

Page, S. T., Amory, J. K., Bowman, F. D., Anawalt, B. D., Matsumoto, A. M., Bremner, W. J., \& Tenover, J. L. (2005). Exogenous testosterone (t) alone or with finasteride increases physical performance, grip strength, and lean body mass in older men with low serum t. Journal of Clinical Endocrinology \& Metabolism, 90(3), 1502-1510. doi: 10.1210/jc.2004-1933

Pass, J. A., Lindenberg, S. M., \& Park, J. H. (2010). All you need is love: Is the sociometer especially sensitive to one's mating capacity? European Journal of Social Psychology, 40(2), 221-234. doi: 10.1002/ejsp.619

Pawlowski, B., Atwal, R., \& Dunbar, R. I. M. (2008). Sex differences in everyday risktaking behavior in humans. Evolutionary Psychology, 6(1), 29-42.

Pawłowski, B., \& Dunbar, R. I. M. (1999). Impact of market value on human mate choice decisions. Proceedings of the Royal Society of London. Series B: Biological Sciences, 266(1416), 281-285. doi: 10.1098/rspb.1999.0634

Pedersen, F. (1991). Secular trends in human sex ratios. Human Nature, 2(3), 271-291. doi: $10.1007 / \mathrm{bf} 02692189$

Penke, L., \& Asendorpf, J. B. (2008). Beyond global sociosexual orientations: A more differentiated look at sociosexuality and its effects on courtship and romantic relationships. Journal of Personality and Social Psychology, 95(5), 1113-1135. doi: 10.1037/0022-3514.95.5.1113

Penke, L., \& Denissen, J. J. A. (2008). Sex differences and lifestyle-dependent shifts in the attunement of self-esteem to self-perceived mate value: Hints to an adaptive mechanism? Journal of Research in Personality, 42(4), 1123-1129. doi: 10.1016/j.jrp.2008.02.003

Penke, L., Todd, P. M., Lenton, A. P., \& Fasolo, B. (2008). How self-assessments can guide human mating decisions. In G. Geher \& G. F. Miller (Eds.), Mating intelligence : Sex, relationships, and the mind's reproductive system (pp.xxiii, 453 p.). New York: Lawrence Erlbaum.

Pennington, R. (2001). Hunter-gatherer demography. In C. Panter-Brick, H. L. Layton \& P. Rowley-Conwy (Eds.), Hunter-gatherers: An interdisciplinary perspective. Cambridge: Cambridge University Press. 
Penton-Voak, I. S., \& Perrett, D. I. (2000). Female preference for male faces changes cyclically: Further evidence. Evolution and Human Behavior, 21(1), 39-48. doi: 10.1016/S1090-5138(99)00033-1

Perrill, S. A., Gerhardt, H. C., \& Daniel, R. E. (1982). Mating strategy shifts in male green treefrogs (Hyla cinerea): An experimental study. Animal Behaviour, 30(1), 43-48. doi: 10.1016/S0003-3472(82)80235-2

Pérusse, D. (1993). Cultural and reproductive success in industrial societies: Testing the relationship at the proximate and ultimate levels. Behavioral and Brain Sciences, 16(02), 267-283. doi: 10.1017/S0140525X00029939

Peters, A. (2000). Testosterone treatment is immunosuppressive in superb fairy-wrens, yet free-living males with high testosterone are more immunocompetent. Proceedings of the Royal Society of London. Series B: Biological Sciences, 267(1446), 883-889. doi: 10.1098/rspb.2000.1085

Peters, M., Simmons, L. W., \& Rhodes, G. (2008). Testosterone is associated with mating success but not attractiveness or masculinity in human males. Animal Behaviour, 76(2), 297-303. doi: 10.1016/j.anbehav.2008.02.008

Pettay, J. E., Kruuk, L. E. B., Jokela, J., \& Lummaa, V. (2005). Heritability and genetic constraints of life-history trait evolution in preindustrial humans. Proceedings of the National Academy of Sciences of the United States of America, 102(8), 28382843. doi: 10.1073/pnas.0406709102

Pickett, K. E., Mookherjee, J., \& Wilkinson, R. G. (2005). Adolescent birth rates, total homicides, and income inequality in rich countries. Am J Public Health, 95(7), 1181-1183. doi: 10.2105/AJPH.2004.056721

Pietrzak, R. H., Laird, J. D., Stevens, D. A., \& Thompson, N. S. (2002). Sex differences in human jealousy: A coordinated study of forced-choice, continuous ratingscale, and physiological responses on the same subjects. Evolution and Human Behavior, 23(2), 83-94. doi: 10.1016/s1090-5138(01)00078-2

Pine, K. J., \& Fletcher, B. (2011). Women's spending behaviour is menstrual-cycle sensitive. Personality and Individual Differences, 50(1), 74-78. doi: 10.1016/j.paid.2010.08.026

Pinker, S. (2011). The better angels of our nature : Why violence has declined. New York: Viking. 
Pollet, T. V., \& Nettle, D. (2008). Driving a hard bargain: Sex ratio and male marriage success in a historical us population. Biology Letters, 4(1), 31-33. doi: $10.1098 / \mathrm{rsbl} .2007 .0543$

Popper, K. R. (1992). 7. Simplicity. The logic of scientific discovery (pp. 121-132). London; New York: Routledge.

Posavac, H. D., Posavac, S. S., \& Posavac, E. J. (1998). Exposure to media images of female attractiveness and concern with body weight among young women. Sex Roles, 38(3-4), 187-201. doi: 10.1023/a:1018729015490

Pound, N., Lawson, D. W., Toma, A. M., Richmond, S., Zhurov, A. I., \& Penton-Voak, I. S. (2014). Facial fluctuating asymmetry is not associated with childhood illhealth in a large British cohort study. Proceedings of the Royal Society B: Biological Sciences, 281(1792). doi: 10.1098/rspb.2014.1639

Pound, N., Penton-Voak, I. S., \& Surridge, A. K. (2009). Testosterone responses to competition in men are related to facial masculinity. Proceedings of the Royal Society B: Biological Sciences, 276(1654), 153-159. doi: $10.1098 /$ rspb.2008.0990

Prasad, A. S., Mantzoros, C. S., Beck, F. W., Hess, J. W., \& Brewer, G. J. (1996). Zinc status and serum testosterone levels of healthy adults. Nutrition, 12(5), 344-348. Prokop, P., \& Fancovicova, J. (2010). Perceived body condition is associated with fear of a large carnivore predator in humans. Annales Zoologici Fennici, 47(6), 417425.

Proverbio, A. M., Brignone, V., Matarazzo, S., Del Zotto, M., \& Zani, A. (2006). Gender and parental status affect the visual cortical response to infant facial expression. Neuropsychologia, 44(14), 2987-2999. doi: 10.1016/j.neuropsychologia.2006.06.015

Provost, M. P., Kormos, C., Kosakoski, G., \& Quinsey, V. L. (2006). Sociosexuality in women and preference for facial masculinization and somatotype in men. Archives of Sexual Behavior, 35(3), 305-312. doi: 10.1007/s10508-006-9029-3

Provost, M. P., Troje, N. F., \& Quinsey, V. L. (2008). Short-term mating strategies and attraction to masculinity in point-light walkers. Evolution and Human Behavior, 29(1), 65-69. doi: 10.1016/j.evolhumbehav.2007.07.007

Prudom, S. L., Broz, C. A., Schultz-Darken, N., Ferris, C. T., Snowdon, C., \& Ziegler, T. E. (2008). Exposure to infant scent lowers serum testosterone in father 
common marmosets (Callithrix jacchus). Biology Letters, 4(6), 603-605. doi: $10.1098 / \mathrm{rsbl} .2008 .0358$

Puts, D. A. (2010). Beauty and the beast: Mechanisms of sexual selection in humans. Evolution and Human Behavior, 31(3), 157-175. doi: 10.1016/j.evolhumbehav.2010.02.005

Pyburn, W. F. (1970). Breeding behavior of leaf-frogs phyllomedusa-callidryas and phyllomedusa-dacnicolor in mexico. Copeia(2), 209.

Quan-Bui, K. H. L., Plaisant, O., Leboyer, M., Gay, C., Kamal, L., Devynck, M.-A., \& Meyer, P. (1984). Reduced platelet serotonin in depression. Psychiatry Research, 13(2), 129-139. doi: 10.1016/0165-1781(84)90056-8

Quinlan, R. J. (2003). Father absence, parental care, and female reproductive development. Evolution and Human Behavior, 24(6), 376-390. doi: 10.1016/S1090-5138(03)00039-4

Quinlan, R. J. (2007). Human parental effort and environmental risk. Proc Biol Sci, 274(1606), 121-125. doi: 10.1098/rspb.2006.3690

Quinsey, V. L., Ketsetzis, M., Earls, C., \& Karamanoukian, A. (1996). Viewing time as a measure of sexual interest. Ethology and Sociobiology, 17(5), 341-354. doi: 10.1016/S0162-3095(96)00060-X

Qvarnström, A. (1997). Experimentally increased badge size increases male competition and reduces male parental care in the collared flycatcher. Proceedings of the Royal Society of London. Series B: Biological Sciences, 264(1385), 1225-1231. doi: 10.1098/rspb.1997.0169

Raleigh, M. J., Brammer, G. L., McGuire, M. T., \& Yuwiler, A. (1985). Dominant social status facilitates the behavioral effects of serotonergic agonists. Brain Research, 348(2), 274-282. doi: 10.1016/0006-8993(85)90445-7

Reede, T. (1995). Life-history shifts in response to different levels of fish kairomones in daphnia. Journal of Plankton Research, 17(8), 1661-1667. doi: 10.1093/plankt/17.8.1661

Regan, P. C., \& Dreyer, C. S. (1999). Lust? Love? Status? Journal of Psychology \& Human Sexuality, 11(1), 1-24. doi: 10.1300/J056v11n01_01

Regan, P. C., Levin, L., Sprecher, S., Christopher, F. S., \& Gate, R. (2000). Partner preferences. Journal of Psychology \& Human Sexuality, 12(3), 1-21. doi: 10.1300/J056v12n03_01 
Regan, P. C., Medina, R., \& Joshi, A. (2001). Partner preferences among homosexual men and women: What is desirable in a sex partner is not necessarily desirable in a romantic partner. Social Behaviour and Personality, 29(7), 625-634. doi: 10.2224/sbp.2001.29.7.625

Rejeski, W. J., Parker, P. E., Gagne, M., \& Koritnik, D. R. (1990). Cardiovascular and testosterone responses to contested dominance in women. Health Psychol, 9(1), $35-47$.

Relyea, R. A. (2002). Local population differences in phenotypic plasticity: Predatorinduced cchanges in wood frog tadpoles. Ecological Monographs, 72(1), 77-93. doi: 10.1890/0012-9615(2002)072[0077:lpdipp]2.0.co;2

Ren, B., Liang, B., Zhang, S., Li, Q., \& Grueter, C. C. (2007). Effects of temporary removal and replacement of the alpha male on social behavior of the captive sichuan snub-nosed monkey Rhinopithecus roxellana. Acta Zoologica Sinica, 53(4), 755-761.

Reznick, D., Bryant, M. J., \& Bashey, F. (2002). R- and k-selection revisited: The role of population regulation in life-history evolution. Ecology, 83(6), 1509-1520. doi: $10.2307 / 3071970$

Rhodes, G., Halberstadt, J., \& Brajkovich, G. (2001). Generalization of mere exposure effects to averaged composite faces. Social Cognition, 19(1), 57-70. doi: 10.1521/soco.19.1.57.18961

Rhodes, G., Simmons, L. W., \& Peters, M. (2005). Attractiveness and sexual behavior: Does attractiveness enhance mating success? Evolution and Human Behavior, 26(2), 186-201. doi: 10.1016/j.evolhumbehav.2004.08.014

Rhodes, G., Yoshikawa, S., Clark, A., Lee, K., McKay, R., \& Akamatsu, S. (2001). Attractiveness of facial averageness and symmetry in non-western cultures: In search of biologically based standards of beauty. Perception, 30(5), 611-625. doi: $10.1068 / \mathrm{p} 3123$

Ridley, M. (2003). The red queen : Sex and the evolution of human nature (1st Perennial ed.). New York: Perennial.

Riem, M. M. E., van IJzendoorn, M. H., Tops, M., Boksem, M. A. S., Rombouts, S. A. R. B., \& Bakermans-Kranenburg, M. J. (2012). No laughing matter: Intranasal oxytocin administration changes functional brain connectivity during exposure to infant laughter. Neuropsychopharmacology, 37(9), 2174-2174. doi: 10.1038/Npp.2012.27 
Robson, S. L., \& Wood, B. (2008). Hominin life history: Reconstruction and evolution. Journal of Anatomy, 212(4), 394-425. doi: 10.1111/j.1469-7580.2008.00867.x

Rockwell, J. A., Rankin, J. W., \& Toderico, B. (2001). Creatine supplementation affects muscle creatine during energy restriction. Med Sci Sports Exerc, 33(1), 61-68.

Rodd, F. H., Reznick, D. N., \& Sokolowski, M. B. (1997). Phenotypic plasticity in the life history traits of guppies: Responses to social environment. Ecology, 78(2), 419-433. doi: $10.2307 / 2266018$

Rodriguez-Llanes, J. M., Verbeke, G., \& Finlayson, C. (2009). Reproductive benefits of high social status in male macaques (macaca). Animal Behaviour, 78(3), 643649. doi: 10.1016/j.anbehav.2009.06.012

Rodriguez-Munoz, R., Bretman, A., \& Tregenza, T. (2011). Guarding males protect females from predation in a wild insect. Curr Biol, 21(20), 1716-1719. doi: 10.1016/j.cub.2011.08.053

Rohwer, S., \& Rohwer, F. C. (1978). Status signalling in harris sparrows: Experimental deceptions achieved. Animal Behaviour, 26, Part 4(0), 1012-1022. doi: 10.1016/0003-3472(78)90090-8

Ronay, R., \& Hippel, W. v. (2010). The presence of an attractive woman elevates testosterone and physical risk taking in young men. Social Psychological and Personality Science, 1(1), 57-64. doi: 10.1177/1948550609352807

Rose, R. M., Bernstein, I. S., \& Gordon, T. P. (1975). Consequences of social conflict on plasma testosterone levels in rhesus-monkeys. Psychosomatic Medicine, $37(1), 50-61$.

Rosenbaum, J., Zenilman, J., Rose, E., Wingood, G., \& DiClemente, R. (2012). Cash, cars, and condoms: Economic factors in disadvantaged adolescent women's condom use. J Adolesc Health, 51(3), 233-241. doi:

10.1016/j.jadohealth.2011.12.012

Rosenberg, M. (1965). Society and the adolescent self-image. Princeton, NJ: Princeton University Press.

Rotman, A. (2005). The robust Australopithecines: Evidence for the genus Paranthropus. Totem: The University of Western Ontario Journal of Anthropology, 13(1), 80-84.

Rowe, D. C. (2000). Environmental and genetic influences on pubertal development: Evolutionary life history traits? In J. Rodgers, D. Rowe \& W. Miller (Eds.), Genetic influences on human fertility and sexuality (pp. 147-168): Springer US. 
Rowe, D. C. (2002). On genetic variation in menarche and age at first sexual intercourse: A critique of the Belsky-Draper hypothesis. Evolution and Human Behavior, 23(5), 365-372. doi: 10.1016/S1090-5138(02)00102-2

Rubin, P. H. (2003). Folk economics. Southern Economic Journal, 70(1), 157-171.

Rucas, S., Gurven, M., Kaplan, H., \& Winking, J. (2010). The social strategy game. Human Nature, 21(1), 1-18. doi: 10.1007/s12110-010-9079-z

Rudolfsen, G., Figenschou, L., Folstad, I., Tveiten, H., \& Figenschou, M. (2006). Rapid adjustments of sperm characteristics in relation to social status. Proc Biol Sci, 273(1584), 325-332. doi: 10.1098/rspb.2005.3305

Ruedy, N. E., \& Schweitzer, M. E. (2010). In the moment: The effect of mindfulness on ethical decision making. Journal of Business Ethics, 95(1), 73-87. doi: 10.1007/s10551-011-0796-y

Ruffle, B. J., \& Sosis, R. (2006). Cooperation and the in-group-out-group bias: A field test on israeli kibbutz members and city residents. Journal of Economic Behavior \& Organization, 60(2), 147-163. doi: 10.1016/j.jebo.2004.07.007

Ruiz-de-la-torre, J. L., \& Manteca, X. (1999). Effects of testosterone on aggressive behaviour after social mixing in male lambs. Physiol Behav, 68(1-2), 109-113. doi: 10.1016/S0031-9384(99)00165-1

Rupp, H. A., \& Wallen, K. (2008). Sex differences in response to visual sexual stimuli: A review. Archives of Sexual Behavior, 37(2), 206-218. doi: 10.1007/s10508007-9217-9

Rusbult, C. E., \& Buunk, B. P. (1993). Commitment processes in close relationships: An interdependence analysis. Journal of Social and Personal Relationships, 10(2), 175-204. doi: 10.1177/026540759301000202

Saad, G., \& Stenstrom, E. (2012). Calories, beauty, and ovulation: The effects of the menstrual cycle on food and appearance-related consumption. Journal of Consumer Psychology, 22(1), 102-113. doi: 10.1016/j.jcps.2011.10.001

Salter Ainsworth, M. D., \& Bowlby, J. (1991). An ethological approach to personality development. American Psychologist, 46(4), 333-341.

Saltzman, W., Digby, L. J., \& Abbott, D. H. (2009). Reproductive skew in female common marmosets: What can proximate mechanisms tell us about ultimate causes? Proceedings of the Royal Society B: Biological Sciences, 276(1656), 389-399. doi: 10.1098/rspb.2008.1374 
Salvador, A., Simón, V., Suay, F., \& Llorens, L. (1987). Testosterone and cortisol responses to competitive fighting in human males: A pilot study. Aggressive Behavior, 13(1), 9-13. doi: 10.1002/1098-2337(1987)13:1<9::aidab2480130103>3.0.co;2-4

Sapienza, P., Zingales, L., \& Maestripieri, D. (2009). Gender differences in financial risk aversion and career choices are affected by testosterone. Proceedings of the National Academy of Sciences of the United States of America, 106(36), 1526815273. doi: 10.1073/pnas.0907352106

Saragusty, J., Hermes, R., Hofer, H., Bouts, T., Göritz, F., \& Hildebrandt, T. B. (2012). Male pygmy hippopotamus influence offspring sex ratio. Nature Communications, 3, 697. doi: 10.1038/ncomms 1700

Sarai, K., \& Kayano, M. (1968). The level and diurnal rhythm of serum serotonin in manic-depressive patients. Psychiatry and Clinical Neurosciences, 22(3), 271281. doi: 10.1111/j.1440-1819.1968.tb01425.x

Sauter, D. A., Eisner, F., Ekman, P., \& Scott, S. K. (2010). Cross-cultural recognition of basic emotions through nonverbal emotional vocalizations. Proceedings of the National Academy of Sciences, 107(6), 2408-2412. doi: 10.1073/pnas.0908239106

Scelza, B. A. (2013). Choosy but not chaste: Multiple mating in human females. Evolutionary Anthropology, 22, 259-269. doi: 10.1002/evan.21373

Scelza, B. A. (2014). Jealousy in a small-scale, natural fertility population: The roles of paternity, investment and love in jealous response. Evolution and Human Behavior, 35(2), 103-108. doi: 10.1016/j.evolhumbehav.2013.11.003

Schaller, M., \& Murray, D. R. (2008). Pathogens, personality, and culture: Disease prevalence predicts worldwide variability in sociosexuality, extraversion, and openness to experience. Journal of Personality and Social Psychology, 95(1), 212-221. doi: 10.1037/0022-3514.95.1.212

Scheepers, D. (2009). Turning social identity threat into challenge: Status stability and cardiovascular reactivity during inter-group competition. Journal of Experimental Social Psychology, 45(1), 228-233. doi: 10.1016/j.jesp.2008.09.011

Scheib, J. E. (2001). Context-specific mate choice criteria: Women's trade-offs in the contexts of long-term and extra-pair mateships. Personal Relationships, 8(4), 371-389. 
Scheib, J. E., Gangestad, S. W., \& Thornhill, R. (1999). Facial attractiveness, symmetry and cues of good genes. Proc Biol Sci, 266(1431), 1913-1917. doi: 10.1098/rspb.1999.0866

Scherr, J., Wolfarth, B., Christle, J., Pressler, A., Wagenpfeil, S., \& Halle, M. (2013). Associations between borg's rating of perceived exertion and physiological measures of exercise intensity. European Journal of Applied Physiology, 113(1), 147-155. doi: 10.1007/s00421-012-2421-x

Schmalt, H. D. (2006). Waist-to-hip ratio and female physical attractiveness: The moderating role of power motivation and the mating context. Personality and Individual Differences, 41(3), 455-465. doi: 10.1016/j.paid.2006.02.008

Schmider, E., Ziegler, M., Danay, E., Beyer, L., \& Bühner, M. (2010). Is it really robust? Reinvestigating the robustness of ANOVA against violations of the normal distribution assumption. Methodology: European Journal of Research Methods for the Behavioral and Social Sciences, 6(4), 147-151. doi: $10.1027 / 1614-2241 / \mathrm{a} 000016$

Schmitt, D. P. (2003). Universal sex differences in the desire for sexual variety: Tests from 52 nations, 6 continents, and 13 islands. Journal of Personality and Social Psychology, 85(1), 85-104. doi: 10.1037/0022-3514.85.1.85

Schmitt, D. P. (2005a). Is short-term mating the maladaptive result of insecure attachment? A test of competing evolutionary perspectives. Personality and Social Psychology Bulletin, 31(6), 747-768. doi: 10.1177/0146167204271843

Schmitt, D. P. (2005b). Sociosexuality from argentina to Zimbabwe: A 48-nation study of sex, culture, and strategies of human mating. Behav Brain Sci, 28(2), 247275. doi: 10.1017/S0140525X05000051

Schmitt, D. P. (2007). Sexual strategies across sexual orientations. Journal of Psychology \& Human Sexuality, 18(2-3), 183-214. doi: 10.1300/J056v18n02_06

Schmitt, D. P. (2012). Sexual strategies across sexual orientations: How personality traits and culture relate to sociosexuality among gays, lesbians, bisexuals, and heterosexuals. In M. R. Kauth (Ed.), Handbook of the evolution of human sexuality. New York: Routledge.

Schmitt, D. P., \& Shackelford, T. K. (2008). Big five traits related to short-term mating: From personality to promiscuity across 46 nations. Evolutionary Psychology, 6, 246-282. 
Schradin, C., König, B., \& Pillay, N. (2010). Reproductive competition favours solitary living while ecological constraints impose group-living in african striped mice. Journal of Animal Ecology, 79(3), 515-521. doi: 10.1111/j.13652656.2009.01651.x

Schradin, C., \& Lindholm, A. K. (2011). Relative fitness of alternative male reproductive tactics in a mammal varies between years. Journal of Animal Ecology, 80(5), 908-917. doi: 10.1111/j.1365-2656.2011.01831.x

Schwagmeyer, P. L., \& Brown, C. H. (1983). Factors affecting male-male competition in 13-lined ground-squirrels. Behavioral Ecology and Sociobiology, 13(1), 1-6. doi: 10.1007/Bf00295069

Seal, D. W., Agostinelli, G., \& Hannett, C. A. (1994). Extradyadic romantic involvement: Moderating effects of sociosexuality and gender. Sex Roles, 31(12), 1-22. doi: 10.1007/bf01560274

Sear, R., \& Mace, R. (2008). Who keeps children alive? A review of the effects of kin on child survival. Evolution and Human Behavior, 29(1), 1-18. doi: 10.1016/j.evolhumbehav.2007.10.001

Setchell, J. M., \& Dixson, A. F. (2001). Changes in the secondary sexual adornments of male mandrills (Mandrillus sphinx) are associated with gain and loss of alpha status. Horm Behav, 39(3), 177-184. doi: 10.1006/hbeh.2000.1628

Shackelford, T. K. (2001). Self-esteem in marriage. Personality and Individual Differences, 30(3), 371-390. doi: 10.1016/S0191-8869(00)00023-4

Shackelford, T. K., Pound, N., \& Goetz, A. T. (2005). Psychological and physiological adaptations to sperm competition in humans. Review of General Psychology, 9(3), 228-248. doi: 10.1037/1089-2680.9.3.228

Shamay-Tsoory, S. G., Fischer, M., Dvash, J., Harari, H., Perach-Bloom, N., \& Levkovitz, Y. (2009). Intranasal administration of oxytocin increases envy and schadenfreude (gloating). Biological Psychiatry, 66(9), 864-870. doi:

10.1016/j.biopsych.2009.06.009

Shan, W., Shenghua, J., Davis, H. M., Peng, K., Shao, X., Wu, Y., . . Wang, Y. (2012). Mating strategies in chinese culture: Female risk avoiding vs. Male risk taking. Evolution and Human Behavior, 33(3), 182-192. doi:

10.1016/j.evolhumbehav.2011.09.001 
Shanks, D. R., Newell, B. R., Lee, E. H., Balakrishnan, D., Ekelund, L., Cenac, Z., . . . Moore, C. (2013). Priming intelligent behavior: An elusive phenomenon. PLoS ONE, 8(4), e56515. doi: 10.1371/journal.pone.0056515

Shariff, A. F., \& Norenzayan, A. (2007). God is watching you: Priming god concepts increases prosocial behavior in an anonymous economic game. Psychological Science, 18(9), 803-809. doi: 10.1111/j.1467-9280.2007.01983.x

Shifren, J. L., Braunstein, G. D., Simon, J. A., Casson, P. R., Buster, J. E., Redmond, G. P., . . Daugherty, C. A. (2000). Transdermal testosterone treatment in women with impaired sexual function after oophorectomy. New England Journal of Medicine, 343(10), 682-688. doi: 10.1056/Nejm200009073431002

Shine, R., Langkilde, T., Wall, M., \& Mason, R. T. (2005). Alternative male mating tactics in garter snakes, Thamnophis sirtalis parietalis. Animal Behaviour, 70, 387-396. doi: 10.1016/j.anbehav.2005.04.001

Shuster, S. M. (2010). Alternative mating strategies. In C. Fox \& D. F. Westneat (Eds.), Evolutionary behavioral ecology (pp. 434-450): Cambridge University Press.

Shuster, S. M., \& Wade, M. J. (2003). Mating systems and strategies. Princeton: Princeton University Press.

Silverman, I., Choi, J., \& Peters, M. (2007). The hunter-gatherer theory of sex differences in spatial abilities: Data from 40 countries. Archives of Sexual Behavior, 36(2), 261-268. doi: 10.1007/s10508-006-9168-6

Simpson, J. A., \& Gangestad, S. W. (1989). Two month test-retest reliability of the sociosexual orientation inventory. Texas A\&M University: Unpublished data.

Simpson, J. A., \& Gangestad, S. W. (1991). Individual-differences in sociosexuality evidence for convergent and discriminant validity. Journal of Personality and Social Psychology, 60(6), 870-883. doi: 10.1037/0022-3514.60.6.870

Simpson, J. A., Gangestad, S. W., Christensen, P. N., \& Leck, K. (1999). Fluctuating asymmetry, sociosexuality, and intrasexual competitive tactics. Journal of Personality and Social Psychology, 76(1), 159-172. doi: 10.1037/00223514.76.1.159

Simpson, J. A., Gangestad, S. W., \& Lerma, M. (1990). Perception of physical attractiveness: Mechanisms involved in the maintenance of romantic relationships. Journal of Personality and Social Psychology, 59(6), 1192-1201.

Simpson, Stephen J., Sword, Gregory A., \& Lo, N. (2011). Polyphenism in insects. Current Biology, 21(18), R738-R749. doi: 10.1016/j.cub.2011.06.006 
Singh, D. (2004). Mating strategies of young women: Role of physical attractiveness. Journal of Sex Research, 41(1), 43-54. doi: 10.1080/00224490409552212

Slatcher, R. B., Mehta, P. H., \& Josephs, R. A. (2011). Testosterone and self-reported dominance interact to influence human mating behavior. Social Psychological and Personality Science, 2(5), 531-539. doi: 10.1177/1948550611400099

Slonim-Nevo, V., \& Al-Krenawi, A. (2006). Success and failure among polygamous families: The experience of wives, husbands, and children. Family Process, 45(3), 311-330. doi: 10.1111/j.1545-5300.2006.00173.x

Smeesters, D., Yzerbyt, V. Y., Corneille, O., \& Warlop, L. (2009). When do primes prime? The moderating role of the self-concept in individuals' susceptibility to priming effects on social behavior. Journal of Experimental Social Psychology, 45(1), 211-216. doi: 10.1016/j.jesp.2008.09.002

Smith, E. A. (2004). Why do good hunters have higher reproductive success? Human Nature, 15(4), 343-364. doi: 10.1007/s12110-004-1013-9

Smith, E. A., Bird, R. B., \& Bird, D. W. (2003). The benefits of costly signaling: Meriam turtle hunters. Behavioral Ecology, 14(1), 116-126. doi: 10.1093/beheco/14.1.116

Smith, R. J., \& Cheverud, J. M. (2002). Scaling of sexual dimorphism in body mass: A phylogenetic analysis of rensch's rule in primates. International Journal of Primatology, 23(5), 1095-1135. doi: 10.1023/a:1019654100876

Smuts, B. (1992). Male aggression against women : An evolutionary perspective. Human Nature, 3(1), 1-44. doi: 10.1007/BF02692265

Snyder, J. K., Kirkpatrick, L. A., \& Barrett, H. C. (2008). The dominance dilemma: Do women really prefer dominant mates? Personal Relationships, 15(4), 425-444. doi: 10.1111/j.1475-6811.2008.00208.x

Soares, P., Ermini, L., Thomson, N., Mormina, M., Rito, T., Röhl, A., . . Richards, M. B. (2009). Correcting for purifying selection: An improved human mitochondrial molecular clock. The American Journal of Human Genetics, 84(6), 740-759. doi: 10.1016/j.ajhg.2009.05.001

Soldan, V. A., deGraft-Johnson, J. E., Bisika, T., \& Tsui, A. O. (2007). Social, economic and demographic determinants of sexual risk behaviors among men in rural Malawi: A district-level study. Afr J Reprod Health, 11(2), 33-46.

Soler, C., Kekäläinen, J., Núñez, M., Sancho, M., Álvarez, J. G., Núñez, J., . . . Gutiérrez, R. (2014). Male facial attractiveness and masculinity may provide 
sex- and culture-independent cues to semen quality. Journal of Evolutionary Biology. doi: 10.1111/jeb.12446

Speizer, I. (1995). Men's desire for additional wives and children. Social Biology, 42(34), 199-213.

Spoor, F., Leakey, M. G., Gathogo, P. N., Brown, F. H., Anton, S. C., McDougall, I., . . . Leakey, L. N. (2007). Implications of new early Homo fossils from ileret, east of lake turkana, kenya. Nature, 448(7154), 688-691. doi: 10.1038/nature05986

Stanton, S. J., Beehner, J. C., Saini, E. K., Kuhn, C. M., \& LaBar, K. S. (2009). Dominance, politics, and physiology: Voters' testosterone changes on the night of the 2008 United States presidential election. PLOS ONE, 4(10), e7543. doi: 10.1371/journal.pone.0007543

Stanton, S. J., Liening, S. H., \& Schultheiss, O. C. (2011). Testosterone is positively associated with risk taking in the Iowa gambling task. Horm Behav, 59(2), 252256. doi: 10.1016/j.yhbeh.2010.12.003

Starling, S. P., Holden, J. R., \& Jenny, C. (1995). Abusive head trauma: The relationship of perpetrators to their victims. Pediatrics, 95(2), 259-262.

Steinmann, A. R., \& Priotto, J. W. (2011). Inter-male aggression in relation to female availability and residence status in corn mice Calomys musculinus. Acta Theriologica, 56(1), 81-89. doi: 10.1007/s13364-010-0003-z

Stewart-Williams, S., \& Thomas, A. G. (2013a). The ape that kicked the hornet's nest: Response to commentaries on "the ape that thought it was a peacock". Psychological Inquiry, 24(3), 248-271. doi: 10.1080/1047840X.2013.823831

Stewart-Williams, S., \& Thomas, A. G. (2013b). The ape that thought it was a peacock: Does evolutionary psychology exaggerate human sex differences? Psychological Inquiry, 24(3), 137-168. doi: 10.1080/1047840X.2013.804899

Stewart, S., Stinnett, H., \& Rosenfeld, L. B. (2000). Sex differences in desired characteristics of short-term and long-term relationship partners. Journal of Social and Personal Relationships, 17(6), 843-853. doi: $10.1177 / 0265407500176008$

Stoehr, A. M., \& Hill, G. E. (2000). Testosterone and the allocation of reproductive effort in male house finches (Carpodacus mexicanus). Behavioral Ecology and Sociobiology, 48(5), 407-411. doi: 10.1007/s002650000247 
Storbeck, J., \& Clore, G. L. (2005). With sadness comes accuracy; with happiness, false memory: Mood and the false memory effect. Psychological Science, 16(10), 785-791. doi: 10.1111/j.1467-9280.2005.01615.x

Storey, A. E., Noseworthy, D. E., Delahunty, K. M., Halfyard, S. J., \& McKay, D. W. (2011). The effects of social context on the hormonal and behavioral responsiveness of human fathers. Horm Behav, 60(4), 353-361. doi: 10.1016/j.yhbeh.2011.07.001

Storey, A. E., Walsh, C. J., Quinton, R. L., \& Wynne-Edwards, K. E. (2000). Hormonal correlates of paternal responsiveness in new and expectant fathers. Evolution and Human Behavior, 21(2), 79-95. doi: 10.1016/S1090-5138(99)00042-2

Suay, F., Salvador, A., González-Bono, E., Sanchís, C., Martínez, M., MartínezSanchis, S., . . Montoro, J. B. (1999). Effects of competition and its outcome on serum testosterone, cortisol and prolactin. Psychoneuroendocrinology, 24(5), 551-566. doi: 10.1016/S0306-4530(99)00011-6

Surbeck, M., Mundry, R., \& Hohmann, G. (2011). Mothers matter! Maternal support, dominance status and mating success in male bonobos (Pan paniscus). Proceedings of the Royal Society B: Biological Sciences, 278(1705), 590-598. doi: 10.1098/rspb.2010.1572

Surbey, M., \& Brice, G. R. (2007). Enhancement of self-perceived mate value precedes a shift in men's preferred mating strategy. Acta Psychologica Sinica, 39(3), 513522.

Suzuki, S., \& Cavanagh, P. (1998). A shape-contrast effect for briefly presented stimuli. Journal of Experimental Psychology: Human Perception and Performance, 24(5), 1315-1341. doi: 10.1037/0096-1523.24.5.1315

Swami, V., Miller, R., Furnham, A., Penke, L., \& Tovée, M. J. (2008). The influence of men's sexual strategies on perceptions of women's bodily attractiveness, health and fertility. Personality and Individual Differences, 44(1), 98-107. doi: 10.1016/j.paid.2007.07.017

Symons, D. (1979). The evolution of human sexuality. New York: Oxford University Press.

Tapper, K., Shaw, C., Ilsley, J., Hill, A. J., Bond, F. W., \& Moore, L. (2009). Exploratory randomised controlled trial of a mindfulness-based weight loss intervention for women. Appetite, 52(2), 396-404. doi:

10.1016/j.appet.2008.11.012 
Taylor, A. B. (1997). Relative growth, ontogeny, and sexual dimorphism in gorilla (Gorilla gorilla gorilla and g. G. Beringei): Evolutionary and ecological considerations. American Journal of Primatology, 43(1), 1-31. doi: 10.1002/(SICI)1098-2345(1997)43:1<1::AID-AJP1>3.0.CO;2-0

Teaford, M. F., \& Ungar, P. S. (2000). Diet and the evolution of the earliest human ancestors. Proceedings of the National Academy of Sciences, 97(25), 1350613511. doi: 10.1073/pnas.260368897

Teuscher, U., \& Teuscher, C. (2007). Reconsidering the double standard of aging: Effects of gender and sexual orientation on facial attractiveness ratings. Personality and Individual Differences, 42(4), 631-639. doi: 10.1016/j.paid.2006.08.020

Thienel, M., Heinrichs, M., Fischer, S., Ott, V., Born, J., \& Hallschmid, M. (2014). Oxytocin's impact on social face processing is stronger in homosexual than heterosexual men. Psychoneuroendocrinology, 39(0), 194-203. doi: 10.1016/j.psyneuen.2013.09.013

Thomas, A. G. (2010). Variability of human mating strategies: Can animal studies lead us down the path to promiscuity? PsyPag Quarterly(75), 5-9.

Thompson, C. W., Moore, I. T., \& Moore, M. C. (1993). Social, environmental and genetic factors in the ontogeny of phenotypic differentiation in a lizard with alternative male reproductive strategies. Behavioral Ecology and Sociobiology, 33(3), 137-146. doi: 10.1007/bf00216593

Thornhill, R. (1981). Panorpa (mecoptera: Panorpidae) scorpionflies: Systems for understanding resource-defense polygyny and alternative male reproductive efforts. Annual Review of Ecology and Systematics, 12(1), 355-386. doi: 10.1146/annurev.es.12.110181.002035

Thornhill, R., \& Gangestad, S. W. (1993). Human facial beauty. Human Nature, 4(3), 237-269. doi: 10.1007/bf02692201

Thornhill, R., \& Gangestad, S. W. (1999). Facial attractiveness. Trends in Cognitive Sciences, 3(12), 452-460. doi: 10.1016/S1364-6613(99)01403-5

Thornhill, R., \& Gangestad, S. W. (2008). The evolutionary biology of human female sexuality. Oxford ; New York: Oxford University Press.

Thornton, B., \& Maurice, J. (1997). Physique contrast effect: Adverse impact of idealized body images for women. Sex Roles, 37(5/6), 433-439. doi: 10.1023/a:1025609624848 
Thornton, B., \& Moore, S. (1993). Physical attractiveness contrast effect: Implications for self-esteem and evaluations of the social self. Personality and Social Psychology Bulletin, 19(4), 474-480. doi: 10.1177/0146167293194012

Thorpe, I. J. N. (2003). Anthropology, archaeology, and the origin of warfare. World Archaeology, 35(1), 145-165. doi: 10.2307/3560217

Tiefer, L. (1970). Gonadal hormones and mating behavior in the adult golden hamster. Horm Behav, 1(3), 189-202. doi: 10.1016/0018-506X(70)90013-9

Tomkins, J. L., \& Hazel, W. (2007). The status of the conditional evolutionarily stable strategy. Trends in Ecology \& Evolution, 22(10), 522-528. doi: 10.1016/j.tree.2007.09.002

Travis, J., \& Woodward, B. D. (1989). Social context and courtship flexibility in male sailfin mollies, Poecilia latipinna (pisces: Poecillidae). Animal Behaviour, 38(6), 1001-1011. doi: 10.1016/S0003-3472(89)80139-3

Treves, A., \& Naughton-Treves, L. (1999). Risk and opportunity for humans coexisting with large carnivores. Journal of Human Evolution, 36(3), 275-282. doi: 10.1006/jhev.1998.0268

Trivers, R. L. (1972). Parental investment and sexual selection. In B. Campbell (Ed.), Sexual selection and the descent of man, 1871-1971 (pp. 136-179). Chicago: Aldine-Atherton.

Trivers, R. L., \& Willard, D. E. (1973). Natural selection of parental ability to vary the sex ratio of offspring. Science, 179(4068), 90-92. doi:

10.1126/science.179.4068.90

Tsapela, I., Fisher, H. E., \& Aron, A. (2010). “Infidelity: When, where, why. In W. R. Cupach \& B. H. Spitzberg (Eds.), The dark side of close relationships ii (pp. 175-196). New York: Routledge.

Tuiten, A., Van Honk, J., Koppeschaar, H., Bernaards, C., Thijssen, J., \& Verbaten, R. (2000). Time course of effects of testosterone administration on sexual arousal in women. Archives of General Psychiatry, 57(2), 149-153. doi: 10.1001/archpsyc.57.2.149

Turner, J. C., Hogg, M. A., Oakes, P. J., Reicher, S. D., \& Wetherell, M. S. (1987). Rediscovering the social group: A self-categorization theory. Cambridge, MA, US: Basil Blackwell. 
Tuttle, E. M. (2003). Alternative reproductive strategies in the white-throated sparrow: Behavioral and genetic evidence. Behavioral Ecology, 14(3), 425-432. doi: 10.1093/beheco/14.3.425

U.S. Census Bureau. (2011). Fertility - 2011 American community survey 1-year estimates. Retrieved May 1st, 2014, from http://factfinder2.census.gov/faces/tableservices/jsf/pages/productview.xhtml?pi d=ACS_11_1YR_S1301\&prodType=table

Udry, J. R., \& Eckland, B. K. (1984). Benefits of being attractive: Differential payoffs for men and women. Psychol Rep, 54(1), 47-56.

Valentova, J. V., Stulp, G., Třebický, V., \& Havlíček, J. (2014). Preferred and actual relative height among homosexual male partners vary with preferred dominance and sex role. PLoS ONE, 9(1), e86534. doi: 10.1371/journal.pone.0086534

van Anders, S. M., Hamilton, L. D., \& Watson, N. V. (2007). Multiple partners are associated with higher testosterone in North American men and women. Horm Behav, 51(3), 454-459. doi: 10.1016/j.yhbeh.2007.01.002

van Anders, S. M., Tolman, R. M., \& Volling, B. L. (2012). Baby cries and nurturance affect testosterone in men. Horm Behav, 61(1), 31-36. doi:

10.1016/j.yhbeh.2011.09.012

van den Hout, J. H. C., Vlaeyen, J. W. S., Peters, M. L., Engelhard, I. M., \& Van den Hout, M. A. (2000). Does failure hurt? The effects of failure feedback on pain report, pain tolerance and pain avoidance. European Journal of Pain, 4(4), 335346. doi: 10.1053/eujp.2000.0195

van der Meij, L., Buunk, A. P., van de Sande, J. P., \& Salvador, A. (2008). The presence of a woman increases testosterone in aggressive dominant men. Horm Behav, 54(5), 640-644. doi: 10.1016/j.yhbeh.2008.07.001

van der Wal, A. J., Schade, H. M., Krabbendam, L., \& van Vugt, M. (2013). Do natural landscapes reduce future discounting in humans? Proceedings of the Royal Society B: Biological Sciences, 280(1773), 1-6. doi: 10.1098/rspb.2013.2295

Veiga, J. P., Salvador, A., Merino, S., \& Puerta, M. (1998). Reproductive effort affects immune response and parasite infection in a lizard: A phenotypic manipulation using testosterone. Oikos, 82(2), 313-318.

Voland, E. (1990). Differential reproductive success within the Krummhörn population (Germany, 18th and 19th centuries). Behavioral Ecology and Sociobiology, 26(1), 65-72. doi: 10.1007/bf00174026 
Volek, J. S., Mazzetti, S., Farquhar, W., Barnes, B., Gomez, A., \& Kraemer, W. J. (2001). Physiological responses to short-term exercise in the heat after creatine loading. Medicine \& Science in Sports \& Exercise, 33(7), 1101-1108.

von Rueden, C., Gurven, M., \& Kaplan, H. (2011). Why do men seek status? Fitness payoffs to dominance and prestige. Proceedings of the Royal Society B: Biological Sciences, 278(1715), 2223-2232. doi: 10.1098/rspb.2010.2145

Wada, M., \& Gorbman, A. (1977). Relation of mode of administration of testosterone to evocation of male sex behavior in frogs. Horm Behav, 8(3), 310-319. doi: 10.1016/0018-506X(77)90005-8

Wade, T. J. (2000). Evolutionary theory and self-perception: Sex differences in body esteem predictors of self-perceived physical and sexual attractiveness and selfesteem. International Journal of Psychology, 35(1), 36-45. doi: $10.1080 / 002075900399501$

Waitt, C., \& Little, A. C. (2006). Preferences for symmetry in conspecific facial shape among macaca mulatta. International Journal of Primatology, 27(1), 133-145. doi: 10.1007/s10764-005-9015-y

Watkins, C. D., DeBruine, L. M., Little, A. C., Feinberg, D. R., \& Jones, B. C. (2012). Priming concerns about pathogen threat versus resource scarcity: Dissociable effects on women's perceptions of men's attractiveness and dominance. Behavioral Ecology and Sociobiology, 66(12), 1549-1556. doi: 10.1007/s00265012-1408-2

Watkins, C. D., Jones, B. C., Little, A. C., DeBruine, L. M., \& Feinberg, D. R. (2012). Cues to the sex ratio of the local population influence women's preferences for facial symmetry. Animal Behaviour, 83(2), 545-553. doi: 10.1016/j.anbehav.2011.12.002

Watson, D., Clark, L. A., \& Tellegen, A. (1988). Development and validation of brief measures of positive and negative affect: The PANAS scales. Journal of Personality and Social Psychology, 54(6), 1063-1070. doi: 10.1037/00223514.54.6.1063

Weaver, J. R., Vandello, J. A., \& Bosson, J. K. (2013). Intrepid, imprudent, or impetuous? The effects of gender threats on men's financial decisions. Psychology of Men \& Masculinity, 14(2), 184-191. doi: 10.1037/a0027087

Weiser, S. D., Leiter, K., Bangsberg, D. R., Butler, L. M., Percy-de Korte, F., Hlanze, Z., . . Heisler, M. (2007). Food insufficiency is associated with high-risk sexual 
behavior among women in Botswana and Swaziland. PLoS Med, 4(10), e260. doi: 10.1371/journal.pmed.0040260

Welling, L. L. M., Jones, B. C., DeBruine, L. M., Conway, C. A., Law Smith, M. J., Little, A. C., . . Al-Dujaili, E. A. S. (2007). Raised salivary testosterone in women is associated with increased attraction to masculine faces. Horm Behav, 52(2), 156-161. doi: 10.1016/j.yhbeh.2007.01.010

Wesselmann, E. D., Butler, F. A., Williams, K. D., \& Pickett, C. L. (2010). Adding injury to insult: Unexpected rejection leads to more aggressive responses. Aggressive Behavior, 36(4), 232-237. doi: 10.1002/Ab.20347

Wikipedia. (2012). Cardiff. Retrieved June 4th, 2012, from https://en.wikipedia.org/wiki/Cardiff

Willer, R., Rogalin, C. L., Conlon, B., \& Wojnowicz, M. T. (2013). Overdoing gender: A test of the masculine overcompensation thesis. American Journal of Sociology, 118(4), 980-1022. doi: 10.1086/668417

Williams, G. C. (1966). Adaptation and natural selection; a critique of some current evolutionary thought. Princeton, N.J.,: Princeton University Press.

Williams, K. D., Cheung, C. K. T., \& Choi, W. (2000). Cyberostracism: Effects of being ignored over the internet. Journal of Personality and Social Psychology, 79(5), 748-762. doi: 10.1037/0022-3514.79.5.748

Wilson, D. R., Nelson, X. J., \& Evans, C. S. (2009). Seizing the opportunity: Subordinate male fowl respond rapidly to variation in social context. Ethology, 115(10), 996-1004. doi: 10.1111/j.1439-0310.2009.01688.x

Wilson, D. S., Hayes, S. C., Biglan, A., \& Embry, D. D. (2014). Evolving the future: Toward a science of intentional change. Behavioral and Brain Sciences, 37(04), 395-416. doi: 10.1017/S0140525X13001593

Wilson II, R. W., Snyder, A. C., \& Dorman, J. C. (2009). Analysis of seated and standing triple wingate tests. Journal of Strength and Conditioning Research, 23(3), 868-873. doi: 10.1519/JSC.0b013e31819d0932

Wilson, M., \& Daly, M. (1985). Competitiveness, risk taking, and violence: The young male syndrome. Ethology and Sociobiology, 6(1), 59-73. doi: 10.1016/01623095(85)90041-x

Wilson, M., \& Daly, M. (2004). Do pretty women inspire men to discount the future? Proceedings of the Royal Society of London. Series B: Biological Sciences, 271(Suppl 4), S177-S179. doi: 10.1098/rsbl.2003.0134 
Wingfield, J. C., Hegner, R. E., Dufty Jr, A. M., \& Ball, G. F. (1990). The challenge hypothesis - theoretical implications for patterns of testosterone secretion, mating systems, and breeding strategies. American Naturalist, 136(6), 829-846.

Wirth, M. M., Welsh, K. M., \& Schultheiss, O. C. (2006). Salivary cortisol changes in humans after winning or losing a dominance contest depend on implicit power motivation. Horm Behav, 49(3), 346-352. doi: 10.1016/j.yhbeh.2005.08.013

Wittemyer, G., Barner Rasmussen, H., \& Douglas-Hamilton, I. (2007). Breeding phenology in relation to ndvi variability in free-ranging african elephant. Ecography, 30(1), 42-50. doi: 10.1111/j.0906-7590.2007.04900.x

Wittman, A. B., \& Wall, L. L. (2007). The evolutionary origins of obstructed labor: Bipedalism, encephalization, and the human obstetric dilemma. Obstetrical \& Gynecological Survey, 62(11), 739-748.

Woll, S. B., \& McFall, M. E. (1979). The effects of false feedback on attributed arousal and rated attractiveness in female subjects. Journal of Personality, 47(2), 214229. doi: 10.1111/j.1467-6494.1979.tb00200.x

Wood, B. (2010). Reconstructing human evolution: Achievements, challenges, and opportunities. Proceedings of the National Academy of Sciences, 107(Supplement 2), 8902-8909. doi: 10.1073/pnas.1001649107

Wood, W., \& Karten, S. J. (1986). Sex differences in interaction style as a product of perceived sex differences in competence. Journal of Personality and Social Psychology, 50(2), 341-347. doi: 10.1037/0022-3514.50.2.341

Woodward, B. D. (1984). Arrival to and location of Bufo woodhousei in the breeding pond: Effect on the operational sex ratio. Oecologia, 62(2), 240-244. doi: 10.1007/bf00379020

Woollaston, V. (2013). Why playing the lottery is so addictive: Our brains can't cope with the odds of winning so we make irrational decisions. Mail Online Retrieved January 12th, 2014, from http://www.dailymail.co.uk/sciencetech/article-2383644/Why-playing-lotteryaddictive-Our-brains-t-cope-little-odds-winning-make-irrational-decisions.html

Workman, L., \& Reader, W. (2004). Evolutionary psychology : An introduction. Cambridge, UK ; New York: Cambridge University Press.

Wrangham, R. W., Wilson, M. L., \& Muller, M. N. (2006). Comparative rates of violence in chimpanzees and humans. Primates, 47(1), 14-26. doi: 10.1007/s10329-005-0140-1 
Yong, E. (2012). Replication studies: Bad copy. Nature, 485(7398), 298-300. doi: $10.1038 / 485298 \mathrm{a}$

Zajonc, R. B. (1968). Attitudinal effects of mere exposure. Journal of Personality and Social Psychology Monograph Supplement, 9(2), 1-27.

Ziegler, T. E., Peterson, L. J., Sosa, M. E., \& Barnard, A. M. (2011). Differential endocrine responses to infant odors in common marmoset (Callithrix jacchus) fathers. Horm Behav, 59(2), 265-270. doi: 10.1016/j.yhbeh.2010.12.001

Zietsch, B. P., Morley, K. I., Shekar, S. N., Verweij, K. J. H., Keller, M. C., Macgregor, S., . . Martin, N. G. (2008). Genetic factors predisposing to homosexuality may increase mating success in heterosexuals. Evolution and Human Behavior, 29(6), 424-433. doi: 10.1016/j.evolhumbehav.2008.07.002

Zilioli, S., Caldbick, E., \& Watson, N. V. (2014). Testosterone reactivity to facial display of emotions in men and women. Horm Behav, 65(5), 461-468. doi: 10.1016/j.yhbeh.2014.04.006

Zillmann, D., Weaver, J. B., Mundorf, N., \& Aust, C. F. (1986). Effects of an oppositegender companion's affect to horror on distress, delight, and attraction. Journal of Personality and Social Psychology, 51(3), 586-594. doi: 10.1037/00223514.51.3.586

Zuk, M., Ligon, J. D., \& Thornhill, R. (1992). Effects of experimental manipulation of male secondary sex characters on female mate preference in red jungle fowl. Animal Behaviour, 44(6), 999-1006. doi: 10.1016/S0003-3472(05)80312-4

Zusi, R. L., \& Gill, F. B. (2009). The marvelous tail of Loddigesia mirabilis (trochilidae). The Auk, 126(3), 590-603. doi: 10.1525/auk.2009.08216 